\title{
NEVADA NATIONAL SECURITY SITE
}

Site-Directed Research \& Development Fiscal Year 2012 Annual Report
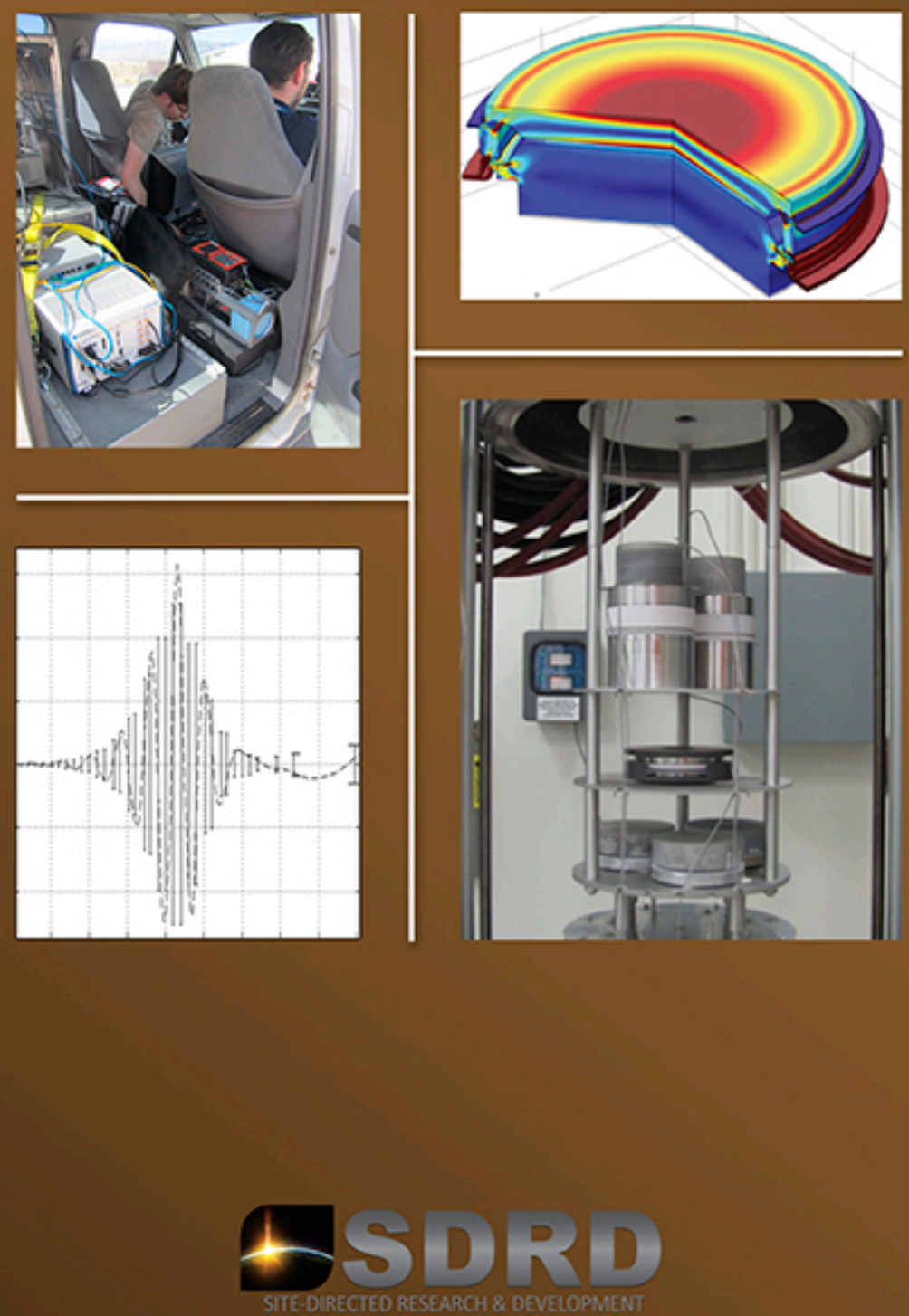

Material Studies and Techniques • Instruments, Detectors, and Sensors • Computational and Information Sciences • Photonics 


\section{Disclaimer}

This report was prepared as an account of work by an agency of the United States Government. Neither the United States Government nor any agency thereof, nor any of their employees, nor any of their contractors, subcontractors, or their employees, makes any warranty, express or implied, or assumes any legal liability or responsibility for the accuracy, completeness or any third party's use or the results of such use of any information, apparatus, product, or process disclosed, or represents that its use would not infringe privately owned rights. Reference herein to any specific commercial product, process, or service trade name, trademark, manufacturer, or otherwise, does not necessarily constitute or imply its endorsement, recommendation, or favoring by the United States Government or any agency thereof or its contractors or subcontractors. The views and opinions of authors expressed herein do not necessarily state or reflect those of the United States Government or any agency thereof.

\section{Availability Statement}

Available for sale to the public from-

U.S. Department of Commerce

National Technical Information Service

5301 Shawnee Road

Alexandria, VA 22312

Telephone: 800.553 .6847

Fax: 703.605.6900

E-mail: orders@ntis.gov

Online ordering: http://www.ntis.gov/help/ordermethods.aspx

Available electronically at http://www.osti.gov/bridge

Available for a processing fee to the U.S. Department of Energy and its contractors in paper, from-

U.S. Department of Energy

Office of Scientific and Technical Information

P.O. Box 62

Oak Ridge, TN 37831-0062

Telephone: 865.576.6401

Fax: 865.576.5728

E-mail: reports@adonis.osti.gov

Online ordering: http://www.osti.gov/reportform.html 


\section{Nevada National Security Site Site-Directed Research and Development} Fiscal Year 2012 Annual Report 



\section{Table of Contents}

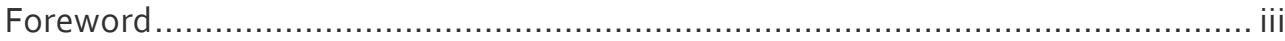

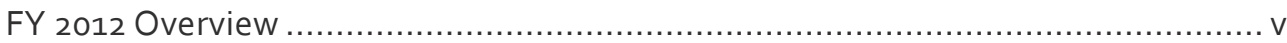

National Security Technologies-Operated Sites ...................................

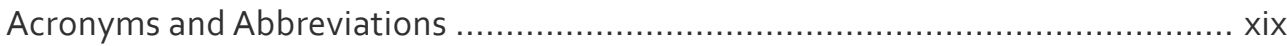

\section{Material Studies and Techniques}

Electron Mobility Study in Scintillator Material, A. Curtis ...................... 1

Low-Cost Cerium Bromide Alloys, P. Guss ....................................... 11

Dynamic Conductivity of Shocked Materials, B. LaLone ......................... 21

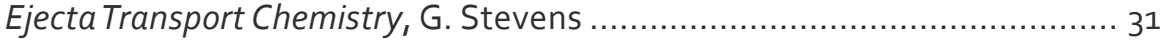

\section{Instruments, Detectors, and Sensors}

Back-Thinned Silicon Radiation Detector Readout, S. A. Baker .................. 39

Pulse-Mode Spiral Neutron Detector, M. Berninger.......................... 47

Ultrafast, High Quantum Efficiency, Microstructured Photocathodes for Advanced Sensors, R. A. Buckles ...................................... 55

Time-Resolved Hyperspectral Fluorescence Spectroscopy Using Frequency-Modulated Excitation-Applications, G. A. Capelle................67

Advanced High-Speed 16-Bit Digitizer System, M. Jones ........................77

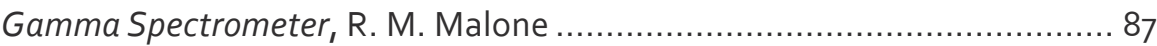

Miniaturized Multiband Antenna via Element Collocation and Inductive Feed Loading, R. Martin ........................................ 97

Electronic Veto and Software Partitioning of Cosmic Neutrons,

S. Mukhopadhyay

High-Efficiency Solid-State Thermal Neutron Detector and Imaging System, M. Raphaelian

Nanoparticle-Based Analytical Biosensor, S. Sawyer Armand

Passive Imaging of Warhead-Like Configurations with Cosmic-Ray Muon Tracking Scanners, D. Schwellenbach

Independently Triggerable LinearTransformer Driver for Short, Multi-Pulse Radiography, A. Smith 135

Radiation Hard AlGaN Imager and Electronics, K.-X. Sun ...................... 145

Large-Area Graphene PANI Neutron Detector, S. Weeks 


\section{Computational and Information Sciences}

An Extensible Architecture for Supporting Next-Generation

Aerial Radiological Measurements, C. Joines

Uncertainty Estimation Techniques for High-Density 1-D

MPDV Imaging Diagnostic, E. Machorro 169

Custom IP Packet Substitution Cyber FrameworkTool, C. Nivens 177

New Hybrid TDMA/CSMA Wireless Mesh Networks (WMN) for Secure Smart Grid Communication, K. Park

\section{Photonics}

Enhanced Accuracy of Shock Arrival Using PDV/Heterodyne

VISAR, B. Marshall.

\section{Unpublished Summary}

Available from SDRD Program Office, Las Vegas, NV 89193-8521

Wi-Fi Exploitation from an Embedded System, S. Gordoni 


\section{Foreword}

As we have engaged over the past year in strategic planning for NNSS and NSTec, one of the enduring themes that has arisen in our deliberations involves the focus that needs to be continually placed on encouraging innovation and creativity in our technology enterprise. This will ensure the most effective and sustained collaborations between NSTec and multiple National Laboratories across the NNSA and DOE, and will encourage other government agencies to seek out the value that accrues by partnering with NSTec at our unique proving ground.

The SDRD program is our innovation vehicle for tackling national security challenges, which continue to change rapidly with the evolving international security environment. Our evolving missions in stockpile stewardship and global security are pushing us into a new era, requiring us to adeptly foresee the technological solutions needed for the future. Clear vision is required for success, and many of our newly instituted strategic efforts are aligned to

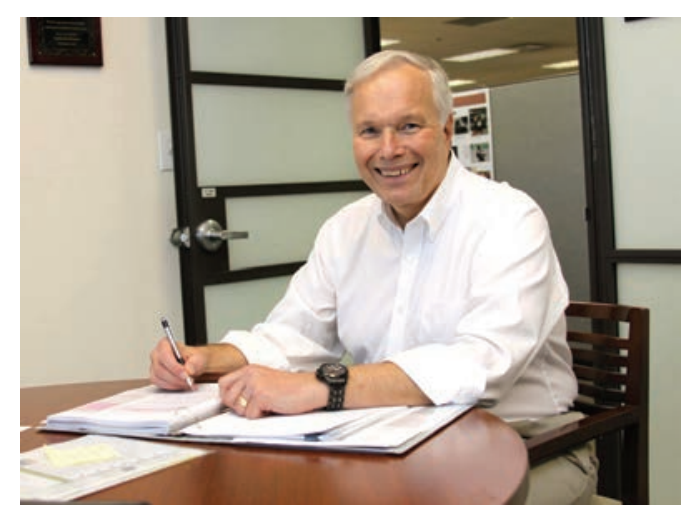
this perspective. SDRD, as such, is one of our most effective tools for realizing technical excellence. The program continues to deliver a high return on investment. A recent culminating success was the Gemini series of subcritical experiments, which demonstrated the transformational capabilities of an SDRD-developed optical velocimetry technique. These capabilities are featured in this fiscal year 2012 annual report. Other successes are being realized in treaty verification and nuclear nonproliferation, enabling our efforts to ensure a safe global landscape in the years to come.

We are actively positioning the NNSS to provide the highest level of support possible for existing and new missions, relying heavily on breakthrough techniques developed through the SDRD program to return maximum value for our customers. Our technical staff is up to the challenge and exploits the research and development (R\&D) program to exercise their creative energy to ultimately fulfill the "unseen possibilities." The program also strives to build strong and effective partnerships - a key attribute in today's complex scientific and technological environment. As illustrated in this report, our R\&D investments are making an impact and benefiting our missions multifold. We see a bright future for even greater outcomes by leveraging SDRD in new strategic ways to support our national and global security missions.

Ray Juzaitis

President

National Security Technologies, LLC 
This page left blank intentionally 


\section{FY 2012 Overview}

Now in the 11th year of Congressional Authorization, the Site-Directed Research and Development (SDRD) program is an essential pillar of the Nevada National Security Site (NNSS) technical enterprise. As a primary source for new discovery and innovation for our national security missions, the program has no equal, and it provides unparalleled return on investment. Based on numerous, high-impact successes coupled with timely conditions, it became clear that the program was well poised to achieve even greater potential. Concurrent with a new emphasis on long-term planning, SDRD became part of the Strategic Development Office (SDO), created in mid-2012. This new venue provides a systematic approach for executing critical long-term strategic initiatives and helps us realize the U. S. Department of Energy's National Nuclear Security Administration (NNSA) vision for the NNSS. Institutionalizing SDRD within the SDO was the first step in our plan to leverage the program to its fullest extent.

Working within the framework of the SDO, we recognized an opportunity to create a separate class of strategically directed R\&D projects. Primarily, SDRD has used an exploratory research concept, soliciting ideas from our staff using R\&D guidance provided by our annual Technology Needs Assessment. Strategic R\&D projects will focus on initiatives that are vital to the long-term success of the NNSS missions. Although the concept of strategic R\&D is not newsimilar directed research is funded at other laboratories-our program also hopes to use this concept to support advanced initiatives and realize strategic outcomes. We anticipate that in 2014 we will implement strategic opportunity research as a new element to the program.

As part of an ongoing commitment to SDRD excellence, we convened an external advisory panel in November 2011 composed of subject matter experts from many national security science areas. The panel sought to critically review the quality and impact of the science and technology

\section{A Defining Year}

Some of the most notable accomplishments to date for the SDRD program occurred in FY 2012. We received an $R \& D 100$ award for our multiplexed photonic Doppler velocimetry system, which recently led to a major success in our stockpile stewardship program. In addition, we launched a new strategic R\&D initiative, made breakthroughs in treaty verification technology, and conducted a highly useful external advisory review meeting.

The introduction of this FY 2012 Annual Report highlights some of the major accomplishments achieved by our SDRD researchers who are ultimately the "intellects behind the innovation." Supported by SDRD, these individuals exercise their creativity and tackle challenging national security issues with far-reaching impact. The flexibility to undertake key technical problems and overcome barriers is the hallmark of advanced $R \& D$, and the SDRD program continues to achieve successes that support not only our missions but also a diverse array of government agencies.

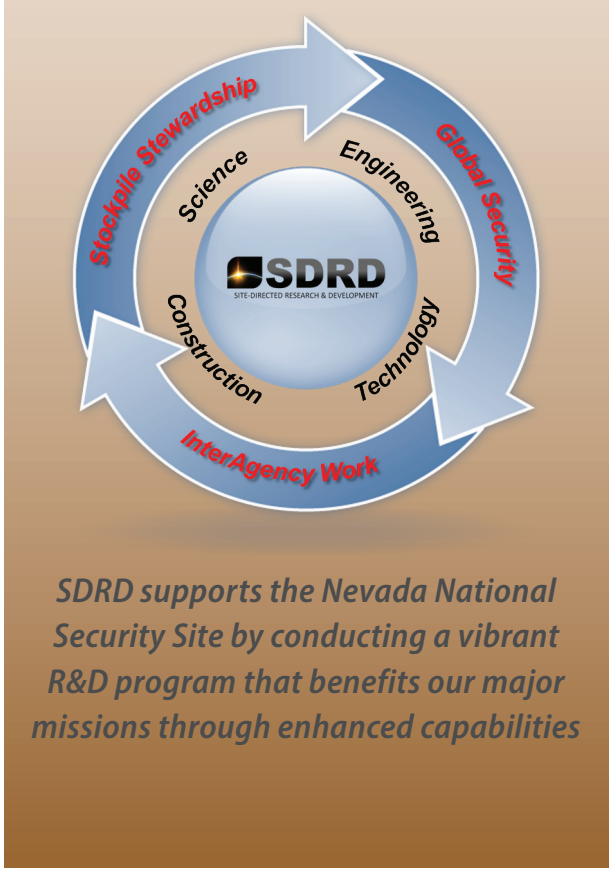


demonstrated by SDRD. In addition, the panel provided guidance regarding how the program could achieve its highest potential in scientific excellence and service to national security. The panel provided a wealth of recommendations that since November we have systematically examined and implemented where possible. Some of the more important recommendations were to (1) strive to push the technical envelope where possible, (2) seek closer ties to universities and develop strong collaborations, and (3) leverage the program fully by funding larger projects. These recommendations have directly contributed to the growth of SDRD over the past year, and many new initiatives are in process that directly stem from panel ideas. External review of SDRD will continue in the future to ensure overall quality, enhance outcome, and provide accountability and assurance of sound R\&D investment. Periodic follow-up reviews have already taken place and more are scheduled for the FY 2013-2014 timeframe.

\section{SDRD Advisory Panel 2013}

The Advisory Panel reconvened in North Las Vegas in January 2013. Unlike the first meeting, which considered the SDRD program in general, the January 2013 panel focused on future directions for the SDRD program. In particular, the panel was charged with providing insights into the technologies that will be needed by customers in the future. The panel consisted of technical personnel from several NSTec locations as well as national laboratory participants and technical advisors.

The panel discussed five strategic thrust areas:

(1) Stockpile Stewardship/Nuclear Weapons,

(2) Treaty Verification/Arms Control,

(3) Nonproliferation/Emergency Response,

(4) Intelligence/Information Security/Cyber

Threats, and (5) Safeguarded Energy/Nuclear

Energy. Strategic challenges of these areas were defined. The panel's deliberations also produced valuable insights into future technological needs and emerging areas.

Contributed by L. Franks

\section{Cosmic-Ray Imaging Update}

We were first introduced to the work Decision Sciences Corporation and Los Alamos National Laboratory (LANL) were doing in cosmic-ray muon imaging in 2010. We saw great potential in this technology, and realized there were some gaps in the experimental work to date. In particular, no classified imaging had been attempted to verify the capabilities or to define the ability to have an information barrier by adjusting integration time. We submitted a proposal to perform this work under SDRD, and our project was funded in FY 2011. Over the 2-year life of the project, we experimentally proved the ability to image warhead configurations using cosmic-ray muons. Further, we demonstrated that one-sided muon imaging in coincidence with fission neutrons was viable.

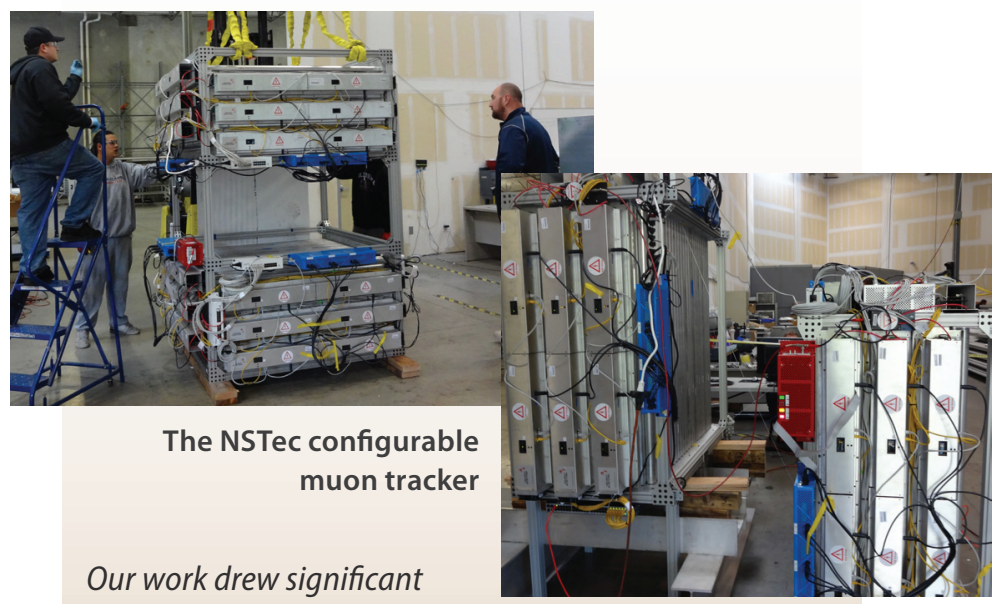

attention from the U.S.

Department of State V-Fund with follow-on interest from other agencies. We are now funded by the DoS to continue studying one-sided muon imaging and by NA-22 under a 3-year life cycle to study the capabilities of imaging using near-horizontal trajectory muons. As of February 2013, NSTec now owns a muon tracker, the configurable muon tracker, which is designed to be adapted to a wide variety of experimental configurations with short setup time. Prior to this, LANL owned the only tracker available for government research. We are pursuing research in muon imaging, and we have grown our collaboration between labs, industry, and academics to expand this work.

Contributed by D. Schwellenbach 


\section{Integrated Sensor and Data Architecture Proves Itself for Advanced Response Mission}

SDRD developed a concept, architecture, and demonstration system to prove the concept of extensible architecture (EA) for radiological aerial measurements. The EA concept is a system structure designed to simultaneously handle input from multiple real-time data sources and seamlessly integrate new sensors and data types. A promising direction is the ability to interface multiple high-purity germanium (HPGe) detectors to complement higher-sensitivity thallium-doped sodium iodide detectors. The EA system is ideal for use as a research platform, as it can rapidly integrate new sensor types. This system is the foundation on which future acquisition system developments for NNSA emergency response programs at the NNSA Remote Sensing Laboratory (RSL) will rest.

As a result of our SDRD program, the EA Next Generation Aerial System includes Microsoft-structured query language data storage for random access, onboard data query using RSL's Advanced Visualization and Integration of Data (AVID) software, simple migration to industry standards, integration of existing detector systems for immediate results, and an expandable platform/

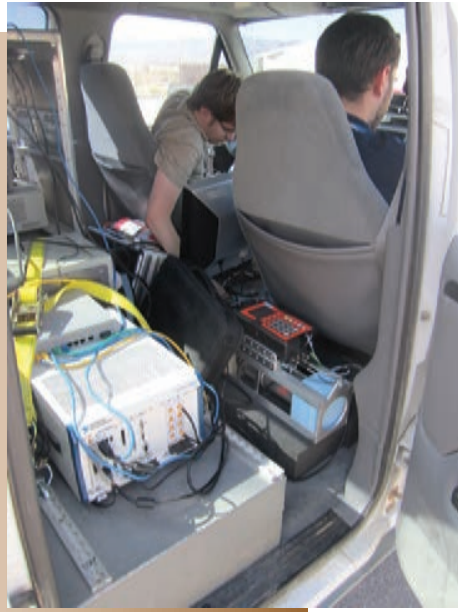

Operational testing was conducted by placing the system in a vehicle and equipping it with several detectors architecture. AVID has an extensible software architecture. The elements added by our SDRD project include a combination of data from individual sensor suites on a single acquisition "server," AVID query and display of data during flight, and an embedded photographic data radiation acquisition system. AVID is a software capability that allows sensor fusion and multi-window extractions in real time. It has full spectral mapping, 3-D spectral simulation, real-time contouring, telemetry capabilities, rapid production of standard products, and significant selection of input/output capabilities. The major innovation of this project is the integration of a diverse range of detector types for use with AVID.
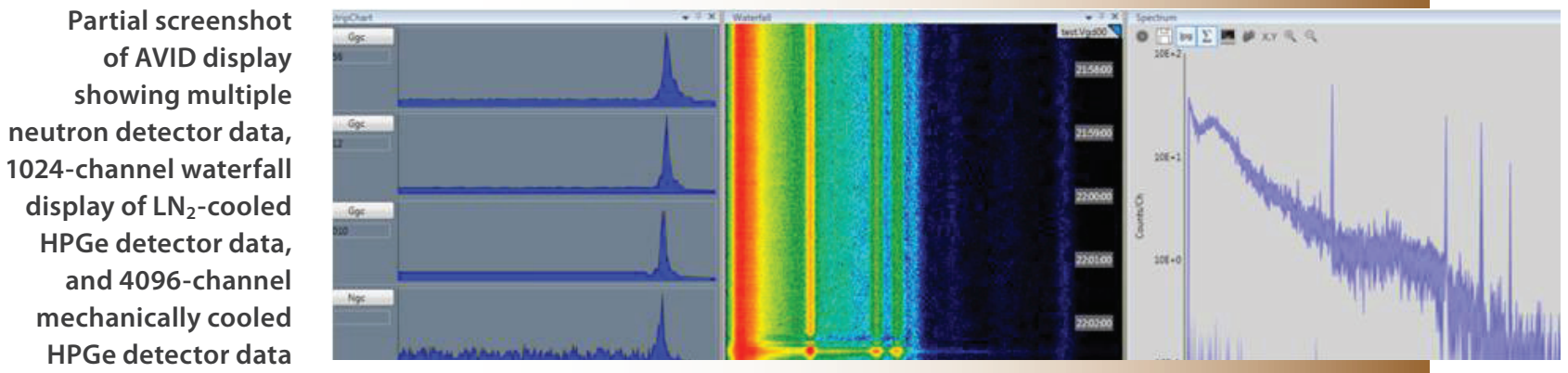

Already, AVID has been integrated into the NNSA Office of Emergency Response operations. The EA system was used for the New Year's Eve Nuclear Response Team (NRT) support to federal and local Las Vegas law enforcement and response officials. Incidentally, the NRT team transferred nuclear data for this New Year's Eve response to command centers using NSTec technology borne of the SDRD-developed MPCD. Further, the EA Next Generation Aerial System was employed to survey the nation's Capitol building and White House just prior to the 2013 presidential inauguration. Finally, the SDRD final report on the EA concept was of interest to Japanese authorities, who are continuing buildup of Japanese technology and capability, with assistance from RSL, to perform long-term monitoring around Fukushima.

Contributed by P. Guss, M. Reed, and C. Joines 


\section{SDRD to Stockpile Success: Development of Multiplexed Photonic Doppler Velocimetry, New Data, and a New Paradigm for Stockpile Stewardship}

The innovation and development of Multiplexed Photonic Doppler Velocimetry (MPDV)

has provided experimenters with a diagnostic tool that heretofore was unavailable, the ability to characterize materials and experimental performance with hundreds of simultaneous optical velocimetry measurements—“"high definition" optical velocimetry. MPDV has its roots in SDRD as an effort that initially intended to investigate "a better way" to do optical velocimetry, then quickly expanded to address a more specific challenge: "How can we make hundreds of high-fidelity velocimetry measurements simultaneously within technical, economical, and logistical constraints?" We met this challenge because of the innovations and technology development that occurred within the SDRD program. This innovation enabled solutions for national security needs, answering questions that in fact had yet to be asked when the MPDV SDRD project was first launched. The ability of SDRD to support high technical risk, high-reward investigations; to provide researchers with the autonomy necessary for success; and to allow flexibility to adjust funding priorities during the course of investigations ultimately enabled this new diagnostic capability. In December of 2012, 128 data channels of Gen-1 MPDVfruition of the original SDRD investigations-allowed experimenters from the national weapons laboratories (NWLs) and NSTec to collect more high-fidelity data from a single integrated experiment than all of the previous comparable experiments combined. And the resulting data

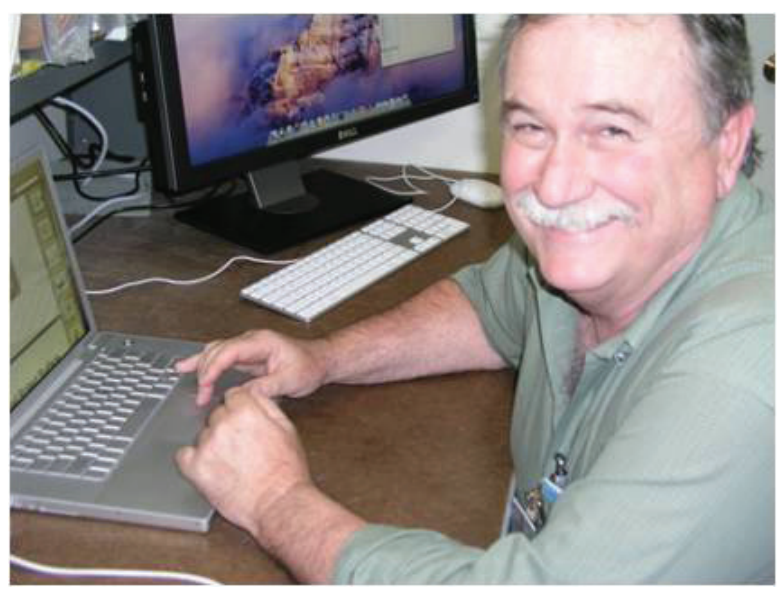

David Holtkamp (LANL) at the Special Technologies Laboratory during MPDV proof-of-concept experiments in April 2011 from these experiments have changed the way the nuclear weapons community views large-scale experiments within the science-based stockpile stewardship strategy.

Over many decades, researchers from the NWLs developed very elegant, capable, and often complex methods to make velocimetry measurements crucial to understanding the physics of materials under shock loading as well as numerous other applications. Collaborative relationships developed between scientists from NSTec, Lawrence Livermore National Laboratory (LLNL), LANL, and Sandia National Laboratories (SNL) were foundational, providing both understanding of the technological needs as well as strengths and weaknesses of the various diagnostic techniques. Historically, techniques in optical velocimetry known as VISAR and Fabry-Perot interferometry were applied to experiments, but with a significant limitation: these techniques could only measure a handful of data channels, typically five to ten, due to their significant complexity and cost. PDV, originally developed at LLNL, leveraged commercially available telecom products, and quickly gained in experimental utility. PDV added more velocimetry measurements, sometimes as many as 20 , within a compact, relatively economical and portable system. The collaborative relationships with NWL principal investigators provided the basis for support of future

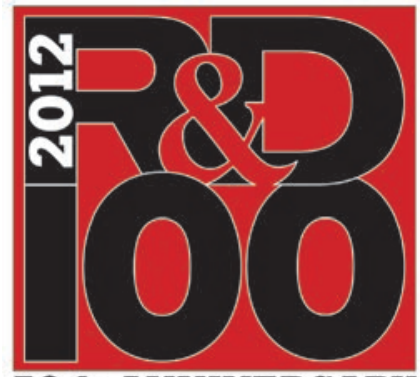

The MPDV won a 2012 R\&D award. The award was the second received for NSTec. 


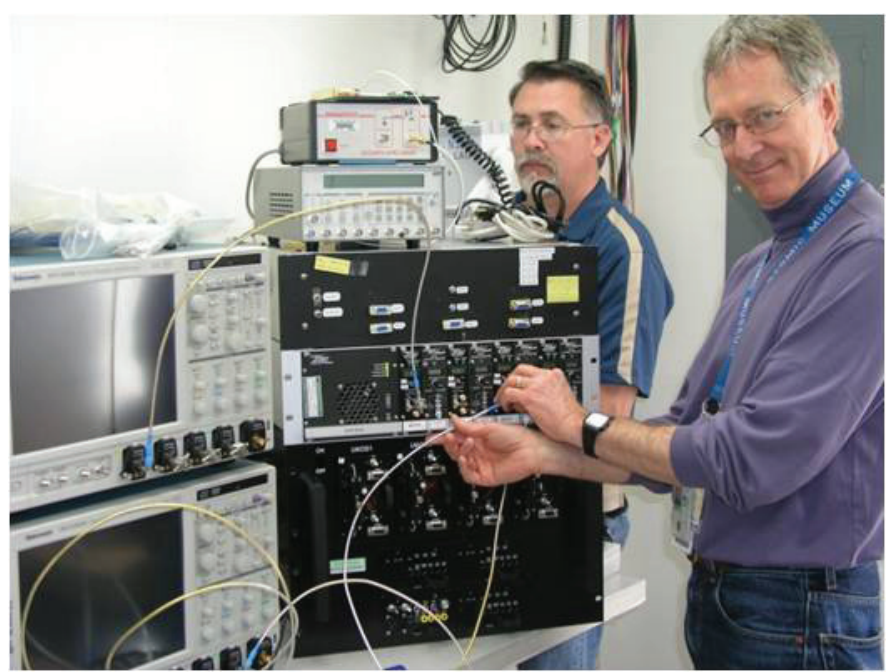

Ted Strand (LLNL, foreground) and Carlos Perez (NSTec) at the Special Technologies Laboratıry with the MPDV demonstration system, April 2011 developmental efforts. These collaborations have been rewarding, enjoyable, and crucial to the success of the MPDV development effort. It has been our pleasure to learn from and work with such distinguished individuals as Wil Hemsing, David Holtkamp, and Matt Briggs of LANL, and David Goosman, Barry Jacoby, and Ted Strand of LLNL, to name just a few.

These collaborative relationships provided us with both technical experiences and an understanding of potential future stockpile needs, and this perspective had a significant effect on the motivation and goals of our SDRD research. Motivation for development of MPDV was as much pragmatic as technical, and the goals of our SDRD investigations evolved as we proceeded. At the beginning of FY 2010, we intended to simply investigate the potential to improve PDV by using interferometric techniques in combination with recently developed telecom products. This stemmed from our observation that PDV data records use only a small portion of the bandwidth and memory available in our expensive digitizers. As we evaluated the feasibility of time and frequency multiplexing, we became convinced mid-way through the SDRD project that some of these techniques were indeed very promising. At that time, two unrelated but quite serendipitous events occurred. Following an SDRD project review, it was decided to significantly increase the investment in the project and effectively "double down" on its future potential. Separately, our collaborative relationship with David Holtkamp came into effect when he stopped by one day to chat about several topics. However, the conversation quickly turned to the promising but rather preliminary results from our SDRD investigations. David immediately recognized the potential impact of these investigations should they prove out, and was extremely supportive during the SDRD and subsequent programmatic development efforts. We continued our SDRD investigations but added an additional challenge: to develop a capability that would enable future stockpile stewardship experiments requiring large channel counts ( 100) by improving the economy — cost per data channel—while maintaining high data fidelity and the portable, robust and operator-friendly aspects of PDV. In the end, it was the SDRD "doubling down" that fueled the rapid advancement of MPDV, and it was the collaborative support of David Holtkamp and Ted Strand that paved the way for successful application into a first-of-its-kind stockpile stewardship experiment.

The adventure that began as a humble SDRD feasibility study had now grown into an accelerated effort to develop an MPDV demonstration system. We expanded our investigations and built a team with the goal of developing an MPDV demonstration system. We subsequently completed the SDRD project in September of $\mathbf{2 0 1 0}$ having successfully demonstrated feasibility in a laboratory environment, and the increased investment also provided for a nearly field-ready MPDV demonstration system. However, more work remained, and further developments were enabled by other collaborative relationships within and outside of NSTec. To complete the MPDV 
demonstration system, funding was provided by the Shockwave Diagnostic Development program, and the accelerated MPDV development effort continued; this provided the means to finalize the demonstration system, develop the control and analysis software, and conduct initial testing of what would eventually become the Gen-1 MPDV. At the same time, the scientific expertise and unique experimental capabilities of the NSTec Special Technologies Laboratory (STL) combined with the support and collaboration of both David Holtkamp (LANL) and Ted Strand (LLNL) to conduct the initial proof-of-concept experiments using explosively driven materials. These experiments both validated the MPDV technique as well as pointed out areas for future improvements. During this time, it had also become clear that this technology would be necessary if we were to accomplish the goals set forth by LANL in response to the imminent experimental challenge initiated by Dr. Cook of the NNSA. Following several weeks of proof-ofconcept experiments in April 2011, we knew three things: first, the MPDV technique could work for the upcoming experiments; second, we needed to design and build an entirely new Gen-1 MPDV system to incorporate the required capabilities; and finally, we needed to get it done in approximately 5 months.

Our research and development team had decades of experience both within the weapons testing community as well as in private industry. At the end of the SDRD project, when the need for this enabling diagnostic technology was becoming clear, it was estimated by our staff that the research and development necessary to produce a "flight ready" diagnostic capability would take about 4 more years under good circumstances. Our team did it in 18 months. This is a testament to our team's selfless contributions and to the gracious contributions and encouragement from our NWL collaborators. The extraordinarily rapid development within the SDRD program, followed by programmatic development, culminated in large-scale demonstration experiments at LANL/ Chamber 8 in August-September 2011. Immediately after, the MPDV was used as a prime diagnostic on high-value experiments at LLNL CFF in November 2011 and then LANL DARHT in December 2011. The exceptionally rapid development of such a complex technology is shown symbolically in the figure.

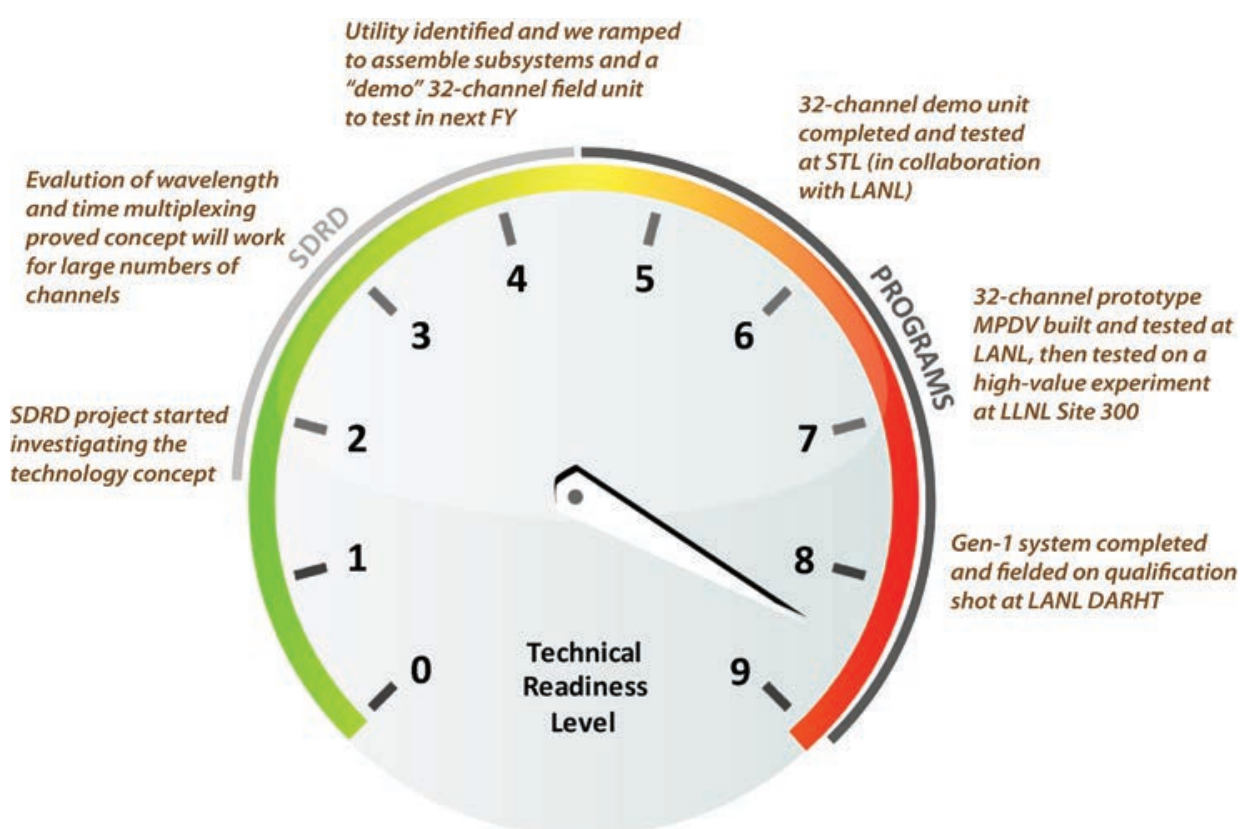

MPDV: TRL 2 to TRL 8 in approximately 24 months 


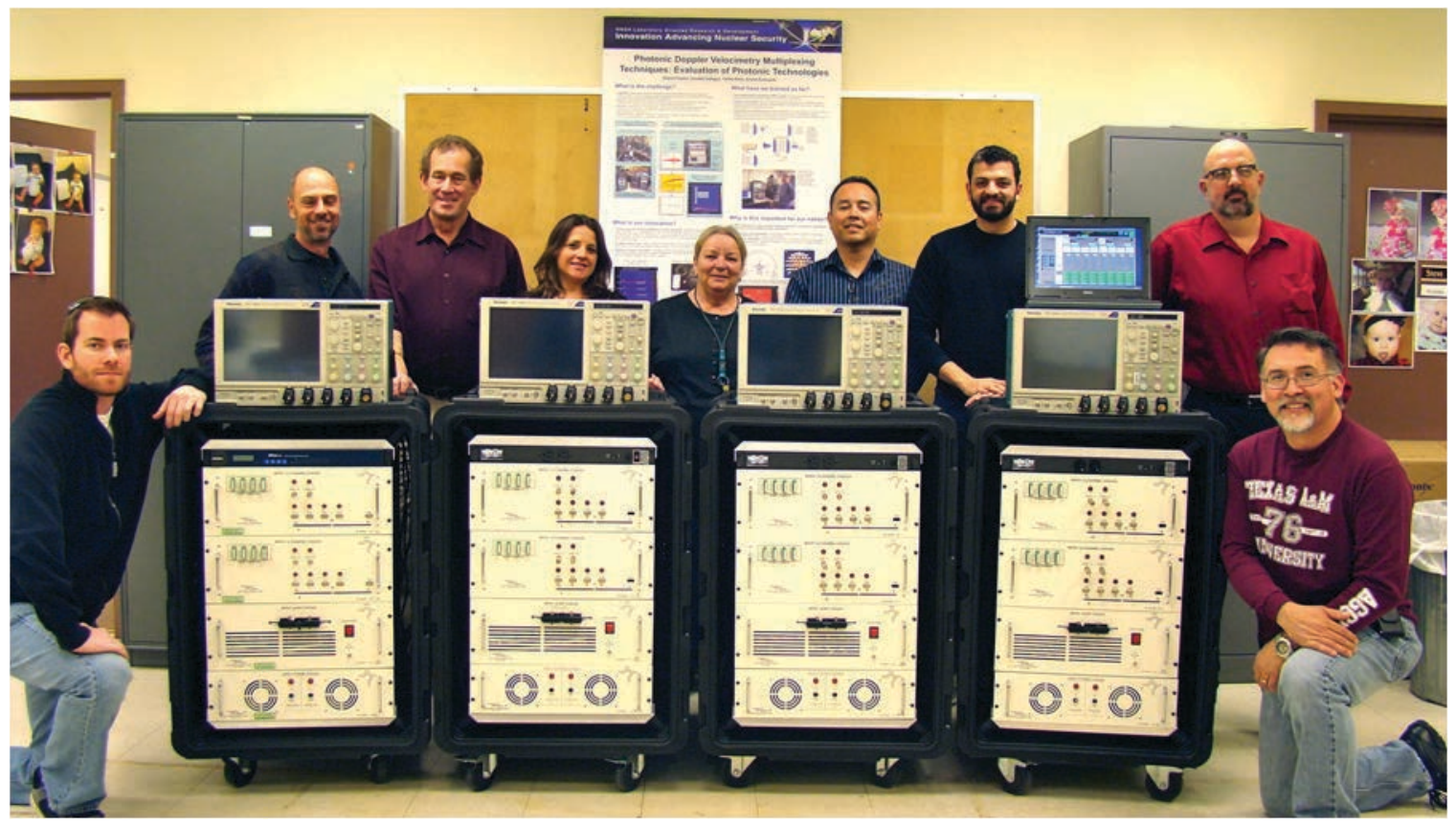

The MPDV development, assembly and fielding team with the 128-channel Gen-1 system fielded on the Gemini experiment in February 2012. From left to right: Ryan Emmitt, Ed Daykin, Martin Burk, Mandy Hutchins, Karen Theuer, Anselmo Garza, Michael Pena, Matthew Teel, and Carlos Perez.

Without the SDRD program, this capability would not exist today. Many factors contributed to this success: the gracious and continuing support from our NWL collaborators; the hard work, long hours and can-do attitude of our team; and the willingness of program leadership to step out in support of a high-risk, high-reward proposition. The ramifications of this technology have gone well beyond anything envisioned when the SDRD proposal was first written. Subsequent recognition of the widespread utility of MPDV has led to several awards, including the 2012 R\&D100. The success of MPDV on the Gemini experiment, providing 128 channels of prime diagnostic data, has significantly affected the future direction of stockpile stewardship programs, changing the paradigm in diagnostics for the weapons program.

Contributed by E. Daykin 


\section{SDRD PIs in the Spotlight}

NSTec researchers publish significant works in peer-reviewed journals, and are occasionally featured on a journal cover. PI Shayla Sawyer Armand and her group are working on advanced sensors that will be used for integrated bio-photonic circuits. Her article, "Photoresponse of indium oxide particulate-based thin films fabricated using milled nanorods grown by the self-catalytic vapor-liquid-solid process," was featured recently in Semiconductor Science and Technology. (L. Qin, P. S. Dutta, and S. Sawyer, Semiconductor Science and Technology, 27 (2012), 045005-1-045005-6.)

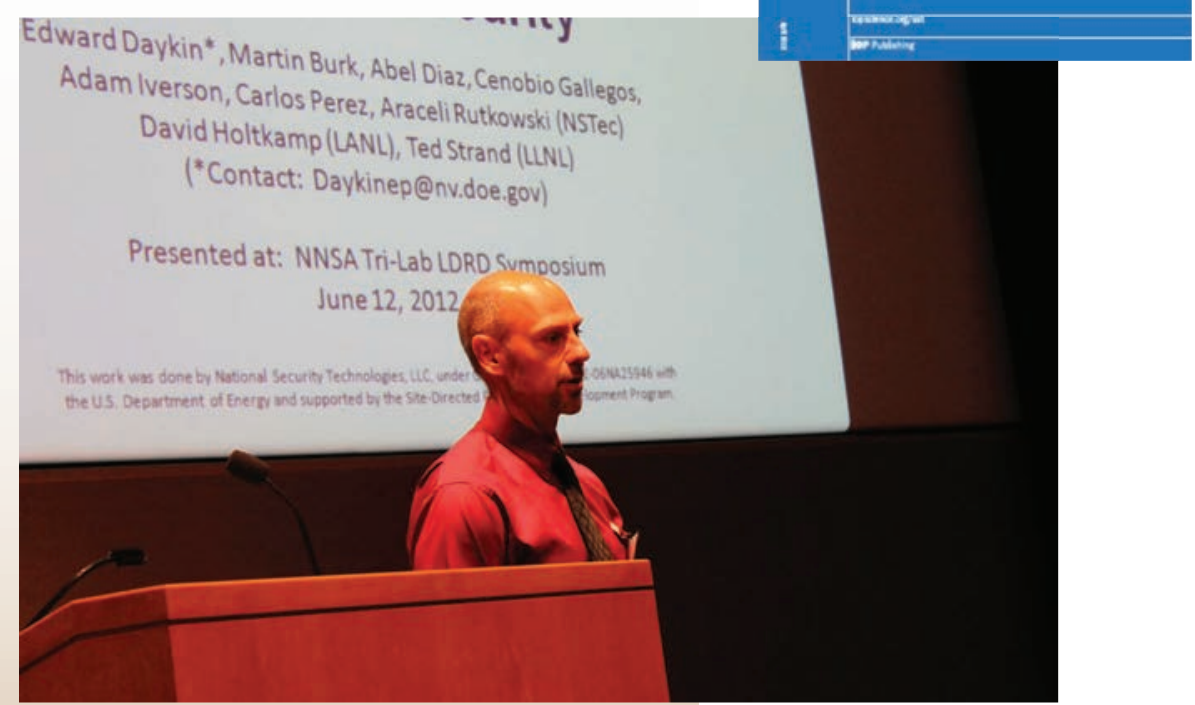

The LDRD symposium in June 2012 concentrated on national security innovation and addressed contemporary topics in stockpile stewardship and homeland security. The symposium was attended by a diverse audience that included NNSA, the NWLs, and the U.S. Departments of Homeland Security and Defense. Ed Daykin, an invited speaker, discussed the multiplexed photonic Doppler velocimetry system, a recipient of a 2012 R\&D100 award. 


\section{Cerium Bromide Breakthrough}

An FY 2012 SDRD project developed ultralow-activity, high-resolution, high-strength, room-temperature scintillators through a program of refining and alloying cerium bromide with aliovalent strengthening agents (substituents with a different valence than the host lattice). Aliovalent substitution, in which a host ion is replaced with an ion of different valence (e.g., $\mathrm{Cd}^{2+}$ for $\mathrm{Ce}^{3+}$ in $\left(\mathrm{CBr}_{3}\right)$ is a far more potent method of strengthening when compared to the usual method of isovalent substitution (i.e., replacing a fraction of ions with like-valence ions). In this approach, the formation of intrinsic defects necessary to maintain charge neutrality results in complexes with long-range interactions in the crystal. The resulting increase in hardening rate can be explained in terms of elastic interaction (tetragonal distortion) with dislocations (Pletka, Physica Status Solidi 39 (1977) 301-311).

SNL, a partner in this work, demonstrated success with this approach, achieving a dramatic reduction in fracture in aliovalent alloys compared with pure $\mathrm{CeBr}_{3}$ crystals. Prototype ingots were compounded with the addition of $2 \%$ of $\mathrm{CeBr}_{2}$ added to a high-purity $\mathrm{CeBr}_{3}$ charge in a closed ampoule before melting and solidification in a gradient-freeze process. Remarkably, this resulted in the measured energy resolution of the $662 \mathrm{keV}$ photopeak for ${ }^{137} \mathrm{Cs}$ to be $3.2 \%$ FWHM at room temperature. Such high energy resolution has never before been achieved with any of the established inorganic $\mathrm{CeBr}_{3}$ scintillators (even in small sizes) at room temperature. Higher light output and proportionality of $\mathrm{CeBr}_{3}: \mathrm{Ca}^{2+}$ (compared to $\mathrm{Nal}: \mathrm{Tl}$ and other inorganic scintillators) are responsible for high energy resolution. Over the energy range from 100 to $1275 \mathrm{keV}$, the nonproportionality of $\mathrm{CeBr}_{3}: \mathrm{Ca}^{2+}$ was measured to be $5 \%$, which is substantially better than that for many established scintillators. As discussed by Cherepy (IEEE Nucl. Sci. Symp. Conf. Rec. (2010) 1288-1291), the false alarm rate (FAR) increases geometrically with resolution, as $\mathrm{R}^{3.4}$; based on Cherepy, we can estimate up to a $97 \%$ reduction in $\mathrm{FAR}$, which is remarkable.

Contributed by P. Guss

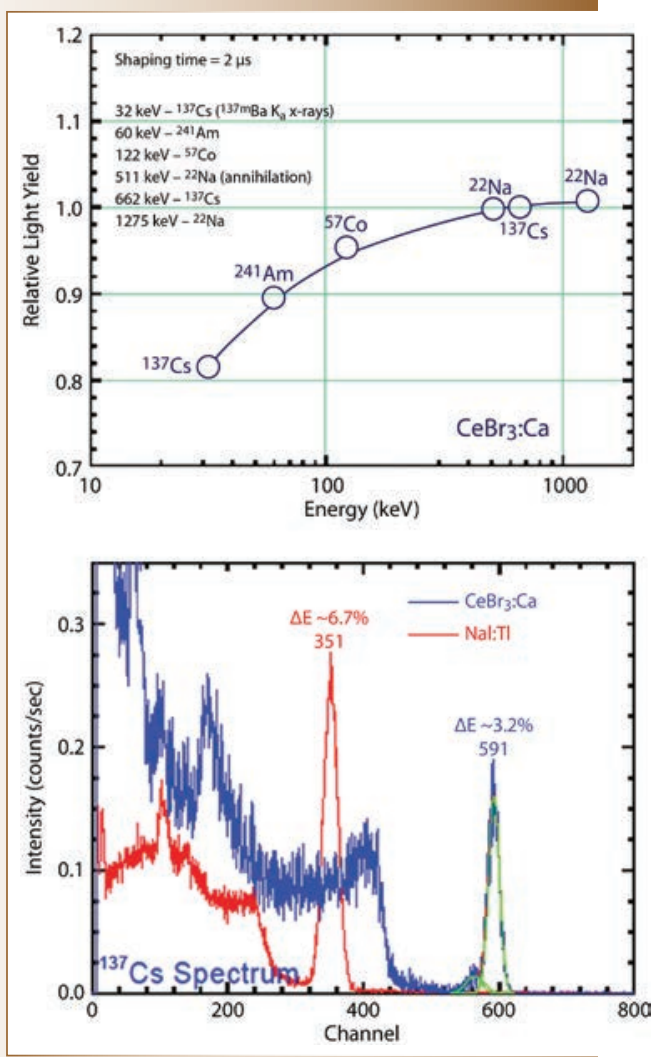

(Top) Relative scintillation light yield proportionality data acquired for the $1.9 \% \mathrm{Ca}^{2+}$-doped $\mathrm{CeBr}_{3}: \mathrm{Ca}^{2+}$ scintillator detector material as a function of gamma-ray energy. Nonproportionality of response for the crystal over $100 \mathrm{keV}$ to $>1 \mathrm{MeV}$ gamma ray energy range for $\mathrm{CeBr}_{3}: \mathrm{Ca}^{2+}$ is $\sim 5 \%$. (Bottom) ${ }^{137} \mathrm{Cs}$ spectra collected with $\mathrm{Nal}: \mathrm{Tl}$ and with $1.9 \%$ $\mathrm{Ca}^{2+}$-doped $\mathrm{CeBr}_{3}$ crystals coupled to a photomultiplier tube. The $662 \mathrm{keV}$ energy resolution peak for $\mathrm{Nal}: \mathrm{Tl}$ (at channel 351) is $\sim 6.7 \%$ (FWHM) and for $\mathrm{CeBr}_{3}: \mathrm{Ca}$ (at channel 591) is $\sim 3.2 \%$ (FWHM). The green curves represent Gaussian fits to the $\mathrm{CeBr}_{3}: \mathrm{Ca}^{2+}$ photopeak at $662 \mathrm{keV}$ and to the low energy tail for this same peak. The reported peak resolution of $3.2 \%$ (FWHM) was derived from this parametric fit to the data. 


\section{FY 2012 Proposals}

The FY 2012 proposal cycle was especially robust; many PIs "pushed the envelope" and focused on emerging technologies, as recommended by our external advisors. Special guidance was given to staff in advance of the proposal call that helped them steer proposals toward transformational research and overcoming scientific and technical limitations. The call also emphasized the importance of collaborations and university-based research partnerships. Our staff responded in kind with 144 high-quality proposals that covered many of our mission areas, such as stockpile stewardship, intelligence, information and cyber security, treaty verification, and monitoring activities. Twenty-four projects were selected and approved for funding, which was a slight decrease over the 26 from FY 2011. The selection rate was comparable to last year's, 1 in 6, thereby ensuring good competition, while maintaining proposers' enthusiasm that their ideas have a reasonable probability for acceptance. Our internal and external peer review system for selecting winning proposals relies on key criteria, which have remained essentially unchanged: high technical innovation, probability of success balanced with technical risk, potential for mission benefit, and alignment with our mission goals to achieve the best possible outcomes. Average cost per project, approximately $\$ 250 K$, was nearly the same as FY 2011. Total funds expended were just under $\$ 7$ million. Administrative and management costs increased slightly due to our investment in external advisory activities. Overall, this modest investment continues to provide exceptional return on investment based on successes and benefits to our mission and programs.

The NNSS Technology Needs Assessment document continues to be an effective tool for proposal submitters and reviewers. It provides a roadmap and guidance for technology gaps and challenges facing mission areas. Our directed research emphasis areas remained unchanged from last year, and they targeted key investment needs, including nuclear security, information security/assurance, high-energy density physics diagnostics, integrated experiments, advanced analysis, and improvised explosive device threat reduction. The needs assessment is developed from a broad base of input from the national security complex, including laboratories, NNSA, and other external agencies. Significant revisions to the assessment were again made in FY 2012, most notably the "Emerging Technologies" section was expanded, as were sections on new challenges in cyber security and materials in the extremes. The needs assessment itself is now in the ninth year of revision, and its utility and effectiveness continues to improve year to year. 


\section{SDRD Performance Metrics}

Metrics such as intellectual property and technology transfer are commonly used to gauge the output and effectiveness of research and development programs. Other factors-follow-on programmatic or external funding received, new methods developed that effectively save costs, and overall increased research staff capabilities-are further indicators of innovation productivity and are also a direct measure of the investment return. SDRD provides our staff with opportunities to explore and exercise creative motivations that ultimately lead to new knowledge and realized technologies. The traditional metrics we have used over the past years are tabulated below and are a broad measure of R\&D performance.

Invention disclosures are the first step in our intellectual property pursuit and are often followed by patent applications when deemed appropriate. Traditionally SDRD has generated well over half of all inventions disclosed company-wide since the program began and continues to do so to this day. On average about one-third of our projects generate new invention disclosures, which is a reasonably high ratio given that projects can vary widely from basic concept, low technical readiness, to much higher more applied development efforts. In fact, our programs benefit from a high rate of technology utilization precisely due to this diverse mix of projects. A relatively high percentage of projects, roughly $40 \%$, have technology that is subsequently adopted by a direct NNSS program. Another measure of program effectiveness and alignment with missions is how well projects address technology needs as identified in the annual NNSS Technology Needs Assessment. The ratio of needs addressed to total projects is also indicative of a trend that aligns efforts strategically with the NNSS mission.

We continue to strive to have SDRD effectively contribute new technology into key programmatic efforts as quickly as possible (as demonstrated by our accelerated TRL development of MPDV). New strategic efforts are also providing greater emphasis on forwardlooking needs and efficiently coupling with long term visionary goals. As always, SDRD looks to be "ahead of our time by design" and push for SDRD innovations to intersect future and evolving missions with the most impact possible.

\begin{tabular}{|c|c|c|c|c|c|c|c|c|c|c|}
\hline \multicolumn{11}{|c|}{ SDRD Performance Metrics } \\
\hline Year & FY03 & FY04 & FY05 & FY06 & FY07 & FY08 & FY09 & FY10 & FY11 & FY12 \\
\hline Number of projects & 41 & 57 & 55 & 50 & 37 & 27 & 23 & 25 & 26 & 24 \\
\hline Invention disclosures & $27 \%$ & $21 \%$ & $38 \%$ & $22 \%$ & $24 \%$ & $22 \%$ & $48 \%$ & $36 \%$ & $27 \%$ & $33 \%$ \\
\hline $\begin{array}{l}\text { Technology adopted } \\
\text { by programs }\end{array}$ & 15 & 18 & 12 & 8 & 8 & 8 & 10 & 10 & 9 & 10 \\
\hline \multirow{2}{*}{$\begin{array}{l}\text { Gap or need } \\
\text { addressed per } \\
\text { NNSS R\&D Technology } \\
\text { Needs Assessment }\end{array}$} & 11 & 14 & 18 & 17 & 18 & 15 & 15 & 13 & 13 & 11 \\
\hline & $27 \%$ & $25 \%$ & $33 \%$ & $34 \%$ & $49 \%$ & $56 \%$ & $65 \%$ & $52 \%$ & $50 \%$ & $46 \%$ \\
\hline
\end{tabular}




\section{FY 2012 Annual Report Synopsis}

The reports that follow are for project activities that occurred from October 2011 through September 2012. These reports describe in detail the discoveries, achievements, and challenges encountered by our talented and enthusiastic principal investigators (PIs). Many of the reports describe R\&D efforts that were "successful" in their pursuits and resulted in a positive outcome or technology realization. As we've stated before, and continue to stress, in some cases the result is a "negative" finding, for instance a technology is currently impractical or out of reach. This can often be viewed erroneously as a "failure," but is actually a valid outcome in the pursuit of high-risk research, which often leads to unforeseen new paths of discovery. Either result advances our knowledge and increases our ability to identify solutions and/or likewise avoid costly paths not appropriate for the challenges presented.

In summary, the SDRD program continues to provide an unfettered mechanism for innovation and development that returns multifold to the NNSS mission. Overall the program is a strong $R \& D$ innovation engine, benefited by an enhanced mission, committed resources, and sound competitiveness to yield maximum benefit. The 23 projects described exemplify the creativity and ability of a diverse scientific and engineering talent base. The efforts also showcase an impressive capability and resource that can be brought to find solutions to a broad array of technology needs and applications relevant to the NNSS mission and national security.

\section{Acknowledgments}

A number of individuals contribute significantly to make SDRD successful year to year and form the real team behind the program. Without their support much of this would not be possible. My sincere gratitude goes out to Michele Vochosky, Sierra Cory, Elizabeth Godfrey, and Bob Watson, for compiling, editing, and publishing this report; to Nancie Nickels for graphic design of the cover and dividers; to Linda Flaugher for efforts in cost accounting and Tom Graves for project management support; to Newell Ramsey and Kathy Gallegos for information system support; to Cathy Cooper and Cindy McIntosh for compiling financial data for reporting requirements; to Larry Franks, Rob Hixson, and Lynn Veeser for exceedingly valuable technical guidance; and to SDRD site representatives and review committee, Frank Cverna, Daniel Frayer, Cathy Snelson-Gerlicher, Paul Guss, Eric Machorro, Michael Mohar, Sanjoy Mukhopadhyay, Christopher Silbernagel, and Gerald Stevens. I would also like to thank the members of our external advisory panel, Larry Franks, Carl Ekdahl, Damon Giovanielli, Ralph James, Glenn Knoll, Dick Lynn, Paul Rockett, and Gerry Yonas, who graciously gave their time and provided valuable recommendations.

Howard A. Bender III

SDRD Program Manager 


\section{National Security Technologies-Operated Sites}

Los Alamos Operations (LAO)

P.O. Box 809

Los Alamos, New Mexico 87544-0809

Livermore Operations (LO)

P.O. Box 2710

Livermore, California 94551-2710

North Las Vegas (NLV)

P.O. Box 98521

Las Vegas, Nevada 89193-8521

Nevada National Security Site (NNSS)

P.O. Box 98521

Las Vegas, Nevada 89193-8521

Remote Sensing Laboratory-Andrews Operations (RSL-A)

P.O. Box 380

Suitland, Maryland 20752-0380

(Andrews Air Force Base)

Remote Sensing Laboratory-Nellis Operations (RSL-N)

P.O. Box 98521

Las Vegas, Nevada 89193-8521

(Nellis Air Force Base)

Sandia Operations (SO)

Sandia National Laboratories

P.O. Box 5800

Mail Stop 1193

Albuquerque, New Mexico 87185

Special Technologies Laboratory (STL)

5520 Ekwill Street

Santa Barbara, California 93111-2352 
This page left blank intentionally 


\title{
Acronyms and Abbreviations
}

\author{
A \\ $A C$ \\ alternating current \\ ACCEPT \\ A Coupled Combinational Electronic Transport (code) \\ ACK \\ acknowledge \\ ADC \\ Al \\ analog-to-digital converter \\ $\mathrm{Al}_{2} \mathrm{O}_{3}$ \\ ALD \\ aluminum \\ aluminum oxide \\ AlGaN \\ Am-Be \\ atomic layer deposition \\ aluminum gallium nitride \\ AMAPS \\ americium-beryllium \\ AMS \\ Advanced Microelectronics and Photonics for Space \\ ANL \\ Aerial Measuring System \\ Argonne National Laboratory \\ AODV \\ ad hoc on-demand distance vector \\ AOFS \\ AP \\ Ar \\ acousto-optic frequency shifter \\ active parallel (programming) \\ argon \\ active serial (programming) \\ AVID \\ Advanced Visualization and Integration of Data \\ AWR \\ Applied Wave Research \\ B \\ ${ }^{10} \mathrm{~B} \quad$ boron-10 \\ $\mathrm{Be} \quad$ beryllium \\ $\mathrm{BF}_{3} \quad$ boron trifluoride \\ BGO bismuth germanate \\ BT back-thinned \\ C \\ $\mathrm{Ca} \quad$ calcium \\ $\mathrm{CaBr}_{2} \quad$ calcium dibromide \\ $\mathrm{CC} \quad$ control center \\ CCD charge-coupled device \\ $\mathrm{Cd} \quad$ cadmium \\ $\mathrm{Ce} \quad$ cerium \\ $\mathrm{CeBr}_{3} \quad$ cerium tribromide \\ $\mathrm{CeBr}_{3}: \mathrm{Ca}^{2+}$ calcium-doped cerium tribromide \\ CFI common flash interface \\ CHN Center for High-Rate Nanomanufacturing \\ (National Science Foundation) \\ CIPS custom Internet protocol packet substitution
}




$\begin{array}{ll}\text { CLEO } & \text { Conference on Lasers and Electronics } \\ \text { CMOS } & \text { complementary metal oxide semiconductor } \\ \text { CMT } & \text { configurable muon tracker } \\ \text { cPs } & \text { counts per second } \\ \text { Cr } & \text { chromium } \\ \text { CS } & \text { cesium } \\ \text { CSI } & \text { cesium iodide } \\ \text { CSI:TI } & \text { thallium-doped cesium iodide } \\ \text { CTH } & \text { hydrodynamic code } \\ \text { CuW } & \text { copper tungsten } \\ \text { CVR } & \text { current viewing resistors } \\ \text { CZT } & \text { cadmium zinc telluride }\end{array}$

D

DARHT Dual-Axis Radiographic Hydrodynamic Test facility

DAQ data acquisition (device)

DC direct current

DD deuterium-deuterium

DDR double data rate

DFT density functional theory

DNA deoxyribonucleic acid

DOE U.S. Department of Energy

DQE detector quantum efficiency

DSA digital spectrum analyzer

DT deuterium-tritium

\section{E}

EA extensible architecture

E. coli

EDM

escherichia coli

EOS

electrical discharge machining

equation of state

$\mathbf{F}$

FAM

fluorescent aptamer molecule

FEM

finite element model

FFT

fast Fourier transform

FM

FPGA

frequency modulated

FS

field-programmable gate array

frequency-shifted (VISAR)

FWHM

full width half maximum

\section{G}

$\mathrm{Gd}$

GGA

GPS

GQD

$\mathrm{GRH}$

GRIN

GUI

gadolinium

generalized gradient approximation

global positioning system

graphene oxide quantum dots

gamma reaction history

gradient index, graded index

graphical user interface 
H

${ }^{3} \mathrm{He}$

HDPE

$\mathrm{HMI}$

HOMO

HPGe

HTML

HVAC

\section{I}

ICCD

ICS

ID

IDC

ID-to-MAC

IEEE

$\mathrm{In}_{2} \mathrm{O}_{3}$

InGaAs

I/O

IP

I/Q

ITMR

ITO

I-V

J

JTAG

L

${ }^{6} \mathrm{Li}$

${ }^{7} \mathrm{Li}$

LA

$\mathrm{LaBr}_{3}: \mathrm{Ce}$

$\mathrm{LaCl}_{3}: \mathrm{Ce}$

LANL

LAO

LED

lidar

LiF

LIFE

linac

$\mathrm{LN}_{2}$

LPA

LPF

LSO:Ce

LTD

LUMO

LVDS helium-3

high-density polyethylene

human-machine interface

highest occupied molecular orbital

high-purity germanium

hypertext markup language

heating, ventilation, and cooling

intensified CCD

industrial control systems

identifier

identifier-based cryptography

identifier-to-medium access control

Institute of Electrical and Electronics Engineers

indium oxide

indium gallium arsenide

input/output

Internet protocol

quadrature-phase

Intelligent Tree Mesh Routing

tin-doped indium oxide

current-voltage

Joint Test Action Group

lithium-6

lithium-7

Lewis acid

cerium-activated lanthanum tribromide

cerium-doped lanthanum chloride

Los Alamos National Laboratory

Los Alamos Operations (NSTec)

light-emitting diode

light detection and ranging

lithium fluoride

Laser Inertial Fusion Energy (facility)

linear accelerator

liquid nitrogen

local polynomial approximation

local polynomial fitting

cerium-doped lutetium oxyorthosilicate

linear transformer driver

lowest unoccupied molecular orbital

low-voltage differential signaling 
M

MAC

MCA

MCNP

MCNPX

MCP

MCPI

MCS

MDA

MMF

MMT

MPDV

MSM

media access control

microchannel analyzer

Monte Carlo N-particle

Monte Carlo N-particle eXtended

microchannel plate

microchannel plate intensifier

multichannel scanner

minimum detectable activity

multi-mode fiber

Mini Muon Tracker

multiplexed photonic Doppler velocimetry

metal-semiconductor-metal

\section{$\mathbf{N}$}

NADH nicotinamide adenine dinucleotide

$\mathrm{Nal}$

Nal:TI

NDA

$\mathrm{Nd}$ YAG

$\mathrm{NI}$

NIF

NNSA

NNSS

NRT

NSTec

NUV

NWL

sodium iodide

thallium-doped sodium iodide

nondisclosure agreement

neodymium-doped yttrium-aluminum-garnet (crystal laser)

National Instruments

National Ignition Facility

National Nuclear Security Administration

Nevada National Security Site

nuclear response team

National Security Technologies, LLC

near ultraviolet

national weapons laboratory

0

1-D

one-dimensional

OAP

off-axis parabolic (mirror)

OGA

other government agencies

OMA

optical multichannel analyzer

OPA

OSI

optical parametric amplifier

open systems interconnection

$\mathbf{P}$

PANI polyaniline

$\mathrm{PANI}_{-} \mathrm{BF}_{3} \quad$ boron trifluoride-doped adduct of polyaniline

PBX

$P C B$

plastic bonded explosive

printed circuit board

PDE

photodetector efficiency

PDV

photonic Doppler velocimetry

PHY physical (layer)

PI principal investigator

PKT Peano kernel theorem 
PLL phase-locked loop

PMT photomultiplier tube

pRad proton radiography

Q

QE quantum efficiency

QoS Quality of Service

$\mathbf{R}$

R\&D research and development

RadHard radiation-hardened

RADIUS remote authentication dial-in user service

$\mathrm{RF} \quad$ radio frequency

$\mathrm{RH} \quad$ reaction history

RIE reactive ion etching

RMD Radiation Monitoring Devices, Inc.

rms root mean square

RNA ribonucleic acid

rpm revolutions per minute

RSL Remote Sensing Laboratory (NSTec)

RSL-A Remote Sensing Laboratory-Andrews (NSTec)

RSI Radiation Solutions, Inc.

RTL register transfer level

RTS/CTS Request-To-Send/Clear-To-Send

\section{$\mathbf{S}$}

SCADA

supervisory control and data acquisition

SDRAM

synchronous dynamic random access memory

SDO

Strategic Development Office

SDRD

Site-Directed Research and Development

SEB

Science and Engineering Building

SELEX

systematic evolution of ligands by exponential enrichment

$\mathrm{SF}_{6}$

$\mathrm{Si}$

$\mathrm{SiC}$

SMF

SND

SNL

SNM

SNR

sulfur hexafluoride

silicon

silicon carbide

single-mode fiber

spiral neutron detector

Sandia National Laboratories

special nuclear material

signal-to-noise ratio

SPICE

Simulation Program with Integrated Circuit Emphasis

SSPM

solid-state photomultiplier

START

Strategic Arms Reduction Treaty

STK

streaked spectrometer

STL

Special Technologies Laboratory (NSTec)

STP

Standard temperature and pressure at $0^{\circ} \mathrm{C}$ and $1 \mathrm{~atm}$ pressure 
T

$\begin{array}{ll}\text { 2-D } & \text { two-dimensional } \\ \text { 3-D } & \text { three-dimensional } \\ \text { TCC } & \text { target chamber center } \\ \text { TCP } & \text { transmission control protocol } \\ \text { TCP/IP } & \text { transmission control protocol/Internet protocol } \\ \text { TMDA/CSMA } & \text { time division multiple access/carrier sense multiple access } \\ \text { TOF } & \text { time of flight } \\ \text { TOPAS } & \text { Traveling-Wave Optical Parametric Amplifier Light Source } \\ \text { TRL } & \text { technical readiness level } \\ \text { TV } & \text { television }\end{array}$

U

UART universal asynchronous receiver/transmitter

$\mathrm{UCl}_{3}$

UCLA

UDP

UNLV

uranium trichloride

University of California, Los Angeles

user datagram protocol

UV

University of Nevada, Las Vegas

ultraviolet

V

VASP

VCO

VDC

VHDL

VHSIC

VISAR

VPF

Vienna Ab-initio Simulation Package

voltage-controlled oscillator

volts direct current

VHSIC hardware description language

very-high-speed integrated circuits

velocity interferometer for any reflector

velocity per fringe

W

WLAN wireless local area network

WMN wireless mesh network

WOFE Workshop on Frontiers in Electronics

$\mathbf{Y}$

Y2F Feynman variance

Z

ZnO zinc oxide 


\section{Material Studies \& Technioues}
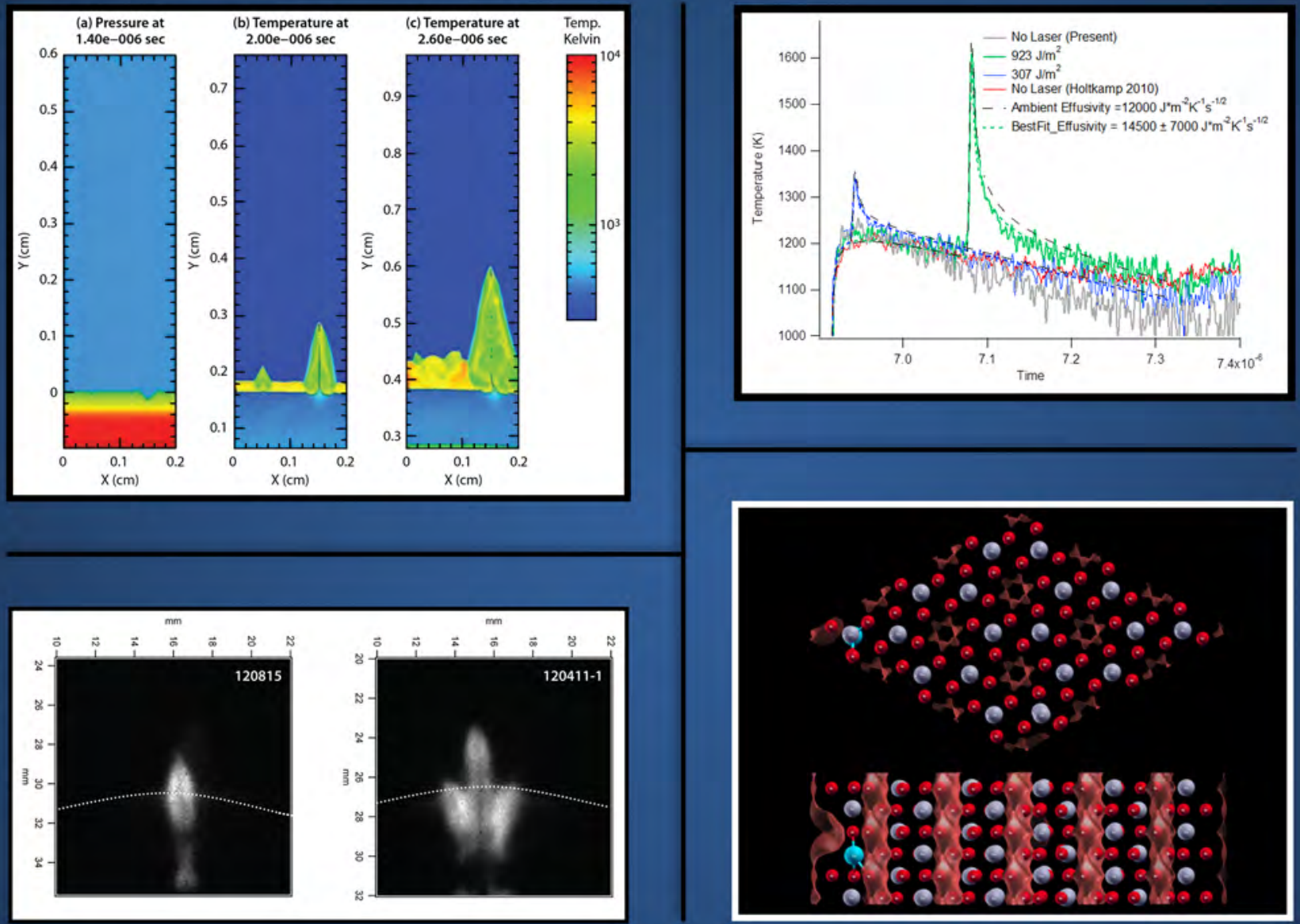


\section{ELECTRON MOBILITY STUDY IN SCINTILLATOR MATERIAL} LAO-38-12 | CONTINUED IN FY 2013 | YEAR 1 OF 2

Alden Curtis, ${ }^{1, a}$ Stuart A. Baker, ${ }^{a}$ Kristina Brown, ${ }^{a}$ Jason Young, ${ }^{a}$ William Quam, ${ }^{b}$ Michael Groza, ${ }^{c}$ Arnold Burger, ${ }^{c}$ and Larry Franks ${ }^{d}$

Understanding the physical mechanisms that cause ionizing radiation to create light in scintillator materials is important in understanding the nonproportionality of scintillator response to different incident particle energies, and for providing a basis for identifying new materials. Charge carrier transport dynamics have been shown to be particularly significant in these interactions. We used several sources of ionizing radiation to create charge carriers in strongly biased, thin scintillator samples and then attempted to measure the transit times of the electrons across the material in order to calculate the electron mobility. We successfully demonstrated the viability of the technique using a cadmium zinc telluride sample. The increase in resistivity by more than six orders of magnitude of the dielectric scintillator over that of the semiconductor sample made measurements using the scintillator more difficult.

${ }^{1}$ curtisah@nv.doe.gov, 505-663-2067

a Los Alamos Operations; ${ }^{\mathrm{b}}$ Special Technologies Laboratory ${ }_{i}{ }^{\mathrm{C}}$ Fisk University; ${ }^{\mathrm{d}}$ Keystone International, Inc.

\section{Background}

Despite the use of scintillators for more than a half century (Hofstadter 1948), the basic physics underlying the operation of inorganic scintillators is poorly understood. To gain insight into their operation, attempts are being made to model the role of a number of processes believed to be involved. A key ingredient is the carrier mobility, which affects the energy transfer process and, in particular, the non-proportionality of light output. The measurement of these critical factors is very difficult and rarely found in the literature. The difficulty arises from the basic nature of carrier mobility in insulators; a process very different than that found in semiconductors. In insulators the carriers move by FrankelPoole mobility-they move by hopping from trap to trap. This movement results in very low mobility, many orders of magnitude less than that of semiconductors, making measurement difficult at best.

\section{Project}

The project consisted of two major tasks. The first task consisted of using different sources of ionizing radiation to measure electron mobility in the semiconductor cadmium zinc telluride (CZT). Electron mobilities measured in the CZT range from 700 to $1350 \mathrm{~cm}^{2} / \mathrm{V} \cdot \mathrm{s}$ (Schlesinger 2001). By comparing our values to the measured values, we were able to gauge whether our techniques were appropriate. The second task was to use these same techniques to measure the electron mobility of cesium iodide (CsI), to be followed by additional scintillator materials if the experiment proved successful.

\section{LaserTests}

Because the physical parameters of CZT vary widely for different samples depending on the manufacturer, we first measured the electron mobility of our sample 


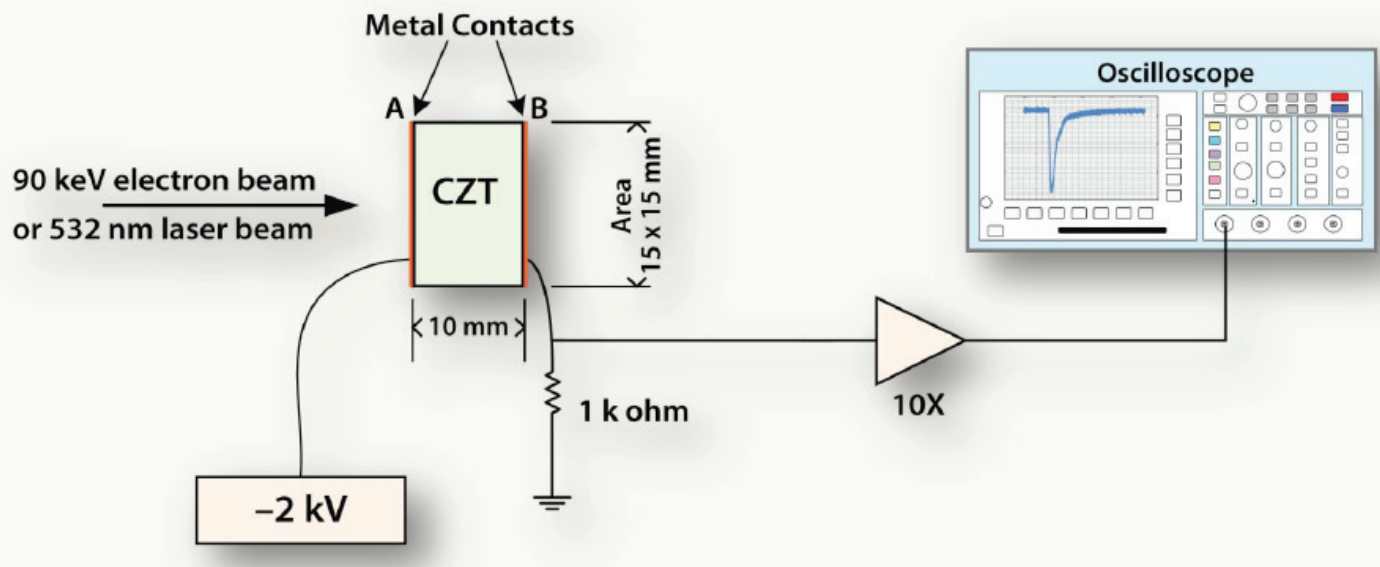

Figure 1. Schematic layout for CZT mobility tests

using laser excitation following the technique demonstrated by Burshtein (1993). By measuring the transit time, $t$, of charge carriers as they travel through the material, we are able to calculate the mobility, $\mu$, using the formula

$$
\mu=\frac{d^{2}}{t \times V^{\prime}},
$$

where $d$ is thickness of the sample and $V$ is the applied bias. Using the formula for different biases, we were able to demonstrate the repeatability of the method.

The schematic layout for the experiment can be seen in Figure 1. In this case, a $10 \mathrm{~ns}, 532 \mathrm{~nm}$ laser pulse impinges on one face of the CZT sample, generating electron-hole pairs. The electrons are then swept across the sample by a negative bias applied to the same face as the laser excitation. The CZT sample is a $15 \times 15 \times 10 \mathrm{~mm}$ crystal mounted in a Pomona shielded box (Figure 2). Both $15 \times 15 \mathrm{~mm}$ sides have been evaporatively coated with $50 \mathrm{~nm}$ of gold soldered to electrical leads for biasing and signal readout. The signal is sent to a Mini-Circuits ZHL-32A power amplifier before being viewed and recorded on an oscilloscope.

The results for the laser-generated electron mobility testing can be seen in Figure 3. The value for the transit time was taken from the first peak to the second peak for each bias value. The spread of the

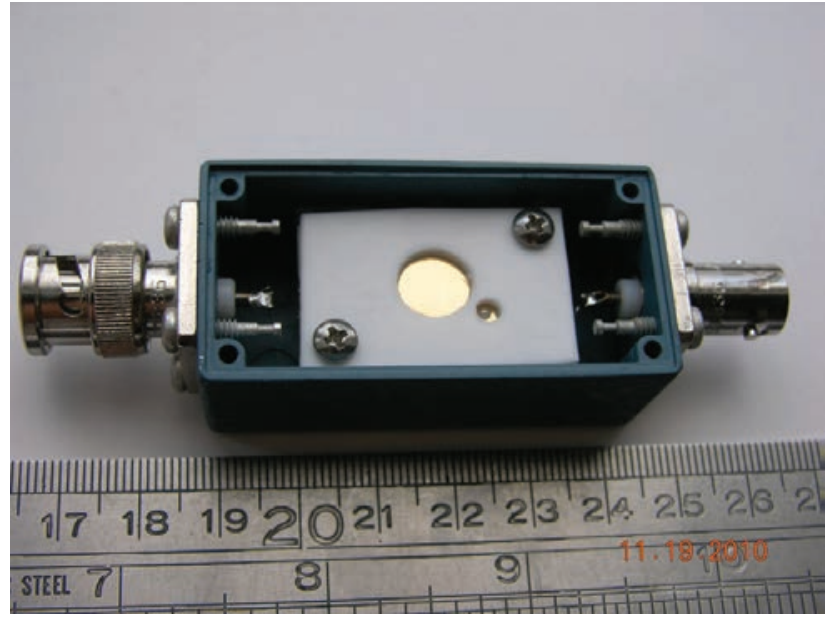

Figure 2. CZT mounted in a Pomona shielded box

later peak is caused by the longitudinal diffusion of the electron pulse as it travels through the material; the peak indicates the highest electron flux at the cathode. With longer transit times, the charge cloud experiences increasing longitudinal dispersion, producing longer tails at lower biases (Fink 2005). Overexposure of the sample to laser intensity causes these tails to become significantly longer, as well as there being a small increase in peak-to-peak duration, indicating space charge saturation effects. The results shown, without the power amplifier in place, exhibit signals in the tens of millivolts.

Measured transit times and a table of calculated mobilities for the sample are shown in Figure 4. 


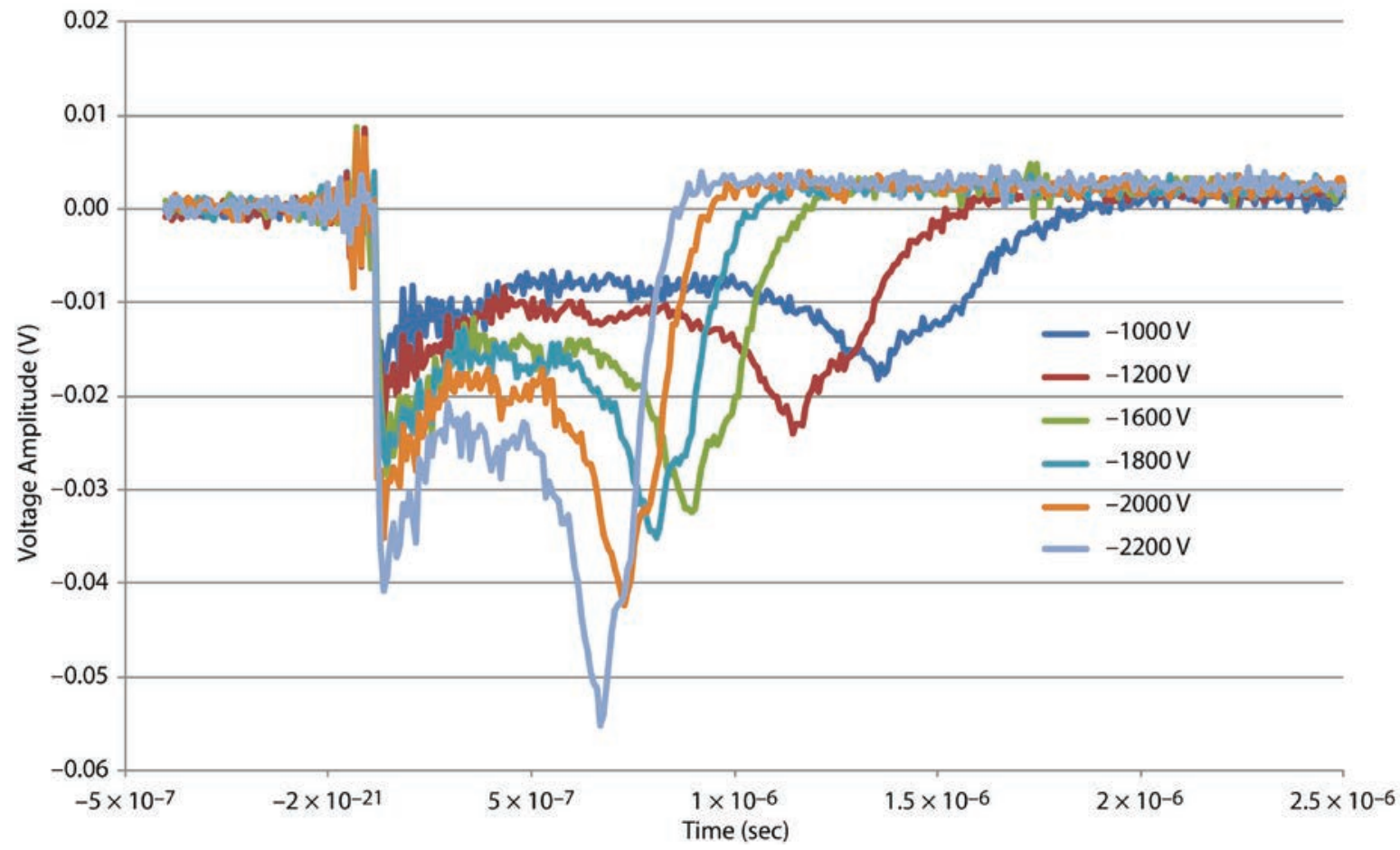

Figure 3. Current waveforms generated in CZT using optical excitation. The duration (mobility) measurements were made between the two peaks of the waveforms. Increasing longitudinal diffusion is also evident at lower biases.

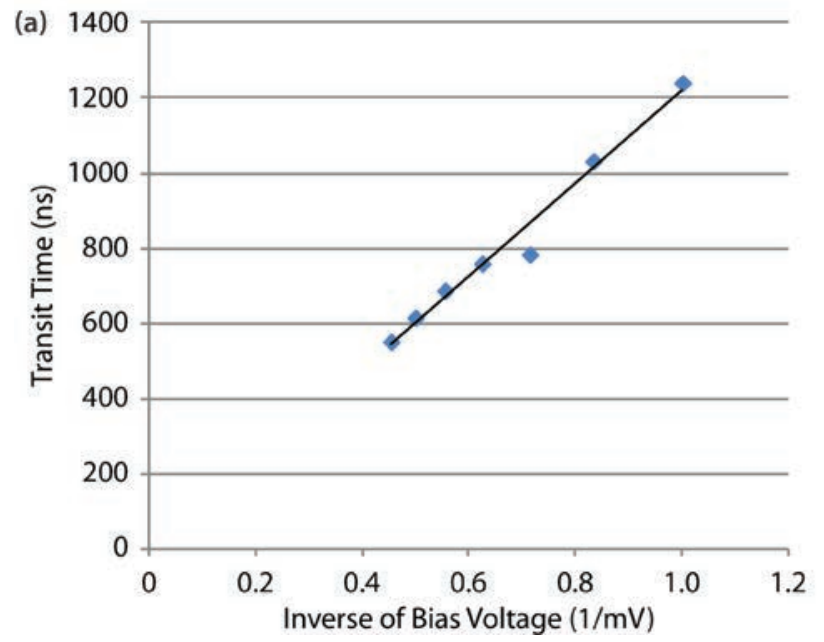

(b) $t_{t}=\frac{L^{2}}{\mu V}$

\begin{tabular}{lcc}
$\begin{array}{l}\text { Electron } \\
\text { Time of } \\
\text { Flight (ns) }\end{array}$ & Bias (-V) & $\begin{array}{c}\text { Electron mobility* } \\
\text { from formula } \\
\left(\mathrm{cm}^{2} / \mathrm{V} \cdot \mathrm{s}\right)\end{array}$ \\
\hline 552 & 2200 & 823 \\
616 & 2000 & 811 \\
688 & 1800 & 807 \\
760 & 1600 & 822 \\
784 & 1400 & 911 \\
1032 & 1200 & 807 \\
1240 & 1000 & 806 \\
*Average electron mobility $=815$
\end{tabular}

Figure 4. (a) A linear fit to the laser testing data of CZT shows good agreement for different biases; (b) the average of the different values is calculated, showing a $4.3 \%$ standard deviation 

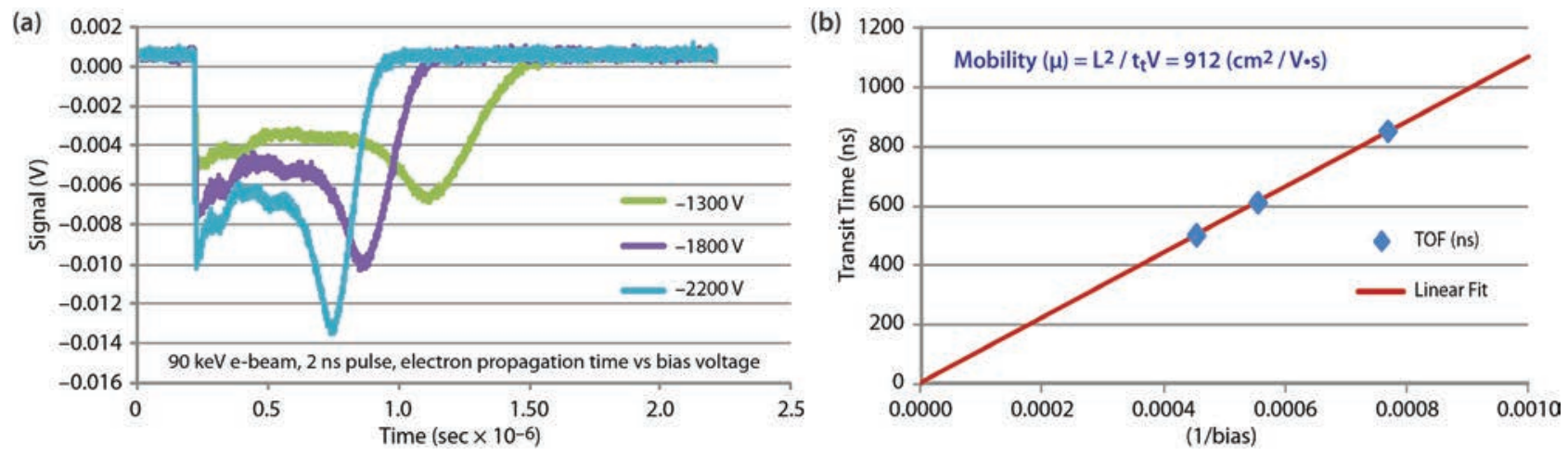

Figure 5. (a) Traces from electron impact CZT tests and (b) a plot showing good linear agreement for the three test points with the electron beam

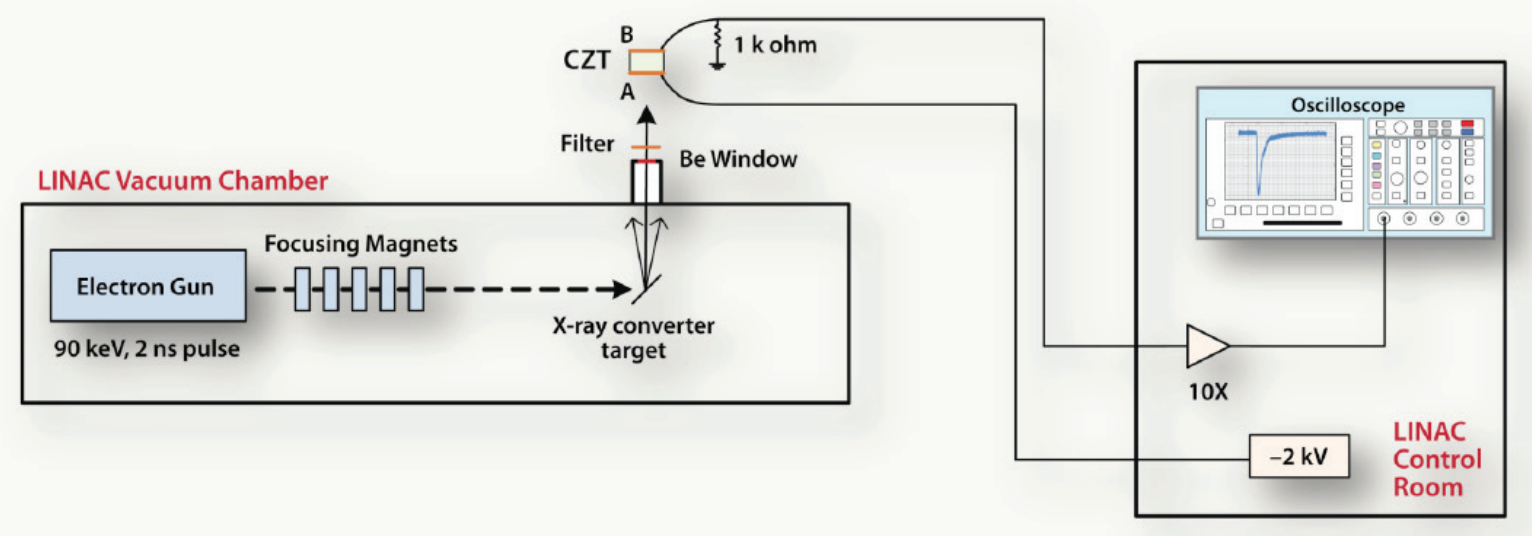

Figure 6. Experimental setup for x-ray tests for CZT

The plot shows that the electron transit time changes approximately linearly with applied bias. The average calculated value for the electron mobility is $815 \mathrm{~cm}^{2} / \mathrm{V} \cdot \mathrm{s}$. The mobility values show good agreement, with a standard deviation of $4.3 \%$, well within the $700-1350 \mathrm{~cm}^{2} / \mathrm{V} \cdot \mathrm{s}$ range reported in the literature.

\section{Radiation Tests}

To demonstrate that our techniques were appropriate for both CZT and scintillators, mobility measurements were taken using a variety of ionizing radiation sources. The number of electron-hole pairs generated in a solid by incident radiation is strongly dependent on the band gap energy: the greater the band gap, the greater the energy required to generate an electron-hole pair. Because insulators such as CsI typically have band gaps of $5 \mathrm{eV}$ or more, compared to CZT with $1.6 \mathrm{eV}$, fewer pairs are generated per unit of energy absorbed. These tests were shown to be repeatable on the CZT with electrons and $x$-rays from an electron linear accelerator (linac), and gamma and alpha radiation from material sources.

The first radiation test was performed using the electron linac located at NSTec Los Alamos Operations (LAO). The sample was inserted directly into the electron beam path. The experimental setup was very similar to that shown in Figure 1 , but the CZT had to be mounted in the vacuum enclosure to be in the electron bunch beam path. The DC portion of the linac produced $2 \mathrm{~ns}, 90 \mathrm{keV}$ electron pulses with around $0.5 \mathrm{~A}$ peak current per pulse. Results for a few different biases are shown in Figure 5. Although there are fewer data points, a linear fit is evident. The 


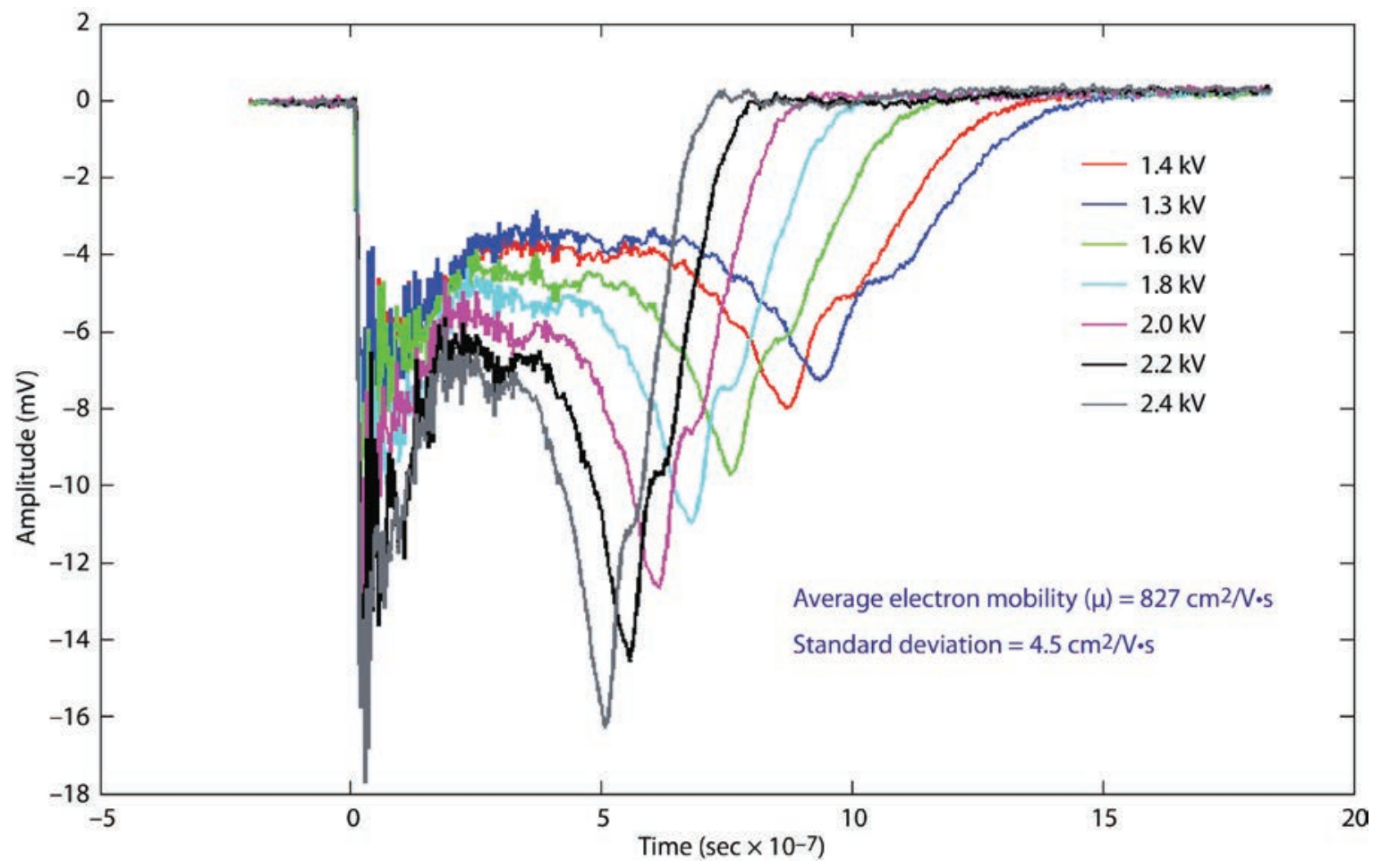

Figure 7. Traces for x-ray tests with CZT show a similar shape and duration to the laser test results shown in Figure 3

mobility value has changed for this measurement by $11 \%$ from 800 to $900 \mathrm{~cm}^{2} / \mathrm{V} \cdot \mathrm{s}$. We believe this may be caused by space charge effects in the material that result from the high peak current of the electron pulses. The LAO linac has limited tunability when less than $0.5 \mathrm{~A}$ of current is used. No amplifier was used to obtain the traces shown in Figure 5.

Another test was done outside the linac target chamber using $\mathrm{x}$-rays generated by the electron pulse as shown schematically in Figure 6 . To generate the $x$-rays, a copper tungsten (CuW) target was put in the path of the electrons, creating bremsstrahlung radiation. The $x$-rays then passed through a 10-mil-thick beryllium window and impinged on the sample. Figure 7 shows the resulting current pulses from the CZT. The mobility value of $827 \mathrm{~cm}^{2} / \mathrm{V} \cdot \mathrm{s}$ agrees well with the laser measurement discussed above, and the standard deviation of less than half of a percent shows good agreement between measurements. Unlike the previous direct electron exposure test, this configuration allowed for easier tunability of the energy deposited on the sample, by changing the distance between target and the sample, allowing for unsaturated operation.

The test was finally performed using a $5 \mu \mathrm{Ci}{ }^{137} \mathrm{Cs}$ gamma source. The schematic for this experiment is shown in Figure 8a. The $662 \mathrm{keV}$ gamma rays produced a very small signal; thus, an integrating amplifier had to be used to acquire the transit time of the electrons. Because of this amplifier we were not able to see the electrical pulse shape that the gamma rays created. The shielding was a piece of aluminum foil electrically grounded to the work bench. Without the foil shielding, we were unable to see a signal above the ambient noise. The calculated mobility value of $817 \mathrm{~cm}^{2} / \mathrm{V} \cdot \mathrm{s}$ is in good agreement with the value obtained using the laser measurement (Figure 8b). These tests were performed at the NSTec Special Technologies Laboratory with the help of Bill Quam and Bob Vogel. A similar test, done with an americium alpha radiation source at Fisk University with the assistance of Mike Groza, produced similar results. 
(a)

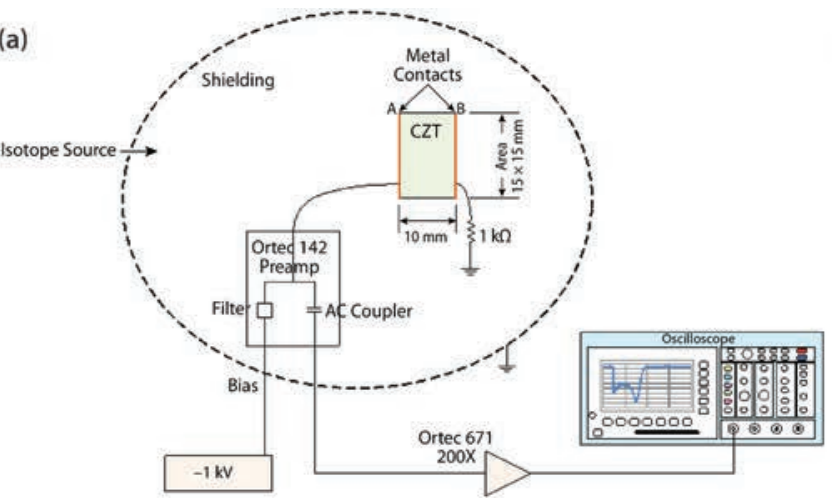

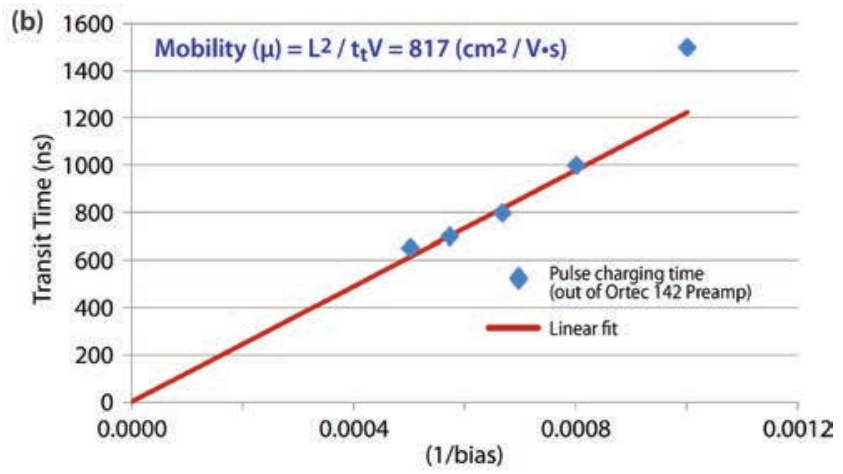

Figure 8. (a) The experimental setup for ${ }^{137} \mathrm{Cs}$ gamma-ray test for CZT; (b) the experimental results show good agreement with previous measurements

(a)

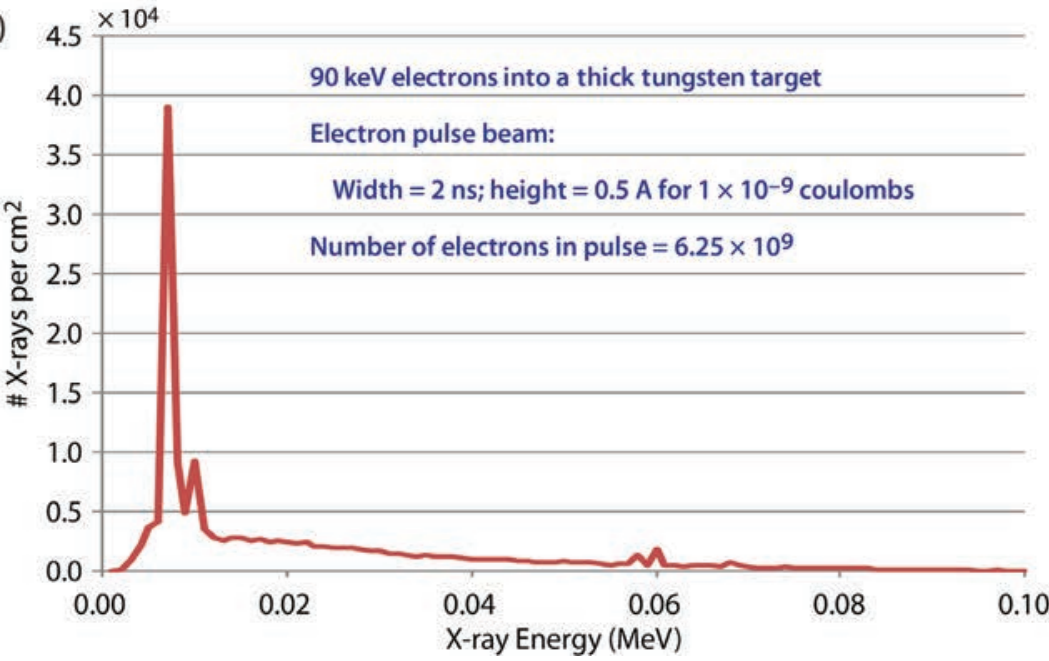

(b)

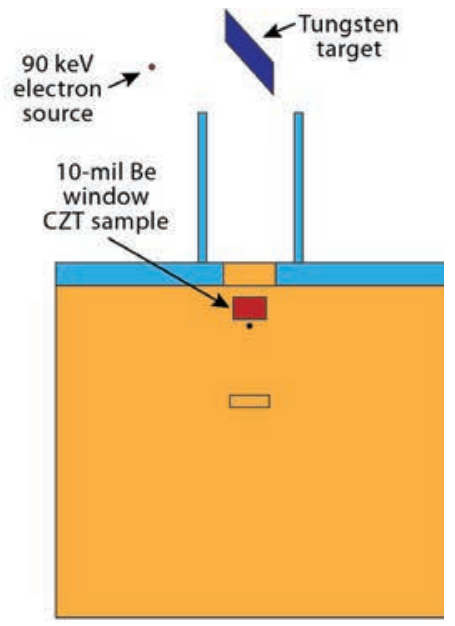

Figure 9. (a) MCNP simulation of x-ray spectrum produced by an electron bunch colliding with a CuW target;

(b) assumed experimental setup used for the simulation

\section{Simulations}

A number of MCNP simulations, mainly to model the $x$-ray production of the linac, were performed by Robert Hilko and Craig Kruschwitz of LAO for various parts of the experiment. A diagram of the model used and the expected $x$-ray spectra produced are shown in Figure 9. The model used is the same as the setup mentioned above, with a thin beryllium (Be) window separating the vacuum chamber from the sample. In the model, a tungsten target is hit with a $90 \mathrm{keV}, 2 \mathrm{~ns}$ electron bunch to produce the $\mathrm{x}$-rays. The predicted spectrum shows a large spike at $10 \mathrm{keV}$ and otherwise bremsstrahlung spectra out to $90 \mathrm{keV}$.
The simulations also examined the photon and scattered electron absorption in CZT and CsI samples, the results of which are seen in Figure 10. Note that for a 90 keV electron pulse, most of the energy per incident particle is absorbed in the first few hundred microns. This is good for samples over $1 \mathrm{~mm}$ thick because we know most of the signal is coming from electrons that must traverse the entire thickness of material, ensuring the correct duration for the transit time. The initial simulations assumed linac $x$-ray excitation because we planned to first implement this method in tests using scintillators. 
(a) Energy deposited in CZT per electron

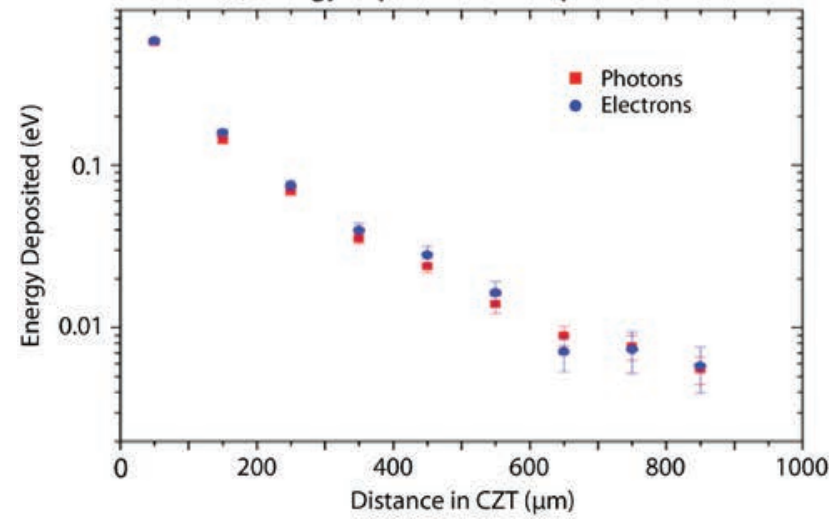

(b) Energy deposited in Csl per $90 \mathrm{keV}$ source electron

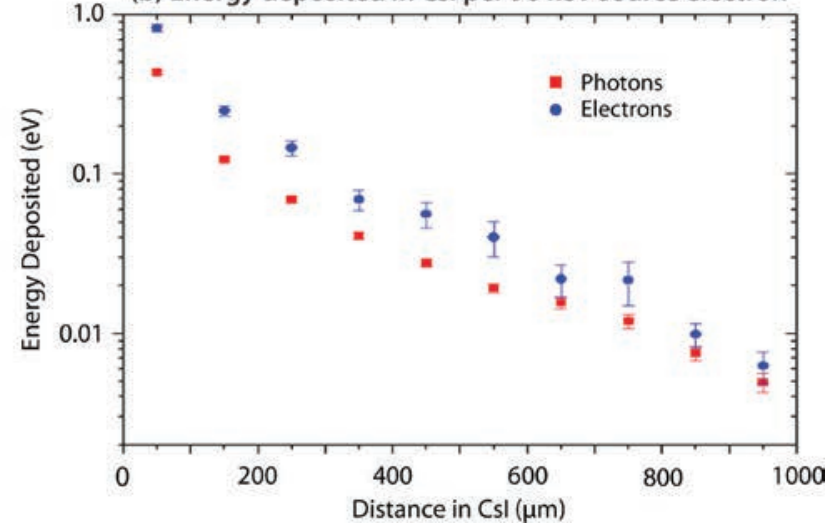

Figure 10. (a) Energy deposited in CZT per electron or photon as a function of sample thickness, and (b) energy deposited in Csl as a function of sample thickness. Both plots show a majority of the energy is deposited on the surface of the sample for the linac tests.

\section{Scintillator Testing}

The scintillator samples were much thinner than the CZT samples. The CsI sample was $250 \mu$ m thick, and the bismuth germanate (BGO) sample was $150 \mu \mathrm{m}$ thick. These thicknesses were based on considerations of the mean free path, $\lambda$, of the carriers and the carrier recombination time, $\tau$, related by

$$
\lambda=\mu|\vec{E}| \tau,
$$

where we have assumed that the values for $\tau$ and $\lambda$ are much smaller in the case of the scintillators because they are insulators and not semiconductors. We posit that if the sample is too thick, the charge carriers will recombine well before they traverse the entire sample thickness, resulting in a recombination measurement rather than a mobility measurement. An unfortunate consequence of this geometry is that the reduced thicknesses will absorb less incident energy, especially if the exciting radiation is highly penetrating. Additionally, there has been some concern about the structural integrity of the samples, which is difficult to evaluate because they are goldcoated and have a protective plastic layer affixed on top of them that holds them to a Teflon mount. When this project continues in FY 2013, tests will be made using slightly thicker samples, which we hope will alleviate these structural concerns.
Although we used both the linac $x$-ray technique and the ${ }^{137} \mathrm{Cs}$ gamma source technique to measure the transit time of carriers across both BGO and Csl, neither technique produced useful results. MCNPX simulations give a good indication of why the $662 \mathrm{keV}$ gamma test failed, as shown in Figure 11: a very small percentage of the gamma energy is deposited in the material. Although some energy is deposited, efforts

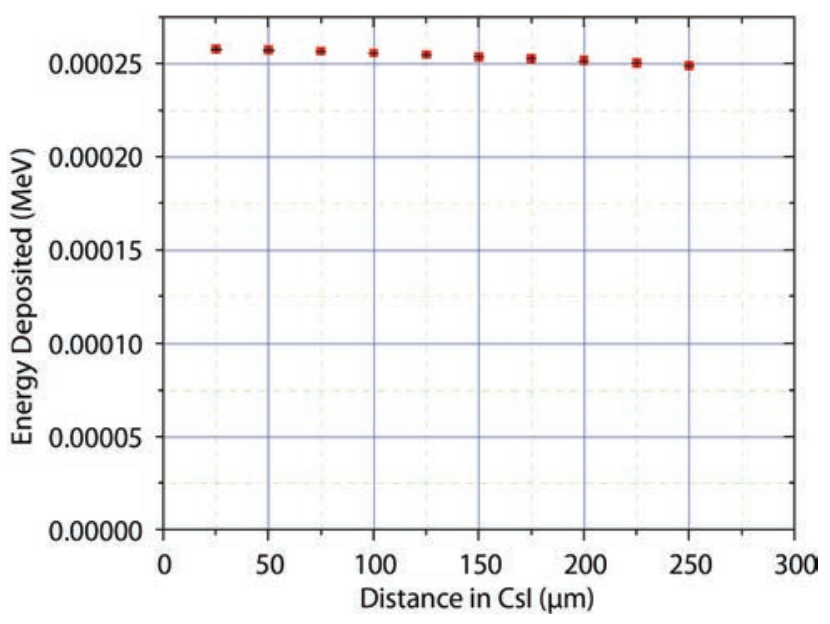

Figure 11. Energy deposited per incident $662 \mathrm{keV}$ gamma ray in a Csl sample. Note that very little energy is deposited on the surface of the sample or even in the bulk material. Clearly, thin samples of the crystal do not have the stopping power to make the Cs source a test source. 
to locate this signal proved unsuccessful. We believe that with such a low activity source and with such high-energy gammas, this technique is not viable.

The LAO linac was also employed to generate a transit time signal using bremsstrahlung-produced $x$-rays. Different amplifier chains were placed after the sample, and different shielding techniques were implemented. However, the presence of high levels of electronic noise ultimately limited our ability to find a signal. More extensive tests will be done in FY 2013 to better determine the source of the noise, and configure more effective shielding methods. We are also considering using shorter, higher peak current electron pulses by engaging the RF portion of the accelerator (for all previously mentioned tests and results, only the DC portion was used) in order to more closely follow the work done by Aduev (1998).

\section{Alternative Approaches}

We are considering three new approaches to making the scintillator measurements. The easiest to implement would be to acquire alpha or beta radiation material sources for a bench-top test. These types of sources have a much lower penetration depth than the ${ }^{137} \mathrm{Cs}$ source we previously used. More energy would be deposited in the material, and most of the energy would be deposited on or near the face of the sample where the radiation is incident, which would be preferable for the experiment.

We have begun collaborating with Professor Pietro Musumeci of the University of California, Los Angeles (UCLA) Pegasus Electron Accelerator facility regarding a different approach. The Pegasus linac uses an ultrashort laser pulse incident on the cathode to create the electron bunch, as opposed to the $100 \mathrm{kV}$ electrical current pulse of the LAO linac. This should reduce noise in the signal. The UCLA linac is also capable of creating cleaner (less jitter, deeper modulation) several hundred picosecond duration pulses than the LAO machine. We plan to test the scintillator samples at UCLA in November 2012.
The advanced photon source electron accelerator at the Argonne National Laboratory could provide another valuable approach to the difficulty of measuring mobility in scintillators. In this scenario, we would be using photons with sufficient energy to promote scintillator electrons into the conduction band without having to collide electrons into a bulk material or the sample electrodes before reaching the sample. It is our suspicion that this will offer a lower noise solution than the linac experimental setups.

\section{Conclusion}

We have conclusively demonstrated that four different types of ionizing radiation measurements agree with laser measurements made to determine the electron mobility of the semiconductor CZT, proving that this is a viable method for measuring material electron mobilities. This is the first time, to our knowledge, that such measurements have been made. Attempts made to measure the mobility in the scintillators CsI and BGO with two of these methods have been unsuccessful so far, due to sample geometries and electronic noise issues. We plan to continue the study with alternate sources, which we hope will mitigate those two obstacles. If the measurements are achievable with the two scintillator samples, we will replicate the measurements on other scintillator materials. These measurements will add to our basic understanding of the material physics of scintillators, and hopefully aid in future scintillator material design.

\section{References}

Aduev, B. P., E. D. Aluker, G. M. Belokurov, V. N. Shvayko, "Radiation-stimulated conductivity of some alkali halides induced by 50 ps electron pulse irradiation," Physica Status Solidi B 208 (1998) 137-143.

Burshtein, Z., H. N. Jayatirtha, A. Burger, J. F. Butler, B. Apotovsky, F. P. Doty, "Charge-carrier mobilities in $\mathrm{Cd}_{0.8} \mathrm{Zn}_{0.2} \mathrm{Te}$ single crystals used as nuclear radiation detectors," Appl. Phys. Lett. 63, 1 (1993) 102-104. 
Fink, J., H. Krüger, P. Lodomex, N. Wermes, "Characterization of charge collection in CdTe and CZT using the transient current technique," Nucl. Instrum. Methods Phys. Res. A 560, 2 (2005) 435-443.

Hofstadter, R., "Alkali halide scintillation counters,"

Phys. Rev. 74 (1948) 100-101.

Schlesinger, T. E., J. E. Toney, H. Yoon, E. Y. Lee, B. A. Brunett, L. Franks, R. B. James, "Cadmium zinc telluride and its use as a nuclear radiation detector material," Mat. Sci.

Eng. R32 (2001) 103-189. 
This page left blank intentionally 


\section{LoW-Cost Cerium Bromide Alloys}

RSLN-18-12 | YEAR 1 OF 1

Paul P. Guss, ${ }^{1, a}$ Bryan Wong, ${ }^{b}$ Michael Foster, ${ }^{b}$ F. Patrick Doty, ${ }^{b}$ Kanai Shah, ${ }^{c}$ Michael Squillante, ${ }^{c}$ Jarek Glodo, ${ }^{c}$ and Ding Yuand

Despite the outstanding scintillation performance characteristics of cerium tribromide $\left(\mathrm{CeBr}_{3}\right)$ and cerium-activated lanthanum tribromide, their commercial availability and application is limited because it is difficult to grow large, crack-free single crystals from these fragile materials. The objective of this investigation was to employ aliovalent doping to increase crystal strength while maintaining the optical properties of the crystal. One divalent dopant $\left(\mathrm{Ca}^{2+}\right)$ was investigated as a dopant to strengthen $\mathrm{CeBr}_{3}$ without negatively impacting scintillation performance. Ingots containing nominal concentrations of $1.9 \%$ of the $\mathrm{Ca}^{2+}$ dopant were grown. Preliminary scintillation measurements are presented for this aliovalently doped scintillator. $\mathrm{Ca}^{2+}$-doped $\mathrm{CeBr}_{3}$ exhibited little or no change in the peak fluorescence emission for $371 \mathrm{~nm}$ optical excitation for $\mathrm{CeBr}_{3}$. The structural, electronic, and optical properties of $\mathrm{CeBr}_{3}$ crystals were investigated using the density functional theory within generalized gradient approximation. The calculated lattice parameters are in good agreement with the experimental data. The energy band structures and density of states were obtained. The optical properties of $\mathrm{CeBr}_{3}$, including the dielectric function, absorption spectra, extinction coefficient, and reflectivity were calculated, and the results were compared with the available experimental data.

${ }^{1}$ gusspp@nv.doe.gov, 702-295-8095

a Remote Sensing Laboratory-Nellis; ${ }^{b}$ Sandia National Laboratories; ${ }^{c}$ Radiation Monitoring Devices, Inc.; ${ }^{d}$ Los Alamos Operations

\section{Background}

Cerium-activated and cerium-based crystals appear to be particularly promising fast scintillators (Rodnyi 1997, Harrison 2010). The discovery of cerium-doped lanthanum tribromide $\left(\mathrm{LaBr}_{3}: \mathrm{Ce}\right)$ and cerium-doped lanthanum chloride $\left(\mathrm{LaCl}_{3}: \mathrm{Ce}\right.$ ) (van Loef 2000, 2001; Dorenbos 2002) and the characterization of their outstanding scintillation performance sparked an interest in the lanthanide halide family of scintillators as candidates for replacing thallium-activated sodium iodide ( $\mathrm{Nal}: \mathrm{Tl})$, the industry standard since its discovery in 1948 (Hofstadter 1948, 1950). Since then, numerous derivatives of this family have been explored, including undoped, self-activated cerium tribromide $\left(\mathrm{CeBr}_{3}\right)$ (Shah 2005). Although the scintillation performance of these cerium-doped and ceriumbased crystals is outstanding, their mechanical properties are not generally conducive to growth of large single crystals (Harrison 2008, 2009, 2010).

Cerium trihalides possess excellent scintillation properties (as do most rare-earth trihalide scintillators), such as high light output, fast decay time, and excellent energy resolution (Shah 2005). Therefore, they are widely used in various fields such as highenergy physics and positron emission tomography, as well as some chemical processes involved in the nuclear industry (Vetere 2000). The structural, electronic, and optical properties of cerium trifluoride $\left(\mathrm{CeF}_{3}\right)$ have been extensively investigated using both experimental and theoretical methods (Wesley 1971, Olson 1978, Joubert 1998, Tsuchiya 1999, Shi 2002). $\mathrm{CeBr}_{3}$ shows preferable optical and scintillation 

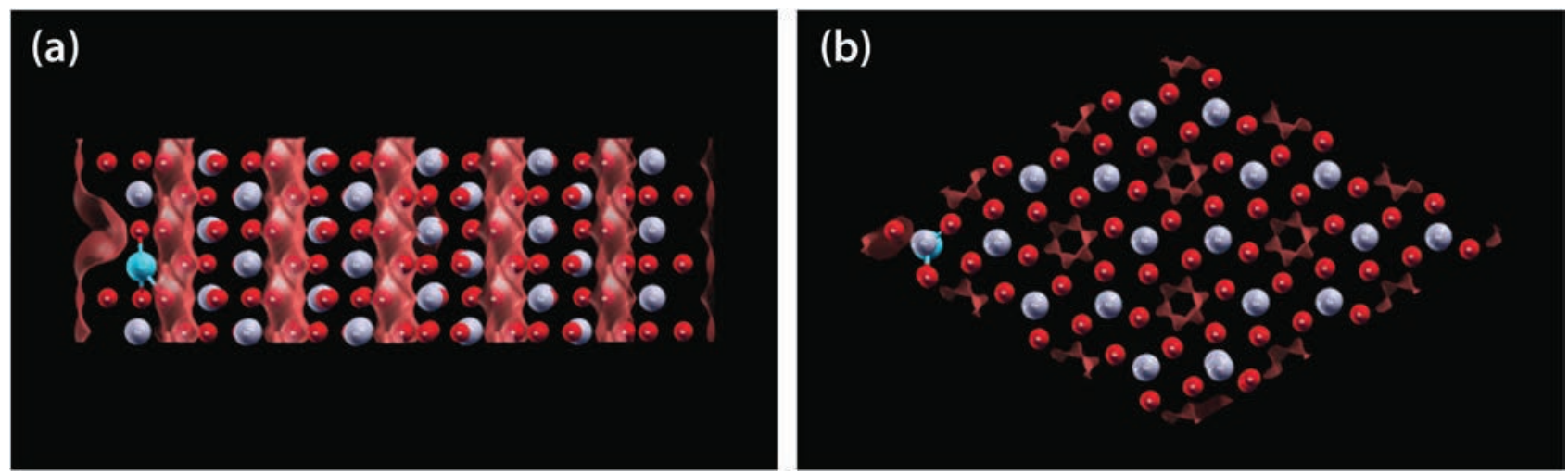

Figure 1. (a) Side view of electron charge density (red lobes) in a $\mathrm{Ca}^{2+}$-doped $\mathrm{CeBr}_{3}$ scintillating material. The electron density is significantly modified and accumulates near the $\mathrm{Ca}$ atom (blue). (b) Top-down view of electron charge density (red lobes) in a Ca ${ }^{2+}$-doped $\mathrm{CeBr}_{3}$ scintillating material. The calcium (blue) lies underneath a $\mathrm{Ce}$ atom in the left corner of the primitive unit cell.

properties; for example, it has a higher light yield (Shah 2005, Li 2007).

$\mathrm{CeBr}_{3}$ is a self-activated lanthanide scintillator, which has received considerable recent attention (Shah 2005) due to proportionality and energy resolution for gamma spectroscopy far superior to Nal:Tl. Because the material possesses no intrinsic radioactivity, $\mathrm{CeBr}_{3}$ has high potential to outperform scintillators such as $\mathrm{LaBr}_{3}$ :Ce or lanthanum-based elpasolites (Guss 2009), making it an excellent candidate for gamma spectroscopy (Shah 2004, Guss 2010). However, due to its hexagonal crystal structure (prototype structure uranium trichloride, $\mathrm{UCl}_{3}$ ), pure $\mathrm{CeBr}_{3}$ can fracture during crystal growth, detector fabrication, and subsequent field use. (The prototype structure notation denotes coordination geometry identical to $\mathrm{UCl}_{3}$.) The fact that this crystal structure fractures easily is expected to impact manufacturing yield, and could compromise reliability for large crystals (Doty 2007). Therefore, significant gains in the practical scale for $\mathrm{CeBr}_{3}$ scintillators will be realized by increasing fracture toughness of the crystals (Harrison 2010). Aliovalent substitution, in which a host ion is replaced with an ion of different valence (e.g., $\mathrm{Ca}^{2+}$ for $\mathrm{Ce}^{3+}$ in $\mathrm{CeBr}_{3}$ ) is a more potent method of strengthening compared to isovalent substitution (i.e., replacing a fraction of ions with like-valence ions). In this approach, the formation of intrinsic defects necessary to maintain charge neutrality results in complexes with long-range interactions in the crystal. The resulting increase in hardening rate can be explained in terms of elastic interaction with dislocations (Pletka 1977).

\section{Project}

\section{Computational Method}

Density functional theory (DFT) calculations were performed to discern the optical properties of the calcium $\left(\mathrm{Ca}^{2+}\right)$-doped $\mathrm{CeBr}_{3}$ compound. Introducing the $\mathrm{Ca}^{2+}$ dopant (Figure 1a) resulted in calculations that provided valuable insight into the increase in crystal strength. These calculations may be used to compare the differences of doping with different dopants or their concentrations and to calculate the optical properties of the resultant crystals. One must accurately account for the electron densities over the space of the crystal. To do this accurately, the calculations were made for a "super-cell" of 200 atoms with well over 1000 electrons (Figure 1). In addition, the electron density of states was calculated. If there is a crystal defect, or an atomic substitution, then the electron density of states will change, leading to different results. The presence of a $\mathrm{Ca}^{2+}$ dopant can significantly affect electronic properties by distorting the electronic charge density near the $\mathrm{Ca}^{2+}$ atom (Figure 1).

The DFT results for both the pure $\mathrm{CeBr}_{3}$ primitive cell and the (divalent) $\mathrm{Ca}^{2+}$-doped super-cell were based 


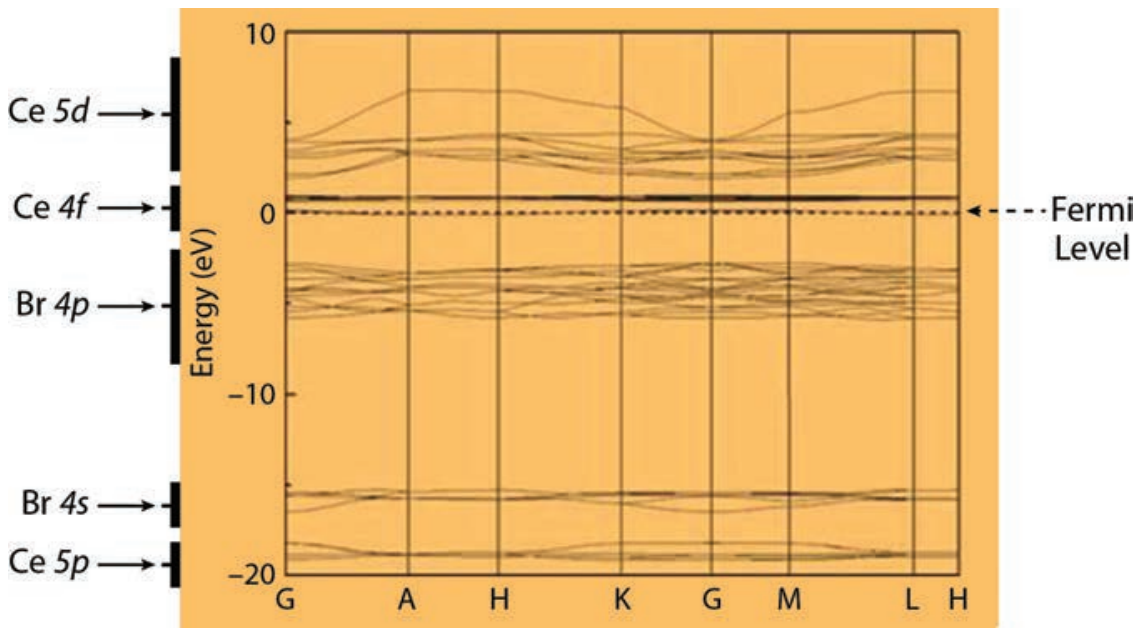

Figure 2. Band structure of $\mathrm{CeBr}_{3}$ in a hexagonal crystal lattice. The band structure corresponds to the Brillouin zone for the $\mathrm{CeBr}_{3}$ hexagonal crystal lattice. The Fermi energy level is shifted to the valence band maximum at zero. on spin-polarized, generalized gradient approximation (GGA) methods using Projector-Augmented-Wave pseudopotentials (Blöchl 1994) calculated by the Vienna Ab-initio Simulation Package (VASP) (VASP 2012). It was found that the inclusion of a Hubbard DFT correction (often denoted as DFT+U) was essential for obtaining accurate band structures and band gaps. This novel DFT method (DFT+U) was used to support experimental efforts in understanding both the structural and electronic properties of aliovalently doped $\mathrm{CeBr}_{3}$ materials. This formalism is essential for the Ce-based materials studied here, as conventional GGAs fail to describe systems with localized (strongly correlated) $d$ and $f$ electrons, which manifests in the form of unrealistic one-electron energies due to spurious self-interaction. This approach has been further modified and applied in many forms by Liechtenstein (1995) and Dudarev (1998). In this work, the rotationally invariant approach by Dudarev is used because the effective on-site Coulomb and exchange parameters ( $\mathrm{U}$ and $\mathrm{J}$ ) do not enter separately, and only their difference $(U-\mathrm{J})$ is required.

Within the Hubbard DFT approach, an on-site penalty function is placed on the $f$ electrons for each of the cerium (Ce) atoms to prevent spurious over-delocalization of the electrons. Based on benchmarks for $\mathrm{Ce}^{3+}$ ions, the Hubbard value (U) for our DFT studies was set at $4.5 \mathrm{eV}$. For the initial study of the primitive cell, a very high cutoff energy of $400 \mathrm{eV}$ was used for the plane-wave basis set, and the Brillouin zone was sampled using a dense $8 \times 8 \times 8$ gamma-centered Monkhorst-Pack grid. In addition to spin-polarization and dispersion effects, a relativistic spin-orbit coupling treatment was used for the valence electrons in the bulk-lattice calculations. Geometry optimizations of both the ions and the unit cell were carried out. The results of the DFT modeling performed at Sandia National Laboratories suggested that a $1.9 \%$ substitution would result in a suitable combination of crystalline structural properties, increased strengthening of the lattice, and metrics for optical properties and emission spectra. The Fermi levels that result at different points in the lattice are also different because of the aliovalent substitution.

\section{Structure Determination and Electronic Properties}

$\mathrm{CeBr}_{3}$ has a hexagonal structure, where cerium and halide occupy $2(\mathrm{a})$ in $(1 / 3,2 / 3,1 / 4)$ and $6(\mathrm{~h})$ in $(0.375$, $0.292,1 / 4)$ sites, respectively (Zachariasen "Crystal" 1948, "UCl ${ }_{3}$ type" 1948; Li 2007). Our structures are in reasonable agreement with experiments

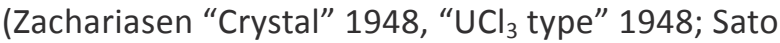
1976, Park 1993). The energy band structure of $\mathrm{CeBr}_{3}$ is shown in Figure 2. The zero energy is arbitrarily taken at the Fermi level. The lowest bands around $-36 \mathrm{eV}$ consist of $5 s$ states of $\mathrm{Ce}$. The $\mathrm{Ce} 5 p$ states are located at around $-19 \mathrm{eV}$. The bands around $-16 \mathrm{eV}$ are derived from the $4 s$ states of $\mathrm{Br}$. The energy bands in the range from -6 to $-2 \mathrm{eV}$ correspond to the $4 p$ 
states of $\mathrm{Br}$ that decide the top of the valence band. These results are in agreement with $\mathrm{CeCl}_{3}$ experimental data (Park 1993). Above the Fermi level, the conduction band consists of the $4 f$ and $5 d$ states of $\mathrm{Ce}$ (Li 2007). The $4 f$ states have a sharp peak due to the strong localization character of the $4 f$ states. If the $4 f$ electron is kept in the core, the calculated result agrees with other experiments (Skorodumova 2001, Li 2007).

\section{Detectors}

The results above were translated into a work authorization for Radiation Monitoring Devices, Inc. (RMD), to build a $\mathrm{CeBr}_{3}$ crystal alloy detector with $2 \%$ substitution using the $\mathrm{Ca}^{2+}$ cation. RMD grew a 1-inchdiameter $\mathrm{CeBr}_{3}$ crystal with $1.9 \%$ calcium dibromide $\left(\mathrm{CaBr}_{2}\right)$, as requested. The vertical Bridgman was used as the crystal growth technique. Materials were purified prior to crystal growth. The charge composition was $222.14 \mathrm{~g}$ of $\mathrm{CeBr}_{3}$ and $2.26 \mathrm{~g}$ of $\mathrm{CaBr}_{2}$. The resulting crystal is polycrystalline white but transparent. The crystal structure is columnar or filamentlike. When RMD cut the crystal, it fell apart. Smaller crystallites were easily separated from the bulk. Samples were then harvested from each ingot as available and tested for fluorescence and scintillation. The detector crystals produced are shown in Figure 3. The small crystallites are clear and transparent, but the large sections of crystal are translucent or whitish-opaque. Small crystallites are typically $\sim 2$ to $3 \mathrm{~mm}$ wide and $10 \mathrm{~mm}$ or more in length. Adding 500 ppm calcium in a prior run (in 2009) did not appear to have a significant impact on the performance; however, there was no cracking of that ingot. In neither run has the calcium concentration in the final ingot been measured.

This investigation involved measurement of the light output, the emission spectrum, and the fluorescent decay time of the crystals. Preliminary results for a select set of samples from the as-grown ingots were obtained. Fluorescence emission curves were recorded to determine the effect of the solute cation on the emission wavelengths of the base compound $\mathrm{CeBr}_{3}$. Initial scintillation characterization was completed as well. The small crystallites were also tested for scintillation properties.
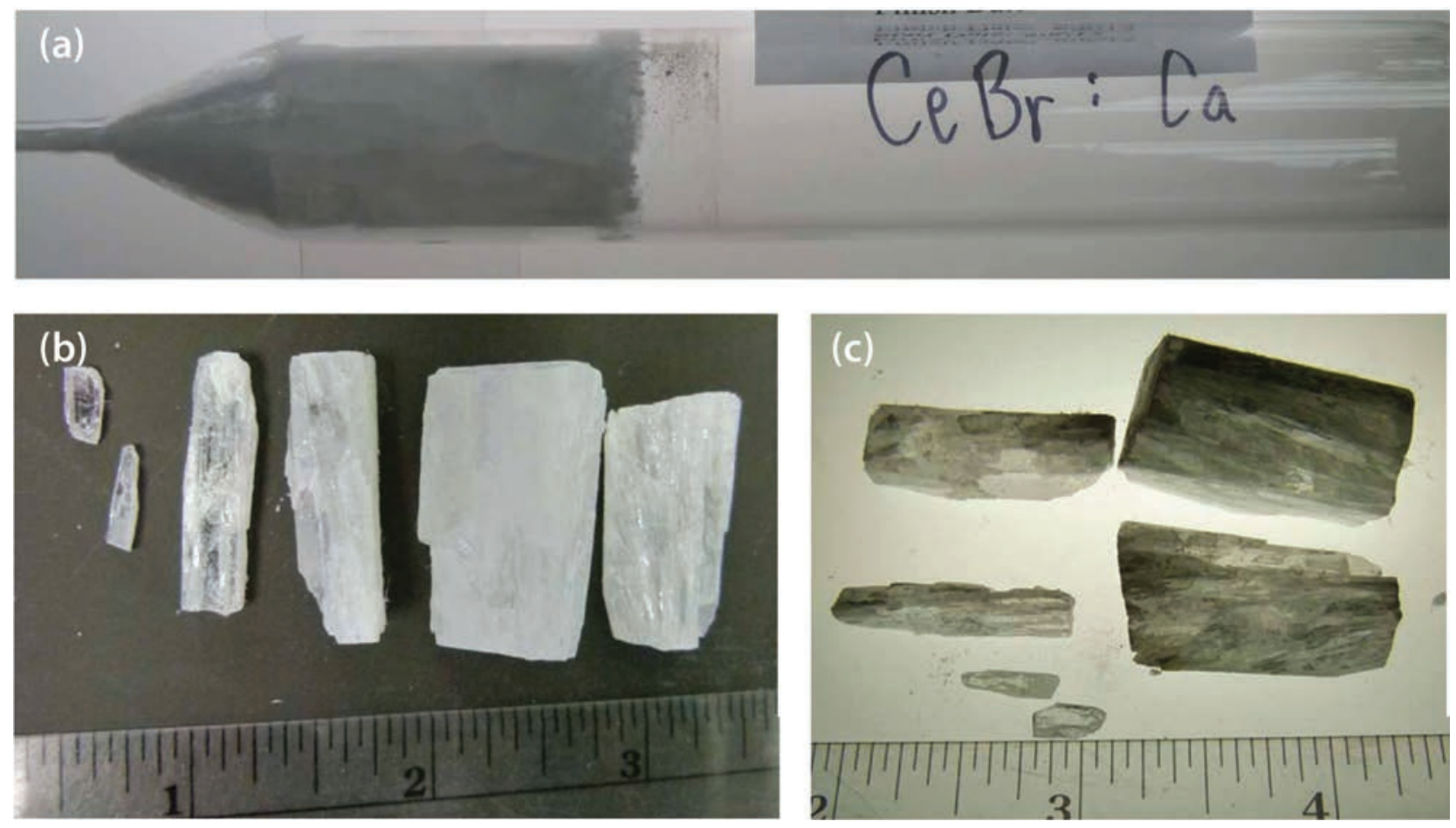

Figure 3. Crystals grown by $\mathrm{RMD}$ : (a) $\mathrm{CeBr}_{3}$ ampoule after growth; note the visible cracking. (b) $\mathrm{CeBr}_{3} \mathrm{crystals}$ with calcium doping. (c) $\mathrm{CeBr}_{3}$ crystals with transmitted light; clear sections are visible. 


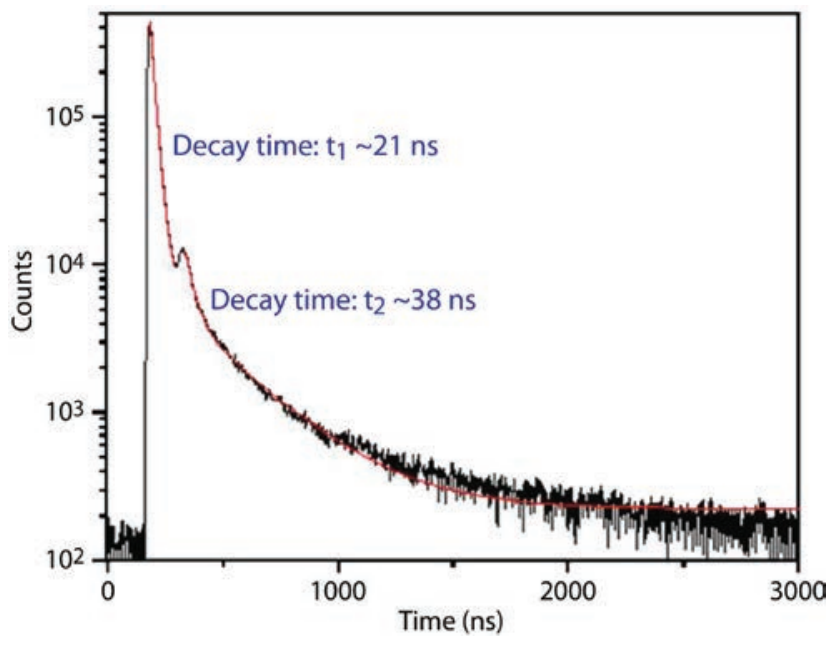

Figure 4. Pulse shape recorded for $1.9 \%$ nominally $\mathrm{Ca}^{2+}$-doped $\mathrm{CeBr}_{3}$. The red line depicts best fit to the data with decay times of $\mathrm{t} 1 \sim 21 \mathrm{~ns}$ and $\mathrm{t} 2 \sim 38 \mathrm{~ns}$.

\section{Pulse Shape}

The time profiles of the $\mathrm{CeBr}_{3}: \mathrm{Ca}^{2+}$ crystals grown at RMD were characterized. Nanosecond time profiles were measured using a standard single photon counting method. A decay-time spectrum of a $\mathrm{CeBr}_{3}: \mathrm{Ca}^{2+}$ crystal was measured using the delayed coincidence method (Bollinger 1961). During measurements, samples were coupled to a photomultiplier tube (PMT) and irradiated with $511 \mathrm{keV}$ gamma rays ( ${ }^{22} \mathrm{Na}$ source). Figure 4 shows the decaytime spectrum recorded for $\mathrm{a} \mathrm{CeBr}_{3}: \mathrm{Ca}^{2+}$ sample along with a fit (to an exponential rise and decay time plus a constant background) to the data. The second pulse in Figure 4 is an artifact; it is an afterpulse from the PMT. The pulse shape has two components, as seen in Figure 4. The decay constants for these two components are $\sim 21$ ns and $\sim 38$ ns. These components cover all of the integrated light output of the sample. The 21 ns decay component can be attributed to optical emission arising from direct capture of electron-hole pairs at the $\mathrm{Ce}^{3+}$ sites (Shah 2005). Using the data shown in Figure 4, the rise time of the scintillation pulse from $\mathrm{CeBr}_{3}: \mathrm{Ca}^{2+}$ is estimated to be $\sim 0.1 \mathrm{~ns}$. The initial photon intensity, a figure of merit for timing applications, is estimated to be $\sim 4,000$ photons/ns/MeV) for $\mathrm{CeBr}_{3}: \mathrm{Ca}^{2+}$, which is higher, compared to all common inorganic scintillators (including $\mathrm{BaF}_{2}$, a benchmark for timing applications).

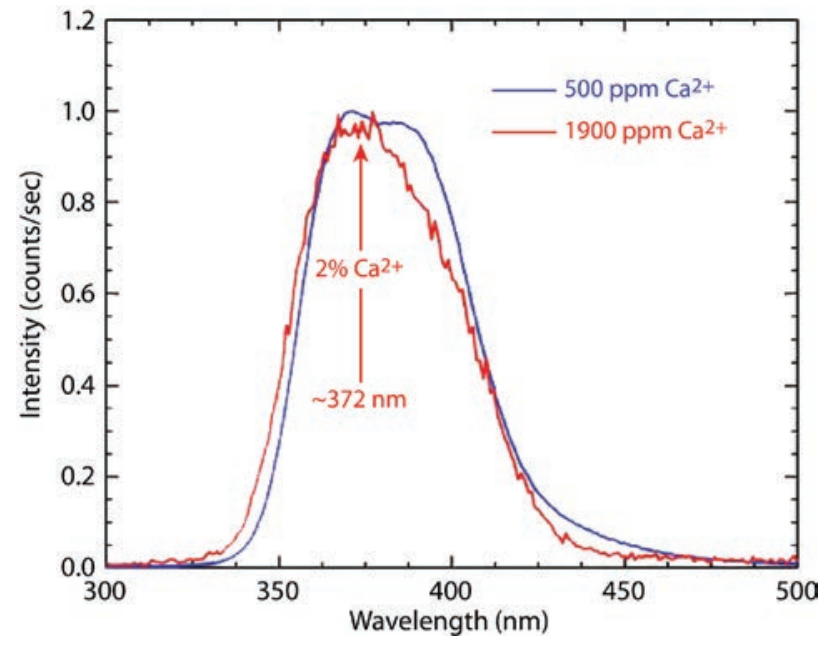

Figure 5. Emission spectra are shown for $\mathrm{a} \mathrm{CeBr}_{3}$ sample doped with 500 ppm Ca ${ }^{2+}$ and 1900 ppm Ca${ }^{2+}$. The emission spectra peak at $\sim 372 \mathrm{~nm}$. There is essentially no change by increasing the $\mathrm{Ca}^{2+}$ content.

\section{Fluorescence Measurements}

The emission spectrum of the $\mathrm{CeBr}_{3}: \mathrm{Ca}^{2+}$ scintillator was measured. Fluorimetry and preliminary scintillation are presented here for this aliovalently doped scintillator. The $\mathrm{CeBr}_{3}: \mathrm{Ca}^{2+}$ samples were excited with radiation from a Philips $x$-ray tube having a copper target, with power settings of $30 \mathrm{kVp}$ and $15 \mathrm{~mA}$. The scintillation light was passed through a McPherson monochromator and detected by a Hamamatsu R2059 PMT with a quartz window. The system was calibrated with a standard light source to enable correction for sensitivity variations as a function of wavelength. A normalized emission spectrum for the $\mathrm{CeBr}_{3}: \mathrm{Ca}^{2+}$ sample $\left(2 \% \mathrm{Ca}^{2+}\right)$ is shown in Figure 5 . The observed bands, peaking at 360 and $380 \mathrm{~nm}$, are characteristic of $\mathrm{Ce}^{3+}$ luminescence. The $\mathrm{Ca}^{2+}$ exhibited little or no change in the peak fluorescence emission for $370 \mathrm{~nm}$ excitation. Fluorescence spectra indicate that the $\mathrm{Ca}^{2+}$ dopant is suitable for strengthening $\mathrm{CeBr}_{3}$. The emission wavelength spectrum peaks at $\sim 372 \mathrm{~nm}$ (Figure 5), indicating a nearly imperceptible red shift from the $370 \mathrm{~nm}$ already documented for $\mathrm{CeBr}_{3}$ (Shah 2005). The peak emission wavelength for the $\mathrm{CeBr}_{3}$ sample is at $\sim 370 \mathrm{~nm}$, and this emission is anticipated to be due to $5 d \rightarrow 4 f$ transition of $\mathrm{Ce}^{3+}$. The peak emission wavelength of $372 \mathrm{~nm}$ for $\mathrm{CeBr}_{3}: \mathrm{Ca}^{2+}$ is attractive for gamma-ray spectroscopy because it matches well 


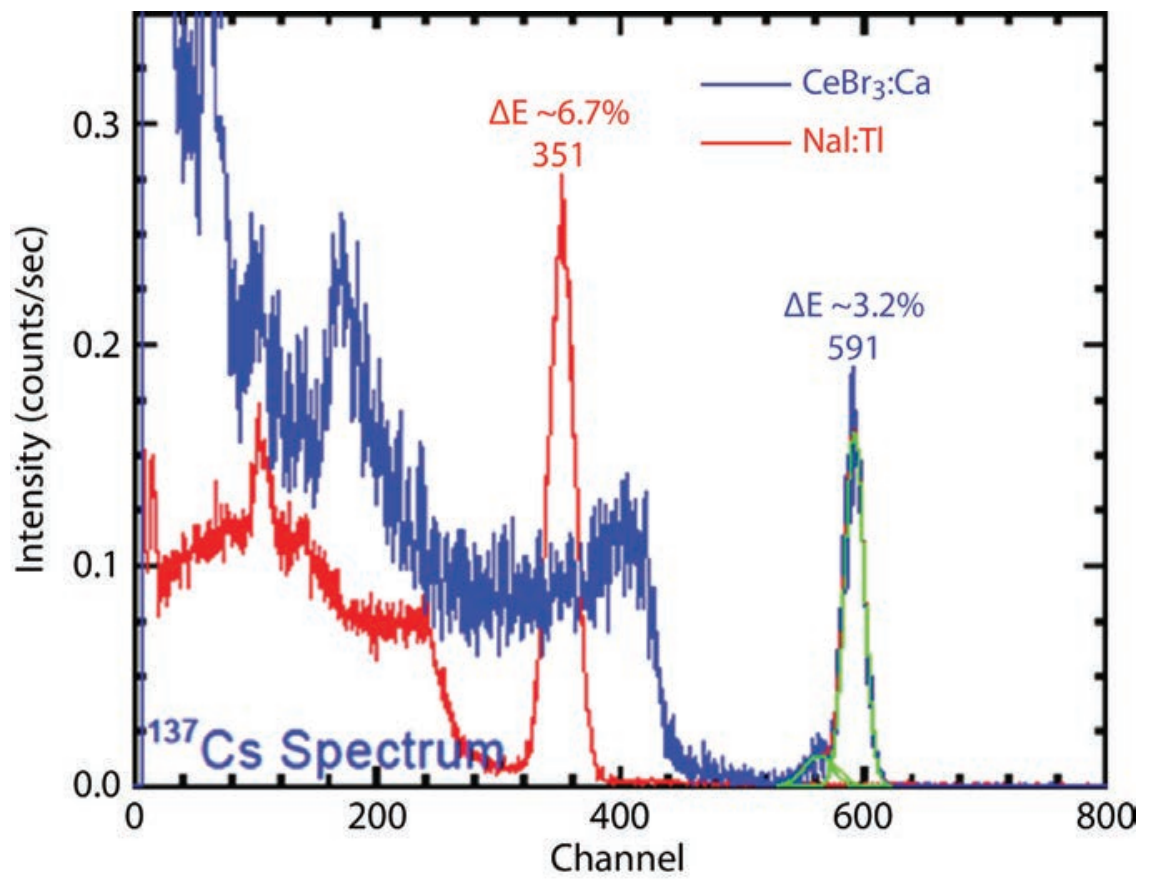

Figure $6 .{ }^{137} \mathrm{Cs}$ spectra collected with $\mathrm{Nal}: \mathrm{Tl}$ and with $1.9 \% \mathrm{Ca}^{2+}$ doped $\mathrm{CeBr}_{3}$ crystals coupled to a PMT. The energy resolution of the $662 \mathrm{keV}$ peak for Nal:TI (at channel 351 ) is 6.7\% FWHM, and for $\mathrm{CeBr}_{3}: \mathrm{Ca}$ (at channel 591) is $\sim 3.2 \% \mathrm{FWHM}$. The green curves represent Gaussian fits to the $\mathrm{CeBr}_{3}: \mathrm{Ca}^{2+}$ photopeak at $662 \mathrm{keV}$ and to the low-energy tail for this same peak. The reported peak resolution of $3.2 \%$ FWHM was derived from this parametric fit to the data. with the spectral response of the PMTs as well as the new generation of silicon photodiodes.

\section{Gamma-RayTotal Light Yield}

Light output (or luminosity) of the $\mathrm{CeBr}_{3}: \mathrm{Ca}^{2+}$ and similarly sized Nal:Tl crystals has been measured at RMD. Total light yield was compared to Nal:Tl by first recording a ${ }^{137} \mathrm{Cs}$ spectrum with a $\mathrm{CeBr}_{3}: \mathrm{Ca}^{2+}$ sample, then replacing the $\mathrm{CeBr}_{3}: \mathrm{Ca}^{2+}$ with $\mathrm{Nal}: \mathrm{Tl}$ and recording a new ${ }^{137} \mathrm{Cs}$ spectrum with the same system settings. Spectra were collected for both the doped $\mathrm{CeBr}_{3}: \mathrm{Ca}^{2+}$ crystal and a Nal:Tl crystal. Samples of doped $\mathrm{CeBr}_{3}: \mathrm{Ca}^{2+}$ were submerged in oil and/or coupled to the photocathode of a PMT. Then the detector system was irradiated with $662 \mathrm{keV}$ photons $\left({ }^{137} \mathrm{Cs}\right.$ source) to record pulse height spectra using standard Nuclear Instrumentation Model electronics. A similar experiment was then performed with a calibrated Nal:Tl crystal under the same conditions. The Nal:Tl crystal was wrapped with a Teflon tape, coupled to a PMT. Comparison of the $662 \mathrm{keV}$ gamma-ray peak position recorded with $\mathrm{CeBr}_{3}: \mathrm{Ca}^{2+}$ and the Nal:TI scintillators (Figure 6) provided an estimate of the light output of $\mathrm{CeBr}_{3}: \mathrm{Ca}^{2+}$. Compared to $\mathrm{Nal}: \mathrm{Tl}$, the doped $\mathrm{CeBr}_{3}: \mathrm{Ca}^{2+}$ ingots produced a full energy peak 1.7 times higher. Light output is estimated by comparison to the known Nal:Tl to be approximately $\sim 58,000$ photons/MeV. Comparison of the measured spectra is shown in Figure 6. Scintillation spectroscopy has thus far indicated the doped crystals still excel over conventional Nal:Tl, despite the fact that total light yield analysis appears to indicate a loss in light production in the measured samples.

\section{Gamma-Ray Scintillation Spectroscopy}

Gamma-ray spectroscopy was also performed. The $\mathrm{CeBr}_{3}: \mathrm{Ca}^{2+}$ crystals grown at RMD were coupled to a PMT, and the scintillator $\left(2 \% \mathrm{Ca}^{2+}\right)$ was irradiated with a ${ }^{137} \mathrm{Cs}$ source (662 keV photons). Note that in the resulting spectrum, shown in Figure 6, the energy resolution of the $662 \mathrm{keV}$ photopeak was measured to be 3.2\% FWHM at room temperature. Such highenergy resolution has never before been achieved with any of the established inorganic $\mathrm{CeBr}_{3}$ scintillators (even in small sizes) at room temperature. Figure 6 also shows a ${ }^{137} \mathrm{Cs}$ spectrum recorded with Nal:Tl. Energy resolution of Nal:Tl crystal was measured to be $6.7 \%$ (FWHM). Thus, the energy resolution of $\mathrm{CeBr}_{3}: \mathrm{Ca}^{2+}$ is twice as good as that for typical Nal:Tl detectors. Higher light output and proportionality of $\mathrm{CeBr}_{3}: \mathrm{Ca}^{2+}$ (compared to $\mathrm{Nal}: \mathrm{Tl}$ 


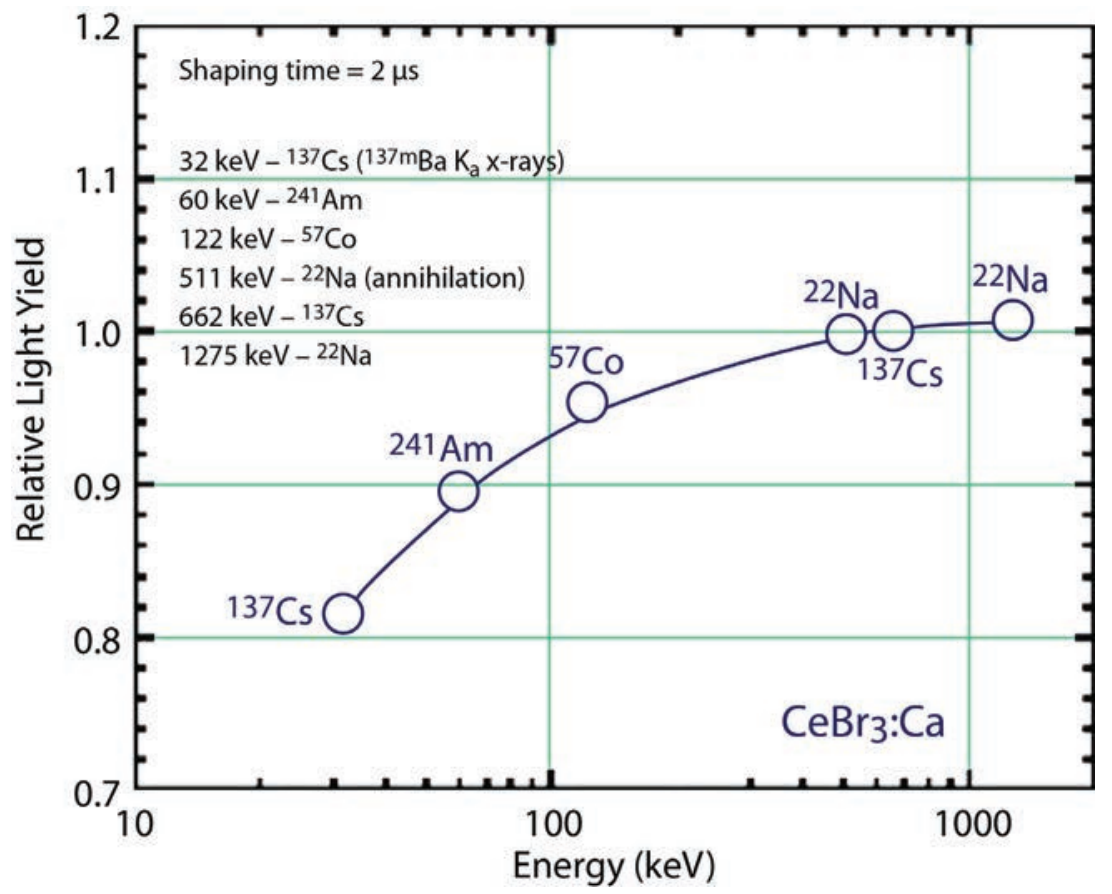

Figure 7. Relative scintillation light yield proportionality data acquired for the $1.9 \% \mathrm{Ca}^{2+}$-doped $\mathrm{CeBr}_{3}: \mathrm{Ca}^{2+}$ scintillator detector material as a function of gammaray energy. Nonproportionality of response for the crystal over $100 \mathrm{keV}$ to $>1 \mathrm{MeV}$ gamma-ray energy range for $\mathrm{CeBr}_{3}: \mathrm{Ca}^{2+}$ is $\sim 5 \%$. Even if the energy range is extended down to $32 \mathrm{keV}$, the nonproportionality is still better than $\sim 20 \%$.

and other inorganic scintillators) are responsible for high-energy resolution obtained with those crystals. It is important to note that energy resolution of $\mathrm{CeBr}_{3}: \mathrm{Ca}^{2+}$ at $662 \mathrm{keV}$ gamma-ray energy is starting to approach that of room temperature semiconductor detectors such as cadmium-telluride and cadmiumzinc-telluride detectors of similar size. The energy resolution is not presently understood. The energy resolution at $662 \mathrm{keV}$ appears to be much better than that typical of $\mathrm{CeBr}_{3}$ (Guss 2009).

\section{Scintillation Light Proportionality}

Proportionality of response for a $\mathrm{CeBr}_{3}: \mathrm{Ca}^{2+}$ sample (with $2 \% \mathrm{Ca}^{2+}$ ) as a function of gamma-ray energy was measured. Relative light yield proportionality was measured for both doped and undoped samples of $\mathrm{CeBr}_{3}$ to ensure that no loss in performance was incurred in aliovalently doping the crystal. Over the energy range from 100 to $1275 \mathrm{keV}$, the nonproportionality of $\mathrm{CeBr}_{3}: \mathrm{Ca}^{2+}$ was measured to be $5 \%$, which is substantially better than that of many established scintillators. For example, over the same energy range, the nonproportionality is about 35\% for LSO:Ce, and about 20\% for Nal:TI and CsI:TI (Guillot-Noël 1999). Proportionality of light yield is one area of performance in which Ce-doped and
Ce-based lanthanide halides excel. Maintaining proportionality is important to producing a strong, high-performance scintillator. The light output and proportionality for $\mathrm{CeBr}_{3}: \mathrm{Ca}^{2+}$, however, appear to be similar to $\mathrm{CeBr}_{3}$-there was a reduced yield at low energy. Relative light yield proportionality measurements suggest that dopants do not significantly affect proportionality at higher energies. A plot of the light yield proportionality for the doped sample is shown in Figure 7.

\section{Conclusion}

Relative light proportionality for $\mathrm{Ca}^{2+}$-doped $\mathrm{CeBr}_{3}$ showed excellent linearity, as expected for undoped $\mathrm{CeBr}_{3}$, implying the dopant does not significantly affect proportionality. $\mathrm{Ca}^{2+}$-doped $\mathrm{CeBr}_{3}$ exhibited little or no change in its fluorescence spectrum, yet produced slightly less light. The dopant may also have introduced an absorption center, which produces an apparent red shift by adding new energy states into the $\mathrm{CeBr}_{3}$ band gap without actually altering the emission spectra. $\mathrm{Ca}^{2+}$ may have produced selfabsorption in the materials as members of the crystal lattice, or perhaps the dopants were present in quantities above their respective solubility limits, creating secondary phase particles within the crystals 
that absorbed scintillation light. Finally, as-grown crystals may have had a large amount of internal strain. Unseen grain boundaries and other discontinuities due to internal strain would necessarily alter light propagation within the crystals, potentially decreasing the observed light yield.

In summary, the crystal structure, band structure, density of states, and optical properties of $\mathrm{CeBr}_{3}$ were calculated by means of DFT within the generalized gradient approximation. Our structural parameters are in good agreement with the experimental results. A 3.2\% energy resolution was measured. Energy resolution at $662 \mathrm{keV}$ appears to be better than $\mathrm{CeBr}_{3}$. This result compares favorably with $\mathrm{LaBr}_{3}: \mathrm{Ce}$, considering that $\mathrm{LaBr}_{3}$ :Ce also has considerable internal selfactivity. A lesson learned is that $1.9 \%$ calcium is too much to incorporate into $\mathrm{CeBr}_{3}$. The light output and proportionality appear to be similar to $\mathrm{CeBr}_{3}$, so the energy resolution is not presently understood. Many dopants have yet to be fully tested, let alone differing concentrations.

A small extension SDRD project was commissioned in FY 2013 to characterize the crystal fragments sent to RSL and to recalculate the crystal properties for a $\mathrm{Ca}^{2+}$ doping of the crystal below the solubility limit using DFT. A draft publication has been prepared and is being reviewed by collaborators. Preliminary results will be presented at the upcoming SPIE conference in August 2013 at San Diego.

\section{Acknowledgments}

The authors thank Michael Lukens and Sanjoy Mukhopadhyay for their contributions.

\section{References}

Blöchl, P. E., "Projector augmented-wave method," Phys. Rev. B, 50, 24 (1994) 17953.

Bollinger, L. M., G. E. Thomas, "Measurement of the time dependence of scintillation intensity by a delayedcoincidence method," Rev. Sci. Instrum. 32 (1961) 1044.
Dorenbos, P., "Light output and energy resolution of $\mathrm{Ce}^{3+}$-doped scintillators," Nucl. Instrum. Methods Phys. Res. A 486 (2002) 208-213.

Dudarev, S. L., G. A. Botton, S. Y. Savrasov, C. J. Humphreys, A. P. Sutton, "Electron-energy-loss spectra and the structural stability of nickel oxide: An LSDA+U study," Phys. Rev. B 57 (1998) 1505-1509.

Doty, F. P., D. S. McGregor, M. Harrison, K. Findley, R. Polichar, P. Yang, "Basic materials studies of lanthanide halide scintillators," MRS Proc. 1038 (2007) 1038-001-03 doi:10.1557/PROC-1038-001-03.

Guss, P. P., M. Reed, D. Yuan, A. Reed, S. Mukhopadhyay, "CeBr 3 as a high-resolution gamma-ray detector," Nucl. Instrum. Methods Phys. Res. A 608, 2 (2009) 297-304.

Guss, P. P., M. Reed, D. Yuan, M. Cutler, C. Contreras, D. Beller, "Comparison of $\mathrm{CeBr}_{3}$ with $\mathrm{LaBr}_{3}: \mathrm{Ce}, \mathrm{LaCl}_{3}: \mathrm{Ce}$, and Nal:TI detectors," Proc. SPIE 7805 (2010) L-1.

Guillot-Noël, O., J. C. van't Spijker, J. T. M. de Haas, P. Dorenbos, C. W. E. van Eijk, K. Krämer, H. U. Güdel, "Scintillation properties of $\mathrm{RbGd}_{2} \mathrm{Br}_{7}$ : $\mathrm{Ce}$ advantages and limitations," IEEE Trans. Nucl. Sci. 46 (1999) 1274-1284.

Harrison, M. J., C. Linnick, B. Montag, S. Brinton, M. McCreary, F. P. Doty, D. S. McGregor, "Radioluminescence and scintillation results of horizontal gradient freeze grown aliovalently-doped $\mathrm{CeBr}_{3}$," IEEE NSS Conf. Rec. (2008) 2850-2855.

Harrison, M. J., C. Linnick, B. Montag, S. Brinton, M. McCreary, F. P. Doty, D. S. McGregor, "Scintillation performance of aliovalently-doped $\mathrm{CeBr}_{3}$," IEEE Trans. Nucl. Sci. 56, 3 (2009) 1661-1665.

Harrison, M. J., P. Ugorowski, C. Linnick, S. Brinton, D. S. McGregor, F. P. Doty, S. Kilpatrick, D. F. Bahr, "Aliovalent doping of $\mathrm{CeBr}_{3}$," Proc. SPIE 7806 (2010) 78060M-78060M-14.

Hofstadter, R., "Alkali halide scintillation counters," Phys. Rev. 74 (1948) 100-101.

Hofstadter, R., "Properties of scintillation materials," Nucleonics 6, 5 (1950) 70-72. 
Joubert, L., G. Picard, J. J. Legendre, "Structural and thermochemical ab initio studies of lanthanide trihalide molecules with pseudopotentials," Inorg. Chem. 37 (1998) 1984-1991.

Li, C., B. Wang, R. Wang, H. Wang, "First-principles studies on the electronic and optical properties of $\mathrm{CeCl}_{3}$ and $\mathrm{CeBr}_{3}$," Solid State Communications 144 (2007) 220-224.

Liechtenstein, A. I., V. I. Anisimov, J. Zaanen, "Densityfunctional theory and strong interactions: Orbital ordering in Mott-Hubbard insulators," Phys. Rev. B 52 (1995) R5467-R5470.

Olson, C. G., M. Piacentini, D. W. Lynch, "Optical properties of single crystals of some rare-earth trifluorides, 5-34 eV," Phys. Rev. B 18 (1978) 5740-5749.

Park, K. H., S. J. Oh, "Electron-spectroscopy study of rare-earth trihalides," Phys. Rev. B 48 (1993) 14833.

Pletka, B. J., T. E. Mitchell, A. H. Heuer, "Solid solution hardening of sapphire $\left(\alpha-\mathrm{Al}_{2} \mathrm{O}_{3}\right)$," Physica Status Solidi 39 (1977) 301-311.

Rodnyi, P., Physical Processes in Inorganic Scintillators, CRC Press, New York, 1997 (Chapter 1).

Sato, S., "Optical absorption and x-ray photoemission spectra of lanthanum and cerium halides," J. Phys. Soc. Japan 41 (1976) 913-920.

Shah, K. S., J. Glodo, M. Klugerman, W. M. Higgins, T. Gupta, P. Wong, "High energy resolution scintillation spectrometers," IEEE Trans. Nucl. Sci. 51, 5 (2004) 2395-2399.

Shah, K. S., J. Glodo, W. Higgins, E. V. D. van Loef, W. W. Moses, S. E. Derenzo, M. J. Weber, " $\mathrm{CeBr}_{3}$ scintillators for gamma-ray spectroscopy," IEEE Trans. Nucl. Sci. 52, 6 (2005) 3157-3159.

Shi, C., G. Zhang, Y. Wei, Z. Han, J. Shi, G. Hu, M. Kirm, G. Zimmerer, "The dynamics properties on luminescence of $\mathrm{CeF}_{3}$ crystals," Surface Review and Letters 9, 1 (2002) 371-374.
Skorodumova, N. V., R. Ahuja, S. I. Simak, A. Abrikosov, B. Johansson, B. I. Lundqvist, "Electronic, bonding, and optical properties of $\mathrm{CeO}_{2}$ and $\mathrm{Ce}_{2} \mathrm{O}_{3}$ from first principles," Phys. Rev. B 64 (2001) 115108, DOI: 10.1103/ PhysRevB.64.115108.

Tsuchiya, T., T. Taketsugu, H. Nakano, K. Hirao, "Theoretical study of electronic and geometric structures of a series of lanthanide trihalides $\operatorname{LnX}_{3}(\operatorname{Ln}=\mathrm{La}-\mathrm{Lu} ; \mathrm{X}=\mathrm{Cl}, \mathrm{F})$," J. Mol. Struct. (Theochem) 461-462 (1999) 203-222.

van Loef, E. V. D., P. Dorenbos, C. W. E. van Eijk, K. Krämer, H. U. Güdel, "High-energy-resolution scintillator: $\mathrm{Ce}^{3+}$ activated $\mathrm{LaCl}_{3}$," Appl. Phys. Lett. 77 (2000) 1467-1468.

van Loef, E. V. D., P. Dorenbos, C. W. E. van Eijk, K. Krämer, H. U. Güdel, "High-energy-resolution scintillator: $\mathrm{Ce}^{3+}$ activated LaBr,", Appl. Phys. Lett. 79 (2001) 1573.

VASP, http://cmp.univie.ac.at/research/vasp/, "Vienna Ab-initio Simulation Package, What is VASP?" accessed April 30, 2012.

Vetere, V., C. Adamo, P. Maldivi, "Performance of the 'parameter free' PBE0 functional for the modeling of molecular properties of heavy metals," Chem. Phys. Lett. 325 (2000) 99-105.

Wesley, R. D., C. W. DeKock, "Geometry and infrared spectra of matrix-isolated rare-earth halides. I. $\mathrm{LaF}_{3}, \mathrm{CeF}_{3}$, $\mathrm{PrF}_{3}, \mathrm{NdF}_{3}, \mathrm{SmF}_{3}$, and $\mathrm{EuF}_{3}, "$ J. Chem. Phys. 55 (1971) 3866.

Zachariasen, W. H., "Crystal chemical studies of the 5f-series of elements. I. New structure types," Acta. Crystallogr. 1 (1948) 265-268.

Zachariasen, W. H., "The UCl 3 type of crystal structure," J. Chem. Phys. 16 (1948) 254. 
This page left blank intentionally 


\section{DYNAMIC CONDUCTIVITY OF SHOCKED MATERIALS}

STL-18-12 | CONTINUED IN FY 2013 | YEAR 1 OF 2

Brandon LaLone, ${ }^{1, a}$ Dale Turley, ${ }^{a}$ Gerald Stevens, $^{a}$ Gene A. Capelle, ${ }^{a}$ and Mike Grover ${ }^{a}$

Our primary objective was to develop a method to determine the thermal conductivity of materials (metals, glue, and transparent windows) that are undergoing shock-wave compression. This project was conceived to help support an ongoing effort to reduce the uncertainty in temperature measurements of shock-compressed materials. Because the interior of metals cannot be measured with optical pyrometry, temperature is typically measured at the interface between the metal and a transparent window. The conductivity is an important parameter for relating the measurement of a metal/window interface temperature to that of the bulk metal temperature, which can differ by $10 \%$ or more due to conduction of heat into the window. A method for measuring the conductivity was selected that utilizes a short laser pulse to rapidly heat the metal-window interface and time-resolved pyrometry to observe the conductivity-dependent temperature decay. The thermal conductivity is determined by fitting the measured temperature-time profiles with a 1-D thermal conduction computer program that was developed specifically for this effort. It was found that this method could not distinguish between changes in thermal conductivity and changes in volumetric heat capacity, so it is a better measure of thermal effusivity than of conductivity alone. The laser heat method was tested under shock-wave loading on tin. Preliminary results indicate a small increase in the thermal effusivity for tin at $\sim 20 \mathrm{GPa}$ shock pressure.

${ }^{1}$ lalonebm@nv.doe.gov, 805-681-2046

a Special Technologies Laboratory

\section{Background}

The temperature of an object is one of the most familiar concepts in everyday life, yet is one of the most difficult variables to measure in shock-wave experiments. This is due, in large part, to a combination of the short time scales of shock-wave measurements and the slow diffusion rates of heat flow. Despite the difficulties in measurement, accurate experimental temperatures of the shock-compressed state are critical to the development and confirmation of complete equations of state (EOS) of materials. Due to its importance, there has been an ongoing effort to improve the accuracy of measured temperatures during shock-wave experiments. Optical pyrometry has been perhaps the most successful tool in shock-wave temperature determination. Advances in emissivity measurements may enable shock-wave pyrometry measurements of temperatures with unprecedented accuracies. However, for opaque materials such as metals, only the thermal radiation emanating from the surface can be collected, hence only the temperature of a surface or interface can be determined using pyrometry. Because a free surface cannot support stress in a shock-wave experiment, a transparent window, such as lithium fluoride (LiF) or sapphire, is often attached to the surface. The window helps to maintain a state of elevated stress and temperature and to permit the transmission of thermal radiation (typically visible and infrared light) emanating from the surface after the shock wave exits the metal sample. For several reasons, the temperature measured at the sample-window interface will differ from the temperature in the interior 
of the sample. The interior temperature is the most useful for EOS studies, and thus developing methods to estimate this value from surface temperature measurements is highly desired.

Grover and Urtiew (Grover 1974) were the first to discuss the multiple effects that the presence of the window has on sample temperature measurements during shock-compression experiments. The effect relevant to this work is related to the thermal transport properties of the metal and window. Following shock interaction from an ideal interface (free of defects/voids), the sample will be at a temperature $T_{S}$ and the window at $T_{A}$. The measured interface temperature, $T$, will take on the value,

$$
T_{l}=T_{S}-\frac{T_{S}-T_{A}}{1+\left(\frac{K_{S} \rho_{S} C_{S}}{\kappa_{A} \rho_{A} C_{A}}\right)^{1 / 2}},
$$

where $k$ is the thermal conductivity, $\rho$ is the density, and $C$ is the specific heat at constant pressure; the subscripts $S$ and $A$ denote sample and window respectively. The quantity $(\kappa \rho C)^{1 / 2}$ is termed the thermal "effusivity." It is clear from Equation 1 that to obtain the sample temperature from a measurement of the sample-window interface, the sample and window thermal effusivities must be known at the elevated temperatures and pressures of the shock-wave experiment. For typical metal samples and LiF windows, it is estimated that the difference between the sample interior and interface temperature is up to $10 \%$ of the sample temperature, but because the effusivities of most materials have not been measured under dynamic loading, the difference is generally unknown.

The group led by T. J. Ahrens (Gallagher 1997, Ahrens 1998, Holland 1998) investigated the thermal transport properties of shock-loaded thin films of iron or steel deposited onto LiF and sapphire windows by measuring the decay time of the temperature of the film with pyrometry. Using the measurements and assumptions about the thermal properties of the films, thermal diffusivity ratios between the windows and films were estimated. They reported dramatic decreases in the thermal diffusivities of the windows at high shock pressures. Aside from the drawback of relying on modeled thermal properties of the metal films, there were other limitations to their method. Because of porosity, the films were shock-heated to far greater temperatures than single-shock loading of bulk samples, and therefore the temperaturepressure state in which the measurements were made was vastly different from the temperaturepressure states of interest. Also, the method was restricted to materials that could be deposited onto windows as thin films, and it is not clear how similar the shock properties of the films are to bulk samples of the same material. Finally, it is also unclear if the effect of extra heat deposited from release and reshocking of the window across a vacuum gap (Grover 1974) was considered.

More recently, Boness (2012) reported preliminary measurements on the thermal relaxation of cesium iodide that had been reshocked across a thin vacuum gap against a LiF window. Although high-quality measurements of temperature decay were obtained, the data were not yet analyzed to extract thermal transport properties, and the author anticipated that only partial or relative quantities could be obtained. We suspect that this method could be successful but will rely heavily on material EOS to simulate the initial temperature profile following shock-wave interactions at the interface, prior to the temperature decay.

The purpose of this project was to develop a method to measure thermal conductivity, and other thermal transport properties, of shocked materials such that the sample interior temperature could be determined from the measurement of an interface temperature in accordance with Equation 1. We sought out a method that was applicable to any material and probed the temperature-pressure states that are relevant to shock-wave experiments. 


\section{Project}

\section{Pulsed-Laser Heating}

Motivated by conversations with Neil Holmes (2012), we decided to pursue a pulsed-laser heating technique to measure thermal conductivity. The technique is based on a method by Beck (2007) for measuring thermal diffusivity in static high-pressure experiments, and is similar to other flash-heating methods for thermal transport measurements (Zammit 2011). A schematic diagram of the method is shown in Figure 1. A thin emissive layer of chromium $(\mathrm{Cr})$ is sandwiched between the sample of interest and a transparent window. A dynamic load (high explosive or projectile impact) sends a shock wave into the sample, which transmits through the $\mathrm{Cr}$ layer into the window, heating the sample, the $\mathrm{Cr}$ layer, and the window. After the shock wave exits the sample, but before it exits the rear surface of the window, a short laser pulse rapidly heats the $\mathrm{Cr}$ layer. Through 1-D thermal heat conduction, the $\mathrm{Cr}$ layer heats and cools at a rate determined by the thermal transport properties of the surrounding sample and window. The temperature of the $\mathrm{Cr}$ layer is monitored throughout the process with time-resolved pyrometry. To determine the thermal transport properties, the measured time-dependent temperature changes are fit to a model of the 1-D thermal conduction process using a computer code (thermalconduct insentropic2.m) that was written for this effort. The laser heat method has advantages over previous

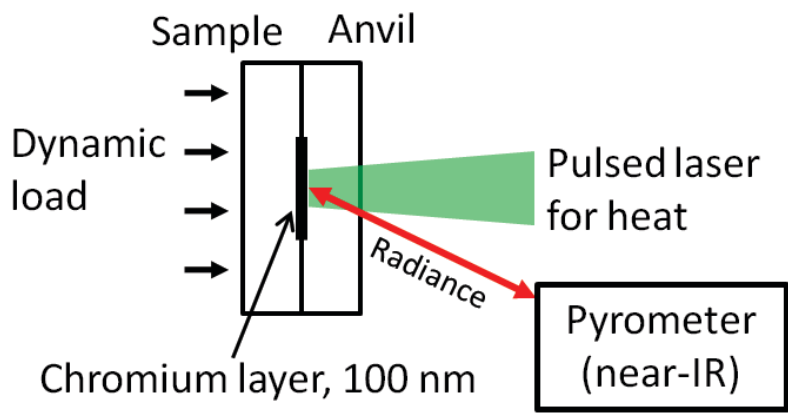

Figure 1. Diagram of the laser heat method used for determining thermal transport properties of shockcompressed materials techniques because the amount of heat energy that enters the system can be accurately known from a measurement of the laser beam energy, and thermal properties can be measured at conditions very near the single-shock state, within $\sim 200 \mathrm{~K}$.

Example temperature-time calculations of pulsedlaser heating of the $\mathrm{Cr}$ layer are shown in Figure 2. The calculations are for a typical sample and window materials shock-heated to $1000 \mathrm{~K}$ and then heated with a $15 \mathrm{~ns}, 100 \mathrm{~mJ} / \mathrm{cm}^{2}$ laser pulse to temperatures from 1200 to $1400 \mathrm{~K}$. The temperature rises quickly then falls over the next several hundred nanoseconds. Calculations were performed with differing values of conductivity, heat capacity, and laser fluence (energy/area) to examine their significance on the temperature-time curves. The calculations demonstrate that the profiles are sensitive not only to thermal conductivity changes, but also to changes in heat capacity and laser fluence. Furthermore, the calculations show that it is difficult to distinguish between a change in thermal conductivity and the same relative change in heat capacity. Therefore, we decided to explore this technique as a method of determining thermal effusivity as it is related to the product of conductivity and heat capacity.

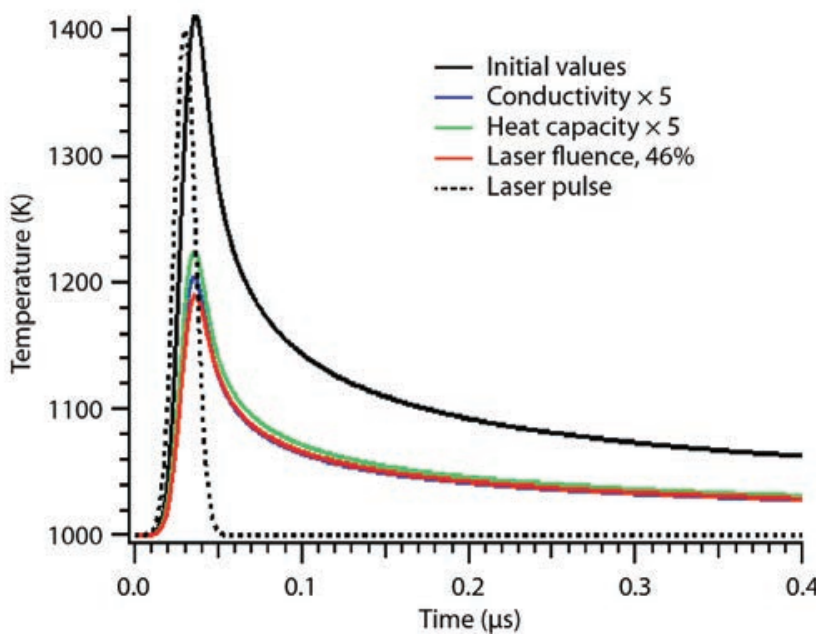

Figure 2. Calculated temperature-time profiles of the $\mathrm{Cr}$ layer surrounded by a typical sample using the laser heat method. Calculations are shown for five-fold increases in conductivity and heat capacity, and reduced laser fluence relative to their starting values. 


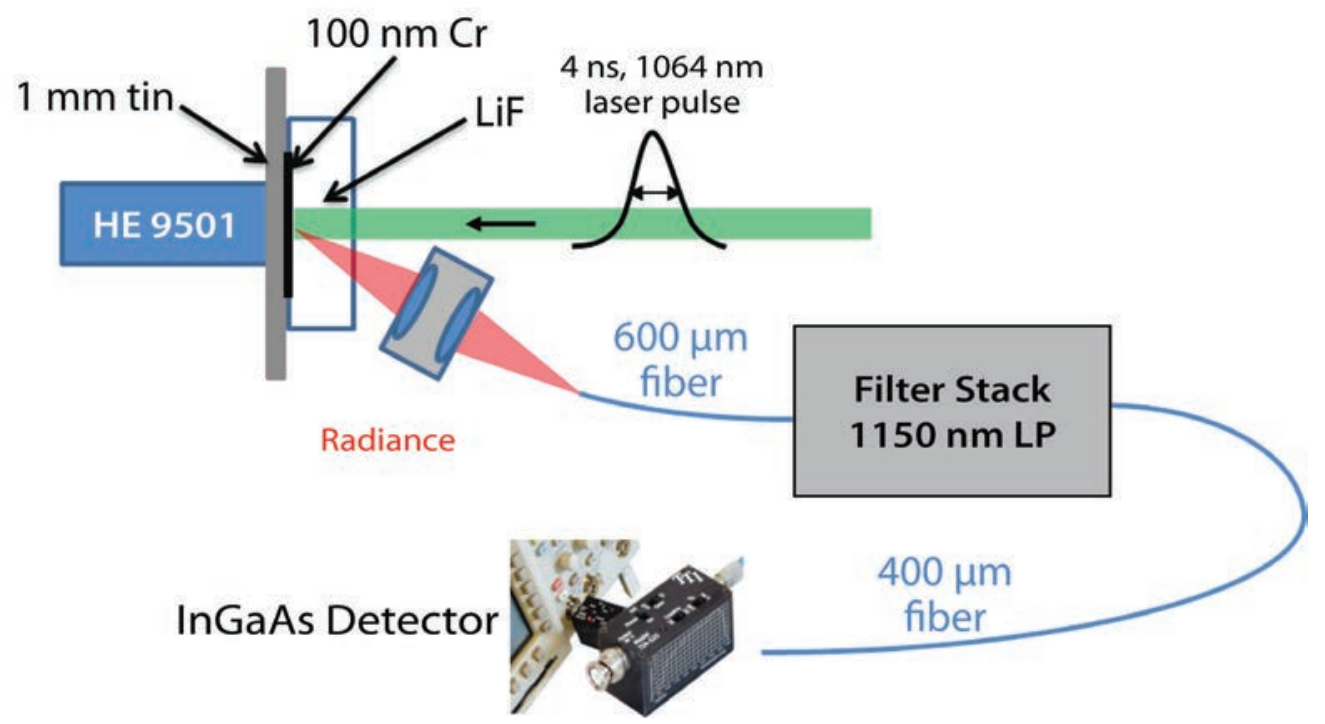

Figure 3. Schematic diagram of the shockwave experiments with laser heating

\section{Ambient Pressure Benchtop Measurements}

Initial testing was performed at ambient pressures on a preheated nickel sample. This testing enabled us to select an appropriate detector and set of optical filters that would comprise the single-channel pyrometer used in the shock-wave experiments. It also allowed us to test various laser configurations and schemes for relaying and attenuating the laser beam. Preheating the nickel to $\sim 1050 \mathrm{~K}$ proved to be invaluable to this intial testing and was done using a hightemperature electric heater. Without preheating, the pyrometer could not detect any thermal radiation, as it is insensitive to temperatures below $\sim 800 \mathrm{~K}$. Therefore, when the laser was pulsed at ambient temperature, any detected signal was a result of laser light reaching the detector, and not from thermal radiation. Contamination from laser light was highly undesirable, and careful selection of optical filters was required to eliminate this nonthermal source of signal. Feasibility of the method was demonstrated by recording a clearly defined pulsed-heating signal when the sample was preheated, whereas no signal was detected when the sample was at room temperature. The final configuration of the benchtop measurements was used in a set of explosively driven shock-wave experiements.

\section{Measurements on Shock-Wave Compressed Tin: Experimental Details}

A schematic diagram of the shock-wave experiments is shown in Figure 3. High explosive, consisting of $\sim 3$ grams of PBX-9501, was used to drive a shock wave into a $1 \mathrm{~mm}$ thick tin sample that was previously coated with $100 \mathrm{~nm}$ of $\mathrm{Cr}$ and glued to a $10 \mathrm{~mm}$ thick LiF window. The Lebow company in Goleta, CA applied the evaporated coating of $\mathrm{Cr}$ onto the tin samples. The thickness of the applied coating was verified to be within several nanometers of the nominal value using a white light interferometer (profilometer). Although porosity is of some concern, we presently do not have any means of determining the porosity of the coatings. The tin sample and LiF window were $\sim 40 \mathrm{~mm}$ in diameter, and the explosive, hence the shock-driven region, was $12.5 \mathrm{~mm}$ in diameter. After the shock wave reached the tin-LiF interface, a pulse from a $1064 \mathrm{~nm}$ laser impinged onto the center of, and normal to, the sample. Thermal radiance from the interface was directed via an imaging probe into a $600 \mu \mathrm{m}$ core optical fiber. The fiber relayed the radiance to a stack of "longpass" optical filters, which filtered out any light with a wavelength shorter than $1150 \mathrm{~nm}$, including the laser light, and then it was coupled into a $400 \mu \mathrm{m}$ core fiber. The intensity of the filtered thermal radiance 
exiting the $400 \mu \mathrm{m}$ fiber was recorded with an amplified indium gallium arsenide (InGaAs) photoreceiver. The detector is insensitive to light with wavelengths longer than $1700 \mathrm{~nm}$; therefore, the filters and detector comprised a single-channel pyrometer operating in the wavelength band from $1150-1700 \mathrm{~nm}$. A photonic Doppler velocimetry (PDV) probe was utilized in every experiment to obtain the tin/ $\mathrm{Cr} / \mathrm{LiF}$ interface velocity.

The pulsed-laser source used for heating in these measurements was the BRILLIANT laser from Quantel. It is a flashlamp-pumped and Q-switched Nd:YAG laser operating at a wavelength of $1064 \mathrm{~nm}$. The output was a $4 \mathrm{~ns}$ long pulse with $\sim 200 \mathrm{~mJ}$ of energy and a $\sim 6 \mathrm{~mm}$ beam diameter. The laser beam was passed through relay optics, including a waveplate and polarizer, to control the energy and direct it onto the target. The beam energy at the target was varied from 9 to $33 \mathrm{~mJ} /$ pulse (300 to $1000 \mathrm{~J} / \mathrm{m}^{2} /$ pulse) depending on the experiment. The beam diameter at the target was measured using photographic burn paper, which was combined with the measurement of the laser energy to obtain a rough estimate of the laser fluence. We assumed a large uncertainty of $50 \%$ in our measurements of fluence with this method. The amount of laser fluence used in these measurements is roughly a factor of ten below the threshold of plasma formation and damage for typical metal surfaces (Benavides 2011), and we did not observe any laser-induced damage to the $\mathrm{Cr}$-coated tin samples during preshot testing with laser pulses.

The optical filter stack consisted of three different filters: an $1100 \mathrm{~nm}$ long-pass filter, an $1150 \mathrm{~nm}$ longpass filter, and a $1064 \mathrm{~nm}$ laser-line notch filter. With all three filters in place, the $1064 \mathrm{~nm}$ light from the laser was adequately rejected from the pyrometer measurement. The transmission spectra of the filters were measured with a double-beam spectrometer and later utilized in the conversion of the detector signals into temperatures.

The InGaAs detector used for these measurements was the TTI-TIA950 made by Terahertz Technologies, Inc. The detector was used in the high-gain,
$12,000 \mathrm{~V} / \mathrm{W}$ setting. The vendor-published detector response curves were used in conjunction with the filter stack transmission measurements for converting the measured signals to temperatures. Calibration of the entire pyrometer, consisting of optics, filters, and the detector, was performed using a blackbody source and an optical chopper.

Prior to the laser heat experiments, a dynamic reflectivity (flash-lamp integrating sphere) shot was performed to obtain the emissivity of the $\mathrm{Cr}$ layer in the present configuration (Shot 120531_1). This was done in order to accurately convert the measured radiance values to temperatures using Planck's law. The emissivity of the layer under shock compression was determined to be 0.54 for wavelengths detected by the pyrometer, and estimated to be 0.58 at the laser wavelength of $1064 \mathrm{~nm}$. We have assumed here that the dynamic emissivity of the $\mathrm{Cr}$ coating measured in this experiment is applicable to all experiments. The emissivity of several $\mathrm{Cr}$-coated samples was measured under ambient conditions with excellent reproducibility, so it is likely that the dynamic emissivity is also reproducible. However, since the emissivity of the $\mathrm{Cr}$ coating under shock compression was only measured in one experiment, this assumption has not been verified. Future experiments are needed to verify that the measured emissivity of the $\mathrm{Cr}$ coatings under shock compression is reproducible.

\section{Measurements on Shock-Wave Compressed Tin: Experimental Results}

A total of five experiments were performed at the Special Technologies Laboratory Boom Box explosive testing facility. Three experiments were performed using the configuration in Figure 3, a fourth experiment used a smaller-diameter pickup fiber, and a fifth experiment was performed without the emissive $\mathrm{Cr}$ layer. The fourth and fifth experiments had very low signal levels with poor signal-to-noise ratios and are not shown here. The first three are discussed below. 


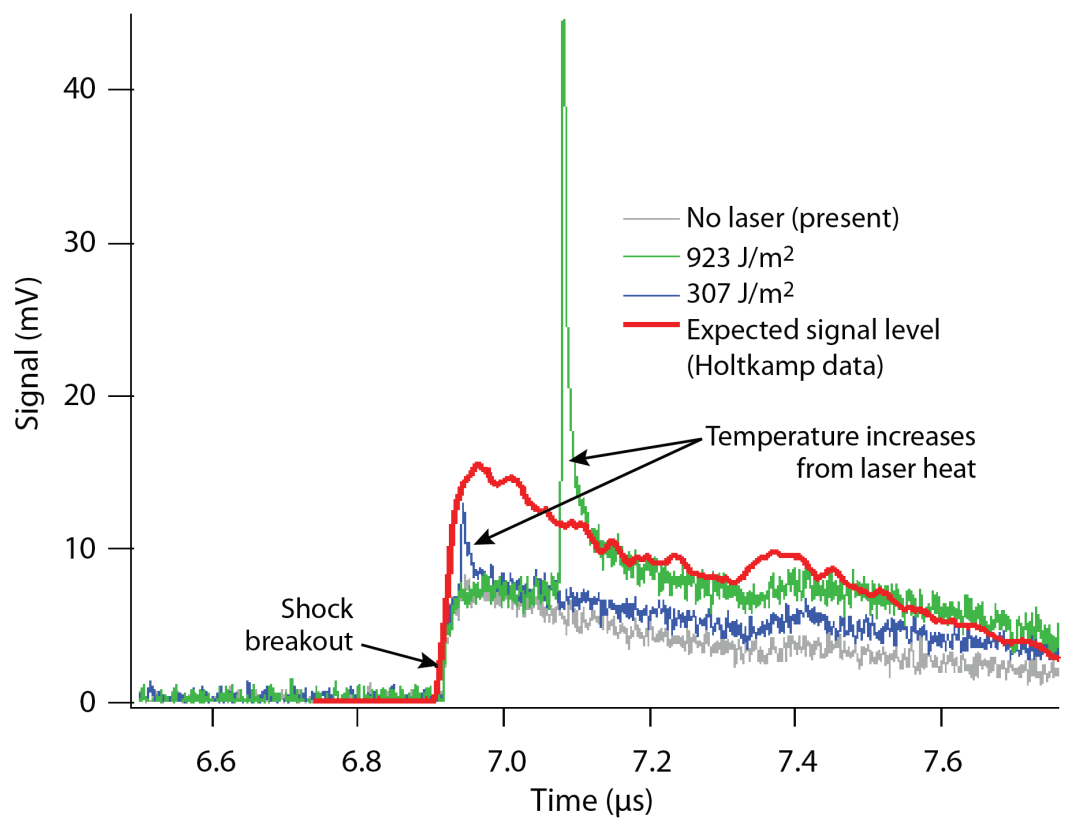

Figure 4. Raw signal voltages from the three experiments performed using the configuration shown in Figure 3. Note: The expected signal from a previous experiment performed by Holtkamp (2010) is shown for comparison.
The raw voltage signals from the three experiments, performed using the configuration in Figure 3 , are shown in Figure 4 . The experiments had laser pulse fluencies of 0,923 , and $307 \mathrm{~J} / \mathrm{m}^{2}$. Also shown are the expected signal levels (for no laser heating) based on a prior pyrometry experiment utilizing the same target configuration (Holtkamp 2010). The three experiments had several qualitative features that were expected: (1) the finite rise time of the signal at shock breakout is due to the shock-wave heated tin raising the temperature of the initially cooler $\mathrm{Cr}$ layer; (2) the overall signal slowly decreases with time after breakout, mimicking the shape of the pressure-time history from the Taylor wave release; and (3) the spikes in signal resulting from rapidly heating the interface with the pulsed laser.

Because the $\mathrm{Cr}$ coatings were initially cooler than the tin at shock breakout, it is unlikely that they have any significant porosity. We estimate that if the $\mathrm{Cr}$ coatings had $10 \%$ porosity ( $90 \%$ full density), additional heat energy comparable to the $300 \mathrm{~J} / \mathrm{m}^{2}$ laser pulse would be deposited into the coating at shock wave breakout. The signal from this deposition of heat would be very obvious and is clearly not present. Therefore, the coatings are likely very near to being fully dense.
A PDV spectrogram from one of the experiments is shown in Figure 5 ; the velocity of the $\mathrm{tin} / \mathrm{Cr} / \mathrm{glue} /$ LiF interface indicates a breakout stress of $24 \mathrm{GPa}$. In addition to the interface velocity, the pulsed-laser heating signal is also clearly seen in the spectrogram because the PDV detectors are sensitive to the $1064 \mathrm{~nm}$ laser light, which allows for accurate cross timing between the radiance and velocity data. This record also shows that the velocity of the interface is not affected by the laser pulse (i.e., there is insufficient laser energy to create a detectable pressure wave).

Although the voltage signals from thermal radiance measurements were qualitatively as expected, there were two problems. First, the signal amplitudes were more than a factor of 2 lower than expected based on a comparison with the Holtkamp (2010) data. Second, after the spike in signal from laser heating in the $923 \mathrm{~J} / \mathrm{m}^{2}$ experiment, the signal does not converge with the other measurements at late times, even though it was expected to do so after several hundred nanoseconds.

Both of these issues were determined to result from poor optical coupling to the TTI detector. We selected a $400 \mu \mathrm{m}$ optical fiber to collect as much of the 


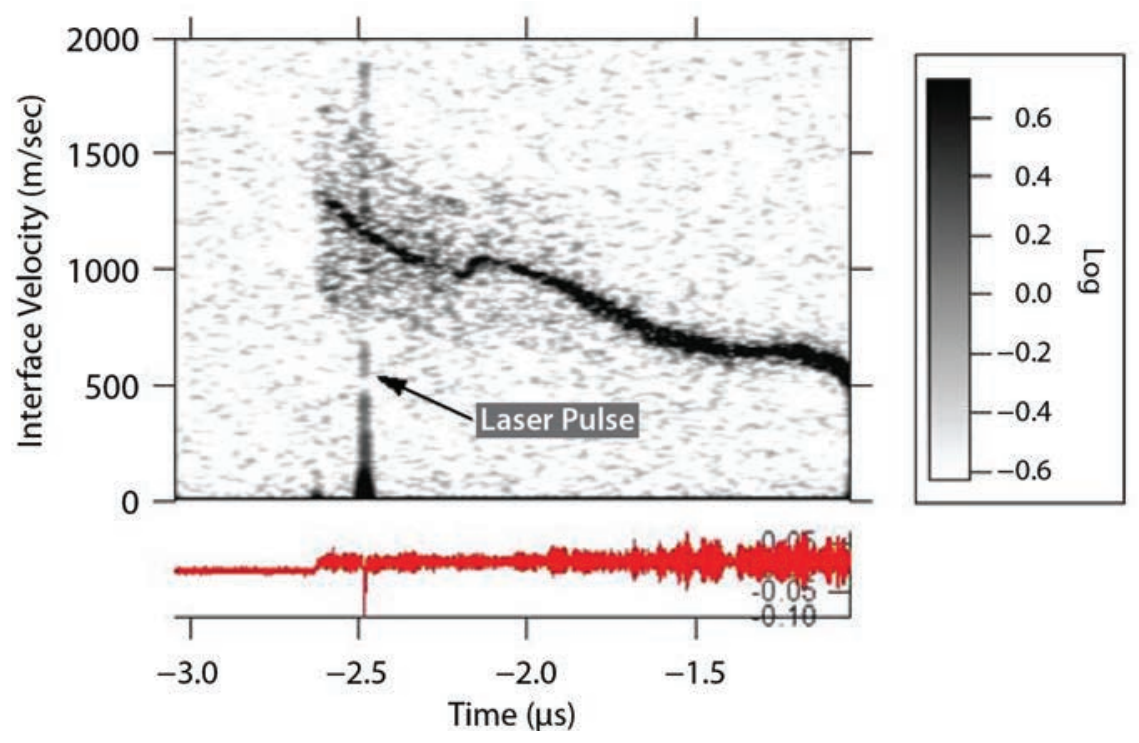

Figure 5. PDV spectrogram showing the velocity of the tin/ $\mathrm{Cr} / \mathrm{glue} / \mathrm{LiF}$ interface and also detecting the pulsed laser used for heating

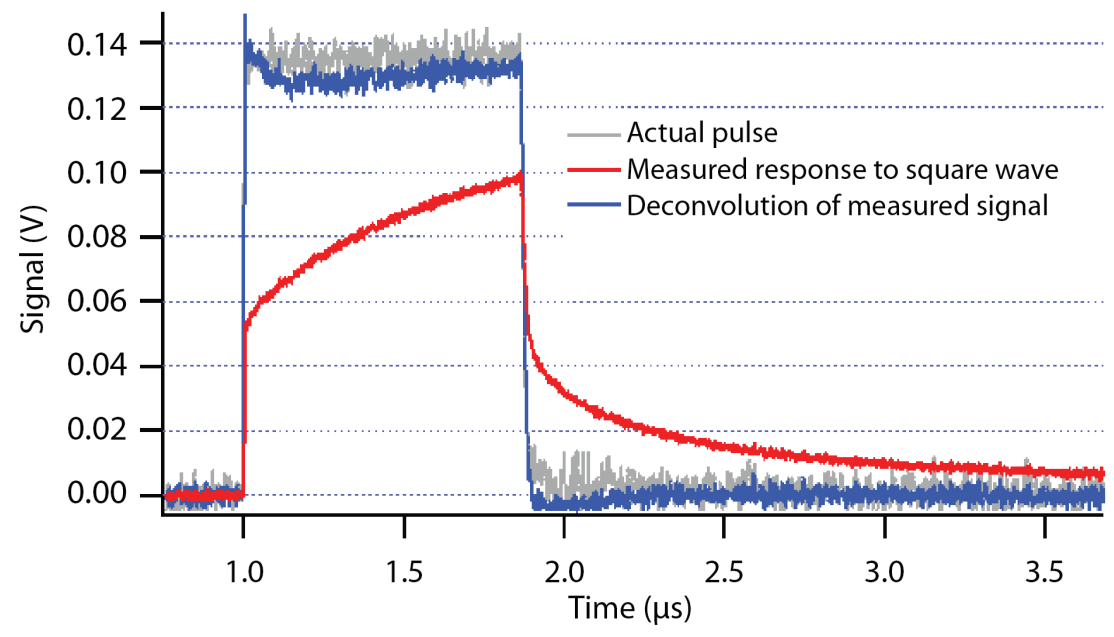

Figure 6. The voltage output of the $\ln G a A s$ detector (red) in response to a $1 \mu$ square-wave optical input (gray). Deconvolution of the output (blue) returns a waveform that is a better representation of the square-wave input.

sample radiance as possible, but the large-diameter fiber overfilled the detector sensor area, complicating its time response.

The detector response was measured by inputting an optical pulse with a square temporal profile of 1 us duration (Figure 6). The voltage output is quite distorted and poorly reproduces the square-wave input. The detector transfer function determined from the measurement in Figure 6 shows that the detector output appears as the sum of a relatively fast ( $0.3 \mathrm{GHz}[300 \mathrm{MHz}]$ ) detector and a relatively slow ( $2 \times 10^{-4} \mathrm{GHz}$ [200 kHz]) detector. This detector transfer function was used to deconvolve the signal output and return a better representation of the square-wave input, also shown in Figure 6.

The measured detector transfer function was used to deconvolve the raw signals from the three laser heat experiments. The deconvolved signals are shown in Figure 7 along with the expected signal from the Holtkamp (2010) data. Unlike the raw data, the deconvolved signals at breakout are now consistent with the estimate from the Holtkamp data, and they appear to more or less converge at late times. The deconvolution seems to have removed the problems 


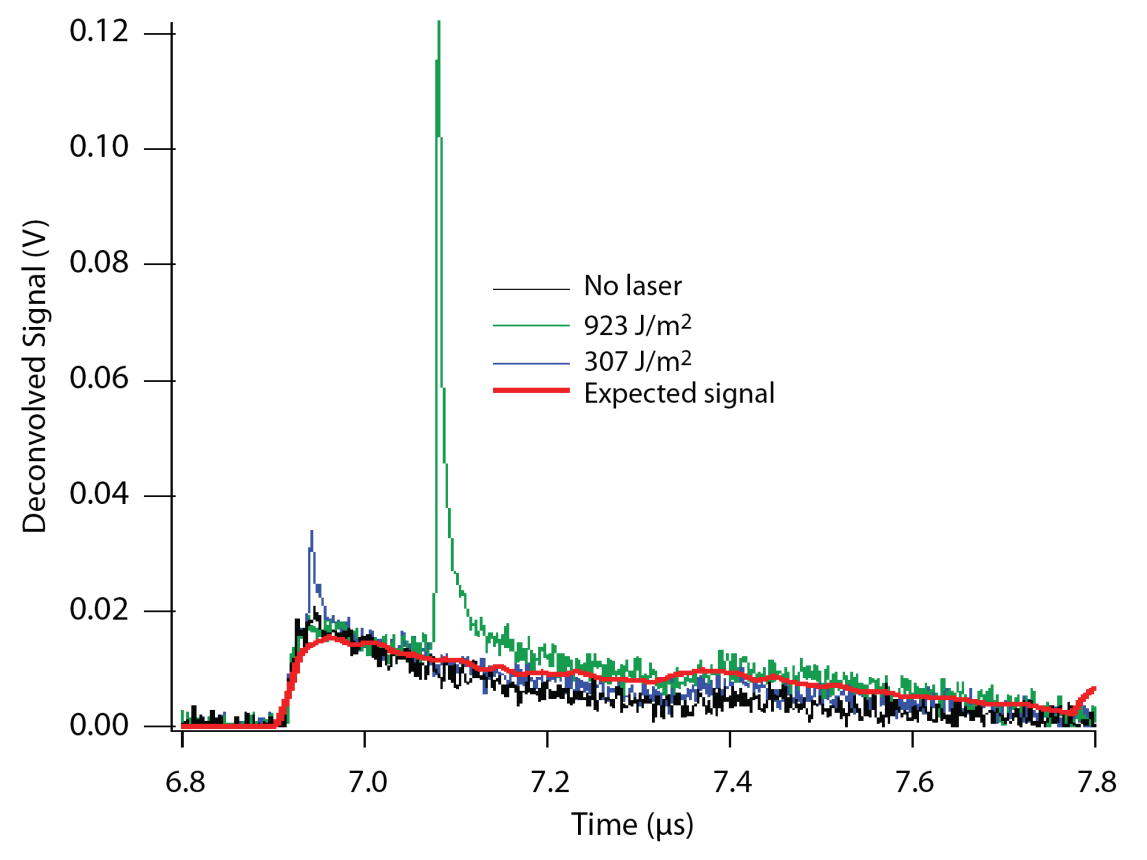

Figure 7. The deconvolved voltage signals from the shockwave experiments with pulsed-laser heating, shown with the expected signal from a previous experiment by Holtkamp (2010)

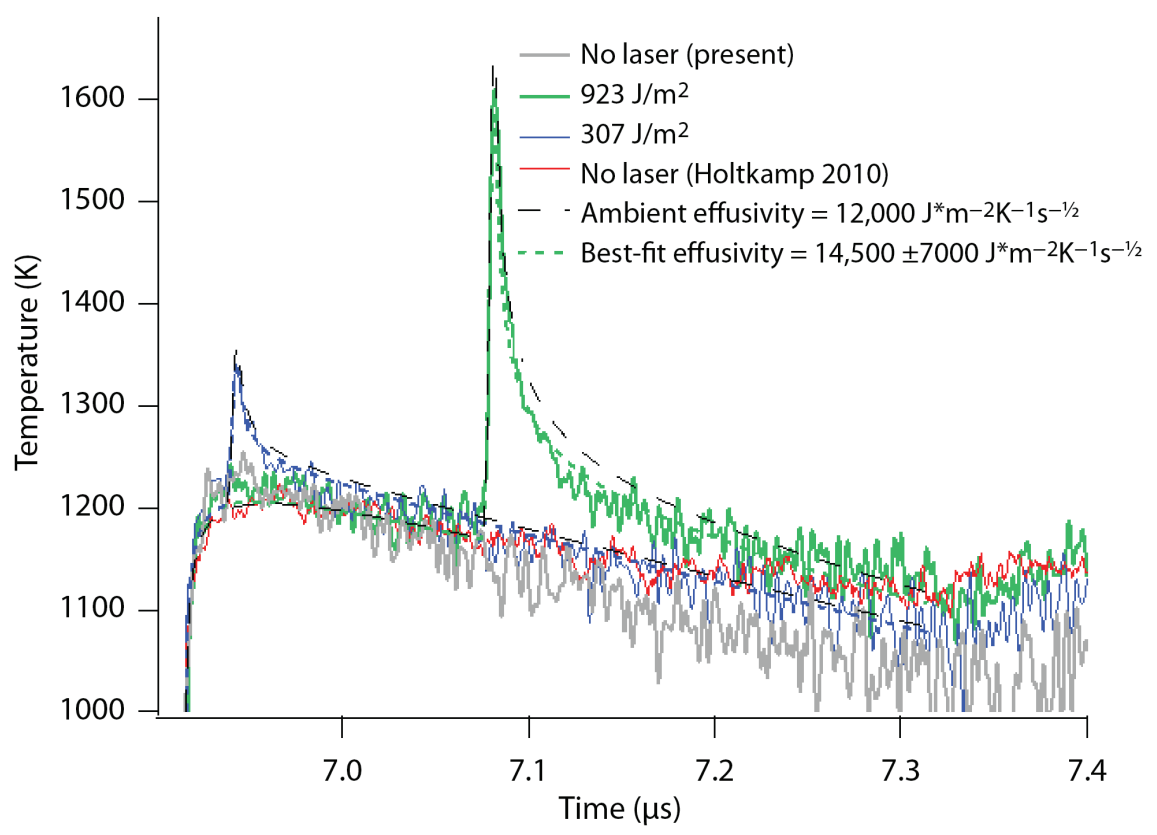

Figure 8. Temperature histories from the shockwave and laser heat experiments on tin (solid lines). The black, long-dashed curves were calculated using the ambient effusivity value for tin of $12,000 \mathrm{~J}^{*} \mathrm{~m}^{-2} \mathrm{~K}^{-1} \mathrm{~s}^{-1 / 2}$. The short-dashed curves are calculated temperature-time profiles for a best fit for the tin effusivity of $14,500 \mathrm{~J} * \mathrm{~m}^{-2} \mathrm{~K}^{-1} \mathrm{~s}^{-1 / 2}$, which is slightly higher than the ambient value but within the experimental uncertainty.

recognized in the raw signals. During the Taylor wave pressure release, there does now appear to be a more rapid decay of signal in the current measurements than was expected. This could be a detector issue, or perhaps the deconvolution of the signals introduced nonphysical artifacts into the data.

The deconvolved signals were converted to temperatures using Planck's law, the blackbody calibration, the spectral response of the filters and detector, and the previously measured dynamic emissivity of the $\mathrm{Cr}$ layer. The experimental temperatures are shown in Figure 8. The shock-wave breakout temperatures from the present experiments and the previous data are all consistent within $\sim 25 \mathrm{~K}$.

The "best-fit" calculations in Figure 8 were obtained by changing the effusivity of tin in the thermal 
conduction code until a reasonable fit (by eye) to both of the laser heat spikes was obtained with the same effusivity value. A more rigorous fitting procedure has not yet been written into the computer code. The calculations shown used a value of $14,500 \mathrm{~J}^{*} \mathrm{~m}^{-2} \mathrm{~K}^{-1} \mathrm{~s}^{-1 / 2}$ for the effusivity of shocked tin, which is similar to the ambient value for tin of $11,800 \mathrm{~J}^{*} \mathrm{~m}^{-2} \mathrm{~K}^{-1} \mathrm{~s}^{-1 / 2}\left(\mathrm{k}=67 \mathrm{~W}^{*} \mathrm{~m}^{-1} \mathrm{~K}^{-1}\right.$, $\left.C=230 \mathrm{~J}^{*} \mathrm{~kg}^{-1} * \mathrm{~K}^{-1}, \rho_{\text {high pressure }}=9100 \mathrm{~kg} / \mathrm{m}^{3}\right)$; ambient value calculations are also shown in Figure 8 . The uncertainty in the best-fit value is estimated to be $7000 \mathrm{~J}^{*} \mathrm{~m}^{-2} \mathrm{~K}^{-1} \mathrm{~s}^{-1 / 2}$; this relatively large uncertainty is mostly due to an estimated uncertainty in laser fluence of $50 \%$. If the laser fluence were accurately measured, we estimate that the uncertainty would reduce to less than $20 \%$. As shown in Figure 8, calculated curves with the best-fit value for the effusivity of tin are only a slightly better match to the measured data than calculations using the ambient effusivity value, indicating that the thermal transport properties of tin do not change significantly under shock-wave stresses near $24 \mathrm{GPa}$.

The temperature-time calculations shown in Figure 8 were not sensitive to changes in the thermal transport properties of the glue or the LiF window. This is because the glue is a good thermal insulator relative to the tin layer and would have to undergo substantial property changes to significantly affect the interface temperature-time profiles.

\section{Conclusion}

We have developed and successfully demonstrated a technique for determining the effusivity of materials subjected to shock-wave compression; effusivity is the key thermal transport parameter used for relating the temperature of a surface to the temperature of the interior of a sample. The technique utilizes a short-pulsed laser to rapidly heat an emissive layer attached to the sample of interest that has been subjected to shock-wave loading. The temperaturetime history of the layer can be directly related to the sample effusivity. The technique was applied to tin that was subjected to an explosively driven shock wave. While several shortcomings in this initial data set have been identified, initial findings indicate that the effusivity of tin does not significantly change under shock-wave loading. With some improvement in diagnostics, the method is capable of effusivity measurement with uncertainties of less than $20 \%$.

\section{Acknowledgments}

Lynn Veeser, David Holtkamp, Rob Hixson, and Neil Holmes are thanked for their contributions to this work.

\section{References}

Ahrens, T. J., K. G. Holland, G. Q. Chen, "Shock temperatures and the melting point of iron," in Shock Compression of Condensed Matter-1997, edited by S. C. Schmidt, J. W. Forbes, and D. P. Dandekar, American Institute of Physics Press, New York, 1998, 133-136.

Beck, P., A. F. Goncharov, V. V. Struzhkin, B. Militzer, H. Mao, R. J. Hemley, "Measurement of thermal diffusivity at high pressure using a transient heating technique," Appl. Phys. Lett. 91, 18 (2007) 181914-181914-3.

Benavides, O., O. Lebedeva, V. Golikov, "Reflection of nanosecond $\mathrm{Nd}$ :YAG laser pulses in ablation of metals," Optics Express 19, 22 (2011) 21842-21848.

Boness, D. A., "Thermal relaxation of Csl shocked to $45 \mathrm{GPa}$, with a LiF window, and optical characterization of LiF shocked to 85 GPa," AIP Conf. Proc. 1426 (2012) 1605-1608.

Gallagher, K. G., T. J. Ahrens, "Ultra-high pressure thermalconductivity measurements of griceite and corundum," in Shock Waves Vol. 2, edited by B. Sturtevant, J. E. Shepherd, and H. G. Hornung, World Scientific, Singapore, 1997, 1401-1406.

Grover, R., P. A. Urtiew, "Thermal relaxation at interfaces following shock compression," J. Appl. Phys. 45, 1 (1974) 146-152.

Holland, K. G., T. J. Ahrens, "Properties of LiF and $\mathrm{Al}_{2} \mathrm{O}_{3}$ to $240 \mathrm{GPa}$ for metal shock temperature measurements," Geophys. Monogr. 101 (1998) 335-343. 
Holmes, N., private communication, 2012.

Holtkamp, D., R. Corrow, M. Grover, B. Marshall, G. Stevens, B. Stone, W. Turley, L. Veeser, J. Young, Shot number BB100531-2, unpublished, Los Alamos National Laboratory, Los Alamos, New Mexico, 2010.

Zammit, U., M. Marinelli, F. Mercuri, S. Paoloni, F. Scudieri, "Invited review article: Photopyroelectric calorimeter for the simultaneous thermal, optical, and structural characterization of samples over phase transitions," Rev. Sci. Instrum. 82, 12 (2011) 121101. 


\section{EJECTA TRANSPORT CHEMISTRY}

STL-14-12 | CONTINUED IN FY 2013 | YEAR 1 OF 2

Gerald Stevens, ${ }^{1, a}$ Brandon LaLone, ${ }^{a}$ Gene A. Capelle, ${ }^{a}$ Mike Grover, ${ }^{a}$ and Dale Turley ${ }^{a}$

Validation and verification of nuclear weapon simulations (specifically transport models) requires ejecta transport measurements for comparison. In addition, refining these transport models requires data concerning the effects of background gas on ejecta, especially where the background gas interacts chemically with the ejecta. We have initiated a study of the effects of chemical reactions with background gas to an ejecta distribution. Results from these dynamic experiments are presented as well as a path forward for future experiments to be executed in FY 2013.

${ }^{1}$ stevengd@nv.doe.gov, 805-681-2219

a Special Technologies Laboratory

\section{Background}

A variety of effects may be observed when a shocked metal releases from a state of compression into a vacuum or background-gas environment. Effects such as material damage and failure lead to macroscopic irreversible changes such as spall that are readily observed via velocimetry and post-shot recovery techniques. Subtler, fine-scale changes and phenomena occur due to surface imperfections. Machine marks, damage layers, material voids, inclusions, and grain boundaries may all interact with a shock-front to cause small quantities of material to be ejected from the metal-free surface, which are commonly referred to as ejecta. Also, macroscopic (many micron scale) surface defects such as machined v-grooves produce ejecta through jetting processes in quantities that scale nominally with the defect volume. Such large defects may be used in conjunction with "integral diagnostics" such as radiography, piezo pins, or Asay foils to facilitate comparison against theoretical models of ejecta formation such as RichtmyerMeshkov instability growth (Buttler 2012). These diagnostics provide information about the time- and area-integrated mass distribution. Initially, theoretical models were developed to describe experiments performed in a vacuum, with the introduction of background gas and particle distribution-evolution being more recent additions (Hammerberg 2009). The effects of chemical reaction with the gas on the production and evolution of ejecta is an additional complication that has not been addressed experimentally or theoretically in open literature to date.

We began this project with the difficult decision of what material system to characterize. One of our primary goals was to perform direct comparisons between reactive and quasi-inert gases. Of the wellknown and common gases, oxygen and nitrogen were good candidates for our experiments. Oxygen is known to strongly react with most metals, and, relative to oxygen, nitrogen is fairly chemically inert but has similar viscosity and thermal conductivity (Lemmon 2004). Most ejecta studies to date have been made using tin. Although tin oxidizes, forms nitrides, and would have been a reasonable starting point, there was limited information on tin oxidation (combustion) reactions in the literature. Therefore, we decided to investigate aluminum ejecta reactions in oxygen and nitrogen background gases. 
We chose to focus on aluminum jets in oxygen for several reasons. Aluminum powders are added to rocket propellants and to explosives to increase their yield, utilizing the highly energetic aluminum-oxygen reactions. Therefore, combustion of aluminum particles has been heavily represented in the literature (Beckstead 2004). Furthermore, the shock properties and equation of state (EOS) of aluminum are well documented. Aluminum jets and the resulting particle distributions had been previously investigated by our Los Alamos National Laboratory (LANL) collaborator at the Pegasus facility using in-line Fraunhofer holography (Sorenson 1996, 2002). Lastly, aluminum is easy to machine, holds a polish well, and does not have multiple high-pressure solid phases that complicate hydrodynamic models.

Aluminum oxidation is a highly exothermic reaction. The combustion heat of an isolated atom is $-2324 \mathrm{~kJ} / \mathrm{mol}$, and for bulk aluminum forming $\mathrm{Al}_{2} \mathrm{O}_{3}$ it is $-1675 \mathrm{~kJ} / \mathrm{mol}$, the difference due to the enthalpy of vaporization. Combustion liquefies then vaporizes the metal, producing gaseous $\mathrm{Al}, \mathrm{AlO}$, and $\mathrm{AlO}_{2}$ in the process. During aluminum combustion, $\mathrm{AlO}$ emits light as unique spectral bands in the visible spectrum near $500 \mathrm{~nm}$ and provides a spectroscopic signature that aluminum combustion has taken place (Miller 2004, Mamen 2005). These combustion products eventually precipitate out of the atmosphere in the form of $\mathrm{Al}_{2} \mathrm{O}_{3}$ (Beckstead 2004). Studies of the oxidation process performed for differently sized particles in various gas mixtures suggest a burning time for a stationary particle of diameter $D$ within a gas at pressure $P$ and temperature $T$ that scales approximately with the square of the diameter (Equation 1).

$$
t_{\text {burn }} \propto \frac{D^{1.8}}{P^{0.1} \times T^{1.2}}
$$

At the low end of the measured burn times, a stationary $10 \mu \mathrm{m}$ particle takes about $250 \mu \mathrm{s}$ to completely burn. At elevated temperature $(6000 \mathrm{~K})$ and pressure (1 kbar of pure $\mathrm{O}_{2}$ ), the same scaling laws predict a burn time of $37 \mu \mathrm{s}$ for a $10 \mu \mathrm{m}$ particle. The temperature dependence of the burn time is considerably weaker than what an Arrhenius law (nearly doubling the reaction rate for every $10^{\circ}$ increase in temperature) would predict. This is because the burn rate is dominated by the rate that the Al vapor can leave the surface of the particle, not by the reaction time of the gaseous $\mathrm{Al}$ and $\mathrm{O}_{2}$ molecules. Equation 1 suggests that combustion occurs and should be observable during the timescale of explosively driven ejecta experiments.

In addition to individual particle burn-up directly impacting the ejecta evolution, the chemical reaction may also influence particle breakup dynamics due to the excess heat that is present. Free particles of aluminum (Friedman 1962) and liquid fuels (Wang 1984) are known to fracture when superheated during the combustion process.

\section{Project}

\section{Experimental Design}

We began our study by characterizing the jet properties produced by various groove geometries imparted on the free surface of an aluminum sample and various background gas mixtures. Our experimental package was composed of a polycarbonate sample holder that holds a $1 \mathrm{~mm}$ thick $\times 40 \mathrm{~mm}$ diameter aluminum sample within a transparent Lexan cylinder that contains the background gas or a vacuum. A half-inch right-cylinder of PBX-9501 high explosive, with an RP-1 detonator (Teledyne RISI, Inc.), is used to drive a shock wave into the aluminum sample. Peak stress in the shock-compressed aluminum is about $30 \mathrm{GPa}$, and is measured by applying the Rankine-Hugoniot jump conditions to half the breakout velocity of the aluminum target, as measured using photonic Doppler velocimetry (PDV); our peak surface velocity at shock-arrival is about $3 \mathrm{~km} / \mathrm{sec}$. A jet of ejecta is formed when the shock wave breaks out of the rear surface of the aluminum sample. Images of the jet were taken with a timegated 9-frame camera, which recorded either selfemission or a shadowgraph of the jet. Backlighting was achieved using a xenon flash lamp. Samples were aligned to look either along or perpendicular to the groove. Jet velocities were determined from the time-gate images. Ten experiments were performed, 
with groove full-angles ranging from $90^{\circ}$ to $110^{\circ}$. Groove depth was varied between 25 and $175 \mu \mathrm{m}$. A summary of experiments performed is in Table 1. Figure 1 is a diagram of a typical experimental package.

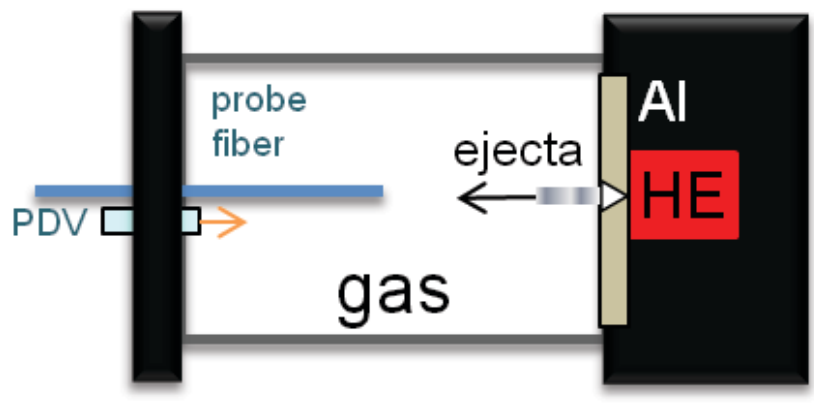

Figure 1. Typical experimental package for experiments in Table 1

In conjunction with the experimental efforts, we built a full 2-D model of the aluminum measurements in the hydrodynamic code, $\mathrm{CTH}$, using a Mie-Grüneisen EOS (with strength) for aluminum, and a SESAME table for the background gas (chosen to be air-CTH does not handle chemical reactions). This model predicts surface and jet velocities close to our observations, with the surface velocity history matching our PDV data quite well. The model also gives us initial temperature and pressure estimates that can be used for burn time and particle breakup estimates.
Figure 2 shows simulated results from one of the CTH models. The image in Figure 2a shows a pressure map of the aluminum sample just before the shock arrives at two defects, a small defect with dimensions taken from shot 120815 , and a larger machined groove defect modeled after shot 120418 . The middle and right images in Figure 2 show temperature maps of the shocked material and background gas at two later times after the shock arrives at the free surface. The model indicates that the temperatures at the base of the defect are at the $933 \mathrm{~K}$ melting temperature of aluminum, and the material at the tip of the inverted defect is just above melt. The model also shows that the background gas is significantly hotter, between 2000 and $4000 \mathrm{~K}$. The right image shows the small jet tip fragmenting at later times and becoming entrained in the heated shocked gas bow-wake. These conditions, small $\sim 1 \mu \mathrm{m}$ melted particles surrounded by $2000-4000 \mathrm{~K}$ heated air, are sufficient to cause significant combustion of aluminum on the timescales of our experiments (Friedman 1962); therefore, combustion of the aluminum ejecta is likely.

Our CTH model predicts jet velocities fairly well. The jet velocities estimated from our model are $4.0 \mathrm{~mm} / \mu \mathrm{s}$ and $5.35 \mathrm{~mm} / \mu \mathrm{s}$ for the two different grooves; the experimental jet velocities for the same grooves were 4.05 and $5.02 \mathrm{~mm} / \mu \mathrm{s}$, respectively (Figure 3).

Table 1. Summary of ejecta experiments performed in this study

\begin{tabular}{|c|c|c|c|c|c|c|c|c|}
\hline Exp. \# & $\begin{array}{c}\text { Background } \\
\text { Gas }\end{array}$ & $\begin{array}{c}\text { Groove } \\
\text { Depth } \\
(\mu \mathrm{s})\end{array}$ & $\begin{array}{c}\text { Groove } \\
\text { Width } \\
(\mu \mathrm{s})\end{array}$ & Half-Angle & $\begin{array}{c}\mathbf{V}_{\text {jet }} \\
(\mathrm{mm} / \mu \mathrm{s})\end{array}$ & $\begin{array}{c}\mathbf{V}_{\mathrm{fs}} \text { at } \\
\text { Breakout } \\
(\mathrm{mm} / \mu \mathrm{s})\end{array}$ & $\begin{array}{c}\text { Peak Stress } \\
(\mathrm{GPa})\end{array}$ & Spectrometer \\
\hline 120315 & Vacuum & 148 & 344 & 49.3 & 5.06 & 2.7907 & 27.2 & - \\
\hline 120319 & $\mathrm{O}_{2}$ & 93 & 269 & 55.3 & 4.14 & - & - & - \\
\hline $120320 \_1$ & $\mathrm{O}_{2}$ & 91 & 256 & 54.6 & 4.68 & 3.032 & 30.2 & - \\
\hline $120320 \_2$ & $\mathrm{~N}_{2}$ & 112 & 300 & 53.3 & 5.35 & 3.092 & 31.0 & - \\
\hline $120411 \_1$ & $\mathrm{O}_{2}$ & 165 & 366 & 48.0 & 5.31 & - & - & OMA \\
\hline $120412 \_1$ & $\mathrm{O}_{2}$ & 175 & 370 & 46.6 & 5.22 & - & - & OMA \\
\hline $120416 \_1$ & $10 \% \mathrm{O}_{2} / \mathrm{N}_{2}$ & 138 & 346 & 51.4 & 4.94 & 3.213 & 32.6 & Late OMA \\
\hline $120418 \_1$ & $10 \% \mathrm{O}_{2} / \mathrm{N}_{2}$ & 156 & 365 & 49.5 & 5.02 & 3.012 & 30.0 & Late OMA \\
\hline 120810 & $10 \% \mathrm{O}_{2} / \mathrm{N}_{2}$ & 44 & 55 & 32.0 & 4.05 & 3.090 & 31.0 & Streak \\
\hline 120815 & $10 \% \mathrm{O}_{2} / \mathrm{N}_{2}$ & 26 & 62 & 50.0 & 4.05 & 3.158 & 31.8 & Streak - Edge \\
\hline
\end{tabular}

The columns are (from left to right) background gas, groove depth from peak to valley, groove width from shoulder peak to peak, calculated groove half angle, jet velocity, free-surface velocity at breakout, calculated peak stress, and what spectrometer (optical multichannel analyzer, [OMA] or streaked spectrometer [Streak]) if any was used. Except for the vacuum experiment, the total background gas pressure was at 1 atm. 


\section{Spectroscopic Measurements}

Several studies that have measured light produced during combustion of aluminum powders indicate that the intermediate oxide, AlO, emits visible light during combustion. Our initial set of experiments was designed to determine whether AlO is produced on short time scales (of several microseconds). Our first such experiment in pure oxygen (number 120411_1) used a $600 \mu \mathrm{m}$ fiber positioned about 1 inch away from the groove to collect light from the entire jet. This light was recorded using a microchannel plateintensified, gated optical multichannel analyzer (OMA). The OMA uses a HORIBA Jobin Yvon CP140 spectrograph with an Andor iStar Intensified CCD, which has $\sim 4 \mathrm{~nm}$ resolution and a 100 ns integration time that was set to open at $1.5 \mu$ s after shock arrival. The light emission was intense, but, surprisingly, the spectrum of the emitted light was broad and structureless, with no indication of the AlO emission lines.

Switching to a $10 \% \mathrm{O}_{2}, 90 \% \mathrm{~N}_{2}$ mixture in a subsequent experiment, we observed line emission.

Figure 4 shows the spectrum recorded on experiment 120418 with a 100 ns gate width, $2.7 \mu$ s after shock breakout. Individual peaks, fit to the spectrum, are shown in red at the bottom of the figure, with the complete multipeak fit shown in blue. Table 2 identifies the peaks shown in the figure alongside peak locations derived from Al flame spectra (Mamen 2005) and imbedded aluminum particles in PBX explosives (Miller 2004). Note that the peak positions shift slightly to higher wavelengths with increasing temperature, and the PBX spectrum is at the high temperature of explosive products ( $3500 \mathrm{~K})$. These emission lines are from transitions between different electronic states ( $\mathrm{AlO}\left(\mathrm{B}^{2} \Sigma^{+} \rightarrow \mathrm{X}^{2} \Sigma^{+}\right)$) with each of the five peaks representing transitions between different vibrational levels with quantum number v. Rotational fine structure within each peak is unresolvable given the resolution ( $10 \mathrm{~nm}$ ) of this system. Based on the spectroscopic analysis, our findings clearly indicate that the aluminum ejecta are reacting chemically with the background oxygen gas.

\section{Time-Resolved Experiments}

The spectrum in Figure 4 was taken with a fiber situated normal to the shocked surface, collecting light from the complete jet and shocked surface simultaneously. The AlO and other radiant, emissive materials in this situation were at a variety of temperatures (see Figure 2 for theoretical details on the temperatures of various features in jets).

In order to better understand the combustion process, we performed two experiments that used a spectrally resolved streak camera recording system. This system was composed of a Hamamatsu C1587 temporal disperser, an M1953 slow-speed streak unit, a C4742-95 digital camera, and a 0.5 m Jarrell-Ash spectrometer with a 150 grooves/mm diffraction grating. A 3:1 reducing lens imaged the diffraction spectrum from a $600 \mu \mathrm{m}$ collection fiber on the streak photocathode, giving a spectral range of $\sim 460-700 \mathrm{~nm}$, which was calibrated using two diode

Table 2. Emission spectrum fit locations and standard deviations from Figure 4. For comparison, flame-spectra peak locations from Mamen (2005) and PBX experiments (Miller 2004) are also listed

\begin{tabular}{|c|c|c|c|c|c|}
\hline Peak & Line Source & $\begin{array}{c}\text { Location } \\
(\mathrm{nm})\end{array}$ & $\sigma$ & $\begin{array}{c}\text { Flame } \\
\text { Spectra }(\mathrm{nm})\end{array}$ & $\begin{array}{c}\text { PBX } \\
\text { Spectra }(\mathrm{nm})\end{array}$ \\
\hline 0 & $\mathrm{Al}(3 \mathrm{P}-4 \mathrm{~S})$ & 393.7 & 3.9 & - & 395.0 \\
\hline 3 & $\mathrm{AlO}\left(\mathrm{B}^{2} \Sigma^{+} \rightarrow \mathrm{X}^{2} \Sigma^{+}\right) \Delta \mathrm{v}=2$ & 454.5 & 0.7 & - & 457.2 \\
\hline $4(\mathrm{~A})$ & $\mathrm{AlO} \Delta \mathrm{v}=1$ & 469.9 & 0.3 & 464.4 & 472.1 \\
\hline $5(\mathrm{~B})$ & $\mathrm{AlO} \Delta \mathrm{v}=0$ & 488.5 & 0.2 & 486.6 & 489.9 \\
\hline $6(\mathrm{C})$ & $\mathrm{AlO} \Delta \mathrm{v}=-1$ & 516.2 & 0.1 & 507.9 & 517.7 \\
\hline 7 & $\mathrm{AlO} \Delta \mathrm{v}=-2$ & 544.1 & 0.2 & - & - \\
\hline 9 & $\mathrm{Na}(3 \mathrm{~S}-3 \mathrm{P})$ & 589.2 & 0.1 & 590.0 & - \\
\hline
\end{tabular}



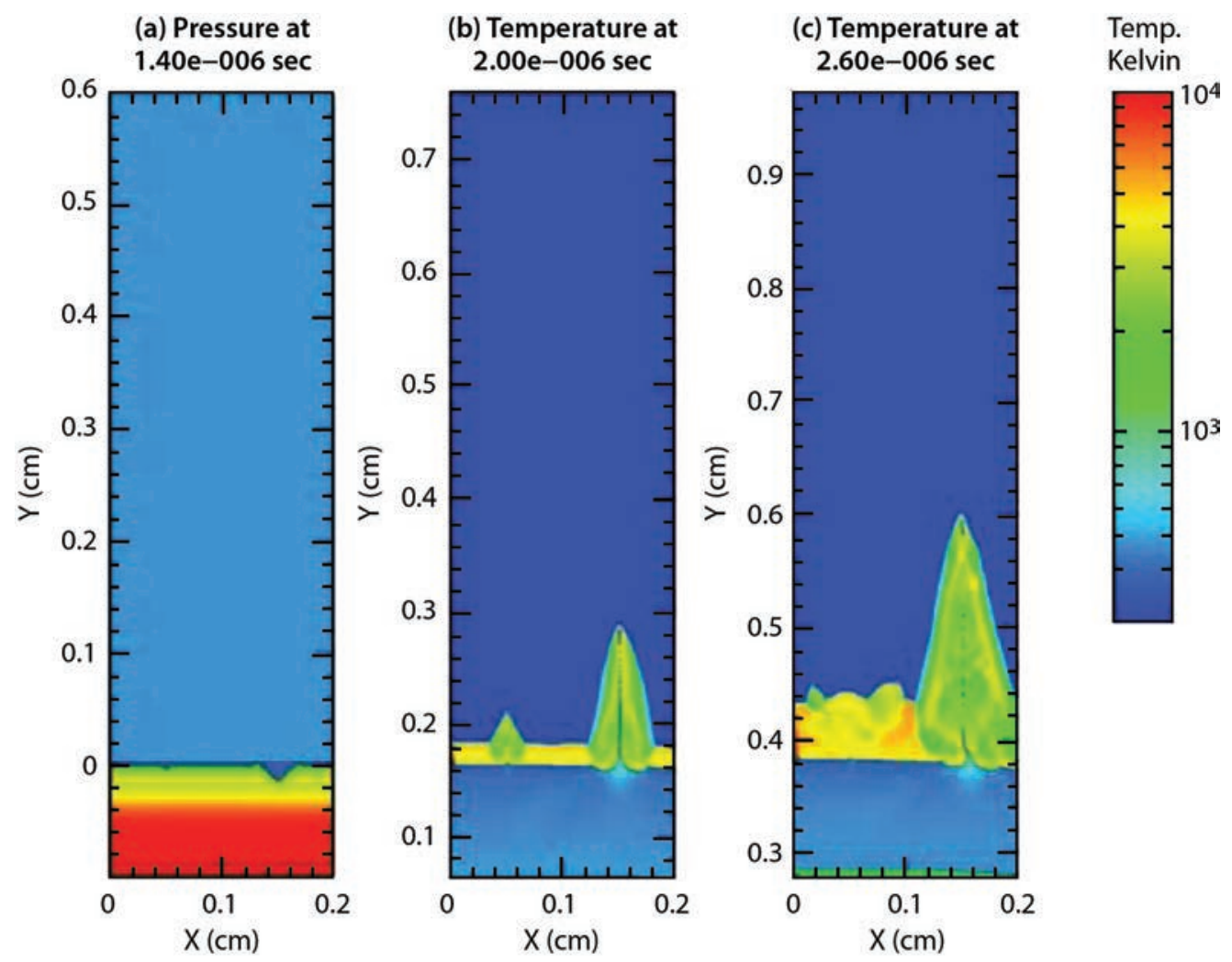

Figure 2. CTH predictions of jet velocities and temperatures for two experimental groove geometries were produced using blade-electrical discharge machining (EDM) (small groove at $0.05 \mathrm{~cm}$ ) and microtooling (large groove with shoulders at $0.15 \mathrm{~cm}$ ) of experiments 120815 and 120418, respectively. (a) A pressure profile prior to breakout (arbitrary color scale), and (b) and (c) material temperatures in Kelvin at two later times (temperature scale at far right).
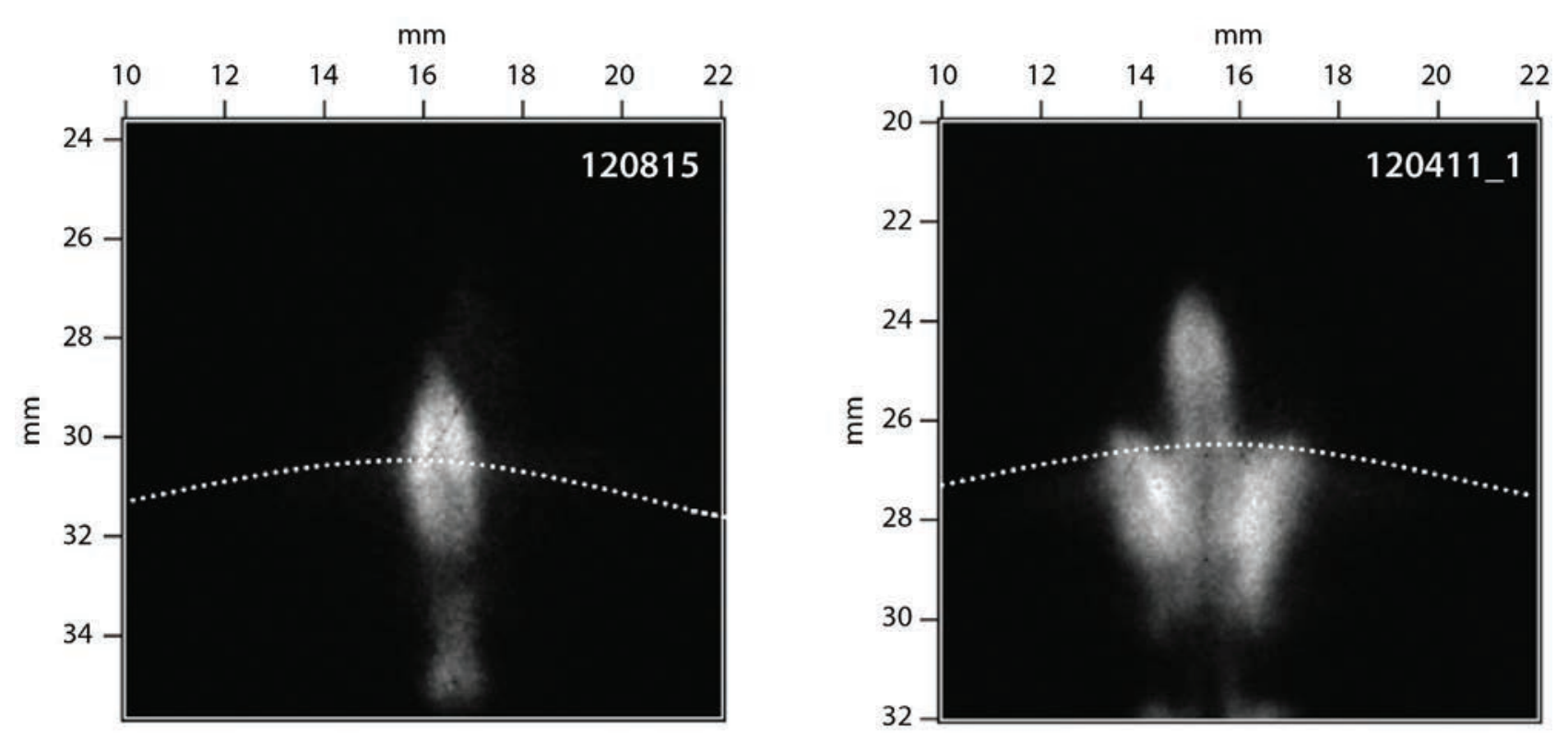

Figure 3. Images (frame 5 of 9) of light emission in the band from 450 to $550 \mathrm{~nm}$ for two experiments (120815 and 120411_1) similar to the model calculations in Figure 2. These images were taken about $1.1 \mu \mathrm{s}$ after shock arrival at the surface. The dashed white line indicates the location of the aluminum target surface. 


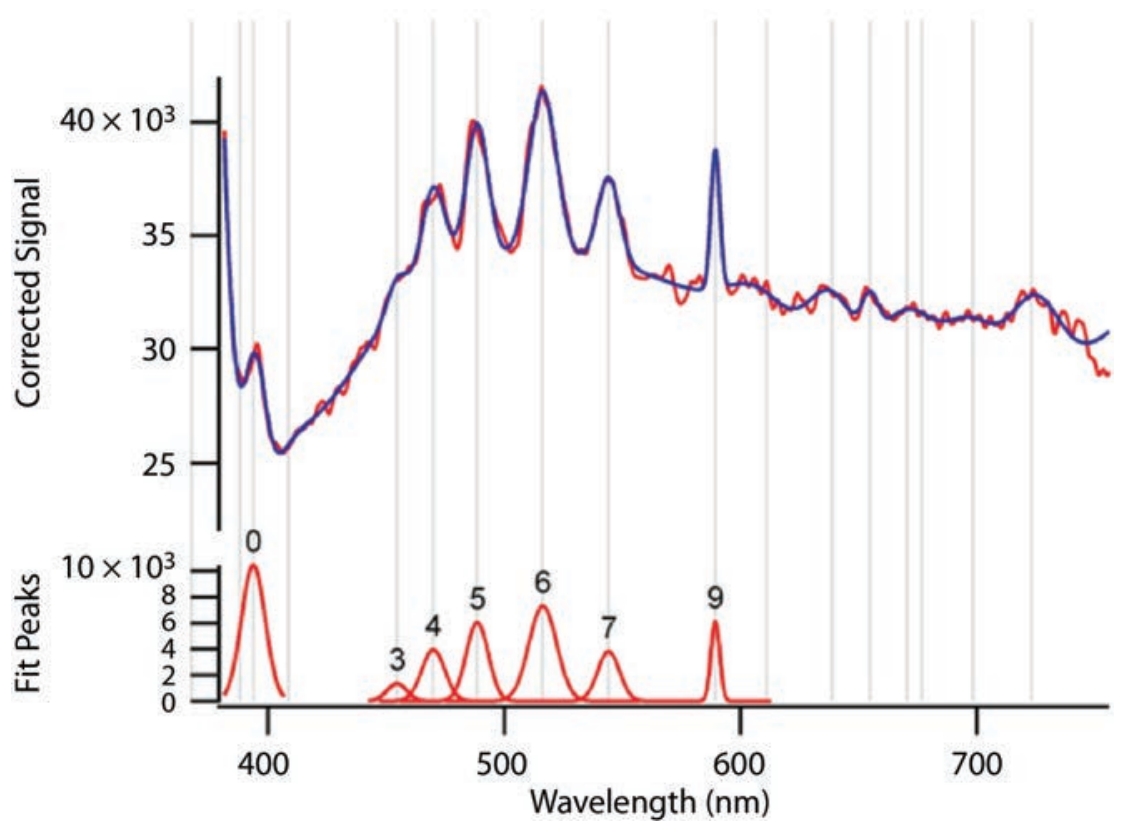

Figure 4. Corrected emission spectrum from experiment 120418 showing line emission from aluminum jet combustion $2.7 \mu \mathrm{s}$ after shock breakout. The blue curve is a multipeak fit of the full spectrum, and selected peaks from this fit are shown in red at the bottom of the figure. lasers of known wavelengths at 408 and $635 \mathrm{~nm}$. We also implemented changes to our sample preparation to reduce the amount of ejecta produced in order to see if we could observe time evolution of the AlO line emission, giving us information about reaction rates over several microseconds of recording. We modified the tool from a Hylozoic electrical discharge machining (EDM) system, using a razor blade to etch a surface defect to a desired depth. The defect angle is fixed by the razor blade geometry, which is manufactured with high tolerances and reproducibility. Experiments 120810 and 120815 (see Table 1 for sample details) recorded the spectrum looking at first normal to the surface as we did with OMA experiments, and then perpendicular to the jet with a small ( $\sim 1 \mathrm{~mm}$ ) sample spot. Temporal profiles from the two shots are shown in Figure 5. The left figure shows light levels decreasing after a peak level near $9.5 \mu$ s. This may indicate cessation of material ejected from the surface, which is something our CTH calculations have suggested may be happening for small defects.

On shot 120810, our spectral streak measurements showed line emission from about 500 ns after shockarrival (at $6 \mu \mathrm{s}$ ) throughout the duration of the streak. To better resolve any temporal changes, we reconfig- ured the probe that collects emission light to collect from the side of the jet front (Figure $5 \mathrm{~b}$ ) set $10 \mathrm{~mm}$ ahead of the aluminum surface. The temporal history of the collapsed spectral streak record is also shown in Figure 5, which agrees with the photodiode record.

Figure 6 shows a collapsed integrated spectrum of light produced between 8 and 10 us on experiment 120815 . Only three of the AlO lines are apparent, and the fit peaks are shown for reference. The rotational-vibrational band intensity is temperature dependent, with states being populated due to collisional excitation by a hot background gas. Relative amplitudes of these lines have been used to infer the temperature of the upper atmosphere from line emission from rocket propellant combustion by-products (Harang 1966). The relative amplitudes of the three peaks shown in Figure 6 ( $A / B$ and $C / B$ ) were used by Servaites (2001) to infer effective temperatures of $\mathrm{AlO}$ combustion in $\mathrm{CO}_{2}$ and $\mathrm{H}_{2} \mathrm{O}$ based on a detailed molecular emission model (Colibaba-Evulet 2000). Applying Servaites' simplified analysis to our measured amplitude ratios, both $\sim 0.5 \pm 0.15$, gives an effective temperature of $3300 \pm 1000 \mathrm{~K}$. This is consistent with the temperatures within the shocked gas bow-wake as calculated by CTH (see Figure 2). 
(a) Experiment 120810

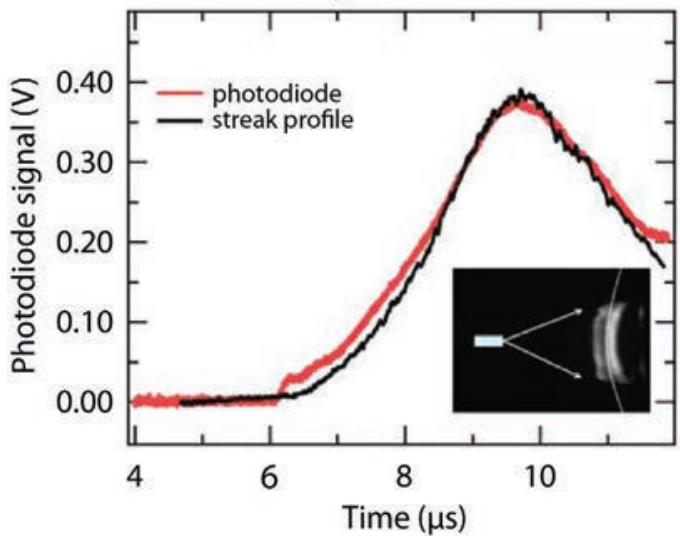

(b) Experiment 120815

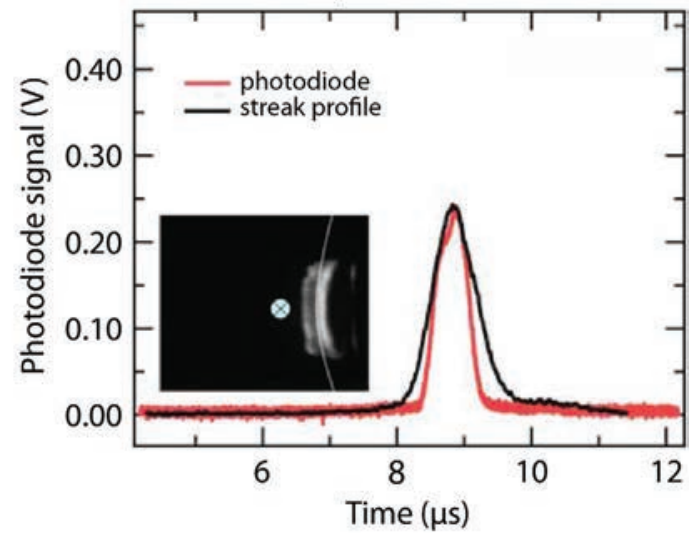

Figure 5. Temporal profiles recorded with a diode detector (red) and by collapsing the streak record image to its time axis (black) for experiments (a) 120810 and (b) 120815. Shock arrives at the surface, producing a jet at $6 \mu \mathrm{s}$ in both experiments. Inset images show placement of a fiber probe that collected emission light either end-on or from the edge (initially $10 \mathrm{~mm}$ ahead of aluminum).

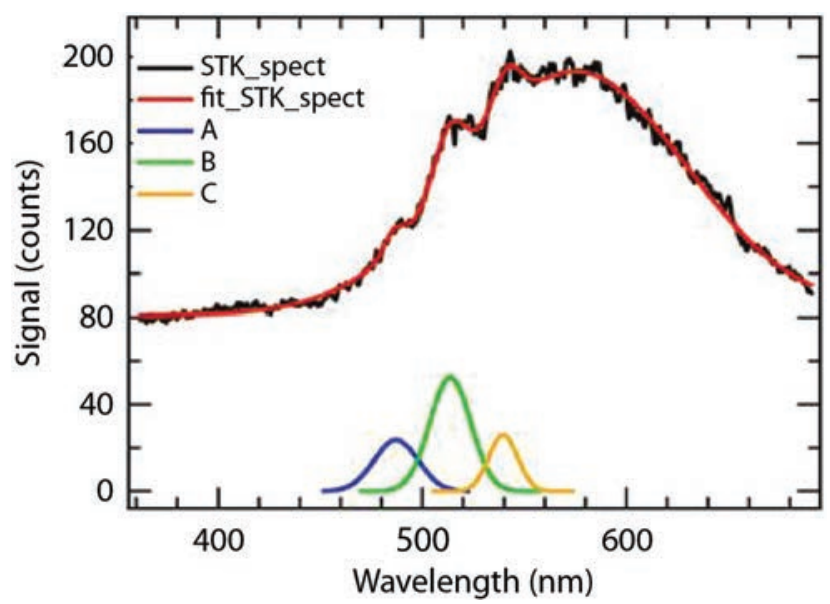

Figure 6. Integrated spectrum from experiment 120815, with multipeak fit and three peaks. The relative amplitudes of peaks $A, B$, and C may be used to infer an effective temperature of the AIO.

\section{Conclusion}

Using spectroscopic techniques, we confirmed that combustion of aluminum ejecta in a mixed $(10 \%$ $\mathrm{O}_{2} / \mathrm{N}_{2}$ balance) gas initiates at early times (within 500 ns of jet formation). Prior work (Friedman 1962) at lower gas pressures indicated that combustion initiation of $\sim 10 \mu \mathrm{m}$ aluminum particles required hundreds of microseconds.
We have developed a simple, reproducible technique for ejecta defect production using a razor-blade EDM process. This technique produces small amounts of ejecta, and our imaging and spectroscopic measurements suggest that the ejecta is breaking up and becoming entrained in shocked gas bow-wake within the short ( $\sigma \mu \mathrm{s}$ ) duration of our experiments. Next year, we will employ multi-exposure high-resolution imaging techniques to observe particle evolution on short time scales in both inert and reactive gases.

\section{Acknowledgments}

The authors would like to thank Billy Buttler of LANL for motivating this work. Danny Sorenson of LANL was also very helpful, sharing details of his holographic ejecta imaging techniques.

\section{References}

Beckstead, M. W., "A Summary of Aluminum Combustion," Brigham Young University, Provo, Utah, USA (2004) NATO Report RTO-EN-023. 
Buttler, W. T., D. M. Oro, D. L. Preston, K. O. Mikaelian, F. J. Cherne, R. S. Hixson, F. G. Mariam, C. Morris, J. B. Stone, G. Terrones, D. Tupa, "Unstable Richtmyer-Meshkov growth of solid and liquid metals in vacuum," J. Fluid Mech. 703 (2012) 60-84.

Colibaba-Evulet, A., A. Singhal, N. G. Glumac, "Detection of AIO and TiO by laser-induced fluorescence in powder synthesis flames," Comb. Sci. and Tech. 157, 1 (2000) 129-139.

Friedman, R., A. Macek, "Ignition and combustion of aluminum particles in hot ambient gases," Combustion and Flame 6, 1 (1962) 9-19.

Hammerberg, J. E., B. Plohr, "Particulate distribution function evolution for ejecta transport," Proceedings NEDPC, Lawrence Livermore National Laboratory, Livermore, California, 2009.

Harang, O., "AlO resonant spectrum for upper atmosphere temperature determination," Environmental Research Paper, No. 192 (1966), re-published by the United States Air Force under Report AFCRL-66-314.

Lemmon, E. W., R. T. Jacobsen, "Viscosity and thermal conductivity equations for nitrogen, oxygen, argon, and air," Internat. J. of Thermophys. 25, 1 (2004) 21-69.

Mamen, J., S. Goroshin, A. Higgins, "Spectral structure of the aluminum dust flame," 20th International Colloquium on the Dynamics of Explosions and Reactive Systems, Montreal, Quebec, July 31-August 5, 2005.

Miller, S., G. I. Pangilinan, "Measurements of aluminum combustion in energetic formulations," Shock Compression of Condensed Matter-2003: AIP Conf. Proc. 706 (2004) 867-870.

Servaites, J., H. Krier, J. C. Melcher, R. L. Burton, "Ignition and combustion of aluminum particles in shocked $\mathrm{H}_{2} \mathrm{O} / \mathrm{O}_{2}$ / $\mathrm{Ar}$ and $\mathrm{CO}_{2} / \mathrm{O}_{2} / \mathrm{Ar}$ mixtures," Combustion and Flame 125, 1 (2001) 1040-1054.

Sorenson, D., R. Malone, B. Frogget, C. Ciarcia, T. Tunnell, R. Flurer, "Particle distribution measurements using in-line Fraunhofer holography," report LA-UR-96-3510, Los Alamos National Laboratory, Los Alamos, New Mexico, October 1996.
Sorenson, D. S., R. W. Minich, J. L. Romero, T. W. Tunnell, R. M. Malone, "Ejecta particle size distributions for shock loaded Sn and Al metals," J. Appl. Phys. 92, 10 (2002) 5830-5836.

Wang, C. H., X. Q. Liu, C. K. Law, "Combustion and microexplosion of freely falling multicomponent droplets," Combustion and Flame 56, 2 (1984) 175-197. 


\section{Instruments, Detectors \& SENSORS}
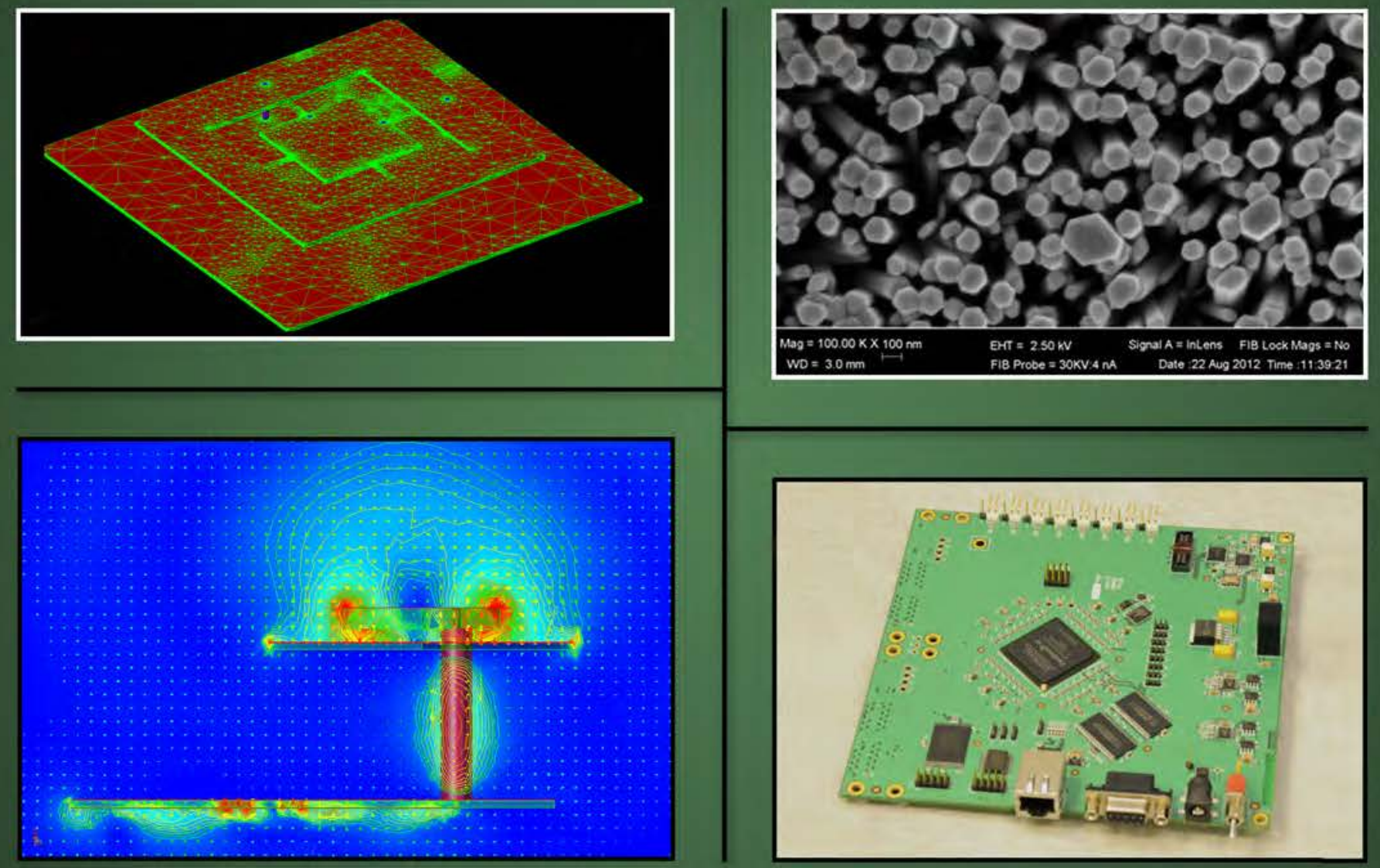


\section{BACK-THINNEd Silicon RADIATION DETECTOR READOUT}

LAO-32-12 | CONTINUED FROM 2011 | YEAR 2 OF 2

Stuart A. Baker, ${ }^{1, a}$ Kristina Brown, ${ }^{a}$ and Alden Curtis ${ }^{a}$

The objective of this effort was to evaluate detector designs to improve gamma radiation detection capabilities applied to homeland security applications. Improvements in gamma radiation detection may also enhance capabilities in high-energy density physics and radiometry diagnostics in programmatic stockpile stewardship applications. In FY 2011 we identified a corporate partner to design a custom solid-state photomultiplier (SSPM) device capable of back-illuminated detection. A small pixel array was designed and wafer fabrication was completed. We also performed optical sensitivity tests on front-illuminated SSPMs. In FY 2012 we performed radiation resolution and sensitivity comparisons with scintillators on front-illuminated SSPM versus PMT detection. We also evaluated results from the SSPM wafer fabrication of FY 2011. Preparations were made to back-thin the SSPM device. This process is expected to be completed in early FY 2013.

${ }^{1}$ bakersa@nv.doe.gov, 505-663-2040

a Los Alamos Operations

\section{Background}

We have been studying the current radiation detection standard of photomultiplier tube/scintillator technology. Initially, we pursued an adaptation of recent developments in hybrid CMOS image sensors. Our past experience with hybrid CMOS imagers applied to radiographic imaging (Douence 2005) led to investigation of back-illuminated CMOS detectors for radiation detection. Our concept is to develop a solid-state detector readout that will modernize optical and radiation detection into a new era of instrumentation. Photomultiplier tubes (PMTs) coupled to sodium iodide Nal:TI are the most widely used gamma radiation detectors, and they have been the detection standard for over 50 years (Knoll 2010). PMTs are available in a variety of sizes and can accommodate large-area gamma radiation detecting scintillators (Mukhopadhyay 2011). Solid-state radiation detection has been shown to be competitive with PMT performance (Konnoff 2011), because it has the potential to improve detection sensitivity and reduce the footprint and power requirements for man-portable and remote monitoring systems. In previous attempts to back illuminate solid-state photomultipliers (SSPMs), performance suffered from poor charge collection efficiency in the silicon pixel (Stapels 2009). Our design attempts to improve detector quantum efficiency and pixel charge collection efficiency.

\section{Project}

To design and fabricate our back-thinned (BT) detector, we partnered with Radiation Monitoring Devices, Inc. (RMD). We developed a basic system performance model to evaluate potential performance gains with this concept. RMD completed the SSPM detailed design and wafer fabrication of the pixel array (Baker, 2011, 2012). This FY 2012 effort supported evaluation of the test pixels fabricated in the wafer run, and back-thinning for evaluation of back-illuminated performance and comparison of the radiation detection performance of traditional PMT detectors with standard front-illuminated SSPMs. 

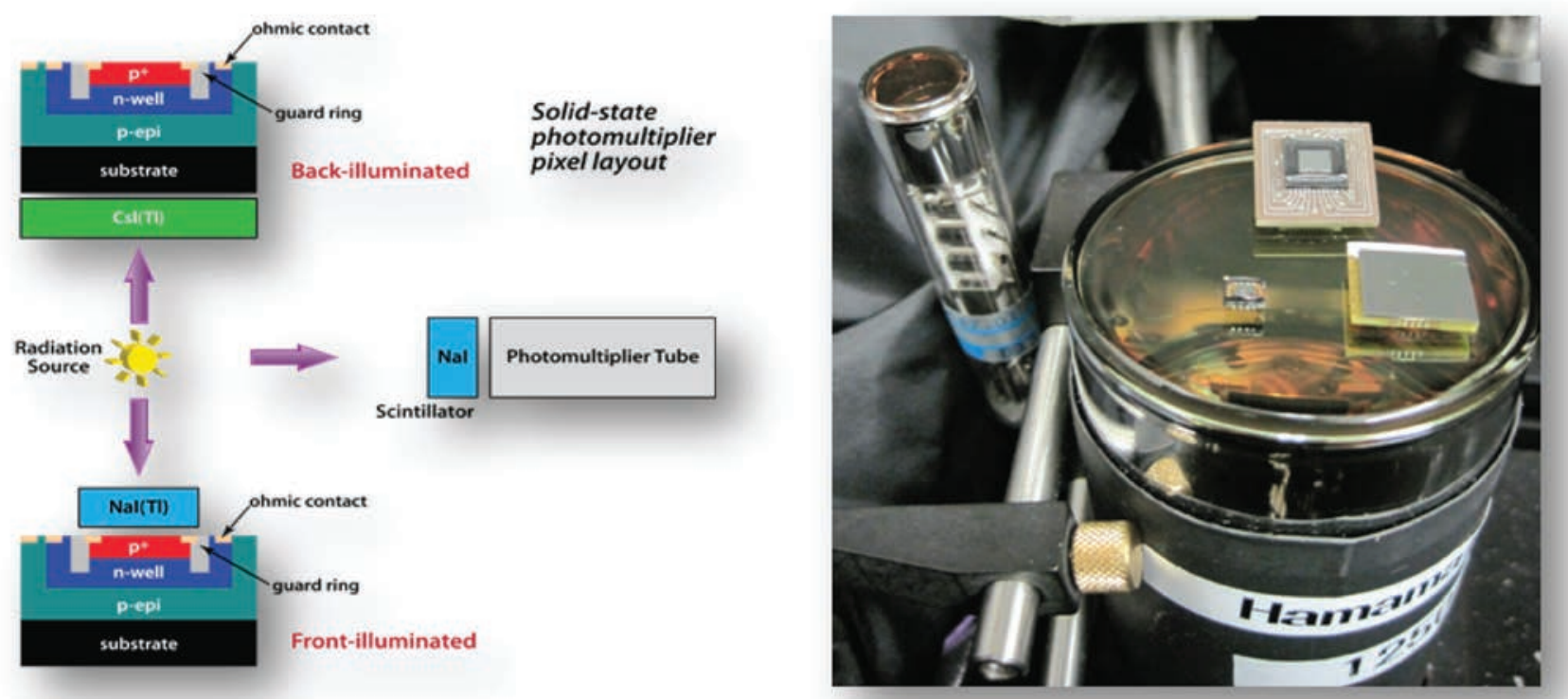

Figure 1. (left) SSPM detector pixel layout compared to PMT detection scheme and (right) a PMT (large tube) with three SSPMs resting on its top and a smaller PMT (upper left corner)

\section{Detector Readout Solid-State Photomultiplier (SSPM) Concept}

Detection schemes employing both front-illumination and back-illumination are compared to traditional PMT detection in Figure 1. Our concept was based on a number of potential advantages unique to back-thinned SSPMs. The emission spectrum of the scintillation material and the absorption spectrum of the readout overlap better, a feature crucial for high radiation detection efficiency. The quantum efficiency (QE) of back-illuminated silicon is well matched to the spectral emission of thallium-doped cesium iodide (CSI:TI). SSPM Geiger mode gain on the order of $\sim 10^{6}$ is similiar to traditional PMTs. This combination predicts a potential for 10X improvement in detection sensitivity. Additionally, PMTs operate at high voltages

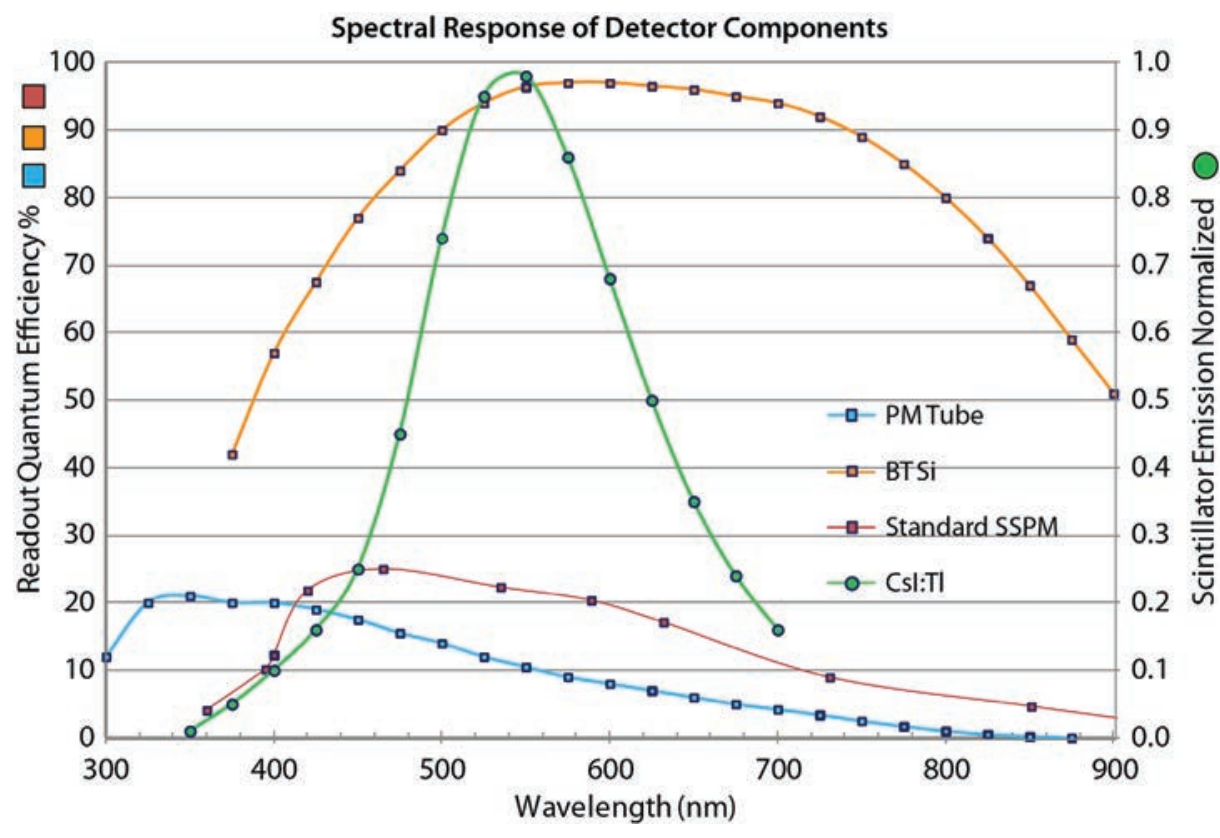

Figure 2. Detector spectral response (squares) is compared to scintillator spectral emission of CsI:TI (circles). Back-thinned CCD QE is superior to current PMT photocathode response for detection of blue or green scintillators. The standard SSPM effective $Q E$ is front-illumination pixel QE $\times$ fill factor. The back-illuminated detector fill factor is expected to be $100 \%$; the front-illuminated fill factor is $\sim 40 \%$. 


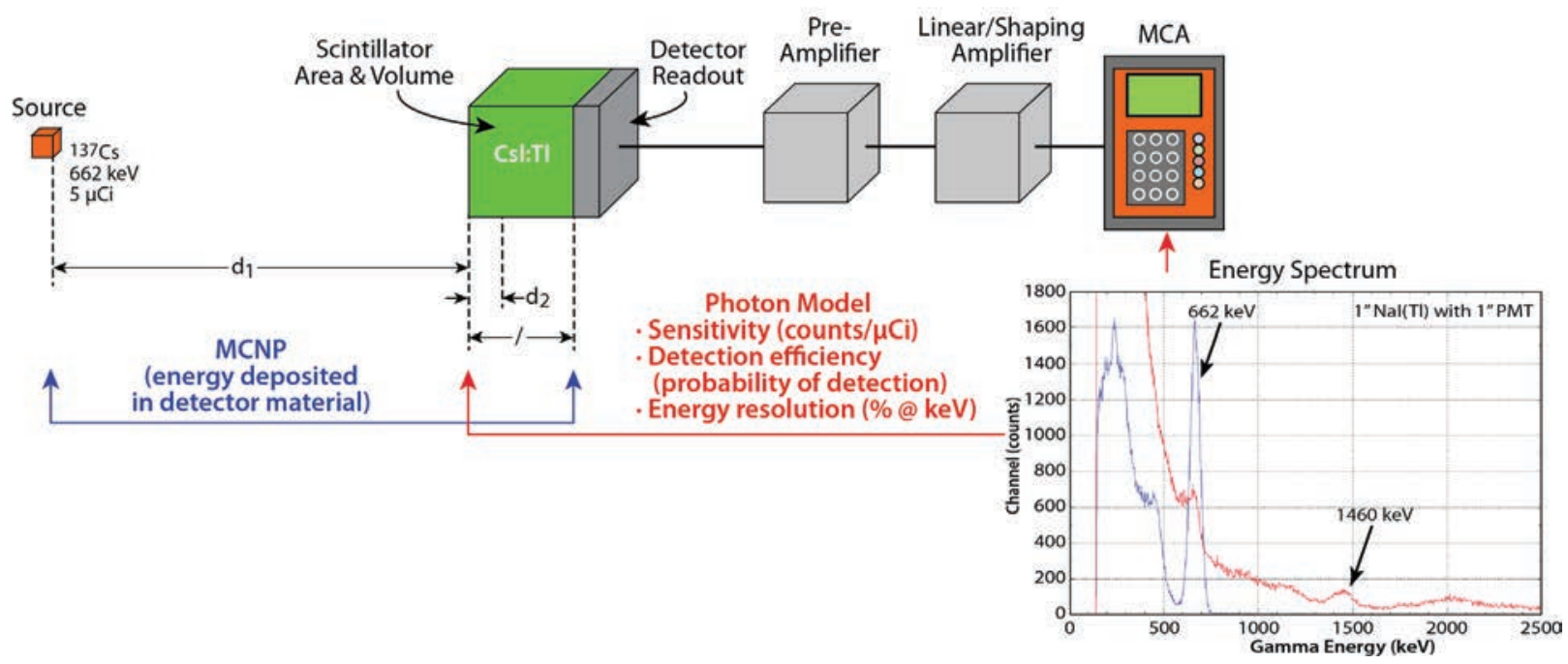

Figure 3. Detector model using MCNP output

and solid-state detectors operate at low voltages, thereby negating power and safety concerns. Finally, the small form factor of the SSPM could allow for a more compact instrument.

\section{Detector Performance Model}

In Figure 2, detector spectral response is compared to the scintillator spectral emission of CsI:Tl, graphically illustrating the significantly higher quantum efficiency of back-thinned CCD relative to current PMT photocathode response for detection of typical blue or green scintillators.

A number of parameters were used in modeling system performance. For modeling the detection system, we used scintillator absorption efficiency, volume, and spectral emission. For the readout, we used optical $Q E$, gain, sensitivity, and area. We limited this detection model to the variable scintillator and readout components for basic sensitivity comparisons.
The figure-of-merit performance model illustrated in Figure 3 is used for first-order approximation of detection efficiency. Sample results are shown in Table 1. CsI:TI has higher radiation-to-photon conversion efficiency, rendering it interesting relative to detection sensitivity, but a longer decay time, potentially limiting energy resolution. Photo detector efficiency (PDE) is the combination of detector QE and fill factor.

\section{Visible Light Measurements}

This year we collected standard front-side SSPMs and PMTs for comparison of sensitivity with visible light, simulating scintillator spectral emission. The PMTs, loaned from the NSTec Remote Sensing Laboratory, were representative of field radiation detection units. SSPMs were purchased from SensL of Ireland.

Table 1. Detection figure of merit

\begin{tabular}{|c|c|c|c|c|c|c|c|c|}
\hline Isotope & $\begin{array}{c}\text { Energy } \\
(\mathbf{k e V})\end{array}$ & Scintillator & Readout & QE & Fill Factor & PDE & $\begin{array}{c}\text { Figure of } \\
\text { Merit }\end{array}$ & $\begin{array}{c}\text { Signal } \\
\text { Ratio }\end{array}$ \\
\hline${ }^{137} \mathrm{Cs}$ & 662 & Nal:TI & PMT & 0.2 & 1 & 0.20 & 2,983 & 1 \\
\hline${ }^{137} \mathrm{Cs}$ & 662 & CSI:TI & SSPM & 0.4 & 0.4 & 0.16 & 5,087 & 1.7 \\
\hline${ }^{137} \mathrm{CS}$ & 662 & CSI:TI & BT-SSPM & 0.95 & 1 & 0.95 & 30,203 & 10.1 \\
\hline
\end{tabular}




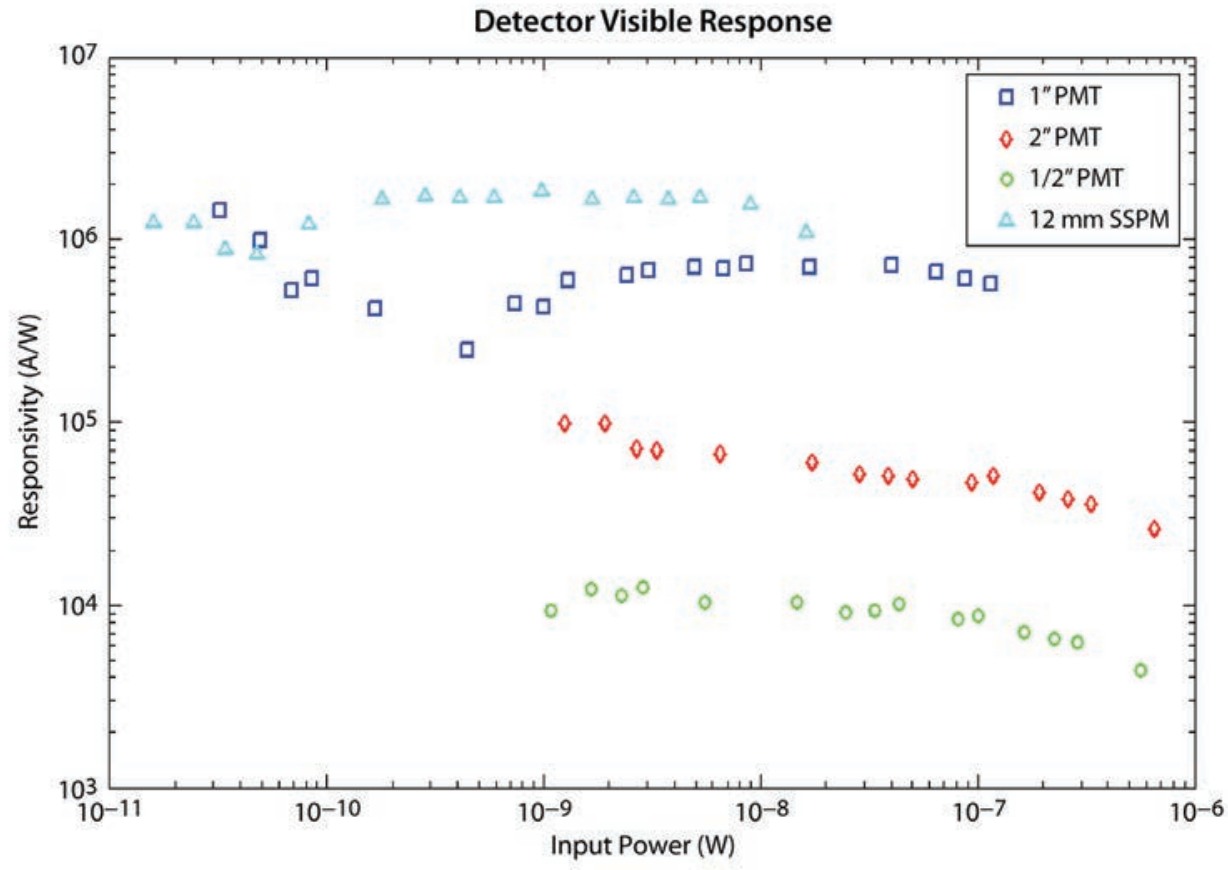

Figure 4. Plot of signal vs photons collected. The signal input is photons collected in the detector area. High-gain, low-noise SSPMs exhibit better sensitivity than larger-area PMTs. The front-illuminated SSPMs exhibit more signal volts per photon collected than some of the PMTs used for this comparison. The units of photons directly relate to the scintillator radiation-tolight conversion efficiency (photons/keV $\gamma$ ).

Figure 4 compares detector sensitivity in amps per watt (A/W) of a front-illuminated SSPM to three varieties of field PMT tubes. Each detector was flood-illuminated with a blue LED array to provide a uniform pulsed illumination over the detector area. The detection area of each detector was accounted for to calculate detector responsivity (A/W). The signal was measured over a range of input light from detector noise level through saturation. The SSPM is shown to operate over a lower range of input light collected, indicating high sensitivity relative to the PMTs. The input power (watts) can be directly related to the scintillator radiation-to-light conversion efficiency.

\section{Sensor Development}

It is challenging to design and manufacture backilluminated SSPM devices, both in the pixel design for efficient charge collection and executing the backthinning process itself. Pixel design for back-illumination requires modification from front-illuminated designs. Some design/process modifications have been incorporated at RMD to improve front-surface QE. Post-processing to reduce the oxide thickness over the Geiger photodiode elements has produced what appears to be measurable improvement in the QE of the SSPM, in preliminary measurements. This QE improvement is most significant at blue wavelengths.

Earlier back-illumination experiments indicate that a near $100 \%$ fill factor is possible (Stapels 2009 ). Back-thinning silicon can allow appropriate surface treatments to achieve high QEs: green wavelengths can reach $90 \%$ QE. Back-thinned devices thus far have shown improved spatial collection, but have not shown improved charge collection from back illumination.

We have worked with RMD to develop a series of prototype designs to test pixel design performance. Approximately 40 chips with 24 small test arrays per chip (Figure 5) have been fabricated for evaluation. Fabrication of BT components in small quantities requires a thinning process on the sensor die after the wafer process. The prototype devices in Figure 6 show the pixel design regions that exhibit Geiger mode operation. Unfortunately the back-thinned devices were not available in time to test, and further work will be necessary to complete detector manufacturing and evaluate performance. 


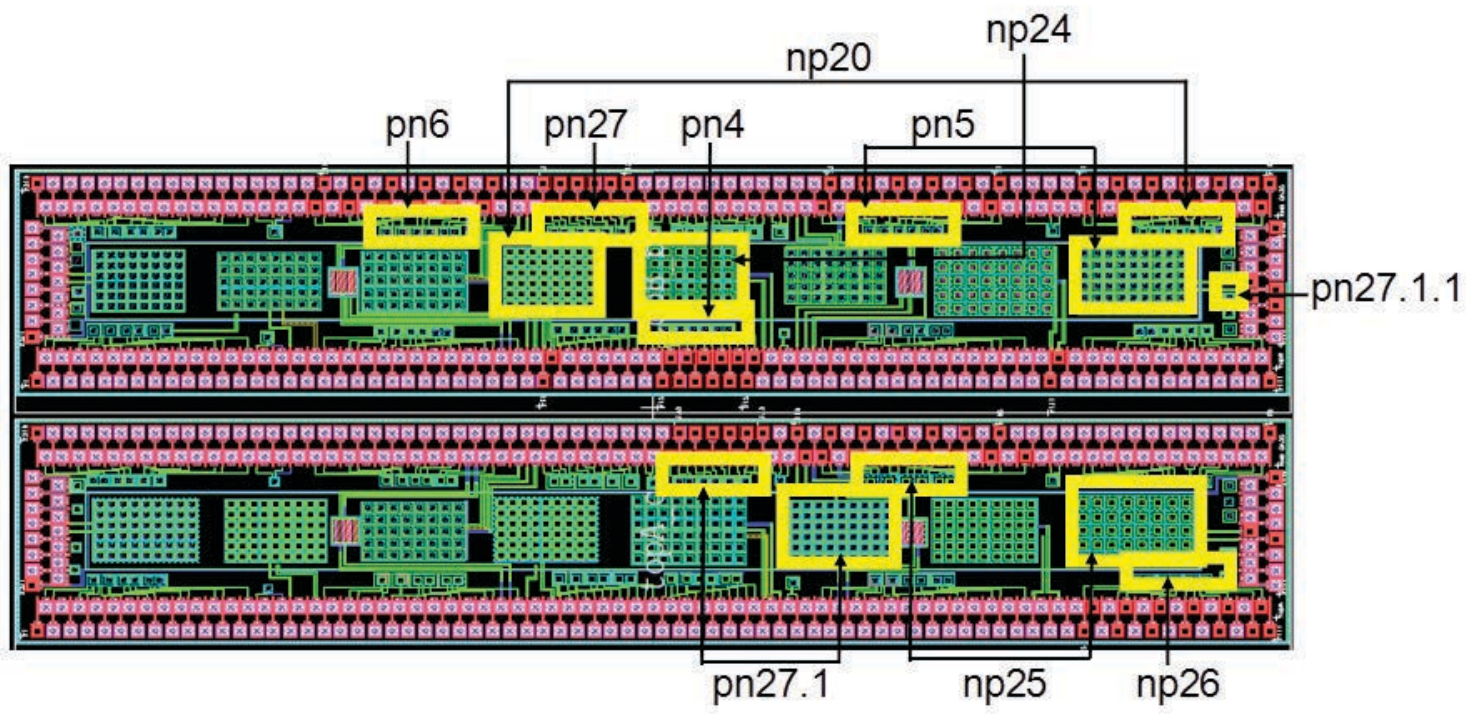

Figure 5. CMOS layout of test structures of Geiger photodiodes and photodiode arrays. The die is $2 \times 5 \mathrm{~mm}$ and is surrounded by 50 -micron contact pads. The designations pn\# and np\# identify the specific pixel design; a sample design is shown in Figure 6. The rectangular shape of the chip allows the maximum number of contact pads, and thus the maximum number of test structures. Table 2 shows RMD measurement of pixel characteristics.

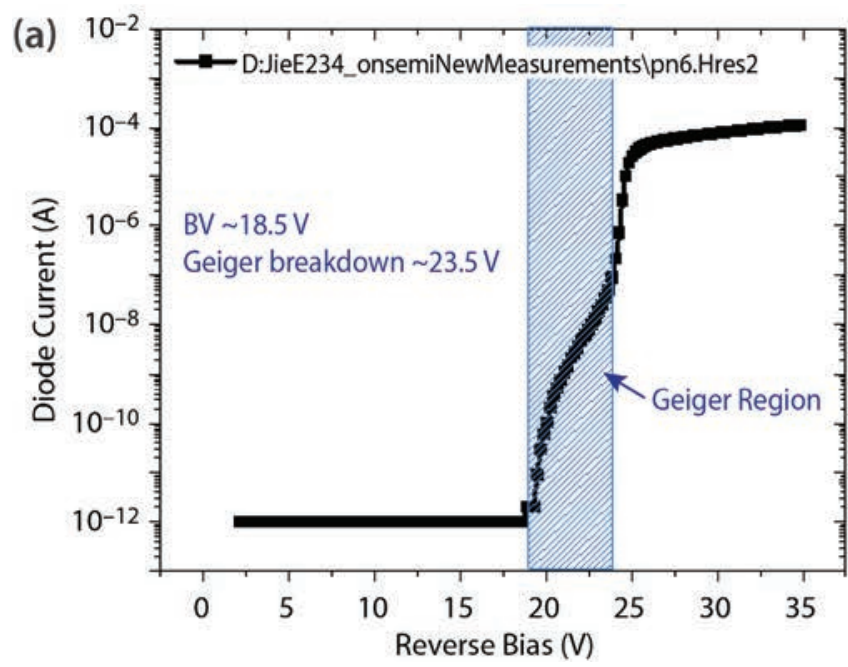

(b)

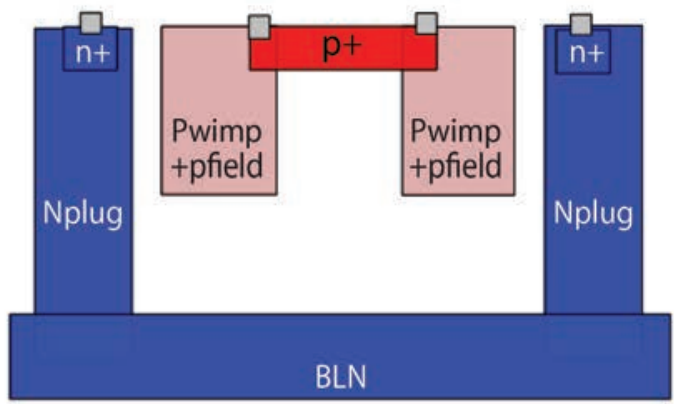

Figure 6. (a) Current-voltage plot indicating Geiger mode operation of pixel design pn6, and (b) the pixel layout

\section{Radiation Detection Comparisons}

We performed many radiation-detection tests to compare the front-side SSPM detection to PMT detection. We performed several laboratory tests with a low-activity ${ }^{137} \mathrm{Cs}, 5.1 \mu \mathrm{Ci}$ button source, recording energy spectra on an Amptek Pocket multichannel analyzer. The PMT was coupled with a Nal:TI scintillator and the SSPM was coupled with CsI:TI. For our low-activity source, we recorded signals at a close distance of $10 \mathrm{~cm}$ to lower integration times.

Table 2. RMD pixel characteristics

\begin{tabular}{|l|l|}
\hline Pixel size & $3 \times 3 \mathrm{~mm}$ \\
\hline Quenching resistance & 139.9 to $143.7 \mathrm{~K}$ ohm \\
\hline Series resistance & $\sim 230 \mathrm{ohm}$ \\
\hline
\end{tabular}




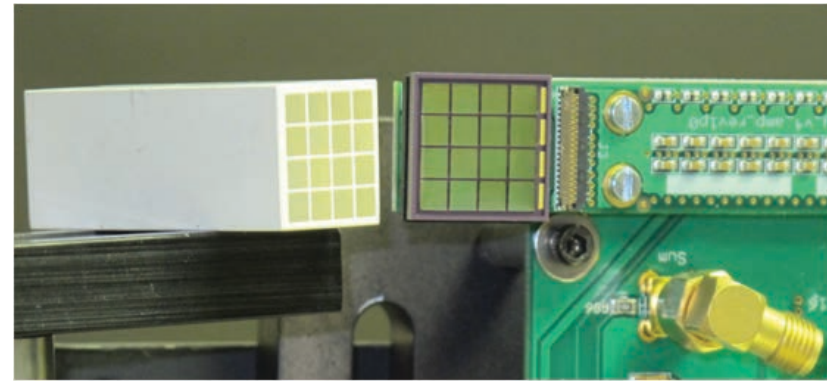

Figure 7. Sample $4 \times 4 \mathrm{Csl}$ :TI scintillator array with $3 \times 3 \mathrm{~mm}$ pixels, $40 \mathrm{~mm}$ long with $4 \times 4$ SSPM readout

Figure 7 shows the SensL SSPM used in our detection comparisons. Some tests were performed with the pixelated scintillator shown here; those results appeared to be susceptible to tight alignment constraints. We felt a better coupling mechanism for pixel alignment was needed to record dependable results.

Figure 8, with monolithic $\mathrm{Csl}$, shows the relative energy spectra in the SSPM, comparing detection of using a $20 \mathrm{~mm}$ thick scintillator to that using a $40 \mathrm{~mm}$ thick Csl:Tl scintillator. The signal counts are normalized to a common integration time of 9000 seconds to show relative signal level over the same integration period. The $20 \mathrm{~mm}$ thick Csl shows better energy resolution, indicated by the narrower FWHM in the energy peak. Shaping time in the amplifier gain stage was tested at $3 \mu \mathrm{s}$ and $6 \mu \mathrm{s}$ for the longish $1.2 \mu \mathrm{s}$ decay Csl:Tl scintillator. The $40 \mathrm{~mm}$ thick Csl detection appears to suffer from detector pileup at either integration time, as described in Knoll (2011).

The background-corrected energy spectra in Figure 9 compare radiation detection ability of the SSPM and the PMT. Their energy resolutions at $662 \mathrm{keV}$ appear to be very similar. The SSPM required more time to build the ${ }^{137} \mathrm{Cs}$ spectrum signature, but the PMT detector suffered significantly higher Compton scattering continua. The Nal scintillator is packaged in an aluminum housing, which may increase the Compton scattering seen with this detector vs the CsI (encapsulated in white epoxy). The high level of the Compton edge in the PMT signal more strongly masks the low-energy $32 \mathrm{keV}{ }^{137} \mathrm{Cs}$ line, while the SSPM required longer integrations to build the signature. The slower signal buildup in the SSPM detector made it difficult to record the very low activity source, such as a ${ }^{40} \mathrm{~K}, 1.42 \mathrm{MeV}$ line of salt substitute, which was seen with several hour-long integrations by the Nal:Tl/PMT detector during project tests.

For the detector performance (Knoll 2010, Quam 2010) in Table 3, we calculate energy resolution, intrinsic detection efficiency, and minimum detectable activity (MDA) as follows:

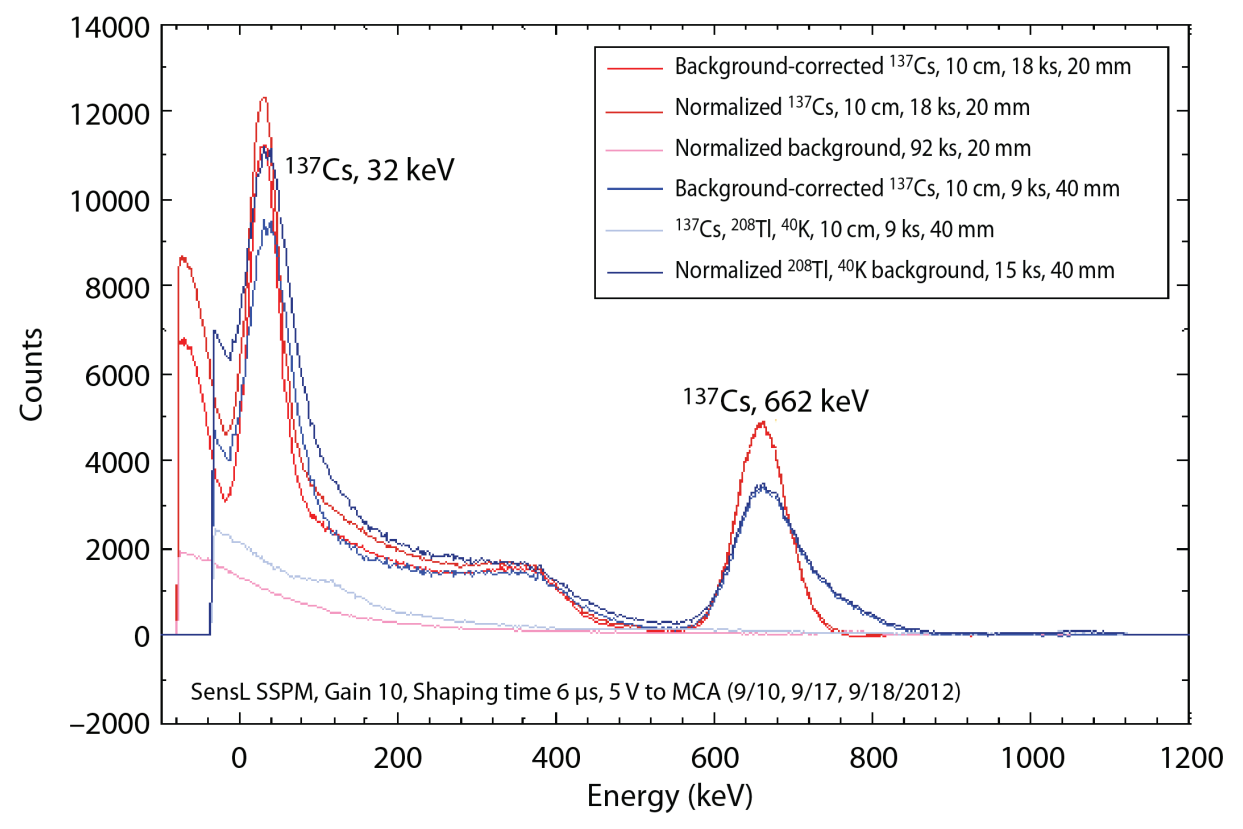

Figure 8. SSPM recording of ${ }^{137} \mathrm{Cs}$ energy spectrum with $20 \mathrm{~mm}$ and $40 \mathrm{~mm} \mathrm{Csl:TI}$ scintillators. The $20 \mathrm{~mm}$ Csl shows better energy resolution. The integration times for signal and background are shown in thousands of seconds (ks). 
Table 3. Radiation detection comparison. MDA at $10 \mathrm{~cm}$ and 1 second. ${ }^{137} \mathrm{Cs}$ photon yield $=0.852$ for $662 \mathrm{keV}$.

\begin{tabular}{|c|c|c|c|c|c|c|c|c|c|}
\hline Isotope & $\begin{array}{c}\text { Energy } \\
(\mathbf{k e V})\end{array}$ & $\begin{array}{c}\text { Activity } \\
(\boldsymbol{\mu} \mathrm{Ci})\end{array}$ & Scintillator & Thickness & $\begin{array}{c}\text { Detector } \\
\text { Area }\left(\mathbf{c m}^{2}\right)\end{array}$ & $\begin{array}{c}\text { Energy } \\
\text { Readout }\end{array}$ & $\begin{array}{c}\text { Peak } \\
\text { Resolution }\end{array}$ & $\begin{array}{c}\text { MDA } \\
\text { Efficiency } \\
(\mathrm{Ci})\end{array}$ \\
\hline${ }^{137} \mathrm{Cs}$ & 662 & 5.1 & $\mathrm{Nal}: \mathrm{Tl}$ & $25.4 \mathrm{~mm}$ & 5.06 & PMT & $9.1 \%$ & 0.110 & $1.66 \mathrm{E}-09$ \\
\hline${ }^{137} \mathrm{Cs}$ & 662 & 5.1 & $\mathrm{Csl}: \mathrm{TI}$ & $20 \mathrm{~mm}$ & 1.44 & SSPM & $11.3 \%$ & 0.120 & $4.92 \mathrm{E}-09$ \\
\hline${ }^{137} \mathrm{Cs}$ & 662 & 5.1 & $\mathrm{Csl}: \mathrm{Tl}$ & $40 \mathrm{~mm}$ & 1.44 & SSPM & $14.5 \%$ & 0.095 & $9.57 \mathrm{E}-09$ \\
\hline
\end{tabular}

$$
\begin{aligned}
& \text { Energy Resolution }=\text { FWHM/Energy Peak, } \\
& \text { Peak Efficiency; } \varepsilon_{\mathrm{ip}}=\left[\mathrm{N}_{\mathrm{i}} / \mathrm{t}_{\mathrm{i}}\right] / \\
& \quad[\text { activity } \times 3.7 \times 1010 \times \text { ield } \times(\Omega / 4 \pi], \\
& M D A \text { for } 3 \sigma_{\mathrm{b}}=\left[3 \sigma_{\mathrm{b}} /(\mathrm{t}) 1 / 2\right] \times 4 \pi \\
& \quad \times\left[\mathrm{d}^{2} /\left(\varepsilon_{\mathrm{ip}} \times \mathrm{A}_{\mathrm{d}} \times 3.7 \times 1010 \times \text { yield }\right)\right] .
\end{aligned}
$$

Intrinsic peak detection efficiency $\left(\varepsilon_{\mathrm{ip}}\right)$ is pulses per second detected per gamma incident on the detector face. $\mathrm{N}_{\mathrm{i}}$ is the total number of pulses detected above background in the energy peak full width to $1 / 20$ th of the peak count level. Signal integration time $\left(t_{i}\right)$ was varied by detector; we integrated signals long enough to record over 1000 counts in the peak. Activity is given in curies $(\mathrm{Ci}), 1 \mathrm{Ci}=3.7 \times 10^{10}$ (disintegrations per second); yield for ${ }^{137} \mathrm{Cs} 662 \mathrm{keV}$ is 0.852 gamma photons emitted per disintegration. Detector collection solid angle $\Omega \sim=A_{d} / d^{2}$; detector area $\left(A_{d}\right)$ divided by distance from source to detector face; $d=10 \mathrm{~cm}$ for each detector.
(1) MDA is presented in $\mu \mathrm{Ci}$ at distance d and time t; $\sigma_{b}$, the standard deviation of the background counts per

(2) second, is given by the square root of the number of counts in the background counts for Gaussian noise distribution. We specify MDA at $3 \sigma_{b}$ for false alarm (3) rejection of $99.7 \%$.

\section{Conclusion}

For this project, the SSPM showed better optical and radiation sensitivity than PMTs used. The roomtemperature, front-illuminated SSPM tested had slightly more noise in signal levels, reflected in the somewhat worse energy resolution and MDA, but the high sensitivity and gain of the SSPM show better overall peak detection efficiency. The overall sensitivity to lower signals appears to allow the SSPM to record better statistics in the lower energy range below a few hundred keV. The SSPM provided adequate identification of the $32 \mathrm{keV}$ line of the ${ }^{137} \mathrm{Cs}$

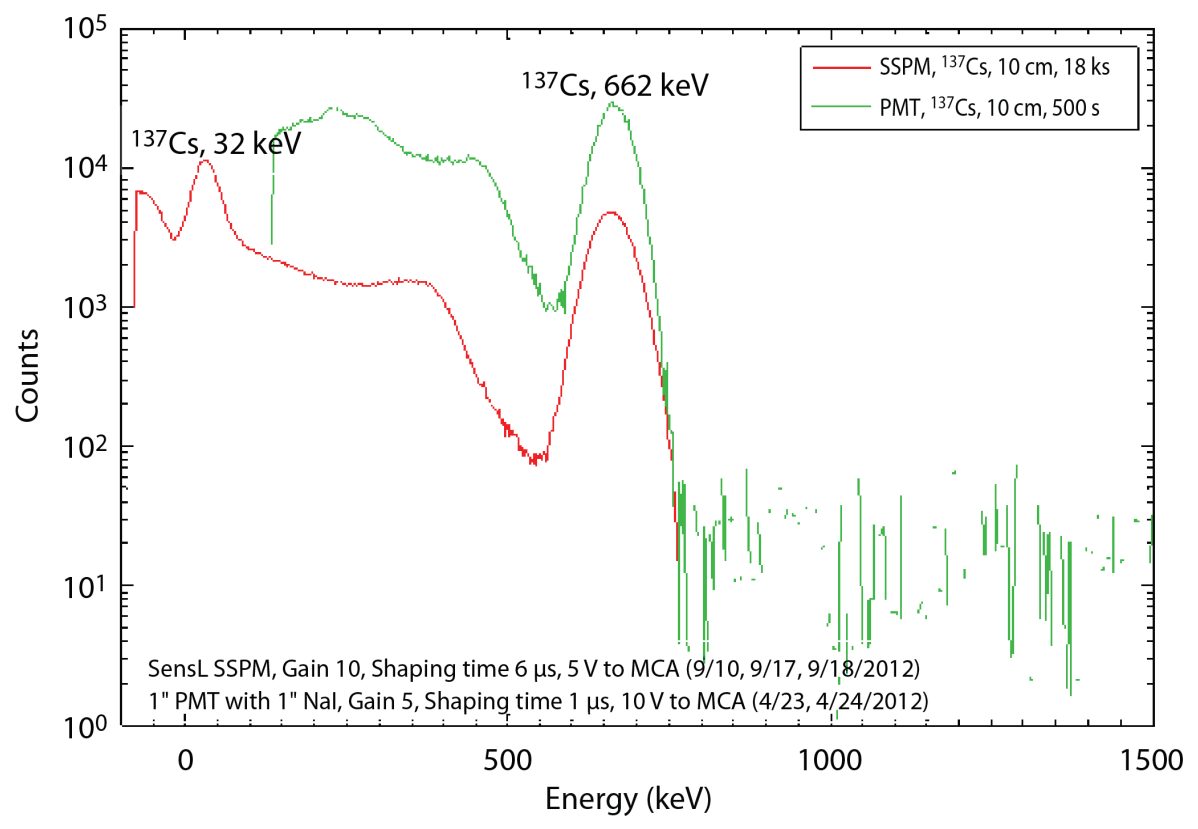

Figure 9. Energy spectra recordings comparing SSPM results with PMT 
source. For this comparison the PMT detector had 3.5X larger detector face area, providing an advantage in reporting energy resolution and MDA. This 1" PMT also had the highest sensitivity when compared to the $1 / 2$ " and 2" PMTs. This further emphasizes the impressive peak efficiency of the SSPM. We have not yet received the BT components from RMD, but hope to report their performance in the coming months.

SSPMs will surely develop into an important tool for radiation detection. They show promise in areas of active field surveillance, and in real-time dosimeters and remote monitoring devices. Using SSPMs will potentially reduce package size and power requirements in field deployment systems. Future work may investigate the performance of cooled SSPM detectors, as we suspect sensor cooling to $\sim 10^{\circ} \mathrm{C}$ would greatly reduce thermal noise and improve sensitivity.

\section{Acknowledgments}

We would like to thank Ronald Guise of the NSTec Remote Sensing Laboratory for supplying radiation detection components for detector development comparisons, and Bill Quam of NSTec's Special Technologies Laboratory for sharing radiation detection techniques.

\section{References}

Baker, S., C. Stapels, J. A. Green, R. E. Guise, J. A. Young, B. Stokes, E. Wendelberger, L. Franks, "SSPM scintillator readout for gamma radiation detection," Proc. SPIE 8144 (2011) 814400.

Baker, S., C. Stapels, L. Franks, J. A. Green, R. Guise, J. Young, "Back-thinned silicon radiation detector readout," Site-Directed Research and Development, FY 2011, National Security Technologies, LLC, Las Vegas, Nevada, 2012, 47-54.

Douence, V. M., Y. Bai, H. Durmus, A. B. Joshi, P.-O. Pettersson, D. Sahoo, K. Kwiatkowski, N. S. P. King, C. Morris, M. D. Wilke, "Hybrid image sensor with multiple on-chip frame storage for ultrahigh-speed imaging,"

Proc. SPIE 5580 (2005) 226-234.
Knoll, G. F., Radiation Detection and Measurement, 4th ed., Wiley \& Sons, New York, 2010, 118, 321.

Knoll, G. F., J. C. Engdahl, Radiation Detection and Measurement, Short Course Notes, NSTec, 2011.

Konnoff, D. C., T. K. Plant, E. Shiner, "SSPM based radiation sensing: Preliminary laboratory and clinical results," Radiation Measurements 46 (2011) 76-87.

Mukhopadhyay, S., P. Guss, R. Maurer, "Current trends in gamma radiation detection for radiological emergency response," Proc. SPIE 8144 (2011) 81440K.

Quam, B., Nuclear Radiation Detection Technology, 6th ed., National Security Technologies, LLC, Special Technologies Laboratory, 2010.

Stapels, C., P. Barton, E. B. Johnson, D. K. Wehe, P. Dokhale, K. Shah, F. L. Augustine, J. F. Christian, "Recent developments with CMOS SSPM photodetectors," Nucl. Instrum. Methods Phys. Res. A 610, 1 (2009) 145-149. 


\section{Pulse-Mode SPIRAL NeUtron Detector}

LAO-34-12 | CONTINUED FROM FY 2011 | YEAR 2 OF 2

Michael Berninger, ${ }_{1}^{1, a}$ Morris Kaufman, ${ }^{a}$ Brian Cox $_{1}{ }^{b}$ Jesus Castaneda,${ }^{a}$ Crystal Maestas, $^{a}$ and Robert O'Brien ${ }^{c}$

The spiral neutron detector (SND), developed in FY 2011, was built to detect thermal neutron radiation from special nuclear materials. The SND used ${ }^{10} \mathrm{~B}$, a replacement for ${ }^{3} \mathrm{He}$, and employed a scalable concentric spiral design that uses thin $(\sim 1 \mathrm{~mm})$ spiral layers, multiple windings, and large surface areas to enhance the overall detection sensitivity. However, the SND was not optimized for low thermal neutron fluxes and where radiation noise can reduce the signal noise ratio. To improve upon the design in the "pulse mode," we investigated how to incorporate wire mesh layers into the SND. A wire grid can improve the time resolution of the detector and, in the proportional regime, enables the intrinsic amplification of weak signals. Two gridded spiral detectors were successfully built but not tested. However, preliminary tests with a planar configuration of the spiral detector components show that when a mesh is used, pulse heights can be amplified $14 \%$ over the ungridded case.

${ }^{1}$ berninmj@nv.doe.gov, 505-663-2032

${ }^{a}$ Los Alamos Operations; ${ }^{b}$ Former Los Alamos Operations employee; ${ }^{c}$ University of Nevada, Las Vegas

\section{Background}

The fundamental mode of the ungridded spiral neutron detector (SND) (Berninger 2012) was the "pulse mode" in which each unit of radiation that interacted in the detector was counted. These radiation interactions occurred when a ${ }^{10} \mathrm{~B}$ atom, excited by a thermal neutron capture, emitted energetic alpha and ${ }^{7} \mathrm{Li}$ particles that ionized a gas along a track in the SND. The time resolution, energy resolution, and signal-to-noise ratio in the SND were limited by a number of factors. The ionization clouds created separate electron and ion signal pulses on the opposite foil electrodes, but the pulses superimposed in the counting circuit, and they were measured as a single time-stretched ( $\sim \mathrm{ms})$ pulse. This superposition lowered the time resolution of the detector. The energy resolution of the detector was diminished because the amplitude and timing of the ion pulses depended on the location and directions of the ionization tracks in the detector. Additionally, the distance between the electrodes was large enough to prohibit the SND from operating in the so-called

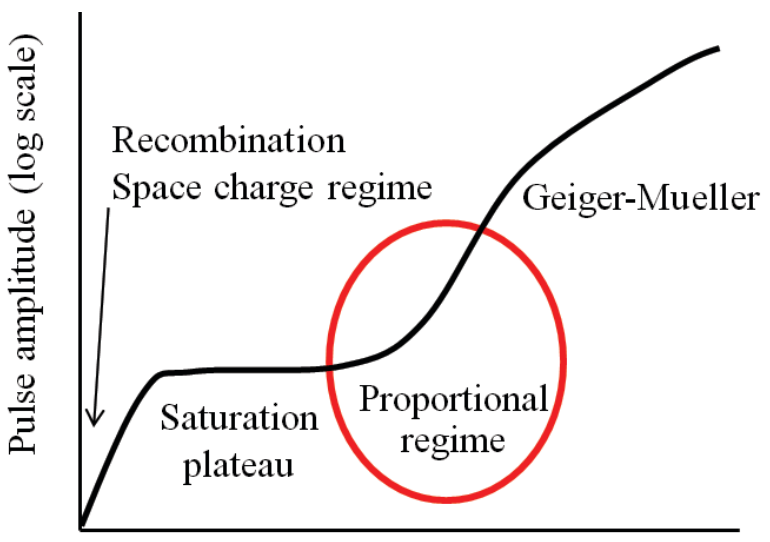

Applied voltage

Figure 1. A schematic plot of voltage amplitude vs applied voltage for an event that ionizes a gas and the electron cloud is measured as an electrical pulse height. The proportional regime occurs with applied voltages above the plateau that signifies minimal effects from charge recombination.

"proportional" regime (Figure 1); thus, the ability of the detector to isolate the alpha signal from environments with significant background radiation noise was limited. 
Our objective was to improve the SND's ability to measure thermal neutron signals in the pulse mode by converting the SND to a gridded detector. Wire grids (or meshes), long known to enhance the operation of vacuum tubes, photomultiplier tubes, and ion chambers for specific applications (Sharpe 1964, Knoll 2010), were installed in the SND to improve the detector's time response and allow the detector to operate in the proportional regime.

The primary advantage of placing a grid between the electrodes of the detector is that it creates a virtual ground. This ground plane improves the detector in two ways: it screens the ion component of the signal, and it masks the initial location of the source track due to the fact that the signal does not start to form on the charge-collection electrode until the charge cloud crosses the grid. The net result is a faster response time and some modification of the signal bandwidth that improves counting statistics. The counting efficiency of the detector is often reduced by the lower transparency of the mesh.

Another advantage is that the close proximity of the mesh and collector can magnify the effect of the voltage bias used to collect the signal, allowing the detector to better distinguish the pulses from background radiation and other sources of noise. When the fields are strong enough, the drifting free electrons obtain enough kinetic energy to ionize additional gas atoms, creating the conditions for an electron avalanche, which amplifies (multiplies) the amplitude of the charge pulse and allows the detector to operate in the proportional regime.

The SND project was motivated by the need to detect neutrons with a material alternative to ${ }^{3} \mathrm{He}$, of which there is a dwindling supply. Candidates for a replacement material were ${ }^{6} \mathrm{Li},{ }^{10} \mathrm{~B}$, and $\mathrm{Gd}$. ${ }^{6} \mathrm{Li}$ and $\mathrm{Gd}$ are typically used in powder form and doped into scintillators that have trouble discriminating gamma rays. Although it is not as efficient as ${ }^{6} \mathrm{Li}$ and $\mathrm{Gd}$ for capturing neutrons, ${ }^{10} \mathrm{~B}$ was chosen because it is readily available from suppliers and can be coated onto surfaces with different geometries and porosities.
The principal goal of creating a spiral-shaped neutron detector rather than simply stacking planar layers is to increase the amount of enriched ${ }^{10} \mathrm{~B}$ in a volume. For a spiral whose foils measure $5 \times 33 \mathrm{~cm}$ where one foil is coated on two sides with $1 \mu \mathrm{m}$ thick layers of ${ }^{10} \mathrm{~B}$, accounting for losses at the edge, we obtain $67 \mathrm{mg}$ of ${ }^{10} \mathrm{~B}$. A detector constructed of stacked planar disks with the same layer spacing and the same cylindrical volume would contain $60 \mathrm{mg}$ of ${ }^{10} \mathrm{~B}$. Therefore, a spiral could ideally include $10 \%$ more boron than a cylindrical detector with stacking planes.

\section{Theory}

When a ${ }^{10} \mathrm{~B}$ atom captures a thermal neutron, it undergoes a ${ }^{10} B(n, \alpha)$ reaction (cross section of 3840 barns) (Sutton 1947, Garber 1976). The ${ }^{10} \mathrm{~B}$ nucleus becomes ${ }^{11} \mathrm{~B}$, which is highly unstable and quickly decays with the following process: ${ }^{10} \mathrm{~B}+{ }^{1} \mathrm{n} \rightarrow\left({ }^{7} \mathrm{Li}+\gamma\right)+\alpha$. The $\alpha$-particle has energy $E_{\alpha}=1.47 \mathrm{MeV}$, the ${ }^{7} \mathrm{Li}$ atom has $840 \mathrm{keV}$ kinetic energy, and the gamma has $480 \mathrm{keV}$ of energy (McGregor 2000).

The neutron capture is detected when the alpha and the ${ }^{7} \mathrm{Li}$ particle ionizes a gas such as argon (Ar). Due to its small cross section with the gas, the gamma passes largely undetected through the detector and contributes only to the background signal. It takes $25.4 \mathrm{eV}$ of energy to ionize an Ar atom (Fermi 1949). The number of electrons liberated in the ionization process is directly proportional to the energy of the $\alpha$-particle or ${ }^{7} \mathrm{Li}$. A $1.47 \alpha$-particle will generate about 40,000 electron and ion pairs if all of its energy goes into ionizing a volume of Ar gas. This charge produces a triangular 1 nA current pulse that typically flows through a resistor and generates a voltage pulse in the counting circuit. The amplitude of the voltage pulse, proportional to the energy deposited in the gas, is then recorded with a pulse-height spectrum.

To identify the proper wire grid for the chamber, we used a gridded ion chamber theory (Bunemann 1949) to analyze the SND with various grid geometries and different voltage biases on opposite sides of the grid. The graph in Figure 2 plots the detector 


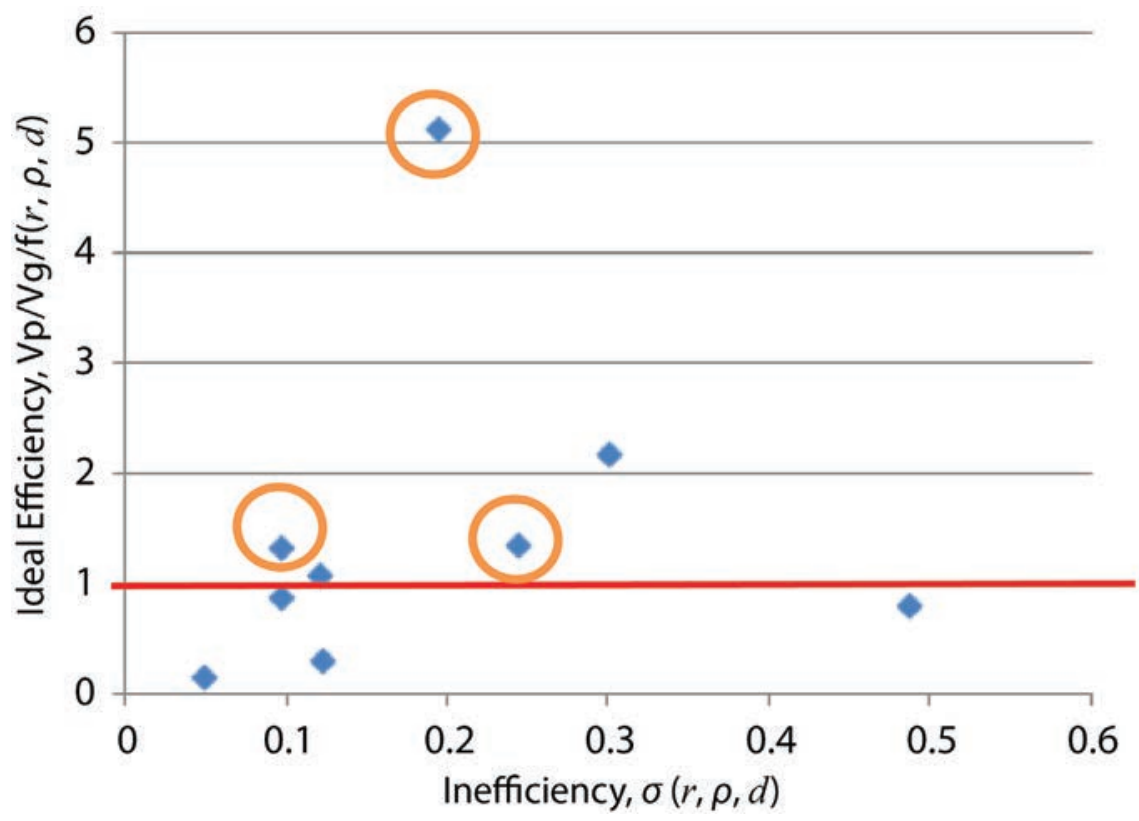

Figure 2. A plot of the fieldline efficiency vs the geometric inefficiency in the SND for a number of voltage ratios and grid geometries. The red line is the threshold for achieving optimal field-line efficiency. The orange circles represent the cases where the SND can minimize the inefficiency of the detector while still achieving the proportional regime. inefficiency-how much signal is blocked by a particular grid geometry-versus the efficiency of the electrical fields for causing a fraction of the electrons to bypass the grid. Each point on the graph represents the efficiency-state of the detector for a different combination of parameters: mesh-hole size, wire diameter, distance between the mesh and collector, and ratio of the voltages on opposite sides of the grid. To operate in the proportional regime, it is necessary for the electrical fields to have a magnitude $E \geq 1 \mathrm{kV} / \mathrm{mm}$. The orange circles in Figure 2 represent the specific cases where the efficiency of the detector is maximized and the field strength between the mesh and collector is greater than $1 \mathrm{kV} / \mathrm{mm}$. Using these more optimal parameters, we built detectors with grids whose predicted inefficiency was $\sigma=0.1$, the voltage ratio was 1.5, and the field strength would be about $1.2 \mathrm{kV} / \mathrm{mm}$.

\section{Project}

The goals were to determine how to best incorporate wire mesh layers in a spiral thermal-neutron detector, and then verify improvements with the detector performance.

After the optimized mesh parameters had been identified with the gridded detector theory, we prepared two grids. The grids were fabricated by
Buckbee-Mears Co. using nickel wire. The first mesh had a square-hole pattern with $0.06 \mathrm{~mm}$ diameter wires and holes $0.5 \mathrm{~mm}$ on each side; it was $80 \%$ transparent. When placed $1 \mathrm{~mm}$ from the collector and biased with a voltage ratio of 1.5 such that the field strength $E=1.2 \mathrm{kV} / \mathrm{mm}$, the predicted inefficiency was $\sigma=0.1$. The second mesh also had a square-hole pattern with $0.11 \mathrm{~mm}$ diameter wires and holes $1.2 \mathrm{~mm}$ on each side, but this mesh was $83 \%$ transparent. When located $1 \mathrm{~mm}$ from the collector with a bias ratio of 1.5 , the field strength was also $E=1.2 \mathrm{kV} / \mathrm{mm}$, but the gridded detector theory predicted the detector would have an inefficiency of $\sigma=0.24$.

The gridded spiral was assembled as follows: Two swatches of mesh were cut $30 \mathrm{~cm}$ long by $5 \mathrm{~cm}$ wide. Two titanium foils $33 \mathrm{~cm}$ long by $5 \mathrm{~cm}$ wide and $50 \mu \mathrm{m}$ thick were also prepared for each spiral. A wire lead was soldered to each of the foils and meshes. One of the titanium foils remained bare. The other titanium foil was coated (by Ktech, Inc.) with a layer of enriched ${ }^{10} \mathrm{~B}$ on two sides, using a $300^{\circ} \mathrm{C}$ electron-beam coating technique. Three different thicknesses were deposited: $225 \mathrm{~nm}, 500 \mathrm{~nm}$, and $1 \mu \mathrm{m}$. This report does not review data collected with the different thicknesses of the ${ }^{10} \mathrm{~B}$ coatings. We also experimented with coating boron onto different metallic substrates: titanium, aluminum, and 

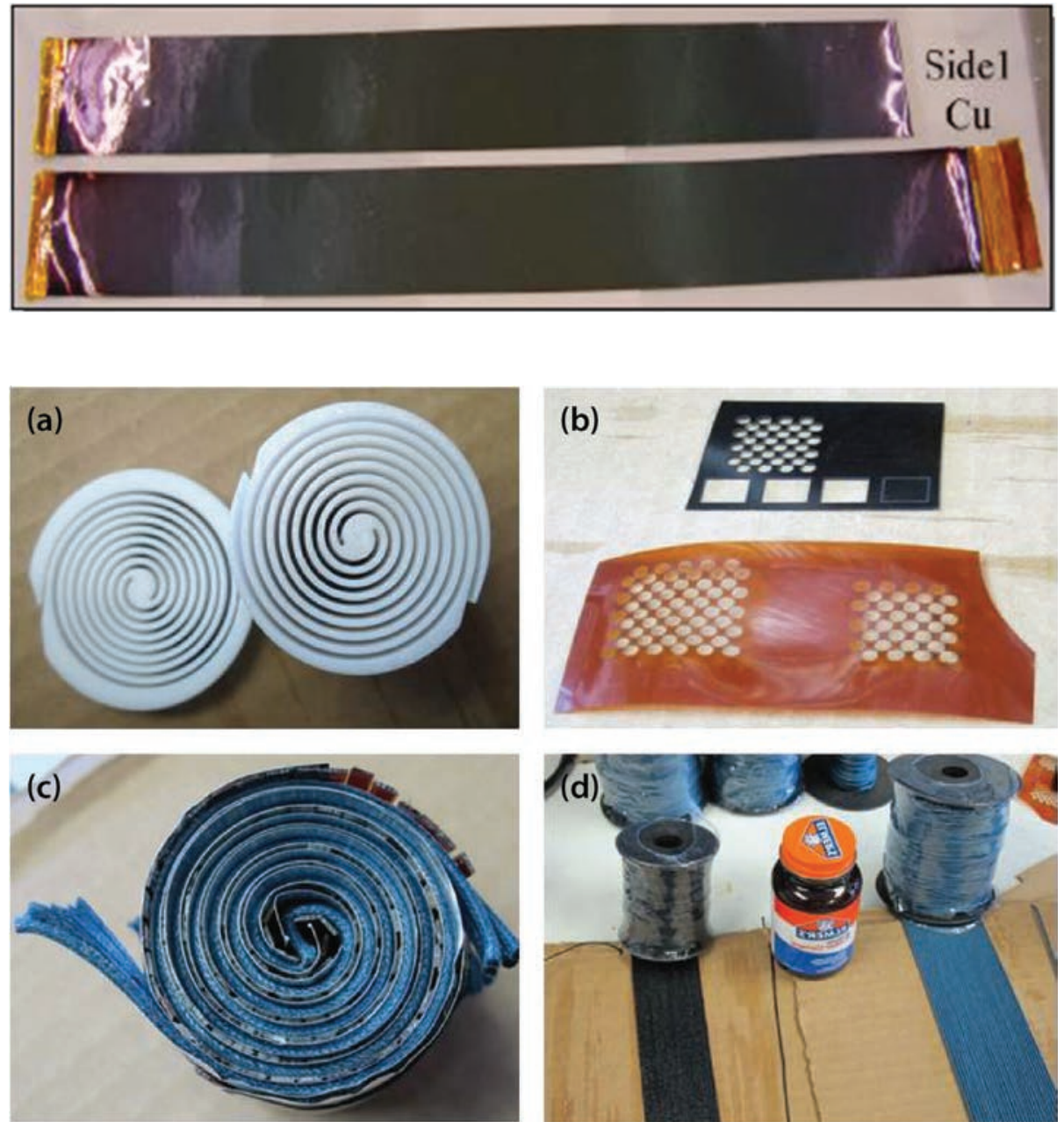

Figure 3. The copper foils

coated with ${ }^{10} \mathrm{~B}$

Figure 4. (a) Spiral forms, (b) Kapton spacers with array of holes, (c) Kapton spacer strips in a "bamboo blind" configuration, and (d) ribbons copper. Each metal proved to be a good substrate for the boron. The coatings had a dark grey or black appearance (Figure 3), and they did not flake in a $90^{\circ}$ bend test, but they could be scratched or rubbed off the substrate if enough force was applied. The copper and aluminum foils tended to be soft and developed numerous dimple and wrinkle defects. In a highvoltage application, these defects collect field lines that increase the chances of an uncontrolled electrical breakdown in the detector gas that would eclipse the neutron signal. The titanium foil was stiffer and remained very smooth. Once spiraled, the titanium had a tendency to sag more, due to its higher tensile strength.

A number of spacer techniques were attempted (Figure 4): spiral frames, permanent spacers with arrays of holes, small strips of Kapton laid out side by side like a "bamboo blind," and ribbons made with shoestring. The most successful technique used three layers of shoestring ribbons. The other techniques failed for various reasons. A number of rapid prototype 3-D printers were employed to print spiral frames into which the foils could be slid, but the edges were too sharp and would have scraped the boron coating off the foil. Two permanent $0.5 \mathrm{~mm}$ thick Kapton sheets were fabricated with close-packed arrays of 0.25- and 0.125-inch holes. The Kapton, too brittle for the tight interior curves of the spiral, began to delaminate and poke holes through the mesh. Small Kapton strips, $1 \mathrm{~mm}$ thick, were laid out in a row and spiraled with the mesh and a shoestring ribbon. These spirals tended to be mechanically unstable, and the mesh sagged and collapsed once the strips were removed.

Using ribbons as spacers, we successfully built two spiral detectors with two mesh layers. Three ribbons 

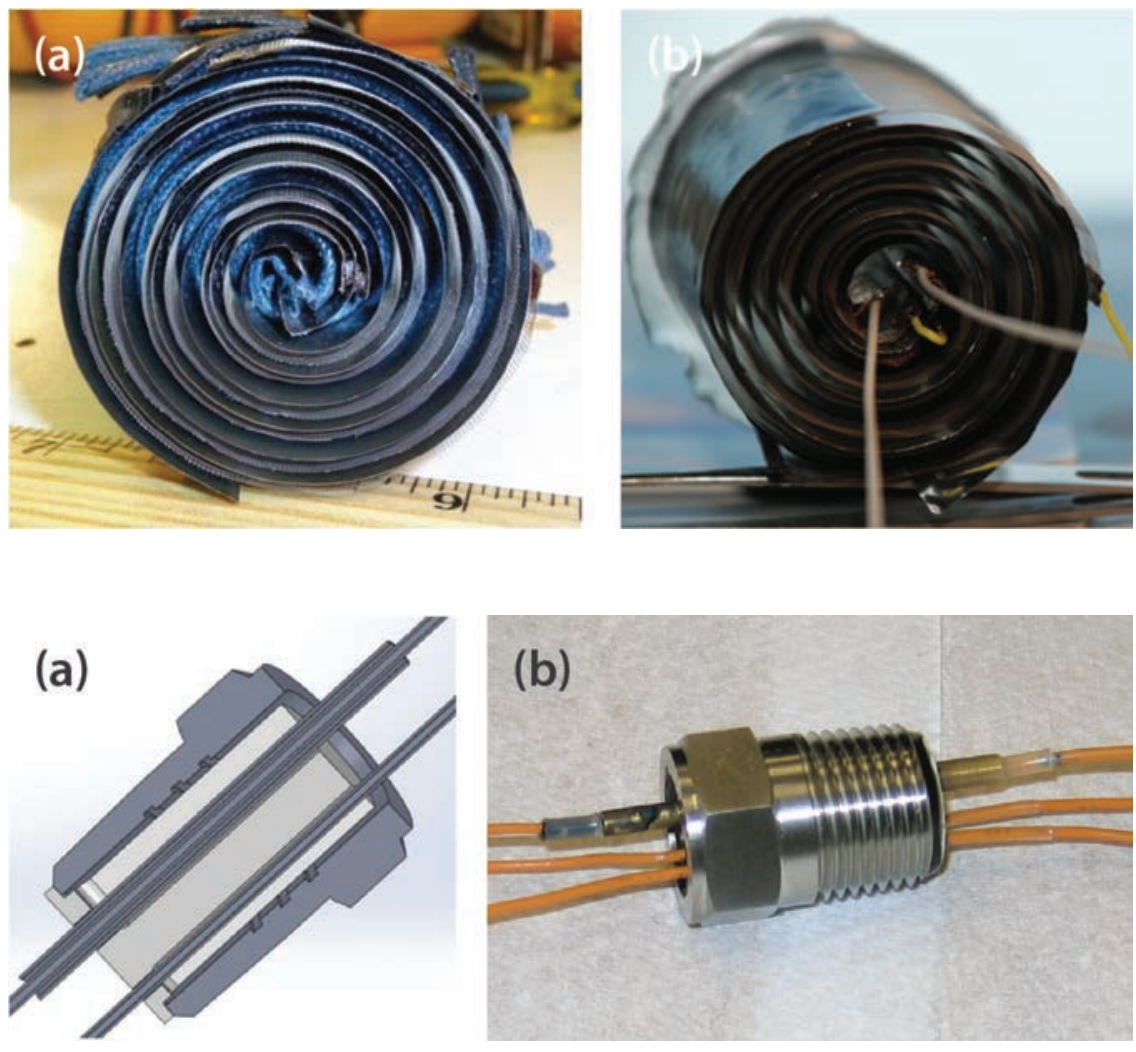

Figure 5. The spiral assembly at two different stages: (a) after $1 / 3$ of the spacer ribbon was removed and (b) after all the ribbon was removed. The uniformity of the spacing is remarkable considering the complexity of the layering and the lack of mechanical strength in the mesh.

Figure 6. (a) A cutaway view of the high-voltage feedthrough. The housing was grounded and the center of the tube was plugged with Macor. (b) A feedthrough after construction. were necessary: one was $66 \mathrm{~cm}$ long by $5 \mathrm{~cm}$ wide and $2 \mathrm{~mm}$ thick; the other two were $33 \mathrm{~cm}$ long by $5 \mathrm{~cm}$ wide and $1 \mathrm{~mm}$ thick. The spiral was created by stacking the foils and meshes with the spacers and clamping them together in a spindle. The stack was then wound around the spindle and secured with tape. After removing the spiraled assembly from the spindle, the spacer ribbon was removed, string by string, until about half the string was removed (Figure 5a). Then the "empty" end of the spiral was epoxied to a depth of about $3 \mathrm{~mm}$. The remaining ribbon was removed, and the other end epoxied (Figure 5b). The distance between boron-coated foils and the mesh averaged $1.8 \mathrm{~mm}$. The distance between the mesh and the collector foil was about $0.8 \mathrm{~mm}$ on average. The total outside diameter of the detector was about $5 \mathrm{~cm}$.

The epoxy used on the SND needed to be mechanically strong enough to hold the foils and mesh together, and dielectrically strong enough to withstand biases greater than $1 \mathrm{kV}$ without arcing and breaking down. The epoxy also had to be a dielectric insulator with resistivities on the order of $10^{14} \Omega$-cm or greater to minimize leakage currents below a satisfactory level. At the time that the first gridded SNDs were fabricated, Duralco 4461 epoxy appeared to be the only epoxy that could satisfy all the mechanical and dielectric requirements. (It had a dielectric strength of $18 \mathrm{k} \Omega / \mathrm{mm}$ with reputed resistivity of $10^{13} \Omega$-cm; however, measurements with an electrometer measured the resistivity at $\geq 1.6 \times 10^{14} \Omega$-cm.) Since building these detectors, we have identified another epoxy, Aremco 2318 (dielectric strength $18 \mathrm{k} \Omega / \mathrm{mm}$ with resistivity $10^{15}$ $\Omega-\mathrm{cm})$, that could potentially be a better candidate to use in building the detectors. The primary drawback with using epoxies is that variable mixing and curing conditions can cause the epoxy properties to change significantly from one batch to the next.

The SND is not structurally sensitive to high pressure; yet, the chamber around the SND must be able to withstand pressures up to 250 psi (this includes a safety margin-operating pressures were below 200 psi). The challenge with building a high-pressure 
chamber was to design and test a high-voltage feedthrough (Figure 6) that would hold the required pressure and meet stringent electrical requirements for minimizing leakage current with its associated noise. The feedthrough materials were required to have a dielectric strength of $19 \mathrm{k} \Omega / \mathrm{mm}$ with resistivity $10^{16} \Omega-\mathrm{cm}$. Two materials satisfied these requirements: Aremco 2315 epoxy $(19 \mathrm{k} \Omega / \mathrm{mm}$ with resistivity $10^{16} \Omega-\mathrm{cm}$ ) and Corning's Macor ceramic (31 $\mathrm{k} \Omega / \mathrm{mm}$ with resistivity $10^{16} \Omega$-cm). Wire-to-wire distances were optimized to reduce field strengths on the metal surfaces and to provide additional noise shielding; the feedthrough was designed with a guard ring. In Figure $6 a$, the light grey plug was made of Macor, the guard-ring tube was made of brass, the gaps were backfilled with Aremco epoxy, and the housing was stainless steel.

Microphonic noise is electrical noise caused by mechanical vibrations. Gridded chambers are especially prone to microphonic noise because the meshes have less mechanical stability than continuous foils, and they tend to vibrate like a drumhead when the ion chamber experiences even slight mechanical disturbances (footsteps and sounds). These vibrations cause the electrical fields in the detector to fluctuate, which in turn appears as low-frequency noise pulses on the counting circuit. This form of noise is mitigated in the SND in the following ways: (1) the long narrow shape of the foils causes the energy in the mechanical vibrations to be weighted with the lower frequencies that are more easily filtered, (2) the vibration amplitudes are damped due to the tensioning caused by the spiral curvature, and (3) the long edges of the foils are fixed with epoxy. Microphonic noise was further diminished by placing the ion chamber on a piece of foam.

\section{Preliminary Tests}

Technical difficulties arose while constructing the ion chamber, and we were unable to collect data with the gridded SNDs, but preliminary tests on the detector components prior to spiraling showed that the gridded SND can operate in the proportional regime.

In these tests, the SND foils and meshes were laid out in a flat configuration inside a box ion chamber (Figure 7). The mesh with larger transparency and holes, $\sigma=0.24$, was installed in the detector in a stainless steel frame. Different thicknesses of G-10 spacers (dielectric strength $22 \mathrm{kV} / \mathrm{mm}$ and resistivity $10^{16} \Omega$-cm) were used to place the mesh at different distances between the ${ }^{10} \mathrm{~B}$-coated ( $500 \mathrm{~nm}$ thick) foil and the uncoated signal collector. The data were collected with the $2 \mathrm{Ci}$ PuBe source in Dr. Culbreth's laboratory at the University of Nevada, Las Vegas.

A sketch of the electrical layout is shown in Figure 8. The detector was powered with a series of $300 \mathrm{~V}$ batteries (Eveready 493 NEDA 722). Great care was taken to design a power-supply circuit that would not fail if a short circuit occurred in the experiment. It was necessary to select passive electrical components that could withstand up to 900 VDC. An ORTEC $142 \mathrm{IH}$ preamplifier was used to precondition the signals because the pulses typically had a current of about $1 \mathrm{nA}$, too small for a standard amplifier to amplify.
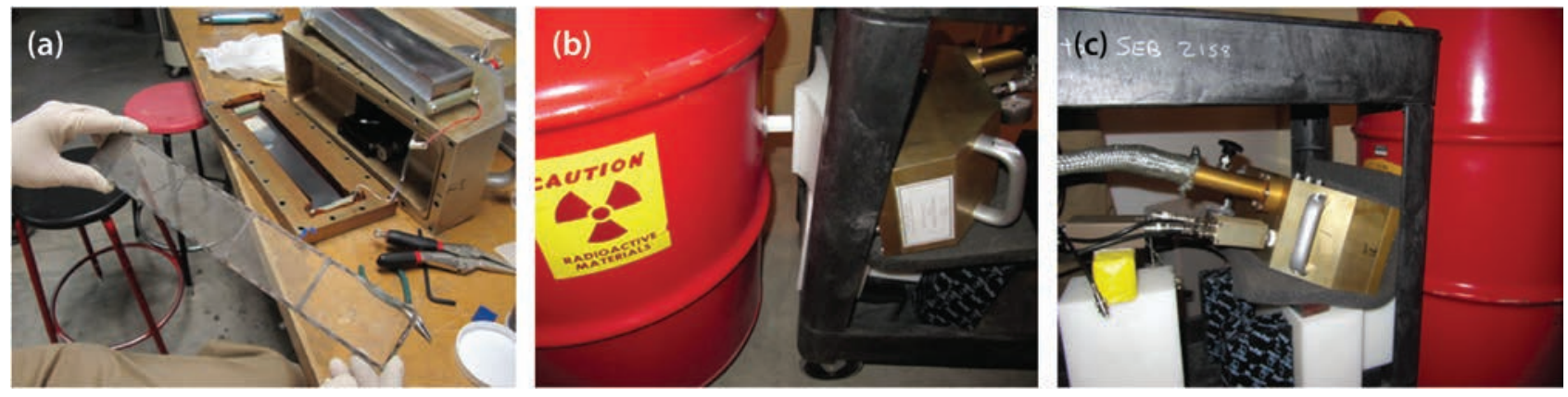

Figure 7. (a) The box chamber is seen with interior exposed. The mesh was installed inside a stainless steel frame. (b) The detector was exposed to the source through a $25.4 \mathrm{~mm}$ diameter porthole. (c) The detector was placed on foam, and moderator blocks were stacked nearby to thermalize the fast neutrons from the PuBe source. 


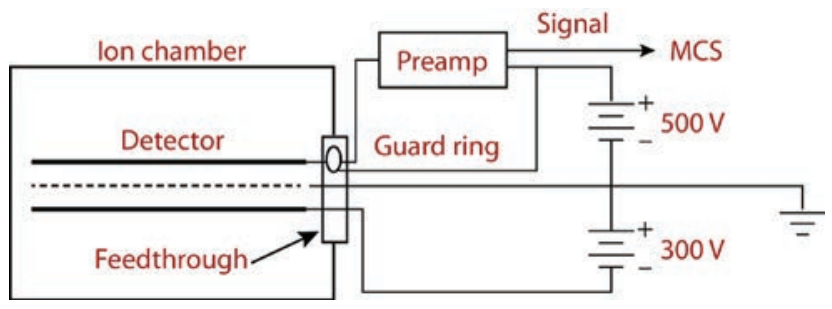

Figure 8. A simplified schematic of the wiring diagram for the gridded chamber

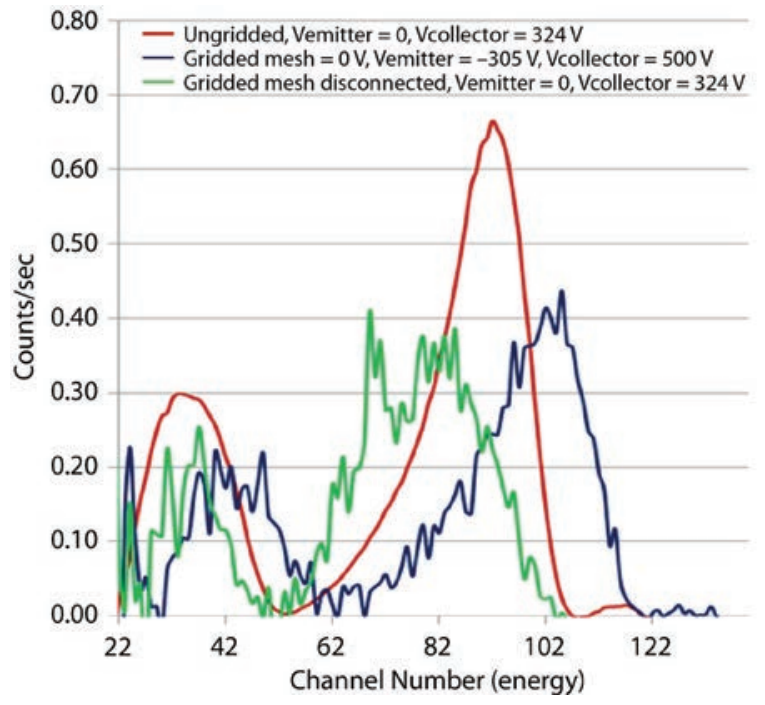

Figure 9. Pulse-height data without the mesh (red) were collected prior to installing the grid. A pulse-height spectrum (green) was collected after the grid was installed, but only the emitter and collector were biased; the mesh wire was left disconnected. Finally a pulse-height spectrum (blue) was collected with the mesh at ground.

A Canberra digital spectrum analyzer (DSA-1000) with a multichannel scaler (MCS) tallied the pulse-height spectrum. Data were displayed on a laptop.

To better demonstrate the effects of adding the mesh to the neutron detector, pulse-height data were collected before and after installing the grid (Figure 9). The ungridded pulse-height spectrum (red line) was a reference spectrum where the emitter and collector were biased with $324 \mathrm{~V}$ (emitter at the ground potential). After the mesh was installed $1.5 \mathrm{~mm}$ away from the emitter (boron-coated foil) and $0.81 \mathrm{~mm}$ away from the collector, two sets of data were collected: first (green line) the detector was biased as in the ungridded case, and second (blue line) the detector had a more optimal (but not

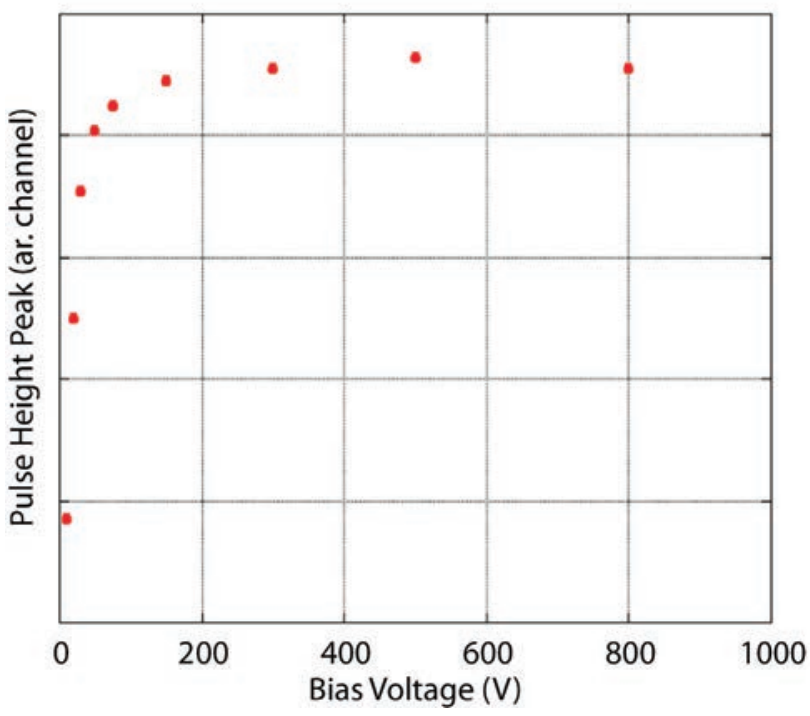

Figure 10. The locations of the peak channel for a range of biases without a mesh installed. The $y$-axis scales with the amplifier gain in the MCS unit; therefore, it is arbitrary. However, the voltage dependence of the ion-current saturation plateau is insensitive to this amplifier gain.

maximized) biasing scheme. The ungridded data (red) was much smoother than the gridded cases (green, blue) because the collection period was significantly longer.

The ungridded detector pulse-height spectrum (red line) shows two well-defined peaks; the taller peak centered on channel 92 is from $\alpha$-particles (1.47 MeV). The energy resolution of the alpha peak is $20 \%$. The ${ }^{7}$ Li peak $(480 \mathrm{keV}$ ) centered on channel 34 has an energy resolution of $70 \%$, worse than the $\alpha$-particle's peak because ${ }^{7} \mathrm{Li}$ loses a larger fraction of its kinetic energy while escaping the boron coating than its less massive partner. As seen in Figure 10, a bias of $324 \mathrm{~V}$ places the ungridded detector in the saturation-current regime where the pulse-height spectrum is relatively insensitive to the detector bias.

When the gridded detector was biased at $324 \mathrm{~V}$ while the mesh was disconnected, the pulse-height spectrum (green) was significantly altered from the ungridded case. Although the ${ }^{7} \mathrm{Li}$ peak appeared in the same location (channel 34), the $\alpha$-peak shifted to a lower position (channel 84 ); $10 \%$ below the ion saturation plateau (Figure 10). The $\alpha$-peak shifted down because the angles of the alpha ionization 
tracks were distributed more uniformly over $2 \pi$ in the detector. Alpha tracks traveling nearly parallel with the emitter surface typically generated the largest pulse heights in the spectrum (i.e., counts in higher channels) because the ions did not interfere with the electron cloud as it drifted to the collection electrode, but the more perpendicular tracks did suffer from recombination and produce smaller-amplitude pulses. With the mesh installed, the parallel electron tracks were reduced by up to $24 \%$ while the pulse heights from the perpendicular tracks were less affected. Thus, the high-energy side of the alpha peak was shifted to lower channels in a greater proportion than the low-energy side, and the net effect was that the entire peak was shifted to a lower channel by $10 \%$.

After connecting the mesh to ground and applying a net $805 \mathrm{~V}$ bias, the pulse-height spectrum (blue) was similar to the ungridded case, but the energy axis (channel number) was scaled-up by a factor of 1.14; this was the expected trend for a detector entering the proportional regime. This $14 \%$ gain is significant because it is much larger than the $\sim 4 \%$ variation in pulse heights seen with the saturation plateau (Figure 10). In this case, the detector had a net field strength of $817 \mathrm{~V} / \mathrm{mm}$, which was strong enough to cause a small percentage of the drifting electrons to produce secondary ionizations near the collector. When biased at $800 \mathrm{~V}$, the ungridded SND was still on the saturation plateau because it only had a field strength of $444 \mathrm{~V} / \mathrm{mm}$, too small to produce an electron-cascade effect.

\section{Conclusion}

In this project, wire grids were added to the SND to improve the time resolution of the detector and amplify weak signals in the proportional mode. Numerous challenges were encountered: high-voltage biases were applied over small distances (1-3 mm), small signal levels $<1 \mathrm{nA}$ required special consideration for minimizing noise, high pressures (250 psi) needed to be contained, and microphonic noise needed to be minimized. Two gridded spiral detectors were successfully built. Preliminary tests with a planar configuration of the detector components show that when a mesh was used, pulse heights were amplified $14 \%$ over the ungridded case. These data strongly suggest that introducing a grid can cause the SND to operate in the proportional regime.

\section{Acknowledgments}

The author would like to thank Buckbee-Mears Co. for fabricating the nickel wire mesh; Dr. Culbreth of the Mechanical Engineering Department at UNLV for use of his $2 \mathrm{Ci}$ PuBe source; Douglas Electrical for fabrication of a prototype high-voltage, high-pressure feedthrough; and Ktech, Inc., for coating the foil.

\section{References}

Berninger, M. "Spiral thermal-neutron detector," Site-Directed Research and Development, FY 2011, National Security Technologies, LLC, Las Vegas, Nevada, 2012, 55-63.

Bunemann, O., T. E. Cranshaw, J. A. Harvey, "Design of Grid Ionization Chambers," Canadian Journal of Research 27, Sec. A (1949) 191.

Fermi, E., Nuclear Physics: A Course Given by Enrico Fermi at the University of Chicago, University of Chicago Press, Chicago, Illinois, 1949, 34.

Garber, D. I., R. R. Kinsey, Neutron Cross Sections, Vol. 2, $3 r d$ ed., Curves, National Neutron Cross Section Center, Brookhaven National Laboratory, BNL-325, Upton, New York, 1976.

Knoll, G. F., Radiation Detection and Measurement, 4th ed., John Wiley and Sons, Inc., Hoboken, New Jersey, 2010, 153.

McGregor, D. S., S. M. Vernon, H. K. Gersch, S. M. Markham, S. J. Wojtczuk, D. K. Wehe, "Self-biased boron10 coated high purity epitaxial GaAs thermal neutron detectors," IEEE Trans. Nucl. Sci. 47, 4 (2000) 1364-1370.

Sharpe, J., Nuclear Radiation Detectors, Methuen \& Co. LTD, London, John Wiley and Sons, Inc., New York, 1964, 181.

Sutton, R. B., B. D. McDaniel, E. E. Anderson, L. S. Lavetelli, "The capture cross section of boron for neutrons of energies from $0.01 \mathrm{eV}$ to 1000 eV," Phys. Rev. 71 (1947) 272. 


\section{Ultrafast, High Quantum Efficiency, Microstructured Photo- CATHODES FOR ADVANCED SENSORS}

LO-01-12 | YEAR 1 OF 1

Robert A. Buckles ${ }^{1, a}$ and Eric Dutra ${ }^{a}$

We developed a metallization process to improve gated imagers by dramatically thickening the photocathode underlayment of fiber-optic faceplates without attenuating the incident light. Metal thickness was increased by as much as $1 \mu \mathrm{m}$ (from the usual $50 \AA$ ) to a full skin depth of the driving electrical gate pulse, thereby allowing much greater charge conduction and ultrafast gate speed, even on large devices. The process we developed employed photolithographic methods of photoresist spin coating, exposure, and etching, but relied on the faceplate pattern itself as an inherent mask. A prototype $75 \mathrm{~mm}$ microchannel plate intensifier with an expected gate speed limited by drive signal, faster than 100 ps for low-impedance high-voltage drivers, was fabricated.

${ }^{1}$ bucklera@nv.doe.gov, 925-960-2520

a Livermore Operations

\section{Background}

There is a growing demand for fast-gate, large-area imagers in several areas of actinide science, highenergy density physics (Buckles 2012), and ignition diagnostics (Fittinghoff 2011), as well as in general high-energy, high-resolution gated particle detection (Beaulieu 2009). For example, high-resolution imaging with neutron time-of-flight spectral resolution can be greatly improved by faster gate speeds, which are presently limited to about $30 \mathrm{~ns}$ for $75 \mathrm{~mm}$ designs. Faster gate speeds offer better energy discrimination and the ability to use shorter drift times and smaller experiments (presently 28 meters at the National Ignition Facility [NIF]).

Historically, nanosecond-gated imaging devices have been limited to small diameters $(18 \mathrm{~mm})$ and diminished quantum efficiency $(<10 \%)$. Photocathode charging on this fast time scale requires more conduction than a thin ( $50 \AA$ ) film can allow, while thicker metal films attenuate the incident light beyond acceptable limits. Thick metal-mesh underlayments are a common fallback; they allow greater bulk conduction, with local charge distribution provided by the thin metal film. However, the mesh obscures the image data. One researcher (Thomas 1990) attempted to resolve this quandary toward the end of the nuclear testing era by fabricating a honeycomb mesh embedded around the individual fiber-optic elements that blocks none of the light and is invisible in the image. However, the demand for fast, highresolution tubes suddenly ceased, and the work was discontinued, until today.

Our aim is to improve on Thomas' process, applying it toward a large-format gated imager. The photo processing, although seemingly straightforward, contains nontrivial limits on contrast, exposure, processing times, and metallization techniques. Although Thomas' photo processing was a totally subtractive one using an acid etch, we find greater contrast with a combined subtractive and additive process, using negative photoresist techniques to employ metal channel shaping during the electroplating process. This microstructuring of the electronemitting cell provides some confinement and control over the electron ballistics and greater resolution in 


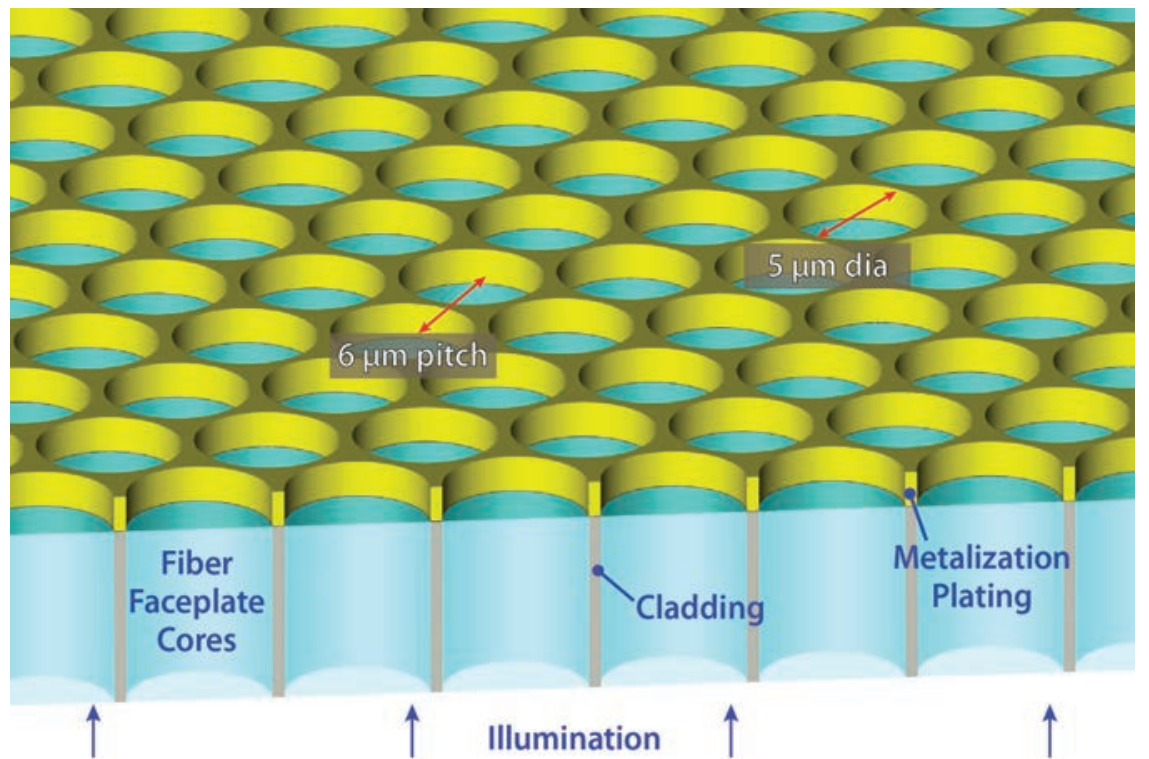

Figure 1. Intagliated photocathode concept: UV light exposes the topsurface photoresist through the optic itself, eliminating a mask and providing a fully clear aperture for the incident light (bottom) a microchannel plate (MCP). For this reason, we have named this method the intagliated photocathode after Thomas' analogous work on phosphor screens.

\section{Project}

\section{Intagliated Photocathode Process}

The intagliated concept uses a thick mesh gold electrode over a $5 \mu \mathrm{m}$ core with $6 \mu \mathrm{m}$ pitch in a hexagonal packing, commensurate with Incom BXI87-6 fiber faceplates. The gold can indeed be sharply intagliated as shown in Figure 1, by using the faceplate itself as the photo exposure mask. In this case, the light is incident from the front of the faceplate (bottom in the figure). The photo-development process is similar to semiconductor and circuit techniques but without a masking and alignment step. Once the photoresist is exposed and developed, we proceed with metallization. Both additive and subtractive metallizations are used.

We also anticipate some ballistic focusing of the photoelectrons due to electric field lines, which must have a component radially inward on each cell. The effect can be beneficial in counteracting the resolution degradation inherent with MCP imagers. After conducting some field modeling, we concluded that the focusing is quite extreme, and that the ideal shape and thickness of the gold wall is somewhat apodized, about 1-2 $\mu \mathrm{m}$ thick. Figure 2 shows a finite element model (FEM) comparison between

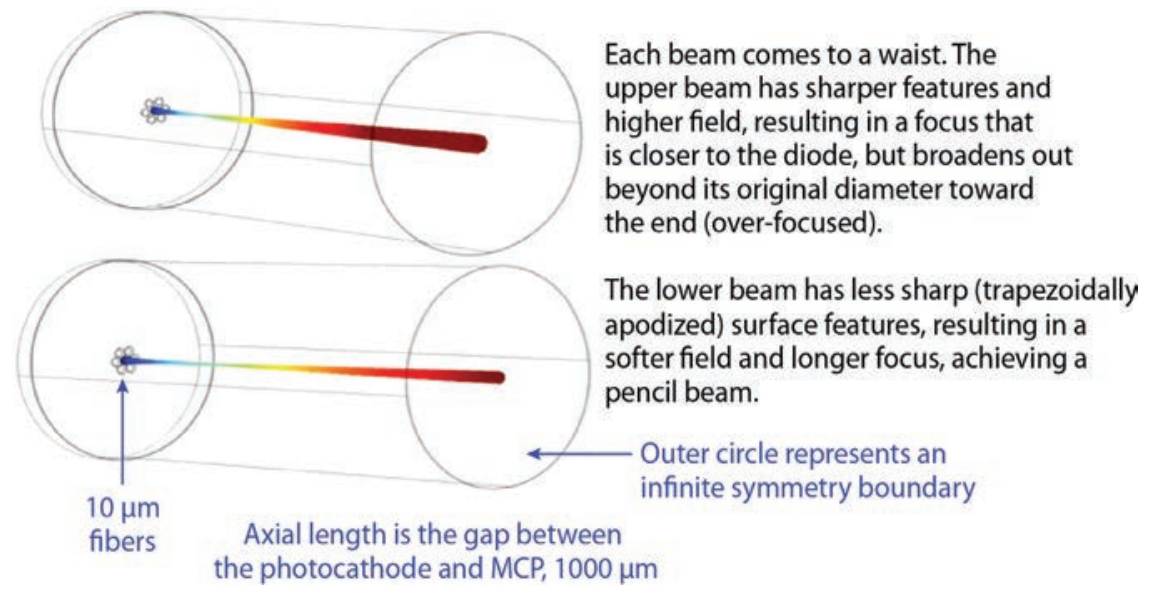

Figure 2. Photoelectron ballistic modeling with Comsol Multiphysics shows ideal collimation with trapezoidal wall profile (lower) and $1 \mu \mathrm{m}$ thickness, as opposed to straight wall, which overfocuses (upper) 


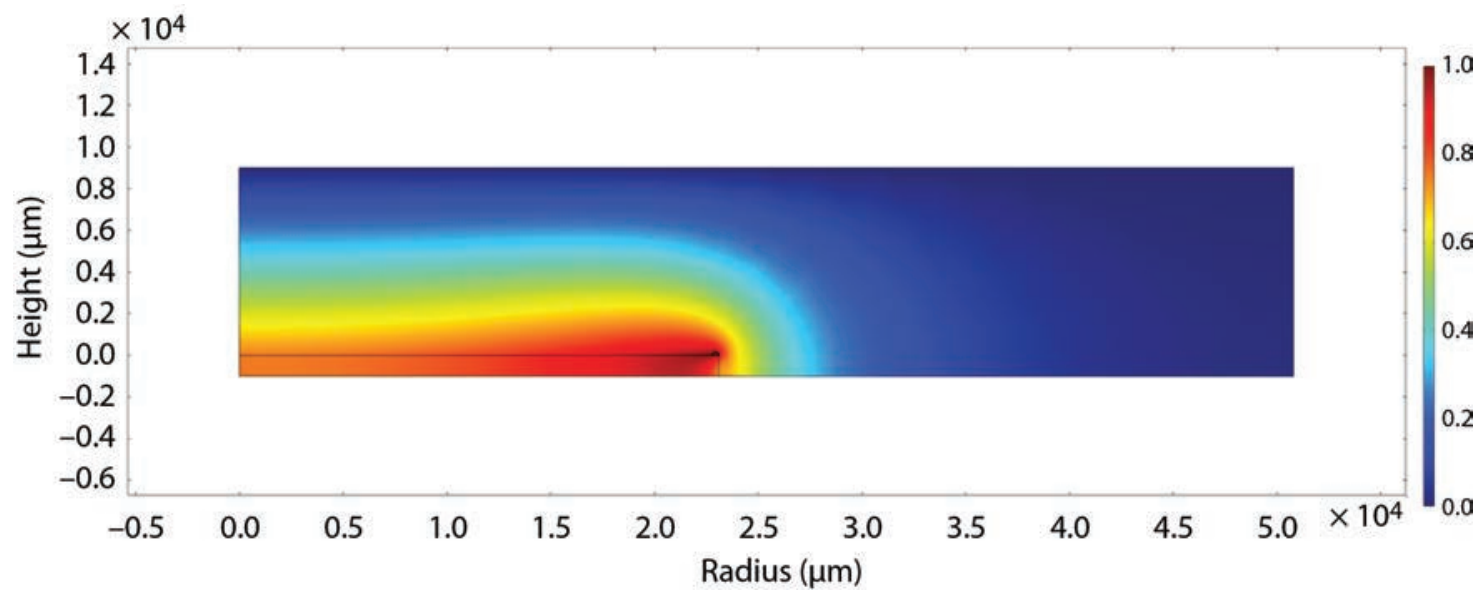

Figure 3. Electroplating voltage distribution around $50 \AA$ layer on $40 \mathrm{~mm}$ diameter glass optic in $20 \Omega-\mathrm{cm}$ bath resistivity (equivalent to sea water).

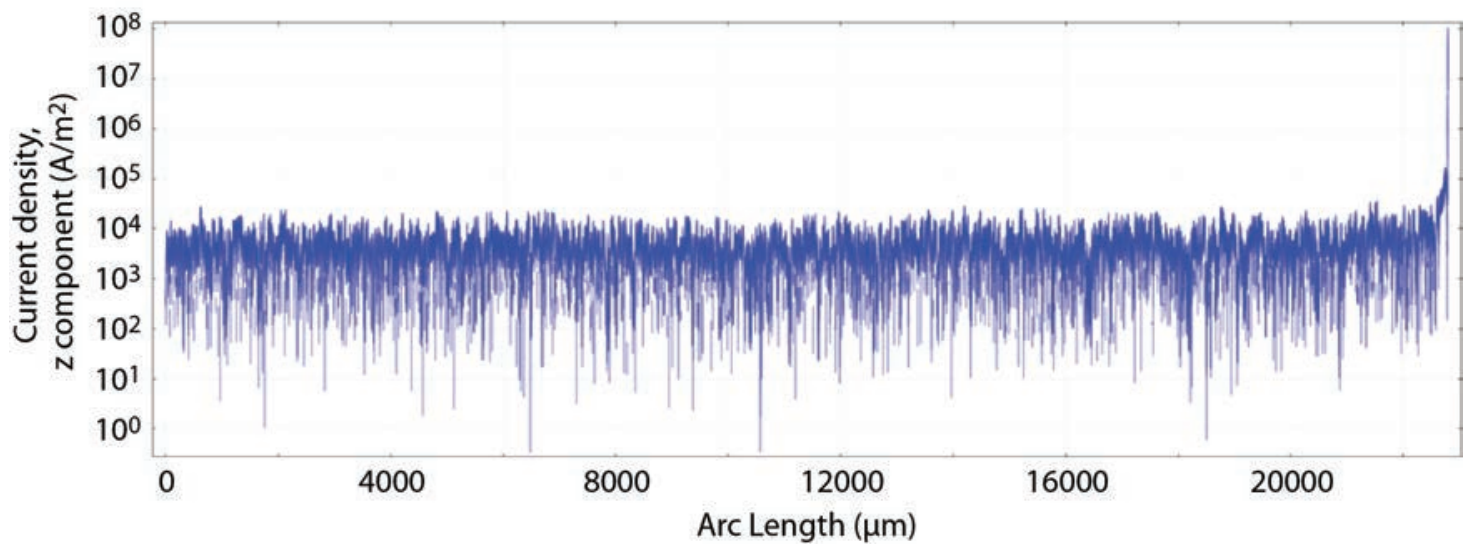

Figure 4. Current density along the optically thin layer, showing good uniformity, at $1 \mathrm{~A} / \mathrm{cm}^{2}$ for a $1 \mathrm{~V}$ deposition. The sharp rise at the right is due to the electrode attachment and is a possible concern. The graininess of the simulation is due to the finite cell size and was the best achievable given the huge aspect ratio in the finite element simulation.

straight wall and trapezoidal wall profiles, where the beam from a straight-walled cell is over-focused and expanding across the photocathode-MCP gap, while a trapezoidal cell has a longer softer focus and achieves collimation. Thicker meshes accentuate the over-focus and are unnecessary in terms of charge conduction, as the skin depth for gold is $2 \mu \mathrm{m}$ at $1 \mathrm{GHz}$.

The select method for achieving thicker metallization involves electroplating onto a thin seed layer of nichrome and nickel. We typically deposit about 10 and $50 \AA$ of each, resulting in 50\% light attenuation. However, within the thick-webbed mesh, we require only a few atomic layers. Some metallization is necessary to provide surface extraction of the photoelectrons, and the $10 \AA$ tie coat is sufficient for good adhesion and surface field, without significantly attenuating the light transmission. However, plating shops prefer to have a $2000 \AA$ ( $0.2 \mu \mathrm{m})$ seed layer, which is too thick for our application. Therefore, we undertook some plating simulations and found a shop to perform trial plating on samples we prepared with optically thin seed layers of $20,30,40$, and $50 \AA$. Figures 3-5 show a Comsol Multiphysics calculation of the voltage and current distribution around a $50 \AA$ thick gold layer on a glass substrate. For a relatively high-conductivity bath ( $20 \Omega$-cm resistivity, sea water 


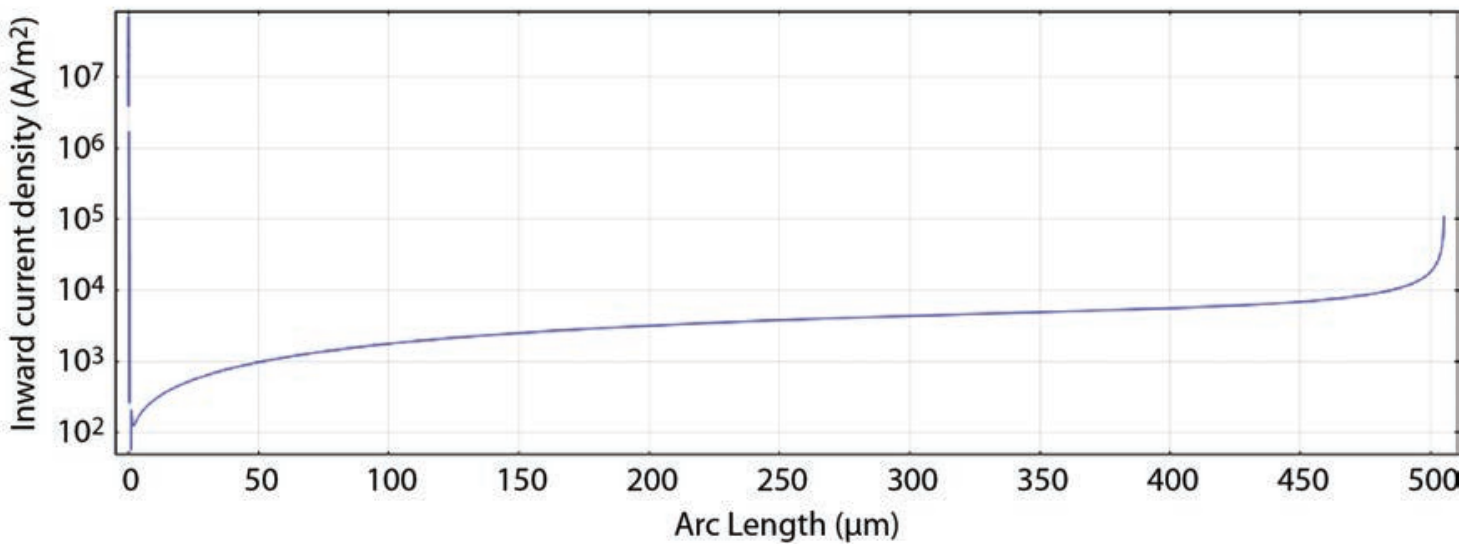

Figure 5. Current density along (bare) electrode path, the circular ring on the top outer edge in Figure 3. The spike at the left is a simulation error at a field convergence where the ring touches the surface, and should have zero field. The increase at the right is truly an increase in the field at the electrode outer diameter, and should be insulated.

equivalent), the field and current density is uniform and not overly large at the plating electrode, which can be insulated. However, the current density ( $1 \mathrm{~A} / \mathrm{cm}^{2}$, at $1 \mathrm{~V}$ discharge) would require 10 hours to apply plating that is $1 \mu \mathrm{m}$ thick.

The vendor, Inta Technologies of Santa Clara, found that it was difficult to electroplate on such a thin seed layer, even after making several plating adjustments, and they did not complete all the samples. We tested the layer thickness of one electroplated sample and measured a $1500 \AA ̊$ gain, but it was not uniform. However, we are determined to find other methods to achieve a thicker seed layer, on the order of 1500-2000 ̊̊ before plating again.

We ran initial photoresist trials using a negative photoresist for metal buildup with two goals in mind: (1) optically thin tie coats and (2) trapezoidal wall profiles. By controlling the exposure of the resist, in principle, we could cause the cured thickness to be conical, cylindrical, or inverted conical with increasing exposure. The cured resist would build up over the fiber cores, and then the walls would be plated up around the resist. We leased a UV exposure source for the project with characteristic mercury-vaporlamp spectra (OAI model LS30/5). We expected enough power to transmit through the fiber-optic faceplates (attenuating below $400 \mathrm{~nm}$ ), for exposures of a few seconds or more. We chose Transene PKP Type II Negative Photoresist, and we used PKP Thinner to determine the correct viscosity for our 1 $\mu \mathrm{m}$ thickness. PKP Developer was used to rinse away the unexposed region of photoresist, and Transene NRR-001 Negative Photoresist Stripper was used to remove photoresist once the process was completed.

Figure 6 shows the class 100 cubicle laboratory setup for mixing, spin coating, and drying the photoresist. All chemicals were mixed in the filtered fumehood, and $2 \mathrm{~mL}$ of solution spun onto $40 \mathrm{~mm}$ optical substrate samples. The optics with photoresist were then baked for 20 minutes at $82^{\circ} \mathrm{C}$, then slowly cooled to avoid cracking the optics. A spin-coating test was performed at $6000 \mathrm{rpm}$ to ascertain the proper concentration of photoresist and thinner to produce a $0.5 \mu \mathrm{m}$ and $1 \mu \mathrm{m}$ layer thicknesses. Concentrations of $20 \%, 30 \%, 40 \%, 60 \%$, and $100 \%$ (by volume) were mixed and placed into Erlenmeyer flasks for the trials. After baking, photoresist film thicknesses were measured using a Tecom profiler. Figure 7 shows the data plot along with regressions. A single power law fit is quite optimal below $70 \%$, transitioning to exponential upwards toward $100 \%$ concentration. Concentrations of $60 \%$ and $40 \%$ seemed ideal targets for $1 \mu \mathrm{m}$ and $0.5 \mu \mathrm{m}$ thickness, respectively. 

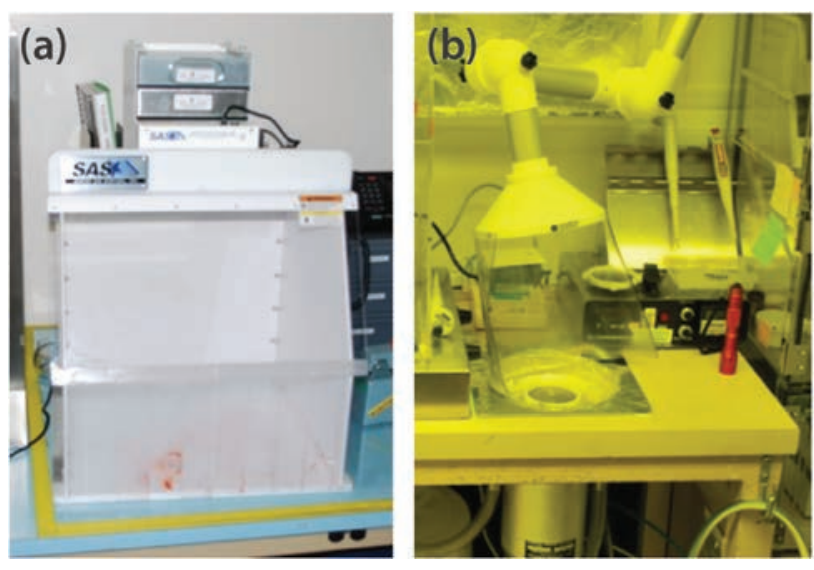

Next, a thickness versus spin speed experiment was performed to better refine the spin rates at specific concentrations. Figure 8 shows thickness for spin rates of $30 \%$ and $60 \%$ concentrations, respectively. A power law fit and second-order regression analysis were performed for each concentration. The error bars indicate random measurement error with the profiler, on the order of $100 \mathrm{~nm}$. Later trials reduced the error with practice.

We anticipated an exposure length anywhere from 2 to 200 seconds to achieve the proper exposure for the inverted conical resist profile. To limit the amount of samples needed to fine-tune the exposure, a linear stage was used as a sliding shutter, varying exposure across a test optic diameter. Using LabVIEW to control and document the different timed exposures and motion profile, we were able to quickly ascertain proper exposure time. Starting with a $1 \mu \mathrm{m}$ thick layer of photoresist, a 10-second timed profile was found.

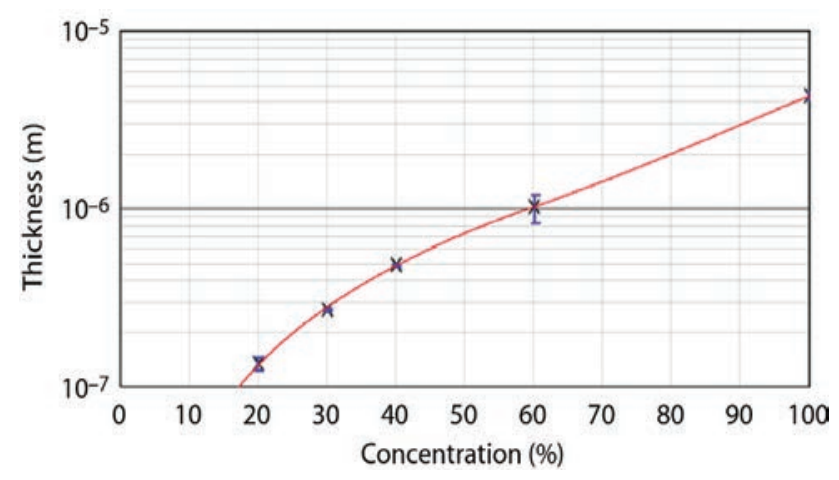

Figure 7. Thickness versus photoresist concentration at $6000 \mathrm{rpm}$

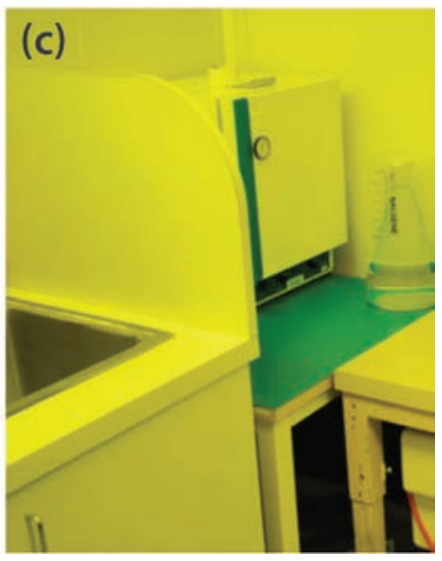

Figure 6. Clean room photo processing equipment included the (a) portable chemical fume hood, (b) photoresist spinner, and (c) process oven

Using the Tecom profiler we were able to measure the thickness of the resist and the height of the individual pillars. This was done with 300,500 , and $800 \mathrm{~nm}$ thick samples as well. Yet, it was discovered that light scatters within the cladding and exposes the photoresist where we wish to deposit gold. As such, only a very short exposure could be used without exposing the cladding and limiting the thickness of the resist layer. The maximum exposure that left the cladding free of negative resist was only 2 seconds long at reduced power $\left(10 \mathrm{~mW} / \mathrm{cm}^{2}\right)$ and would only create a $300 \AA$ A pillar. Any longer of an exposure would cure the photoresist in the cladding region, and we would not be successful using a multistep process with such thin coverage. Therefore, we adopted an alternative (positive resist) process to enhance the contrast between cladding and core; in making this change, we departed from the process used by Thomas (1990) and, we believe, improved it.

Our second process was subtractive, starting with a uniform gold vapor deposition, a spin coating of positive resist, and relatively long UV exposures through the fiber core. We used AZ Materials AZ 1505 Positive Photoresist to protect the gold deposition, and AZ 300 MIF Photoresist Developer to develop and rinse the exposed resist from above the fiber cores. The contrast between core and clad was greatly enhanced with thicker gold coatings and longer exposure. Based on past experiments it was known that blue and green light would still transmit thick layers but with significant attenuation. To experiment, we coated three optics with varied thicknesses of 

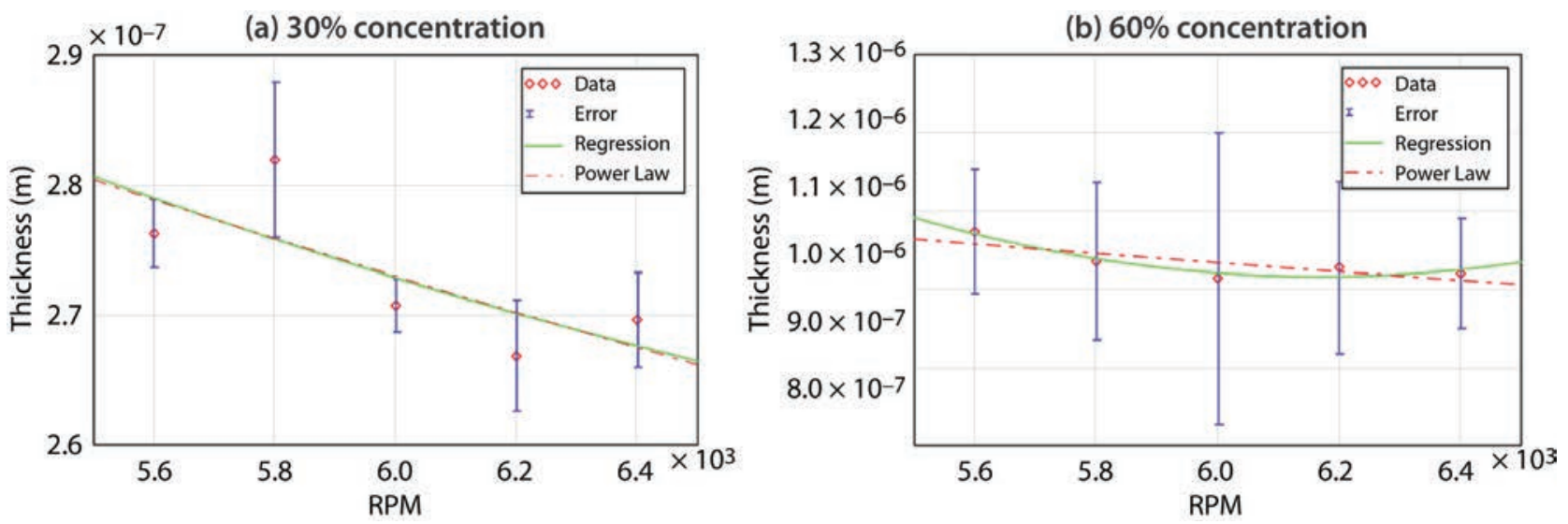

Figure 8 . Thickness versus spin rate for (a) $30 \%$ and (b) $60 \%$ photoresist concentrations indicate the spin speed preferable for this work

gold: 1000,1500 , and $2000 \AA$. The optics were coated with a $0.5 \mu \mathrm{m}$ layer of positive photoresist and then exposed through the optics. The optic coated with $1000 \AA$ of gold was able to transmit enough light to pattern the optic within 22 minutes at full intensity. The thicker gold coatings required exposures of 2 and 8 hours, respectively. However, we discovered that too long a process would allow the resist to self-cure, even without exposure, leaving a thin skin of cured photoresist, which would clump, tear, or bubble instead of rinsing away, and damage the gold pattern. Once exposed, developed, and rinsed, we applied, for approximately 70 seconds, type TFA gold etchant, purchased from Transene Company. This would completely remove all gold from above the core region, leaving the photoresist-covered gold protected. The unexposed photoresist was then stripped with AZ KWIK STRIP, leaving just the gold honeycomb structure in the cladding regions (and the original nickel tie coating over the cores).

Figure 9 shows a resulting gold microstructure. The effort was quite successful in that a continuous thick film of gold could be deposited without obscuring the input light. However, we would desire a perfectly uniform honeycomb structure. We surmise the reason for the apparent defects are misshapen fibers in the glass fiber array, mostly at the boundaries of the hexagonal bundles, causing bright scattering spots. These misshaped fibers can be pressed so close to their neighboring fiber that the clad region almost disappears. Other bright spots exist as well, and one can see these visually in the glass, likely due to internal defects, but these spots only cover about $0.01 \%$
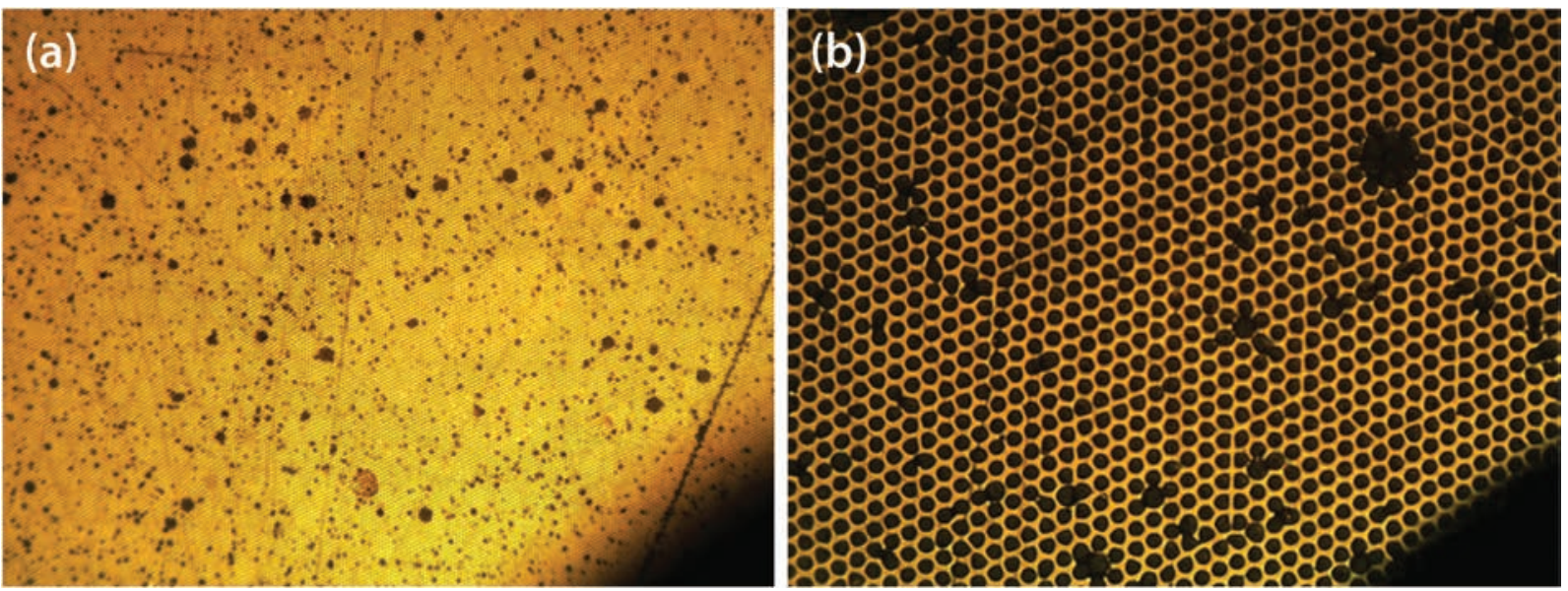

Figure 9. Gold microstructure (a) broad view and (b) fine view 
of the surface. The large holes appear to be defects in the gold coating process. The gold is significantly thinner in these areas, thereby allowing more light through during the photo exposure. These overexposed regions are then rinsed away, leaving a larger area for the etchant to remove the gold. We believe irregularities in the vapor deposition apparatus to be the culprit, but another possibility is surface contamination. Given further effort, we believe the defects can be suppressed with optimal exposure, but the metallization should be fully functional as is.

The final microstructure with $1 \mu \mathrm{m}$ thickness and trapezoidal wall profile should then be achieved with the negative resist process developed earlier. The 1000-2000 A vapor deposited film provides the necessary contrast for exposing the negative resist just within the core, and exposure duration is no longer critical. During electroplating, the resist prevents gold from filling in the core holes and maintains optimum clearance and transmission.

\section{Large Microchannel Plate Intensifiers (MCPIs) Design and Construction}

Subnanosecond 50, 40, and $18 \mathrm{~mm}$ designs of large MCPIs were made in prior years, but a $75 \mathrm{~mm} \mathrm{MCPI}$ has not been attempted. Legacy $75 \mathrm{~mm}$ intensifiers are proximity-focused diodes that cannot be gated, and due to their large area and $500 \mathrm{pF}$ capacitance, they have no temporal specification. However, 75 mm MCPIs, sold by Photek, Ltd., are currently used in large-area gated detection applications such as the NIF neutron imager. Faster speeds on the order of $1 \mathrm{~ns}$ are desired, and this project sought to demonstrate the high-speed concept with the most demanding load. Furthermore, it seemed simpler to start from scratch on a new design rather than adjusting a work in progress. This also gave us a chance to correct some obvious mistakes in the existing larger tube designs.

One deficiency we have noted in commercial tubes is a strict adherence to form factor between quartz window designs and fiber-coupled designs in which the photocathode thickness is much thinner than the phosphor faceplate, leading to poor coupling

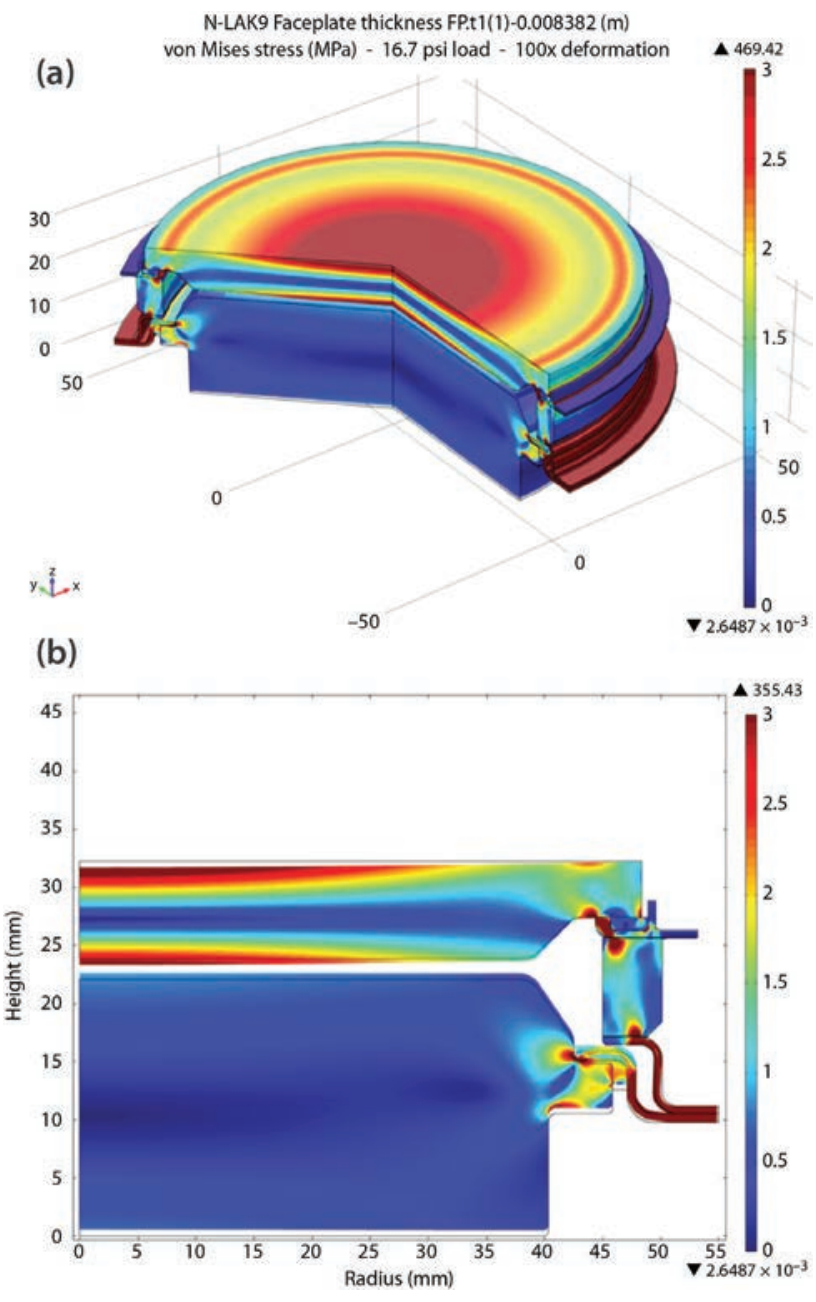

Figure 10. Stress analysis and deflection of legacy $75 \mathrm{~mm}$ image intensifier without the MCP, where (b) photocathode deflection at center is $1 \mu \mathrm{m}$

and mechanical failure when butt-coupled to fiberoptic systems. Partly, this is an oversight of the faceplate mechanical requirements, made correctly for quartz windows, but applied incorrectly to fiber faceplates. One cannot simply remove atmospheric deflection of the faceplate by repolishing. The physical coupling should add 1-2 psi and further distort the faceplate. Rather, one should adopt the same mechanical requirements for both the phosphor and photocathode faceplates. Therefore, we performed a stress analysis of the legacy $75 \mathrm{~mm}$ image intensifier, using the FEM program Comsol Multiphysics. Figure 10 shows the calculated von Mises stress and deflection for our legacy $75 \mathrm{~mm}$ image intensifier for 16.7 psi loading (atmosphere + coupling force), and a net deflection of nearly $3 \mu \mathrm{s}$, which is several 

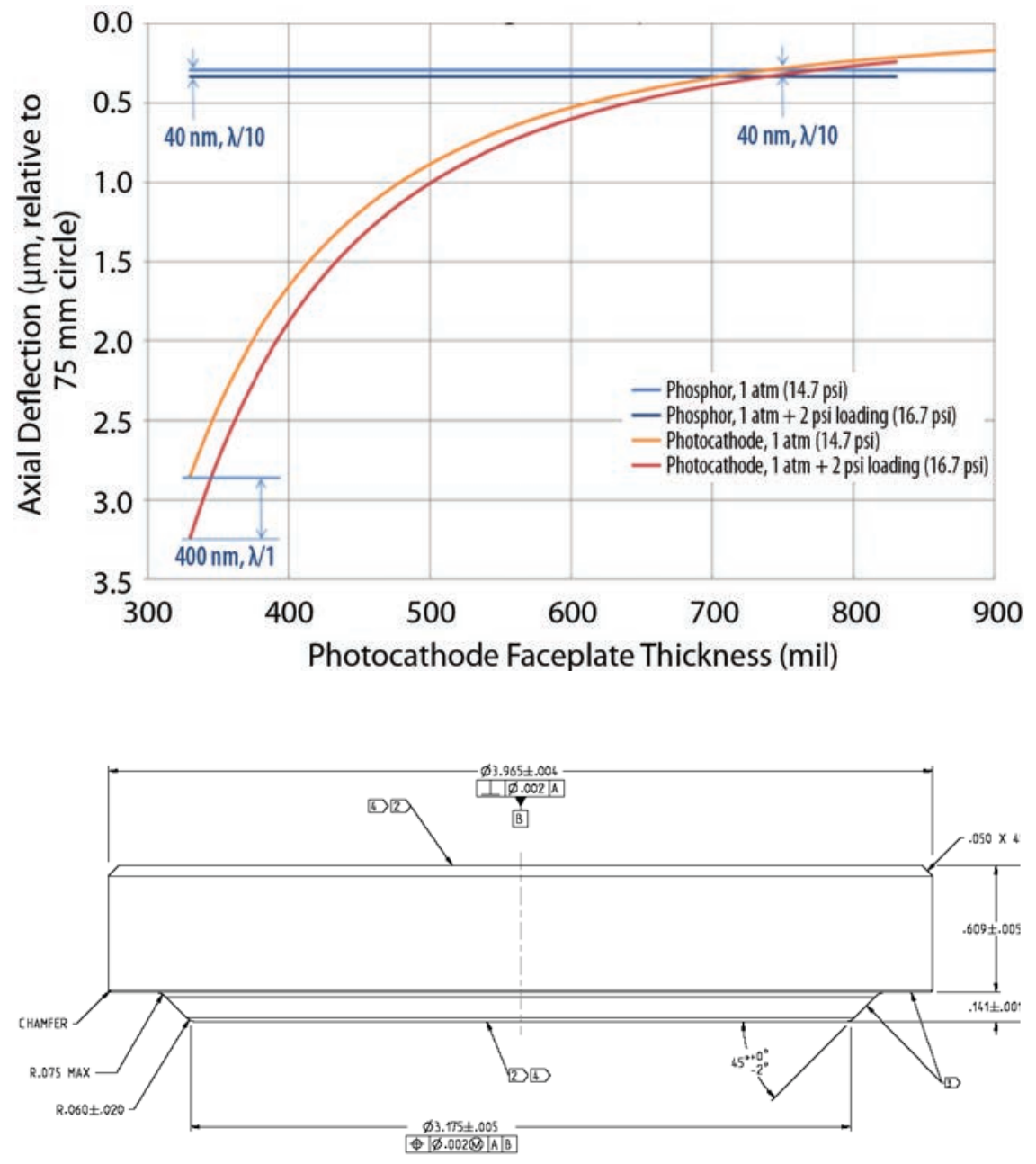

Figure 11. Calculated deflection curves of the older $75 \mathrm{~mm}$ intensifier with thin photocathode faceplates. The phosphor is designed for fiber-optic buttcoupling, but the photocathode is only intended for lens coupling.

Figure 12. Schematic of $75 \mathrm{~mm}$ MCPI faceplate LOP12-355-022, with $3 / 4$-inch thickness, designed for butt-coupling to fiber-optic system like the phosphor output interference fringes. We repeated for $14.7 \mathrm{psi}$, revealing (Figure 11) a net loading deflection of $400 \mathrm{~nm}$, a full wavelength. This seems slight, but surface flatness can be on the order of $\lambda / 10$ with good polishing. Coupling stability is even more important than actual deflection. To meet this stability requirement the phosphor faceplate specified in our new design has a thickness of $3 / 4$ inches (Figure 12 ).

Also noted in Figure 10b are high stress points in the glass. In fact the stress seems to exceed the yield stress, and could explain why leakage failure modes occur in large-area intensifiers, where helium leak detection has been noted around these points. To reduce the possibility of leakage, we thickened the ceramics and radiused the metal parts to take up the deflection without building up extreme stress.
The rest of the tube design, from the tabbed electrodes (for low-impedance parallel-plate transmission line drive) to the spring-finger contact (Figure 13a) are fair extrapolations from a previous design; however, two improvements were made:

(1) a thicker indium cup electrode to relieve the glass stress, and (2) a lower-profile MCP retainer ring, employing 00-90 countersunk flat-head socket cap screws, which should provide a flush MCP drive structure and eliminate parasitic resonances.

We made parts for three tube bodies expecting less than unity yield, but, remarkably, the tube bodies assembled without difficulty, and all three passed a leak check. Self-indexing parts, fixtures, and attention to tolerance stack-up are essential to achieving good yield. Figures 13b-13d show the MCPI fixtures and parts in various stages of assembly. Aside from long 


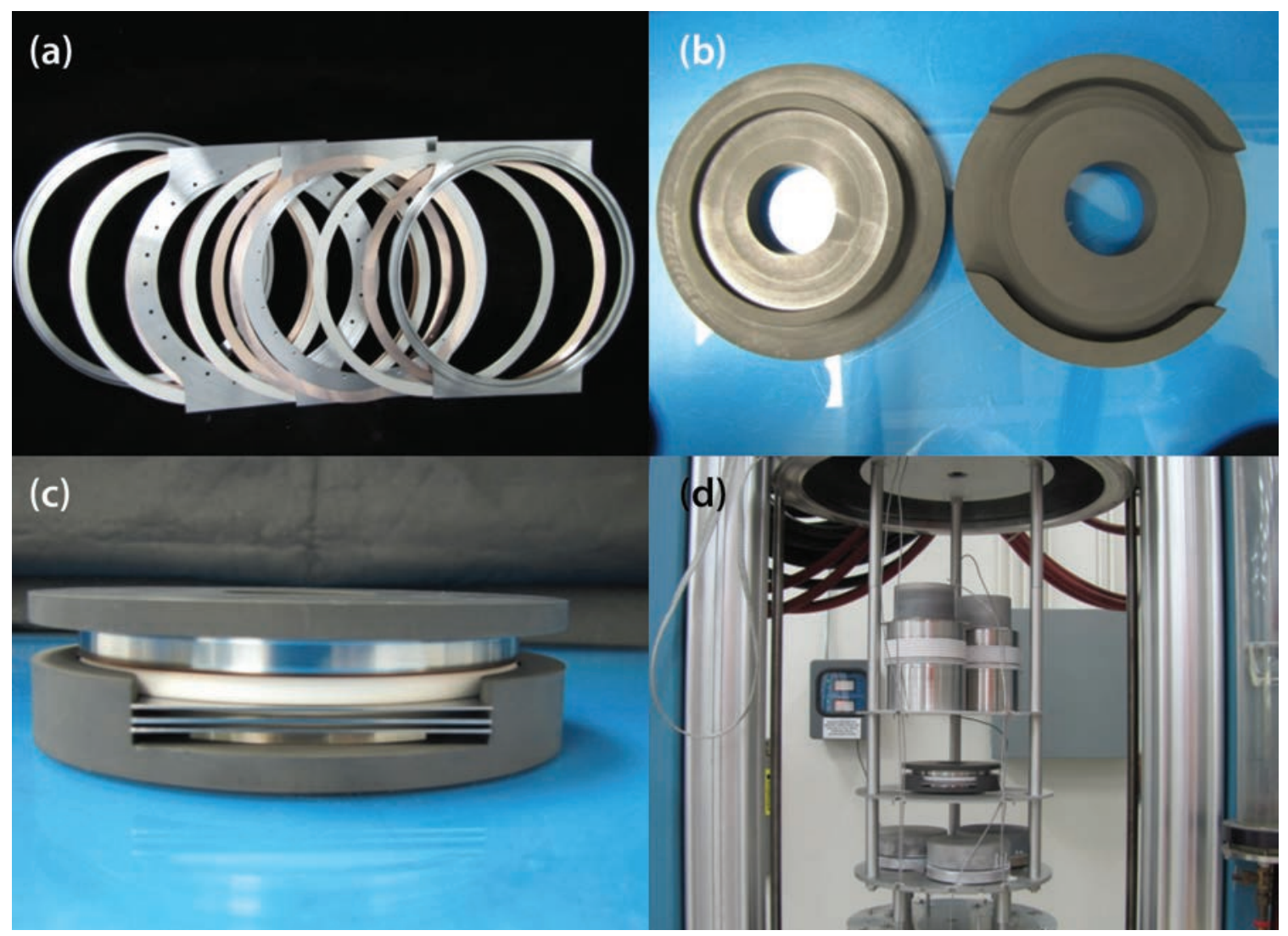

Figure 13. The $75 \mathrm{~mm}$ MCPI (a) ceramic and metal parts, (b) graphite assembly fixture, (c) assembled parts, and (d) body parts in the hydrogen furnace

delivery schedules, there were no difficulties in the construction.

The MCP was another area for improvement, and we chose to compare novel atomic layer deposition (ALD) microcapillary MCPs (Mane 2012) to conventional lead silicate MCPs. Figure 14 shows microscope views of two designs, a standard 60:1 aspect ratio and a 46:1 aspect ratio, but both made from microcapillary with individually optimized conductive and secondary electron emission layers. Figure 15 is a conventional lead-silicate glass MCP; an aspect of 60:1 was the only type we could purchase in the required diameter. Some advantages of ALD include higher gain and broader range of design parameters. The ALD MCP can be made quite large and thin as well without suffering from warping. Note, however, that the surface quality of the ALD MCPs could use some improvement, but we insisted on having them in time for the project completion, even though polishing was non-ideal. We intended to compare gain images in our phosphor test station, but could not obtain the phosphor welding heat sink fixture needed for assembly.

\section{Conclusion}

Great strides were made in developing a new intagliated process, which uses both positive and negative photoresists, vapor deposition, and electroplating. We completed the process on sample optics but not on the production faceplates. We designed and constructed a $75 \mathrm{~mm}$ MCPI body, incorporating the latest design advances for minimal faceplate deflection and wideband response. We also procured one standard and two advanced-prototype ALD MCPS for comparative tests in the three bodies. Further work will be required to complete the assembly of the processed photocathode and phosphor faceplates onto the tube bodies and test the gate speed. We 


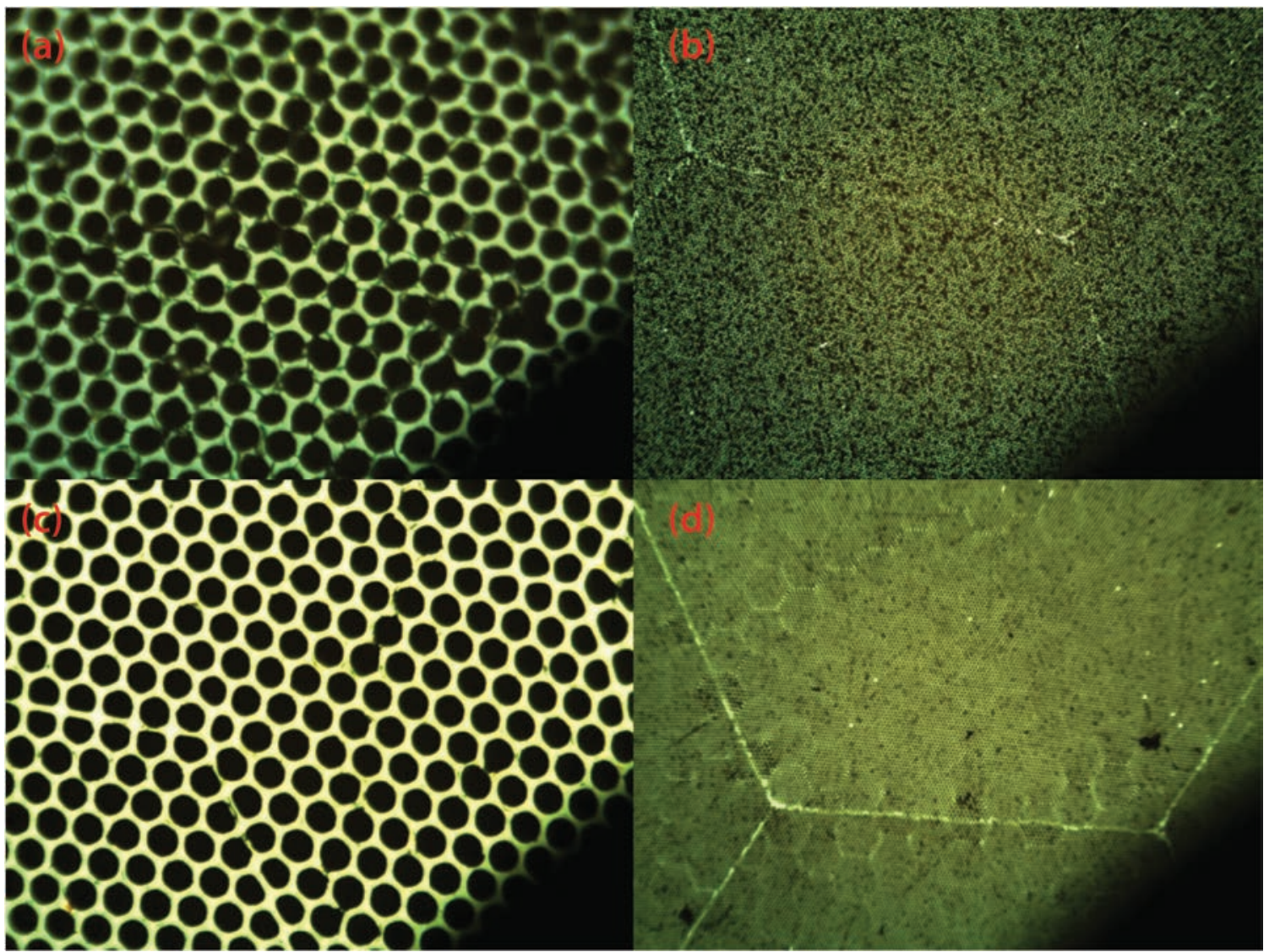

Figure 14. Initial samples of ALD MCPs: (a) fine and (b) broad views of 46:1 L/D, and (c) fine and (d) broad views of 60:1 L/D. Later samples promise greatly improved polish and uniformity.

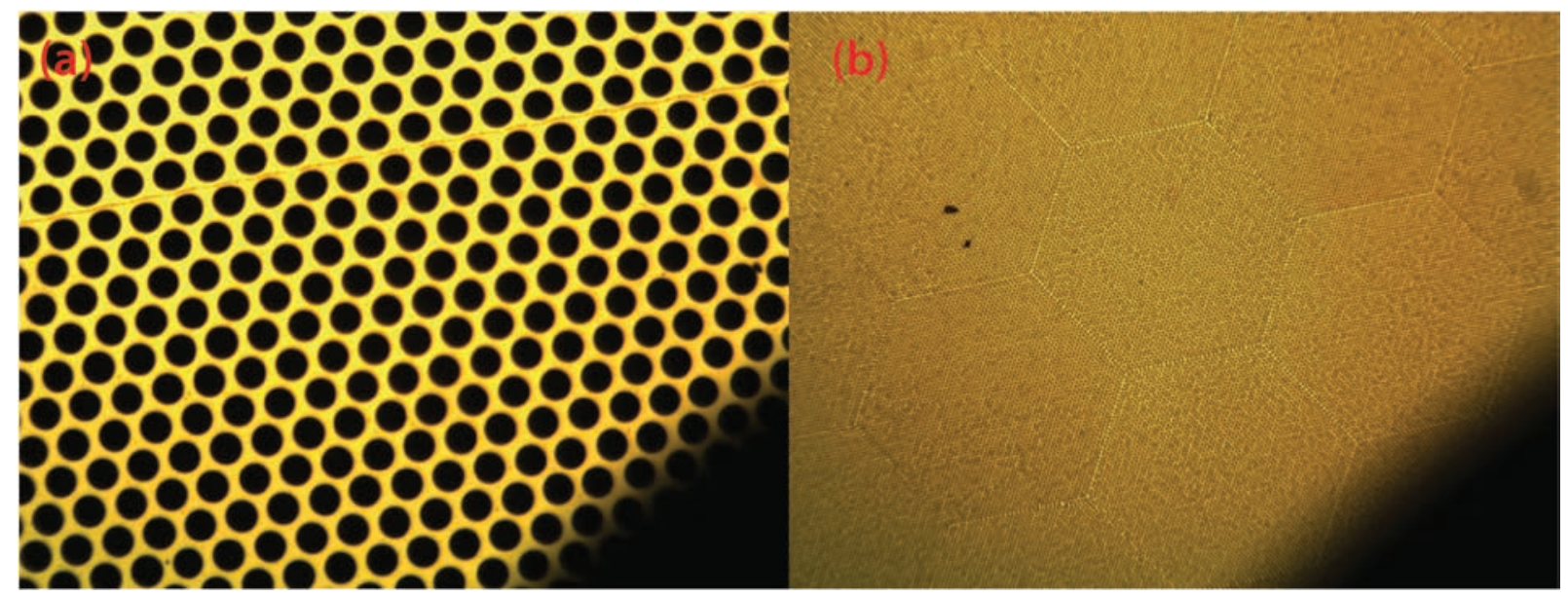

Figure 15. A conventional lead-silicate glass MCP, 60:1 L/D MCP (a) fine and (b) broad views 
expect the large tubes to have subnanosecond response, only limited by drive current and propagation speed across the tube diameter.

\section{References}

Beaulieu, D. R., D. Gorelikov, P. de Rouffignac, K.

Saadatmand, K. Stenton, N. Sullivan, A. S. Tremsin, "Nano-engineered ultra high gain microchannel plates," Nucl. Instrum. Methods Phys. Res. A 607, 1 (2009) 81-84.

Buckles, R. A., R. L. Guyton, P. W. Ross, "Picosecond optical MCPI-based imagers," Proc. SPIE 8505 (2012) 850502.

Fittinghoff, D. N., D. E. Bower, O. B. Drury, J. M. Dzenitis, R. Hatarik, F. E. Merrill, G. P. Grim, C. H. Wilde, D. C. Wilson, O. Landoas, T. Caillaud, J. Bourgade, R. A. Buckles, J. Lee, "Performance Improvements to the Neutron Imaging System at the National Ignition Facility," LLNL-TR-503351, Lawrence Livermore National Laboratory, Livermore, California, October 6, 2011.

Mane, A. U., Q. Peng, J. E. Elam, D. C. Bennis, C. A. Craven, M. A. Detarando, J. R. Escolas, H. J. Frisch, S. J. Jokela, J. McPhate, M. J. Minot, O. H. W. Siegmund, J. M. Renaud, R. G. Wagner, M. J. Wetstein, "An atomic layer deposition method to fabricate economical and robust large area microchannel plates for photodetectors," Physics Procedia 37 (2012) 722-732.

Thomas, R., J. Tevino, "Picosecond Intensifier Gating with a Plated Webbing Cathode Underlay," UCRL 102843, Lawrence Livermore National Laboratory, Livermore, California, July 1990. 
This page left blank intentionally 


\section{TIME-RESOLVED HYPERSPECTRAL FLUORESCENCE SPECTROSCOPY USING FREQUENCY-MODULATED EXCITATION-APPLICATIONS}

STL-41-12 | CONTINUED FROM FY 2011 | CONTINUING IN FY 2013 | YEAR 2 OF 3

Gene A. Capelle, ${ }^{1, a}$ John Di Benedetto, ${ }^{a}$ and Mary O'Neilla

During the first phase of this project in FY 2011, the use of modulated light sources and synchronous detection was demonstrated as an alternative to the use of pulsed-laser systems for time-resolved hyperspectral applications. An intensity-modulated excitation source was used together with a microchannel plate-intensified CCD detector gated at a slightly different frequency to generate a beat frequency. The addition of a spectrograph produced a hyperspectral data product where the resulting beat frequency can be detected with the low frame rate-CCD camera. The measured beat wave gives not only spectral data, but also information on the radiative lifetime of a particular emission feature. In FY 2012, we demonstrated the applicability of this new "optical lock-in" technique to several optical challenges in the Special Technologies Laboratory's current suite of activities from the nuclear nonproliferation, work for others, and environmental portfolios. Specifically, we demonstrated applications in microscopic analysis, remote sensing measurements, and remote serial number detection. The results of these experiments are described and discussed in this report.

${ }^{1}$ capellga@nv.doe.gov, 805-681-2252

a Special Technologies Laboratory

\section{Background}

Time-resolved systems are used for trace detection and/or identification in a multitude of applications (Lakowicz 2006). Time-resolved fluorescence spectroscopy techniques can generally be categorized as either time domain or frequency domain. For most remote sensing applications, pulsed-laser time domain systems are used. These systems require high peak-power lasers and short integration times to detect weak returns in high ambient light-level backgrounds. The second general approach towards fluorescence detection is based on frequency-domain techniques (Lakowicz 2006). Sine or square-wave intensity modulation of the excitation light causes a corresponding modulation of the returned fluorescence light that can be phase shifted and intensity demodulated relative to the excitation wave. Advantages over pulsed laser systems include greatly decreased nonlinear effects due to lower peak excitation power; smaller size, weight, and power requirements; and excellent optical background rejection. Standard (and now commercially available) methods exist to detect frequency-modulated (FM) fluorescence spectra using a single detector. These systems can operate over a large frequency range, obtaining lifetimes in the picosecond range. However, they require a scanning monochromator and acquisition times unsuitable for remote sensing applications. In this work, the beat pattern for the entire spectrum was recorded over multiple CCD camera acquisitions, and the lifetime of the spectrally dispersed emission was calculated both from the phase shift and (independently) from the demodulation of the beat wave. The absolute phase shift was determined by measuring the phase shift of the emission spectrum 
relative to the unshifted excitation peak that is also recorded through the spectrograph. The FM method described in this report records the modulation as a beat pattern generated when an excitation source and a spectral detector gate are operated at slightly different frequencies. As the excitation source and the detector gate slowly pass out of phase and then back into phase again, a beat wave is produced within multiple (wavelength versus intensity) images collected by the spectrograph/intensified CCD (ICCD). The beat pattern for the entire spectrum is recorded over multiple CCD camera acquisitions, and the lifetime of the spectrally dispersed emission can be calculated both from the phase shift and (independently) from the demodulation of the beat wave.

In the FY 2011 project (Di Benedetto 2012), the basic experimental technique was proven to work very well in the laboratory, and various algorithms and data processing techniques were tested, leading to the development of an effective method for extracting the decay time of the material under study. This year, experimental efforts applied the new technique to three separate problems of interest that are part of NSTec's current portfolio of business. First, the technique was applied to microscopic analysis of very small particles: part of the NA-22 nonproliferation effort that requires identification of the emission characteristics (such as lifetime and emission spectrum). Second, and also part of the NA-22 nonproliferation effort, is detection/identification (again through measurement of optical radiative decay time and emission spectrum) of materials at large standoff distances (remote sensing). Third, and related to NA-22 safeguards and other efforts, is remote serial number detection (marking applications), specifically to demonstrate 3-D serialized codes (excitation/emission/lifetime) measured at a distance. Each of these applications is discussed.

\section{Project}

\section{Theory}

A detailed theoretical discussion may be found in the FY 2011 SDRD report (Di Benedetto 2012); however, a brief review follows. In most optical frequency domain systems, the primary method for determining the lifetime of a sample is to measure the phase shift and/or modulation of the fluorescence return with respect to the laser excitation. Figure 1 shows the change in depth of modulation and the phase shift of fluorescence relative to the excitation, where $\phi$ is the phase shift of fluorescence return relative to the excitation signal, $\omega$ is the modulation frequency in radians/second, $\tau$ is the lifetime in seconds, and $m$ is the modulation, with signals as defined in Figure 1.

The equations (Lakowicz 2006) used in determination of fluorescence lifetime from modulation and phase shift are

$$
m=\frac{B / A}{b / a}=\frac{1}{\sqrt{1+\omega^{2} \tau^{2}}}
$$

and

$$
\tan =(\phi)=\omega \tau .
$$

For our hyperspectral data, the phase shift of the beat wave at the fluorescence wavelength of interest is measured relative to the (unshifted) beat waveform at the excitation wavelength, $405 \mathrm{~nm}$ for the experiments to be described.

\section{Experiments}

Figure 2 is a simplified schematic that represents the technique. All demonstrations carried out this year were variations on this schematic. The first demonstration uses a microscope between the exciter/ detector system, and the target and the distance is very small; the second demonstration uses a telescope between the exciter/detector system, and the distance is very large.

\section{Microscopic Analysis}

The first experimental demonstration was built around a Leica Ergolux AMC microscope with a 10X objective. The microscope was modified to accept a $405 \mathrm{~nm}$ diode laser excitation source. The laser output beam was diffused (1500 grit diffuser) 

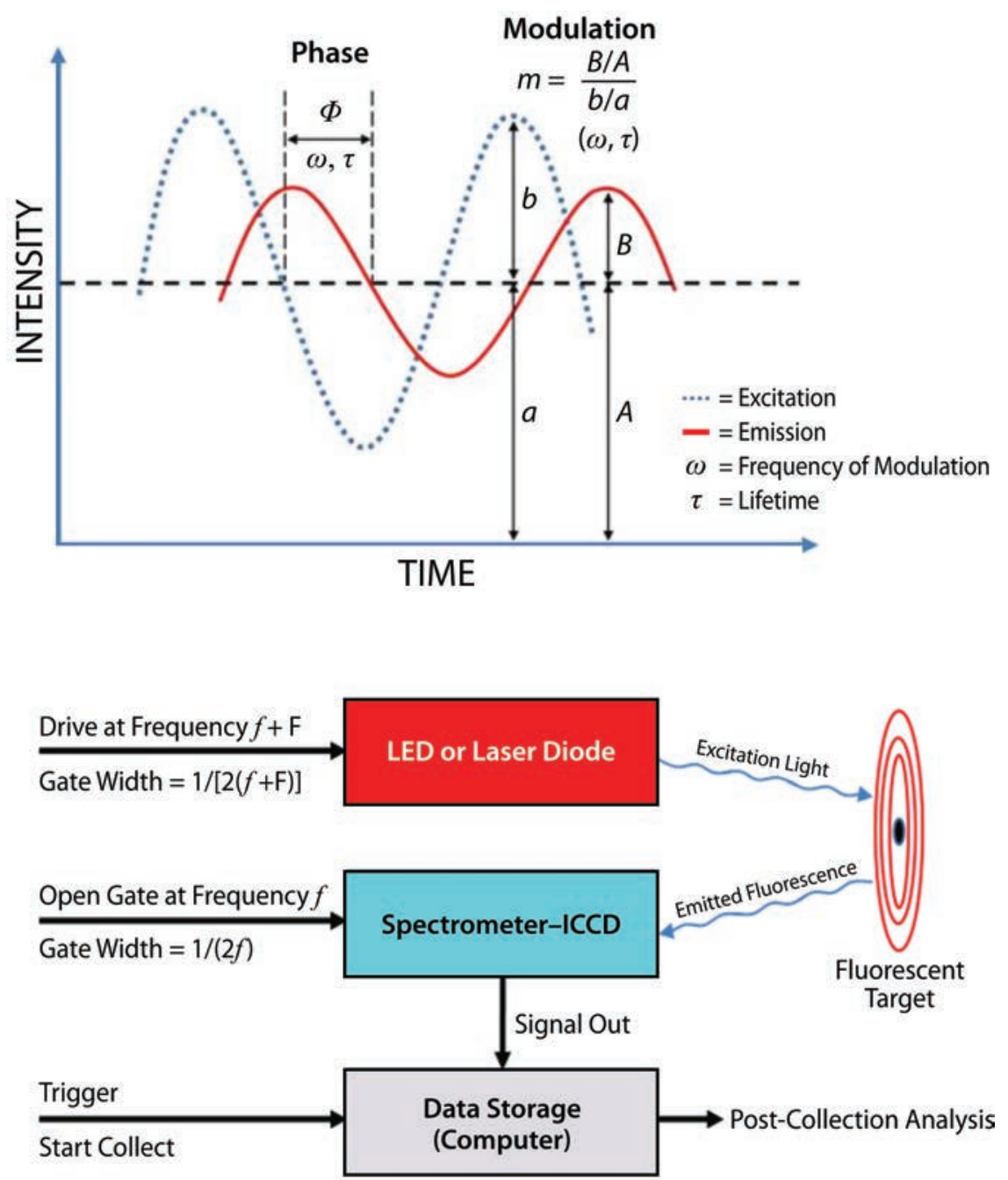

Figure 1. Phase shift and change in modulation depth of the fluorescence return relative to the excitation

Figure 2. Experimental setup just before injection by means of a $45^{\circ}$ mirror reflective at $405 \mathrm{~nm}$ into the microscope's optical path.

The beam was thereby ultimately imaged through the microscope objective and onto the sample slide. The laser light completely illuminated the field of view. The microscope had a flip mirror to allow light collected from the sample slide to be sent to port 1 (for viewing with the eye or a camera) or port 2. Parts were machined to allow a fiber-optic probe to be connected with an SMA905 connector and positioned at a real image plane in port 2 (optics were placed at the port in front of the fiber). This fiber, a single $400 \mu \mathrm{m}$ core fiber, carried light collected from a $70 \mu \mathrm{m}$ (measured) spot on the sample slide back through a lens assembly (to convert the beam to parallel space so optical filters could be inserted as needed) and then back into a fiber bundle that led into the spectrometer/ICCD detector. For better resolution, some measurements were made with a $200 \mu \mathrm{m}$ core fiber; this change gave a $35 \mu \mathrm{m}$ observation spot with the $10 \mathrm{X}$ objective. A more powerful objective could accomplish the same results.

A mount was made to hold a filter in the microscope return optical path (above the point of laser injection) to block most of the back-scattered $405 \mathrm{~nm}$ light from reaching the detector and the observer. A Semrock BLP01-405R was initially used for setup, but it excessively attenuated the $405 \mathrm{~nm}$ return light needed as a reference for the beat frequency technique, so it was 
replaced by a $2 \mathrm{~mm}$ thick GG-420 filter for the actual measurements. The excitation source was normally operated at an average output power near $100 \mathrm{~mW}$ onto the diffuser; the power on target was much less. The laser and detection system were operated as described previously (Di Benedetto 2012).

Targets were made using standard $1 " \times 3$ " glass microscope slides. The first sample slide was made by shattering a small ruby crystal with a hammer, then securing some of the smaller chips to a blank slide with cellophane tape. The second slide was made using ruby spheres from diamondanvils.com, measuring approximately $14 \mu \mathrm{m}$ in diameter, and the third slide was made from powder collected from a sample of autunite $\left[\mathrm{Ca}\left(\mathrm{UO}_{2} \mathrm{PO}_{4}\right)_{2} \cdot 10 \mathrm{H}_{2} \mathrm{O}\right]$, a naturally occurring mineral containing uranium oxide.

\section{Remote Sensing}

The second experiment was built around an existing in-house remote sensing light detection and ranging (lidar) system. This system consisted of a gimbal-mounted 14-inch diameter telescope with a large, pulsed Nd:YAG laser source and various detectors, all mounted in a trailer. The optical train behind the telescope was reconfigured to include a through-the-telescope TV camera and optics to launch telescope-collected light (with a 1.5 milliradian field of view) into a fiber-optic bundle. The bundle in turn carried light to the standard (i.e., common to all these experiments) spectrometer/ICCD detector. A modification was added at the fiber-optic input to add filters as needed to adjust the intensity of the returned excitation light on the detector, to keep both the collected excitation light and the collected fluorescence light on scale. When modifications were completed, the trailer was moved to the Special Technologies Laboratory (STL) outdoor laser range, and measurements were made on the range midday (sunny days) with targets at a distance of 43 meters.

A rigid frame was added to the telescope, to which five $405 \mathrm{~nm}$ lasers were mounted. Each laser was fitted with elevation-azimuth adjustment capability (for alignment) and custom optics, which were built in-house, to reduce each laser's divergence to less than 1 milliradian. The lasers were all connected to a common power supply and were controlled by a single square-wave signal generator.

A number of different targets were used at the 43-meter standoff (Figure 3): (1) a frame containing uranium glass tubing (approximately $12 " \times 14$ " active area), (2) a 1" $\times 3$ " glass microscope slide with three ruby crystals held in place by black photographic tape, and (3) a large target (three 4-inch squares), one containing a phosphor (PhosphorTech YOSR05, 5 grams mixed in a clear epoxy [EPO-TEK 301-2] and flowed onto the surface), one a blank, and the third ruby sand ( 15 grams of ruby, crushed, mixed in clear epoxy, and flowed onto the surface).
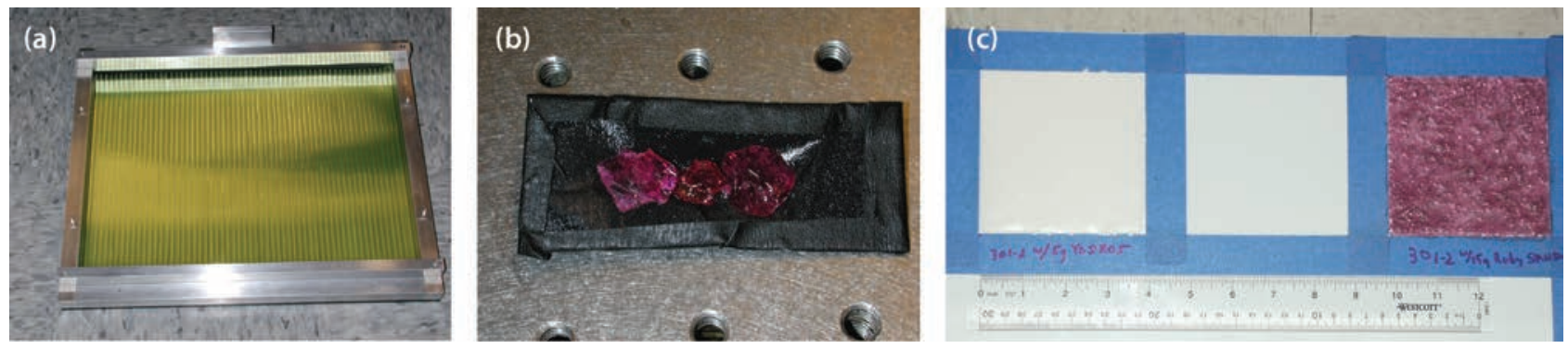

Figure 3. Remote sensing targets: (a) uranium glass tubing, (b) three small ruby crystals on 1 " $\times 3$ " microscope slide, and (c) three 4-inch squares on white painted aluminum honeycomb containing (left) phosphor, (center) blank, and (right) ruby sand. 
(a)
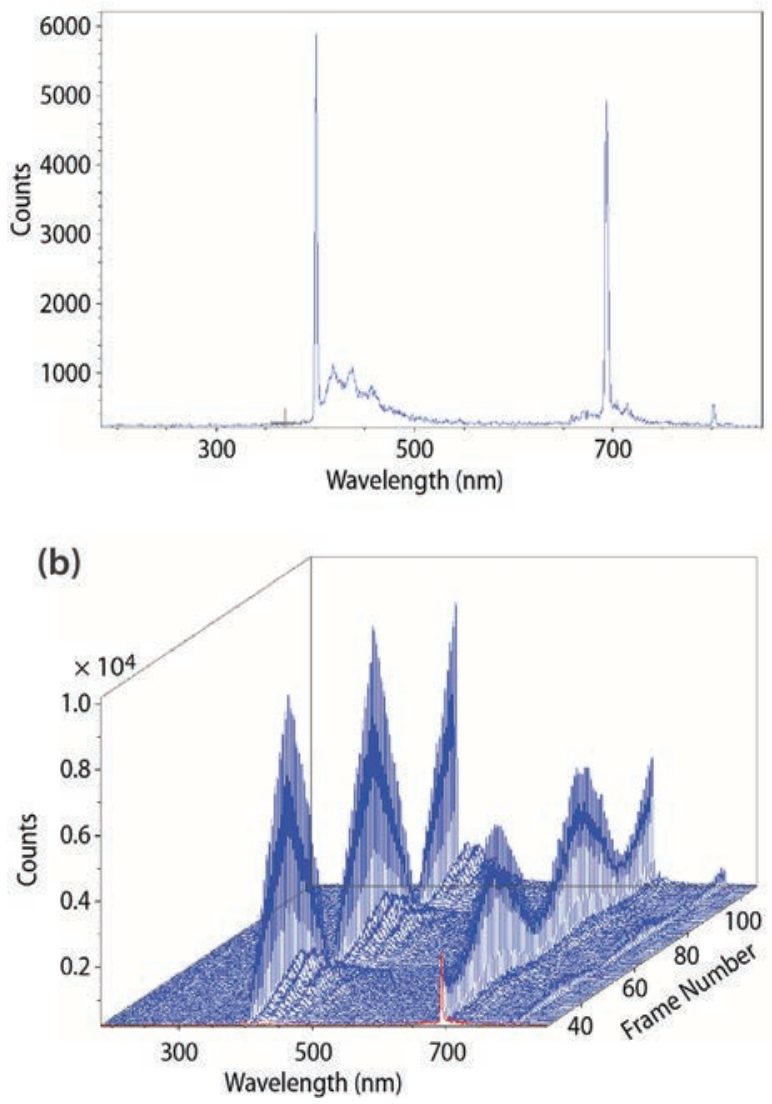

Figure 4. (a) Single CCD frame showing $405 \mathrm{~nm}$ excitation light (left peak) and ruby fluorescence signal (right peak); (b) frames 27 through 107 of the same collection

\section{3-D Serialized Codes}

For this demonstration, the remote sensing system from the second experimental setup was used, as well as the phosphor target, placed at the 43-meter position. The primary difference was that a $355 \mathrm{~nm}$ laser excitation source was used alternately with the $405 \mathrm{~nm}$ source.

\section{Results and Analysis}

As the $405 \mathrm{~nm}$ excitation source and the microchannel plate (MCP) gate (the electronic shutter on the detector) slowly pass out of phase and then back in phase again, a beat wave is produced. At the $405 \mathrm{~nm}$ readout wavelength of the detected excitation source, the beat wave is a triangle wave (the convolution of the two square waves passing over one another), which serves as a built-in zero phaseshift reference wave. An experimental data set for a particular target material is collected at several experimental excitation frequencies, which range from well below $f^{\prime}=1 / \tau$ (where $\tau$ is the radiative lifetime of the sample) to approximately $f^{\prime}$.

Parameters were normally adjusted to achieve nearly 40 frames ("snapshots") per cycle of the beat wave to ensure a reasonable representation of the wave, and data were collected for multiple cycles of the beat wave, typically 800 frames. Because our ICCD is limited to a frame rate of 20 to 40 frames per second, we were limited to beat frequencies of $1 \mathrm{~Hz}$ or less, and collection times were tens of seconds. The results of applying this beat frequency technique to a $70 \mu \mathrm{m}$ ruby crystal chip on a microscope slide are shown in Figure 4, taken at a frequency $f=64 \mathrm{~Hz}$ and a beat frequency $F=0.2 \mathrm{~Hz}$. Figure $4 \mathrm{a}$ is a single CCD frame of the data collection. Both the excitation pulse around $405 \mathrm{~nm}$ and the ruby emission near $694 \mathrm{~nm}$ are clearly visible. The structure between 400 and $500 \mathrm{~nm}$ is fluorescence from the translucent cellophane tape we first used; switching to clear tape reduced this problem. Figure $4 \mathrm{~b}$ shows frames 27 through 107 of the same data collected through the microscope, and the effects of the ruby lifetime are quite evident. While the excitation light peak shows the fully modulated triangle-wave pattern as the frames advance, the fluorescence light signal has become more rounded, the modulation depth has decreased, and there is already a clear delay or phase shift with respect to the excitation wave. These 3-D data are reduced to two dimensions by taking a slice through multiple successive CCD frames at a single wavelength.

Examples of intensity at a single wavelength versus frame number are plotted in Figure 5 (data from FY 2011) for both the excitation light at $405 \mathrm{~nm}$ (the triangle wave) and for several wavelengths around the maximum ruby emission. Data at this wavelength are shown at four different excitation frequencies. Note that the phase shift increases and the modulation depth decreases as frequency increases. 
(a) $16 \mathrm{~Hz}$

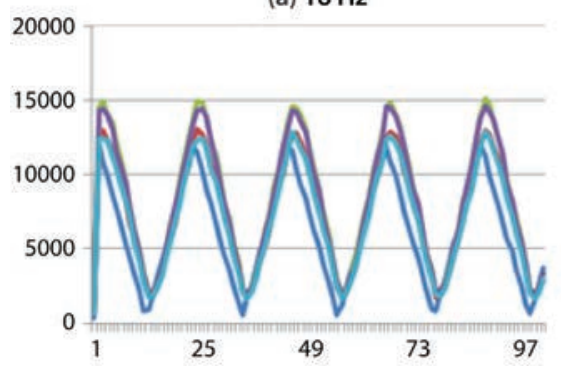

(c) $256 \mathrm{~Hz}$

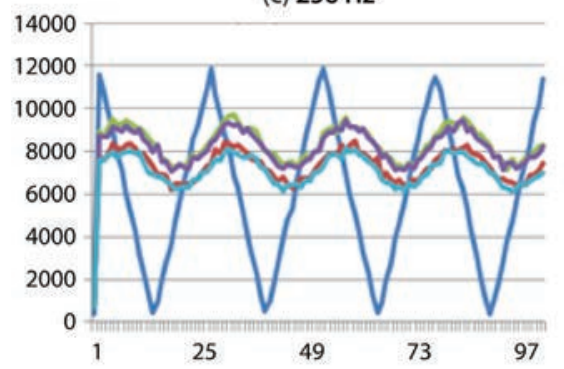

(b) $64 \mathrm{~Hz}$

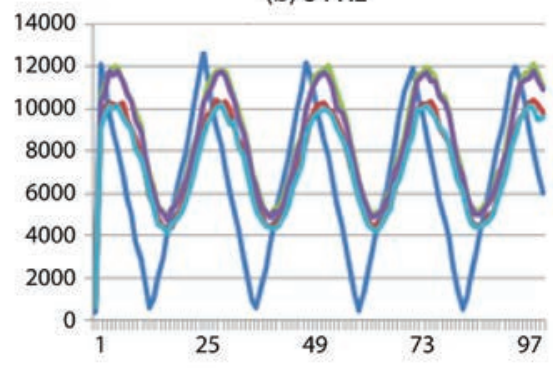

(d) $1024 \mathrm{~Hz}$

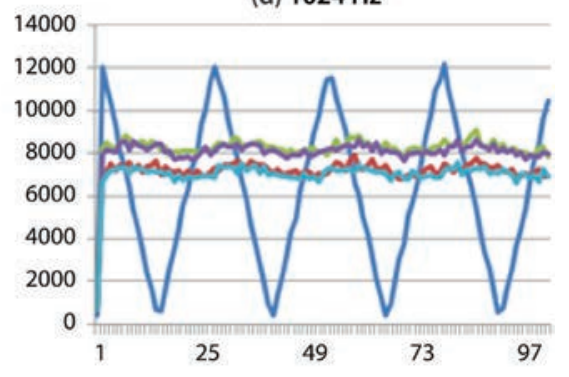

Figure 5. Superposition of signal collected at $405 \mathrm{~nm}$ (unshifted sawtooth) and at several wavelengths around the maximum ruby emission; data are shown at four different frequencies
Reduction of these waveforms into target material lifetimes was done using a digital implementation of the common quadrature-phase $(I / Q)$ phase detection method (Lakowicz 2006) as shown in Figure 6. The incoming signal (in our case the fluorescence return with phase shift, $\phi$, relative to the excitationwavelength beat wave) is mixed with both in-phase reference (excitation return) and quadrature-phase reference (excitation return shifted by $90^{\circ}$ ) signals.

The low-pass filter removes the high-frequency components such that the $D C$ levels of $I$ and $Q$ are measured. The ratio of $Q$ to $/$ is directly related to the phase shift $\phi$ and the lifetime $\tau$ by

$$
\tan (\phi)=\frac{Q}{I}=\omega \tau
$$

and

$$
\tau=\frac{Q}{l \cdot \omega}=\frac{\tan (\phi)}{\omega} .
$$

This analysis requires knowledge of the excitation wavelength and the beat frequency. The data analysis method results in a single lifetime even if multiple lifetimes are present. Note that for $I / Q$ calculations, the $A C$ coupling of the signals removes offsets that would skew the phase calculation. Also, data must be full cycles or there will be a skew in the I or $Q$ value, resulting in an incorrect phase calculation. In addition to using phase to compute lifetime, the lifetime from the modulation was also computed (based on Lackowicz 2006) from the demodulation as

$$
\tau_{m}=\frac{\sqrt{1-m^{2}}}{\omega \cdot m} .
$$

However, the I/Q method was found to give better results and is now the exclusive method used for data analysis.

\section{Microscopic Analysis}

Several different samples were analyzed with our new technique; all samples were small particles mounted on $1 " \times 3$ " slides and placed under the microscope. The first sample measured was a $70 \mu \mathrm{m}$ ruby chip; data collected from this particle are shown in Figure 4. A lifetime near $5 \mathrm{~ms}$ was measured from the data; for our purposes (i.e., to separate materials by lifetime) this is in sufficiently good agreement with the true lifetime of around $4 \mathrm{~ms}$. We were also able to collect usable data from a $14 \mu \mathrm{m}$ diameter ruby sphere. 


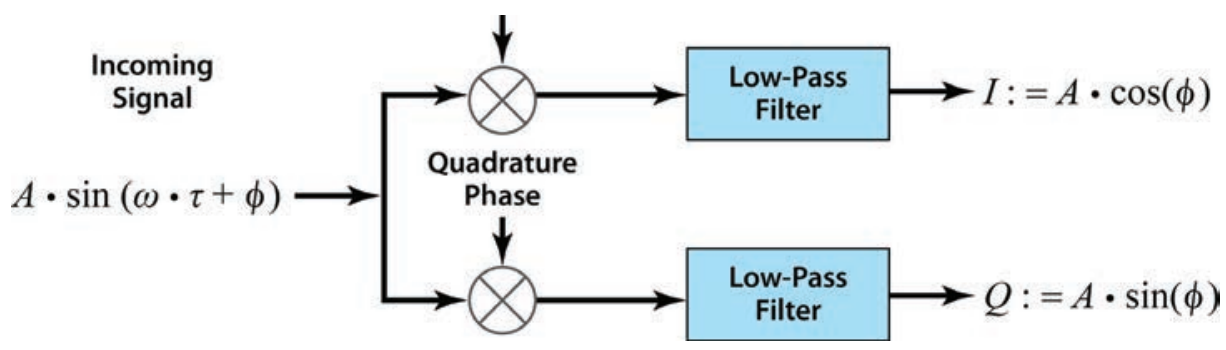

Figure 6. I/Q block diagram. The incoming signal with a phase shift, $\phi$, is mixed (multiplied) by in-phase and quadrature-phase reference signals resulting in $/$ and $Q$ respectively. By definition, $\tan (\phi)=Q / I=\omega \tau$, where $\omega$ is the frequency in radians/sec and $\tau$ is the lifetime.
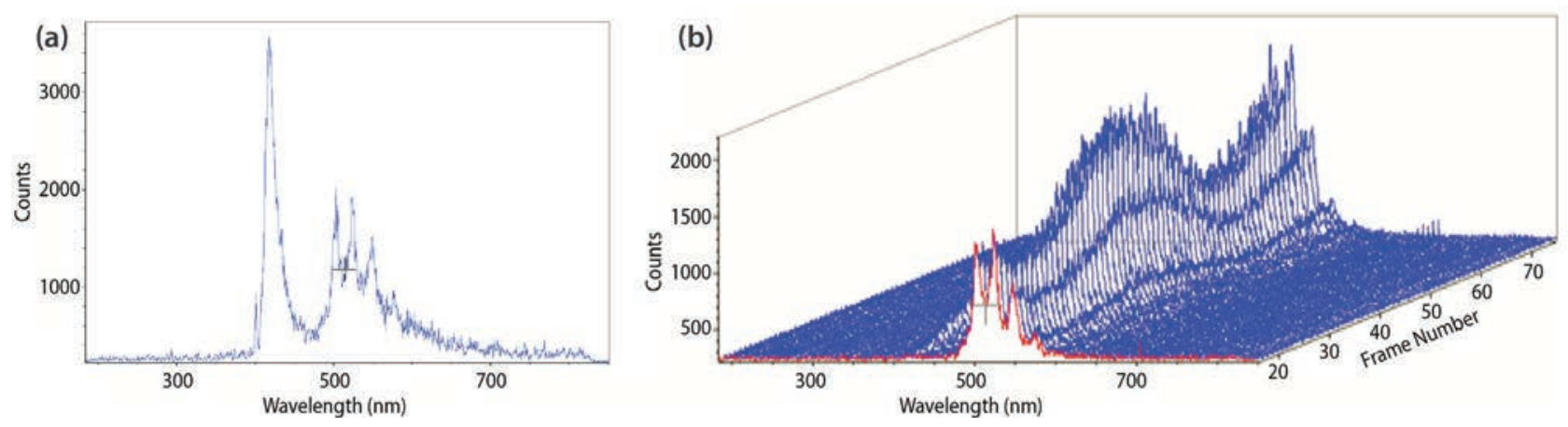

Figure 7. (a) A single CCD frame from the 45-micron autunite particle; (b) 60 CCD frames of 100-micron autunite flake, taken through a microscope at $16,380 \mathrm{~Hz}$ and $1 / 2 \mathrm{~Hz}$ beat frequency

Autunite was our second test sample; very acceptable and usable data were collected from a $100 \mu \mathrm{m}$ flake of autunite, and later from a $45 \mu \mathrm{m}$ flake. Autunite differs greatly from ruby in two ways. First, the fluorescence emitted is spread out over a number of bands and over a broad spectral range, rather than concentrated primarily in a single spectral line as is the case for ruby, so it is more difficult to discern the signal in the presence of background noise. Second, the radiative lifetime is about three orders of magnitude shorter than ruby, or about $5 \mu$ s (Geipel 1999), which was slightly beyond the limits of our instrumentation to measure comfortably. The short lifetime means that a much faster frequency must be used to achieve an observable phase shift in the collected data. For these measurements, data were collected at frequencies up to $25 \mathrm{kHz}$, which is the upper limit of our instrumentation (specifically, the MCP repetition rate). Figure $7 a$ shows a single frame collected from the $45 \mu \mathrm{m}$ autunite flake; the characteristic $\mathrm{UO}_{2}$ bands in the green are quite prominent, while the small peak near $405 \mathrm{~nm}$ is the laser excitation light (a filter was used to reduce the intensity of the signal from this laser light and thus keep it from saturating). The broader blue feature (highest peak in Figure 7a) at slightly longer wavelength than the laser is due to the clear cellophane tape used to hold the autunite chunks in place; it had a very fast lifetime compared to autunite, so could actually also be used as a reference beat if the laser signal became too weak to measure accurately.

Figure $7 \mathrm{~b}$ shows 60 frames of a data collection taken on the $100 \mu \mathrm{m}$ sample at an excitation frequency of $16,380 \mathrm{~Hz}$, with a $1 / 2 \mathrm{~Hz}$ beat frequency. Note the demodulation of the fluorescence beat wave, as well as its phase delay (in the figure, the excitation at $405 \mathrm{~nm}$ is zero and beginning to rise, while the fluorescence peaks in the green region of the spectrum are still decreasing in intensity as the frames advance over the next five or so frames). While it was not possible to take data at a high enough frequency to achieve a $45^{\circ}$ phase shift, it was still possible to establish magnitude estimate of lifetime, which is the goal for these experiments. 
Table 1. Microscope autunite data analyzed at various frequencies

\begin{tabular}{|c|c|c|c|c|}
\hline $\begin{array}{c}\text { Frequency } \\
(\mathbf{k H z})\end{array}$ & $\begin{array}{c}\text { Expected Phase } \\
\text { for } 5 \boldsymbol{\mu \text { Lifetime }} \\
\text { (degrees) }\end{array}$ & $\begin{array}{c}\text { Measured Phase } \\
\text { (degrees) }\end{array}$ & $\begin{array}{c}\text { Apparent Lifetime } \\
(\mu \mathrm{s})\end{array}$ & $\begin{array}{c}\text { Lifetime Error } \\
(\mu \mathrm{s})\end{array}$ \\
\hline 2.0 & 4.0 & 9.1 & 12.8 & \pm 10.4 \\
\hline 4.0 & 7.2 & 15.9 & 11.3 & \pm 4.8 \\
\hline 16.0 & 26.7 & 36.8 & 7.4 & \pm 2.0 \\
\hline 25.0 & 38.1 & 39.5 & 5.2 & \pm 1.4 \\
\hline
\end{tabular}

(a) Ruby

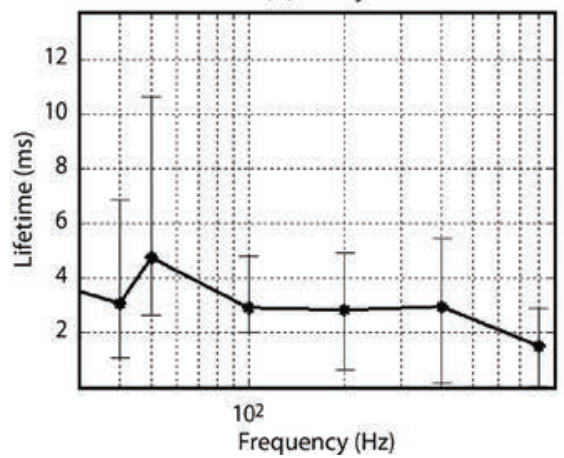

(b) Glass tubing

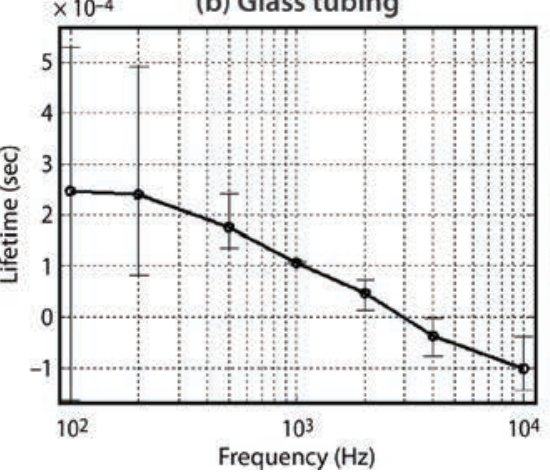

(c) Phosphor target

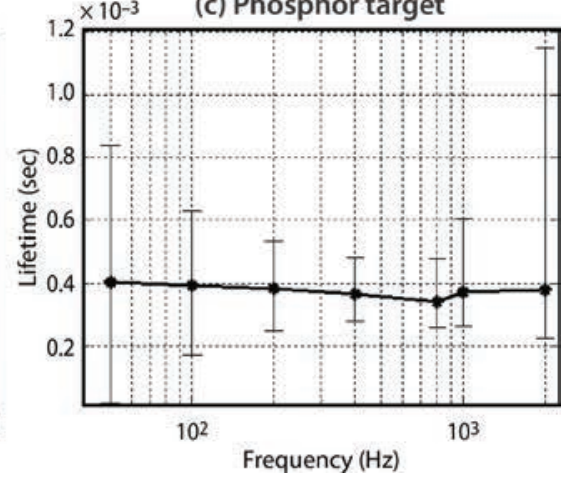

Figure 8. Results calculated from data taken at a 43-meter standoff from (a) ruby, (b) glass tubing, and (c) phosphor targets

Table 1 shows the phase shift expected at a given experimental frequency (based on the literature lifetime value of $5 \mu \mathrm{s})$, the actual phase shift measured from our data, and the lifetime calculated using the $I / Q$ method.

\section{Remote Sensing}

Data from a number of different targets placed 43 meters down the laser range were collected between 10 a.m. and 2 p.m. on sunny days, but with targets placed in the shade. Figure 8 a shows the lifetimes calculated for the microscope slidesized ruby target located 43 meters away; agreement with the nominal 4 ms decay time is good. Similarly, for the uranium glass tubing target (Figure $8 b$ ), the calculated lifetimes are in agreement with the $200 \mu$ s range we have measured in the past using other methods. Results calculated from the data collected from the phosphor target are shown in Figure 8c; the calculated values of just below $0.4 \mathrm{~ms}$ agree with the "true" value stated by the supplier (Menkara 2012) to be around $350 \mu \mathrm{s}$. Figure 9 shows the signal returned from the phosphor target.

\section{3-D Serialized Codes}

The idea of 3-D serialized codes involves measurements of three "orthogonal parameters," and for remote serial code detection, this measurement is preferably made at a significant standoff distance. The measurements described under Remote Sensing, above, demonstrate the technique in two dimensions: they are made at a distance, and the two "dimensions" characterized are spectral output and radiative lifetime. While there was insufficient time to procure a diode laser of shorter wavelength than the $405 \mathrm{~nm}$ that has been used in the foregoing measurements (although they are available commercially), the $355 \mathrm{~nm}$ pulsed Nd:YAG laser that was part of the lidar system was available for use, and it provided a demonstration of the third "dimension" of the (emission, lifetime, excitation) matrix. Figure 10 shows the variation that can be achieved by changing the excitation wavelength. The data of that figure were collected from the phosphor target at the 43-meter standoff distance during midday using two different excitation wavelengths. 


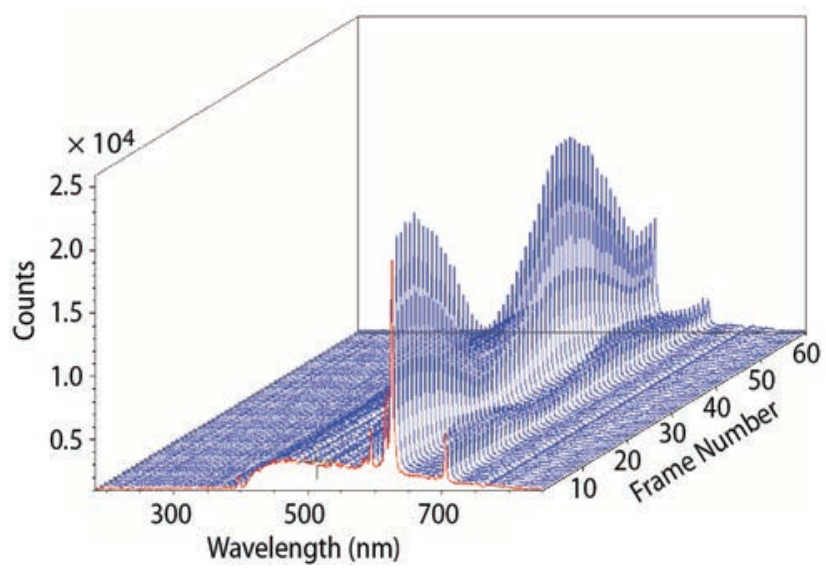

Figure 9. Signal as collected from phosphor target at 43 meters; $400 \mathrm{~Hz}, 60 \mathrm{CCD}$ frames, $1 / 2 \mathrm{~Hz}$ beat frequency

(a)

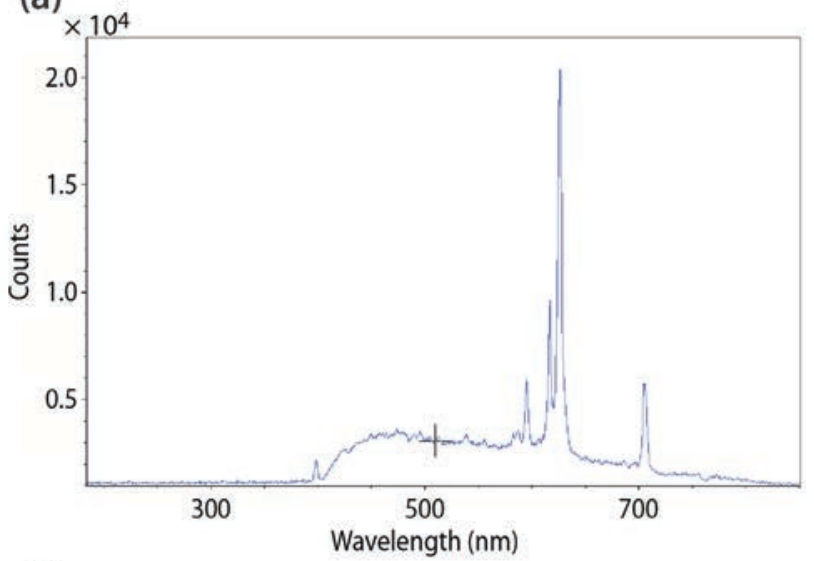

(b)

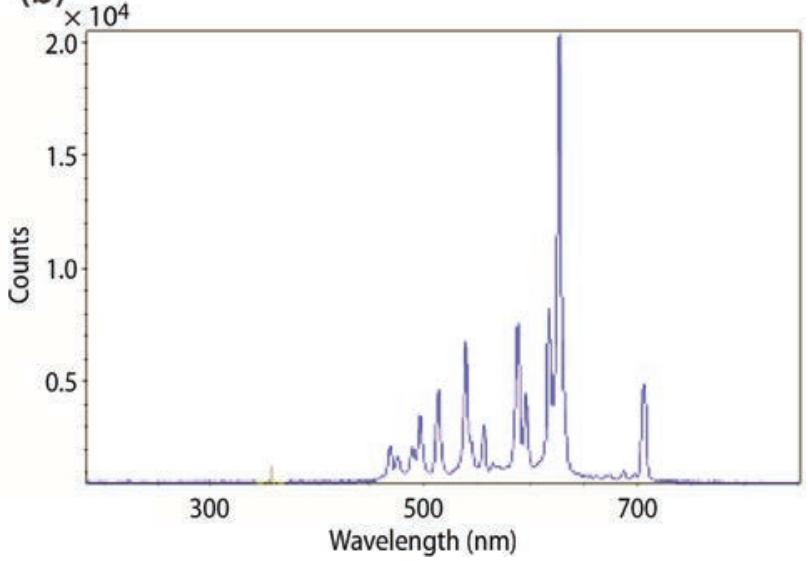

Figure 10. Emission spectrum of phosphor target collected using (a) $405 \mathrm{~nm}$ excitation and (b) $355 \mathrm{~nm}$ excitation

\section{Conclusion}

This year's project successfully demonstrated the application of the hyperspectral beat frequency technique to three different challenges in NSTec's portfolio. We have shown that emission spectra and lifetimes of microscopic particles can be measured using the technique through a microscope, avoiding the high peak power of pulsed systems that can damage the sample. While the smallest particles we measured were $16 \mu \mathrm{m}$ in diameter, this was not an inherent limit, but was only dictated by the available equipment. The technique was also demonstrated to be capable of measuring a range of radiative lifetimes from $4 \mathrm{~ms}$ down to the $5 \mu$ s region with the existing hardware; shorter lifetimes would be measurable with a faster system, or perhaps with the existing system with more complex analysis algorithms.

We also demonstrated that spectra and lifetimes of materials can be measured using the hyperspectral beat frequency technique in a remote sensing scenario, with a standoff of 43 meters under daylight conditions. Again, this is not an inherent limit, but was dictated by the maximum length of STL's outdoor laser test range. All indications are that the technique should work at much longer standoff distances. (Note: At the time of writing this report, the instrumented trailer was transported to the Mojave Desert, and data were collected at longer standoff distances at an outdoor range there. Targets were large [40" $\times 40 "$ "] versions of the $4 " \times 4$ " phosphor and ruby sand targets. Analysis of the data has just begun.) Finally, 3-D serialized measurements (excitation, emission, and lifetime) at a distance have been demonstrated with the technique; this has obvious implications to remote serial code detection work.

For remote sensing (outdoor) measurements, a much faster CCD detector system would permit increasing the beat frequency to mitigate the effects of some noise, because background noise (solar, atmospheric fluctuations, etc.) is worst at lowest frequencies. A lock-in could also be used to recover weak signals (Gardner 2005). A faster detector would also extend the lifetime measurement range to shorter lifetimes. 
For purposes of material characterization, this method gives a reasonable measurement of the radiative lifetime of a sample; the accuracy is sufficient to provide discrimination between fluorescent targets of reasonably different lifetimes. By collecting data at multiple frequencies, target materials can be analyzed not only by spectral output, but also simultaneously by their radiative lifetime and also by response to different excitation wavelengths.

\section{References}

Di Benedetto, J., G. A. Capelle, M. D. O’Neill, "Time-resolved hyperspectral fluorescence spectroscopy using frequency-modulated excitation," Site-Directed Research and Development, FY 2011, National Security Technologies, LLC, 2012, 87-96.

Gardner, F. M., Phaselock Techniques, 3rd ed., Wiley Interscience, John Wiley \& Sons, Hoboken, New Jersey, 2005.

Geipel, G., G. Bernhard, H. Nitsche, "Time-resolved laser-induced spectroscopy of uranium minerals - Part I: Earth alkaline uranyl phosphates," Annual Report 1998, Institute of Radiochemistry, Forschungszentrum Rossendorf Research Institute, 1999, 6.

Lakowicz, J. R., Principles of Fluorescence Spectroscopy, 3rd ed., Springer Science+Business Media, LLC, New York, 2006, 160-163.

Menkara, M., Phosphortech Corp., private communication, 2012. 


\section{AdVANCEd High-SPEed 16-Bit Digitizer SySTEM}

LAO-33-12 | CONTINUED FROM FY 2011 | YEAR 2 OF 2

Michael Jones, ${ }^{1, a}$ Andrew Smith, ${ }^{a}$ Sean Leffler, ${ }^{a}$ Bradley Delamarter, ${ }^{a}$ and Dennis Martin ${ }^{b}$

In FY 2011, this project began development of a single-channel, PCB-level prototype digitizer system capable of digitizing frequencies greater than $1 \mathrm{GHz}$ with a throughput of $\sim 1 \mathrm{GS} / \mathrm{s}$. This in-house solution may pave the way to creating low-cost, expendable data acquisition systems. It can be used for collecting data in a variety of traditional (stockpile stewardship) and nontraditional mission applications. Three PCBs (analog front end, clock distribution, and clock phase shifting) were designed in FY 2011. Testing these PCBs in FY 2012, we found that the frequency response of the analog front end was not as flat as expected, the clocking circuits failed to generate a clock output, and problems exist implementing the Marvell 88E1111 PHY. The PHY is the physical layer, or layer one, in the seven-layer open systems interconnection model of computer networking. A new analog front end was developed in addition to new clocking circuits, and a Nios II processor system was programmed into a field-programmable gate array (FPGA). Two-way communication was established between the FPGA and a web browser running on a computer. All major pieces required to create the advanced 16 -bit digitizer system have been designed and tested; however, the PCB-level prototype was not achieved.

1 jonesmj@nv.doe.gov, 505-663-2126

${ }^{a}$ Los Alamos Operations; ${ }^{\text {b }}$ Great Basin Technology, Inc.

\section{Background}

Historically, the NNSS and government laboratories rely on large numbers of high-speed oscilloscopes or digitizers to perform analog-to-digital conversion and data storage. These oscilloscopes only offer 8-bit resolution, can cost over $\$ 100,000$ each, and are large and bulky. Companies such as Tektronix Component Solutions, formerly Maxtec, offer stand-alone digitizer modules, but these 8 - to 10 -bit resolution units require additional circuitry and packaging, and cost over $\$ 75,000$. Migrating to 16 -bit technology theoretically improves sensitivity by a factor of 256 ; however, commercially available 16-bit analog-to-digital converter (ADC) chips only operate at $200 \mathrm{MS} / \mathrm{s}$. In order to achieve a reasonable sample rate, multiple ADC chips would have to be interleaved. This method requires an analog fan-out circuit to impedancematch the input signal to each ADC chip, a complex clock circuit to phase shift the ADC sample points, digital processing to realign the digital data into a single time domain, a storage medium to store the digital data, and a mechanism to retrieve the digital data.

\section{Project}

The initial 16-bit digitizer concept (Figure 1) was developed in FY 2011 (Jones 2012) in addition to three PCBs. The PCBs include the analog front end, clock distribution, and clock phase shifting (Figure 2c). The analog front end accepts a 50-ohm analog input signal, splits it eight ways, and is impedance-matched for the ADC chips. The clock distribution circuits are responsible for creating eight low-jitter $160 \mathrm{MHz}$ clocks that are in phase with each other. The clock 


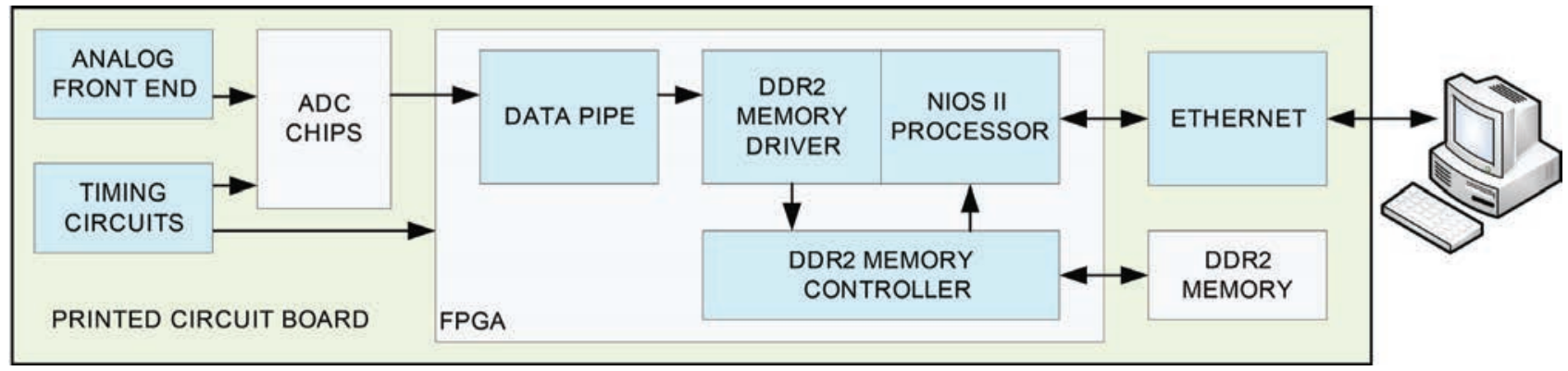

Figure 1. Conceptual system block diagram for the advanced high-speed 16-bit digitizer system. The analog front end takes a single input signal and fans it out to multiple ADC chips. The timing circuit creates multiple phase-shifted, low-jitter clocks to offset the ADC chips' sampling point, thereby allowing multiple time-shifted samples to be digitized within a single clock period. The data pipe realigns the interleaved data back into a single time domain to be stored into memory. The Nios II processor provides the web-based server for system control and data retrieval.
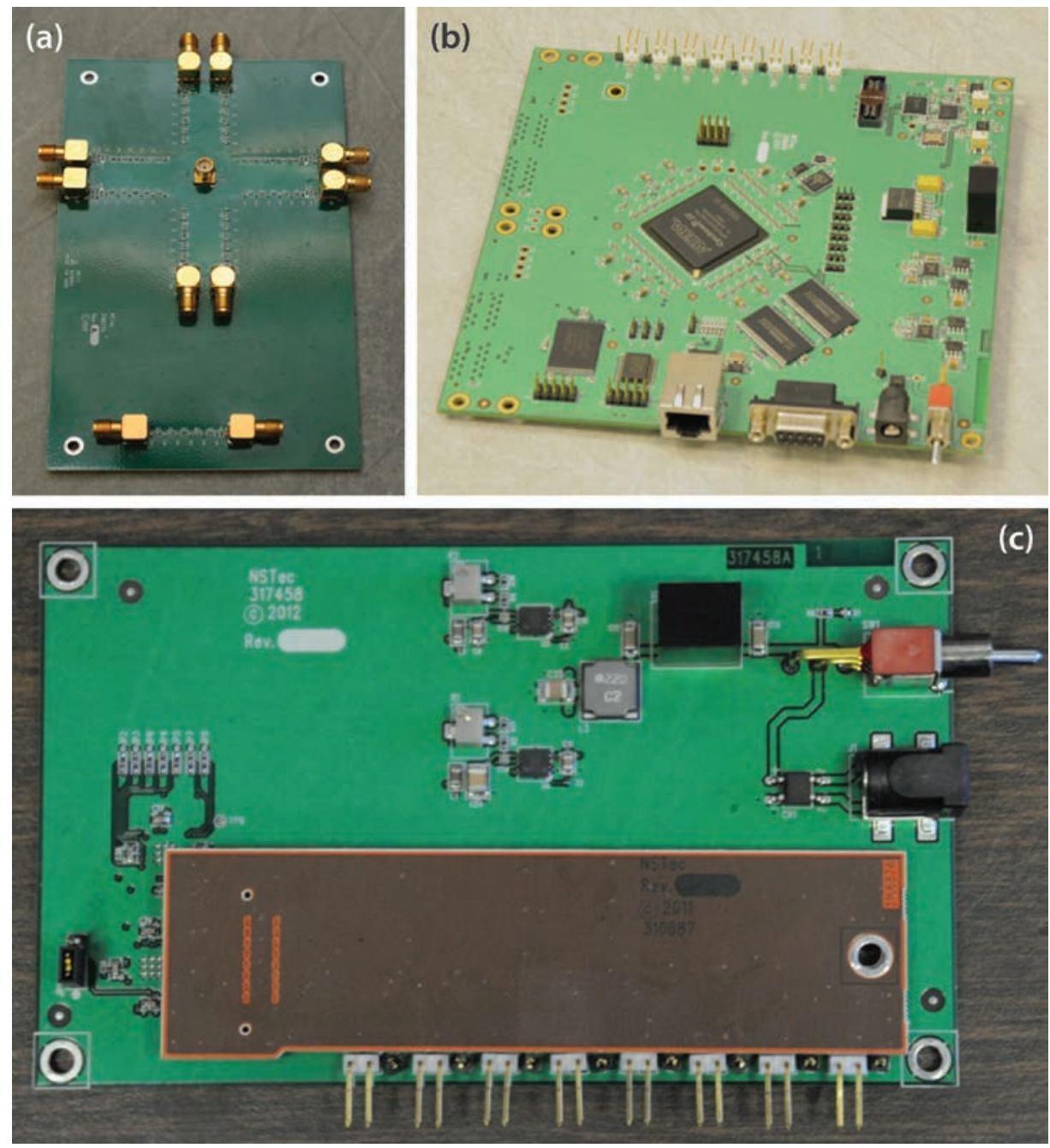

Figure 2. (a) The analog front end designed using the Besser method (Besser 1993) fans out a single analog input signal to eight analog outputs; its 50-ohm input impedance drives a load of $6.8 \mathrm{pF}$ series capacitance and 20 ohms to ground (the $6.8 \mathrm{pF} 20$-ohm load is the calculated input impedance of the ADC chips). (b) The FPGA clocking board (shown without the phase delay PCB) was designed to test clocking circuits, Nios II processor systems inside the FPGA, and, ultimately, Ethernet via FPGA. (c) The redesigned clocking circuit PCB (green) with the attached phase delay PCB (orange); together these boards generate eight unique phase-shifted clocks at $160 \mathrm{MHz}$. 
phase shifting board, also called a phase delay board creates a $45^{\circ}$ phase shift from channel to channel across all eight clocks. Testing of these PCBs began in FY 2012.

Three different methods of programming the FPGA, Joint Test Action Group (JTAG), active serial (AS), and active parallel (AP), were tested, and all modes performed as expected. JTAG and AS are relatively standard ways to program an FPGA, but this was the first time AP mode was used by the team. The MSEL pins on the FPGA select which programming method is used, the power-on-reset delay, and the configuration voltage standard. The downsides of using the AP method are that the mode is not supported by all FPGAs, and the interface to the configuration memory is distributed across four different inputoutput $(\mathrm{I} / \mathrm{O})$ banks in the FPGA. These I/O banks must use the same voltage standard, which may be a concern in a design where multiple I/O voltages are required. Using this type of FPGA programming strategy is very appealing when using a processor inside the FPGA that uses a common flash interface (CFI). By selecting a configuration memory device larger than what is needed to configure the FPGA, the designer is provided with non-volatile memory that can be used to store system configuration parameters, processor instruc-tion code, read-only file systems, or web pages. The FPGA configuration file is a known size and well documented by the manufacturer; this information is very helpful in determining minimum memory device requirements.

The FPGA clocking board was designed to handle multiple types of memory. Two IS42S16320B synchronous dynamic random access memory (SDRAM) chips (Synchronous 2013) are configured in parallel to achieve $128 \mathrm{MB}$ of high-speed memory used by the Nios II processor to execute programs and for general purpose memory. The heap, stack, and exceptions for the processor are normally mapped to this memory. The 16 MB P33 flash memory (P33 2013) uses a single memory chip to store the FPGA configuration in AP mode, processor code, the read-only file system, and web page code. The 8 MB S29 flash memory (S29 2013) uses a single memory chip and was intended for an alternate development path in case problems were encountered using the P33 flash memory beyond FPGA configuration. Two different development paths for creating a system for Altera FPGAs are readily available within the Quartus II (Quartus 2013) development suite. They are Qsys and SOPC Builder. A simple Nios II processor was developed to test all of the various memory devices. This processor system was designed with Qsys (Altera 2013) and operated from memory internal to the FPGA. SOPC Builder can still be used to develop processor systems. Both Qsys and SOPC Builder are still part of the Quartus II software from Altera as of version 11.0 service pack 1; however, it is important to note that SOPC Builder will soon be obsolete. After the processor system was designed, testing of the memory devices proved very difficult. Each memory interface has unique problems, which are described below.

A -3 ns phase shift on the $50 \mathrm{MHz}$ clock to the SDRAM chips was required to make them function properly. The $-3 \mathrm{~ns}$ phase shift also worked when clocking the SDRAM at $100 \mathrm{MHz}$. Additional problems were encountered using the Qsys component declaration template for VHDL code. The read, write, and chip select signals for each flash memory device were defined as "std_logic_vector (0 downto 0)" (Figure 3). This prevents the signals from being mapped to the top level of the hierarchy. This problem seems to only affect VHDL code and does not appear to affect Verilog code. This problem can be seen by using the register transfer level (RTL) netlist viewer in Quartus II, which will show the signal pins on the Qsys system component not connecting to the top level of the FPGA project. To correct this issue, we changed the signal type in the component declaration from "std_ logic_vector(0 downto 0)" to "std_logic". With all changes in place, all memory chips were thoroughly tested and passed successfully.

When the circuits for the FPGA clocking board were initially developed, very little was understood about how the Nios II processor communicates to a terminal device. Given this lack of understanding, an RS-232 port was included in the design. We now know that the terminal connection to the Nios II processor is 


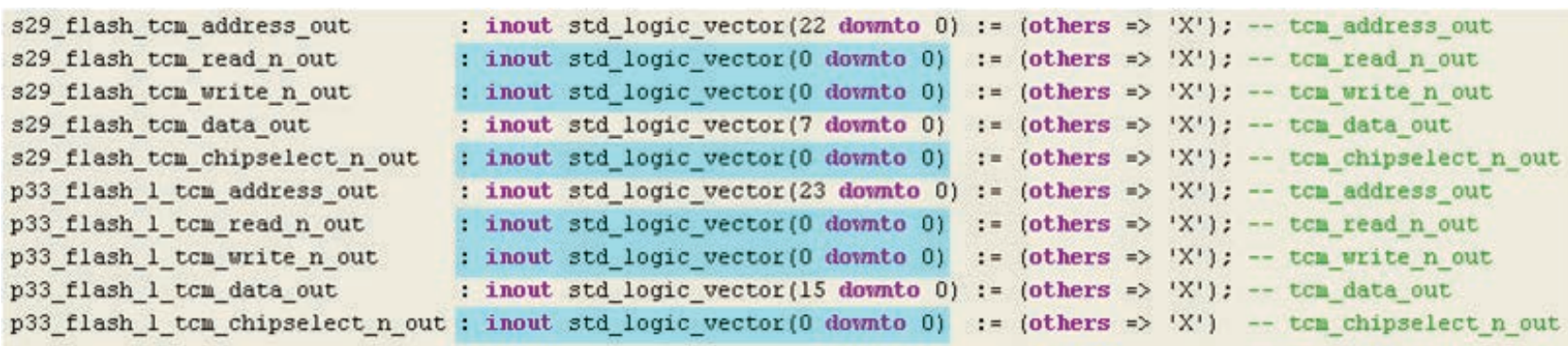

Figure 3. The highlighted areas on this code fragment from the Qsys system component declaration template are not correct, which caused the signals associated with the two different flash memory components not to be mapped to the top level of the VHDL code. To correct this problem we changed "std_logic_vector(0 downto 0)" to "std_logic."

typically established through a JTAG-UART [universal asynchronous receiver/transmitter]. The FPGA programmer is attached to the FPGA JTAG port, and a JTAG-UART is defined in the Nios II system. The Nios II software application, which is part of the Quartus II software, is used to develop software for the processor. It has a terminal program that uses this JTAG-UART to communicate with the Nios II processor. Even though the RS-232 circuits are not needed, they were tested successfully with and without hardware flow control.

A new FPGA program was written to test the clocking circuits. This program implemented an $\mathrm{I}^{2} \mathrm{C}$ interface to configure the clocking circuits. We found that a critical connection needed for the voltage-controlled oscillator (VCO) was missing in the design. Given the technology used, we were unable to correct this issue on the PCB. We isolated the VCO from the clock fan-out chip via the coupling capacitor and injected $1.28 \mathrm{GHz}$ directly into the clock fan-out chip. This allowed us to continue testing. Changes were required and implemented into the FPGA to correct configuration issues with the clock fan-out chip. As we continued testing, we discovered that the clock fan-out chip was introducing unpredictable phase shifts of $180^{\circ}$ each time the circuit was powered. This behavior is unacceptable in this design, and a new solution would be required. Even though the clocking circuits did not function as desired, they did allow us to do some initial testing on the clock phase delay board.
The clock phase delay board was designed to take eight identical clocks at $160 \mathrm{MHz}$ and phase shift them $45^{\circ}$ from channel to channel. Initial measurements showed that we achieved a phase delay of

(a) 8-way splitter simulation

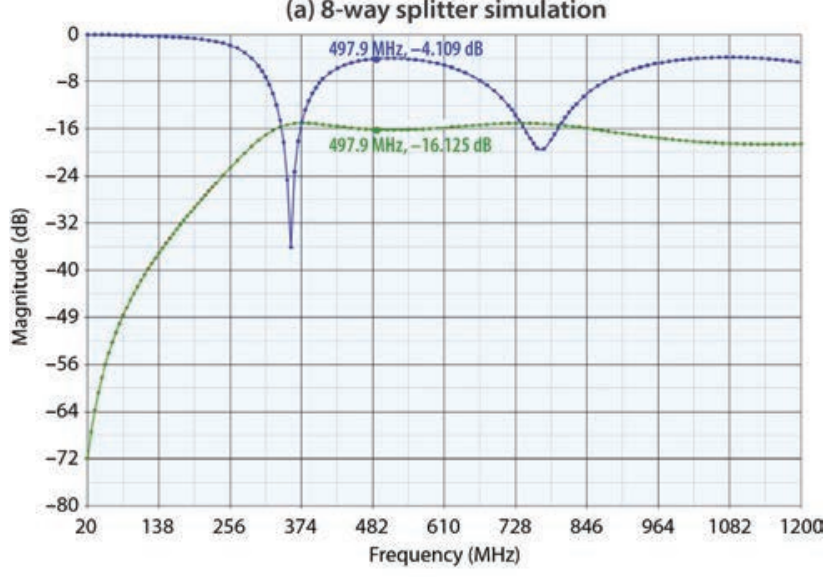

(b) 8-way splitter characterization

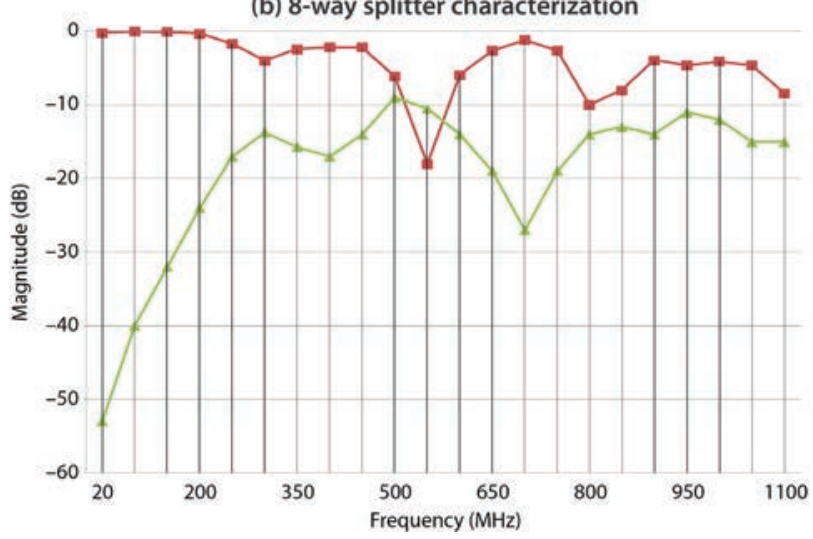

Figure 4. The simulated (a) and measured (b) characteristics of the 8-way splitter designed using the Besser method (Besser 1993). The frequency response shown in green is not as flat as we had hoped. 
$52.7^{\circ}$. The four-layer phase delay board was manufactured using R03010 PCB material, which is typically used for one- or two-layer PCBs. During manufacturing of the $P C B$, the glue used to add the additional layers to the PCB had a dielectric constant that was different than the RO3010 material. This ultimately changed the dielectric constant of the entire PCB and caused the phase shift of the clocks to change by an additional $7.7^{\circ}$. This problem can be resolved by shortening the $\mathrm{PCB}$ traces or redesigning this $\mathrm{PCB}$ in a different material where the dielectric constant of the glue and PCB material are more closely matched. Either way, achieving the consistent channel-tochannel phase shift proved this approach to be viable.

Testing of the 8-way splitter using the Besser method (Besser 1993) (Figure 4) was disappointing. We had predicted the frequency response to be $\pm 4 \mathrm{~dB}$ between $100 \mathrm{MHz}$ and $1 \mathrm{GHz}$. We cannot explain the difference between our simulated and measured results at this time. Given the unexpected results for the analog front end and clocking circuits that failed, new circuits were designed.

The new clocking circuit (Figure 2c) utilizes the CDCM61002 two-output, integrated-VCO, low-jitter clock generator (CDCM61002 2013) from Texas Instruments that is driven with a $24 \mathrm{MHz}$ highstability oscillator (24 MHz oscillator 2013). The clock generator produces a low-jitter $160 \mathrm{MHz}$ clock that is fed into the CDCLVD1208RHDT 2:8 low-additive jitter, low-voltage differential signaling (LVDS) buffer (CDCLVD1208RHDT 2013) from Texas Instruments. This buffer outputs eight nearly-identical $160 \mathrm{MHz}$ clocks. These clocks enter the phase delay PCB, which creates the final eight unique phase-shifted clocks (Figure 5). Jitter measurements show the new clocking circuit has 3.248 ps of random jitter (Figure 6), a significant improvement over the 300 ps jitter specification on the phase-locked loops located in the FPGA.

A new analog front end was designed using the Lumped Element Wilkinson Power Divider method (Lumped 2013). The assembled PCB is shown in Figure 7 along with the schematic for a single 1:2 power splitter. The measured output characteristics
(Figure 8) of this circuit yield a $-3 \mathrm{~dB}$ bandwidth from 190 to $762 \mathrm{MHz}$. Additional efforts would improve the low-frequency response. One of the challenges in quantifying this circuit is the load requirement of the $6.8 \mathrm{pF}$ series capacitance with 20 ohms resistance to ground, the calculated input impedance for the ADC. If this impedance is used in the circuit and then connected to the Agilent 8702E network analyzer for characterization, an impedance mismatch results. The impact of this impedance mismatch is not fully understood at this time. If the output impedance of the circuit is set to 50 ohms (Figure 9), then there is no impedance mismatch with the Agilent network analyzer; however, this configuration is not representative of the designed configuration. A reliable characterization of this circuit would require it to drive the ADC chips as designed and then characterize the digital output, which may contain errors in the analog-to-digital conversion.

The final piece in the puzzle for the advanced 16-bit digitizer system is an Ethernet connection via the FPGA. Solving this challenge required substantial effort. Communication was eventually established between an FPGA and a web browser beginning with the establishment of a simple web server on the Cyclone III 3C120 Embedded Systems Development Kit (part number DK-EMB-3C120) (Cyclone 2013). This kit is very similar to the Cyclone III FPGA Development kit (part number DK-DEV-3C120). At this time, the files related to DK-DEV-3C120 are out of date and should be avoided. Both of these kits have much support on the Altera forums and function with the simple web server (RGMII) template within the Nios II application.

Two different Ethernet systems were tested on the DK-EMB-3C120 kit. One system was designed with SOPC Builder, and the other was designed with Qsys. Each system is implemented differently inside the FPGA and uses completely different clocking schemes, rendering their inclusion highly valuable for learning purposes. Both systems served web pages to a web browser using gigabit Ethernet (often referred to as Gig-E or T-1000). Working with these two Ethernetbased systems greatly enhanced our ability to create 


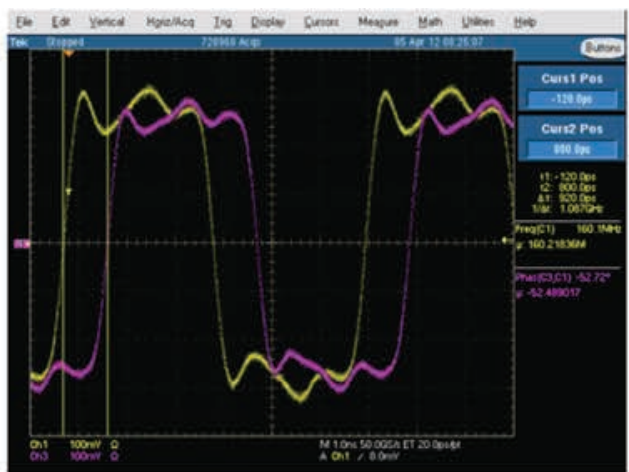

Channel 1 - 2

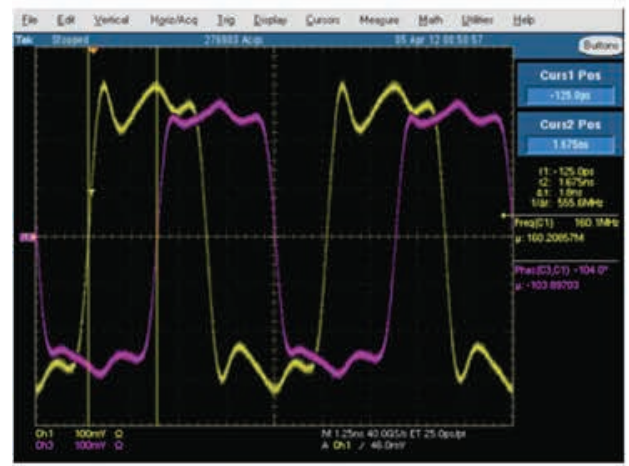

Channel 1 - 3

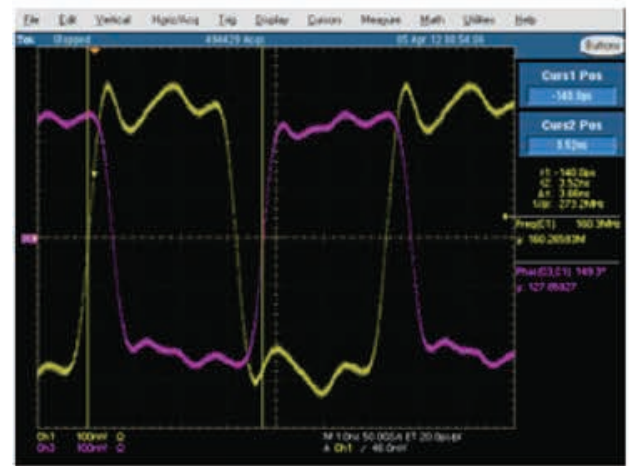

Channel 1-5

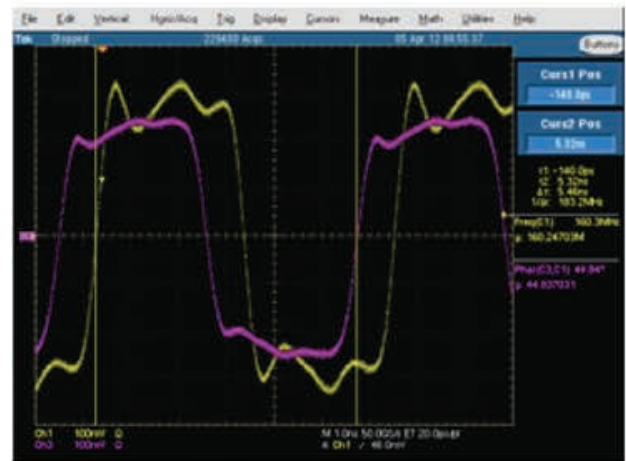

Channel 1-7

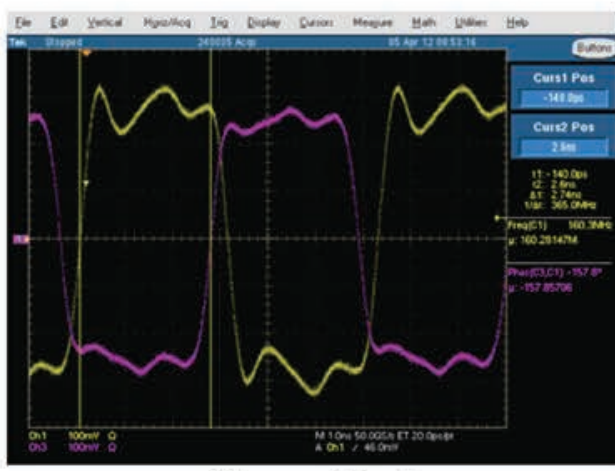

Channel 1-4

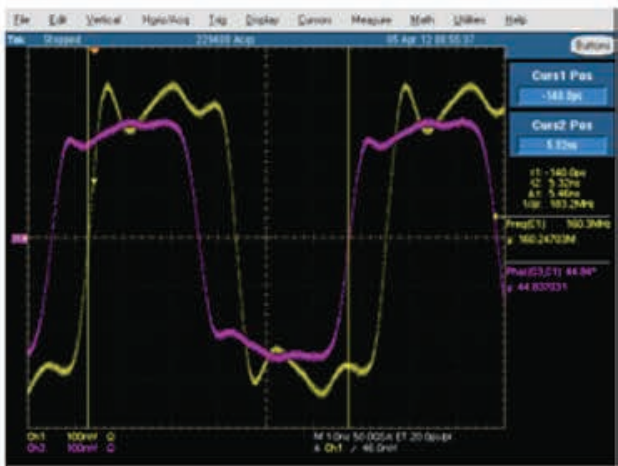

Channel 1-6

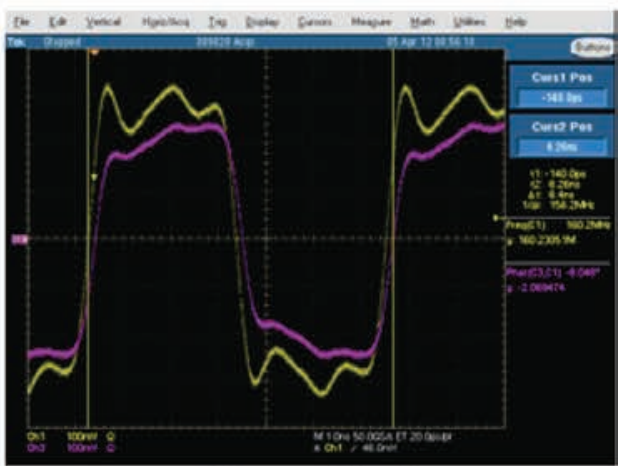

Channel 1 - 8
Figure 5. A channelto-channel phase shift of $52.7^{\circ}$ generated through the clock delay board. Each measurement was taken in reference to channel 1. The desired phase shift is $45^{\circ}$; however, given the channel-to-channel phase shift is the same, the concept of using PCB traces to perform phase shifts was proven viable. 


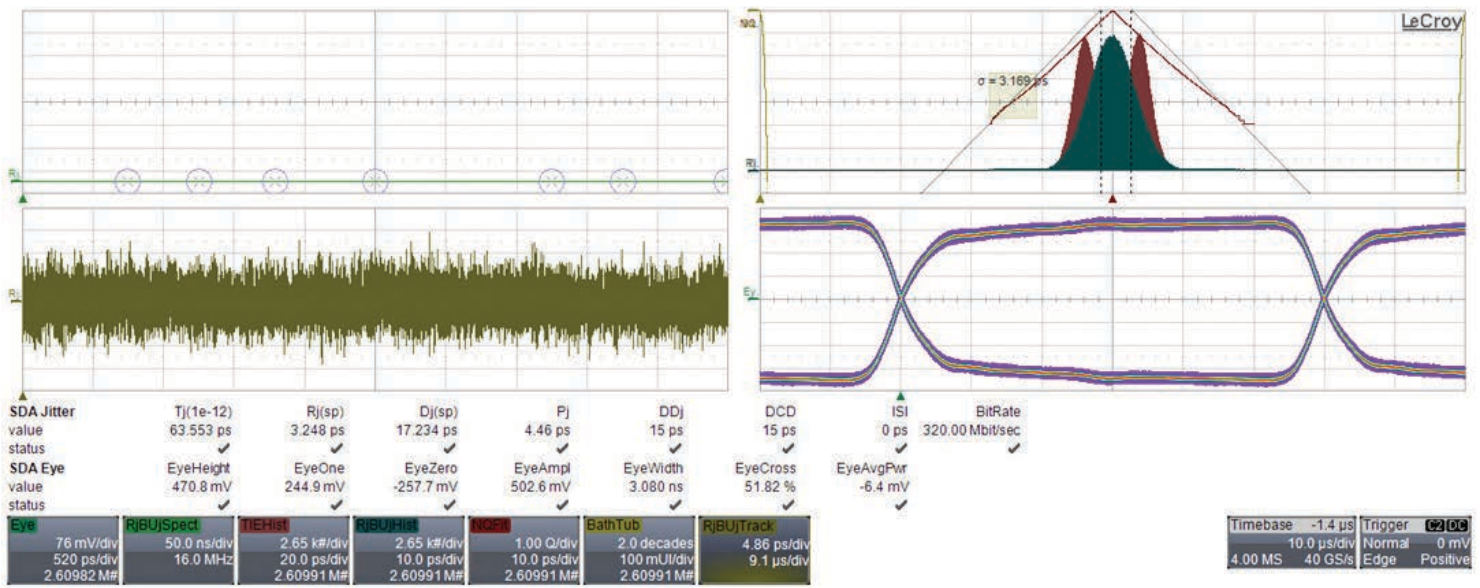

Figure 6. The new clocking circuit has 3.248 ps of random jitter. This is a significant improvement over the 300 ps jitter specification on the PLLs in the FPGA.

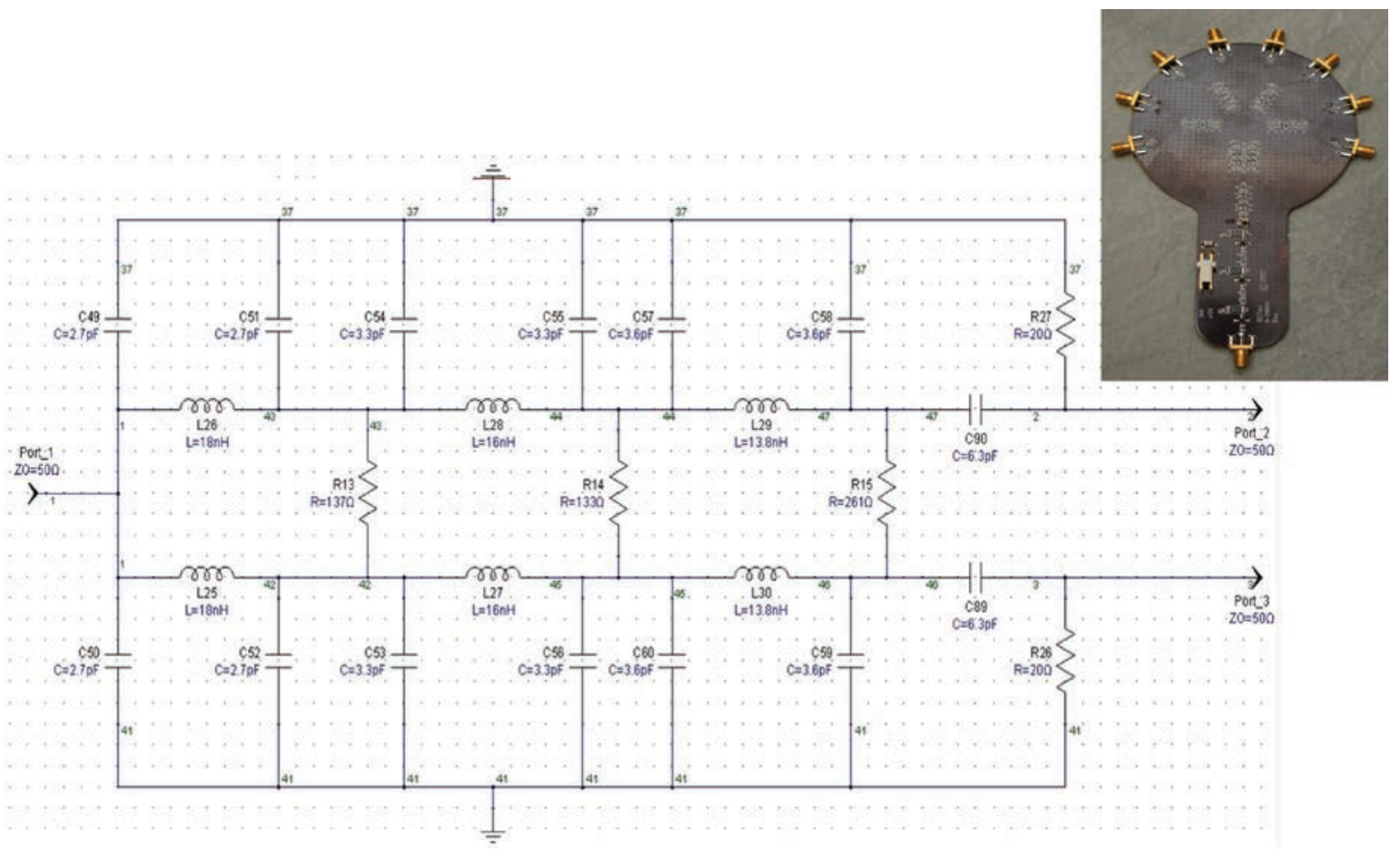

Figure 7. (inset) The assembled 8-way splitter circuit is designed with 50-ohm input impedance, and impedancematched to drive a load of $6.8 \mathrm{pF}$ series capacitance and $20 \mathrm{ohms}$ to ground. The $6.8 \mathrm{pF} 20$-ohm load is the calculated input impedance of the ADC chips. The schematic for a single 1:2 stage used in the 8-way splitter uses a 1:8 power divider with amplifiers to boost the low-end frequencies.

the Nios II processor system. With the information learned here, we were able to establish an Ethernet connection on the Altera DE2-115 development and education board, which contains two Ethernet ports, ETHO and ETH1. Solid Ethernet connections were established on the ETH1 port. The ETH0 port has issues that were confirmed in testing and lack good documentation. All of these Ethernet systems have limited functionality. The most important features that need to be improved are detecting whether an 
(a) Simulation, frequency response and return loss

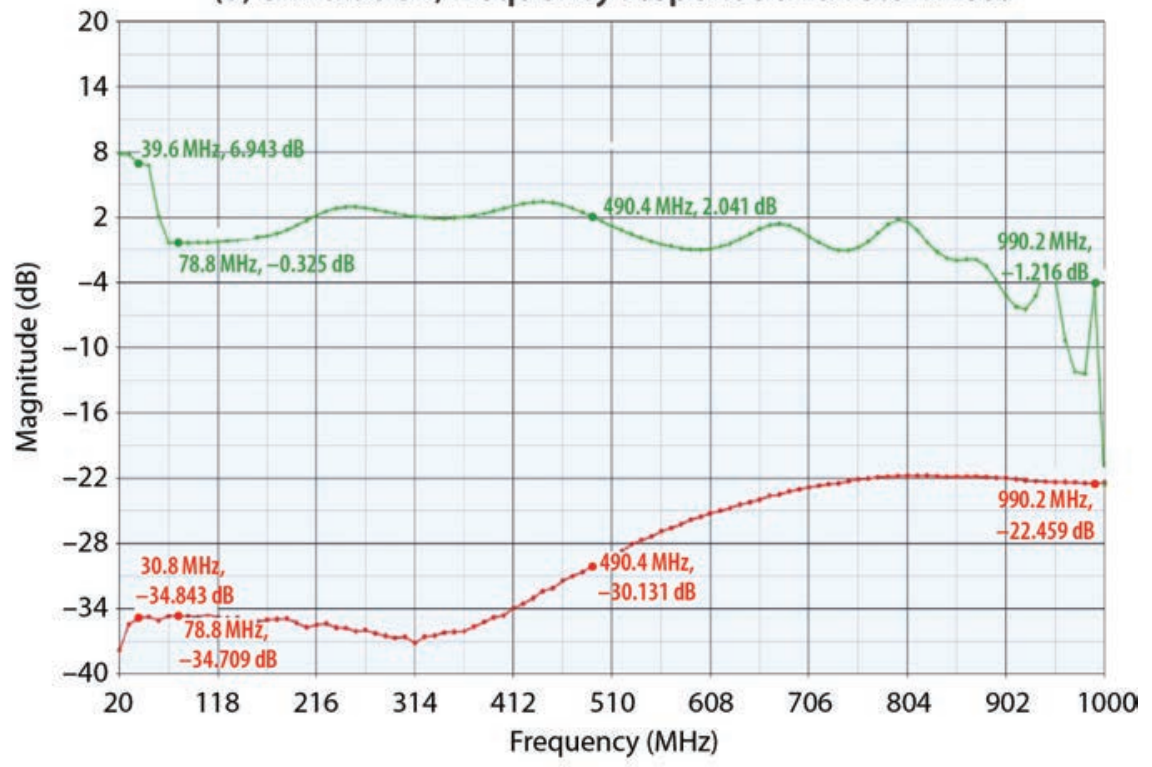

(b) Measured frequency response of Lumped Element Wilkinson Power Divider front-end circuit

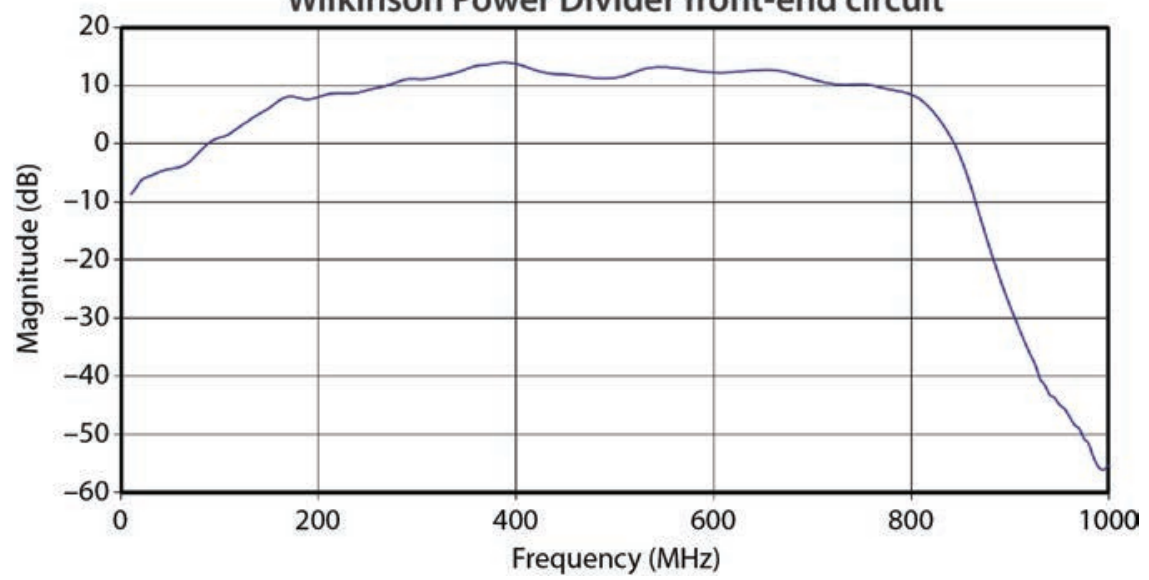

Figure 8. The simulated (a) and measured (b) characteristics of the 8-way splitter. These results may be flawed, given the Agilent 8702E network analyzer requires a 50-ohm impedance.
Ethernet cable is plugged in and enabling all three supported speeds: T-10, T-100, and T-1000. Currently the design only supports T-1000 and the Ethernet cable must be plugged in before the development kits are powered up. The Ethernet system that was used on the DE2-115 kit was modified for our SDRD-developed FPGA clocking board. An Ethernet connection could not be established on our board; however, the MAC [media access control] inside the FPGA successfully communicated with the Marvell 88E1111 PHY. A nondisclosure agreement (NDA) is required from Marvell to obtain a datasheet for the 88E1111 Ethernet PHY. We are in the process of obtaining the NDA. We know that there are issues with the component footprint that we used on our PCB. Without the data sheet it is difficult to get the correct footprint or to confirm if there are any other challenges. Given that the PHY could not properly drive the LEDs connected to it, the PCB footprint and actual component are in question.

At this point the code inside the Nios II processor was modified to allow the 18 switches on the DE2-115 board to be read and displayed on the web page. Using dojo.js, a free Javascript program, we retrieved information on the switch positions, which were then displayed on the web page. We successfully modified the code again with Adobe Flex to retrieve the switch 
(a) Simulation, frequency response and return loss

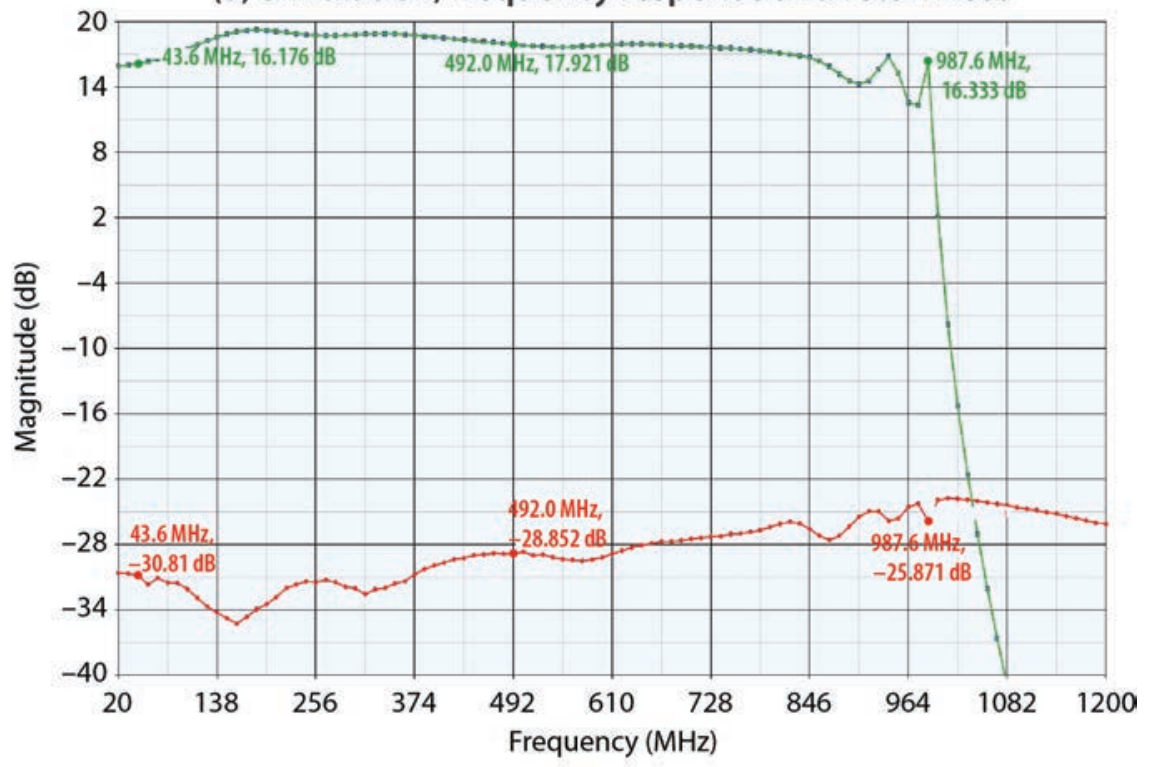

(b) Measured frequency response of Lumped Element Wilkinson Power Divider front-end circuit with 50-ohm load

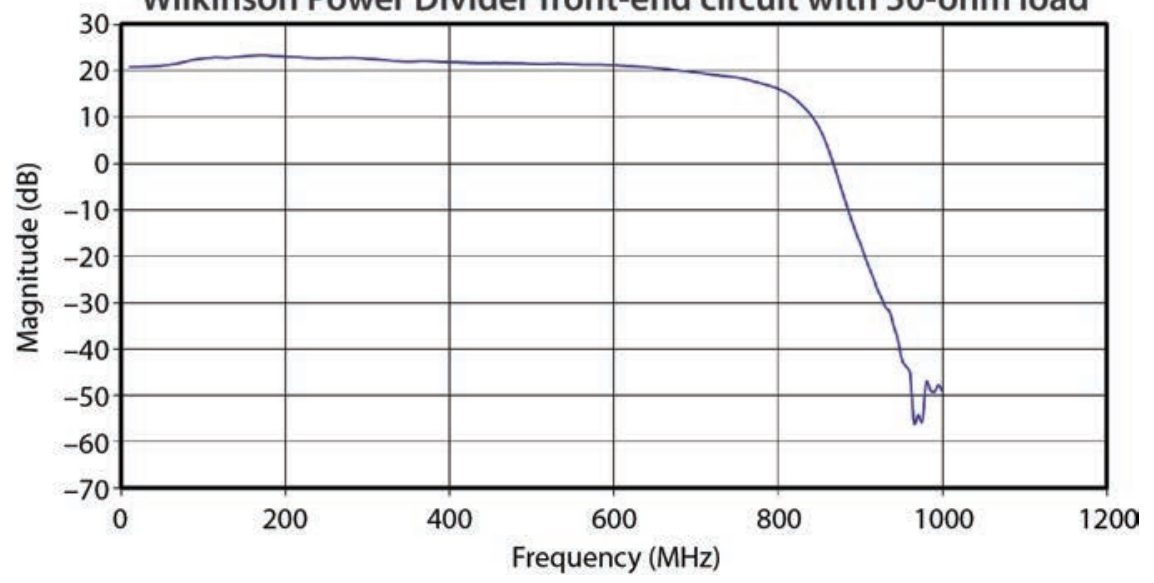

Figure 9. The simulated (a) and measured (b) characteristics of the 8-way splitter designed to have a 50-ohm load and a $-3 \mathrm{~dB}$ bandwidth of $<10$ to $782 \mathrm{MHz}$ positions. Both of these code modifications required designing new web pages and modifying the readonly file system for the Nios II processor.

Finally, we were able to prompt the web browser to automatically open a "save" dialog box. When a web browser requests a particular file from the Nios II processor, a function is called to create the required HTTP header that is sent back to the web browser along with the file. This function was modified to create a HTTP_CONTENT_TYPE of "application/ $x$-download" for all files containing an .exe extension. This trap can be applied to almost any file extension, but only .exe was tested. The web browser does not understand what "x-download" is, so it presents the save dialog box.

\section{Conclusion}

We engineered the analog front end, clocking circuits with phase delay, and an FPGA processor system to provide an Ethernet connection: three key components of the advanced 16-bit digitizer system. All PCBs were tested in FY 2012. The analog front end using the Besser method gave disappointing results. The frequency response of the circuit was not as flat as we had hoped, so a better analog front end was designed using the Lumped Element Wilkinson 
Power Divider. This front end has a $-3 \mathrm{~dB}$ bandwidth from 190 to $762 \mathrm{MHz}$. We are confident that the low-frequency response of this analog front end can be improved; however, we believe that fast apertures that are used in track-and-hold circuits would reap even larger benefits and should be explored. The clocking circuits failed to function, and new clocking circuits were developed and tested. The circuits yield 3.248 ps of random jitter, which is very good. The target phase shift of $45^{\circ}$ was off by $7.7^{\circ}$. Even though the phase shift is off, we proved our concept given the channel-to-channel phase shift was $52.7^{\circ}$ across all channels. The PCB manufacturing process and the materials used were found to significantly impact the dielectric properties of the PCB; an understanding of this is required to correct the phase delay error. The Ethernet circuits that we designed failed to make an Ethernet connection, but the MAC was able to communicate to the PHY, which tells us that we were very close to achieving the goal. We had an incredible level of success using two different FPGA development kits to implement, test, and modify three unique Ethernet systems. Even though these systems have limited functionality, their foundation is stable to continue development. With the information that should be contained in the datasheet for the Marvell 88E1111 PHY, we should be able to correct our Ethernet problems with a new PCB.

We believe that sufficient progress has been made on all major fronts to warrant further effort to build a PCB-level board for the advanced 16-bit digitizer system, a goal that we did not quite achieve this year. Ultimately this SDRD project greatly expanded our ability to design and build Nios II processer systems inside FPGAs. This knowledge and experience increases the complexity and capabilities of FPGA designs that NSTec can now offer its customers.

\section{References}

"Altera DE2-115 development and education board," http://www.terasic.com.tw/cgi-bin/page/archive. pl? Language=English\&No=502, accessed January 29, 2013.
Besser, L. "Reactive transformation of resistances," Applied Microwave (Winter 1993) 104-110.

“CDCLVD1208RHDT, 2:8 low-additive jitter LVDS buffer," http://www.ti.com/lit/ds/symlink/cdclvd1208.pdf, accessed January 29, 2013.

"CDCM61002, two output, integrated VCO, low-jitter clock generator," http://www.ti.com/lit/ds/symlink/cdcm61002. pdf, accessed January 29, 2013.

"Cyclone III 3C120 Embedded Systems Development Kit," http://www.altera.com/products/devkits/altera/kit-embdev-cyc3.html, accessed January 29, 2013.

Jones, M., A. Smith, W. Dreesen, B. Delamarter, D. Martin, "Advanced high-speed 16-bit digitizer system," Site-Directed Research and Development, FY 2011, National Security Technologies, LLC, Las Vegas Nevada, 2012, 111-120.

"Lumped Element Wilkinson power divider," http:// mobiledevdesign.com/images/archive/0802Noriega18.pdf, accessed January 29, 2013.

"P33 flash memory," TE28F128P33T85A, http:// www.micron.com/parts/nor-flash/parallel-nor-flash/ te28f128p33t85a, accessed January 29, 2013.

"Quartus II software," https://www.altera.com/download/ software/quartus-ii-se, accessed January 29, 2013.

"S29 flash memory," S29GL064N90BFI040, http://www. spansion.com/Support/Datasheets/s29gl-n_01.pdf, accessed January 29, 2013.

"Synchronous DRAM," IS42S16320B, http://www.issi.com/ pdf/42S16320B-86400B.pdf, accessed January 29, 2013.

"24 MHz oscillator," ECS-2532HS-240-3-G, http://www. ecsxtal.com/store/pdf/ECS_2532HS.pdf, accessed January 29, 2013. 


\section{GAMMA SPECTROMETER}

LAO-37-12 | YEAR 1 OF 1

Robert M. Malone, ${ }^{1, a}$ Carl S. Young, ${ }^{b}$ and Joseph M. Mack ${ }^{b}$

Better diagnostics are needed to observe the fusion process during the National Ignition Facility fuel burn cycle. Fusion gamma-ray diagnostics are relatively unobtrusive, but they do not presently provide adequate energy resolution. The current NIF gamma reaction history diagnostic uses a cluster of four instruments, each with a different detection threshold obtained by varying the fill gas and its pressure. It is possible to obtain very limited understanding of the gamma-ray spectra by subtracting the signals from pairs of these detectors. To improve the resolution we have designed a time-integrated gamma spectrometer that uses a multiple-ring detector to discriminate different energy bins of gammas.

${ }^{1}$ malonerm@nv.doe.gov, 505-663-2014

${ }^{\text {a }}$ Los Alamos Operations; ${ }^{\mathrm{b}}$ Keystone International, Inc.

\section{Background}

Time-resolved recording of gamma radiation at the National Ignition Facility (NIF) provides fusion reaction history (bang time and burn width) and yields of various nuclear reactions (total deuteriumtritium [DT] neutron yield and plastic ablator areal density [or]). Bang time is used to establish laser energy coupling to the target. Burn width provides tuning information. Since the branching ratio for DT fusion gammas is extremely low, about $4.2 \times 10^{-5}$ relative to $14 \mathrm{MeV}$ neutron emission (Kim 2012), DT fusion gammas have been studied only rarely, but the large NIF fluxes make fusion gammas a useful diagnostic with far less time-of-flight dispersion than for the $14 \mathrm{MeV}$ neutrons. In this project we address a need for a spectrometer to monitor the different energy ranges of the gammas.

The NIF gamma reaction history (GRH) diagnostic (Malone 2008, 2009, 2010 "Overview," 2010 "Design") converts some of the gammas to highenergy electrons in a Compton converter at the entrance to the diagnostic. Interactions of these electrons in the fill gas produce $0^{\circ}-6^{\circ}$ Cerenkov light. The GRH can detect gammas ranging in energy

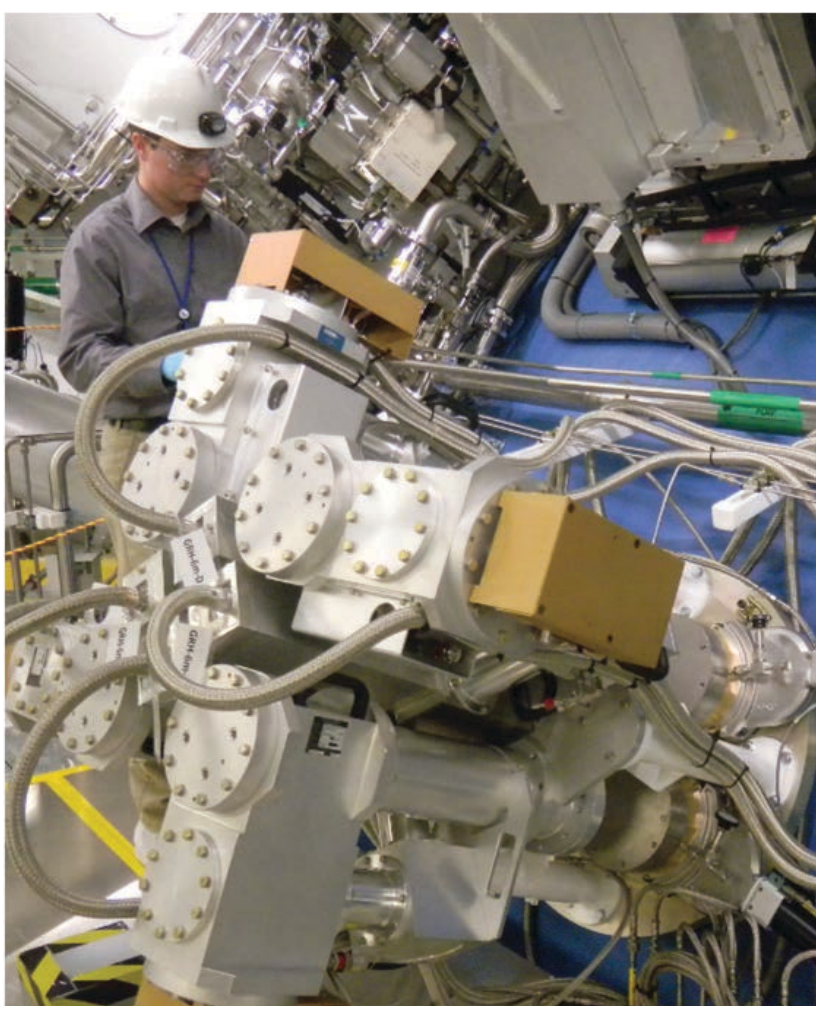

Figure 1. Four GRH instruments are clustered together onto the NIF target chamber 


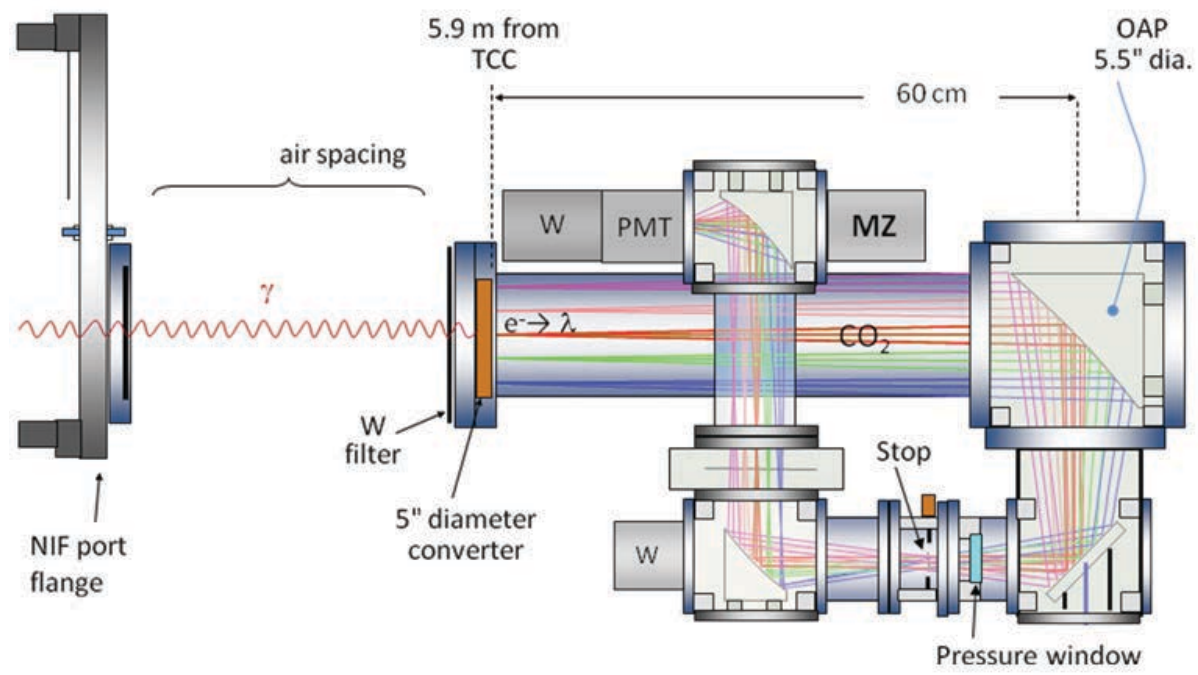

Figure 2. Ray tracing through the NIF GRH diagnostic. $W$ is tungsten, OAP is off-axis parabolic mirror, TCC is target chamber center. The converter is beryllium or aluminum.

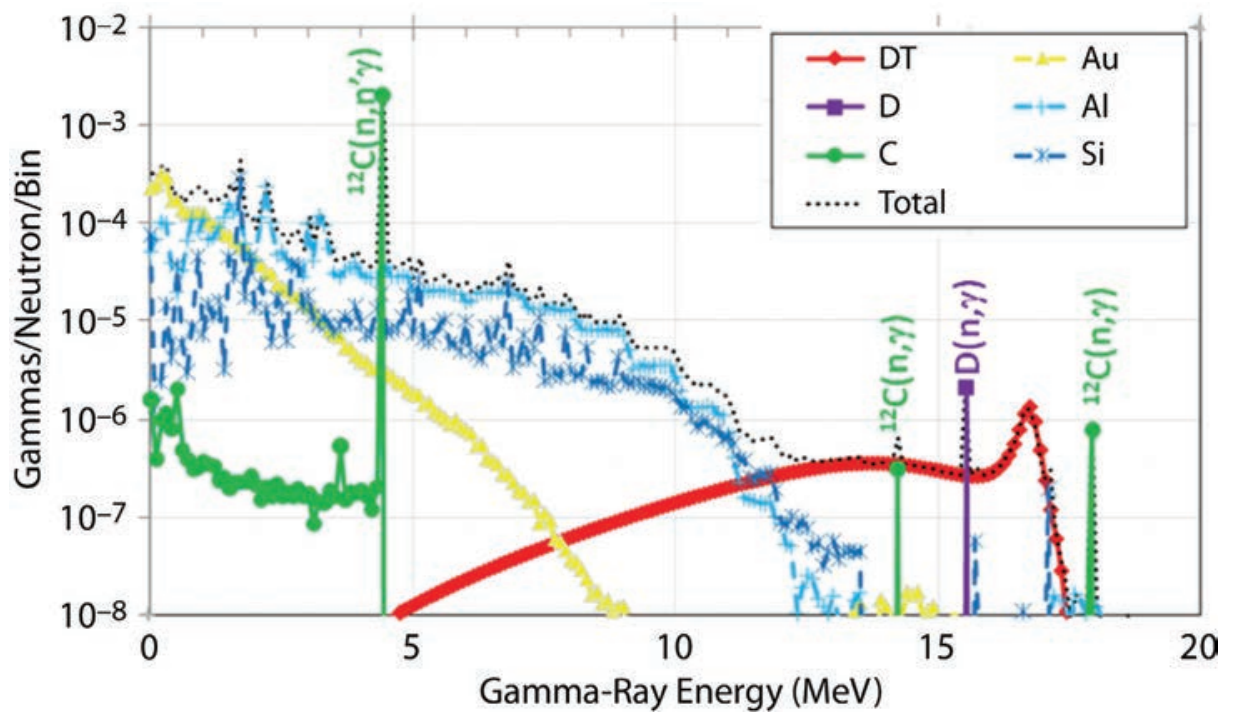

Figure 3. The calculated prompt gamma-ray energy spectrum from indirect-drive, cryo-layered implosions is complicated. Components of the target assembly produce neutron-induced gammas.

from 3.5 to $20 \mathrm{MeV}$, depending on the fill gas and its pressure. Each of four diagnostic channels is set to detect gammas above a different energy threshold. For example, 200 psi of $\mathrm{SF}_{6}$ gives a $3.0 \mathrm{MeV}$ Cherenkov threshold, and 100 psi of $\mathrm{CO}_{2}$ has a 6.3 MeV Cherenkov threshold. Each channel measures all gammas above its threshold. By normalizing the sensitivities of a pair of channels and then subtracting one channel from another, the number of gammas in the energy range between their two thresholds can be determined. In this way we achieve three energy bins from the differences between adjacent detector pairs plus a fourth bin comprising data for the highest-threshold detector. The four GRH units are attached to a support frame attached to a NIF chamber port, as in Figure 1.
Cherenkov light in each channel is collected into the $1 \mathrm{~cm}$ diameter PMT detector as shown in Figure 2. This sample ray trace shows five gamma rays originating from a point at the target chamber center (TCC), located 5.9 meters from the detector. The gamma rays hit five field positions on the converter, producing Compton electrons shown at $-2^{\circ}, 0^{\circ}$, and $+2^{\circ}$. Cherenkov light is emitted along each of these electron trajectories, and the detector collects light within $\mathrm{a} \pm 1^{\circ}$ cone centered about each electron trajectory. Three off-axis parabolic (OAP) mirrors and one flat turning mirror relay the Cherenkov light into a $1 \mathrm{~cm}$ diameter, high-speed PMT detector. (The PMT detectors for the four GRH channels are arranged in 


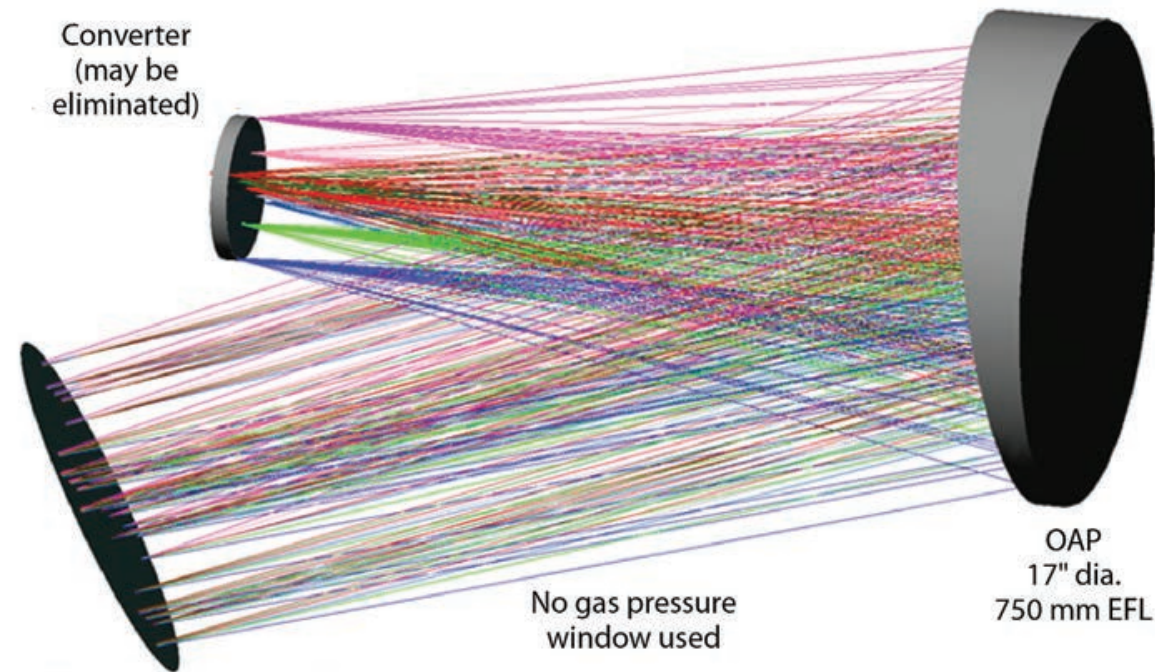

Figure 4. Ray tracing of a $20^{\circ}$ OAP used to collect light for the gamma spectrometer

Tilted ring detector, 12.6 " dia. (detector inside pressure volume)

the middle of the cluster so they experience similar shielding environments.) The combination of mirrors OAP2 and OAP3 is used to compress light from the large emission volume onto the small detector. This 10:1 demagnification limits the collection angle within the gas to $\pm 3^{\circ}$, which can be summed up from any combination of electron and photon angles Figure 2 shows $\pm 2^{\circ}$ electrons and $\pm 0^{\circ}$ photons.

Figure 3 shows the calculated gamma spectra that need to be measured. More channels of gamma energies are needed for an adequate spectral measurement. Currently there is no instrument that can resolve more than four energy bins on the same DT experiment.

\section{Project}

Cherenkov light is produced with an angular distribution that is a function of the Compton electron energy, which is itself a function of the energy of the gamma ray which produced Compton electrons. In an imaging optical system, angular information from emitting light sources is imaged at the stop position, which is insensitive to the positioning of these emitting sources. Therefore, a multiple-ring detector positioned at the stop will measure the angular distribution of the Cherenkov photons and, so, infer the gamma energies.
The form of the proposed diagnostic was similar to the first part of the existing $\mathrm{GRH}$, with a gas volume and an OAP. We performed many design studies to determine the optimum angle for the OAP and the most compact geometry for light collection. The best design is shown in Figure 4. For the redesigned detector we eliminated the large pressure window shown in Figure 2 because it is a source of unwanted Cherenkov light. This places the detector inside the pressurized gas.

Detailed ray tracings in Figure 5 show an overlay of Cherenkov light generated from six different source planes of the gas volume. Light from five electron tracks (in different colors) is traced. The ray trace includes the effect of gammas striking the converter at a range of angles from $-0.4^{\circ}$ to $+0.4^{\circ}$, and the electrons emitted by the converter at a range of angles from $-2^{\circ}$ to $+2^{\circ}$. Both angular spreads cause broadening of the angular discrimination at the ring detector.

Angular blurring produced by the gammas depends on the diameter of the converter. Making the effective converter size smaller reduces the blur, but it also reduces the overall signal available for recording; therefore, this blurring cannot be completely eliminated. However, if the electron scatter can be 


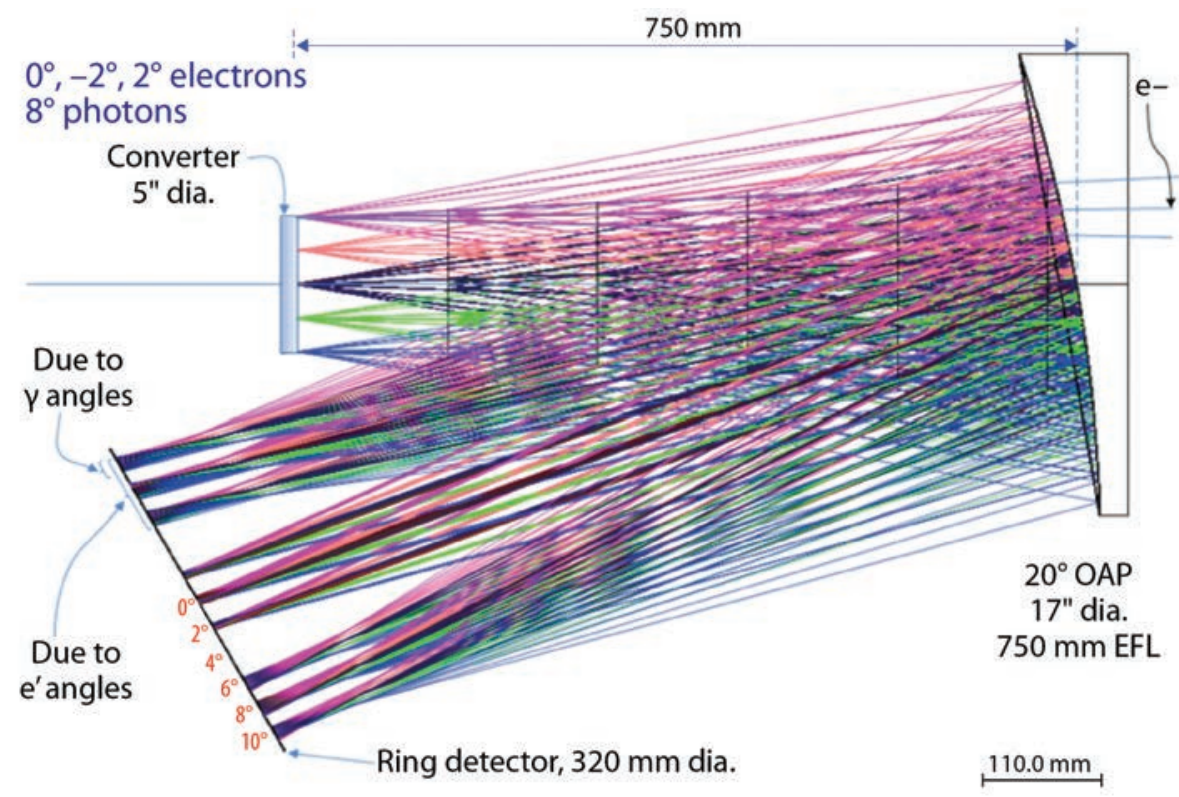

Figure 5. Overlaying

Cherenkov light

emitted by six

different source

planes. Compton

electrons scatter

at $-2^{\circ}, 0^{\circ},+2^{\circ}$.

reduced to zero, then excellent angular discrimination at the ring detector is possible, as shown in Figure 6. In addition, if there is minimal electron scattering, then the outer edges of the OAP allow larger angles of Cherenkov light to be recorded.

The time delay between prompt gammas hitting the gated-off detector and the arrival of the Cherenkov light ranges from 5.5 to $6.0 \mathrm{~ns}$. The delay is useful in allowing the detectors to recover from the gamma flash before the Cerenkov arrives. The range is due to the detector tilt relative to the optical axis.

\section{Electron Scattering Issues}

A charged particle traversing a medium is deflected by many small angle scatterings (Highland 1975, Li 1995). These scatterings are due to the coulomb field of the individual electrons on the atoms and are assumed to be elastic. In each scattering the energy of the particle remains nearly constant, but the particle direction changes. Thus the charged particle beam, the Compton electrons in our case, is gradually broadened.

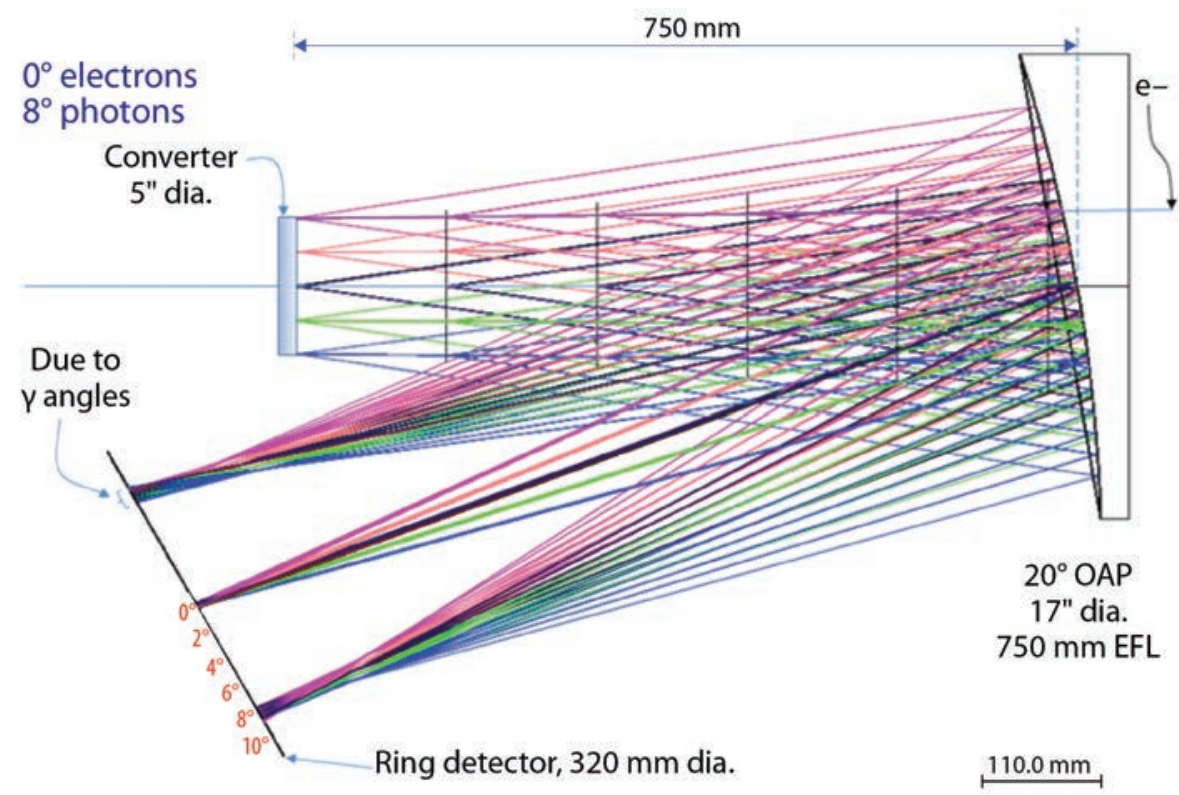

Figure 6. Overlaying

Cherenkov light emitted by six different source planes. Electron scatter is ignored. 
The radiation length is an important quantity describing energy loss of electrons traveling through material. An expression accurate to within a few percent is:

$$
L_{r}=\frac{716.4 A}{Z(Z+1) 1 \mathrm{n}(287 / \sqrt{Z})}\left(g-c m^{-2}\right),
$$

where $Z$ is the atomic number for the absorber material and $A$ is its atomic weight.

In the simplest model of multiple scattering, we ignore large angle scatters. In this approximation, the distribution of electron scattering angle, $\theta_{\text {plane, }}$ after traveling a distance $x$ through a material with radiation length $L r$, is approximately Gaussian,

$$
\frac{d P\left(\theta_{\text {plane }}\right)}{d \theta_{\text {plane }}}=\frac{1}{\theta_{0} \sqrt{2 \pi}} \exp \left[-\frac{\theta_{\text {plane }}^{2}}{2 \theta_{0}^{2}}\right],
$$

with

$$
\begin{aligned}
\theta_{0}= & \frac{13.6 \mathrm{MeV}}{\beta p c} z \sqrt{x(\text { density } \times \text { pressure } / 14.7) / L_{\nu}} \\
& \times\left(1+0.0381 \mathrm{n}\left\{x(\text { density } \times \text { pressure } / 14.7) / L_{\gamma}\right\}\right) .
\end{aligned}
$$

In Equation 3,

$p=$ momentum of incident particle,

$z=$ charge of incident particle,

$x=$ electron path length,

$$
\begin{aligned}
& \text { pressure }=\text { gas pressure }(\mathrm{psia}), \\
& \text { density }=\text { density at } \mathrm{STP}\left(\mathrm{g} / \mathrm{cm}^{3}\right), \\
& \beta \equiv v / c=\sqrt{1-1 / \mathrm{v}^{2}}, \\
& \gamma=\mathrm{E}+0.511 / 0.511, \text { where } \mathrm{E} \text { is the kinetic energy } \\
& \text { of the electron in } \mathrm{MeV} \text {, and } \\
& p c=\sqrt{(\mathrm{E}+0.511)^{2}-(0.511)^{2}} \text {. }
\end{aligned}
$$

The average scattering angle $\left\langle\theta_{\text {plane }}>=0\right.$, but the RMS scattering angle $<\theta_{\text {plane }}^{2}>^{1 / 2}=\theta_{0}$.

Equation 3 is still an approximation, accurate to a few percent. Equation 1 was input into Excel to plot a family of curves relating the electron scatter angle versus gas pressure for $\mathrm{He}, \mathrm{CO}_{2}$, and $\mathrm{SF}_{6}$. Figure 7 shows such curves for $\mathrm{He}$ gas at different gas pressures. To minimize the electron scatter, lower gas pressure is required. However, high gas pressures are required for detection of low-energy gammas.

Cherenkov light is emitted whenever the velocity of a charged particle exceeds that of light in the medium through which it is passing, or $\beta n>1$. In Cherenkov generation (Figure 8), a particle (red arrow) travels in a medium with speed $v_{p}$, and we define the ratio between the speed of the particle and the speed of light as $\beta=v_{p} / c$, where $c$ is speed of light. The refractive index of the medium is $n$, and so the emitted light waves (blue arrows) travel at speed $v_{e m}=c / n$.

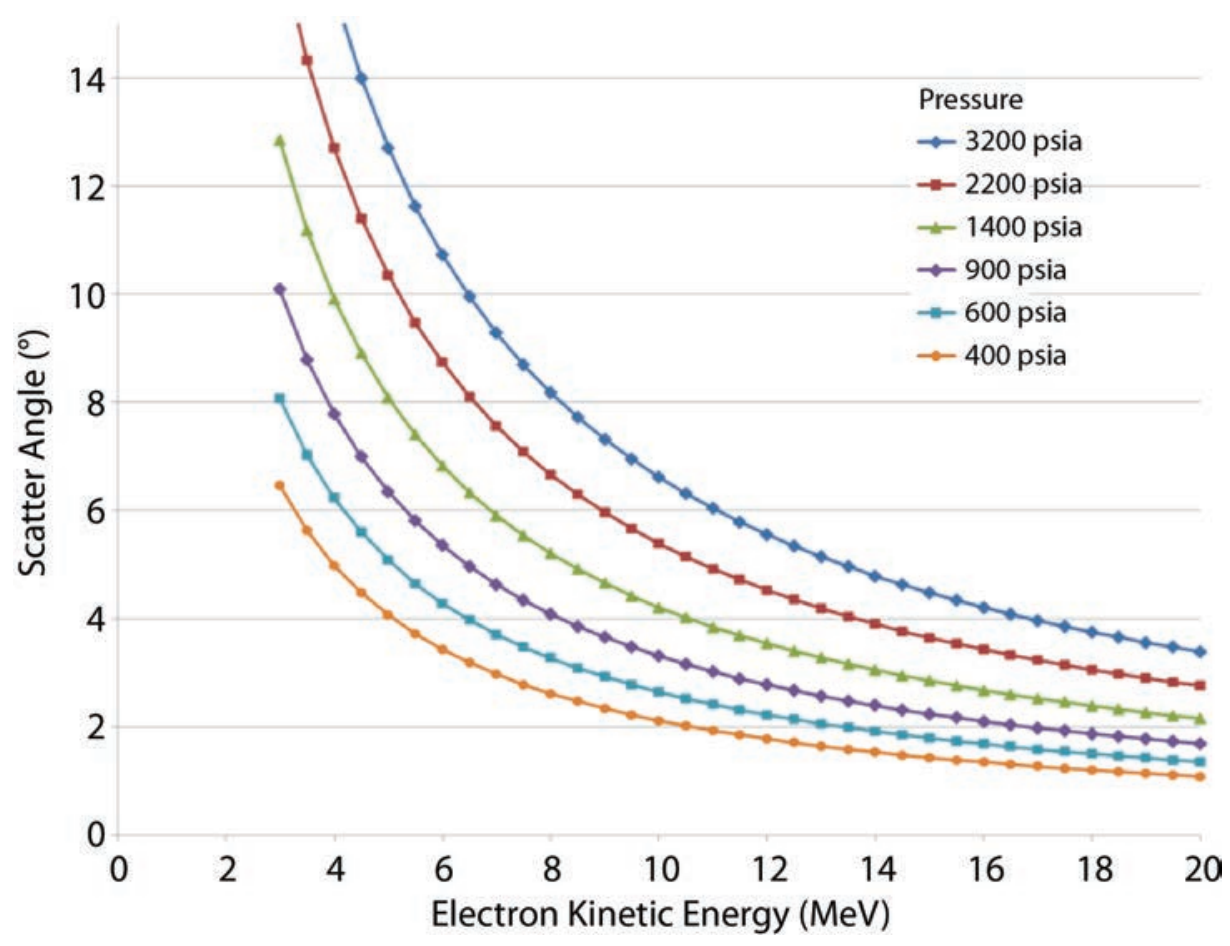

Figure 7. Plotting Compton electron scatter angles vs their energy as they transit pressurized He gas 


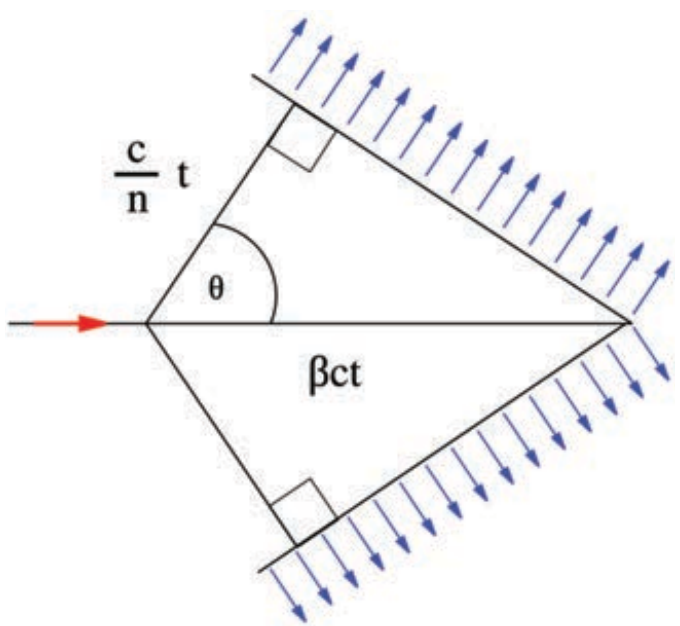

Figure 8. Diagram of Cherenkov generation. During the same time interval, the electron travels a distance $\beta c t$ and the photon travels a distance $\mathrm{ct} / \mathrm{n}$. If the electron velocity increases, the Cherenkov photon angle increases.

Therefore,

$$
\cos \theta=\frac{1}{n \beta} \text { or } \cos \theta=\frac{c}{n v_{p}} .
$$

Equation 4 states that as the electron velocity increases, the Cherenkov light angle increases.

Inputting Equation 4 into Excel yielded a family of curves relating the Cherenkov angle versus gas pressure. Figure 9 shows an example of $\mathrm{CO}_{2}$ gas at pressures ranging from 31 to $247 \mathrm{psia}$. The family of curves is independent in the type of gas used and only depends on the index of refraction.

Ideally, light angle would be a linear function of electron energy for constant angular resolution. However, as shown in Figure 9, actual curves are far from linear, indicating a need for multiple channels, as in the GRH, to sufficiently resolve gamma energy across the total energy range of 3.5 to $20 \mathrm{MeV}$. Figure 9 indicates that a 3-10 MeV spectrum can be measured using $\mathrm{CO}_{2}$ at $247 \mathrm{psia}$, and the 10-20 MeV spectrum can be covered by $\mathrm{CO}_{2}$ at 46 psia.

\section{ACCEPT Calculations}

A Coupled Combinatorial Electron Photon Transport (ACCEPT) is a Monte Carlo code that includes multiple elastic electron scattering. The entire spectrometer design was verified using this code (used by both Sandia National Laboratories and Los Alamos National Laboratory). The code ran Monte Carlo simulations to model gamma rays emitted at the target chamber center and traveling to the converter material, the conversion of these gamma rays into Compton electrons, electron transport and multiple scattering in the convertor material and gas cell, conversion of electrons into UV/visible photons by Cherenkov radiation, and ray tracing of these photons through the system optics into the detector. The code provided direction cosines, angles, wavelengths, and probabilities of the Cherenkov photons generated and detected.

New Cherenkov visualization capabilities were created and added to the output of the ACCEPT code to enable 3-D viewing of the Cherenkov ring patterns. An example is shown in Figure 10. The ACCEPT code was modified to allow turning the electron multiple scattering on and off to observe how it broadens the Cherenkov angles arriving at the detector plane. After comparing electron multiple scattering calculations, we found that hydrogen gives better discrimination than either helium or $\mathrm{CO}_{2}$.

Figures 11 and 12 show example outputs from the ACCEPT code; a $6.5 \mathrm{~cm}$ beam of electrons is passed from the converter through a $330 \mathrm{psia}_{2}$ gas toward the OAP, using the geometry shown in Figures 4 and 5. Figure 11 provides results for 13.0 $\mathrm{MeV}$ electrons and Figure 12 for $16.5 \mathrm{MeV}$ electrons. Both figures plot the number of Cherenkov photons versus the radius of the detector. These are timeintensive calculations with coarse bins on the detector plane. Even with this crude binning, there is adequate angular discrimination between these two electron energies. Other gas pressures need to be calculated. 

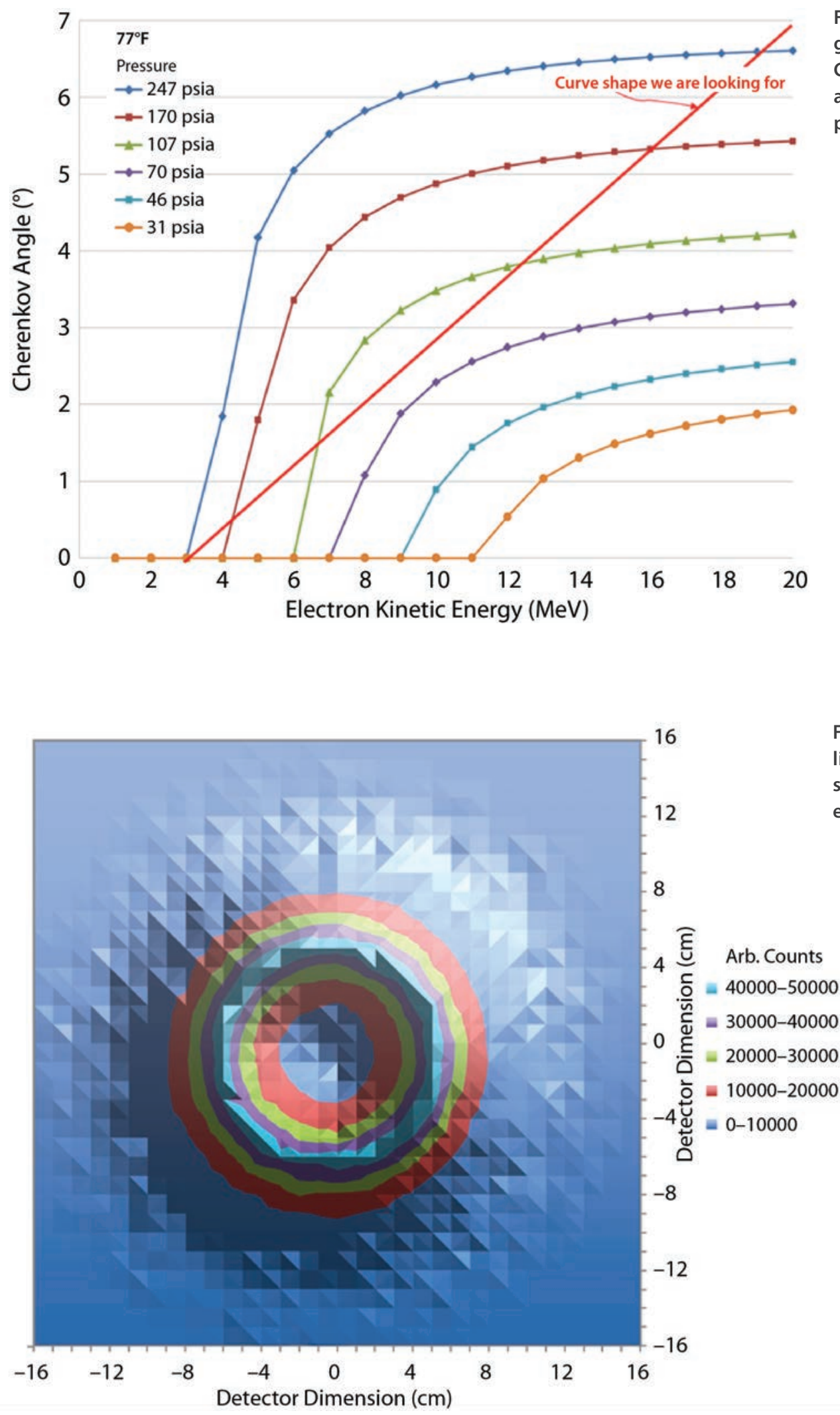

Figure 9. Plotting Cherenkovgenerated angles versus Compton electron energy as the electrons transit pressurized $\mathrm{CO}_{2}$ gas.

Figure 10.3-D intensity map of light at the detector plane. This shows $\mathrm{H}_{2}$ gas at 330 psia with electron scattering turned on. 


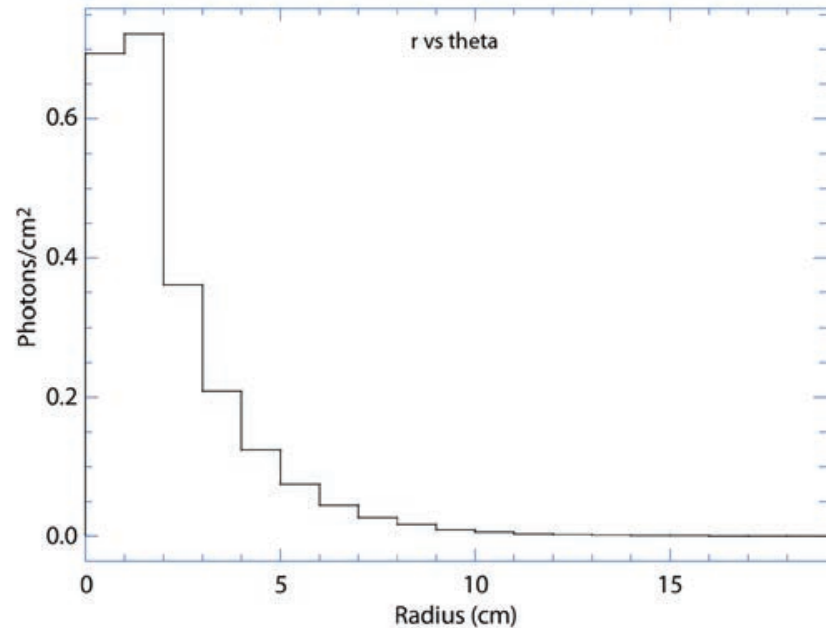

Figure 11. Number of Cherenkov photons created by 13.0 MeV electrons passing through a 330 psia $\mathrm{H}_{2}$ gas that arrive onto the detector surface.

\section{Conclusion}

Significant progress was made towards defining the design of a gamma spectrometer. We designed and optimized a single instrument operating as a timeintegrated gamma spectrometer able to discriminate fewer than five different energy bins of gammas. This instrument would utilize a multi-ring integrating detector to provide the capability to record spectroscopic data available at the stop position of a modified GRH. A real system utilizing this design would likely have to be entirely contained within a large pressure vessel and employ baffling to block Cherenkov light generated outside the region of interest.

Models using pressurized $\mathrm{H}_{2}$ gas were found to exhibit the best angular discrimination in the presence of multiple electron scatter. We examined $\mathrm{H}_{2}, \mathrm{He}_{2}, \mathrm{CO}_{2}$, $\mathrm{SF}_{6}$ gases; although $\mathrm{C}_{2} \mathrm{~F}_{6}$ is currently being evaluated for a new design of the Gas Cherenkov Detector for the Omega laser facility, we did not evaluate this gas. Future work would require further modeling using $\mathrm{H}_{2}$ gas and development of calculations needed

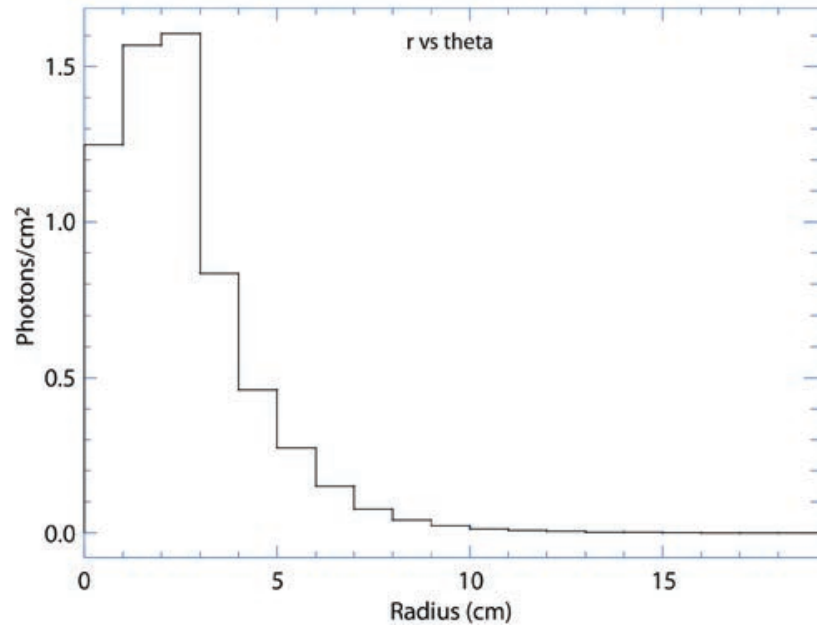

Figure 12. Number of Cherenkov photons created by 16.5 MeV electrons passing through a $330 \mathrm{psia}_{2}$ gas that arrive onto the detector surface.

for better gamma spectrometer designs. Modeling using finer energy resolution would be necessary to fully characterize and potentially mitigate multiple electron scatter issues. Other programs are benefitting from the work done on this SDRD project. The electron scatter calculations are currently being applied to the "Gamma Compton Spectrometer" LDRD project being conducted at Los Alamos National Laboratory and will continue in 2013.

Our optimized design for a single instrument was unable to achieve the desired angular discrimination of greater than 10 bins in the energy range of 3.5 to $20 \mathrm{MeV}$. However, further discussions with principal investigators from NIF now suggest that a minimum of 30 bins of energy discrimination will be necessary to adequately resolve the gamma spectrum for the DT burn cycle. It is possible that a system employing four channels, similar to the current GRH diagnostic, could provide the initially required ten bins; further work would need to be done to determine whether it could achieve thirty. 


\section{References}

Highland, V. L., "Some practical remarks on multiple scattering," Nucl. Instrum. Methods Phys. Res. A 129 (1975) 497-499.

Kim, Y., J. M. Mack, H. W. Herrmann, C. S. Young, G. M. Hale, S. Caldwell, N. M. Hoffman, S. C. Evans, T. J. Sedillo, A. McEvoy, J. Langenbrunner, H. H. Hsu, M. A. Huff, S. Batha, C. J. Horsfield, M. S. Rubery, W. J. Garbett, W. Stoeffl, E. Grafil, L. Bernstein, J. A. Church, D. B. Sayre, M. J. Rosenberrg, C. Waugh, H. G. Rinderknecht, M. G. Johnson, A. B. Zylstra, J. A. Frenje, D. T. Casey, R. D. Petrasso, E. K. Miller, V. Y. Glebov, C. Stoeckl, and T. C. Sangster, "D-T gamma-to-neutron branching ratio determined from inertial confinement fusion plasmas," Physics of Plasmas 19 (2012) 056313.

Li, X. A., D. W. O. Rogers, "Electron mass scattering power: Monte Carlo and analytical calculations," Medical Physics 22 (1995) 531-541.

Malone, R. M., H. W. Herrmann, W. Stoeffl, J. M. Mack, C. S. Young, "Gamma bang time/reaction history diagnostics for the National Ignition Facility using $90^{\circ}$ off-axis parabolic mirrors," Rev. Sci. Instrum. 79 (2008) $10 \mathrm{E} 532$.

Malone, R. M., B. C. Cox, B. C. Frogget, M. I. Kaufman, T. W. Tunnell, H. W. Herrmann, S. C. Evans, C. S. Young, W. Stoeffl, "Design of a gamma reaction history diagnostic for the National Ignition Facility," ICOPS IP3H-48 (2009).

Malone, R. M., B. C. Cox, S. C. Evans, B. C. Frogget, H. W. Herrmann, M. I. Kaufman, Y. H. Kim, J. M. Mack, K. D. McGillivray, M. Palagi, W. Stoeffl, A. Tibbitts, T. W. Tunnell, C. S. Young, "Design and construction of a gamma reaction history diagnostic for the National Ignition Facility," J. Phys.: Conf. Ser. 244 (2010) 032052.

Malone, R. M., Z. A. Ali, B. C. Cox, S. C. Evans, B. C. Frogget, H. W. Herrmann, M. I. Kaufman, Y. H. Kim, K. D. McGillivray, J. M. Mack, E. K. Miller, M. J. Palagi, W. Stoeffl, A. Tibbitts, T. W. Tunnell, C. S. Young, "Overview of the gamma reaction history diagnostic for the National Ignition Facility (NIF),"

Proc. SPIE 7652 (2010) 76520Z-1-10. 
This page left blank intentionally 


\section{Miniaturized Multiband ANTEnNa Via Element Collocation AND INDUCTIVE FEED LOADING}

RSLN-04-12 | CONTINUED FROM FY 2009 | YEAR 2 OF 2

Ryan Martin ${ }^{1, a}$

Although much research has been performed on a driven element parasitically loaded by another element shorted to ground for dual frequency operation, the novel concept of two or more coplanar-driven elements in close proximity designed for multiple frequency operation has not been represented in the literature. Because each higher-frequency antenna is built into the lowerfrequency elements, the largest element controls the structure's total size. Furthermore, by using the self-resonant frequency inherent in reactive elements due to device packaging, the aperture of each antenna, due to a low insertion loss path at the frequency of the larger element, will include that of all smaller radiators. This configuration provides a large standing wave ratio at the shorter wavelengths via several series capacitive-inductive connections. Therefore, each antenna element provides the required surface area for the frequency of operation while being isolated from the larger radiators. For this study, a dual $2.45 / 5.8 \mathrm{GHz}$ microstrip patch encompasses a small surface area of 9 square inches and provides circularly polarized electromagnetic radiation in excess of $6 \mathrm{dBi}$. A further investigation into the inclusion of additional radiators covering the frequency ranges of $9.3-10.8 \mathrm{GHz}$ and $24.05-24.25 \mathrm{GHz}$ was explored with exceptional results.

${ }^{1}$ martinrp@nv.doe.gov, 702-295-8758

a Remote Sensing Laboratory-Nellis

\section{Background}

In the FY 2009 SDRD project, "Advanced Microwave Antenna Array and Multiband Receiver" (Martin 2010), four separate antennas were designed to receive signals of interest covering a broad range of frequencies. While the elements provide excellent gain, beam width, impedance bandwidth, and tolerance to objects in the near field, the antenna array footprint is substantial at $3.5^{\prime \prime} \times 6 "$. Research performed by the University of Colorado Microwave Active Antenna Group led by Zoya Popovic, in collaboration with the Remote Sensing Laboratory (RSL), showed promise in realizing a reduced structure (Martin 2010). This work, which expands on this previous research, challenges the limits of the antenna aperture while maintaining acceptable directivity.

\section{Project}

The resonant frequency of a microstrip patch antenna may be further reduced by adding slots as in Chen (1998). Expanding upon this concept in favor of a significant reduction in the tuned width of the radiator, nearly $60 \%$ of the antenna metallization is removed as seen in Figure 1, where the radiating element and ground plane are represented in red and green, respectively. To facilitate an increase in the gain of the antenna as in Guha (2010), the radiator is suspended over the ground plane by an air substrate at a height of 0.250 inches while being mechanically supported by 0.030 -inch-thick Rogers RO4003 laminate in the same profile as the element. 

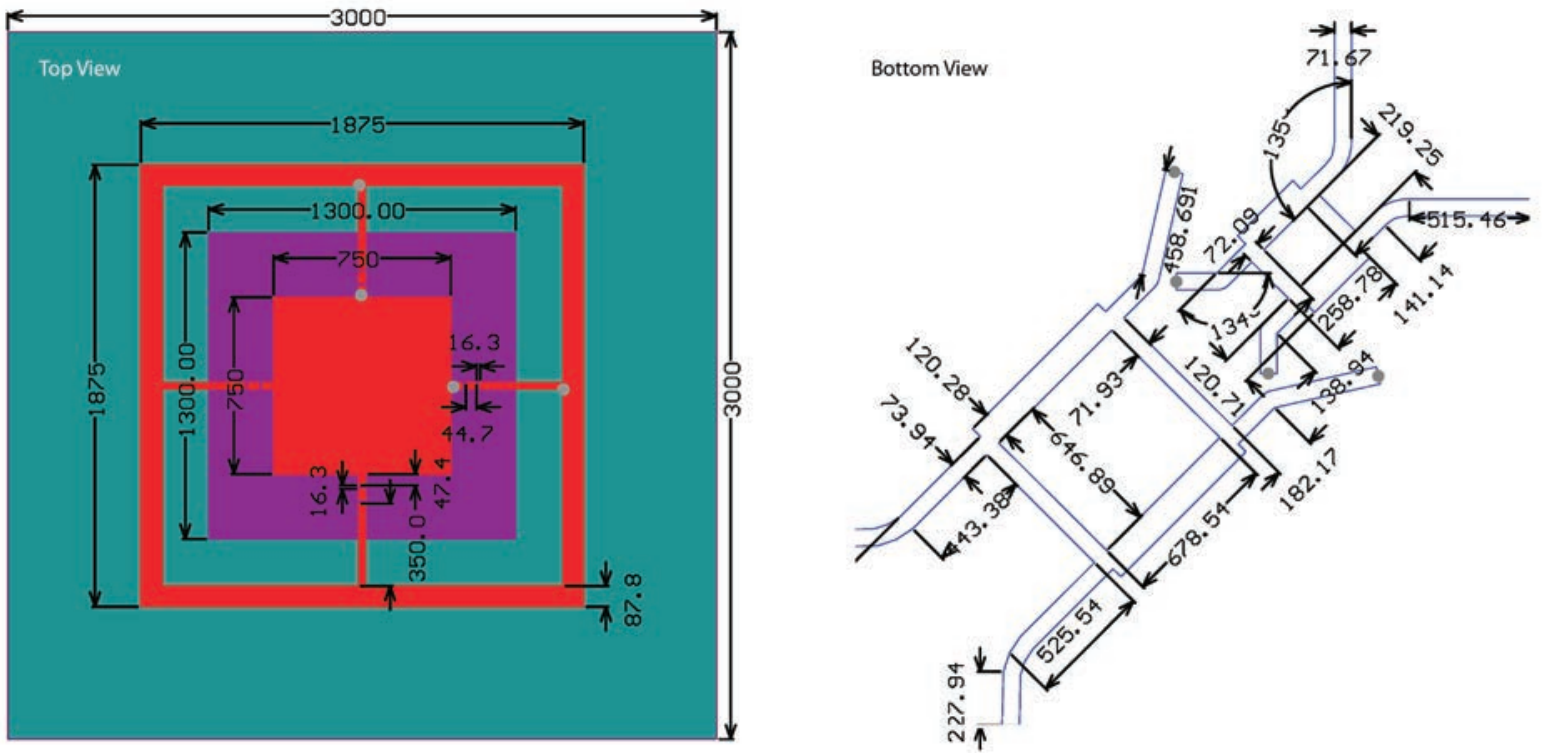

Figure 1. The coplanar dual band antenna negates the requirement for a separate antenna

(a) Inductance Self-Resonance Effect

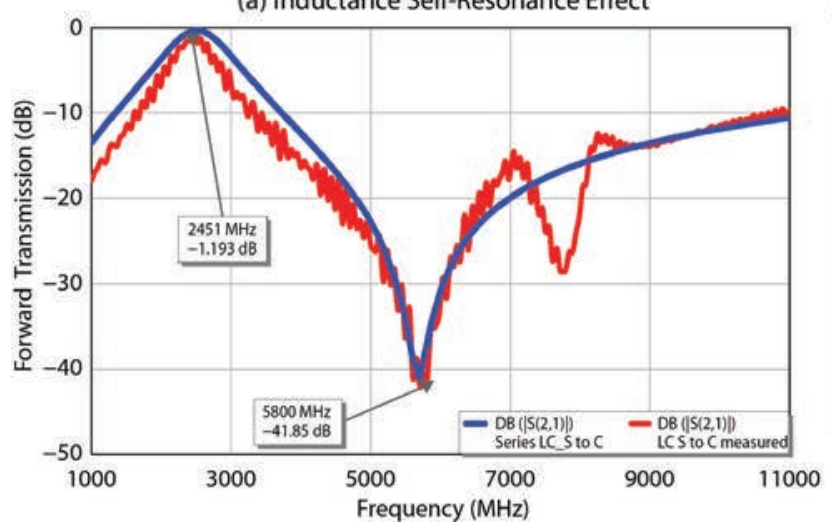

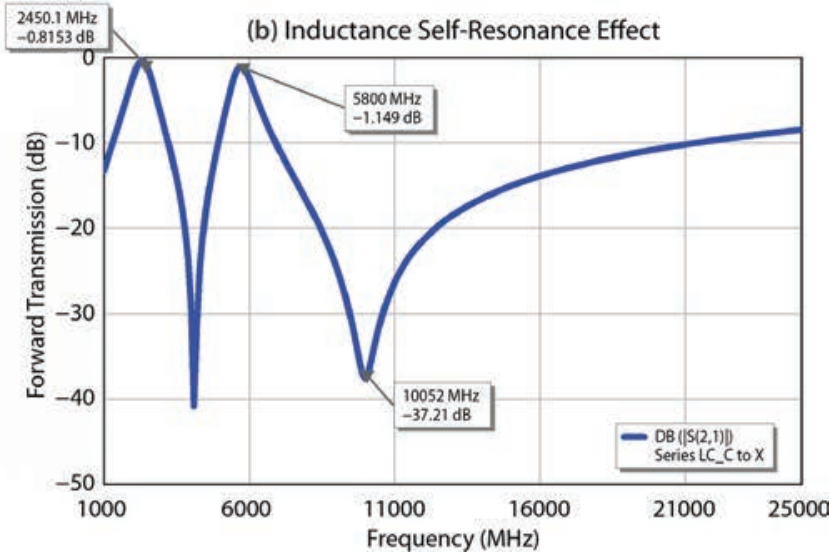

Figure 2. Less than $1 \%$ of the signal is transferred at (a) $5.8 \mathrm{GHz}$ with only $1 \mathrm{~dB}$ of loss at $2.45 \mathrm{GHz}$ and (b) $10.05 \mathrm{GHz}$ with 0.8 and $1.1 \mathrm{~dB}$ of loss at $2.45 \mathrm{GHz}$ and $5.8 \mathrm{GHz}$, respectively

While the entire surface of the antenna (shown in red) provides $2.45 \mathrm{GHz}$ operation with insignificant negative effects on performance due to the material removal, the smaller square microstrip in the middle must be isolated from the additional aperture in order to afford higher-frequency operation. A low insertion loss path centered at $2.45 \mathrm{GHz}$ may simultaneously provide considerable attenuation at additional frequencies through the implementation of a series-parallel resonant reactive path. As seen in the graph in Figure 2a, the resonance at $5.8 \mathrm{GHz}$ of a $12 \mathrm{nH}$ inductor with its parasitic selfcapacitance presents isolation in excess of $40 \mathrm{~dB}$; this, however, permits a lower-frequency energy to propagate across the intended discontinuity. To mitigate this, a $0.2 \mathrm{pF}$ capacitor is introduced in series with the inductor, generating a path at $2.45 \mathrm{GHz}$ with minimum forward transmission loss. Four of these reactive pairs are placed between the coplanar elements shown in Figure 1. Therefore, the aperture of the lower-frequency outer segment includes the smaller radiator while the higher-frequency section is isolated from the additional material. Extrapolating this technique to other frequency ranges of interest, the series connection of a $0.3 \mathrm{pF}$ capacitor, a $6.2 \mathrm{nH}$ inductor in parallel with $0.2 \mathrm{pF}$ capacitance, and a 
$6.2 \mathrm{nH}$ inductor present a low insertion loss path at 2.45 and $5.8 \mathrm{GHz}$ while providing significant attenuation at $X$ Band $(9.3-10.8 \mathrm{GHz})$, as can be seen in Figure 2b. Excellent correlation between the measured results in red and the simulation in blue is exhibited in Figure 2a. Modeling was performed with the Applied Wave Research (AWR) Microwave Office software package.

In order to avoid cross-polarization losses due to the orientation of a transmitter or receiver in reference to the antenna, circular polarization is realized by a quadrature coupler for each collocated antenna as seen in the bottom view in Figure 1. To generate electromagnetic radiation concentrically rotating about the direction of propagation, ideally one-half of the power must be delivered to the output of each branch with a phase shift of $90^{\circ}$ and identical in amplitude. These conditions are met by placing the lines $\lambda / 4$ apart and from the design equations of James (1989),

$$
\frac{Z_{0}}{Z_{a}}=a \quad \frac{z_{0}}{Z_{b}}=b \quad Z_{a} \approx Z_{0} \quad Z_{b}=\frac{z_{0}}{\sqrt{2}}
$$

where $Z_{0}$ is the characteristic impedance of the line, $Z_{a}$ is the impedance of the shunt line, and $Z_{b}$ is the impedance of the series line. In this instance, an impedance of $50 \Omega$ on a 0.030-inch-thick RO4003 results in a $\sim 0.074$-inch $\left(Z_{0}\right)$ wide line. Similarly, the shunt and series elements are roughly 0.072 inch and 0.120 inch, representing 48 and $33 \Omega$ impedances,

(a) $\mathrm{X}$ Band $|\mathrm{S} 11|$ vs Frequency

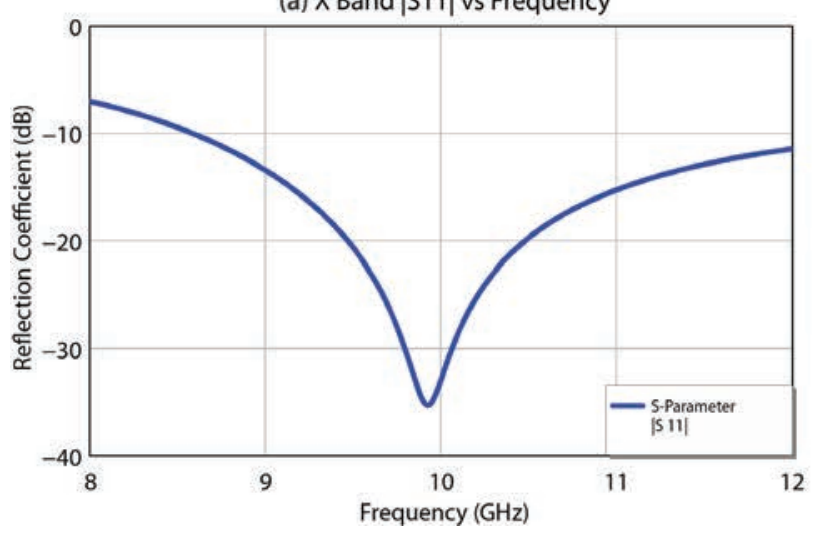

respectively. From Equation 1, we can calculate the normalized admittances as 0.98 for the value of $a$ and 1.39 for the value of $b$. From the matched and coupling conditions of Equation 2, the output of the coupler is well matched to the characteristic impedance of $50 \Omega$ with a value of 0.97 , and $50 \%$ of the energy is coupled to each port with a value of 0.498 .

$$
\begin{gathered}
b^{2}-a^{2}=1 \rightarrow(\text { matched condition) } \\
\frac{P_{3}}{P_{1}}=\left(\frac{a}{b}\right)^{2}=0.498 \rightarrow \text { coupling. }
\end{gathered}
$$

From the results of the AWR simulation, these conditions are indeed met at $2.45 \mathrm{GHz}$ and the ports are in phase quadrature with a phase of $33.084^{\circ}$ and $-57.185^{\circ}$ at ports two and three, respectively. Similar results follow for the $5.8 \mathrm{GHz}$ frequency band. By choosing one of the two feed locations, each antenna can present either right- or left-hand circularly polarized radiation, depending on the input port.

However, geometrically scaling this concept up in frequency while obtaining a wide bandwidth of 9.3 to $10.8 \mathrm{GHz}$ will not be effective, due to the narrow regime where $90^{\circ}$ phase deviation is kept at a minimum. This is due to the quarter wavelength separation of the feed lines. Through the addition of shunt elements or open/short circuited stubs, one may increase the impedance bandwidth of the quadrature coupler. In this study, the widths of the series lines from the ideal design equations were increased by $22.4 \%$ from 42.93 to 52.583 mils

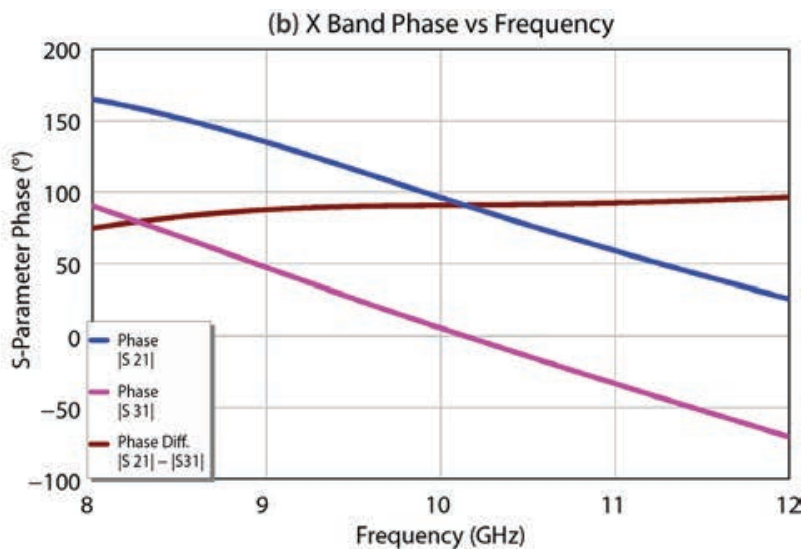

Figure 3. Modifying the width of the coupler's shunt elements increases the frequency response 

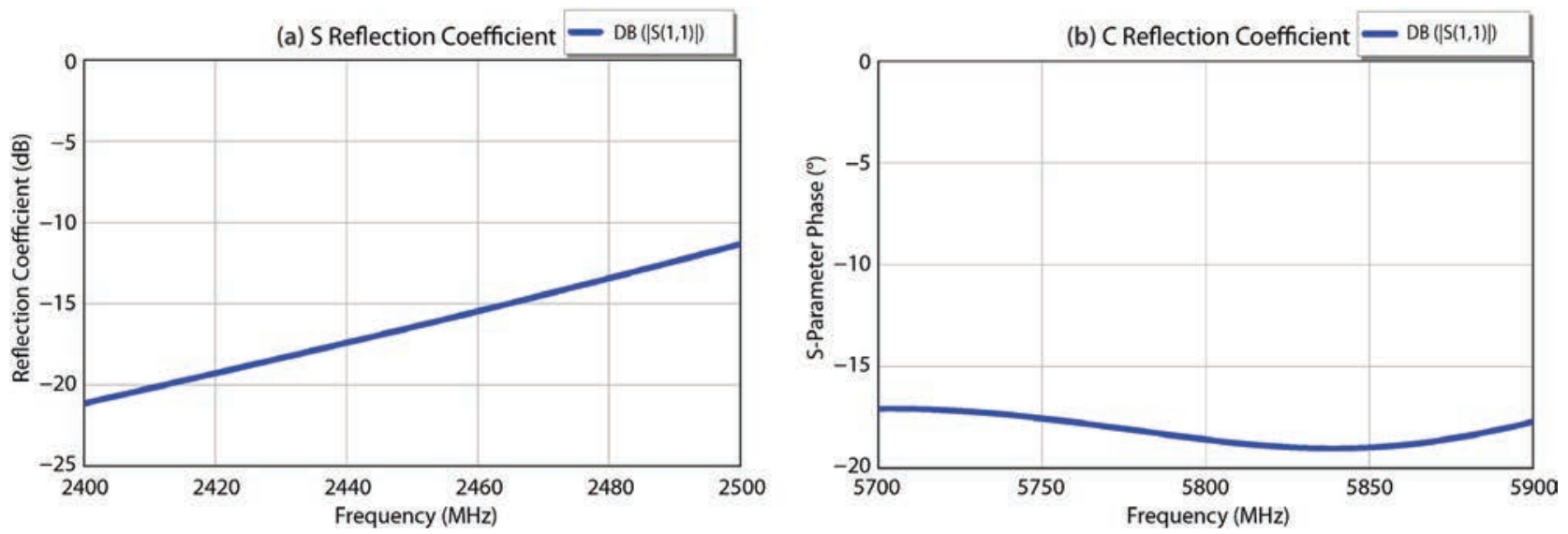

Figure 4. Simulated reflection coefficient for the two antennas shows at least $90 \%$ of the available power is delivered to the antenna

with the shunt and $Z_{0}$ lines having a width of 24.8 mils (near $50 \Omega$ impedance) on a 0.010-inchthick R04003 substrate. Figure 3a illustrates the impedance bandwidth of nearly $35 \%$ from 8.5 to $12 \mathrm{GHz}$ while maintaining phase quadrature over the frequency band as shown in Figure $3 b$.

The antenna is connected to the feed lines by means of a 0.047 -inch-diameter coaxial cable 0.250 inches in length at the circular drill locations as illustrated in Figure 1. Accordingly, one can see the antenna is well matched to the system impedance by examining the reflection coefficient expressed in $\mathrm{dB}$ from Figure 4, where over $95 \%$ of the power is delivered to the load at $2.45 \mathrm{GHz}$ and $5.8 \mathrm{GHz}$, corresponding to a voltage standing wave ratio of less than 1.4. By connecting the S Band antenna to the coupler, the impedance moved to the capacitive region of the Smith chart. This was resolved by adding a parallel inductor between the feed lines and ground, resulting in a counterclockwise rotation on the constant-conductance circle of the admittance Smith chart, restoring the reactive match to approximately $50 \Omega$.

As shown in Figure 5, the simulated axial ratio is at an acceptable level, below $3 \mathrm{~dB}$ for the $5.8 \mathrm{GHz}$ antenna, revealing that the antenna is circularly polarized. The deviation from circular polarization is at most $2 \mathrm{~dB}$ over the frequency range of interest with an acceptable simulated absolute gain in excess of $8 \mathrm{dBi}$, as (a) Axial Ratio (C Radiator)

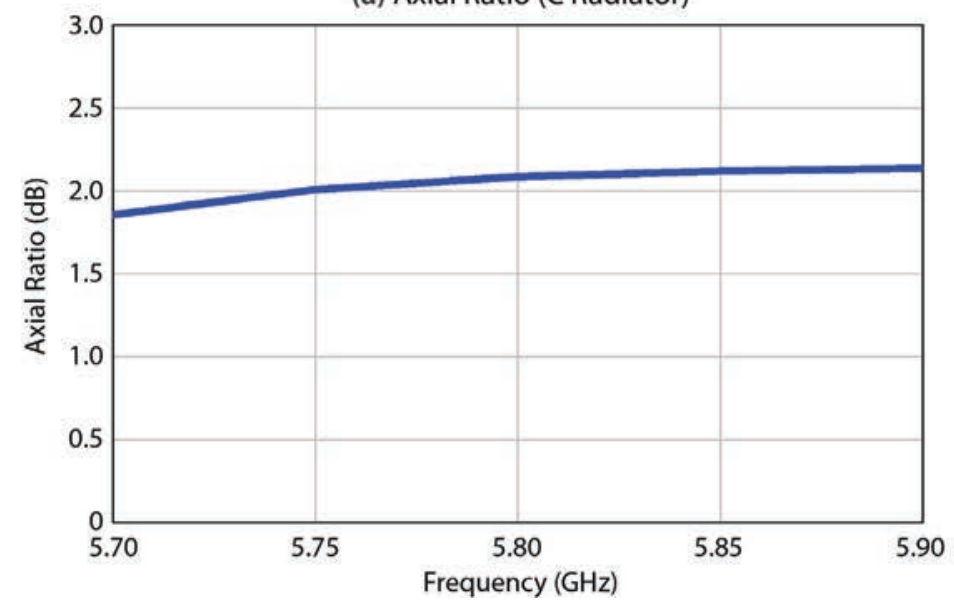

(b) Gain Pattern

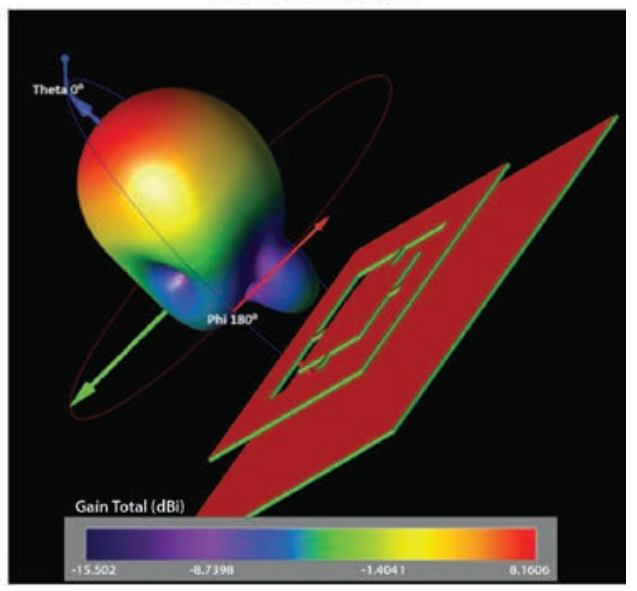

Figure 5. Simulated axial ratio and gain pattern for the $C$ Band antenna 

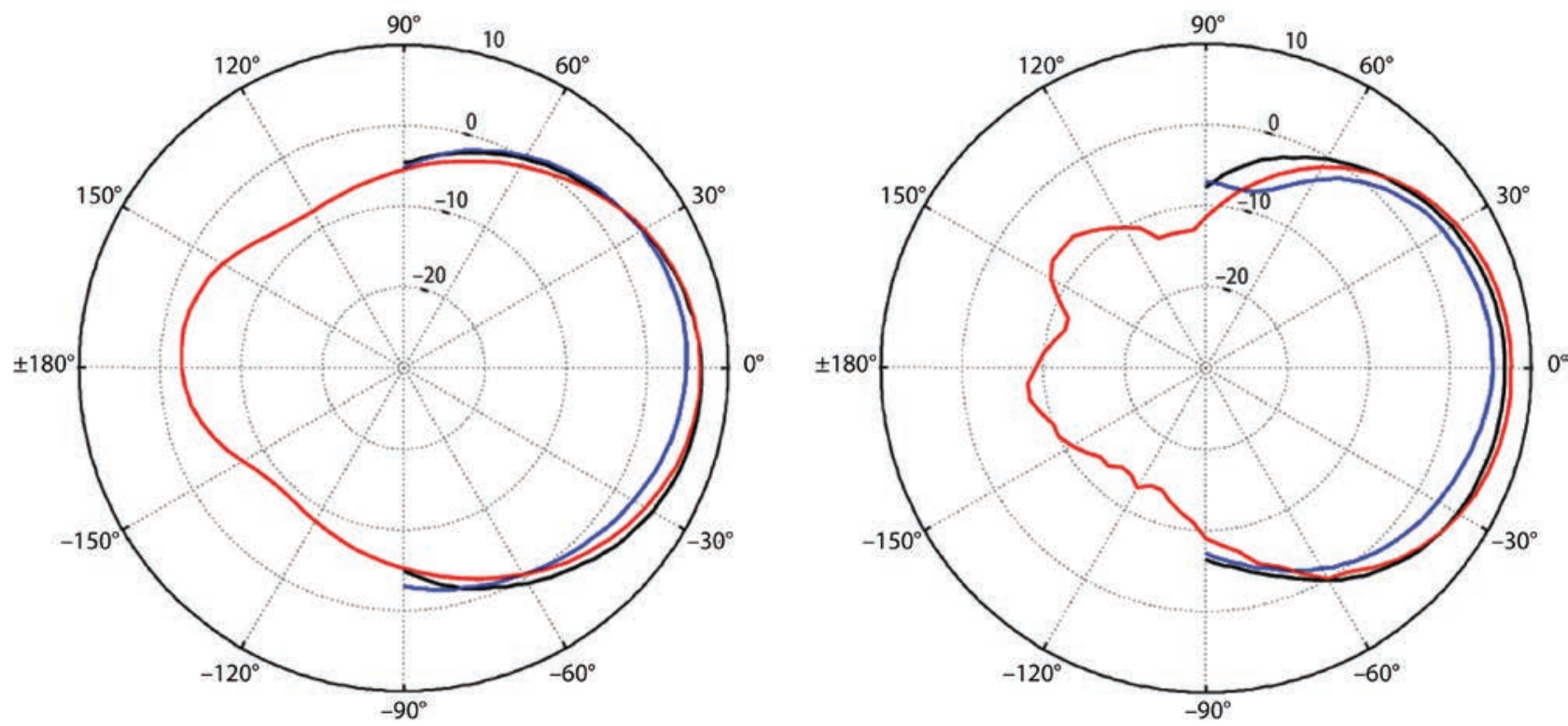

- Measured relative gain magnitude $\left(\phi=0^{\circ}\right)$

Measured relative gain magnitude $\left(\phi=90^{\circ}\right)$

Simulation $\left(\phi=0^{\circ}\right)$

Figure 6. The simulation matches the measurements with less than $1 \mathrm{~dB}$ variation

shown in the 3-D model in Figure $5 \mathrm{~b}$. Similar results follow for the $\mathrm{S}$ Band antenna. Furthermore, Figure 6 illustrates that the measured results of the divergence from circular polarization is $1.8 \mathrm{~dB}$ for the $\mathrm{S}$ Band and $1.5 \mathrm{~dB}$ for the $\mathrm{C}$ Band when rotating the antenna from the co-polarization to the cross-polarization orientations.

In order for the fringing fields at the exterior of the higher-frequency element to proceed to an appropriately placed ground potential (Balanis 1982) and not perturb the radiation pattern, a local square ground plane of 1.3 inches on a side (as seen in Figure 1) was placed 0.185 inches below the antennas. The ground plane was then soldered to the outer conductor of the interior coaxial cables. This improved the directivity of the C Band antenna with little to no effect on the $S$ band antenna.

Prior to the incorporation of additional higherfrequency radiators, it is prudent to observe the electric field vectors in the proximity of the coplanar antenna design in its simplest form. By representing the electric field vector, $\vec{E}$, as a traveling wave in phasor form as

$$
E(x, y, z, t)=\Re\left[E(x, y, z) e^{j \omega t}\right],
$$

one can derive a solution to $\vec{E}$ in free space by solving the time-domain differential form of Ampère's circuital law with Maxwell's correction. The curl of the magnetic field intensity $(H)$ is a function of time equal to the sum of the current density $(J)$ and the derivative of the electric flux density $(D)$, with respect to time. Equivalently, this can be stated as

$$
\begin{aligned}
& \nabla \times H(t)=J(t)+\frac{\partial}{\partial t} D(t) \\
& \quad \text { (time domain representation). }
\end{aligned}
$$

Because the structure is excited at a discrete frequency or at $\omega=2 \pi f$ with $f$ as the frequency of oscillation, the time-domain representation can be converted to a phasor form as

$$
\begin{aligned}
\nabla & \times H e^{j \omega t}=J e^{j \omega t}+\frac{\partial}{\partial t} D e^{j \omega t} \\
& =J e^{j \omega t}+J \omega D e^{j \omega t} \Rightarrow \nabla \times H=J+j \omega D .
\end{aligned}
$$



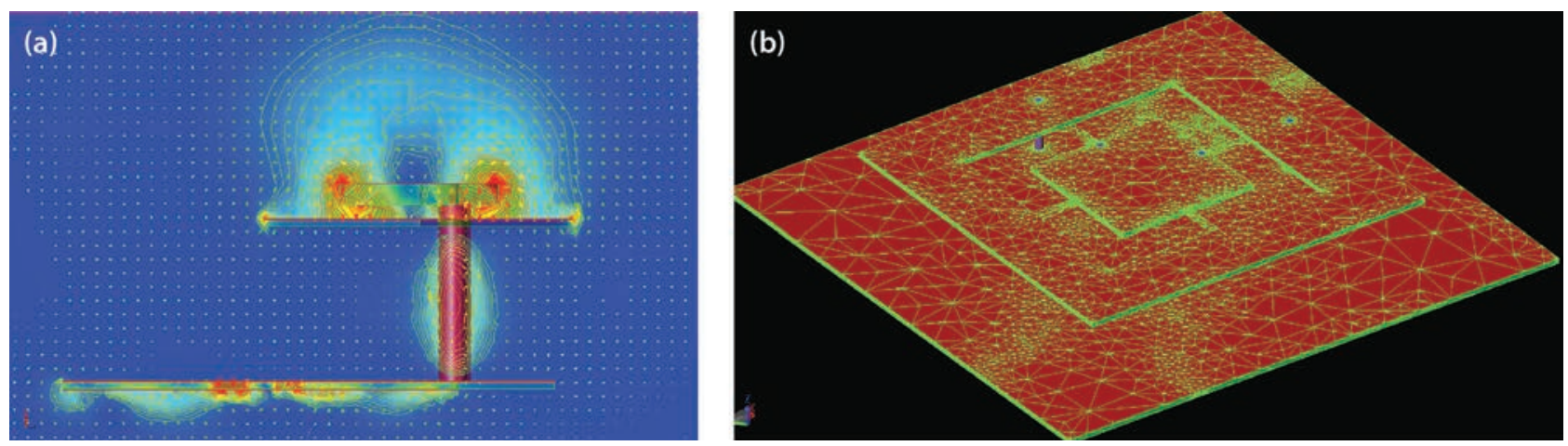

Figure 7. (a) Electric field vectors are computed from the (b) tetrahedral mesh via the finite element method
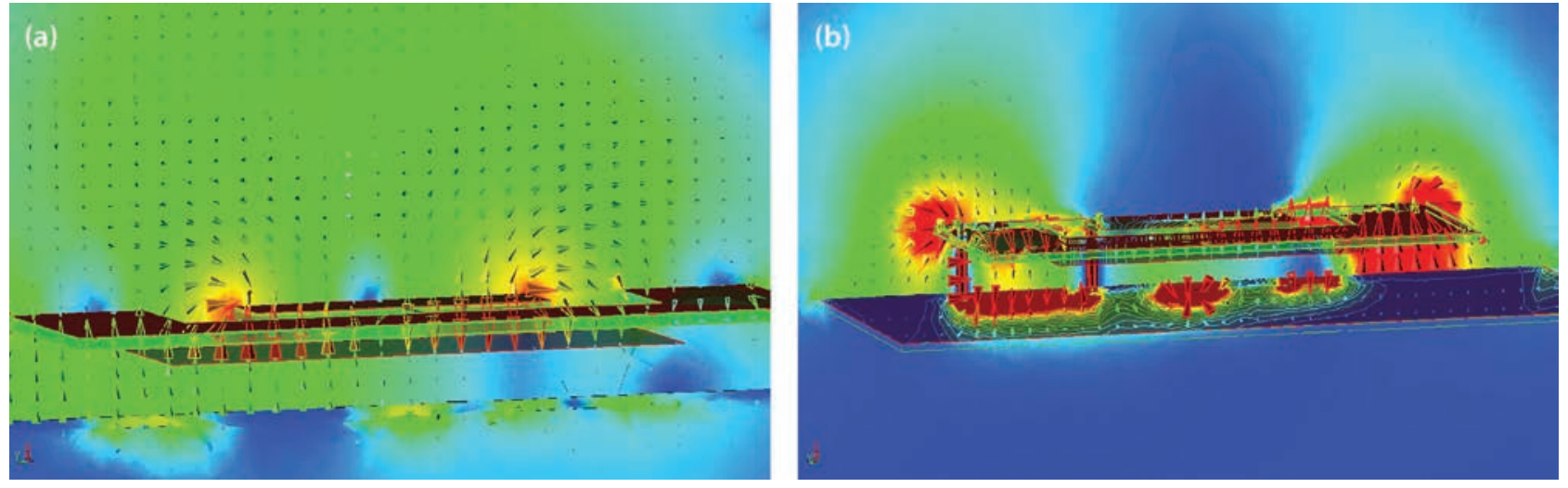

Figure 8. Placing additional metallization exterior and coplanar to the single patch does not greatly disturb the electric field vectors when the antenna is excited from either the (a) interior C Band or (b) exterior S Band antenna feed locations

Considering that the electric flux density is equal to the complex permittivity multiplied by the electric field strength $(D=\varepsilon E)$, and that the current density is equal to the multiplication of the conductivity with $\mathrm{E}$ $(\mathrm{J}=\sigma \mathrm{E})$, one arrives at

$$
\begin{aligned}
& \nabla \times H=\sigma E+j \omega \varepsilon E \Rightarrow \nabla \times H=j \omega \varepsilon E \\
& \quad(\text { for } \sigma=0) .
\end{aligned}
$$

In Equation 6 one can approximate the conductivity as zero because we are calculating the result for free space or interior to non-conductive material (e.g., the substrate dielectric). Finally, expressing $\vec{H}$ as a function of $\vec{E}$ and utilizing the free space wave number $\left(k_{0}=\omega \sqrt{\mu_{0} \varepsilon_{0}}\right)$, leads to one equation with one unknown: the electric field vector.

$$
\begin{array}{r}
\nabla \times \vec{H}=\nabla \times\left(-\frac{1}{j \omega \mu} \nabla \times \vec{E}\right)=j \omega \varepsilon \vec{E} \\
\Rightarrow \nabla \times\left(-\frac{1}{\mu} \nabla \times \vec{E}\right)=j^{2} \omega^{2} \varepsilon \vec{E} \Rightarrow
\end{array}
$$

$$
\begin{aligned}
& \nabla \times\left(-\frac{1}{\mu_{0} \mu_{r}} \nabla \times \vec{E}\right)=(-1) \omega^{2} \varepsilon_{0} \varepsilon_{r} \vec{E} \\
& \quad \Rightarrow \nabla \times\left(-\frac{1}{\mu_{r}} \nabla \times \vec{E}\right)=(-1) \omega^{2} \mu_{0} \varepsilon_{0} \varepsilon_{r} \vec{E} \Rightarrow \\
& \nabla \times \\
& \quad=\left(-\frac{1}{\mu_{r}} \nabla \times \vec{E}\right)-\omega^{2} \mu_{0} \varepsilon_{0} \varepsilon_{r} \vec{E} \\
& \quad 0 \therefore \nabla \times\left(\frac{1}{\mu_{r}} \nabla \times \vec{E}\right)-k_{0}^{2} \varepsilon_{r} \vec{E}=0 .
\end{aligned}
$$

By dividing the three-dimensional antenna structure into hundreds of thousands of tetrahedra as seen in Figure $7 \mathrm{~b}$, the wave equation can be solved at each tetrahedal node. To facilitate this, the resultant nodal equations are transformed into a matrix and solved using traditional numerical methods for all points enclosed by the boundary conditions. Using this methodology, the $\vec{E}$ field was calculated for a single patch antenna in the image in Figure 7a. One can see the $\vec{E}$ field density is greatest at the ends of the patch with a null about the center. Due to the truncation 


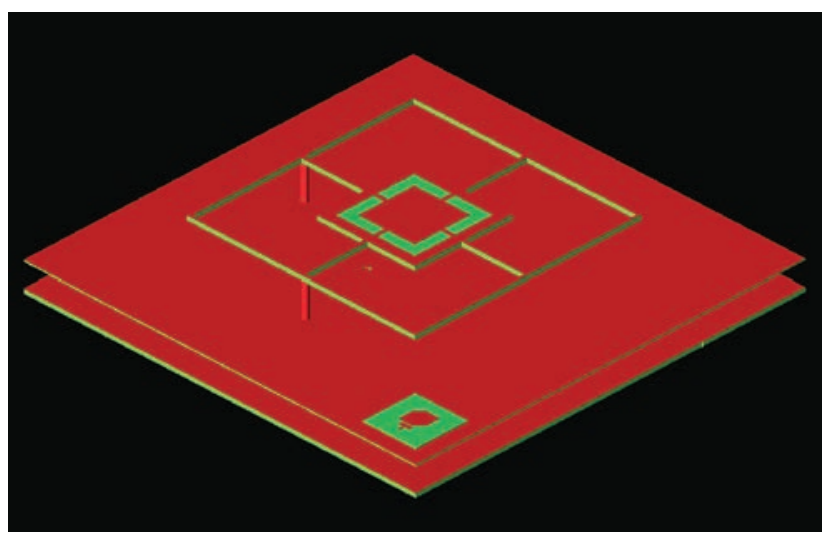

Figure 9. A truncated corner patch antenna was placed at the edge of the $\mathrm{S}$ Band ground plane to achieve quad band performance

of the metallization at the edge, the fields fringe and create large standing waves at the boundaries. This results in the transmission of electromagnetic energy orthogonal to the surface.

Furthermore, an additional radiator is placed coplanar and exterior to the smaller higher-frequency radiator previously simulated as seen in Figure 8. As shown in Figure $8 \mathrm{a}$, the interior $5.8 \mathrm{GHz}$ antenna is driven via the associated quadrature coupler. The $\vec{E}$ field density appears similar to that of the isolated microstrip patch antenna. However, in Figure 8b the exterior lower-frequency antenna has several perturbations when viewing the electric field vectors. Consequently, the radiation pattern is diminished somewhat from nominal with a loss in total gain

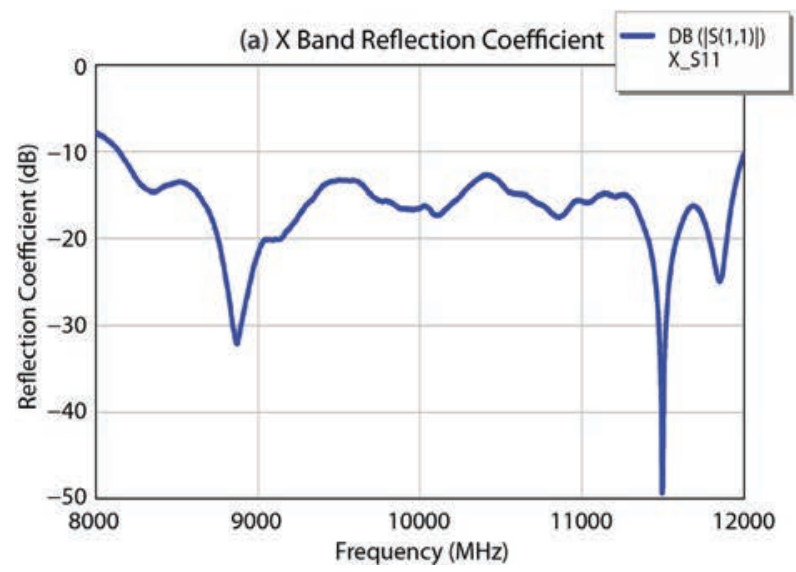

at the lower frequency. Moreover, the $\vec{E}$ field has a rather large magnitude where the null is ideally maintained. Due to this result, not all four antennas could be located coplanar to one another as shown in the quad band layout of Figure 9. With the collocation of more than three radiators in the same plane, the electric field density located at the null region becomes prominent and disturbs the directivity and radiation pattern. In the figure, the lower copper plane provides a ground reference for both the $S$ and C Band quadrature couplers. The upper substrate's metallization performs the same function for both the $\mathrm{X}$ Band coupler and the $\mathrm{S}$ Band radiator as well as for a truncated corner patch antenna with a center frequency of $24.15 \mathrm{GHz}$. For the $\mathrm{C}$ and $\mathrm{X}$ coplanar radiators, a local ground plane is incorporated at the appropriate height beneath and concentric with the antenna elements.

The $\mathrm{S}$ and $\mathrm{C}$ Band reflection coefficient measurements were similar to the previously mentioned results and are not repeated for brevity. However, as can be seen in Figure 10a, the X Band antenna is very well matched to $50 \Omega$ while increasing the broad impedance bandwidth to nearly $40 \%$. Likewise, the K Band antenna accepts nearly $99 \%$ of the available power from the driving source.

With a measured axial ratio less than $0.5 \mathrm{~dB}$, as illustrated in Figure $11 \mathrm{~b}$, the $X$ Band antenna is circularly polarized nearly approaching the theoretical limit of

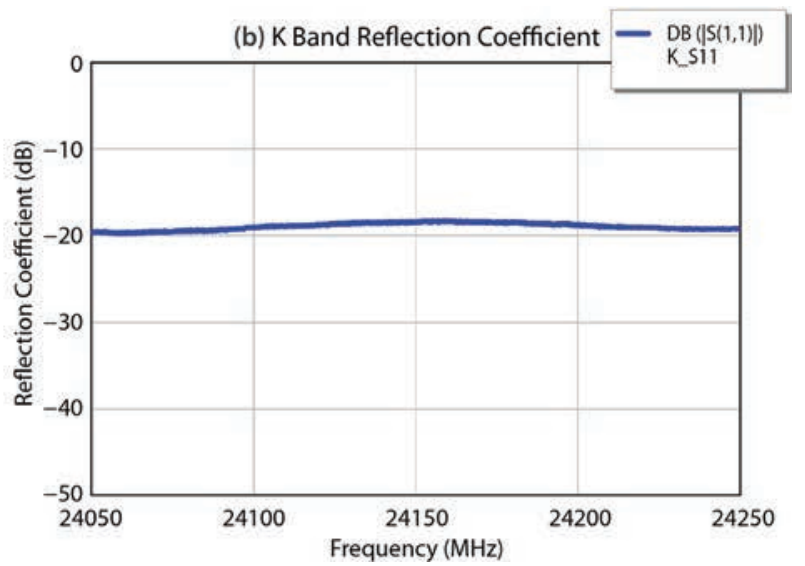

Figure 10. All four antennas are well matched to $50 \Omega$ as exhibited by a return loss less than $-10 \mathrm{~dB}$. Similar results were found for the $S$ and C Band antennas (not shown). 
(a) C Band Relative Antenna Gain - Rev 0 (dBi)

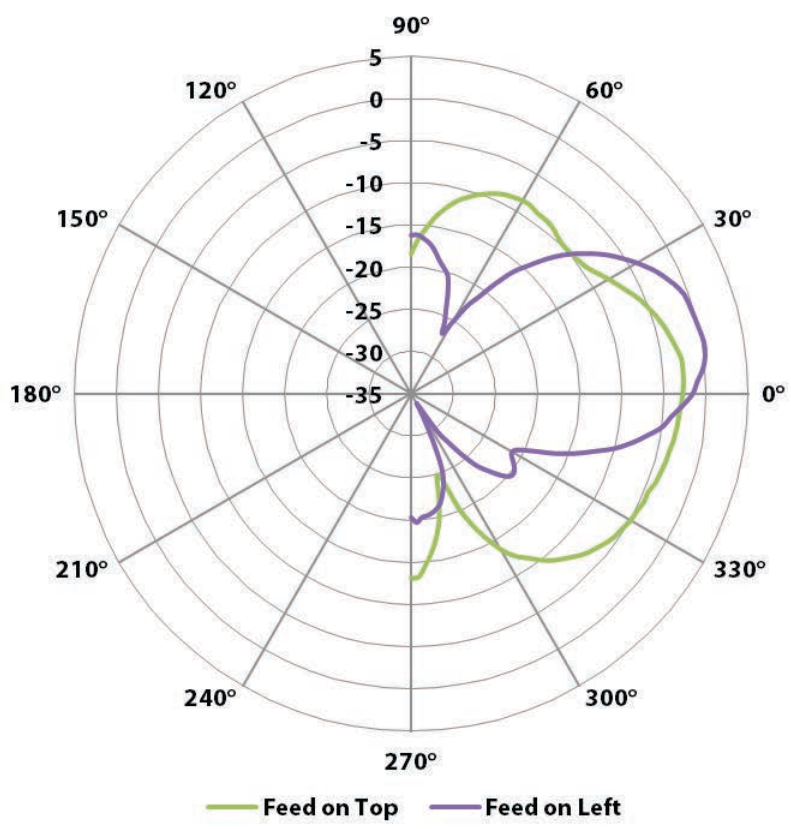

(b) X Band Relative Antenna Gain - Rev 0 (dBi)

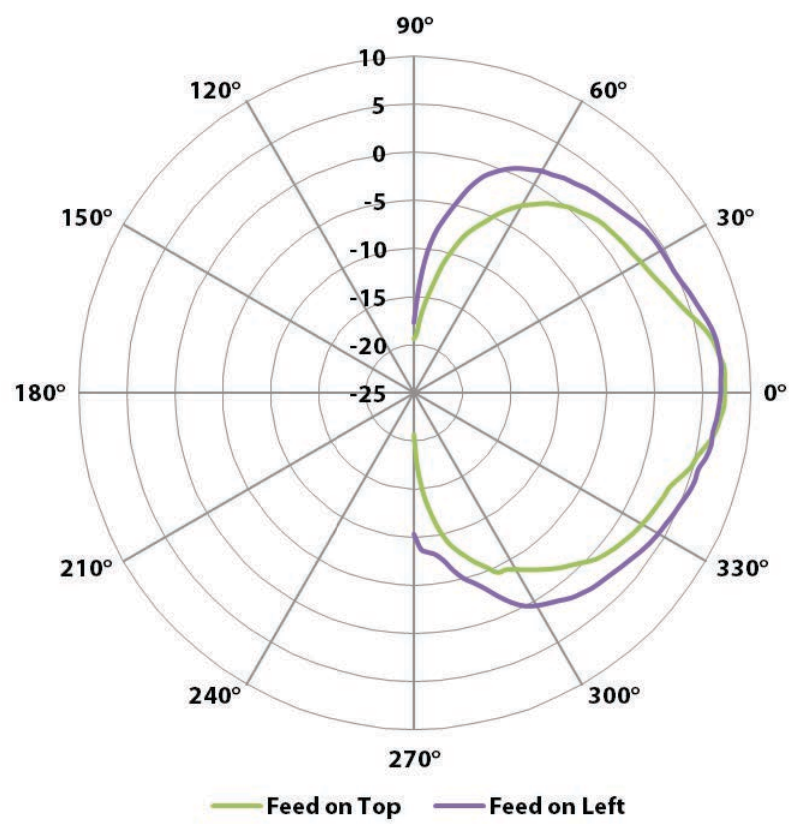

Figure 11. As seen in the previous analysis of the electric field vectors, the (a) C Band patch radiation pattern is slightly skewed from receiving maximum signal at $0^{\circ}$ while the (b) most interior X Band patch exhibits the best cross- and co-polar radiation patterns

$0 \mathrm{~dB}$. As one would expect from the aforementioned results, the $\mathrm{C}$ Band main lobe, as shown in Figure $11 \mathrm{a}$, is somewhat skewed but still performs acceptably for direction-finding purposes. Similar results to the measured $C$ Band radiation pattern are observed for the $\mathrm{S}$ Band. The K Band antenna maintains good directivity orthogonal to the antenna similar to that demonstrated by the $\mathrm{X}$ Band, as the $\mathrm{K}$ element is merely collocated on the total system ground plane and not coplanar to the other radiators.

\section{Conclusion}

It has been shown that coplanar elements can be collocated in the reactive near field and provide excellent directivity and absolute gain in excess of $6 \mathrm{dBi}$ while maintaining an acceptable impedance match. However, with the addition of more than three coplanar elements, the absolute gain and the shape of the radiation pattern is diminished from ideal. This is the result of a high $\vec{E}$ field density located at the center of the structure between the radiating element and ground. In this investigation, a quad band antenna was simulated in software, fabricated to the model, and evaluated for impedance match, gain, and axial ratio in a $3^{\prime \prime} \times 3^{\prime \prime} \times 0.5^{\prime \prime}$ design space.

\section{References}

Balanis, C. A., Antenna Theory, John Wiley and Sons, Inc., New York, 1982, 727-728.

Chen, W. S., C. K. Wu, K. L. Wong, "Compact circularly polarized microstrip antenna with bent slots," Electronics Letters, 34, 13 (June 1998) 1278-1279.

Guha, D., S. Chattopadhyay, J. Y. Siddiqui, "Estimation of gain enhancement replacing PTFE by air substrate in a microstrip patch antenna," IEEE Antennas and Propagation Magazine, 52, 3 (June 2010) 92-95.

James, J. R., P. S. Hall, Handbook of Microstrip Antennas, Institution of Engineering and Technology, London, 1988, 854-856.

Martin, R. P., K. Thomas, "Advanced microwave antenna array and multiband receiver," Nevada Test Site-Directed Research and Development, FY 2009, National Security Technologies, LLC, Las Vegas, Nevada, 2010, 113-120. 


\title{
Electronic Veto and Software Partitioning of Cosmic Neutrons
}

\author{
RSLA-10-12 | YEAR 1 OF 1
}

Sanjoy Mukhopadhyay, ${ }^{1, a}$ Ronald S. Wolff, ${ }^{a}$ Ryan B. Detweiler, ${ }^{a}$ Ethan Smith, ${ }^{b}$ Stephen Mitchell, ${ }^{c}$ Paul P. Guss, ${ }^{d}$ and Richard Maurer ${ }^{a}$

Neutron counting using large arrays of pressurized helium-3 $\left({ }^{3} \mathrm{He}\right)$ proportional counters in an aerial system or maritime environment suffers from the background counts from the primary cosmic neutrons and secondary neutrons caused by cosmic ray-induced mechanisms like spallation and charge-exchange reaction. This report describes work and results obtained in attempting two different methods to reduce the cosmic neutron background in real time. Both methods used shielding materials with a high concentration (up to $30 \%$ by weight) of neutronabsorbing materials, such as natural boron, to remove the low-energy neutron flux from the cosmic background as the first step of the background reduction process. The goal of the first method was to design, prototype, and test an up-looking plastic scintillator to tag the cosmic neutrons and then create a logic pulse of a fixed time duration $(\sim 120 \mu \mathrm{s})$ to block the data taken by the neutron counter (pressurized ${ }^{3} \mathrm{He}$ tubes run in proportional counter mode). The second method examined the time correlation between the arrivals of two successive neutron signals to the counting array and calculated the excess of variance in the neutron count distribution from Poisson distribution. The first method removes approximately $87 \%$ of the cosmic neutrons, while the second method constantly partitions between cosmic and non-cosmic neutrons qualitatively.

${ }^{1}$ mukhops@nv.doe.gov, 301-817-3319

a Remote Sensing Laboratory-Andrews; ${ }^{\text {b }}$ Keystone International, Inc.; ${ }^{c}$ North Las Vegas; ${ }^{d}$ Remote Sensing Laboratory-Nellis

\section{Background}

When using passive detection of special nuclear materials in aerial or maritime environments from large standoff distances, we focus on neutron signals because neutrons penetrate through common (high atomic number, $Z_{\text {eff }}$ construction materials like lead, tin, and aluminum and have a high probability of reaching the detectors. Fast neutrons from a plutonium source can be detected at a far distance because scattered in air their free path is long (>100 m for $2 \mathrm{MeV}$ neutrons) (Forman 2003). The fundamental detection limit is usually dictated by the neutron background production by cosmic-ray interactions. The neutron background varies with geomagnetic latitude, atmospheric pressure, solar activity, and the altitude of the observation location.
In maritime environments, neutron counting is made difficult because of the "ship effect," the excess production of neutrons by secondary cosmic-induced interaction (spallation) of high-Z materials (e.g., steel structures, containers, large engine blocks on board a ship).

The current project attempted to reduce the cosmic neutron background on board a ship or in flight.

A typical cosmic neutron background at sea level will be 120 neutrons per second per square meter (nps/ $\mathrm{m}^{2}$ ); ship effect would add an additional $25 \%$ on board a ship, for a total of $150 \mathrm{nps} / \mathrm{m}^{2}$ (latitude dependent). In terms of ways to reduce cosmic neutron background, material shielding would 


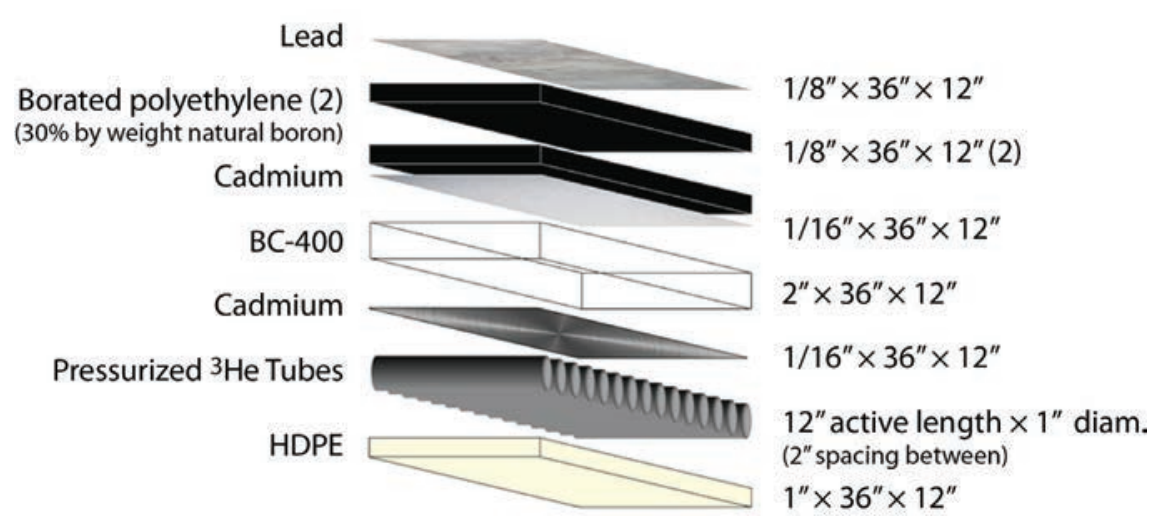

Figure 1. From top to bottom are a lead sheet, two borated polyethylene sheets, a cadmium sheet, a BC-400 scintillator, a cadmium sheet, an array of 15 cylindrical pressurized ${ }^{3} \mathrm{He}$ (2.7 atmosphere) tubes spaced 2 inches apart, and a high-density polyethylene (HDPE) layer remove more than $55 \%$ of the flux, leaving about $68 \mathrm{nps} / \mathrm{m}^{2}$ remnant neutrons; the electronic veto method would take out $70 \%$ of these later fluxes, leaving only about $13 \%$ of the original cosmic neutrons to pass through. The general approach used in this project to reduce cosmic neutron background was to (1) use effective shielding materials to remove neutron flux, (2) tag the remnant neutrons and use an electronic veto for not counting them, and ( 3 ) use cumulative Feynman variance $\mathrm{Y} 2 \mathrm{~F}(\mathrm{t})$ to mark the presence of a man-made neutron source. Figure 1 is a schematic of the experimental setup. All the elements above the helium-3 $\left({ }^{3} \mathrm{He}\right)$ tube array help remove cosmic background neutron flux, and the elements below the ${ }^{3} \mathrm{He}$ tube array contribute to the real-time analysis of the neutron counts ( $\mathrm{Y} 2 \mathrm{~F}$ calculations) from any neutron source present on the ground. The functional form of Feynman variance $\mathrm{Y} 2 \mathrm{~F}(\mathrm{t})$ as a function of the time bin width of neutron correlation measurements is

$$
Y 2 F(t)=\frac{A}{\alpha C_{n}}\left[1-\frac{1-e^{-\alpha t}}{\alpha t}\right],
$$

where $\alpha^{-1}$ is die-away time (average time a neutron spends from the time of origination until it reaches the detector) in $\mu \mathrm{s}, t$ is the time bin windows in $\mu \mathrm{s}$, $C_{n}$ is the total neutron count, and $A$ is a normalizing constant. Die-away times are determined primarily by the size, shape, composition, and efficiency of the neutron counter, but also are slightly affected by scattering, moderation, or neutron-induced fission within the sample being assayed.

\section{Project}

Besides shielding, we proposed to use two additional methods to address the cosmic neutron background problem: (1) electronic vetoing of tagged neutrons and (2) studying time-resolved measurements of neutrons from a man-made source to find intervals between counts that are not random. Any deviation from the Poisson distribution of the neutron time correlation would prove the existence of a noncosmic source. The two methods are described in detail below.

In method 1, a 2-inch-thick BC-400 paddle covered the entire active area of the ${ }^{3} \mathrm{He}$ tube array. A photomultiplier signal above a preset threshold indicated existence of the cosmic background. The ${ }^{3} \mathrm{He}$ tube array electronics were free running and counting neutrons. Whenever the plastic paddle detected a pulse above a preset threshold, it created an $\sim 120 \mu$ s logic pulse to block the neutron counting by the ${ }^{3} \mathrm{He}$ array. These pulses blocked a large portion of the cosmic background-a signal-to-noise gain of a factor of 2 was obtained. The veto pulse width was between 2-3 times greater than the die-away time for the detector. It is interesting to note that a commercially available fission meter has a die-away time of $40 \mu \mathrm{s}$.

Method 2 is a calculational procedure in which the Feynman variance (excess of variance over Poisson distribution of neutron counts), $Y 2 F$, is calculated constantly in the background for a series of 
measurements from 1 to $512 \mu$ s and repeated. For cosmic neutrons this value should be a constant and slightly positive. Any introduction of a neutron source would disturb this correlation, and the measure of the correlation function Y2F would be lower. Using a commercially available fission meter, it has been shown that it is possible to partition the neutron counts into two groups, cosmic and non-cosmic, by establishing empirical rules relating neutron count rate and Y2F. This method does not exclude cosmic neutrons from being counted, but shows what percentage of neutrons belongs to cosmic origin. This method involves understanding the characteristics of the neutron counter in great detail, whereas the first method is straightforward. We tested both methods to increase the counting sensitivity for man-made neutrons.

\section{Shielding Materials and Thickness}

Choosing shielding materials requires considerable understanding of neutron interactions with materials like neutron converters $\left({ }^{3} \mathrm{He},{ }^{6} \mathrm{Li},{ }^{10} \mathrm{~B}\right)$, moderators $\left({ }^{1} \mathrm{H}, \mathrm{CH}_{2}, \mathrm{H}_{2} \mathrm{O}, \mathrm{D}_{2} \mathrm{O}\right)$, and absorbers $\left({ }^{10} \mathrm{~B},{ }^{113} \mathrm{Cd}\right.$, ${ }^{157} \mathrm{Gd}$ ). Neutron shielding is effectively accomplished by initially using good moderating materials followed by neutron absorber materials. Sometimes these absorbing materials $\left({ }^{113} \mathrm{Cd}\right.$ or $\left.{ }^{157} \mathrm{Gd}\right)$ are uniformly distributed in a matrix made out of the moderating material. Effective neutron moderators must be low cost and machineable, with high moderating power $\left(\Sigma_{s} \varsigma\right)$, defined as the product of macroscopic scattering cross section $\left(\Sigma_{s}\right)$ and the average logarithmic decrement of kinetic energy of neutron in each scattering process, c. A good neutron moderator will also have to have a high moderating ratio, defined as $\left(\Sigma_{\mathrm{s}} \mathrm{s} / \Sigma_{\mathrm{a}}\right)$, where $\Sigma_{\mathrm{a}}$ is the absorption cross section. From the table of moderating power and moderating ratio (Rinard 1991), it is easy to understand why polyethylene with highly absorbing boron dopant followed by a thin cadmium sheet would make an effective cosmic neutron shielding block. The highdensity $\left(1.19 \mathrm{gm} / \mathrm{cm}^{3}\right)$ borated polyolefin (SWX-210 manufactured by Shieldwerx, a division of Bladewerx, LLC) that was used for this project had hydrogen and boron atomic density per $\mathrm{cm}^{3}$ of $6.07 \times 10^{22}$ and $1.99 \times 10^{22}$, respectively; both had a macroscopic thermal neutron cross section of $14.5 \mathrm{~cm}^{-1}$ (represented as the inverse of the mean free path of thermal neutrons in the bulk medium).

The cosmic-ray shield was constructed of two layers of 1-inch-thick borated high-density polyethylene (HDPE) with $30 \%$ natural boron by weight fabricated into a five-sided cover for two moderated neutron modules. A second thermal neutron absorber, cadmium, in the form of a 1/16-inch-thick sheet of ${ }^{113} \mathrm{Cd}$ composed of $12.2 \%$ of natural cadmium was

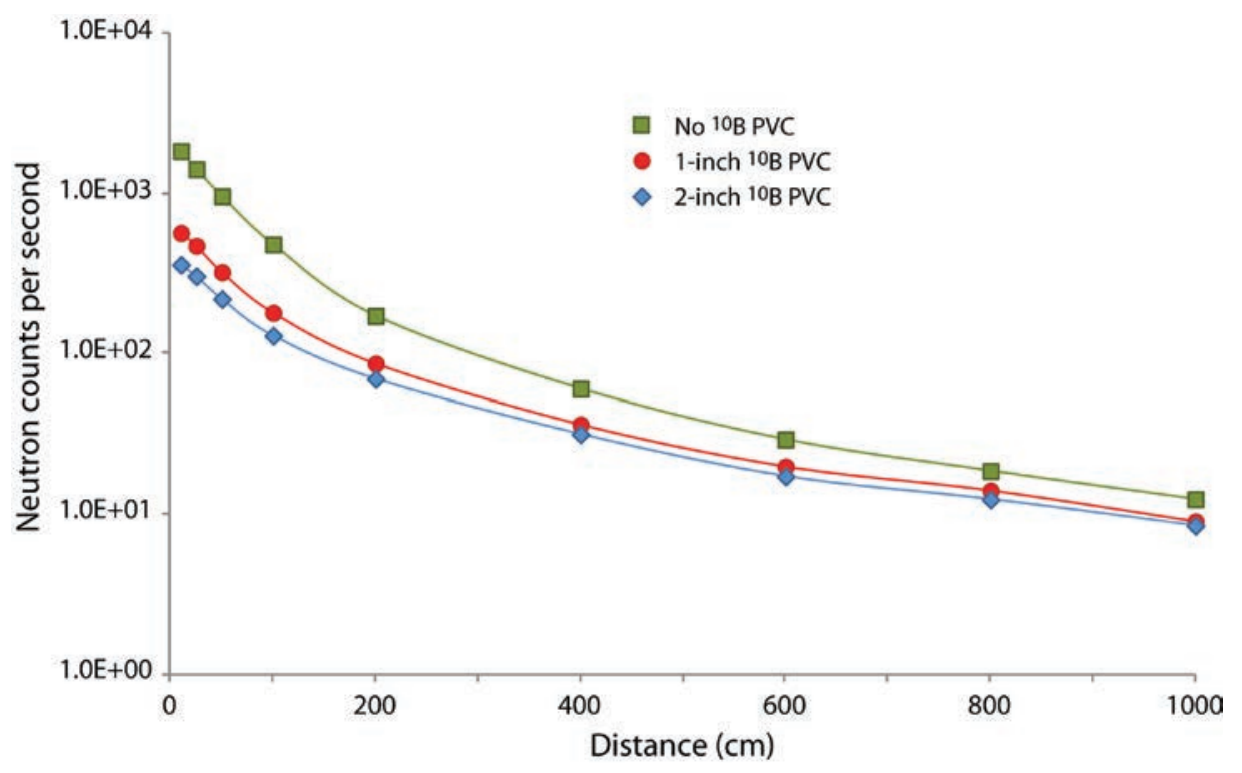

Figure 2. MCNPXsimulated neutron counts per second measured by a large array of 16 pressurized ${ }^{3} \mathrm{He}$ tubes (72" $\times 2$ " diameter). The neutron sensitivity (counts per second) as simulated by MCNPX is shown as a function of distance between the source and the detector for unshielded (green squares), shielded with 1 -inch (red dots), and 2-inch (blue diamonds) shielding materials. 


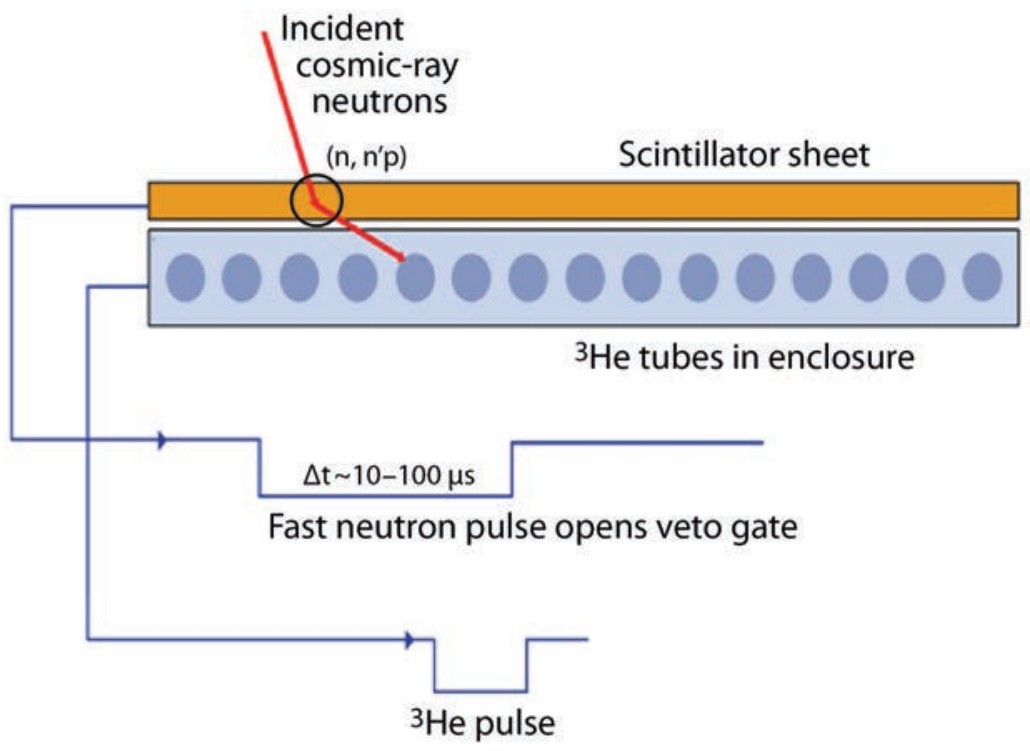

Figure 3. The incident cosmic neutrons undergo $(n, n),\left(n, n^{\prime} p\right)$ and charge-exchange reactions within BC-400 (predominantly made of $\mathrm{H}\left[5.23 \times 10^{22} / \mathrm{cm}^{3}\right]$ and $\mathrm{C}\left[4.74 \times 10^{22} / \mathrm{cm}^{3}\right]$ atoms) and create light pulses. Neutron pulses are discriminated against gamma-ray and other charged particle pulses and tagged by creating a transistor-totransistor logic pulse that signals to open a $120 \mu$ s veto gate that is sent to the anticoincidence unit to block the neutrons from being counted by the ${ }^{3} \mathrm{He}$ counter electronics.

Time

placed between the neutron detector modules and the shield. The MCNPX code (Pelowitz 2005), version 2.5.0, was used to simulate neutron transport and to generate sensitivity shown in Figure 2. A ${ }^{252} \mathrm{Cf}$ source of $2 \mu \mathrm{Ci}$ strength was used to simulate the response function for the 16-element 72 " $\times 2$ " cylindrical pressurized ${ }^{3} \mathrm{He}$ tube array (2.7 atmospheric pressure with $1 / 4$-inch moderating sleeves).

\section{Y2F Measurements}

A high-speed field-programmable gate array (FPGA) SBRIO-9602 manufactured by National Instruments, Inc., has been used to exploit the time correlations between neutrons from different sources (National Instruments 2008). For example, cosmic or background neutrons in a maritime environment are only mildly correlated, primarily because of the high rates of spallation neutrons created by cosmic interactions. Neutrons from the $(\alpha, n)$ channel are completely uncorrelated, and neutrons from fission are very highly correlated, particularly when multiplications are taking place following spontaneous or induced fission. By following the detected neutron counting distributions with very narrow time gates ranging from 1 to $512 \mu \mathrm{s}$, one can partition between fission and cosmic neutrons within a very short time, on the order of 10 minutes. This approach provides a unique solution to discriminate against cosmic neutrons in a maritime search environment (in real time) and enables effective measurement of a neutron source on the ground from a large standoff distance.

\section{BC-400 Plastic Response to Neutron and Electronic Veto}

In low-rate multiplicity measurement conditions (like those present in maritime searches for special nuclear materials), even though mildly time-correlated, the cosmic-coincidence neutron backgrounds are the biggest challenge. Essentially all neutron-coincidence background counts in a ${ }^{3} \mathrm{He}$ thermal neutron detector originate from the cosmic-ray spallation events. To reduce the neutron-coincidence background, the incident cosmic rays must be reduced both by shielding and by electronic means. Material shielding cannot reduce the coincidence background rate. By using a $5 \mathrm{~cm}$ thick plastic scintillator slab as a veto trigger, a reduction of $72 \%$ of coincidence background has been reported (Miller 1992). A similar technique has been employed in this project. The conceptual design of the experiment is shown in Figure 3 , in which an array of 15 pressurized ${ }^{3} \mathrm{He}$ tubes is covered on one side (cosmic-ray incidence side) by a 2 -inchthick rectangular slab of BC-400 plastic, measuring 
$36 "$ (length) $\times 12$ " (width) with an extended light guide used to collect light pulses using a photomultiplier tube (RCA 8575). An Ortec Model 265 tube base was placed on one end. A coincidence unit was used as the gate. This nuclear instrumentation module unit accepts as inputs the logic signal output from the ${ }^{3} \mathrm{He}$ detector; the veto pulses are accepted through an anticoincidence gate. The output of this unit is then sent to the FPGA for time-correlation counting of the ${ }^{3} \mathrm{He}$ signals. When the anticoincidence gate input is positive, the coincidence unit gives a zero (null) output; in the absence of an anticoincidence pulse, the ${ }^{3} \mathrm{He}$ signal passes through the unit to the FPGA with minimal signal distortion.

\section{Results}

\section{Shielding}

A series of shielding measurements were conducted using an americium-beryllium (Am-Be) neutron source (RAM-95048, $47.6 \mathrm{mCi}$ ) and a series of californium-252 $\left({ }^{252} \mathrm{Cf}\right)$ neutron sources (ranging in strength from 1.8 to $96.4 \mu \mathrm{Ci}$ ) to determine the effectiveness of the shielding block, which consisted of two highly borated polyethylene sheets, each 1 inch thick; the second layer away from the source had a sheet of 1/16-inch-thick Cd glued onto it to capture the moderated neutrons. The neutron counting equipment used to perform the tests was from the Remote Sensing Laboratory-Andrews (RSL-A). The equipment used included a neutron drop sensor, an infield backpack, RSL mobile neutron pods, and a 6 -foot ${ }^{3} \mathrm{He}$ tube array. Figure 4 shows the source detector and shielding block orientation and configurations for the shielding measurement campaign.

Long-dwell static data were collected to obtain stable and statistically valid neutron count rates from the ${ }^{3} \mathrm{He}$ tube detector arrays, first without shielding in the presence of a neutron source, and then by bringing in the shielding layers one at a time. First, a single 1-inch layer of the borated polyethylene, then two layers of 1-inch polyethylene panels, and finally 2 -inch polyethylene plus a 1/16-inch-sheet (away from the source side) were used. Effective shielding power was calculated (in percentile form) as

$$
P_{S}=100 \times \frac{N_{u}-N_{s}}{N_{u}},
$$

where $P_{s}$ is the shielding power, and $N_{u}$ and $N_{s}$ are neutron count rates measured by the ${ }^{3} \mathrm{He}$ tubes in unshielded and shielded configurations, respectively. Table 1 shows the shielding power $\left(P_{s}\right)$ for various deployable RSL-A neutron counters, which vary greatly in terms of their size (solid angle coverage) relative to neutron count rates.

Table 1. Shielding measurements count rate data in counts per second for various neutron counters

\begin{tabular}{|c|c|c|c|c|}
\hline $\begin{array}{l}\text { Equipment Type } \\
\text { (Generic Name) }\end{array}$ & $\begin{array}{l}\text { Active Counting } \\
\text { Element }\end{array}$ & $\begin{array}{l}\text { Unshielded Neutron } \\
\text { Count Rate (cps) }\end{array}$ & $\begin{array}{l}\text { Shielded Neutron } \\
\text { Count Rate (cps) }\end{array}$ & Shielding Power $\left(P_{S}\right)$ \\
\hline Drop Sensor & $\begin{array}{l}\text { Single }{ }^{3} \mathrm{He} \\
(2.7 \text { atm }) \text { tubes } \\
\left(12^{\prime \prime} \times 1 " \text { diameter }\right) \\
\text { with moderator }\end{array}$ & $59.30 \pm 0.45$ & $\begin{array}{c}30.8 \pm 0.32 \\
\text { Background }=0.17\end{array}$ & $48 \%$ \\
\hline Infield Backpack & $\begin{array}{l}\text { Five }{ }^{3} \mathrm{He} \text { tubes } \\
\left(12^{\prime \prime} \times 1 " \text { diameter }\right) \\
\text { with moderator }\end{array}$ & $214.3 \pm 4.9$ & $\begin{array}{c}54.1 \pm 2.3 \\
\text { Background }=0.7\end{array}$ & $75 \%$ \\
\hline Mobile Neutron Pods & $\begin{array}{l}\text { Sixteen }{ }^{3} \mathrm{He} \text { tubes } \\
\left(36^{\prime \prime} \times 2 \text { " diameter }\right) \\
\text { with } 1 / 4 \text { " polyethylene } \\
\text { sleeves }\end{array}$ & $216.8 \pm 14.7$ & $\begin{array}{c}102.7 \pm 11.1 \\
\text { Background }=5.1\end{array}$ & $53 \%$ \\
\hline $\begin{array}{l}6^{\prime}{ }^{3} \mathrm{He} \text { Tubes for Aerial } \\
\text { Measurements }\end{array}$ & $\begin{array}{l}\text { Sixteen }{ }^{3} \text { He tubes } \\
\left(72^{\prime \prime} \times 2^{\prime \prime} \text { diameter }\right) \\
\text { with } 1 / 4 " \text { moderator } \\
\text { sleeves }\end{array}$ & $174.0 \pm 22.7$ & $\begin{array}{c}137.4 \pm 20.7 \\
\text { Background }=135.6\end{array}$ & $\begin{array}{l}21 \% \text { (all counts } \\
\text { above background } \\
\text { removed) }\end{array}$ \\
\hline
\end{tabular}




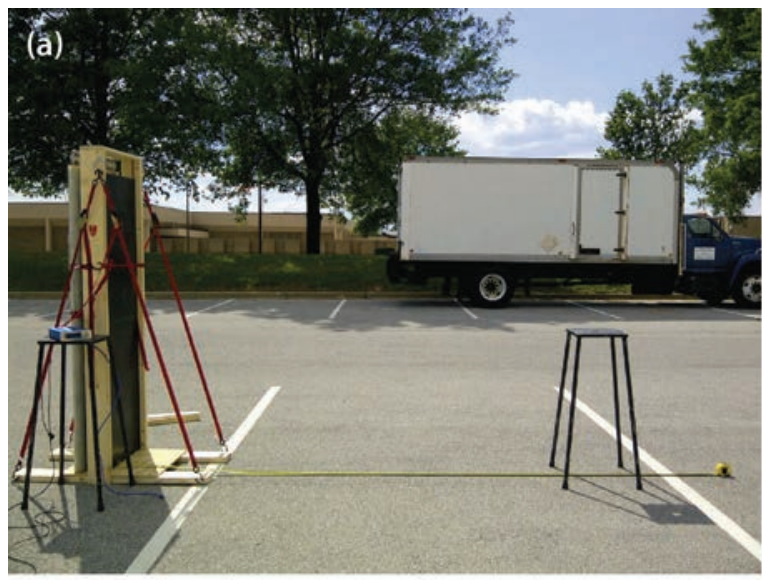

(c)

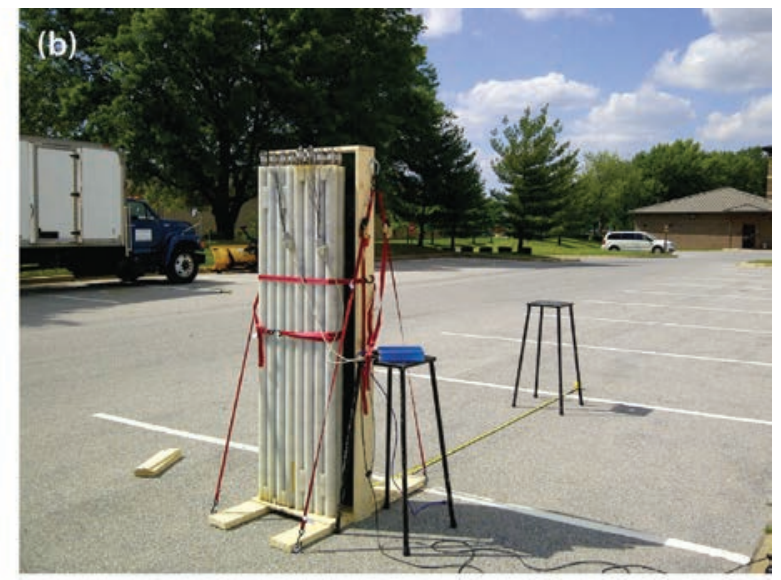

(d)
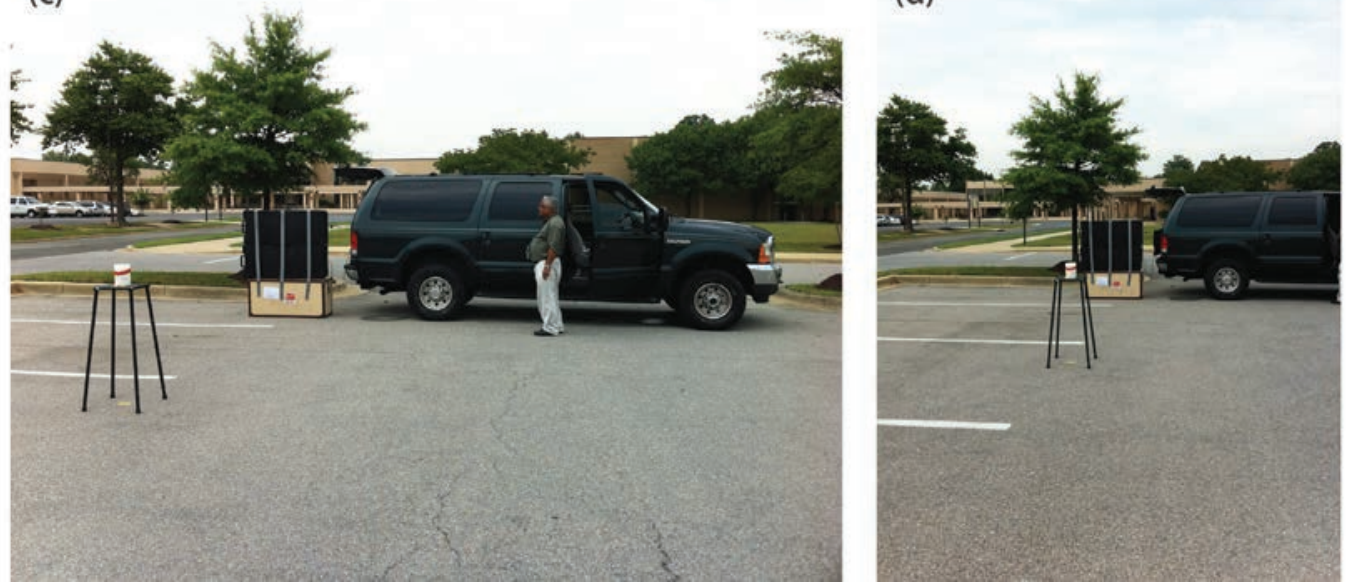

Figure 4. (a) The shielding side of the 72 " $\times 2^{\text {" }}{ }^{3} \mathrm{He}$ tube array, (b) the detector side of the 72 " $\times 2$ " tube array, (c and d) two mobile neutron pods facing the source. The detectors, shielding elements, and the source were always centered about 1 meter above ground.

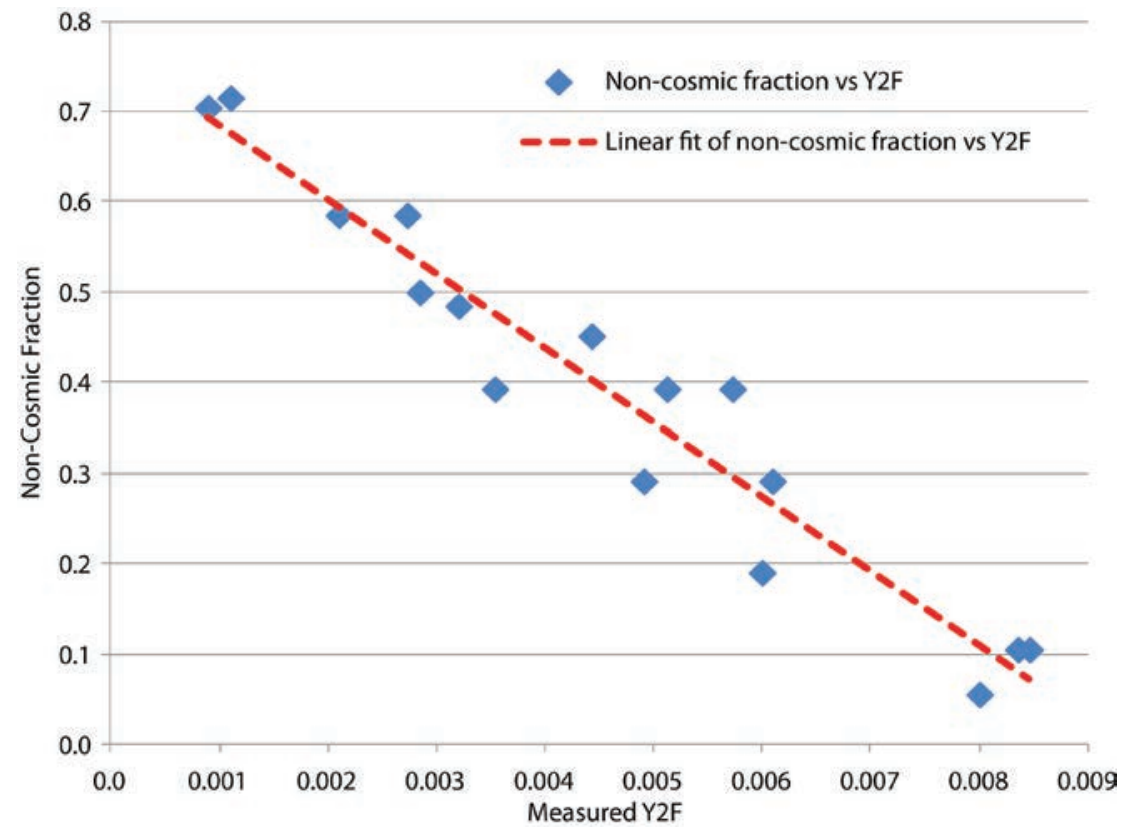

Figure 5. Non-cosmic fraction of the total neutron counts are plotted for measured $\mathrm{Y} 2 \mathrm{~F}$ values for various count rates. The red line shows a weighted mean slope (linear fit of the data points) of negative magnitude, meaning the increasing non-cosmic counts results in a decrease in Y2F. 

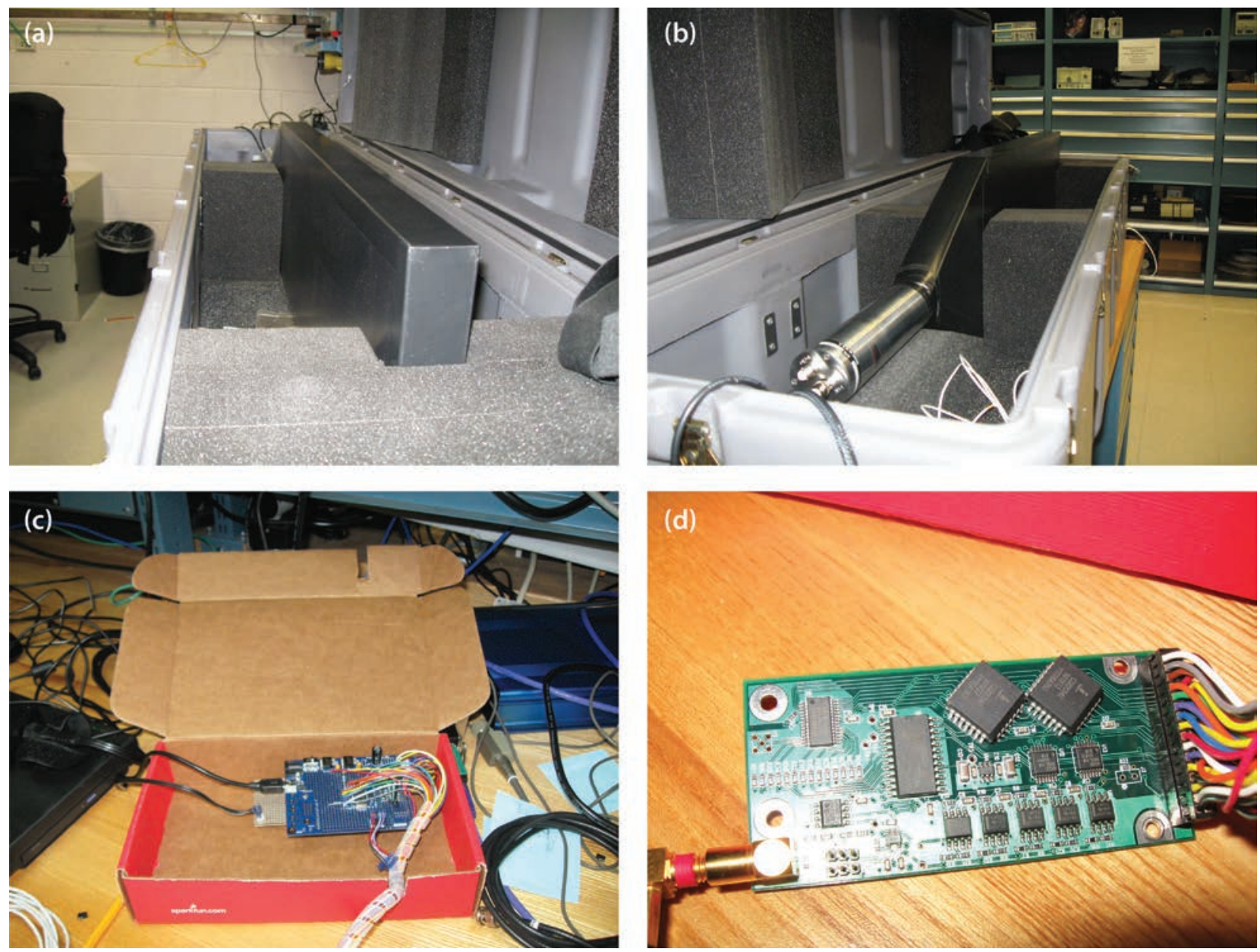

Figure 6. ( $\mathrm{a}$ and $\mathrm{b}) \mathrm{A}$ 2-inch-thick BC-400 (12" $\times 36$ " length without the light guide) with photomultiplier tube at one end was used along with the (c) high-voltage pre-amplifier and (d) amplifier board to tag the cosmic neutrons and separate them by stopping them from being counted by the ${ }^{3} \mathrm{He}$ proportional counter array

\section{Parameterization of Non-Cosmic Neutron Content in Terms of Measured $\mathrm{Y}_{2} \mathrm{~F}$}

One of the goals of this project was to parameterize the known percentile component of man-made neutrons in a mixed neutron flux as a function of the cumulative value of $\mathrm{Y} 2 \mathrm{~F}$ as measured by the freerunning ${ }^{3} \mathrm{He}$ proportional counter system. A large amount of data were collected to provide a varied range of neutron counting rates so that a stable, statistically valid algebraic relation could be established between the $\mathrm{Y} 2 \mathrm{~F}$ and corresponding man-made neutron component. Figure 5 shows the plot of stable $\mathrm{Y} 2 \mathrm{~F}$ values versus the fractional composition of the man-made neutrons.

\section{$B C-400$ Electronic Veto}

The electronic veto system shown in Figure 3 was set up using the BC-400 plastic paddle shown in Figure 6. The gamma-ray and neutron pulses generated by the plastic are shown in Figure 7. The pulse height difference in them is clear; on the basis of pulse height separation, we can tag the neutrons inside the BC-400 and start the anticoincidence window. If the threshold is set at $2.1 \mathrm{VDC}$, a cosmic reduction of $68 \%$ is expected (Miller 1992), which would translate into an improvement of detector sensitivity by a factor of 1.8 . 
(a) Large neutron signal, $3.14 \mathrm{~V}$

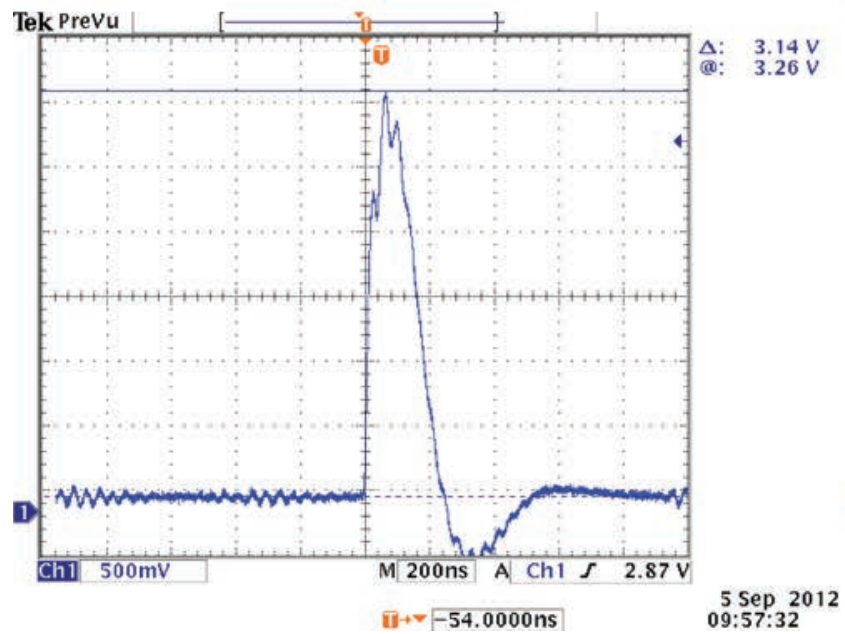

(b) Smaller, broader gamma signal, $\sim 2.10 \mathrm{~V}$

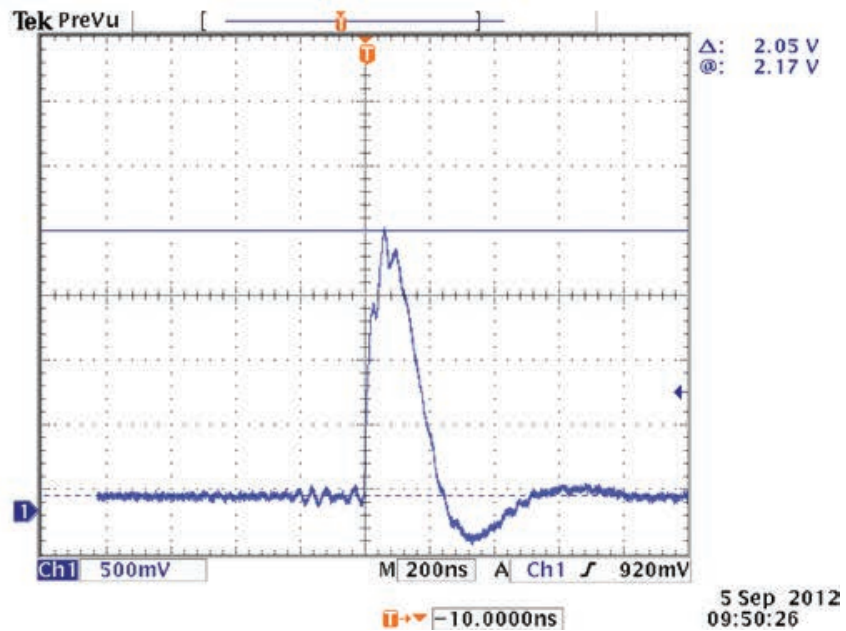

Figure 7. (a) The neutron pulses at 3.14 VDC amplitude are distinctly higher than the (b) gamma-ray pulses at 2.10 VDC and are, therefore, separable. Pulse shape discrimination was not applied because that would have caused unacceptable dead time.

\section{Conclusion}

A set of neutron moderating and absorbing materials in the form of rectangular slabs and sheets have been arranged to form a shielding package that successfully removes neutron flux from a source at a rate above 55\%. A 2-inch-thick and 36-inch-long (length without the light guide) rectangular plastic scintillator BC-400 (acting as an active electronic veto) was configured successfully to tag incident cosmic neutrons and inhibit them from being counted by an array of ${ }^{3} \mathrm{He}$ tubes. This veto system removes another $70 \%$ of the remaining cosmic neutrons, effectively eliminating $87 \%$ of the incident cosmic background neutron flux. The efficiency of this veto counter has not been experimentally benchmarked. A factor of $2.8 \mathrm{improvement}$ in detection sensitivity is accomplished with $70 \%$ veto efficiency (in conjunction with the shielding mechanism).

A real-time algorithm has been developed that determines the percentile man-made neutron component in a neutron flux from the real-time measured values of the excess of variance (Feynman variance, Y2F), providing software partitioning of neutrons. Because the two methods can run in parallel without interference, simultaneous application of these combined methods is most beneficial in maritime search operations where one is trying to find small correlated neutron signals.

\section{Acknowledgments}

The authors acknowledge the support provided by Joshua Jahn and Michael Taylor of RSL-A for providing specialized electronic cables and making shielding measurements. Support provided by John Meade in the area of graphic drawings and designs is greatly appreciated.

\section{References}

Forman, L., P. E. Vanier, K. Welsh, "Fast neutron source detecting at long distances using double-scatter spectrometry," SPIE Hard X-Ray and Gamma-Ray Detector Physics, San Diego, California, August 3, 2003.

Miller, D. W., H. O. Menlove, "Cosmic-ray-veto detector system," LA-12469-MS (DE93 004787), Los Alamos National Laboratory, Los Alamos, New Mexico, 1992. 
National Instruments product data sheet "NI Single Board RIO Embedded Control and Acquisition Devices," http://www.ni.com/pdf/products/us/cat_sbRIO_96xx.pdf, 2008, accessed August 30, 2012.

Pelowitz, D. B., editor, "MCNPXTM User's Manual," Version 2.5.0., LA-CP-05-0369, Los Alamos National Laboratory, Los Alamos, New Mexico, April 2005.

Rinard, P., "Neutron Interactions with Matter," in Passive Nondestructive Assay of Nuclear Materials, edited by T. D. Reilly, N. Ensslin, H. A. Smith, U.S. Nuclear Regulatory Commission NUREG/CR-5550, March 1991. 
This page left blank intentionally 


\section{High-Efficiency Solid-State Thermal Neutron Detector and IMAGING SYSTEM}

LO-14-12 | YEAR 1 OF 1

Mark Raphaelian ${ }^{1, a}$ and Michael McElfresh ${ }^{a}$

A design for a solid-state neutron detector was previously developed using MCNP calculations based on a two-phase system of silicon-diode and boron that incorporates geometrical features patterned into the semiconductor. MCNP models were used to explore possible performance improvements resulting from (1) systematically changing the size and distribution of the geometrical features and (2) varying the density of the boron phase. For this project, doped silicon wafers were used to produce a deep depletion region and then were patterned with geometrical features in a range of sizes in order to study the trends observed in the MCNP models. New boron10-enriched boron cluster phases were also synthesized to introduce more chemical stability and better control the boron matrix density. Boron nanoparticle incorporation by way of newly developed nanomanufacturing methods was also studied as a method to control matrix density and incorporate the boron phase uniformly into the structure. Detectors were built and tested with neutron radiation sources. An understanding of current limits for building a high-efficiency, low-cost neutron detector using standard commercial methods was developed, and many in the science community are developing strategies to operate within, and overcome, those limits.

${ }^{1}$ raphaeml@nv.doe.gov, 925-960-2549

a Livermore Operations

\section{Background}

For over a decade more efficient solid-state neutron detectors have been developed by altering the geometrical arrangement in which the boron transmutation phase is integrated with the semiconductor diode device (Nikolić 2005, Shultis 2006, Nikolić 2007, Bellinger 2007, Solomon 2007, Nikolić “Pillar structured thermal neutron detector with 6:1 aspect ratio" 2008, Nikolić "Pillar structured thermal neutron detector" 2008, Conway 2009, McGregor 2009, Shultis 2009, Bellinger "High efficiency" 2010, Bellinger "Characteristics" 2010, Brickner 2010, Nikolić 2010, Raphaelian 2010, McElfresh 2011, Nikolić 2011, Bellinger 2012). When the ${ }^{10} \mathrm{~B}(n, \alpha)^{7} \mathrm{Li}$ reaction is used for neutron detection, the key is to capture as many of the $\alpha$-particles, and as much of the initial particle energy, as possible. The simplest geometry is a layer of a boron- $10\left({ }^{10} \mathrm{~B}\right)$-enriched material on top of a silicon diode wafer. In this geometry over half of the $\alpha$-particles are lost, and, depending on the thickness of the boron matrix layer, much of the neutron energy will be lost as the $\alpha$-particle traverses the boron phase on its way to the silicon-diode detection phase. Alternative geometries for the boron matrix were explored in order to identify structures that would produce more efficient recovery of the $\alpha$-particles and their energy.

In our previous work with MCNP (McElfresh 2011), hexagonal arrays of cylindrical pillars, as shown schematically in Figure 1, were studied as a function of pillar diameter (d) over a range of 100 to $2000 \mathrm{~nm}$, pillar spacing $(L)$ from $1.05 d$ to $5.00 d$, and the density 
Top Profile

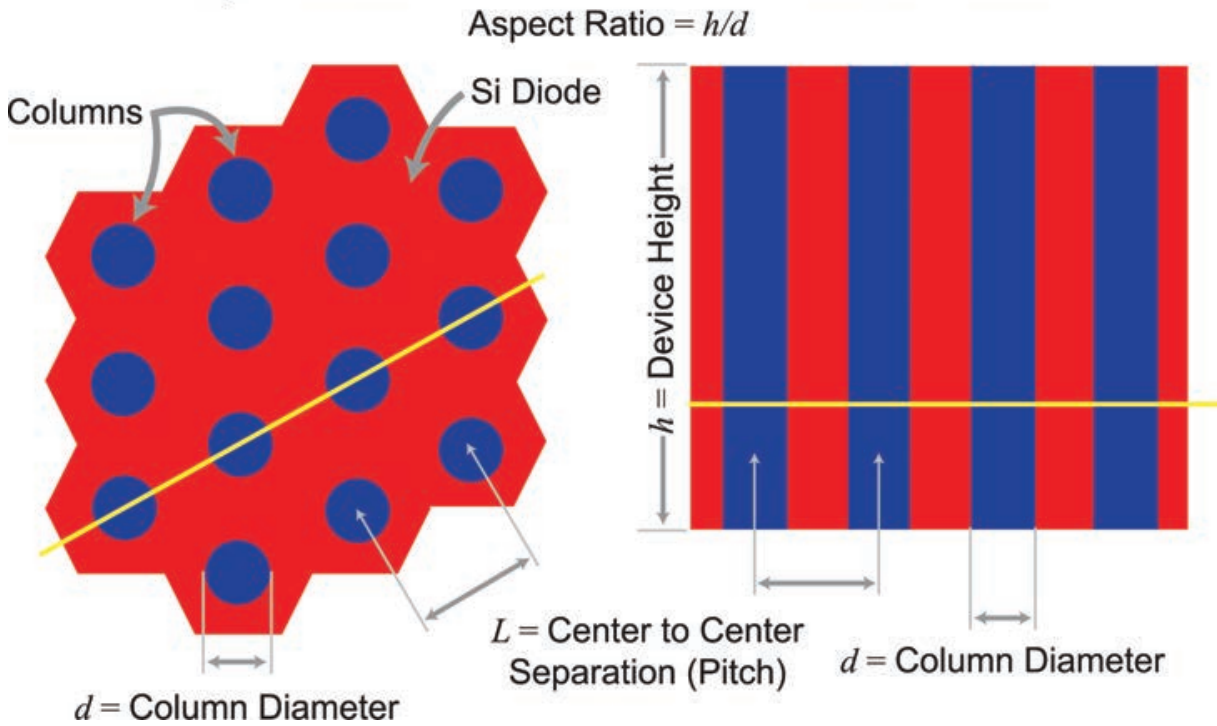

Figure 1. The devices are a hexagonal array of silicon pillars in a boron-based matrix or boron-based cylinders in a silicon matrix. The geometrical variables are $d$ the diameter of the pillars, $L$ the distance center to center of the pillars, and $h$ the height of the pillars. of a fully ${ }^{10} \mathrm{~B}$-enriched boron phase from $10 \%$ to $100 \%$ of theoretical density.

Figure 2 displays the major trends observed in these calculations for boron cylinders embedded in a silicon diode matrix. In Figure 2, each plot is the energy in a bin versus the energy of the bin. For all plots, $d$ changes in the horizontal direction and $L$ changes in the vertical direction. Colors refer to specific boron matrix densities. The curves become narrower as $d$ is reduced, and they move to higher energy and narrow further as the density is reduced. The peak in the distribution of deposited energy into the silicon matrix decreases in energy the smaller the pitch becomes.

The trends in Figure 2 for decreasing $d$ can be explained by considering that the various potential $\alpha$-particle paths become increasingly similar in energy, leading to a narrowing of the distributions. The trends associated with decreasing density can be explained by realizing that more and more energy is delivered to the silicon, both increasing the energy and narrowing the energy spread. The trend with $L$ is related to the $\alpha$-particle traversing more boron as $L$ decreases, thereby reducing the amount of energy available for deposition into the silicon matrix.

\section{Project}

A primary goal of this project was to test the ability of current manufacturing methods for producing these detectors. The other important goal was to determine if the trends observed in MCNP calculations could be observed in real systems.

Silicon wafers with a low level of n-type 10 to $20 \Omega$ doping were used as the initial material for devices. On one side of the wafer, high p-doping (on the order of $5 \times 10^{16}$ ) was done via ion implantation. The wafers were then annealed so that the doping reached $100 \mathrm{~nm}$ in depth to form the pn-junction. This results in the wafers having a deep depletion region exceeding the depth of the etch features (maximum $20 \mu \mathrm{m})$. The opposite side of the wafer required a high $n$-doping profile $\left(5 \times 10^{16}\right.$ to $\left.10^{19}\right)$ to function as an electrical contact. Three different doping profiles were tested. Shown in Figure 3 are current-voltage $(\mathrm{I}-\mathrm{V})$ curves for $1 \mathrm{~cm}$ square devices for $d=500 \mathrm{~nm}$ and $L=1000 \mathrm{~nm}$ (i.e., $2 d$ ). Solid-state radiation detectors are normally operated at as large a reverse bias as the device will support in order to maximize the depth of the depletion region and reduce recombination. Under ideal circumstances, silicon devices can be operated at up to 40 volts of reverse bias. Figure 3 shows that these devices are showing reverse-bias 


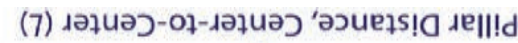
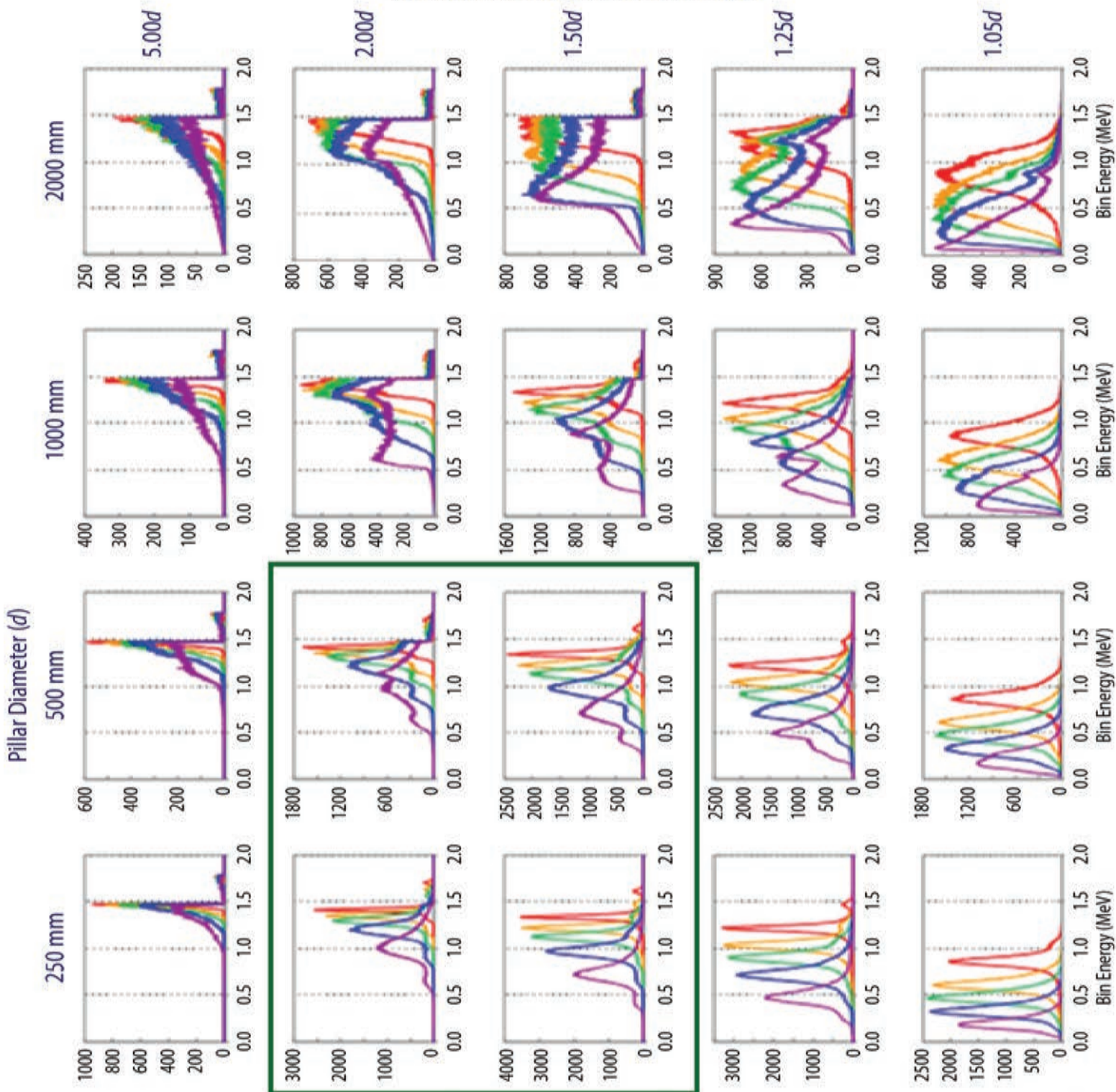

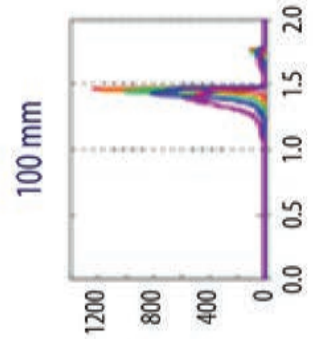

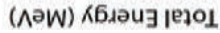

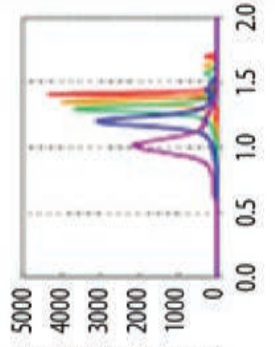

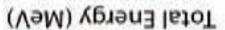

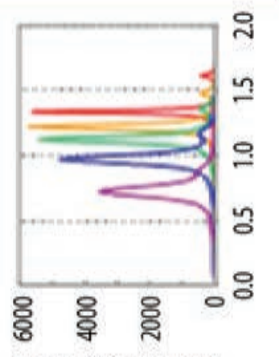

(
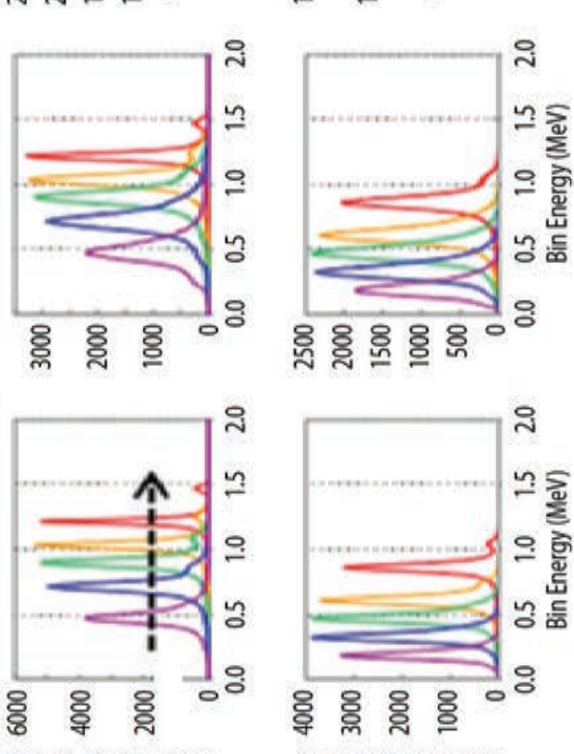

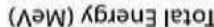

Figure 2. Each curve is a histogram detailing the energy deposition profile from alpha particles into the silicon as a function of energy. The area outlined in green identifies where the best trade-offs between manufacturability/cost and detector performance exist. A lower density boron matrix (heavy black dotted arrow) produces higher energy and sharper peaks. Both smaller $d$ and $\rho$ produce sharper peaks. Each color corresponds to a particular boron matrix density: purple $=100 \%$, blue $=50 \%$, green $=30 \%$, yellow $=20 \%$, and red $=10 \%$. 


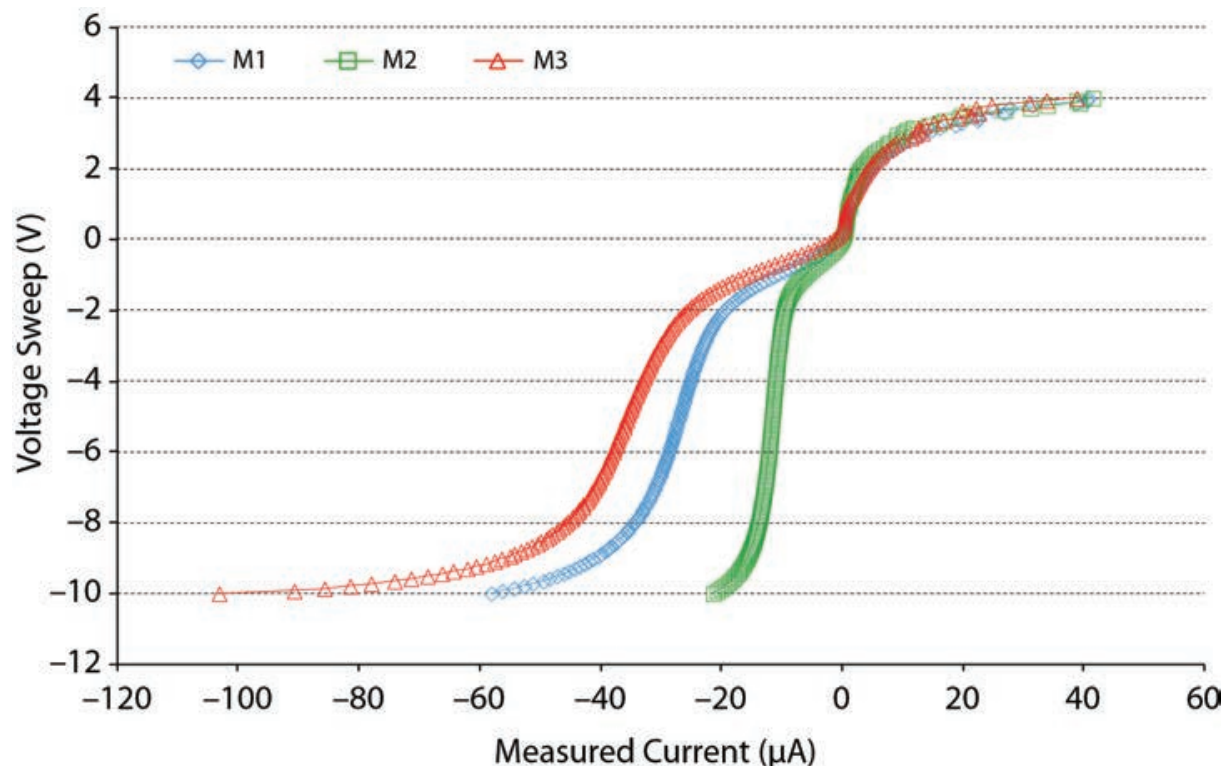

Figure 3. Current-voltage (I-V) curves for manufactured devices taken with and without external illumination

breakdown at voltages of as little as 2 or 3 volts. Previous successful devices could be operated at reverse bias voltages of 25 volts; however, compared to ours, these devices were much smaller in size (i.e., a few $\mathrm{mm}^{2}$ versus $100 \mathrm{~mm}^{2}$ ) (Klann 2011). The best explanation available for the low reverse bias is the nonuniformity of the doping, where curves like those seen in Figure 3 are characteristic. Other features of the I-V curves that support nonuniform doping were the effects of light exposure and leakage currents. Much better contacts will also be required to get optimal pn-junction behavior.

Figure 4 shows $500 \mathrm{~nm}$ structures that have been etched using standard reactive ion etching (RIE). Two different lithographic procedures were employed, one with a standard spin-on resist and another using a hard mask. The hard mask allows for deeper etching. Due to the small size of the features, $<1000 \mathrm{~nm}$, a step projection method of exposing the resist was used. Small large-aspect-ratio feature sizes present an etching challenge. The current state-of-the-art etch is held by Applied Materials, which has shown that they can etch $350 \mathrm{~nm}$ holes with an aspect ratio of 100:1.

Several boron matrix materials were explored. Two stable boron cluster materials, both carboranes, were synthesized in the fully ${ }^{10} \mathrm{~B}$-enriched form. The synthetic route for one of these carboranes is shown

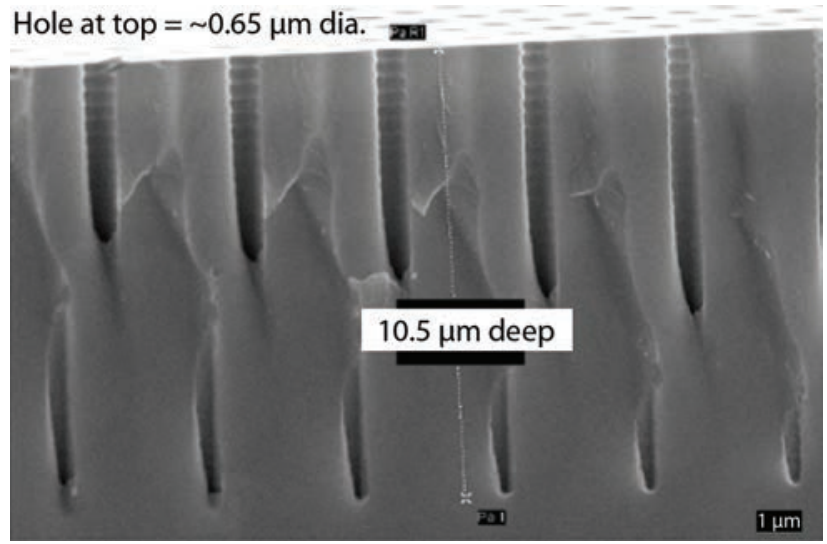

Figure 4. The cross section of a reactive ion etched (RIE) wafer showing the cylindrical profiles etched into the silicon prior to boron material filling

in Figure 5 . While any atoms other than ${ }^{10} \mathrm{~B}$ in the boron matrix will only increase energy loss to the matrix and broaden the energy curves, the gains of adding other atoms must be weighed against the losses. Specifically, the additional atoms in these compounds stabilize the matrix with regard to chemical decomposition and create avenues for varying the density. Incorporation of these compounds into small-diameter holes remains a challenge that will take time to learn to control and has caused problems with many of the earlier attempts to make these types of devices. Two carboranes were synthesized, including the ionic compound shown in Figure 5, 
${ }^{10} B-E n r i c h e d$ Borane Cluster Synthesis: $\left[\mathrm{C}^{10} \mathrm{~B}_{11} \mathrm{H}_{12}\right]^{-}$ $\mathrm{H}_{3}{ }^{10} \mathrm{BO}_{3}$<smiles>CC(CO)CO</smiles>

$\left(\mathrm{H}_{3} \mathrm{CO}\right)_{3}{ }^{10} \mathrm{~B}$<smiles>[13NH2][Mg]</smiles>

$\mathrm{Na}^{10} \mathrm{BH}_{4}$

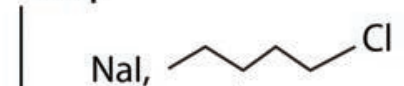
Heat, $\mathrm{H}_{2} \uparrow$ $\mathrm{H}_{3} \mathrm{PO}_{4}$

\section{$\left.{ }^{10} \mathrm{~B}_{11} \mathrm{H}_{14}\right]^{-}$}

$\mathrm{CHCl}_{3}$

$\mathrm{NaH}$

$\mathrm{EtOH}$

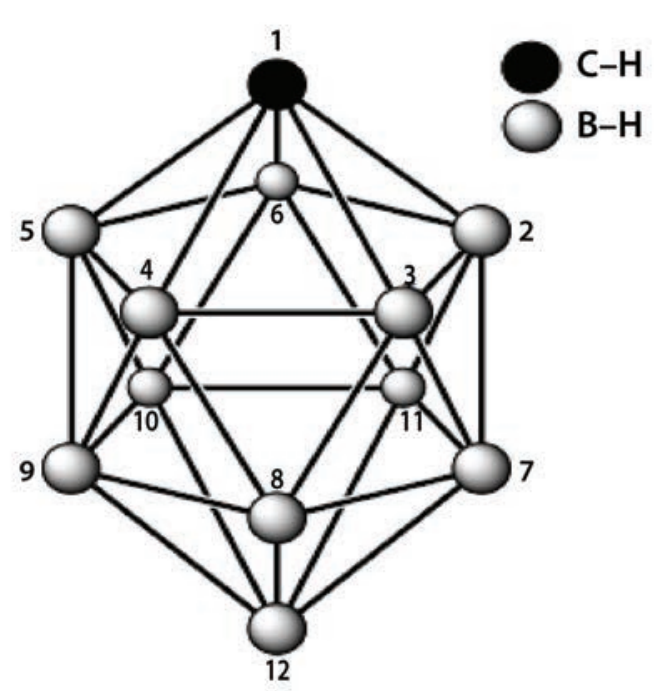

Figure 5. The steps in the synthesis of the ionic carborane cluster material. The structure of the synthesized cluster is also shown.

\section{$\mathrm{Na}+\left[\mathrm{C}^{10} \mathrm{~B}_{11} \mathrm{H}_{12}\right]^{-}$}

which is fairly water soluble, and another nonionic compound that is more soluble in nonpolar solvents. This approach was intended to provide a range of options for addressing surface tension issues.

Another method to incorporate boron in a controlled manner involved methods developed at the National Science Foundation Center for High-Rate Nanomanufacturing (CHN) at Northeastern University.
CHN has developed two methods for incorporating nanoparticles into small-diameter holes: one is a dielectrophoretic method and the other a turbulent mixing method. We used the latter with commercially obtained $\mathrm{B}_{4} \mathrm{C}$ nanoparticles, and the results are shown in Figure 6 . The holes are filled to the top with particles. In some regions, some material is also on the top. This material would be removed before coating with encapsulating contact surfaces.
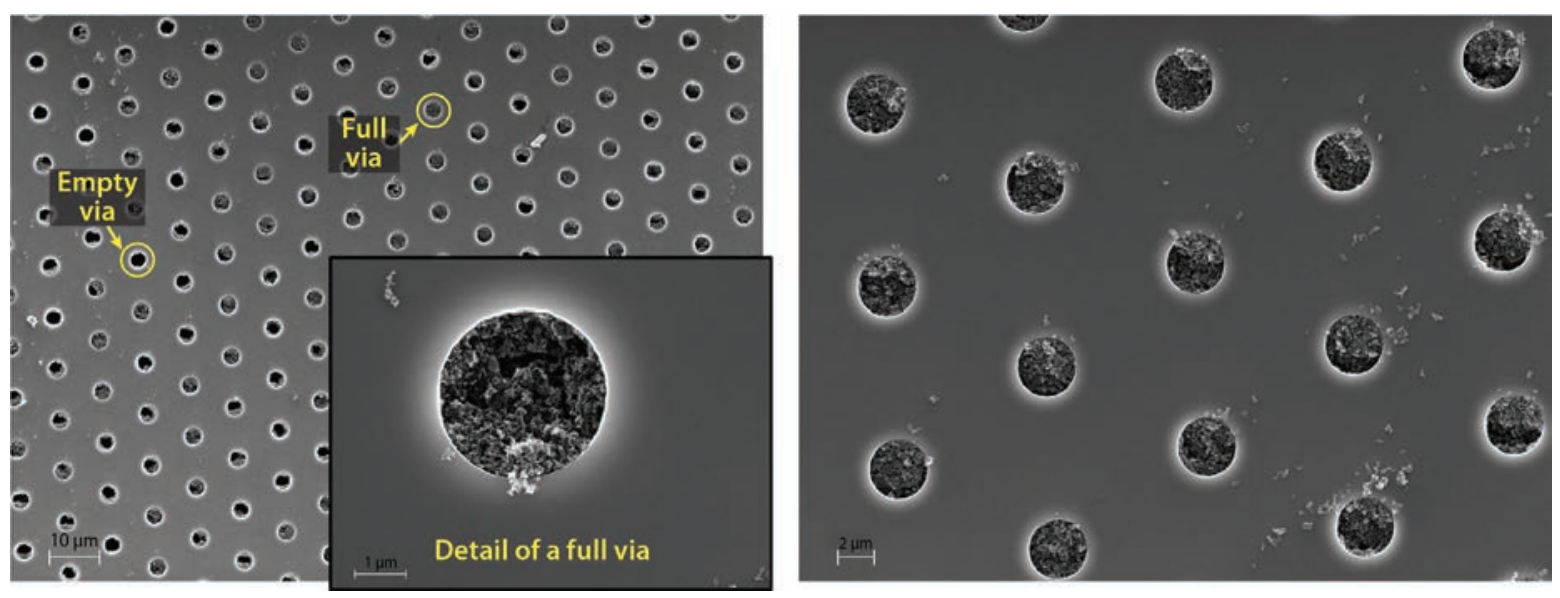

Figure 6. $\mathrm{B}_{4} \mathrm{C}$ nanoparticles fill the high aspect ratio cylindrical holes in the silicon wafer/chip 

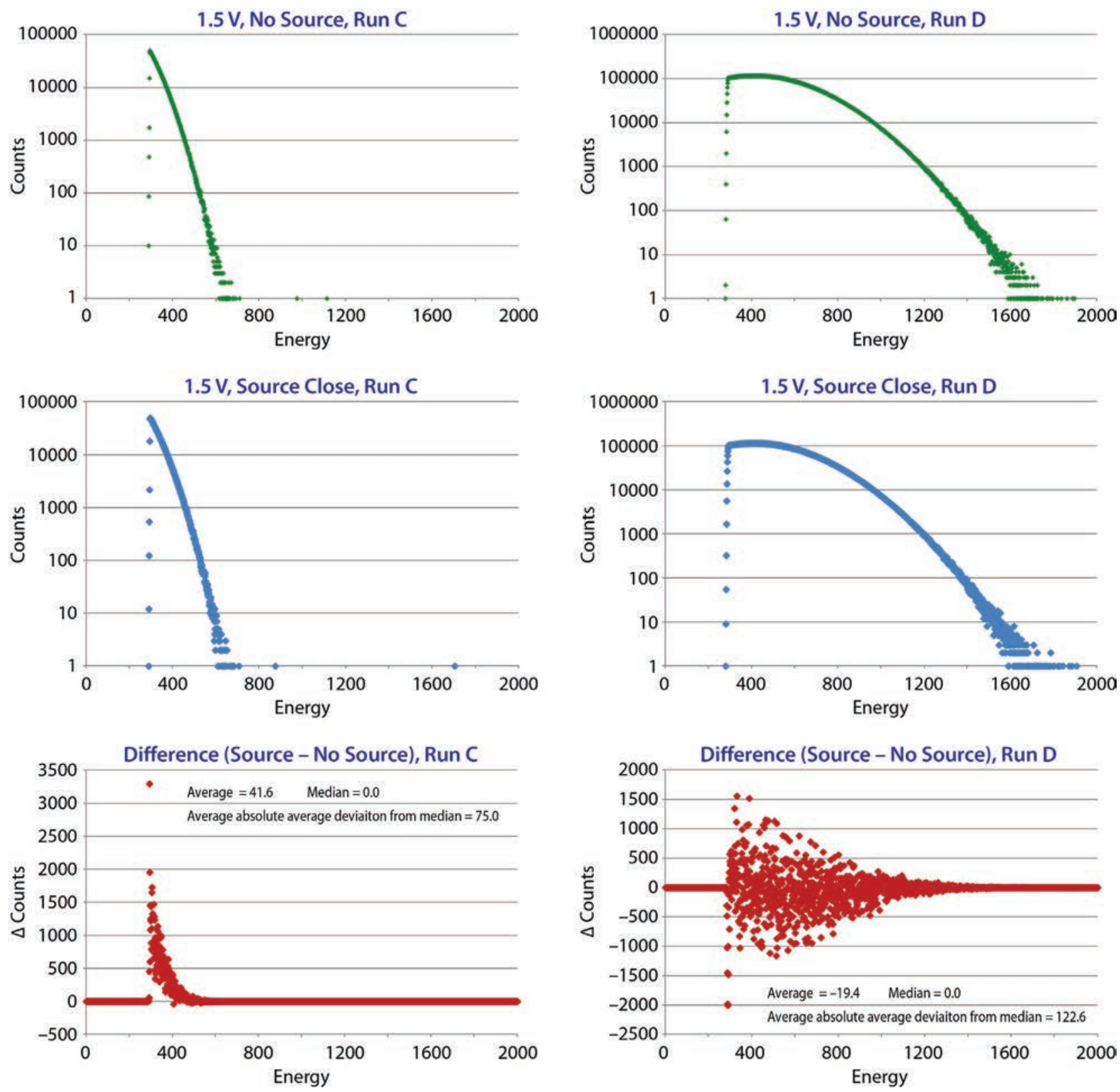

Figure 7. The manufactured detector response: top plots show the response without a source (i.e., background), middle plots show the response with a source, bottom plots show the response difference between with and without source exposure.

A first round of radiation detection testing was done with early stage devices. As noted above, the I-V curves were not well suited for radiation detection; however, there were many lessons to be learned using these early stage devices. Because our Argonne National Laboratory (ANL) collaborators had done work on some of the earliest designs of this general type of sensor, it was possible to make some direct comparisons between the current device and previous devices that were not yet described in the literature. Some detection results using neutron sources at ANL are shown in Figure 7. The detected signals are associated with small differences observed between the curves. The signal-to-noise ratio was poor. Devices that ANL had previously tested were retested and required very large bias voltages before they produced a significant and meaningful detector response. 


\section{Conclusion}

Our work has allowed us to identify several of the current barriers to increasing the detector size and performance of geometrically structured two-phase neutron detectors, and has also allowed us to develop a strategy for moving this technology forward.

It seems clear that uniform doping is critical to obtaining suitable devices. The size of any device will be limited by the uniformity of the doping. For some commercial applications, fairly uniform doping has produced high-quality diode materials over the full expanse of 6-inch wafers. Having good electrical contacts are also critical to getting high performance and high efficiency. Uniform and reproducible incorporation of the boron matrix at controlled densities needs to be perfected, possibly by working with specialized vendors or by obtaining suitable commercial materials.

Two ${ }^{10} \mathrm{~B}$-doped carboranes were developed, one suitable for polar solvents and the other for nonpolar solvents. There are very few additional non- ${ }^{10} \mathrm{~B}$ atoms in these compounds, which are mostly low- $Z$ hydrogen. Chemical stability of the boron phase has been a problem for boron-based detectors, and these compounds should directly address this issue and provide more flexibility in methods for incorporating the boron phase into the device.

Nanoparticles are another material that may allow control over the density and chemical stability of the boron phase. Very stable $\mathrm{B}_{4} \mathrm{C}$ particles were incorporated into the structure using a micro-turbulence method. Excellent results were obtained using this method.

\section{References}

Bellinger, S. L., W. J. MacNeil, T. C. Unruh, D. S. McGregor, "Angular response of perforated silicon diode high efficiency neutron detectors," IEEE Nucl. Sci. Symp. Conf. Rec. 3 (2007) 1904-1907.

Bellinger, S. L., R. G. Fronk, W. J. MacNeil, T. J. Sobering, D. S. McGregor, "High efficiency dual-integrated stacked microstructured solid-state neutron detectors," IEEE Nucl. Sci. Symp. Conf. Rec. (2010) 2008-2012.

Bellinger, S. L., R. G. Fronk, W. J. MacNeil, T. J. Sobering, D. S. McGregor, "Characteristics of the stacked microstructured solid-state neutron detector," Proc. SPIE 7805 (2010) 78050N-1-78050N-15.

Bellinger, S. L., R. G. Fronk, W. J. MacNeil, T. J. Sobering, D. S. McGregor, "Improved high efficiency stacked microstructured neutron detectors with nanoparticle ${ }^{6} \mathrm{LiF}, "$ IEEE Trans. Nucl. Sci. 59 (2012) 167-173.

Brickner, N. M., M. W. McElfresh, M. L. Raphaelian, "Lightweight High Efficiency Thermal Neutron Detector," presented at the 2010 American Nuclear Society Winter Meeting and Nuclear Technology Exposition, Las Vegas, Nevada.

Conway, A. W., T. F. Wang, N. Deo, C. L. Cheung, R. J. Nikolić, "Numerical simulations of pillar structured solid state thermal neutron detector: Efficiency and gamma discrimination," IEEE Trans. Nucl. Sci. 56, 5 (2009) 2802-2807.

Klann, R. T., Argonne National Laboratory, Argonne, Illinois, private communication, July 2011.

McGregor, D. S., W. J. MacNeil, S. L. Bellinger, T. C. Unruh, J. K. Shultis, "Microstructured semiconductor neutron detectors," Nucl. Instrum. Methods Phys. Res. A 608, 1 (2009) 125-131.

McElfresh, M. W., M. R. Raphaelian, N. M. Brickner, "Lightweight high-efficiency thermal neutron detection," Nevada National Security Site-Directed Research and Development, FY 2010, National Security Technologies, LLC, Las Vegas, Nevada, 2011, 105-114.

Nikolić, R. J., C. L. Cheung, C. E. Reinhardt, T. F. Wang, "Roadmap for high-efficiency solid-state neutron detectors," Proc. SPIE 6013 (2005) 36-44.

Nikolić, R. J., A. M. Conway, C. E. Reinhardt, R. T. Graff, T. F. Wang, N. Deo, C. L. Cheung, "Fabrication of pillarstructured thermal neutron detectors," IEEE Nucl. Sci. Symp. Conf. Rec. 2 (2007) 1577-1580. 
Nikolić, R. J., A. M. Conway, C. E. Reinhardt, R. T. Graff, T. F. Wang, N. Deo, C. L. Cheung, "Pillar structured thermal neutron detector with 6:1 aspect ratio," Appl. Phys. Lett. 93 (2008) 133502-133505.

Nikolić, R. J., A. M. Conway, C. E. Reinhardt, R. T. Graff, T. F. Wang, N. Deo, C. L. Cheung, "Pillar structured thermal neutron detector," Proc. IEEE 93 (2008) 2361-2364.

Nikolić, R. J., A. M. Conway, R. Radev, Q. Shao, L. Voss, T. F. Wang, J. R. Brewer, C. L. Cheung, L. Fabris, C. L. Britton, M. N. Ericson, "Nine element Si-based pillar structured thermal neutron detector," Proc. SPIE 7805 (2010) 780500-780500-9.

Nikolić, R. J., Q. Shao, L. F. Voss, A. M. Conway, R. Radev, T. F. Wang, M. Dar, N. Deo, C. L. Cheung, L. Fabris, C. L. Britton, M. N. Ericson, "Si pillar structured thermal neutron detectors: Fabrication challenges and performance expectations," Proc. SPIE 8031 (2011) 803109-803109-11.

Raphaelian, M. L., N. M. Brickner, M. W. McElfresh, "Transducer density variation using aerogels and silicon diode geometrical factors affecting solid-state thermal neutron detection efficiency and performance," presentation, 2010.

Shultis, J. K., D. S. McGregor, "Design and performance considerations for perforated semiconductor thermalneutron detectors," Nucl. Instrum. Methods Phys. Res. A 606, 3 (2009) 608-636.

Shultis, J. K., D. S. McGregor, "Efficiencies of coated and perforated semiconductor neutron detectors," IEEE Trans. Nucl. Sci. 53, 3 (2006) 1659-1665.

Solomon, C. J., J. K. Shultis, D. S. McGregor, "Angular design considerations for perforated semiconductor detectors," IEEE Nucl. Sci. Symp. Conf. Rec. 2 (2007) 1556-1559. 


\title{
NANOPARTICLE-BASEd ANALYTICAL BIOSENSOR
}

\author{
STL-69-12 | CONTINUED FROM FY 2011 | YEAR 2 OF 2
}

Shayla Sawyer Armand, ${ }^{1, a, b}$ Irina Barash, ${ }^{b}$ Sydney Halperin, ${ }^{b}$ Kevin Kyle, ${ }^{a}$ Kethia Mathieu, ${ }^{b}$ Liqiao Qin, ${ }^{b}$

Christopher Shing, ${ }^{b}$ and Stephan Weeks ${ }^{a}$

In this FY 2012 SDRD project, we explored bioaffinity methods of detection using aptamers.

Biorecognition layers (aptamers) are deposited on both flat surfaces and metal oxide nano-

structures for comparison. Specifically, indium oxide and zinc oxide nanostructures were investigated by using a chemical protocol that facilitates thiol/silica binding. The results show that both $\mathrm{ZnO}$ and $\mathrm{In}_{2} \mathrm{O}_{3}$ can be used with a similar chemical process for immobilization of aptamers on the surface. Progress was made toward a rugged, lower-power, compact, multipurpose device array.

${ }^{1}$ sawyersm@nv.doe.gov, 805-681-2262; sawyes@rpi.edu, 518-276-2164

${ }^{\text {a }}$ Special Technologies Laboratory; ${ }^{\text {b }}$ Rensselaer Polytechnic Institute

\section{Background}

The FY 2010 investigation of colloidal nanoparticleenhanced, UV photodetectors presented the potential for a hybrid material sensor. The objective was to directly couple scintillator light into a visible blind, UV nanoparticle detector by multilayer deposition of materials with different functions (Sawyer 2011). In FY 2011, a new functional layer was introduced: aptamers, as a biorecognition element used to achieve selective biosensing (Sawyer Armand 2012). The intended biosensor will detect intrinsic fluorescence for signal transduction in the UV-blue range of the electromagnetic spectrum. Specific wavelengths within this region correspond to intrinsic aptamer fluorescence peaks that can be used for biodetection, as shown in Figure 1.

Biorecognition elements with high specificity such as antibodies or enzymes have dominated applications in biomedical research and medical point-of-care systems. DNA and RNA aptamers are engineered to bind to various molecular targets by repeated rounds of in vitro selection, otherwise known as the SELEX [systematic evolution of ligands by exponential enrichment] process. They provide important

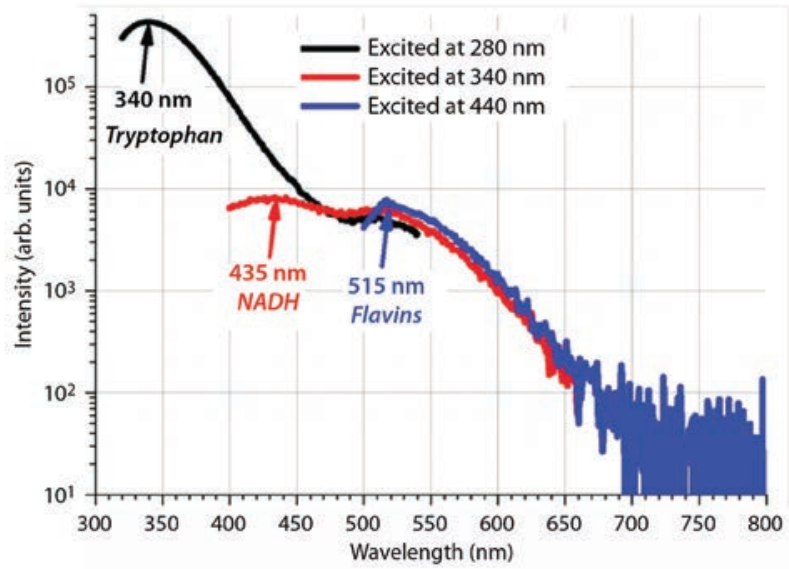

Figure 1. Intrinsic fluorescence emission spectrum of Enterobacter aerogenous when excited at specific wavelengths (Sawyer Armand 2012)

advantages over antibodies (Que-Gewirth 2007, Dollins 2008, Song 2008, Han 2010), including:

- A markedly broadened range of potential targets, demonstrating high affinity to both microand macromolecules, beyond capabilities of antibodies. 
- Bulk synthesis by chemical processes, which significantly reduces the cost of fabrication.

- The ability to be created in the exact environment of its operational conditions.

- Ease of chemical modifications, creating unique biosensing opportunities for probes, quenchers, fluorophores, etc.

- Greater stability at elevated temperatures.

- The ability to be regenerated and reused by chemical or thermal denaturation, improving longevity and reducing cost.

- The ability to interact with other DNA or RNA molecules, leading to hybridization of structures with differing functionality.

Ultimately, a microbial biosensor can be created using an aptamer specifically created for a target such as Escherichia coli (E. coli) and then verified by its tryptophan, nicotinamide adenine dinucleotide (NADH), or flavin intrinsic fluorescence response.

Low-dimensional, metal-oxide semiconductor nanomaterials have stimulated great interest and extensive research due to their novel electronic and optical properties in addition to their biocompatibility. Specifically, colloidal nanoparticles and nanowires are attractive as they take advantage of quantum confinement effects, are easy to fabricate, have a high surface-to-volume ratio, and are relatively low cost. Furthermore, our research to create metalsemiconductor-metal (MSM) devices demonstrated that the current carrying mechanism creates a high internal photoconductive gain due to the presence of oxygen-related hole-trap states at the nanoparticle's surface and the high resolution of interdigitated electrodes (Qin “Comparison" 2010, Qin “Metalsemiconductor-metal” 2010, Qin 2011, Qin 2012, Sawyer 2011, Shao 2011, Shao "Optical" 2012, Shao "High Responsivity" 2012). Recently, detectors using zinc oxide $(\mathrm{ZnO})$ nanowires were demonstrated (Shao "Heterojunction" 2012).
The material results presented here include three metal oxide structures: indium oxide $\left(\mathrm{In}_{2} \mathrm{O}_{3}\right)$ nanoparticles, $\mathrm{ZnO}$ nanoparticles, and $\mathrm{ZnO}$ nanowires. $\mathrm{In}_{2} \mathrm{O}_{3}$ material was chosen for its band gap properties that correspond with the detection of NADH intrinsic fluorescence from potential biohazards. It is a wideband semiconductor material with a documented band gap value of $2.9 \mathrm{eV}$. $\mathrm{ZnO}$ is best suited for tryptophan intrinsic fluorescence detection in the $\mathrm{UV}$ range. $\mathrm{ZnO}$ is a direct, wide band gap semiconductor material (documented values range from 3.2 to $3.4 \mathrm{eV}$ ). These materials fill a significant niche where inexpensive and traditionally used photomultiplier tubes (PMTs) and silicon (Si)-based photodetectors show a marked reduction in responsivity. Nanowires were investigated as nanostructures bound to the substrate. The nanowires will not be removed if a washing procedure is used (part of the experiment's validation process). To our knowledge, the immobilization of aptamers using this surface modification method with the above nanostructures for use in optical detection has not been investigated.

\section{Project}

In this project, the process to select the biorecognition layer included the following steps: (1) identify an aptamer sequence for an E. coli target, (2) investigate surface immobilization approaches for flat surfaces and nanostructures for comparison, and (3) characterize aptamer binding.

\section{Biorecognition Layer Selection and Immobilization}

In FY 2011, as a proof-of-concept, the chemical tryptophan was chosen as the target. A similar immobilization process was applied to the final target, E. coli. The aptamer sequence was found through a literature search. The following sequence for $E$. coli was obtained from Professor Sung-Kun Kim at Baylor University for our experiments (Shipley 2010): 
5- /5ThioMC6-D/GGC GAC CAA ACC AAT CAC GAC GTC AATTGG CGATGC CTA C -3

Two versions of the above aptamer sequence were ordered from Integrated DNA Technologies, Inc. The first was terminated only by a thiol molecule, leaving the other end of the aptamer for binding to $E$. coli. The second was terminated by a fluorescent aptamer molecule 6-FAM (6-carboxyfluorescein) and used as a preliminary binding and characterization step. The expected emission from 6-FAM is at $520 \mathrm{~nm}$ under $495 \mathrm{~nm}$ excitation. The surface modification process for thiol/silica can be found in the FY 2011 SDRD report (Sawyer Armand 2012).

E. coli aptamer binding was measured using a spectrofluorimeter Spex Fluorolog Tau-3 (HORIBA Jobin Yvon, Inc.) or an LED-based setup in which the sample was excited by a $280 \mathrm{~nm}$ LED and detected by a band-pass filtered ( $340 \mathrm{~nm}$ and $20 \mathrm{~nm}$ FWHM) photon-counting PMT. On flat surfaces, only a small peak from the expected fluorescence appeared in each experiment, indicating that the concentration of aptamers bound to the surface is very small. The marginal success of binding aptamers to a flat substrate indicated a need to increase the surface area for binding sites by using nanostructures.

(a) Flat surface: ITO-coated glass processed for FAM thiol

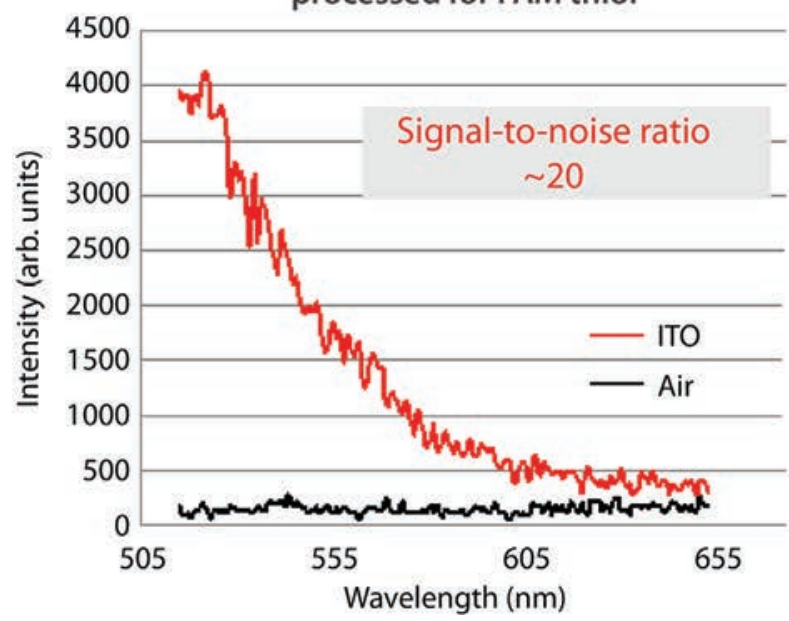

ITO-coated glass was modified using the same chemical protocol as $\ln _{2} \mathrm{O}_{3}$ nanoparticles. The aptamer used in Figure 2 was designed for tryptophan with a FAM-terminating molecule. The E. coli aptamer sequence was used on $\ln _{2} \mathrm{O}_{3}$ nanoparticles using the same procedure. This aptamer terminated with FAM showed similar results, with a signal-to-noise ratio (SNR) of the same order of magnitude.

Next, the E. coli aptamer was tested with the intended target. Though a large signal was found, the results were inconclusive. The results could not confirm whether the signal was caused by $E$. coli residue lying on the surface of the sensor or by E. coli bound to the aptamer. At this time we added a step to the process: simply washing the sample at the end of the protocol. This wash process addressed the issue, but it removed the nanoparticles from the surface. For this reason, we sought a nanostructure material that was bound to the substrate. Nanowires are difficult to make with $\mathrm{In}_{2} \mathrm{O}_{3}$; however, $\mathrm{ZnO}$ nanowires (Figure 3 ) were readily available and part of an ongoing investigation of another research project at Rensselaer Polytechnic Institute (Shao "Heterodyne" 2012).

(b) $\mathrm{In}_{2} \mathrm{O}_{3}$ nanoparticles processed for FAM thiol aptamer, then spin-coated on quartz

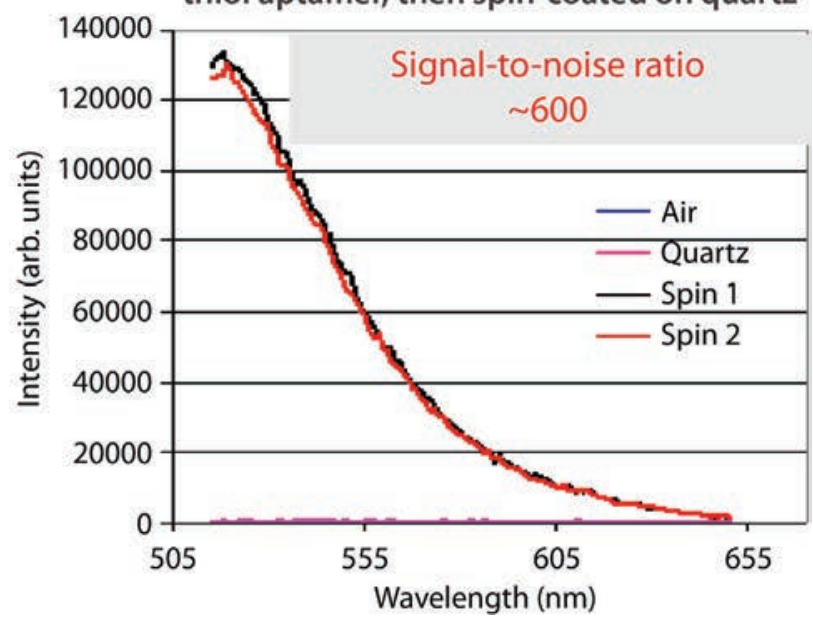

Figure 2. (a) The signal-to-noise ratio (SNR) on an indium oxide doped with tin (ITO) flat surface was 20 , and (b) was 600 on $\ln _{2} \mathrm{O}_{3}$ nanoparticles 

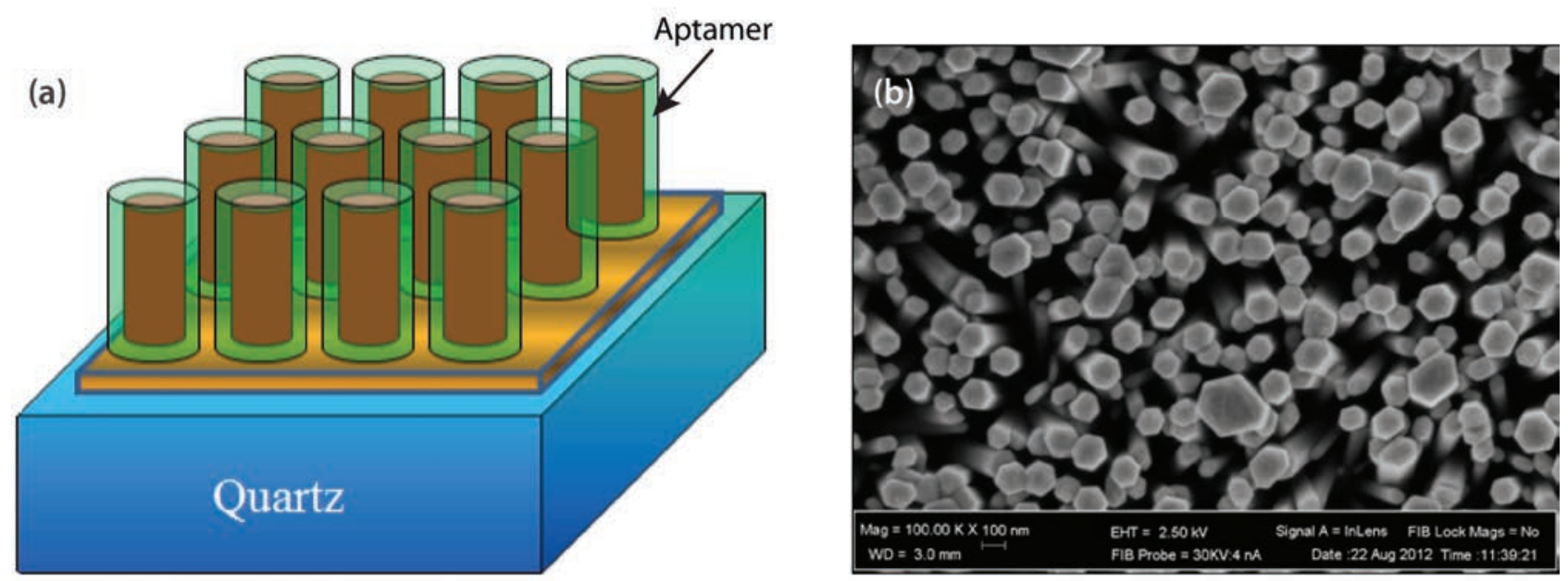

Figure 3. (a) Schematic of $\mathrm{ZnO}$ nanowires with bound aptamers on a quartz substrate; and (b) a scanning electron microscope image of ZnO nanowires (Shao “Heterodyne" 2012)

Notice in Figure 4 that the expected peaks $(340 \mathrm{~nm}$ and $440 \mathrm{~nm}$ ) for the washed slides (blue lines) are either greatly diminished or gone. Furthermore, both EA and EC fluorescence was detected. This led to the conclusion that the bacteria may only lie on the surface.

The deposition method for ITO-based substrates was attempted for $\mathrm{ZnO}$ nanoparticles and nanowires. These nanoparticles demonstrate a similar SNR to that of $\operatorname{In}_{2} \mathrm{O}_{3}$, but the $\mathrm{ZnO}$ nanowires showed improvement over nanoparticles by six times.

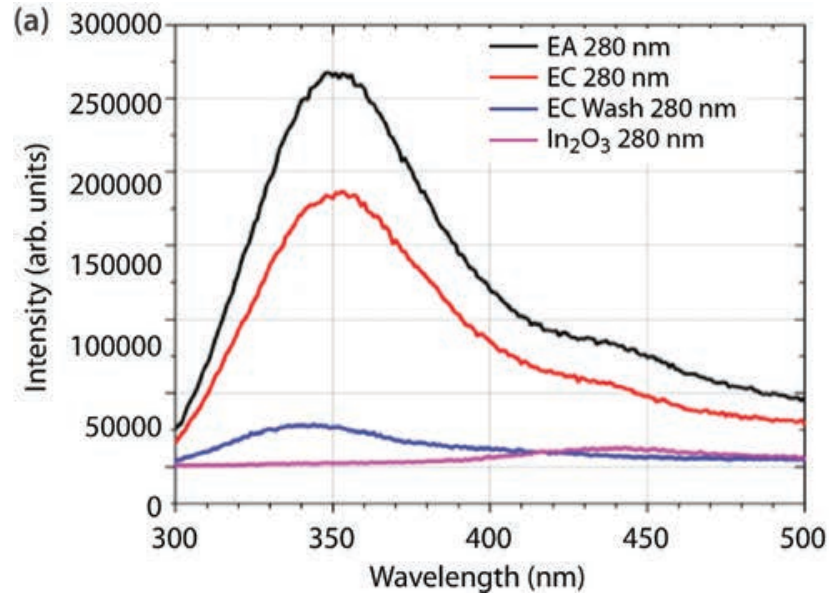

Furthermore, the surface modification procedure was changed to exclude sonication of the sample, which further loosens particles from the surface. As a result, the fluorescent response increased by five times, which also supports our assumption of reduced fluorescence due to removed nanoparticles.

The fluorescence response (Figure 5) using ZnO nanowires is six times that of $\mathrm{ZnO}$ nanoparticles. The peak of the reference sample at $531 \mathrm{~nm}$ is due to the common parasitic photoluminescence due to defects in $\mathrm{ZnO}$.

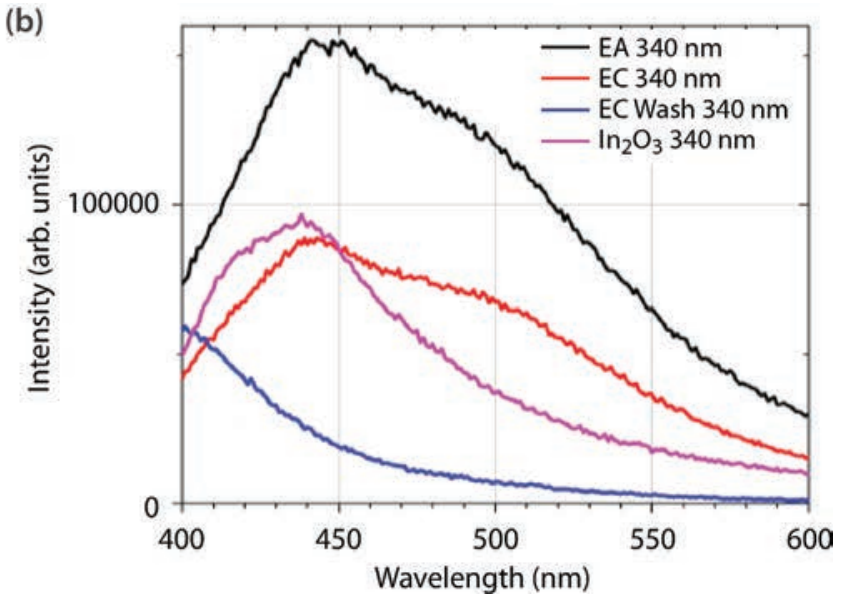

Figure 4. (a) Tryptophan intrinsic fluorescence of E. coli (EC) and Enterobacter aerogenous (EA) with $280 \mathrm{~nm}$ excitation before and after washing. (b) NADH intrinsic fluorescence of E. coli (EC) and Enterobacter aerogenous (EA) with $280 \mathrm{~nm}$ excitation before washing and after washing. 


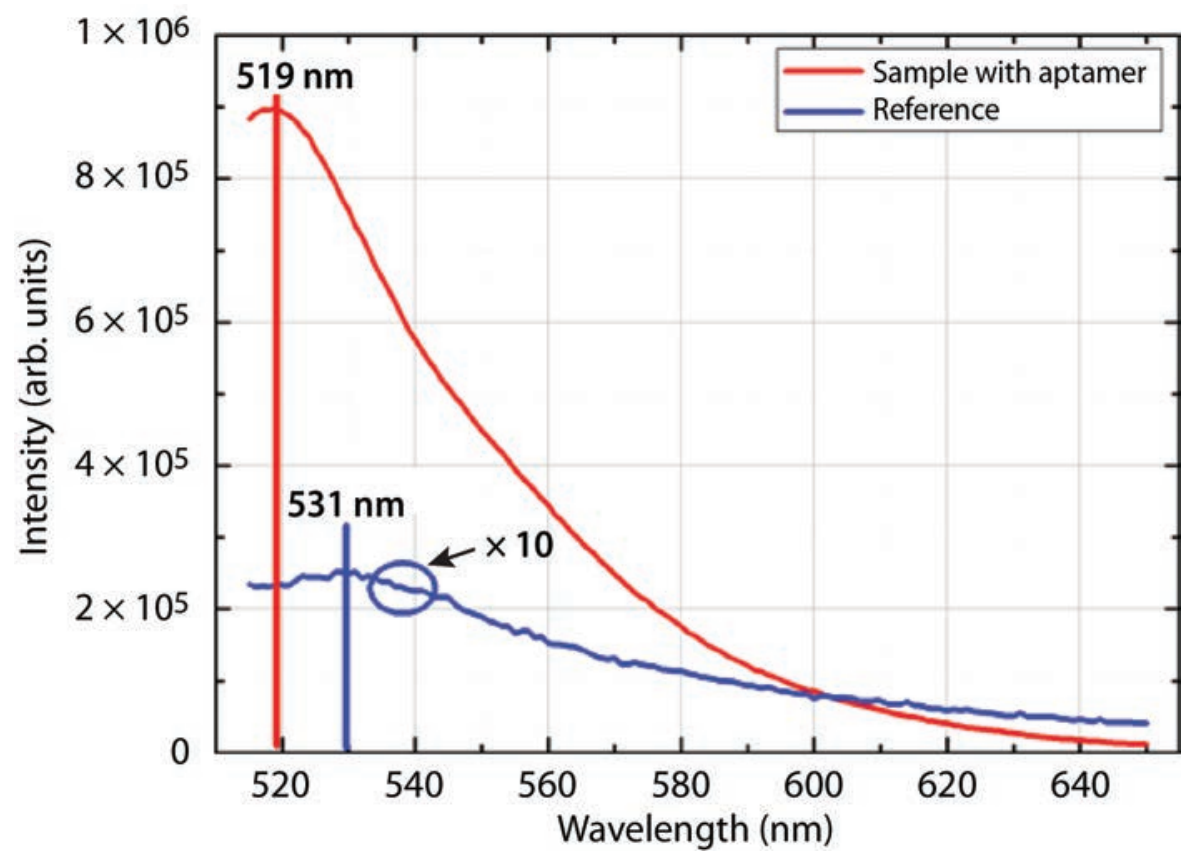

Figure 5. The fluorescent response of FAM-terminated aptamers conjugated to $\mathrm{ZnO}$ nanowires is six times that of ZnO nanoparticles

\section{Conclusion}

Three different metal oxide nanostructures were used to increase the binding surface area for aptamers. The results show that both $\mathrm{ZnO}$ and $\ln _{2} \mathrm{O}_{3}$ can be used with a similar chemical process for immobilization of aptamers on the surface. Work that will be performed in the investigator's lab includes measurement and comparisons of the $E$. coli aptamer with the intended target. A nanostructurebased device will be deposited on the other side of the sample substrate, making a complete, compact biosensor.

\section{Acknowledgments}

We would like to thank Professor Partha Dutta, Professor Linda McGown, and Professor Jie Lian, and the Connection One National Science Foundationfunded Industry/University Cooperative Research Center.

\section{References}

Dollins, C. M., S. Nair, B. Sullenger, "Aptamers in immunotherapy," Hum. Gene Ther. 19, 5 (May 2008) 443-450.
Han, K., Z. Liang, N. Zhou, "Design strategies for aptamerbased biosensors," Sensors 10, 5 (May 2010) 4541-4557.

Qin, L., C. Shing, S. Sawyer, "Comparison of UV optical properties of ZnO nanoparticles dispersed in traditional organic and novel bio-molecular solvents," Physica Status Solidi C 7, 10 (June 2010) 2463-2466.

Qin, L., C. Shing, S. Sawyer, "Metal-semiconductor-metal ultraviolet photodetectors based on zinc-oxide colloidal nanoparticles," IEEE Electron Device Lett. 32, 1 (December 2010) 51-53.

Qin, L., C. Shing, S. Sawyer, "Low-pass and band-pass alternative ultraviolet photoconductor based on zinc oxide nanoparticles on intrinsic gallium nitride-based substrate," IEEE Photon. Technol. Lett. 23, 7 (January 2011) 414-416.

Qin, L., P. Dutta, S. Sawyer "Photoresponse of indium oxide particulate-based thin films fabricated using milled nanorods grown by the self-catalytic vapor-liquid-solid process," Semicond. Sci. Technol. 27 (February 2012) 045005-1-045005-6.

Que-Gewirth, N. S., B. A. Sullenger, "Gene therapy progress and prospects: RNA aptamers," Gene Ther. 14 (February 2007) 283-291. 
Sawyer, S., L. Qin, C. Shing, "Zinc oxide nanoparticles for ultraviolet photodetection," International Journal of High Speed Electronics and Systems 20, 1 (2011) 183-194.

Sawyer Armand, S., I. Barash, S. Halperin, K. Kyle, K. Mathieu, L. Qin, C. Shing, S. Weeks, "Nanoparticlebased analytical biosensor," Site-Directed Research and Development, FY 2011, National Security Technologies, LLC, Las Vegas, Nevada, 2012, 169-180.

Shao, D., L. Qin, S. Sawyer, "High responsivity, bandpass near-UV photodetector fabricated from PVA- $\mathrm{In}_{2} \mathrm{O}_{3}$ nanoparticles on a GaN substrate," IEEE Photon. J. 4, 3 (June 2012) 715-720.

Shao, D., L. Qin, S. Sawyer, "Near ultraviolet photodetector fabricated from PVA coated $\ln _{2} \mathrm{O}_{3}$ nanoparticles," Appl. Surf. Sci. 261 (November 2012) 123-127.

Shao, D., M. Yu, J. Lian, S. Sawyer, "Heterojunction photodiode fabricated from hydrogen treated $\mathrm{ZnO}$ nanowires grown on p-silicon substrate," Appl. Phys. Lett. 101, 21 (November 2012) 211103.

Shao, D., L. Qin, S. Sawyer, "Optical properties of polyvinyl alcohol (PVA) coated $\ln _{2} \mathrm{O}_{3}$ nanoparticles," Optical Materials 35 (January 2013) 563-566.

Shipley, S. L., E. White, S.-K. Kim, "Selection of aptamers against live E.coli cells using cell SELEX," FASEB J. 24, Meeting Abstracts (April 2010) 907.14.

Song, S., L. Wang, J. Li, J. Zhao, C. Fan, "Aptamer-based biosensors," Trends Anal. Chem. 27, 2 (February 2008) 108-117. 


\title{
PASSIVE IMAGING OF WARHEAD-LIKE CONFIGURATIONS WITH COSMIC-RAY MUON TRACKING SCANNERS
}

\author{
LAO-17-12 | CONTINUED FROM FY 2011 | YEAR 2 OF 2
}

David Schwellenbach, ${ }^{1, a}$ Derek Aberle, ${ }^{a}$ Jeffrey Bacon, ${ }^{b}$ Konstantin N. Borozdin, ${ }^{b}$ Wendi Dreesen, ${ }^{a}$ J. Andrew Green, ${ }^{c}$ Steven Greene, ${ }^{b}$ George Glen McDuff, ${ }^{d}$ Christopher Morris, ${ }^{b}$ John Perry, ${ }^{b}$ Adam Scott, ${ }^{b}$ and Michael Sossong ${ }^{e}$

Experiments to develop an imaging system for warheads or warhead-like configurations based on cosmic-ray muon counting and tracking along with gamma and neutron measurement capability were conducted. The proposed technology offers an innovative, efficient, portable, robust, and inexpensive solution as a passive imaging system. The project reconfigured an existing muon detector system and developed algorithms specifically to scan for special nuclear material (SNM) in deployed configurations for applications such as treaty verification. Experiments have shown conclusively that passive cosmic-ray muon tracking technology can detect and image SNM in warhead-like configurations in both tomographic and telescopic modes.

${ }^{1}$ schweldd@nv.doe.gov, 505-663-2017

a Los Alamos Operations; ${ }^{\mathrm{b}}$ Los Alamos National Laboratory ${ }^{\mathrm{c}}$ Remote Sensing Laboratory-Nellis; ${ }^{\mathrm{d}}$ Keystone International, Inc.;

e Decision Sciences International Corporation

\section{Background}

A new, passive imaging technology was previously developed by Los Alamos National Laboratory (LANL) and commercially by Decision Sciences International Corporation (Decision Sciences) that utilizes cosmicray muons to detect special nuclear material (SNM) in shielded configurations such as cargo containers. This is the second year of a project designed to adapt this technology to warhead monitoring and treaty verification applications. High-energy protons bombarding our atmosphere collide with other particles, creating showers that decay into many different particles, including muons (Figure 1). At sea level the muon flux is approximately $10,000 / \mathrm{min} / \mathrm{m}^{2}$. Muons have a mean energy of about $3 \mathrm{GeV}$, which means that they have an effective lifetime of $\nu \tau=(28) \times(2.2 \mu \mathrm{s})=61.6 \mu \mathrm{s}$ and are highly penetrating. The distribution of these particles is roughly $\cos ^{2}(\theta), \theta$ from zenith, with $55 \%$ of the muons positively charged $\left(\mu^{+}\right)$and $45 \%$ negatively charged $\left(\mu^{-}\right)$. A muon's properties make it a great candidate for passive imaging for several reasons. Muons are similar to electrons but are 200 times more massive $\left(m_{\mu}=105 \mathrm{MeV}\right)$. Unlike protons and neutrons, muons are subject only to weak and electromagnetic forces. At high energies, the muon is highly penetrating through matter because of its high mass. After scattering has reduced the energy, muons can replace electrons in an atom, forming muonic atoms resulting in high-energy x-rays. Muon-induced fission is an event that occurs when a negatively charged muon $\left(\mu^{-}\right)$is captured by the nucleus of an atom; these negatively charged muons are of great interest in this project.

In general, two modes of operation can be used for cosmic-ray muon tracking and imaging: tomographic and telescopic. Tomographic mode relies on multiple coulomb scattering, and has been shown to detect and image high-Z material in shielded configurations. Tomographic mode requires detectors above and below the object of interest, because it tracks incoming and outgoing scattered muons. In the first 


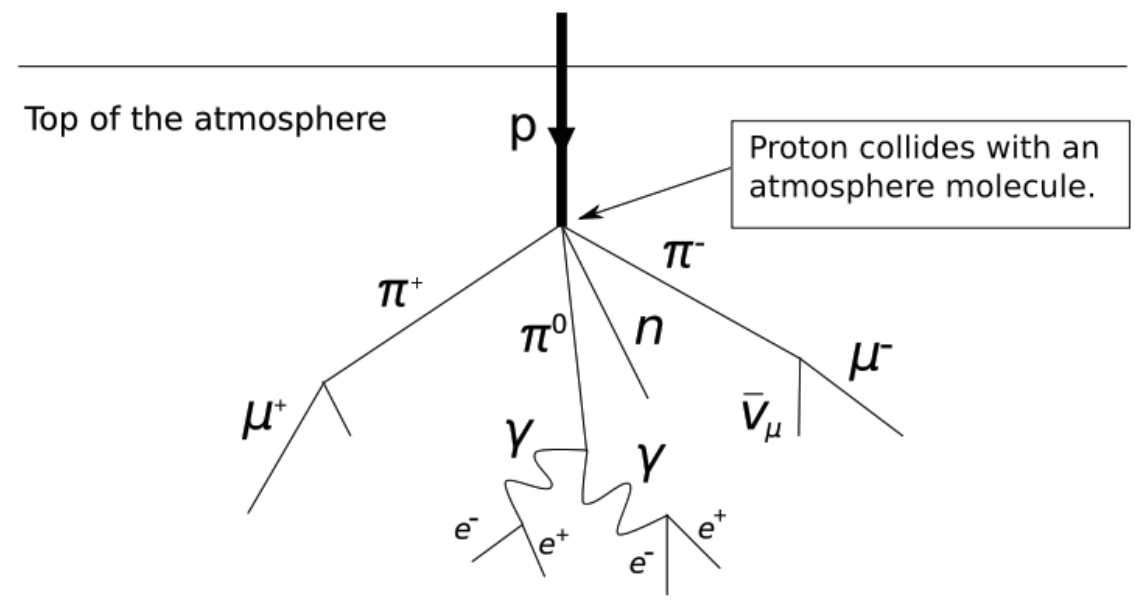

Figure 1. Decay tree for an inbound cosmic ray entering the atmosphere

year of this project (Schwellenbach 2012), it was shown that imaging dense materials from one side was feasible through stopped-track imaging. Stoppedtrack imaging requires that muons be tracked into and out of a scene, such as in tomographic mode; however, incoming muons that cannot be correlated with an outgoing muon are used to generate images. Earlier proof-of-concept experiments (Borozdin 2010) indicate that cosmic-ray muons will induce fission in high-Z materials, leading to emission of secondary particles (fission neutrons and gamma rays) that are in time coincidence with stopped muons. Using these neutrons along with the stopped muon tracks, the telescopic mode of muon imaging can be developed for a variety of warhead monitoring and standoff detection applications. Telescopic mode tracks the incoming muons that are seen in coincidence with gamma rays or neutrons, which are a signature of the muon-induced fission. Because of this ability, the telescopic mode can be very sensitive to SNM and requires only single-sided detectors.

The New START treaty limits the Russian and U.S. nuclear arsenals to a maximum of 1,550 nuclear warheads, down from the current ceiling of 2,200. With this decrease in the number of allowable warheads, the approach for confirmation of warhead numbers becomes more important, dictating the development of new methods for warhead counting. The existing process for inspection is intrusive and requires considerable handling and protection of sensitive/classified material, while providing only limited inspector transparency; some handling is done out of view of the inspectors. Additionally, future treaties will require an enhanced ability to monitor or count warheads. Treaty requirements include minimal intrusiveness on operations, shareable technology, and information barriers (intrinsic or built-in) that provide confidence to all parties that warheads are being counted without revealing sensitive or classified information. These requirements also imply the need for transparency and simplicity of data collection and processing methods to minimize hidden weapons capabilities. Systems that can count warheads in situ are particularly needed, primarily in storage containers at storage sites. X-ray radiography (Mozley 1990) and fast-neutron counting (Ewing 1990, Byrd 1992) have been proposed to address this need, as well as neutron (Zalyubovskii 1993) or gamma-ray interrogation. So far these methods have not been adopted as part of standard treaty verification protocol because they are too invasive or unreliable in the presence of variable background signals. Muon tracking technology satisfies the requirements, as it is based on nonintrusive imaging of the warheads, enabling their reliable identification with low sensitivity to background.

Potential applications of muon imaging include vehicle-mounted detectors that could look for buried SNM or SNM hidden inside buildings; detectors that could monitor stored fuel rods outside of containment vessels or even externally to the storage facility; and monitoring of transportation systems (ships, 


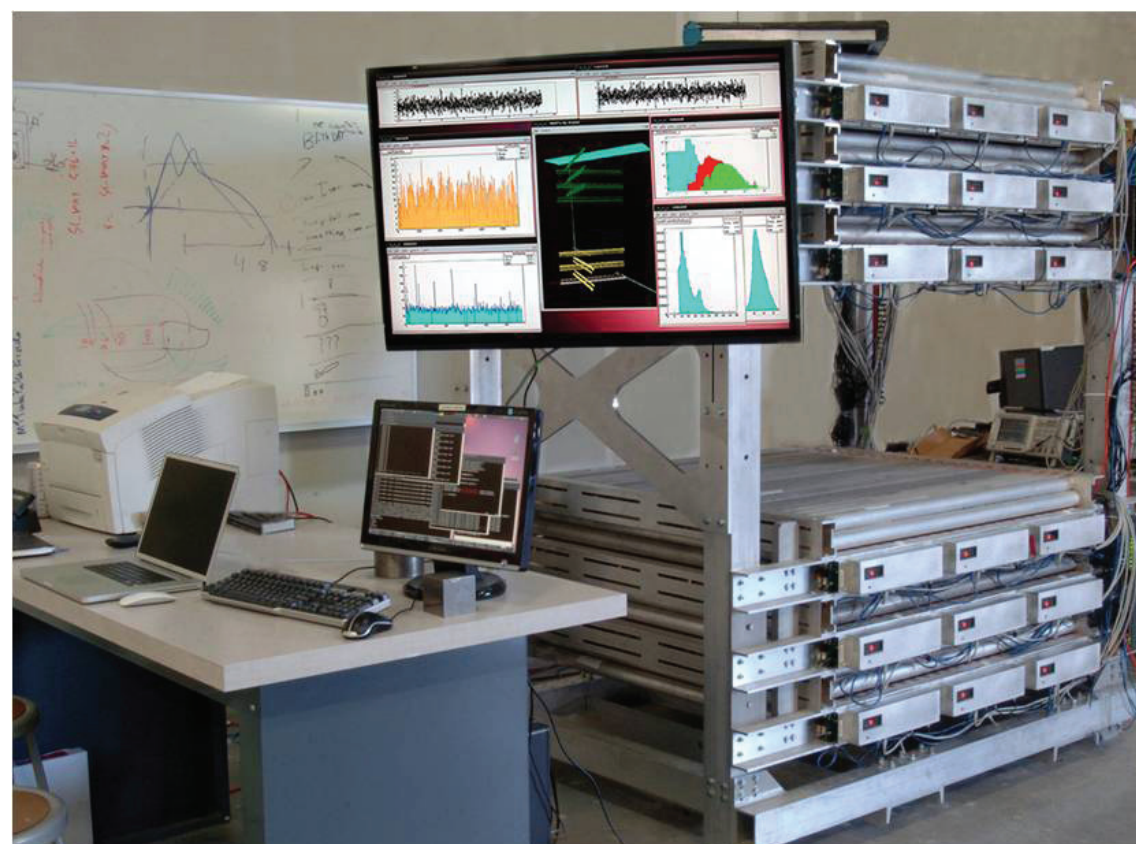

Figure 2. The mini muon tracker (MMT) was built at Los Alamos for muon tomography applications. There are 24 planes of 4 -foot long drift tubes to track muons in $x$ and $y$ directions.

trains, and trucks), without exposure or knowledge by the occupants, to detect movement of SNM. Only one-sided access to the object is required to identify fissile material, but two-sided access enables faster detection and higher-resolution imaging.

\section{Project}

This project's goal was to prove concepts toward the development of advanced imaging systems using cosmic-ray muons designed for applications such as warhead monitoring and standoff detection. Muon tomography has been developed at LANL to address the problem of nuclear smuggling (Borozdin 2003, Morris 2008). LANL has constructed several muon tracking detectors; the most recent detector is the Mini Muon Tracker (MMT), shown in Figure 2, which was used in this project.

\section{Advancements in Tomographic Mode}

In terms of material detection, research prior to this SDRD has emphasized categorizing materials to be low, medium, or high Z, where the latter is consistent with SNM or shielded SNM. Simple categorization of material shortens the scan time, which is important for an application such as monitoring a border crossing. Longer scan times give the additional data

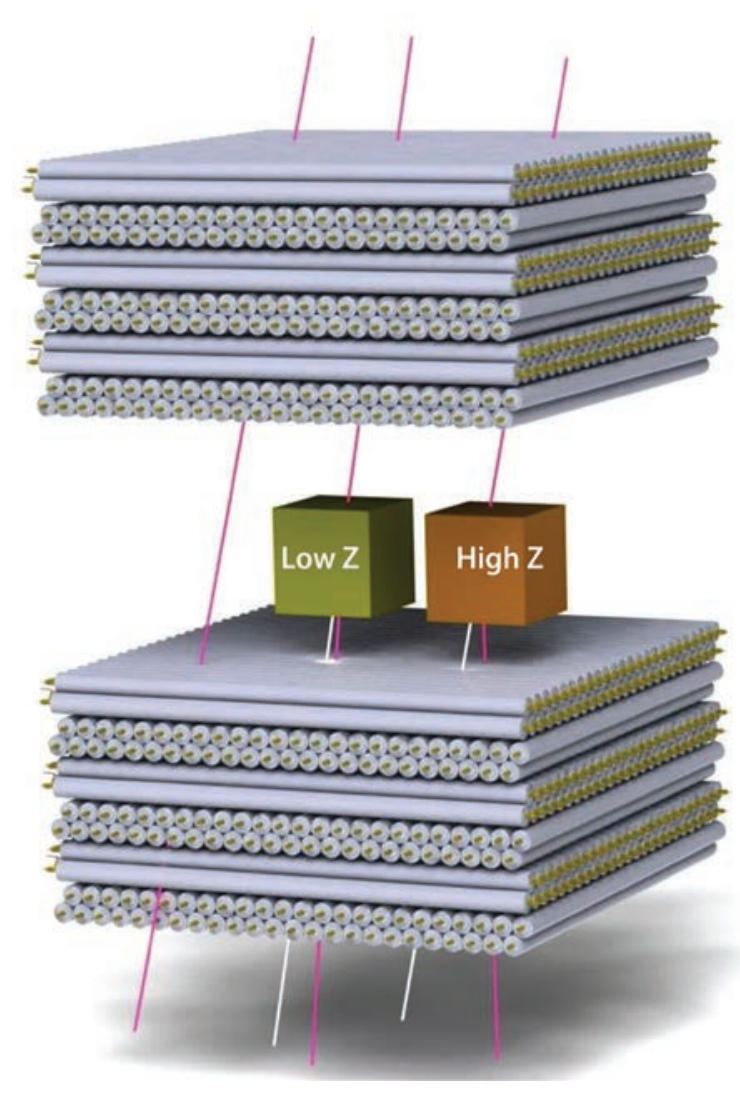

Figure 3. Concept of imaging in tomographic mode: muons are tracked both into and out of a scene with higher deflection being measured for high-Z materials. The magenta lines indicate the tracked muon trajectories while the white lines are a projection of an undeflected muon path. 

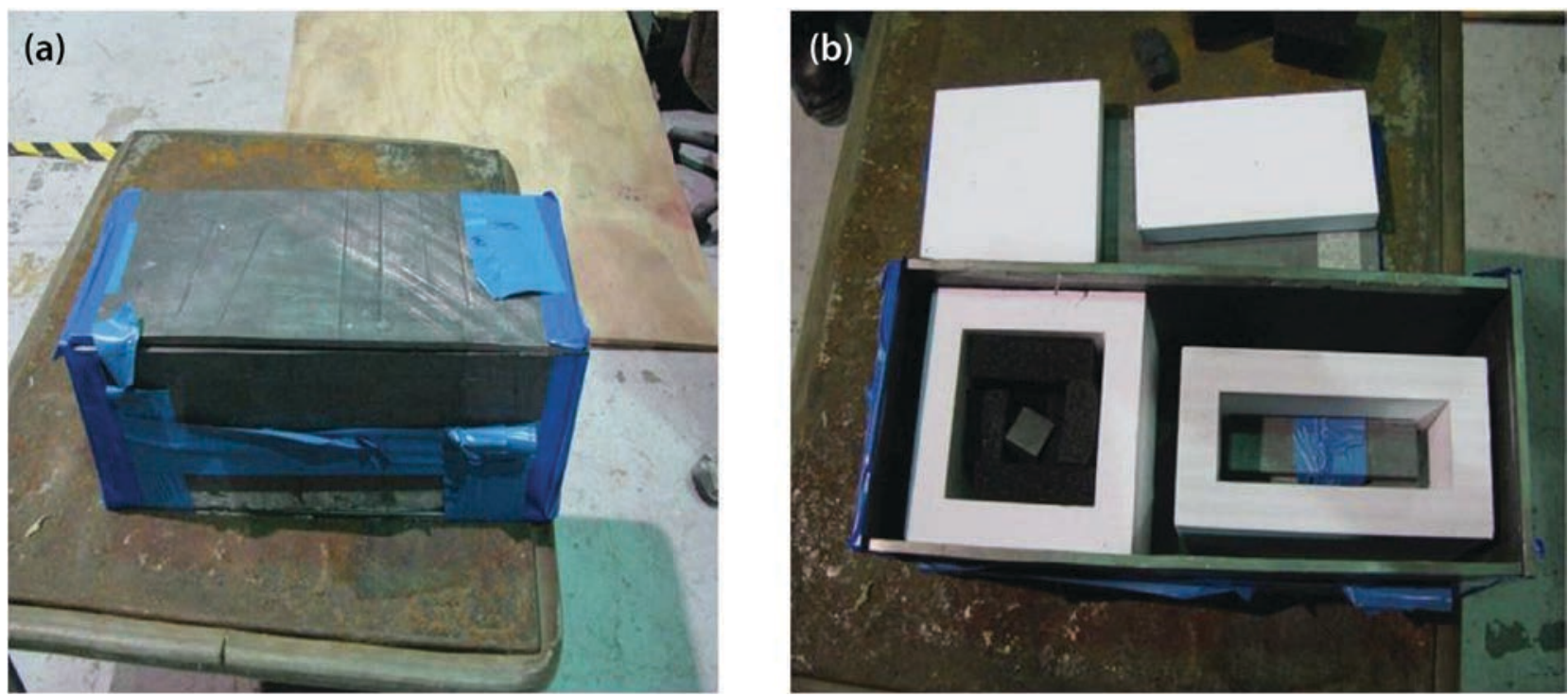

Figure 4. (a) The shield box is made of 0.25 -inch lead used to conceal objects. (b) The compartment on the left contains a 1 -inch cube of tungsten, and the compartment on the right contains a $5 " \times 1.75^{\prime \prime} \times 0.5$ " lead sample.

needed for warhead monitoring, allowing shape recognition through 3-D reconstructions based on the muon scattering angles through materials (Figure 3). For this project, we developed algorithms that allow for real-time 3-D imaging of materials of interest as well as for higher-resolution, post-processed imaging.

To test the algorithms, we placed materials of various sizes inside a shield box (Figure 4) having 0.25 -inch lead walls designed to conceal the materials, and then imaged the box. Tomographic images shown in Figure 5 reveal the multiple shielded shapes detected. This test setup was also used for proof-of-concept telescopic imaging tests.

The algorithms and experimental methods developed using the shield box were applied to the imaging of objects of interest. Successful imaging has been documented in a separate report (Schwellenbach 2013).

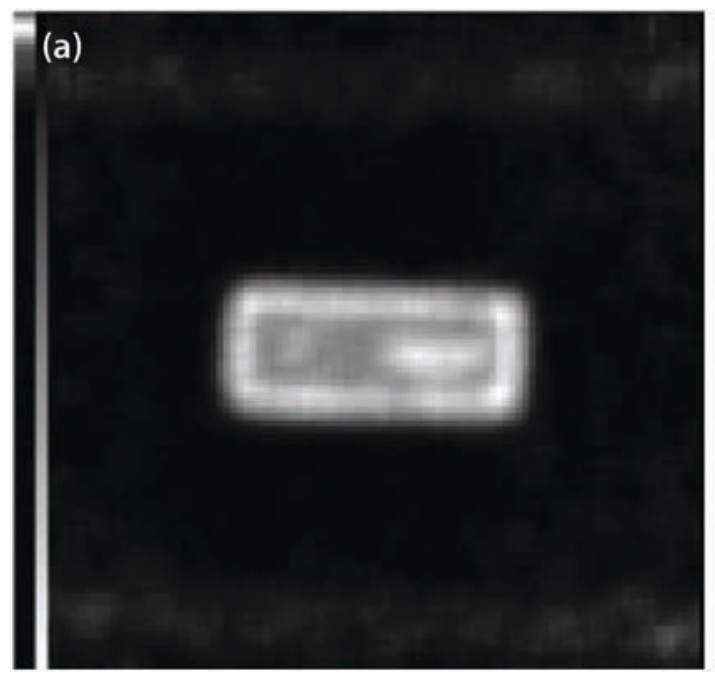

(b)

Figure 5. Tomographic images of the shield box reveal multiple objects. Shown are (a) the results of real-time imaging of $\sim 1$ hour of data and (b) a post-processed, single Z-slice image demonstrating the system's high-resolution capability. 


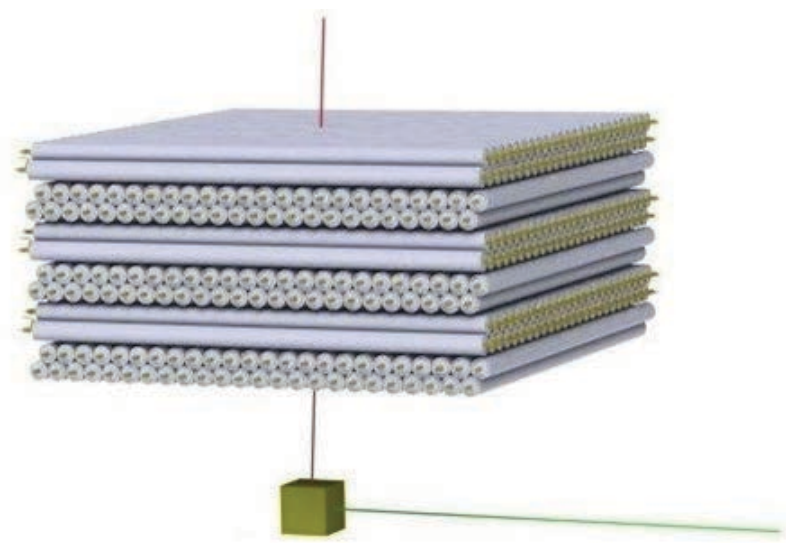

Figure 6. In telescopic mode imaging, the muon is tracked from the top (red line) and used for imaging if it is seen in coincidence with a fission neutron. The green line shows an outgoing neutron/gamma detected by the system.

\section{Telescopic Mode}

In tomographic mode, the tracking system detects the muon path before and after it passes through the object. This mode is only possible when the scanned object can be placed between two muon tracking detectors. This geometry provides the most expedient 3-D information available, but using this method is not always practical. Telescopic mode tracks incoming muons before interacting with the object and then correlates with muon-induced fission neutrons or gammas in time coincidence. Figure 6 shows the detector scenario in telescopic mode. After several muon tracks produce coincident fission neutrons/ gammas, sufficient information exists to locate a SNM candidate. Longer integration times improve statistics and enable us to image the SNM.

Before demonstrating telescopic mode imaging, stopped-track imaging experiments were performed to verify that one-sided imaging was feasible.

Stopped-track imaging is like tomographic imaging except that it keeps only incoming tracks that lack an associated outgoing track. These selected incoming tracks, seen on the top detector, do not have a corresponding outgoing track at the bottom of the detector. These stopped tracks are candidates for muon-induced fission. The image shown in Figure 7 shows a stopped-track image of a tungsten cube and a lead bar concealed by the shield box. This image demonstrates that one-sided imaging is feasible, and it gives confidence that images can be produced in telescopic mode.

Having validated the concept of one-sided imaging, the MMT was modified by adding various types of neutron detectors. These detectors allowed us to simultaneously track cosmic-ray muons into objects and detect muon-induced fission neutrons for telescopic mode studies. We saved the muon data in event mode, which preserves geometric information along with a time stamp for each event, and

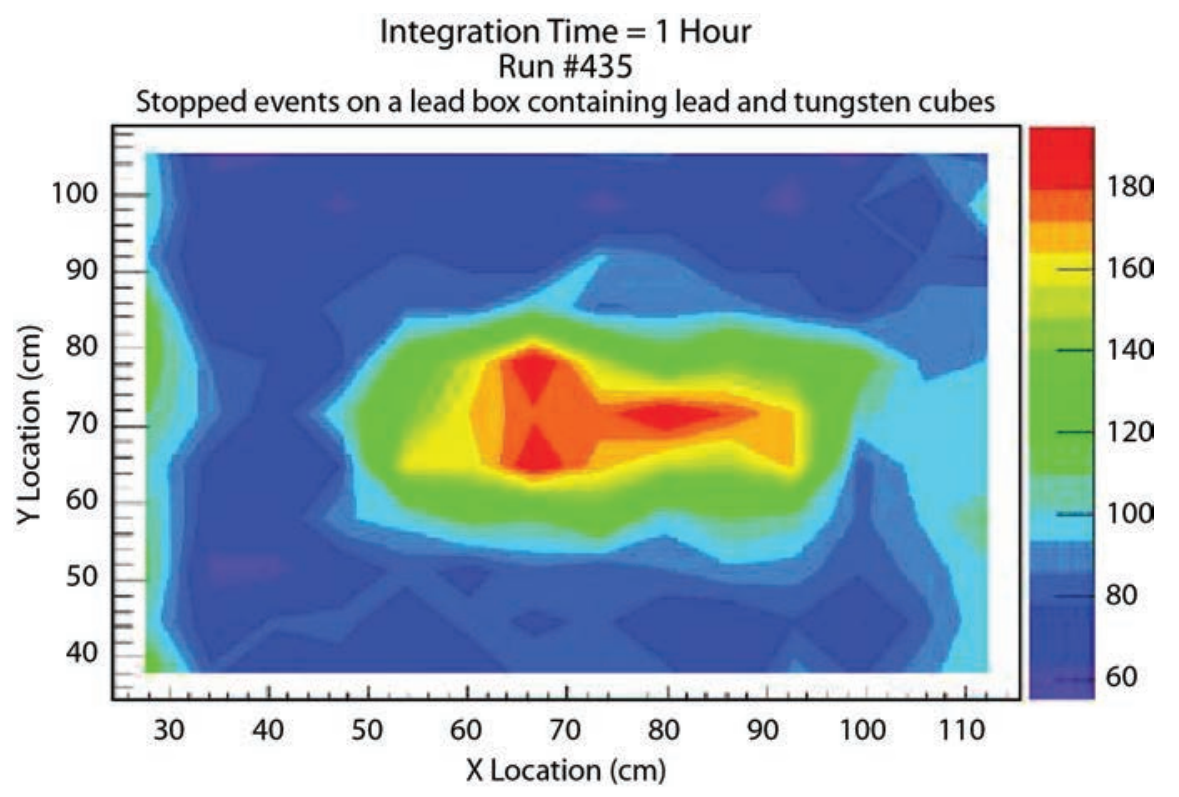

Figure 7. Stopped-track image of the shield box showing the ability to estimate the location of objects with low resolution using muons from only one side 
saved neutron event timestamps. Using various time correlation windows, we studied the resulting muon induced fission images.

${ }^{3} \mathrm{He},{ }^{4} \mathrm{He}$, and liquid scintillator (Eljen EJ301) neutron detectors were used simultaneously to detect the fission neutrons from an object. The hardware and software developed in this project allowed imaging based on coincidence on any or all of the detector channels. Real-time telescopic mode imaging was successfully performed on objects of interest (Schwellenbach 2013), demonstrating conclusively that telescopic mode imaging can be performed and has promise for treaty verification applications.

\section{Conclusion}

Cosmic-ray muon imaging has shown promise in applications such as scanning of cargo containers and other border security applications. This project demonstrated conclusively that tomographic and telescopic modes of imaging using cosmic-ray muons are feasible for applications in treaty verifications and related fields. Continued improvements to the software will be required to tailor this technology to the specific needs of treaty verification. Small area neutron detectors were used in this study, and larger detectors will need to be used in similar experiments to determine standoff detection capabilities of telescopic mode muon imaging. Further work is required to demonstrate that the techniques established here can be applied to fieldable detector configurations for treaty verification.

\section{References}

Borozdin, K. N., G. E. Hogan, C. Morris, W. C. Priedhorsky, A. Saunders, L. J. Schultz, M. E. Teasdale, "Surveillance: Radiographic imaging with cosmic-ray muons," Nature 422 (2003) 277.

Borozdin, K. N., C. Morris, A. V. Klimenko, R. Spaulding, J. Bacon, "Passive imaging of SNM with cosmic-ray generated neutrons and gamma-rays," IEEE Nucl. Sci. Symp. Conf. Rec. R05-70 (2010) 3864-3867.
Byrd, R. C., G. F. Auchampaugh, C. E. Moss, W. C. Feldman, "Warhead counting using neutron scintillators: Detector development, testing, and demonstration," IEEE Trans. Nucl. Sci. 39, 4 (1992) 1051-1055.

Ewing, R. I., K. W. Marlow, "A fast-neutron detector used in verification of the INF Treaty," Nucl. Instrum. Methods Phys. A 299 (1990) 559-561.

Morris, C. L., C. C. Alexander, J. D. Bacon, K. N. Borozdin, D. J. Clark, R. Chartrand, C. J. Espinoza, A. M. Fraser, M. C. Galassi, J. A. Green, J. S. Gonzales, J. J. Gomez, N. W. Hengartner, G. E. Hogan, A. V. Klimenko, M. F. Makela, P. McGaughey, J. J. Medina, F. E. Pazuchanics, W. C. Priedhorsky, J. C. Ramsey, A. Saunders, R. C. Schirato, L. J. Schultz, M. J. Sossong, G. S. Blanpied, "Tomographic imaging with cosmic ray muons," Science \& Global Security 16 (2008) 37-53.

Mozley, R., "Verifying the number of warheads on multiplewarhead missiles through on-site inspections," Science \& Global Security 1 (1990) 303-321.

Schwellenbach, D. D., D. Aberle, J. Bacon, K. N. Borozdin, W. Dreesen, J. A. Green, S. Greene, G. G. McDuff, E. C. Milner, C. Morris, J. Perry, M. Sossong, "Passive imaging of warhead-like configurations with cosmic-ray muon tracking scanners," Site-Directed Research and Development, FY 2011, National Security Technologies, LLC, Las Vegas, Nevada, 2012, 191-200.

Schwellenbach, D., "Passive imaging of warhead-like configurations using cosmic-ray muons," report, National Security Technologies, LLC, Las Vegas, Nevada, 2013.

Zalyubovskii, I. I., A. A. Lomako, O. N. Morgun, V. V. Chernyi, "Method for active remote detection of nuclear warheads," Atomic Energy 74, 6 (1993) 464-467. 


\section{INDEPENDENTLY TRIGGERABLE LINEAR TRANSFORMER DRIVER FOR SHORT, MULTI-PULSE RADIOGRAPHY \\ LAO-14-12 | YEAR 1 OF 1}

Andrew Smith, ${ }^{1, a}$ Glen McDuff, ${ }^{b}$ Nicholas Wilcox, ${ }^{a}$ and Nick Kallas ${ }^{c}$

We describe the concept, design, construction, and test results of an independently triggered, multi-pulse, linear transformer driver (LTD). Our multi-pulse LTD concept enables variable pulse timing delay for each specific expected event, potentially enhancing data quality for hydrodynamic experiments, and possibly leading to the development of smaller, more affordable multi-pulse radiographic systems. This concept uses a single, independently triggered primary winding on each of multiple cores; each primary is switched by a triggered spark gap. Energy storage is provided by discrete capacitors. The driver also contains a single secondary common to all cores. The system can provide up to $100 \mathrm{kV}, 2 \mathrm{kA}$ pulses from 50 to $100 \mathrm{~ns}$. SPICE circuit simulations on various circuit designs concurred with measured data. We have produced multipulse output on the secondary of a two-stage modulator using a trigger module and a delayed trigger that produced a pulse spacing of about $200 \mathrm{~ns}$. We then used two independent triggers with variable pulse spacing to produce a multi-pulse output under various charge voltage conditions. This report also introduces options for continued work and areas of investigation.

${ }^{1}$ smithas@nv.doe.gov, 505-663-2074

${ }^{a}$ Los Alamos Operations; ${ }^{b}$ Keystone International, Inc.; ${ }^{c}$ New Mexico Tech, Socorro, New Mexico

\section{Background}

Most radiographic machines, such as Cygnus at the Nevada National Security Site and the Radiographic Integrated Test Stand at Sandia National Laboratories (SNL), use a single pulse and an x-ray diode output. Multi-pulse radiography has an advantage over singlepulse radiography in that it delivers multiple images in a dynamic environment, which is useful for capturing a series of events, and interactions between, as they unfold. These images can be combined to produce a short movie. Multi-pulse radiography is done at the Dual-Axis Radiographic Hydrodynamic Test facility (DARHT) and the Proton Radiography facility (pRad) at Los Alamos National Laboratory.

DARHT uses a pulse-forming network combined with a linear induction accelerator to perform $x$-ray radiography. The multi-pulse capability is a magnetic switch ("kicker") that sends the beam energy to a target or a beam dump. DARHT can vary $x$-ray dose and spacing within a $2 \mu$ s window (Scapetti 2007). The pRad facility performs radiography by using high-energy protons generated by a radio frequency linear accelerator. These protons are "bunched" into specific sizes and collected in the proton storage ring. These bunches are then delivered to the target via a kicker magnet. The number of pulses is limited by the storage capacity. There are limitations to the DARHT and pRad facilities. The approximately $2 \mu$ s pulse width of DARHT excludes events of interest that occur after the $2 \mu \mathrm{s}$ window. The pRad system is a complex machine, measuring one-half mile in length, requiring many operators. Additionally, accessing the device for experiments is very competitive, and conducting experiments on the system can be very expensive. 
A new approach to multi-pulse radiography may provide machines that are smaller and more affordable, while providing more capabilities than current technologies. Variable pulse spacing is a desire of many hydrodynamic test researchers (Toury 2012). If the pulse spacing can be varied, then an experimenter could time the pulses to predicted events, and possibly obtain a complete set of data on a single-shot event.

\section{Project}

The pulse modulator design for our multi-pulse linear transformer driver (LTD) is loosely based on the proven LTD technology originated at the High Current Electronic Institute in Tomsk, Russia, and refined at SNL (Mazarakis 2010). We set out to design and build a pulse modulator that had reasonable requirements for a radiography system for hydrodynamic testing. Each pulse modulator is required to trigger independently. Basic requirements are listed in Table 1.

We investigated challenges to multi-pulsing. Consultations with SNL revealed sympathetic triggering as the greatest challenge, with isolation networks to decouple the multiple modulators needed. We needed to ensure that only the selected module fires at the required time. We had concerns about the effect of non-triggered stages on secondary current, and the effect of triggered stages on subsequent secondary current pulses. We also had concerns about inter-stage coupling due to the close proximity of the modulator cores and the fact that they shared a common secondary.

\section{Circuit Simulations}

We performed circuit modeling based on initial calculations and estimates of parasitic elements using the SPICE program. We developed two circuits, a singlestage modulator and a two-stage modulator with a common secondary. The single-stage circuit and the two-stage circuit simulations validated the circuit design and verified the saturable core model.
Table 1. Modular system requirements

\begin{tabular}{|l|l|}
\hline Requirement & Specification \\
\hline Voltage & $100 \mathrm{kV}$ \\
\hline Current & $2000 \mathrm{~A}$ \\
\hline Pulse width & 50 to $100 \mathrm{~ns}$ \\
\hline Pulse spacing & Variable $(\mu \mathrm{s}$ range) \\
\hline
\end{tabular}

Table 2. Metglas core specifications

\begin{tabular}{|l|l|}
\hline Core outer diameter & $203.20 \pm 2.0 \mathrm{~mm}$ \\
\hline Core inner diameter & $101.60 \pm 0.25 \mathrm{~mm}$ \\
\hline Ribbon alloy width & $25.4 \mathrm{~mm}$ \\
\hline Insulator width & $28.5 \mathrm{~mm}$ \\
\hline Layers & 800 \\
\hline
\end{tabular}

We performed single-stage SPICE simulations depicting the effect of core saturation on circuit response for 100, 200, 300, and $500 \mathrm{kV}$. Results are shown in Figures $1 \mathrm{a}-1 \mathrm{~d}$. Note that as the voltage goes up, the pulse length becomes shorter, because the core can only support the voltage for a limited time. This is indicative of a core going into saturation. Permalloy, a commercial magnetic core material, was used as the core material in the simulations, as it had the best available data. However, such high-frequency data were not available for the Metglas cores used in the investigation. The accuracy of our models could be improved with such data.

We also performed two-stage modulator circuit simulations with a charge voltage of $50 \mathrm{kV}$. The voltage results in Figure 2 show each primary pulsing at approximately $100 \mathrm{~ns}$ spacing, with the secondary capturing both pulses. The waveforms are underdamped with nearly $50 \%$ reversal. The current waveforms, shown in Figure 3, indicate a large reversal current. This characteristic will be discussed in the Results section. The current in the secondary has a low magnitude due to the loading effects of the first modulator in the system. Based on the results of these circuit simulations, we have confidence that the prototype design will be able to meet the multipulsing requirements stated in Table 1. 
(a) Charge voltage $=100 \mathrm{keV}$

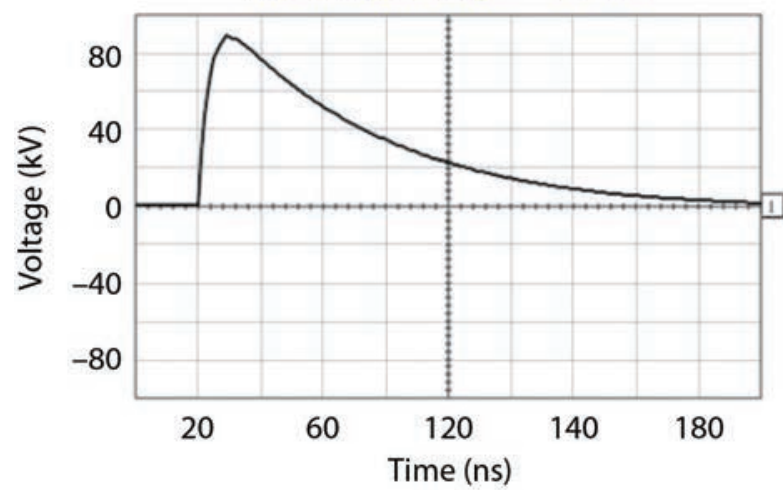

(c) Charge voltage $=300 \mathrm{keV}$

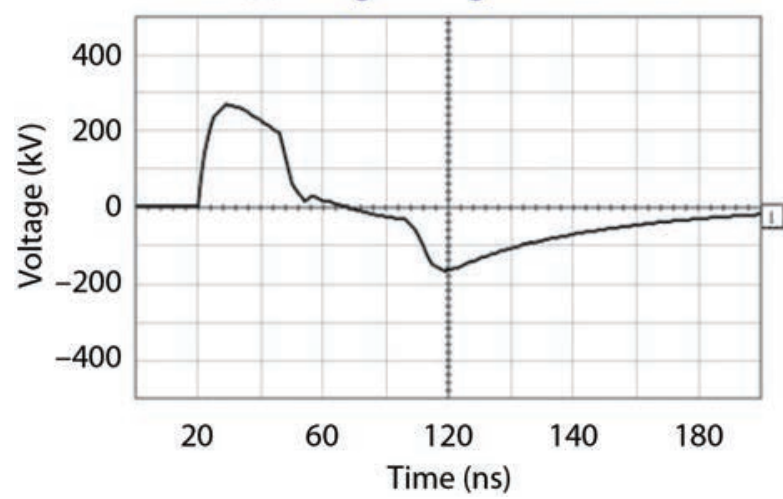

(b) Charge voltage $=200 \mathrm{keV}$

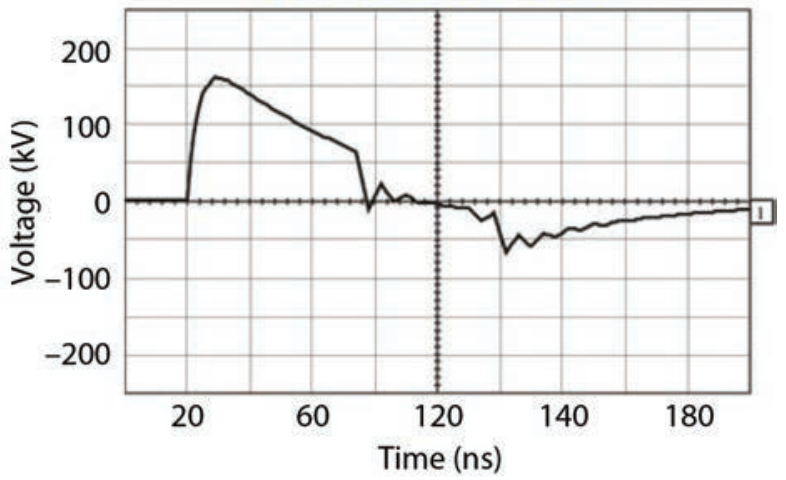

(d) Charge voltage $=500 \mathrm{keV}$

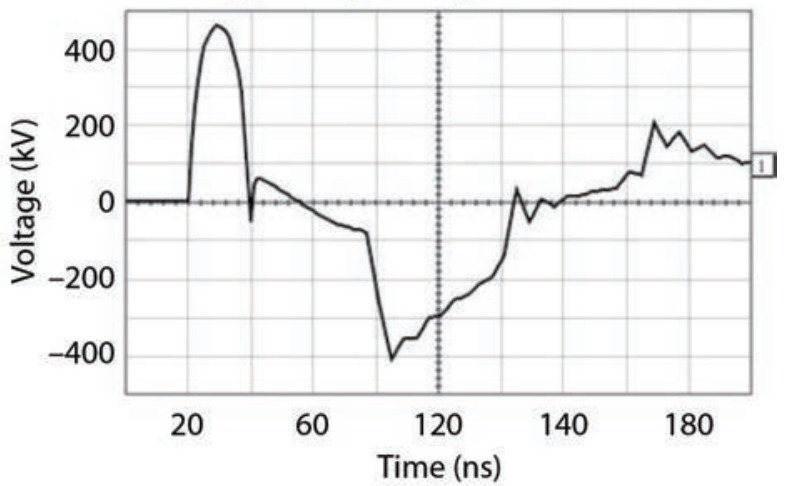

Figure 1. SPICE simulations at four charge voltages show that pulse length becomes shorter because the core can only support the voltage for a limited time before reaching saturation

\section{Construction of Modulators}

There were two options for energy storage:

(1) designing and building a coaxial capacitor or

(2) using commercially available discrete capacitors; we chose to use four parallel strings of three series 2.7 nF, discrete, ultra-high voltage capacitors (TDK model UHV-6A). The energy storage capacity for the capacitor network is $18 \mathrm{~J}$ with $100 \mathrm{kV}$ charge voltage.

A T670 spark gap was employed for the switching element because it met the $100 \mathrm{kV}$ requirement, had fast switching times, and was readily available. We pressurized the spark gap with various gases including sulfur hexafluoride $\left(\mathrm{SF}_{6}\right)$, nitrogen, and a combination of $15 \% \mathrm{SF}_{6}$ and $85 \%$ argon. The argon/SF $\mathrm{SF}_{6}$ mixture worked best for our spark gaps because of the holdoff ability of the $\mathrm{SF}_{6}$ and the lower resistance $(20 \%$ less) of the argon compared to nitrogen (air). We switched to pure $\mathrm{SF}_{6}$ for its higher hold-off properties during our higher voltage testing.

A Spellman model SL100P10/DPM4 power supply provided the high voltage for the energy storage capacitors. Transformer windings were 0.1 -inch aluminum sheet bent to fit the cores and instrumentation. We used toroidal Metglas 2605HB1M material cores with dimensions shown in Table 2 for the final design. The entire modulator system was submerged in Shell diala transformer oil. Initially, TM-11 trigger generators were used; however, in our final system design, we used Maxwell model 40168 trigger generators to trigger the spark gaps. 

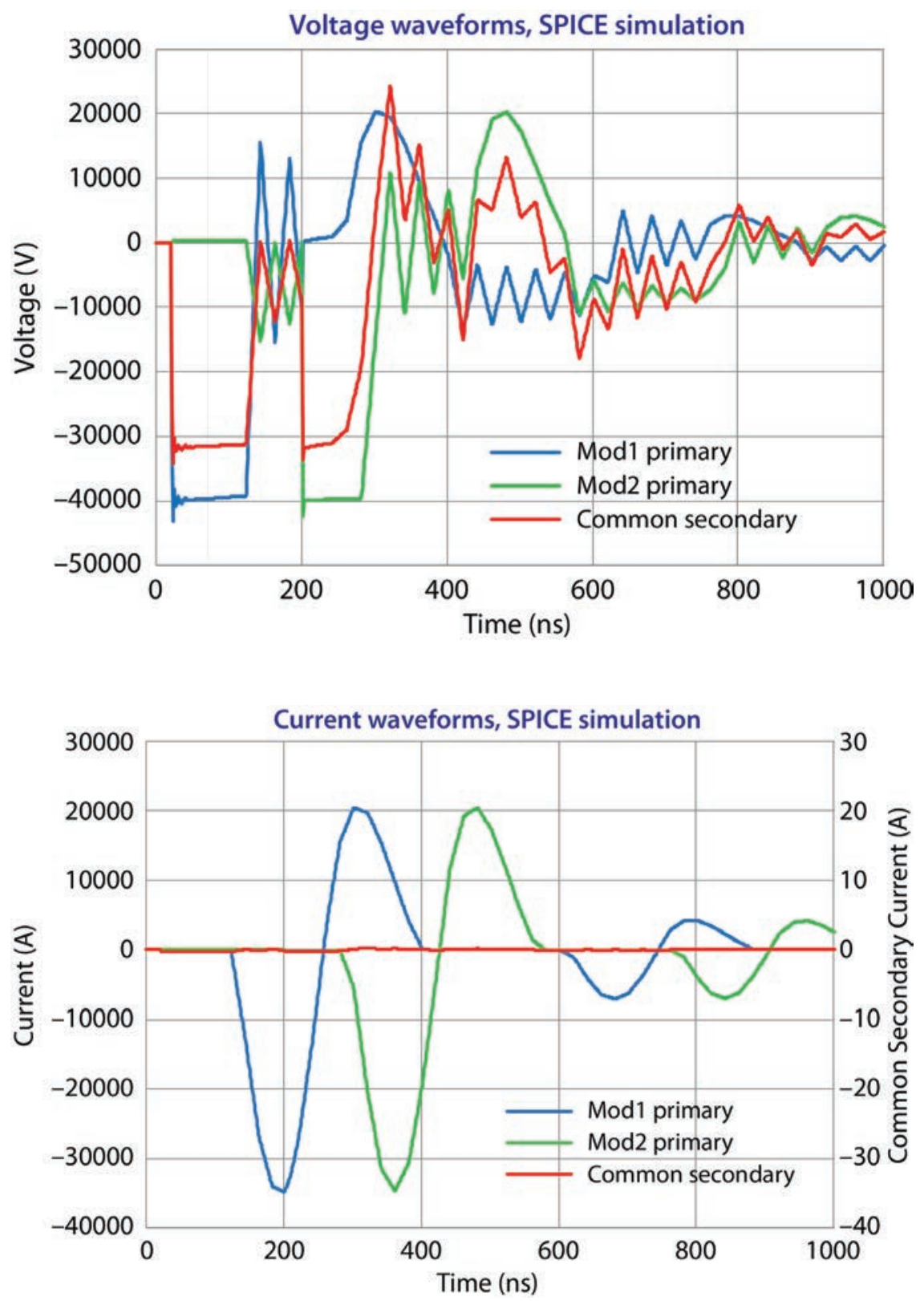

Figure 2. Two-stage SPICE voltage simulation output of the two primaries and the common secondary shows two pulses in the secondary, where it is desired

Figure 3. Two-stage SPICE current simulation output of the two primaries and the common secondary. Note that the common secondary current is much lower than the primary currents.
Two T \& M Research W-2-001-6FC current viewing resistors (CVRs) monitored primary currents, and Tektronix P6013A high-voltage probes measured voltage. All components were installed on a Tektronix model DPO 4104 four-channel, $1 \mathrm{GHz}$ digital oscilloscope, as shown in Figure 4.

\section{Results}

To gain insight into the high-frequency behavior of the cores, we measured the core frequency response with an Agilent 8702 network analyzer. The frequency response plot, shown in Figure 5, indicates that up to $50 \mathrm{MHz}$ the core loss was about $-7 \mathrm{~dB}$, with a sharp increase in loss above $50 \mathrm{MHz}$. The maximum loss of $-21 \mathrm{~dB}$ at $75 \mathrm{MHz}$ is substantial and may indicate material property interactions.

Preliminary measurements of the magnetization curves were made using the modulator circuits as a source of drive power. These measurements were plagued by spurious noise in the voltage and current 


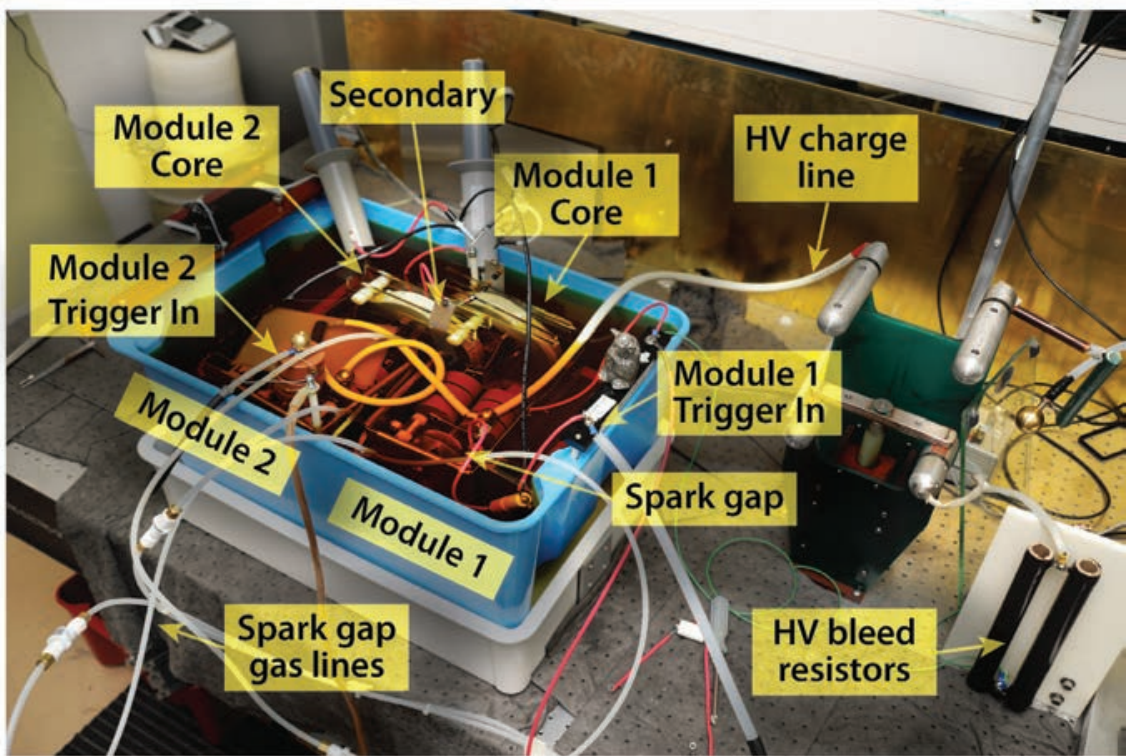

Figure 4. Two-stage

modulator test setup

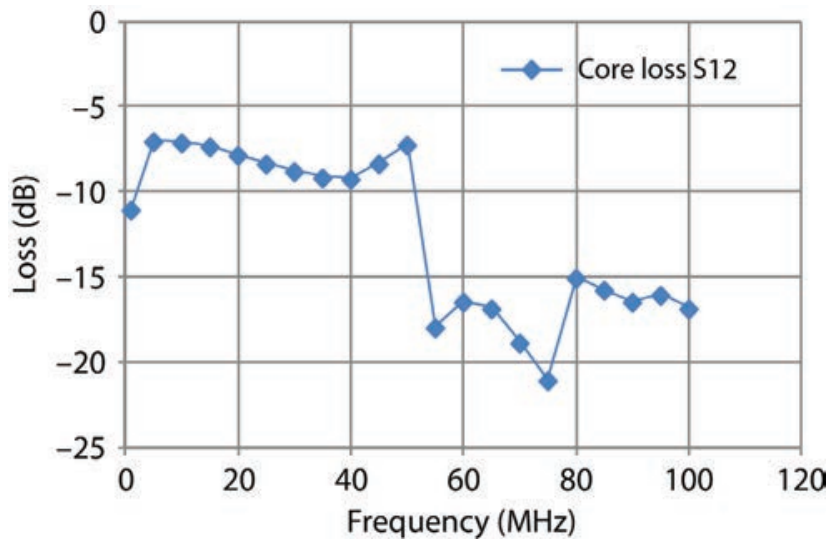

Figure 5. High-frequency core loss in Metglas core using a 1:1 turns ratio transformer, showing the sharp increase in loss at $50 \mathrm{MHz}$

signals, making acquisition of good data difficult on our large cores. The high-power, high-performance oscillators required to take these data correctly are not readily available. The use of smaller core samples makes data acquisition easier, but is often not representative of large core behavior (McDonald 2003).

One of the first tests performed on the two-stage system was triggering one module to detect whether the second module triggered sympathetically. This test indicated no sympathetic triggering (Figure 6). The two circuits could not be made to sympathetically trigger until physically connected, an unexpected result, as closely coupled circuits that share the same enclosure, the same electrical common structure, and the same power supply with essentially no decoupling networks are expected to sympathetically trigger.

After this, we performed multi-pulse testing using an inductively coupled delay circuit connected from the output of one modulator's primary to the spark gap trigger of a second modulator. The second trigger was fired from the primary of the first modulator, and delayed using a capacitive network. We observed independent output pulses separated by the expected delay (approximately $200 \mathrm{~ns}$ ) as shown in Figure 7.

When both modulators were triggered, with the second delayed relative to the first, energy loss was observed in the second. It was expected that if the first discharged, and its core was unsaturated, essentially all of the energy from the second would flow back into it instead of into the high impedance load, but this was not observed to be the case, with either a saturated or unsaturated core. In the case of a saturated core, the observed behavior indicates negligible residual core magnetization. In the unsaturated case, this behavior may indicate another mechanism or combination of mechanisms affecting the circuit response. 

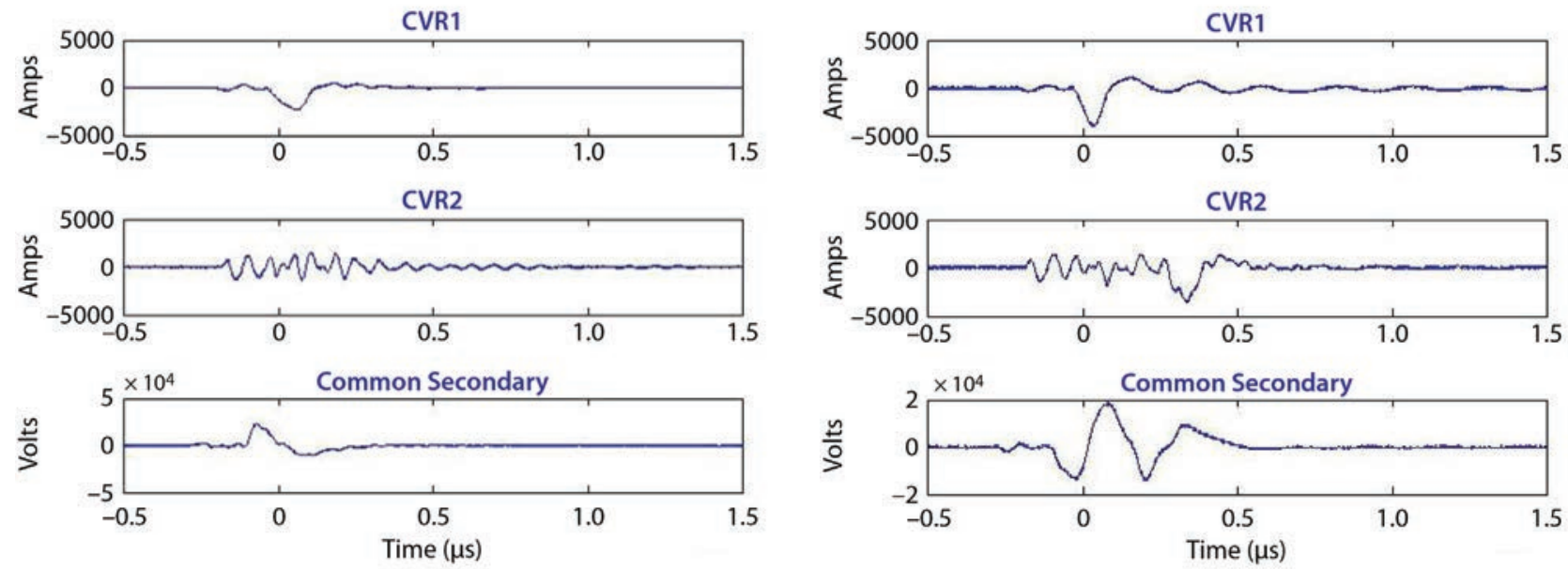

Figure 6. Plot of test results showing no sympathetic triggering of second module when triggering the first. CVR1 is primary current of modulator (triggered). CVR2 is primary current of modulator two (not triggered). Common secondary shows the voltage at the load.

We offer two observations of circuit characteristics: (1) the transient impedance of the primary appears to be far greater than calculated, and (2) energy from the second modulator is driving the first core into saturation. The extreme nonlinearity of the Metglas may contribute to this effect. Previous measurements indicated that some frequencies are greatly attenuated in Metglas relative to others, distorting diagnostic results and making energy flow tracking difficult. Investigation of the Metglas' frequency dependence may provide insight into this issue.

Further investigation of this effect requires two independent triggers such that the time interval between the two modulator pulses can be set precisely. We reconfigured the two-stage modulator as such and performed experiments varying the pulse spacing. We also performed experiments varying the charge voltage at points above, below, and at the

estimated saturation point of our cores as shown in Table 3. A sample waveform is shown in Figure 8.

We were able to obtain pulse spacing from 770 ns to $27.69 \mu \mathrm{s}$, thus proving the concept of arbitrary pulse spacing in a multi-pulsed system. Variable pulse spacing was achieved with no sympathetic triggering. We observed some energy loss in the pulses. The highest voltage output pulse was around $15 \mathrm{kV}$. Power supply loading along with voltage drop across charge resistors accounts for some loss. With a power supply limit of $100 \mu \mathrm{A}$, leakage of only a few microamperes can result in a large percentage loss. The voltage drop from the power supply output to the capacitor charge line at a power supply voltage of $30 \mathrm{kV}$ is $4500 \mathrm{~V}$ (approximately 15\%). Even the Tektronix high-voltage probe loads the circuit sufficiently to preclude its use as a diagnostic. The rest of the loss appears to be in the core. The simulations and measured data agree

Table 3. Pulse data from selected shots above, below, and at the estimated saturation point of our cores

\begin{tabular}{|c|c|c|c|c|c|c|}
\hline Shot Number & $\begin{array}{c}\text { Charge Voltage } \\
(\mathbf{k V})\end{array}$ & $\begin{array}{c}\text { Pulse 1 Voltage } \\
(\mathbf{k V})\end{array}$ & $\begin{array}{c}\text { Pulse 2 Voltage } \\
(\mathbf{k V})\end{array}$ & $\begin{array}{c}\text { Pulse 1 Width } \\
(\mathbf{n s})\end{array}$ & $\begin{array}{c}\text { Pulse 2 Width } \\
(\mathbf{n s})\end{array}$ & $\begin{array}{c}\text { Pulse Spacing } \\
(\boldsymbol{\mu})\end{array}$ \\
\hline 95 & 67.5 & 15 & 12 & 80 & 90 & 0.77 \\
\hline 99 & 35 & 10 & 8 & 140 & 80 & 27.69 \\
\hline 111 & 49 & 10 & 10 & 74 & 74 & 8.80 \\
\hline
\end{tabular}




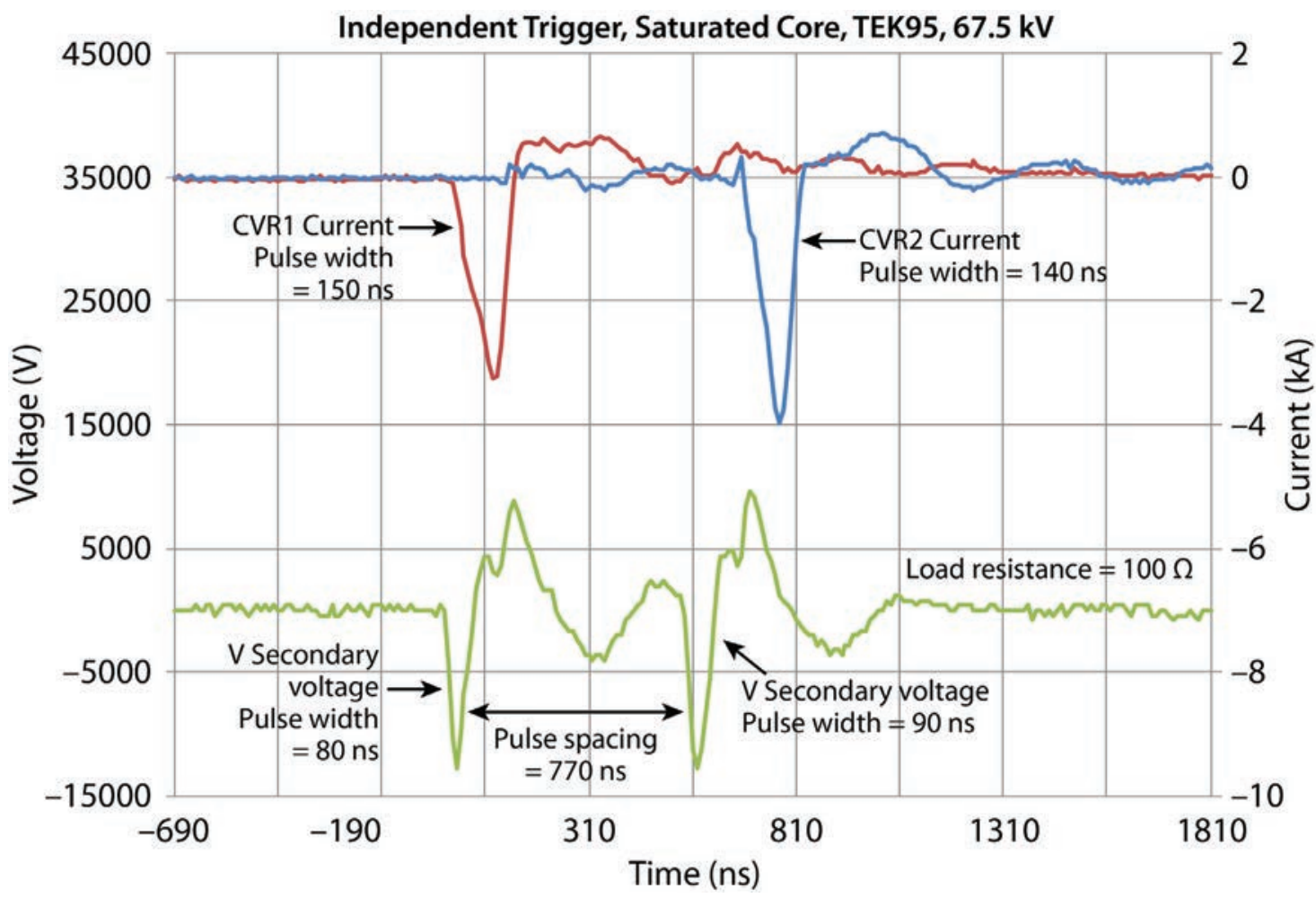

Figure 8. Independently triggered waveforms at charge voltage of $67.5 \mathrm{kV}$ show the independent current pulses creating two voltage pulses. The voltage pulses have a much shorter pulse width due to the effects of saturation in the cores.

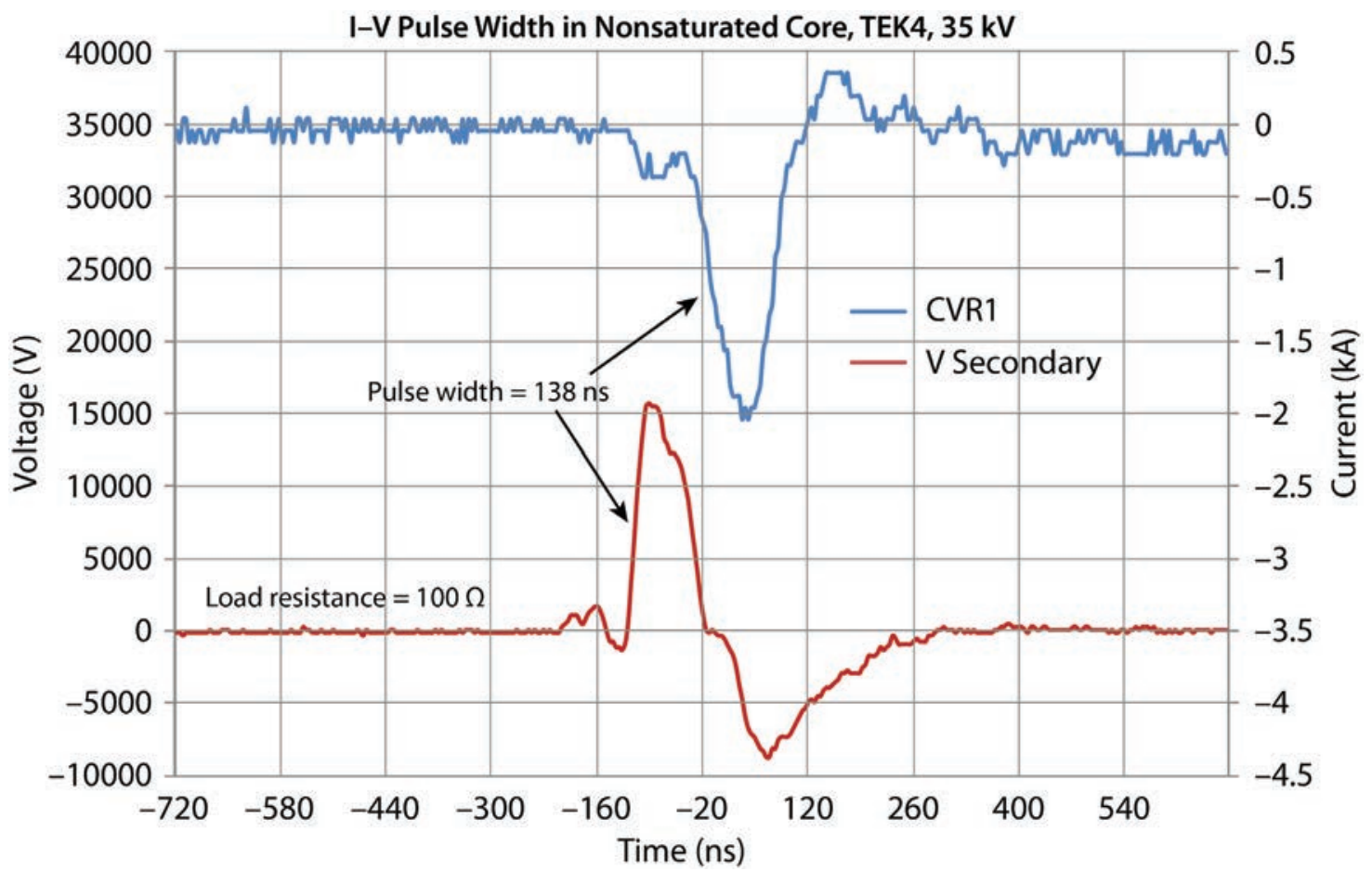

Figure 9. Voltage pulse width of core below saturation. Charge voltage is $35 \mathrm{kV}$. Note that the voltage and current pulse width are the same, which indicates saturation of the core. 


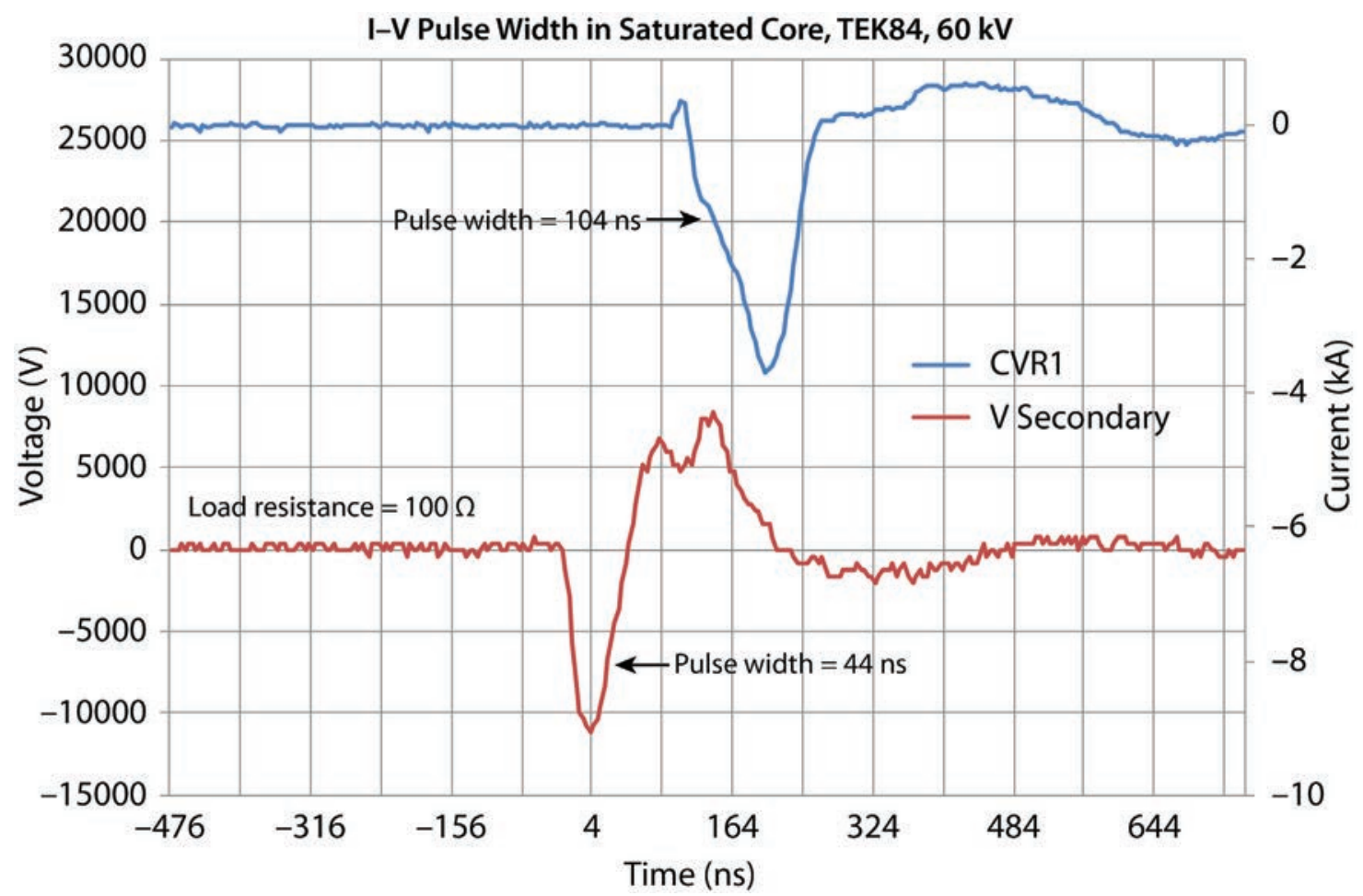

Figure 10. Voltage pulse width of core above saturation at a charge voltage of $60 \mathrm{kV}$. In this case the voltage pulse is much shorter than the current pulse, which indicates saturation of the core.

on circuit quality factor and frequency response.

After performing an energy inventory, the difference between simulated and measured data shows about $40 \%$ less in the measured data. Because the circuit quality factor and frequency response are similar, this indicates a resistive (real) loss in the system. The high-frequency behavior of the core material, which is not completely understood, is the only circuit element about which detailed electrical characteristics are not known, and the only element for which loss cannot be calculated.

We varied charge voltage to observe modulator behavior above and below calculated saturation values for the Metglas cores. Voltage plots show that secondary voltage pulse width changed as the charge voltage increased, as shown in Figures 9 and 10. The voltage pulse width decreased as voltage increased above the expected saturation point, indicating core saturation and validating design calculations.
The results validate the circuit is working. Two important features were not part of the initial design.

First, ringing in the current pulses and a first-reversal magnitude of approximately $500 \mathrm{~A}$ is more than ten times the required reset current to drive the core out of saturation. Circuit damping occurs in five to six cycles, which is more than adequate to randomize the core so that pulse repetition without a reset circuit is possible. Second, the impedance looking back toward the primary of the transformer circuit is far greater than the load; therefore, little energy is transferred back to the primary circuit of the first modulator when the second modulator is fired. Because the second modulator is under-damped, the secondary current randomizes the cores of both modulators. If the circuit were critically damped, this would not be the case. Whether ultimately the reversal is detrimental to the output characteristics is not clear. The reversal can be modified by the addition of snubber and/or clamp circuits, which are both passive techniques. 


\section{Conclusion}

We designed and built a two-stage LTD with individual primaries and a common secondary. We ran SPICE circuit simulations on various circuit designs, and the results agreed with measured data. We produced multi-pulse output on the secondary of a two-stage modulator, using a trigger module and a delay trigger that was inductively coupled from the primary of the first modulator to the trigger of the second modulator's spark gap. We saw no sympathetic triggering in this mode, and generated a pulse spacing of about 200 ns. We also successfully performed independently triggered multi-pulse tests at various charge voltages. We observed behavior below and above core saturation. Closer investigation is needed to explain the core behavior at high frequencies, the observed energy losses, and the effects of the voltage reversal on a diode or electron beam.

\section{Acknowledgments}

We would like to thank Del Anderson for the initial idea; Chris Hagen for his advice on safety issues; Josh Leckbee and Bryan Oliver for their consulting expertise; William Reass for providing equipment, spare parts, and advice; and Dave Schwellenbach for use of his lab space and expertise.

\section{References}

Mazarakis, M. G., W. E. Fowler, K. L. LeChien, F. W. Long, M. K. Matzen, D. H. McDaniel, R. G. McKee, C. L. Olson, J. L. Porter, S. T. Rogowski, K. W. Struve, W. A. Stygar, J. R. Woodworth, A. A. Kim, V. A. Sinebryukhov, R. M. Gilgenbach, M. R. Gomez, D. M. French, Y. Y. Lau, J. C. Zier, D. M. VanDevalde, R. A. Sharpe, K. Ward, "High-current linear transformer driver development at Sandia National Laboratories," IEEE Trans. Plasma Sci. 38, 4 (2010) 704-713.

McDonald, K., R. Curry, R. O'Connell, P. Melcher, R. Ness, C. Huang, "Evaluation of magnetic materials and insulation systems for repetition-rate pulse compression applications," 14th IEEE International Pulsed Power Conference Digest of Technical Papers 1 (2003) 603-606.
Scapetti, R. D., S. Nath, J. Barraza, C. A. Ekdahl, E. Jacquez, B. T. McCuistian, K. Nielsen, M. Schulze, J. Seitz, G. J. Caporaso, Y.-J. Chen, G. Logan, F. Bieniosek, "Status of the DARHT 2nd axis accelerator at Los Alamos National Laboratory," IEEE Particle Accelerator Conference (2007) 831-835.

Toury, M., F. Cartier, M. Caron, J. Leckbee, B. Oliver, "Two pulse tests with a single LTD cavity," poster, 4th Euro-Asian Pulsed Power Conference/19th International Conference on High-Power Particle Beams, October 2012. 
This page left blank intentionally 


\section{RADIATION HARD AIGaN IMAGER AND ELECTRONICS}

LO-07-12 | CONTINUED FROM FY 2011 | YEAR 2 OF 2

Ke-Xun Sun ${ }^{1, a, b}$

Continuing SDRD work begun in FY 2011, we have demonstrated extreme radiation-hard AlGaN optoelectronics devices. These devices largely retain normal functionality with the irradiation of $3 \times 10^{12}$ protons $/ \mathrm{cm}^{2} 65 \mathrm{MeV}$ proton flux. This may convert to $\sim 3 \times 10^{16}$ neutrons $/ \mathrm{cm}^{2}$ $14 \mathrm{MeV}$ neutron radiation hardness, sufficient for sustained diagnostics operation at the National Ignition Facility. Further, we have demonstrated the successful design and fabrication of a $2 \times 2 \mathrm{AlGaN}$ photodiode array. This is an encouraging step towards develoing an AIGaN radiation-hard imager with the higher pixel counts required by the high-energy density physics diagnostics community who employ these arrays as imaging layer elements in fast imagers. Due to the complexity and support level required for developing the AIGaN imager and researching $\mathrm{GaN}$ electronics, this project also established a research collaboration with the University of Nevada, Las Vegas.

${ }^{1}$ sunke@nv.doe.gov, 925-960-2514; Ke-Xun.Sun@unlv.edu, 702-774-1486

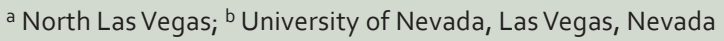

\section{Background}

NSTec pioneered proton radiation hardness studies of AIGaN optoelectronics devices, UV LED, and UV photodiodes (Sun 2006, "UV LED" 2009, "Space" 2009, "Radiation hardness" 2010, "GaN radiation" 2010, "Extreme" 2010). This work is important for high-energy density activities. The need for radiationhardened (RadHard) detectors, detector arrays, and imagers has become more evident in recent years. The National Ignition Facility (NIF) can reach a neutron yield of up to $10^{15}$ per shot or even higher, but achieving this is not without complications: damage to diagnostic instrumentation can cause lower image quality and diagnostic malfunctions, but relocation of diagnostics leads to data blanks for highyield shots; complex $x$-ray optics are needed to route the signal beam to the relocated diagnostics input; and there are still potentially serious worker safety issues.

Since FY 2010, we have been conducting an experimental demonstration of the radiation hardness of the AlGaN photodetector up to a fluence of $3 \times 10^{12}$ protons $/ \mathrm{cm}^{2}$. Because proton damage is typically more severe than neutron damage, this result may indicate higher neutron hardness of $10^{15}-10^{16}$ neutrons $/ \mathrm{cm}^{2}$, which is equivalent to $1,000 \sim 10,000$ high-yield shots if the AIGaN detector is placed 1 meter away from the target chamber center. This will radically solve the radiation damage problems for NIF high-yield shots, and even for future Laser Inertial Fusion Energy (LIFE) facility experiments, which will yield neutrons 2 to 3 orders of magnitude higher than NIF.

This project continues work begun in FY 2011 (Sun 2012), during which we developed two types of new RadHard AlGaN devices: photodiode arrays and a RadHard photodiode with a built-in calibration LED. Second, we laid out the development path for RadHard AlGaN imagers considered this year. Third, we successfully acquired, installed, and commissioned TOPAS, which provides a powerful tool for 


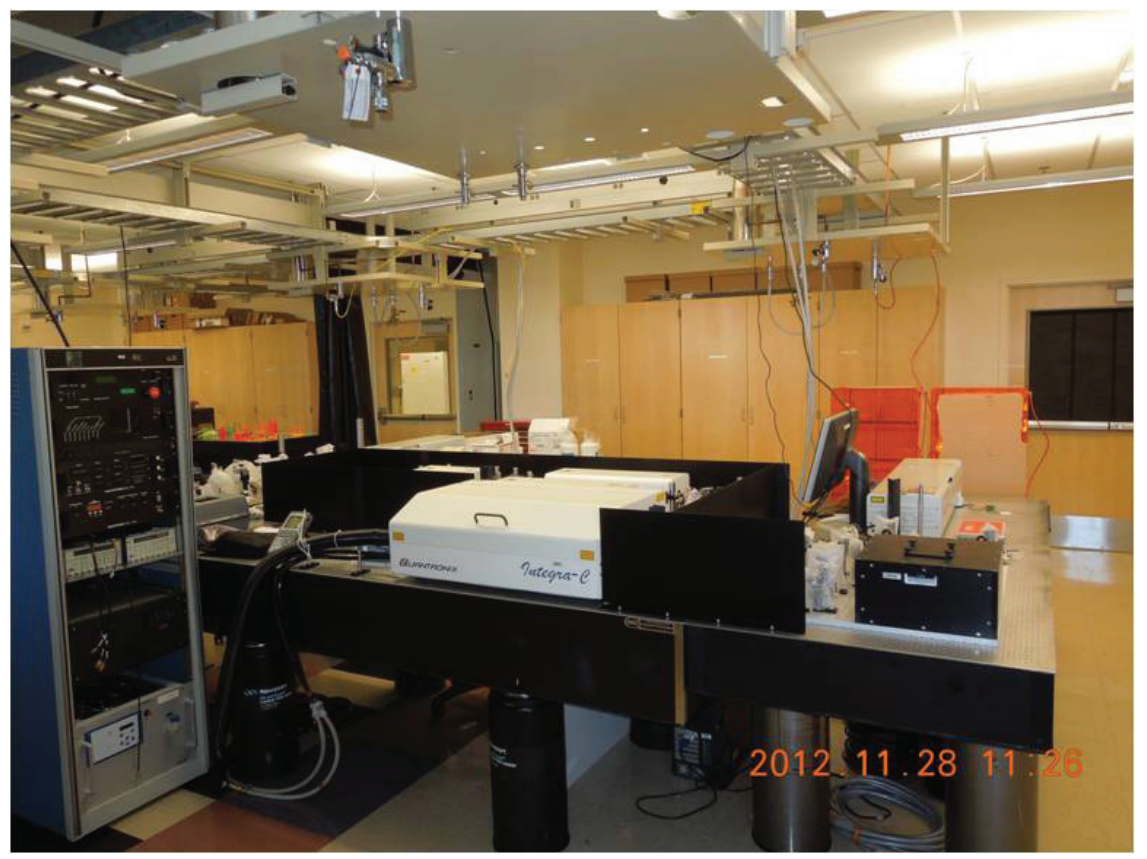

Figure 1. The UNLV laboratory for AIGaN device characterization. TOPAS, a femtosecond widely tunable optical parametric amplifier, is installed in the lab.

temporal and spectral characterization of AlGaN optoelectronics devices. We also accomplished architectural studies for larger AlGaN detector arrays and CCDs.

The level of radiation hardness that we demonstrated in FY 2011 challenged the current theoretical understanding of radiation hardness. We gave presentations at several semiconductor research conferences, including the Conference on Lasers and Electronics (CLEO) (Sun CLEO 2011), the Advanced Microelectronics and Photonics for Space 2011 symposium (Sun AMAPS 2011), and the Workshop on Frontiers in Electronics (Sun WOFE 2011). Our CLEO presentation was reported by Nature Photonics (Pile 2011). The FY 2012 SDRD project built on these accomplishments.

\section{Project}

\section{NSTec-UNLV Collaboration}

Integral to this project was establishing an electrooptical laboratory at the University of Nevada, Las Vegas (UNLV) (Figure 1). As part of an ongoing collaboration, we relocated NNSS equipment, including the TOPAS tunable optical parametric amplifier (OPA), the Quantronix pump laser, Thales lasers, and many electronic instruments, including a semiconductor $40 \mathrm{GHz}$ spectrum analyzer and a $40 \mathrm{GHz}$ sweep signal generator. These instruments are important for use in radiation hardness studies in unexplored territories, as follows:

- The semiconductor parameter analyzer will efficiently and precisely measure the currentvoltage (I-V) curve characteristics, thus identifying the changes before and after radiation, and even in real time. The I-V curve indicates the characteristics and the quality of semiconductor materials and junctions. The I-V curve family can be modeled using SPICE tools, and quantify the radiation hardness in terms of I-V curve variations versus irradiation flux.

- The femtosecond tunable OPA source will be used for temporal response characterization. For example, the pulse response before and after the proton and neutron irradiation is used to fully characterize the radiation hardness.

\section{Neutron Damages to Silicon CCD Imagers Revisited}

Neutron damage to silicon CCD imagers has been assessed very differently, and varies according 


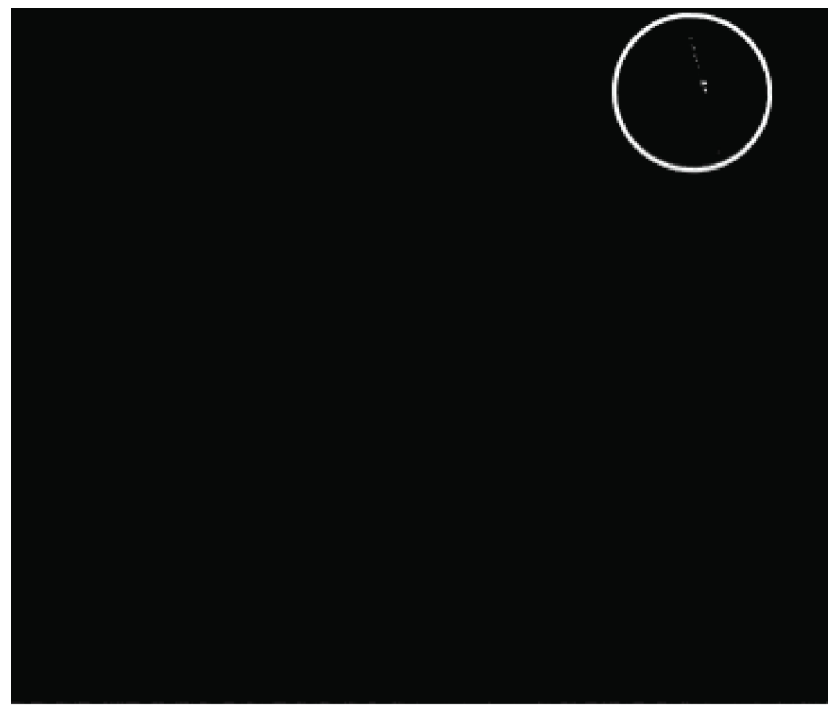

$\overline{600 \mu \mathrm{m}}$

Figure 2. Transient noises of CCD image sensor (DT neutron flux: $10^{4}$ neutrons $/ \mathrm{cm}^{2}$ )

to testing methods and characterization criteria. Especially at NIF, high neutron yield shots are scarce because of neutron damage concern. There is a lack of quantitative data on neutron damaging to diagnostic instruments. It is therefore necessary to review these results to understand the necessity and urgency of producing neutron RadHard imagers.

The neutron damage to silicon and germanium electronics actually occurs at fairly moderate neutron flux and fluence levels (Tanimura 1998, Giauque 1999). Careful observation with deuterium-deuterium (DD) and deuterium-tritium (DT) neutrons revealed that silicon CCD dynamic images degrade at irradiation neutron flux as low as $10^{4}$ neutrons $/ \mathrm{cm}^{2}$. Figure 2 shows the neutron transient tracks. Figure 3 shows that permanent, irrecoverable damages occur at $\sim 10^{6}-10^{7}$ neutrons $/ \mathrm{cm}^{2}$. These values are much lower than some common estimates, but now are considered to be explanatory to

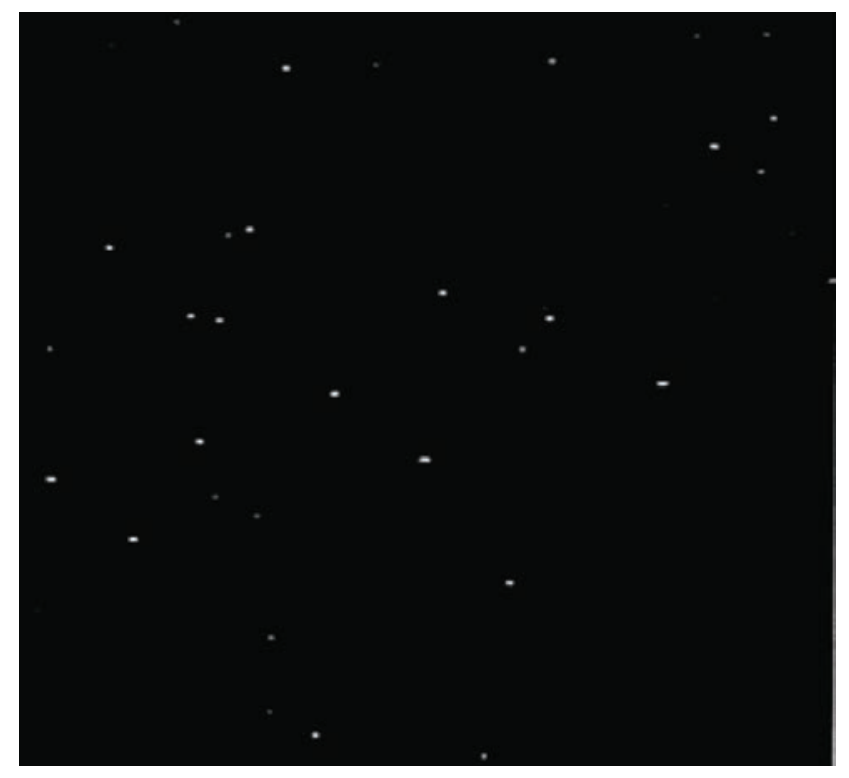

Figure 3. Unrecoverable specks of CCD image sensor (DT neutron fluence: $8 \times 10^{7}$ neutrons $/ \mathrm{cm}^{2}$ )

observation at NIF (Izumi 2012). At NIF, the neutron damage is more substantial due to intensified neutron fluxes and fluencies.

A further observation yet to be confirmed is the comparable degrees of damages caused by $2.4 \mathrm{MeV}$ DD neutrons and $15 \mathrm{MeV}$ DT neutrons. As shown in Table 1 (Tanimura 1998), the damage constants only differ by $10 \%$ for DD and DT neutron irradiations. If these observations are correct, the neutron damages at NIF must take into account the large amount of down-converted neutrons at all lower energies too, not only the high-energy DT neutrons.

Therefore, the neutron damage problem is more severe than previously estimated. As such, our research on radiation hardness of $\mathrm{GaN}$ devices are more significant and timely. There is an escalated urgency to develop the GaN radiation hard imagers.

Table 1. Neutron damage to Si on CCDs (Tanimura 1998)

\begin{tabular}{|l|c|c|c|}
\hline \multirow{2}{*}{} & \multicolumn{2}{|c|}{ Damage Constant $\left(\mathrm{cm}^{2} / \mathrm{n}\right)$} & \multirow{2}{*}{ Correlation Factor } \\
\cline { 2 - 4 } & DT Neutron & DD Neutron & 1.1 \\
\hline Experiment & $6.5 \times 10^{-7}$ & $5.6 \times 10^{-7}$ & 1.2 \\
\hline Calculation & $1.1 \times 10^{-6}$ & $8.9 \times 10^{-7}$ & 1.2 \\
\hline
\end{tabular}




\section{Neutron Damage Mechanisms}

Although neutron damage mechanisms have been studied, the literature does not fully explain observational data. A more plausible summary is given by Carron (2006). He posited that neutron damages occur in two situations: (1) when the neutron energy is below $3 \mathrm{MeV}$, the nuclei interaction, and thus the atom displacement, is the main damage mechanism; and (2) when the neutron energy is above $3 \mathrm{MeV}$, ionization process-induced damages will dominate. In the second situation, neutrons will collide with protons. The proton movement, as charged particles, will further break the electron bonding structure.

However, this theory cannot explain some aspects of the NIF neutron damages to electronics and optics. For example, based on this theory, the $15 \mathrm{MeV}$ DT neutron damage will be recoverable, inconsistent with the fact that NIF diagnostics instruments suffer most from high-yield DT neutron shots. There is much work to be done to understand the relevant mechanisms of neutron damagers, to explain the $\mathrm{GaN}$ radiation hardness, and to improve the materials and devices.

\section{Neutron Equivalence of Proton Radiation Hardness}

It has been easier for us to access proton sources rather than neutron sources. Our radiation hardness tests were conducted using a proton beam at $\sim 65 \mathrm{MeV}$. The DT fusion reaction generates neutrons with energies of $14.8 \mathrm{MeV}$, and DD reaction neutron energies of $2.45 \mathrm{MeV}$. Therefore, we sought to discover if proton damage is equivalent to neutron damage. Because of the importance of this question, proton and neutron damage equivalence to semiconductors has been a research topic since the 1960s (Bilinski 1963). The conclusion at the time was that there was not enough data to study the problem.

Physically, protons are charged particles that have a higher interaction cross section than neutrons. However, the equivalent neutron doses have been wild guesses, ranging from 1:1 to 1:1000. It is clear that 1:1 estimates do not reflect the observational reality at NIF. The large span of the proton-neutron damage equivalence demonstrated the needs of further studies. Subsequently, this problem was investigated using kerma data (Caswell 1980), which reflects energy loss in particle interactions. The initial conclusion is that at $15 \mathrm{MeV}$, the neutron damage for equivalent proton damage ratio is 1:10,000 (Izumi 2012). This is quite encouraging. If the $1: 10,000$ ratio is correct, our results of $3 \times 10^{12}$ protons $/ \mathrm{cm}^{2}$ radiation hardness results will be equivalent to $3 \times 10^{16}$ neutrons $/ \mathrm{cm}^{2}$ fluence. When the GaN detector is placed 1 meter away from the NIF target chamber center, this is equivalent to $3.8 \times 10^{21}$ neutron yield, much greater than today's highest neutron yield of $\sim 10^{14}$ neutrons per shot. As such, GaN-based devices promise to provide the ultimate solution for radiation hard NIF/LIFE diagnostics.

\section{GaN Imager Architecture Studies-Sensing Pixels}

The SDRD project has studied GaN imager architectures quite broadly in anticipation of device design, fabrication, and equipment preparation.

Since the late 1990s, GaN imagers have been attempted by several groups: (1) GaN photoconductive elements, which used doping to change AlGaN photoconductivity (Huang 1999); (2) GaN photocathodes (Siegmund 2003); (3) GaN CCD pixels (SBIR Programs); and (4) GaN photodiodes (Long 2002, Barkusky 2009, Malinowski 2009). Each type has merits and particular applications that we can use. For the arrayed GaN imagers, the latest development of $\mathrm{GaN}$ photodiodes is a promising path, thanks to its potential wavelength selectivity and fast response time.

\section{GaN Imager Architecture Studies-Substrate Selection}

Substrate selection is another important step for developing the GaN imager and selecting equipment. Several materials have been used for substrates. Sapphire, the earliest used, is still a viable solution, due to its lattice match, radiation hardness, and 
transparency. Silicon carbide (SiC), another latticematched material, is particularly popular in GaN electronics development. SiC substrates provide better heat conductivity. Silicon is the most recently popular substrate used because of its potential convenience to be interfaced to existing electronics. Finally, $\mathrm{GaN}$ is the native material on which a GaN imager should be grown. Once its fabrication technique is perfected, it will be the most promising substrate.

\section{GaN Imager Architecture Studies-Readout Electronics}

Readout electronics is an important component of GaN imager architecture. Silicon-integrated circuits are still used as readout electronics, and will be for years to come. However, the lack of radiation hardness is a downfall. GaN electronics, which will be most appropriate and promising, will take time to develop. CCD-type readout circuits provide flexibility in interconnection design but are slow in data fan out. CMOS-type readout circuits will be fast but require a more complicated flip-chip connection fabrication technique. The analog-to-digital circuit in CMOS-type imagers requires more in-depth studies, as it may address our special requirements in collecting data at fast rates.

\section{GaN Processing}

The UNLV group plans to develop in-house capability in its central campus laboratory. We have been designing a GaN material, substrate, and device fabrication line. Among the equipment that will be part of this system are a metal organic chemical vapor deposition machine, high-temperature furnaces, a plasma sputtering system, a lithography system, electron beam lithography devices, an x-ray diffractometer, and instruments for optical elipsometry. More equipment will be added. The UNLV group is well connected to the industry experts who can help this exciting effort.

\section{Conclusion}

In summary, we have further assessed neutron damage to Si- and AlGaN-based devices and imagers. The Si-based devices can be permanently damaged at neutron fluences as low as $1 \times 10^{8} / \mathrm{cm}^{2}$. The GaN/ AlGaN devices can maintain normal functionality at neutron fluences up to $1 \times 10^{15}-10^{16} / \mathrm{cm}^{2}$. Therefore, it is important to develop GaN/AIGaN devices for fusion diagnostics. A prototype design and fabrication for a $2 \times 2 \mathrm{AlGaN}$ array was also completed although future work is required to scale this device to higher pixel counts. A successful collaboration has been established with UNLV with the lead principal investigator for this SDRD project now part of the faculty and establishing a new center for national security science. It is anticipated that future work will evolve within this center for advanced GaN science and development of specialized imaging technologies to serve broader high energy density physics applications.

\section{Acknowledgments}

I am indebted to my new colleagues at UNLV, especially my collaborators on the HEDP Diagnostics Proposal: Biswajit Das, Yingtao Jiang, Pushkin Kachroo, Eunja Kim, Brendon O’Toole, Tao Pang, Emma Regentova, Robert Schill, Mohamed Trabia, Rama Venkat, Woosoon Yim, Keith Evans, Yuri Bilenko, and Remis Gaska. In addition, helpful discussions with Bob Cauble, Joe Kilkenny, David Fittinghoff, Marilyn Schneider, Niko Izumi, and Andrew MacPhee at Lawrence Livermore National Laboratory and Ray Leeper at Sandia National Laboratories are gratefully acknowledged. Finally, I am grateful to my new group at UNLV, to my students Angsuman Roy, Sean Crippen, Stryder Loveday, Mary West, Tu Anh Tran, Thomas Castro, and to my special colleagues Rob Buckles and Jared Gordon in my group.

\section{References}

Barkusky, F., C. Peth, A. Bayer, K. Mann, J. John, P. E. Malinowski, "Radiation damage resistance of AIGaN detectors for applications in the extreme-ultraviolet spectral range," Rev. Sci. Instrum. 80 (2009) 093102. 
Bilinski, J. R., E. H. Brooks, U. Cocca, R. J. Maier, "Protonneutron damage equivalence in $\mathrm{Si}$ and Ge semiconductors," IEEE Trans. Nucl. Sci. 10 (1963) 71-86.

Carron, N. J., An Introduction to the Passage of Energetic Particles through Matter, Taylor \& Francis, Boca raton, Florida, 2006.

Caswell, R. S., J. J. Coyne, M. L. Randolph, "Kerma Factors for Neutron Energies below $30 \mathrm{MeV}$," Radiation Research 83, 2 (1980), 217-254.

Giauque, M. S., "Fusion neutron transient effects to charge coupled device camera images," thesis, Monterey, California, Naval Postgraduate School, 1999, http:// www.dtic.mil/dtic/tr/fulltext/u2/a367146.pdf, accessed September 30, 2012.

Huang, T. Z. C., D. B. Mott, A. T. La, “Development of 256x256 GaN ultraviolet imaging arrays," Proc. SPIE 3764 (1999).

Izumi, N., personal communication, 2012.

Long, J. P., S. Varadaraajan, J. Mathews, J. F. Schetzina, “UV detectors and focal plane array imagers based on AIGaN p-i-n photodiodes," Opto-Electronics Rev. 10, 4 (2002) 251-260.

Malinowski, P. E., J. John, J. Y. Duboz, G. Hellings, A. Lorenz, J. G. R. Madrid, C. Sturdevant, K. Cheng, M. Leys, J. Derluyn, J. Das, M. Germain, K. Minoglou, P. De Moor, E. Frayssinet, F. Semond, J.-F. Hochedez, B. Giordanengo, R. Mertens, "Backside-illuminated GaN-on-Si Schottky photodiodes for UV radiation detection," IEEE Electron Device Letters 30, 12 (2009) 1308-1310.

Pile, D., "View from CLEO 2011: Ultraviolet goes solidstate," Nature Photonics 5 (2011) 394-395.

Siegmund, O. H. W., A. S. Tremsin, A. Martin, J. Malloy, M. P. Ulmer, B. Wessels, "GaN photocathodes for UV detection and imaging," Proc. SPIE 5164, 134 (2003).

Sun K.-X., B. Allard, S. Buchman, S. Williams, R. L. Byer. "LED deep UV source for charge management for gravitational reference sensors," Class. Quantum Grav. 23 (2006) S141-S150.

Sun, K.-X., N. Leindecker, S. Higuchi, J. Goebel, S. Buchman, R. L. Byer, "UV LED operation lifetime and radiation hardness qualification for space flights," J. Phys. Conf. Ser. 154 (2009) 012028.

Sun, K.-X., N. Leindecker, J. Goebel, S. Buchman, R. Byer, "Space qualification for radiation hard UV LED," 3rd NASA EJSM Workshop, Applied Physics Lab, Johns Hopkins University, July 7-9, 2009.

Sun, K.-X., L. MacNeil, "Radiation hardness of AlGaN photodiodes," 4th NASA/ESA EJSM Workshop, Jet Propulsion Laboratory, July 26-29, 2010.

Sun, K.-X., L. MacNeil, "GaN radiation hard properties and detectors," SPIE Hard X-ray, Gamma Ray, and Neutron Detection, San Diego, August 1-5, 2010.

Sun, K.-X., L. MacNeil, K. Balakrishnan, E. Hultgren, J. Goebel, Y. Bilenko, J. Yang, W. Sun, M. Shatalov, X. Hu, R. Gaska, "Extreme radiation hardness and space qualification of AIGaN optoelectronic devices," International Workshop on Nitride Semiconductors 2010, Tampa, Florida, September 2010.

Sun K.-X., "Applications of robust, radiation hard AIGaN optoelectronic devices in space exploration and high energy density physics," Proc. CLEO: $A$ and T, Baltimore, Maryland, May 2011.

Sun, K.-X., "Applications of robust, radiation hard AIGaN optoelectronic devices in space exploration," Advanced Microelectronics and Photonics for Space (AMAPS) Review \& SBIR Forum, XII 2011, San Diego, California, June 2011.

Sun, K.-X., K. Balakrishnan, E. Hultgren, Y. Bilenko, J. Yang, W. Sun, M. Shatalov, X. Hu, R. Gaska, "Characteristics and applications of radiation hard, high reliability AIGaN optoelectronics devices and imagers for harsh environments," Workshop on Frontiers in Electronics (WOFE) 2011, San Juan, Puerto Rico, December 2011.

Sun, K.-X., A. Behnke, R. Buckles, "Radiation-hardened semiconductor detectors and detector arrays," Site-Directed Research and Development, FY 2011, National Security Technologies, LLC, Las Vegas, Nevada, 2012, 201-209.

Tanimura, Y., T. lida, "Effects of DD and DT neutron irradiation on some Si devices for fusion diagnostics," J. Nucl. Mat. 258-263, Part 2 (1998) 1812-1816. 


\title{
LARge-Area Graphene PANI Neutron Detector
}

\author{
STL-62-12 | YEAR 1 OF 1
}

Stephan Weeks, ${ }^{1, a}$ Kevin Kyle, ${ }^{a}$ William Quam, ${ }^{a}$ Rusty Trainham, ${ }^{a}$ Robert Vogel, a Shayla Sawyer Armand, ${ }^{a, b}$

Peter Dinoflo, ${ }^{b}$ Christopher Shing, ${ }^{b}$ and Harold McHugh ${ }^{c}$

The purpose of the project was to develop a thermal neutron detector using a chemically bonded neutron capture isotope in a polymer matrix, such as a boron trifluoride Lewis adduct of polyaniline (PANI-BF3 adduct). Because the neutron absorption with the resulting generation of charged particle reaction products and their subsequent cascade of secondary ion products occur directly within the active detector material, the theoretical intrinsic efficiency for neutron detection approaches unity. Graphene, a highly conductive allotrope of carbon, was proposed to be used to enable large-area, flexible sheets with phenomenal charge transport. The extraction of carriers (and/or detecting any changes in conductivity) as a result of neutron capture events was of primary focus in creating a viable solid-state semiconductor. In this work, multiple devices were created with various conductive materials including graphene. These devices attempt to

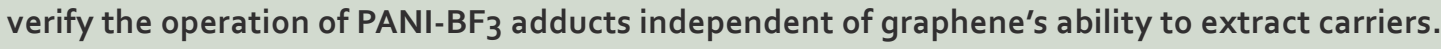
Results were compared. Although we could not confirm a detected signal from any of the devices, Schottky diodes were demonstrated, holding promise for charge carrier extraction.

${ }^{1}$ weekssj@nv.doe.gov, 805-681-2262

a Special Technologies Laboratory; ${ }^{\text {b Rensselaer Polytechnic Institute } ; ~}{ }^{c}$ Keystone International, Inc.

\section{Background}

\section{Review: Solid-State Neutron Detectors}

Solid-state neutron detectors are the subject of intense research for their potential use in, for example, remote screening of shipping containers, nonproliferation of special nuclear materials, and neutron forensics (Osberg 2006, Caruso 2010). Because they are compact and rugged, and their constituents are easy to obtain, they offer significant advantages compared to currently used gas- and liquid-based neutron detectors such as ${ }^{3} \mathrm{He}$ devices. Two categories of solid-state devices have been demonstrated in literature: indirect conversion and direct conversion heterostructures. Indirect conversion device geometries place a thin film of neutronsensitive material within range of, but external to, an adjacent space-charge layer (LiCausi 2008, Caruso
2010, Melton 2011, Dahal 2012, Murphy 2012). In contrast, direct conversion heterostructures contain a neutron-sensitive material that can also be the space-charge layer (Robertson 2002; Caruso 2006, 2010; Hong 2010). As a result, direct conversion is a straightforward method that has high total efficiencies. However, the number of materials capable of efficient neutron capture and electron-hole pair separation is limited. Because of their rarity, their processing, electrical properties, and carrier transport mechanisms are poorly understood. Therefore, addressing the trade-off between the use of mature semiconductor technologies with indirect conversion materials and the high total efficiency of immature direct conversion materials is the primary challenge in solid-state neutron detection research. In this work, a direct conversion heterostructure using a potentially 
flexible semiconductor is proposed. Because the boron in the boron trifluoride Lewis adduct of the

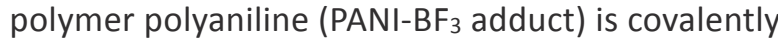
bonded, a semiconductor device is created to both capture neutrons and extract carriers with a Schottky diode device geometry by sandwiching the PANI-BF 3 adduct between two conducting electrodes.

${ }^{10} \mathrm{~B}$ captures thermal neutrons $\left[{ }^{10} \mathrm{~B}(\mathrm{n}, \alpha)^{7} \mathrm{Li}\right]$, releasing energetic particles into the surrounding medium, creating electron-hole pairs,

$$
{ }^{10} \mathrm{~B}+\mathrm{n} \rightarrow{ }^{7} \mathrm{Li}+\alpha(1.47 \mathrm{MeV}) .
$$

The optimal thickness of a ${ }^{10} \mathrm{~B}$ coating is $\sim 2-3 \mu \mathrm{m}$ before self-absorption of the alphas limits detection. $A{ }^{10} \mathrm{~B}$ coating of approximately $30 \mu \mathrm{m}$ would stop approximately $95 \%$ of incident thermal neutrons, but few alphas would escape to be detected. A Schottky diode device using a ${ }^{10} \mathrm{~B}$ adduct of PANI material could potentially possess the equivalent of $30 \mu \mathrm{m}$ of ${ }^{10} \mathrm{~B}$ and still permit most of the alphas to be detected inside of the diode because the ${ }^{10} \mathrm{~B}$ would be distributed internally. This configuration avoids the limitations of a simple coating, an indirection conversion, and may lead to more efficient devices that could be used to replace ${ }^{3} \mathrm{He}$ in some applications.

\section{Neutron Detection Mechanisms in PANI Polymer Device}

PANI with a ${ }^{10} \mathrm{~B}$ adduct can be used to form a semiconductor device (Yakuphanoglu 2008) that can detect thermal neutrons. There are two primary mechanisms by which the boron adduct of PANI polymers can detect thermal neutrons. In the first mechanism, electron-hole pairs are created in the $\mathrm{PANI}-\mathrm{BF}_{3}$ adduct when a $1.47 \mathrm{MeV}$ alpha and a $0.84 \mathrm{MeV}{ }^{7} \mathrm{Li}$ ion are released due to a thermal neutron capture. A reverse bias creates a depletion layer in the PANI-BF 3 adduct Schottky diode with an electric field that separates the carriers for collection of a single neutron capture event before recombining. The second mechanism is manifested by an integrated change in conductivity in the polymer due to altered crystal structure. The energetic particles that are released by the neutron interaction with the boron create defects that affect carrier movement throughout the material. This mechanism is not ideal because it does not provide the desired real-time measurement capability; however, the conductivity change could be an indirect measurement of the accumulated neutron exposure. If the latter mechanism is found, an increase of resistance is expected.

\section{PANI Polymer Characteristics}

The discovery of intrinsically conducting polymers occurred in 1960 and gained popularity in research because PANI is easily synthesized, is a low-cost monomer with good processiblity, has high conductivity, and has good chemical and environmental stability. Several oxidized forms of PANI exist, but the emeraldine base form is the only one that conducts in a doped state with different protonic acids. Doping is an acid-base reaction that occurs in two forms. The Brönsted reaction consists of protonation of the base form of emeraldine with a strong protonic acid. The Lewis adduct occurs due to the presence of the lone electron pair of the imine and amine sites, which reacts with a Lewis acid (LA). Lewis acids, such as $\mathrm{BF}_{3}$, are defined as molecules with an electron deficit capable of forming a coordination bond with emeraldine via its lone pair of electrons, as shown in Figure 1 (Basavaiah 2012). For this project, only acids containing boron were of interest.

With doping, PANI's conductivity can be altered to create materials that are insulators, semiconductors, or metals. The ability of PANI to form semiconductors depends upon the degree of unsaturation in the carbon-carbon chain and the nature of oxidative or reductive processing, the content and identity of dopants, and the purity of the material.

The preparation of PANI as a semiconductor follows a process (Basavaiah 2012) where the reaction mechanism of the PANI-BF 3 adduct is as given in Figure 2 . 
<smiles>CNc1ccc(Nc2ccc(N=C3C=CC(=Nc4ccc(C)cc4)C=C3)cc2)cc1</smiles><smiles></smiles>

Figure 1. Chemical structure of a Lewis acid $\left(\mathrm{BF}_{3}\right)$-adduct emeraldine base PANI

\section{PANI Schottky Diode}

As discussed, the junction of PANI and a metal layer creates a Schottky-type diode at the interface. The injection of carriers at metal contacts to organic semiconductors such as PANI is central to the semiconductor operation. At the metal-organic interface, the hole and electron barriers depend on the relative positions of the highest occupied molecular orbital (HOMO) and the lowest unoccupied molecular orbital (LUMO) with respect to the Fermi level of the metal (Hill 1998). Aluminum (Al) is the most commonly used metal for creation of a PANI Schottky diode; other metals also work.

The diagram in Figure 3 describes the behavior of the band gap mechanism of a PANI Schottky diode. Figure 3a illustrates a theoretical Schottky-Mott band

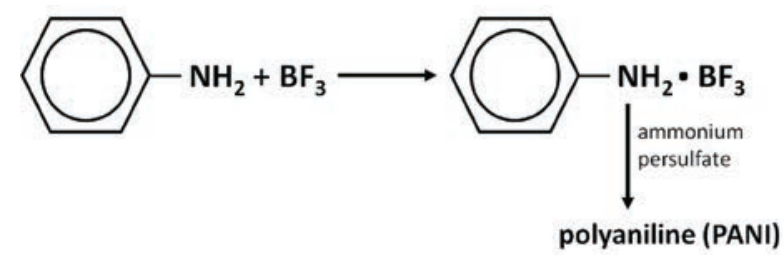

Figure 2. Reaction mechanism of $\mathrm{BF}_{3}$ adduct of PANI

gap model for an organic semiconductor where

$\Phi_{\mathrm{M}}=$ metal work function,

$\Phi_{\mathrm{Be}}=$ electron voltage barrier $=\mathrm{LUMO}-\mathrm{E}_{\mathrm{F}}$,

$\Phi_{\mathrm{Bh}}=$ hole barrier voltage $=\mathrm{E}_{\mathrm{F}}-\mathrm{HOMO}$,

$\mathrm{EVAC}_{\mathrm{VA}}$ = voltage at which the onset of photoemissions occurs under a vacuum for the metal,

$E_{\text {VAC (O) }}=$ voltage at which the onset of photoemissions occurs under a vacuum for the PANI, and

$E_{F}=$ voltage at the top of the occupied band of the electrons in the metal or Fermi level.

Note the alignment of the EVAC levels of the metal and the semiconductor in the ideal diode, shown in Figure 3a, as specified by the Schottky-Mott limit, (a) Ideal Diode

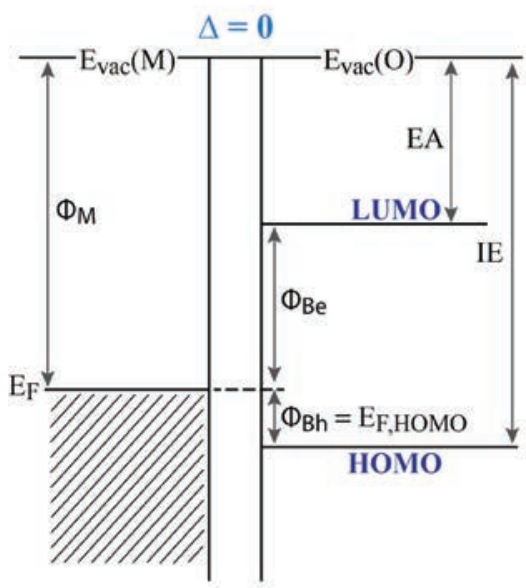

(b) Actual Diode

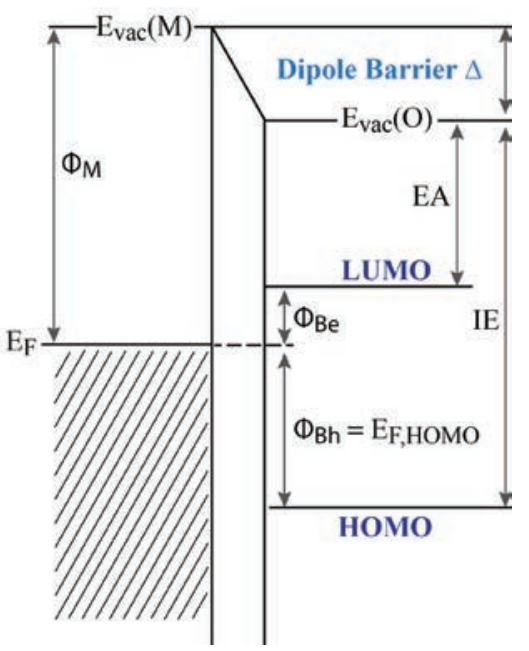

Figure 3. PANI-Al interface energy diagram (a) without interface dipole and (b) with dipole barrier $\Delta$ 
resulting in a large $\Phi_{\mathrm{Be}}$. Diodes that exhibit this behavior tend to have ideal diode behavior.

Most organic Schottky diodes have an extra band, called the dipole barrier (Figure $3 b$ ). The dipole barrier mechanism is not well understood at this time. The dipole voltage greatly reduces $\Phi_{\mathrm{Be}}$, which significantly changes the performance of the diode, particularly in the reverse bias depletion mode. Typically, a charge collection diode is operated with as large a reverse bias voltage as possible to remove as much charge from the depletion layer as possible. However, with a low $\Phi_{\mathrm{Be}}$, it is difficult to significantly deplete the junction layer. Increasing the reverse bias voltage beyond the breakdown point merely causes more current to flow, making charge collection difficult.

The adduct level of PANI and the amount of surface defects are two major contributors to the height of the dipole barrier and hence the resulting Schottky barrier voltage $\left(\Phi_{\mathrm{Be}}\right)$. For an ohmic junction, forward and reverse bias current conduction is symmetrical; in a Schottky junction, a forward bias permits a large current at low voltages, while a reverse bias typically restricts current flow to a small value. A depletion region is formed when a reverse bias is applied to the device, creating a strong electric field that is used to separate the carriers before they can recombine. The height of the Schottky barrier determines the amount of reverse bias that can be applied and the resulting width of the depletion layer within the PANI. The shot current noise is proportional to the reverse bias current, reducing the signal-to-noise characteristics of the device.

\section{Project}

\section{Scope and Objectives}

The project scope required fabrication of the $\mathrm{PANI}_{-} \mathrm{BF}_{3}$ adduct, its deposition on a conductive layer or substrate, deposition of conductive electrodes (contacts) on top, and preliminary characterization to determine its viability as a Schottky diode. The devices were subsequently exposed to a neutron source to determine if they produced a change in current from its exposure.

The project objectives below are detailed individually.

- Objective 1: Determine method(s) to produce $\mathrm{PANI}-\mathrm{BF}_{3}$ adduct semiconductor.

- Objective 2: Fabricate devices using various conductive materials for material comparison.

- Objective 3: Evaluate electrical properties of materials/devices.

- Objective 4: Measure thermal neutron response of devices.

\section{Objective 1: Fabrication of the PANI-BF3 Adduct}

Researchers Yakuphanoglu and Senkal successfully created a Schottky diode using a tin-doped indium oxide (ITO)-coated glass substrate, polyaniline, $\mathrm{BF}_{3}$, and aluminum contacts (Yakuphanoglu 2008). In our work, the devices with Al and graphene conductive layers were synthesized following their method. Then they were drop-cast from a saturated polymer solution in N-Methyl-2-pyrrolidone.
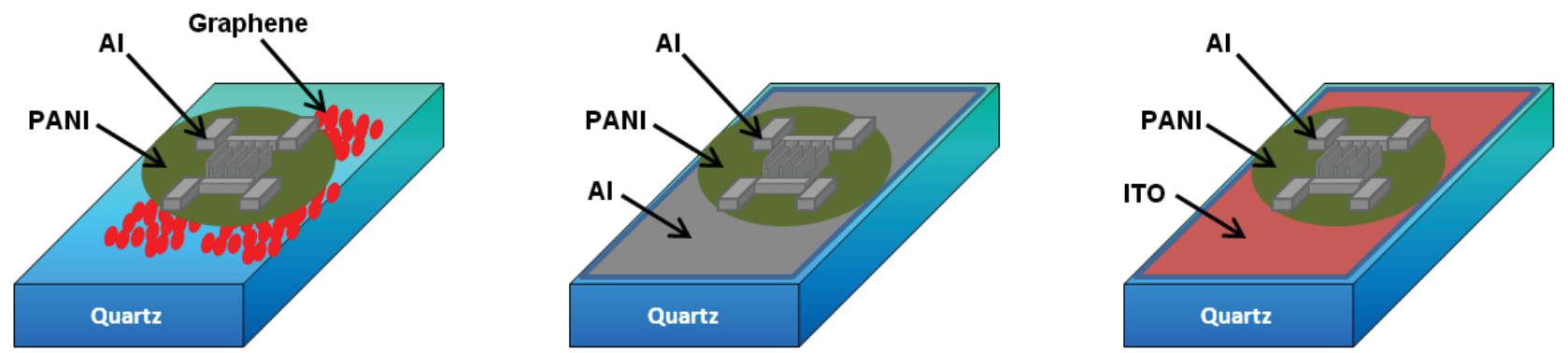

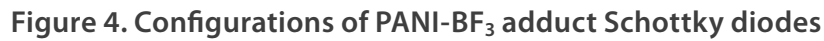




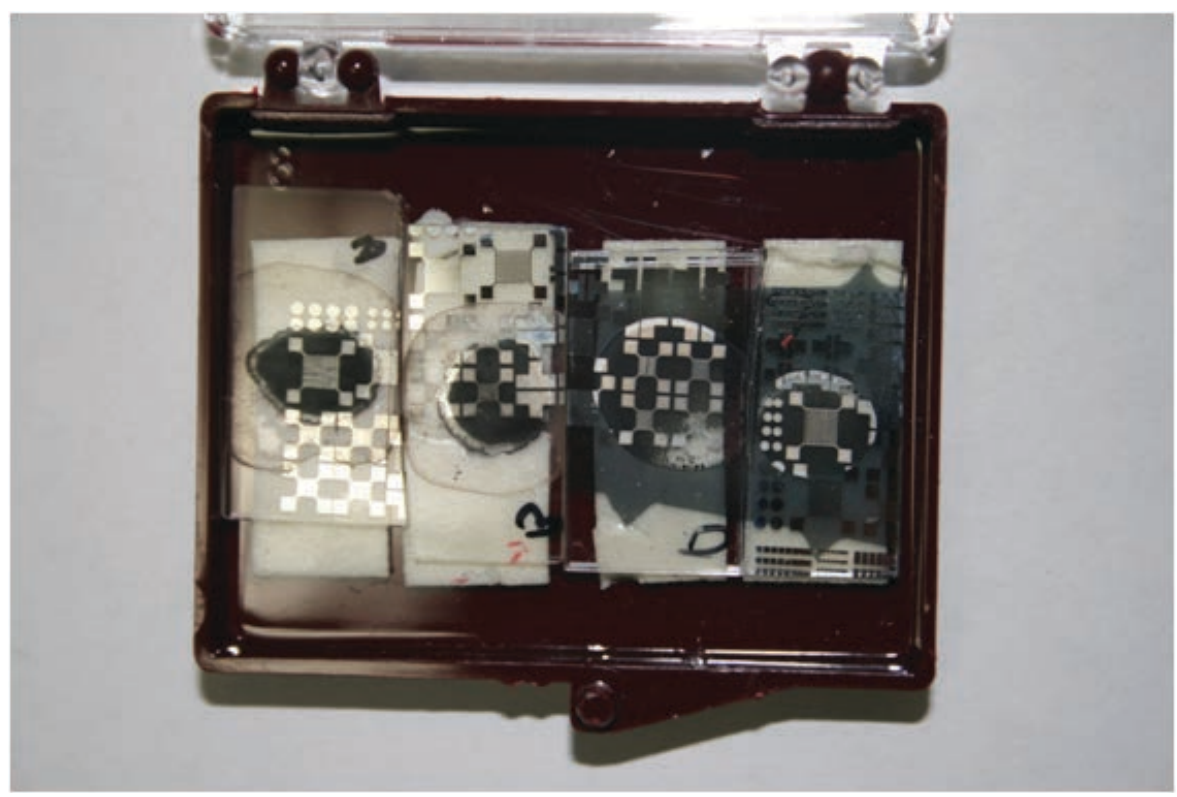

Figure 5. Box holding Al/ PANI/GQD and AI/PANI/AI devices

The $\mathrm{PANI}-\mathrm{BF}_{3}$ adduct samples on the ITO glass slides were deposited by electropolymerization in hydrochloric salt form. After electropolymerization, the slides were soaked in aqueous ammonium hydroxide solution to deprotonate the polymer, then the slides were dried under vacuum and, finally, soaked in an ether solution of $\mathrm{BF}_{3}$.

\section{Objective 2: Device Fabrication with Conductive Materials}

Various Schottky diodes are illustrated in Figure 4. A Schottky junction is formed whenever the $\mathrm{BF}_{3-}$ adducted PANI comes into contact with a metal or other conductor such as graphene. A conductive contact must be applied to both sides of the PANI, a semiconductor, to create a working device. Thus, each device has a pair of back-to-back Schottky diodes. When a voltage bias is applied to the device, one diode will be forward biased and the other will be reverse biased. The forward-biased diode will conduct freely; the current flowing in the device will be determined by the reverse-biased diode.

Different configurations of devices were fabricated with the following metal-semiconductor-metal contact configurations: Al/PANI/graphene, Al/PANI/ $\mathrm{Al}$, and $\mathrm{Al} / \mathrm{PANI} / \mathrm{ITO}$. The Al/PANI/ITO devices were made using 1,10 , and $100 \mu \mathrm{m}$ thicknesses of PANI. The sample devices had quartz slides as a structural base. The Al and ITO contacts were deposited using e-beam evaporation through a shadow mask. The contact configuration of the mask used existing designs for convenience and had little effect on these experiments, as conduction is vertical through the metallization. Examples of the devices are shown in Figure 5. The graphene was applied in the form of graphene oxide quantum dots (GQD) and deposited using a drop-cast method. The graphene can be seen in Figure 5 as a light yellow coating on the slide located on the left in the box. The PANI, also dropcast, appears as black discs in the photograph. The aluminum bottom electrode appears dark gray in the rightmost two devices.

\section{Objective 3: Material/Device Electrical Properties}

A current-voltage (I-V) curve was measured for each device. A picoammeter measured the reverse leakage currents of the PANI Schottky diodes. The I-V plot for the Al/PANI/GQD device (Figure 6) shows that this device exhibits the characteristic rectification curve with the expected rise in current with forward bias above a certain threshold and a small current below it. The reverse leakage currents are reasonably low for this device, making it potentially useful 

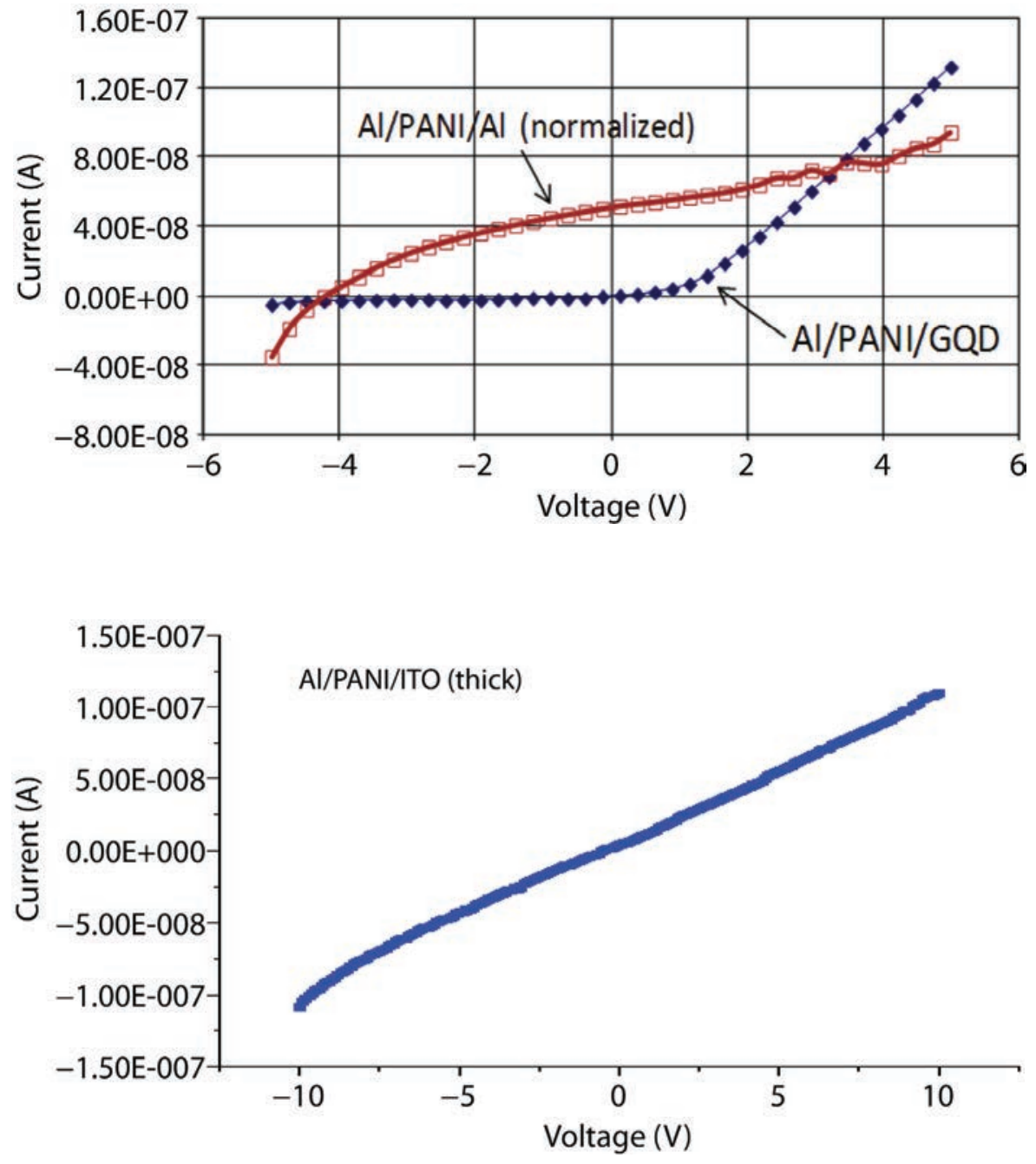

Figure 6. I-V curves for AI/PANI/GQD and AI/PANI/AI diodes. The reverse leakage current is reasonably low for the AI/PANI/GQD device, making it potentially useful as a detector. The AI/PANI/AI device diode did not show diode-like behavior.

Figure 7. Thick AI/PANI/ITO diode I-V curve shows a linear response, which is not the expected diode behavior, that may be due to surface defects or the deposition method as a detector, depending upon boron content. An I-V curve for the AI/PANI/AI device is also included in Figure 6. This curve is offset for clarity. It does not show a Schottky characteristic and is, in fact, a poor diode.

The plot for a thick (100 $\mu \mathrm{m})$ PANI device on ITO (Figure 7) shows that this device did not exhibit diode behavior. Conduction in the forward direction was reasonable, but no rectification or reduced current flow in the negative direction was evident. This sample device was created using electropolymerization rather than drop-casting due to severe cracking that occurred when drop-casting was attempted. The method of deposition may be the cause of the lack of a significant Schottky voltage barrier being formed. It is important to determine whether the method of deposition is the reason for the difference in I-V characteristics because thick devices are advantageous in that they contain more boron and are therefore theoretically more sensitive.

\section{Objective 4:Thermal Neutron Response of Devices}

Preliminary neutron response testing was initiated using a moderated neutron source (approximately 3 thermal neutrons $\mathrm{cm}^{-2} \mathrm{~s}^{-1}$ at the $17.5 \mathrm{~cm}$ distance in the probe station) to irradiate the PANI devices while the DC reverse bias diode current was monitored in the probe station. This simple test attempted to discern if there were any gross effects due to the neutron irradiation. It was during this testing that inconsistencies in the characteristics of the devices were encountered. The reverse bias 

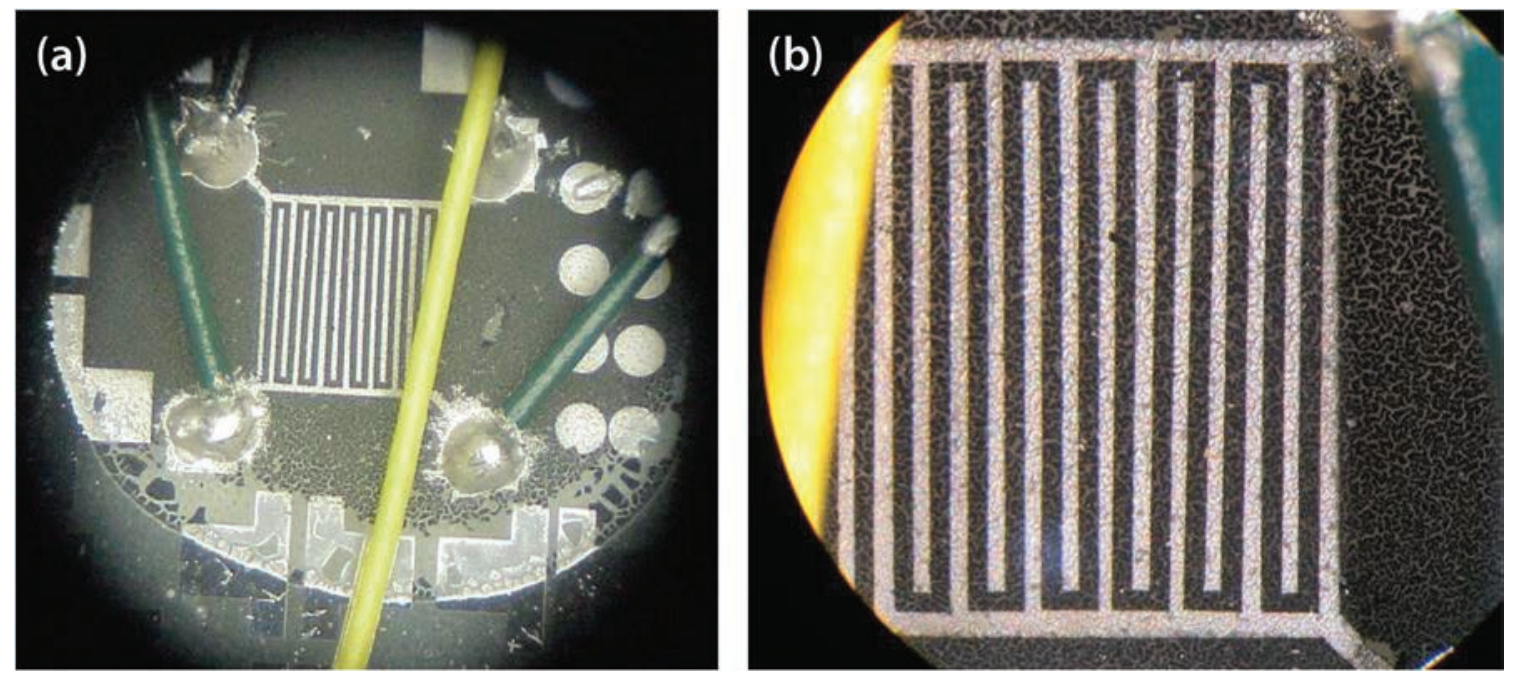

Figure 8. (a) PANI device showing lead wires and cracking, and (b) cracking seen on the PANI and Al electrodes

currents were changed dramatically over time; these changes were at first attributed to static electric or noise rectification issues. The reverse bias current vs time plot for Al/PANI/Al showed a fluctuating background current before irradiating with the source. A differentiation between the current when exposed to the bare source and after removing the source could not be confirmed through further testing. The fluctuation of current seemed to reduce significantly over the measurement time.

\section{Fabrication Difficulties Encountered}

A decision was made to attach leads to the devices and place the devices in a shielded metal enclosure to eliminate the inconsistencies mentioned above. While attaching lead wires, it became evident that the PANI had become severely cracked. We assumed that the cracking was progressing with time; it is probable that the cracking had started during the fabrication drying cycle. The change of resistance could be evidence of the progressing deterioration. Due to this condition, we were unable to take any more measurements to confirm the ability of any devices to detect neutrons. Subsequently, it was discovered that the PANI was cracking after the solvent went through the vacuum drying cycle. This cracking can be seen in Figure 8 . The aluminum metallization on the PANI also cracked, destroying electrical continuity of the traces. The first iteration of material and device fabrication provided some information about the characteristics of the materials. Iterative changes to the fabrication process, such as eliminating the vacuum drying cycle and drop-casting PANI on ITO, need to be done to improve device performance.

Measurements of the response to thermal neutrons for the Schottky diodes did not, therefore, show a signal above noise. Though changes of resistance may have been found for the AI/PANI/Al device, they could have been caused by the deterioration of the material rather than neutron source exposure. The latter could not be confirmed by further experiment because of cracking.

\section{Conclusion}

We have demonstrated that $\mathrm{PANI}^{-\mathrm{BF}_{3}}$ adduct materials can form Schottky diode devices with both aluminum and graphene contacts. Using GQD appears to form the higher quality diode. This work has shown potential for developing a solid-state, flexible largearea neutron detector, using new materialschemically-bonded boron within a polymer matrixto both capture neutrons and extract carriers with a Schottky diode device geometry.

The material cracking made the placement of electrodes and, therefore, the area from which charge collection could occur, problematic. Maximizing the 
embedded ${ }^{10} \mathrm{~B}$ concentration (loading) in the polymer (e.g., PANI) material, then developing methods to apply the material to devices that show uniformity and stability, plus the ability to extract signal from neutron capture events (e.g., Schottky diode) will allow the characterization of the devices. The working devices could then be placed in our shielded amplifier box (existing) to make pulse-counting measurements. Upon demonstration of feasibility, further planned work to develop multilayered devices and study other neutron capture elements $\left({ }^{6} \mathrm{Li},{ }^{157} \mathrm{Gd}\right.$ ) could proceed. The role of graphene to enable large-area detection devices and the ability to detect fast neutrons using the ${ }^{12} \mathrm{C}(\mathrm{n}, \alpha){ }^{9} \mathrm{Be}$ reaction could also be pursued.

\section{Acknowledgments}

We would like to thank Linda Chandos, Tony Botello, Rod Tiangco, Roger Bernal, Chusia Moua, and Glen Anthony for their support on this project.

\section{References}

Basavaiah, K., D. Samsonu, A. V. Prasada Rao, "Synthesis and characterization of boron trifluoride doped high performance polyaniline," E-Journal of Chemistry 9, 4 (2012) 2332-2337.

Caruso, A. N., P. A. Dowben, S. Balkir, N. Schemm, K. Osberg, R. W. Fairchild, O. Barrios Flores, S. Balaz, A. D. Harken, B. W. Robertson, J. I. Brand, "The all boron carbide diode neutron detector: Comparison with theory," Mater. Sci. Engin. B 135 (2006) 129-133.

Caruso, A. N., "The physics of solid-state neutron materials and geometries," J. Phys. Condens. Mater. 22 (2010)

443201-1-443201-32.

Dahal, R., K. C. Huang, J. Clinton, N. LiCausi, J.-Q. Lu, Y. Danon, I. Bhat, "Self-powered micro-structured solid state neutron detector with very low leakage current and high efficiency," Appl. Phys. Lett. 100, 24 (2012) 243507-1-243506-4.

Hill, I. G., A. Rajagopal, A. Kahn, Y. Hu, "Molecular level alignment at organic semiconductor-metal interfaces," Appl. Phys. Lett. 73, 5 (1998) 662-664.
Hong, N., J. Mullins, K. Foreman, S. Adenwalla, "Boron carbide based solid state neutron detectors: The effects of bias and time constant on detection efficiency," J. Phys. D: Appl. Phys. 43 (2010), 275101-1-275101-11.

LiCausi, N., J. Dingley, Y. Danon, J.-Q. Lu, I. B. Bhat, "A novel solid state self-powered neutron detector," Proc. SPIE 7079 (2008) 707908-1-707908-12.

Melton, A. G., "Development of wide bandgap solid-state neutron detectors," Dissertation, Georgia Institute of Technology, 2011.

Murphy, J. W., G. R. Kunnen, I. Mejia, M. A. Quevedo-Lopez, D. Allee, B. Gnade, "Optimizing diode thickness for thin-film solid state thermal neutron detectors," Appl. Phys. Lett. 101 (2012) 143506-1-143506-5.

Osberg, K., N. Schemm, S. Balkir, J. I. Brand, M. S. Hallbeck, P. A. Dowben, M. W. Hoffman, "A handheld neutrondetection sensor system utilizing a new class of boron carbide," IEEE Sensors Journal 6, 6 (2006) 1531-1538.

Robertson, B. W., S. Adenwalla, A. Harken, P. Welsch, J. I. Brand, P. A. Dowben, J. P. Claassen, "A class of boron-rich solid-state neutron detectors," Appl. Phys. Lett. 80 (2002) 3644-3646.

Yakuphanoglu, F., B. F. Şenkal, "Current-voltage and capacitance-voltage characteristics of the ITO/polyaniline doped boron trifluoride/Al Schottky diode," Polym. Adv. Technol. 19, 12 (2008) 1882-1886. 


\section{COMPUTATIONAL \& INFORMATION SCIENCES}
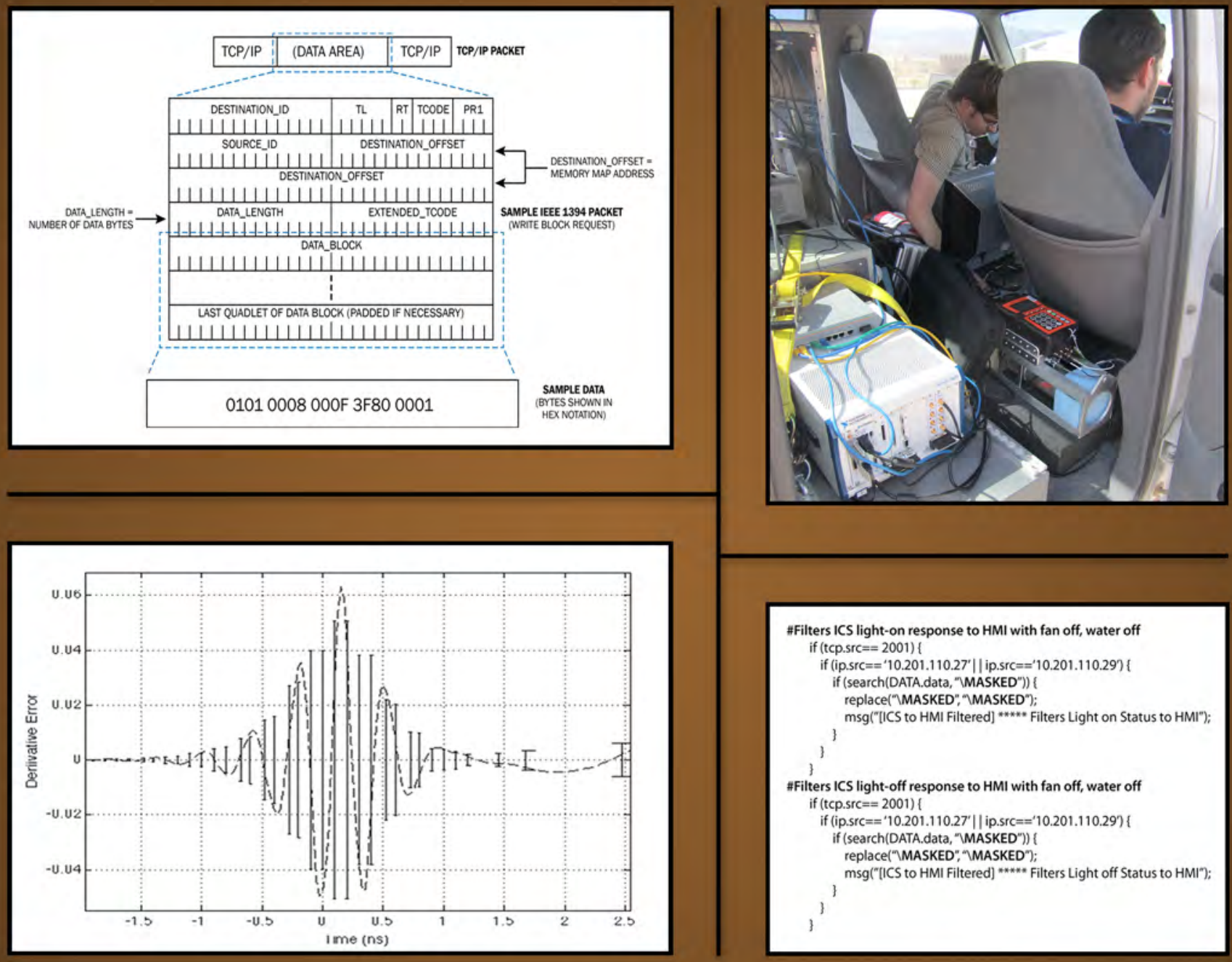


\section{An EXTENSIBLE ARCHITECTURE FOR SUPPORTING NeXt-GenERATION AERIAL RADIOLOGICAL MEASUREMENTS \\ RSLN-34-12 | YEAR 1 OF 1} Christopher Joines, ${ }^{1, a}$ Michael Reed, ${ }^{a}$ Ron Guise, ${ }^{a}$ Justin Schmidhofer, ${ }^{a}$ Ray Yost, $^{a}$ Matt Kiser, ${ }_{1}^{b}$ David Schwellenbach, ${ }^{c}$
Krikor Hovasapian, ${ }^{a}$ Paul Ainsworth, ${ }^{a}$ Ken Braithwaite, ${ }^{a}$ and James Essex ${ }^{a}$

A need exists for mobile and aerial data acquisition systems that can integrate additional sensors, handle and store more sensor data, exploit data from multiple instruments, and provide real-time displays for operators and analysts. For this effort we conceptualized a proof-ofprinciple data acquisition system, focusing on "sensor fusion" and real-time capabilities. The benefits of such an architecture would enable sensor fusion (more cohesive data management leading to a better answer faster), expand possibilities for accurate real-time processing, allow a more progressive algorithm development cycle, and introduce a data acquisition system that is more adaptable to non-remote sensing aircraft platforms. As a byproduct of this research, a number of significant new capabilities were established for integrating previously unsupported sensor formats into an emerging standard data acquisition software.

${ }^{1}$ joinescj@nv.doe.gov, 702-295-8071

a Remote Sensing Laboratory-Nellis; ${ }^{\mathrm{b}}$ Remote Sensing Laboratory-Andrews; ${ }^{\mathrm{c}}$ Los Alamos Operations

\section{Background}

During the decades that the Remote Sensing Laboratory (RSL) has performed aerial measurements in mission areas such as radiological emergency response, baseline radiological surveys, and periodic area monitoring, most detection capabilities improvements have been associated with industry developments in detector technology or improved electronics and computational systems. Detector technology improvement has been slow, and what has been produced is usually initially more for laboratory use and impractical for field operations; the most operationally significant enhancement opportunities relate to integrating modern data acquisition components and best-available data acquisition and display software.

Our goal was to develop a proof-of-principle data acquisition system that would integrate data from numerous sensors (a capability we are calling "sensor fusion") and provide real-time capabilities. To demonstrate the concept, a prototype system capable of collecting gamma, neutron, and photographic data was assembled and tested. The Extensible Architecture Next Generation Aerial System was conceived with the NSTec-designed Aerial Measuring System (AMS 2012, 2013) in mind but also with the intention that it could be scaled to support broader efforts such as R\&D. The term extensible architecture (EA) implies a system structure designed to be able to simultaneously handle input from multiple real-time data sources and also to somewhat seamlessly integrate new sensors and data types. A visualization of EA appears in Figure 1. This figure shows the laptop display screens that a test operator may view as high-resolution gamma, high-sensitivity gamma, neutron, and photographic data are being acquired and collectively displayed. 


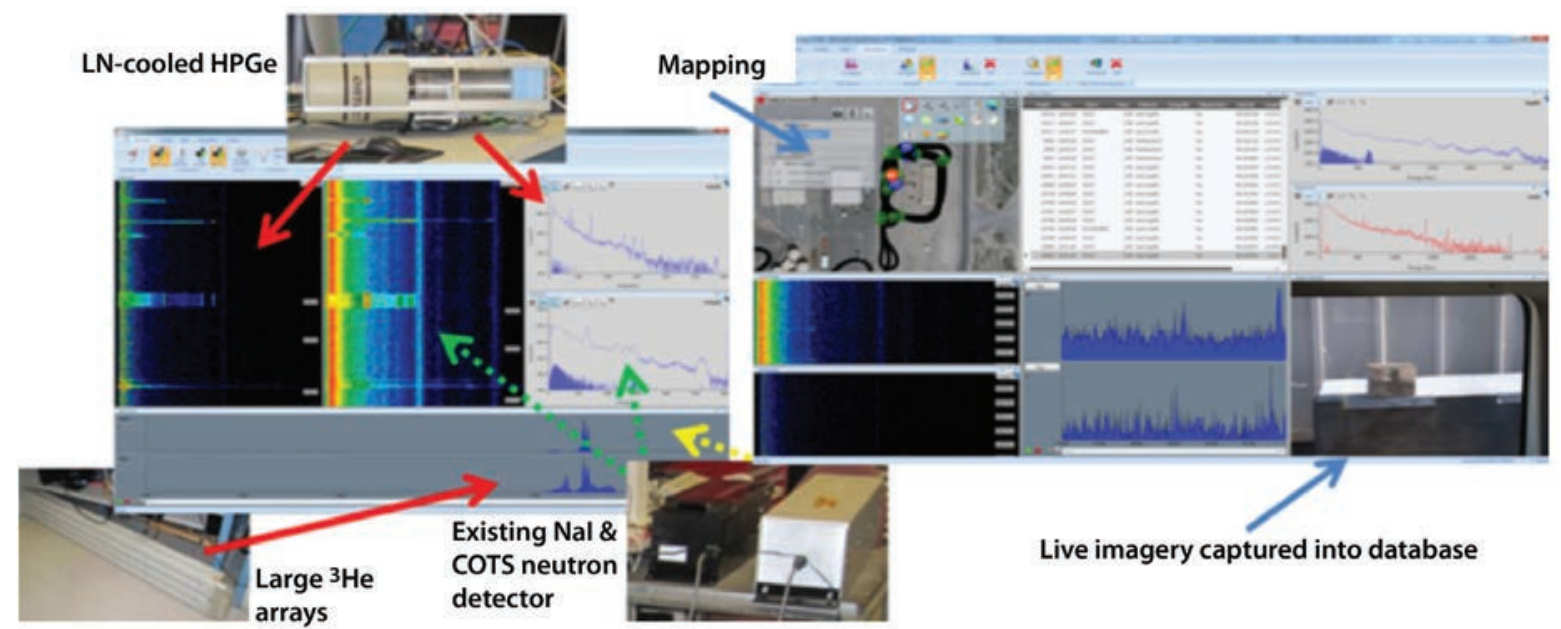

Figure 1. The extensible architecture (EA) concept is a system structure designed to simultaneously handle input from multiple real-time data sources and seamlessly integrate new sensors and data types

This way of merging and displaying data is an example of "data fusion," but the true power of the data fusion achieved here is not just the data display, but rather the ability for the multiple data types to be processed in real time by algorithms (such as alarming and mapping algorithms) that are highly beneficial during field operations.

\section{Project}

The Extensible Architecture Next Generation Aerial System includes components already available: Microsoft SQL data storage for random access and RSL's Advanced Visualization and Integration of Data (AVID) software for onboard data query. This approach would allow an expandable platform/architecture that would simplify the migration to industry standards and the integration of existing detector systems for immediate results. The elements added by our project include (a) a combination of data from individual sensor suites on a single acquisition "server," (b) AVID query and display of data during flight, and (c) an embedded photographic data radiation acquisition system.

AVID is an RSL-developed software capability that allows multi-window extractions in real time. It has full spectral mapping, 3-D spectral simulation, realtime contouring, telemetry capabilities, rapid production of standard products, and significant selection of input/output capabilities. AVID has an extensible software architecture, and this can be brought to the hardware level. AVID is database driven, which ensures a high degree of generalization, and it is relatively easy to add other platforms to it. The modular architecture allows end users to construct their own modules for inclusion into the framework, and it promotes efficient reusability of code. Because each AVID module spawns its own set of threads, the software is scalable, and its processing resources are more effectively managed across all concurrent modules. The basic graphical user interface (GUI) provided by the framework is a set of tiled window panes. Each pane may be arbitrarily resized, docked in a single window, undocked and allowed to float, or tabbed behind existing panes.

The system used existing gamma and neutron detectors. The sodium iodide ( $\mathrm{Nal}$ ) detector is integral to gamma detection and mapping operations, and the commercial Radiation Solutions, Inc. (RSI), detection system flown aboard RSL's Bell 412 helicopter includes twelve 16-inch Nal detectors. Data from the RSI system are available as a TCP/IP socket data stream that also provides GPS data. A stream manager has been created to allow AVID to support the legacy RSL mobile Nal system, which allows expansion of the concept to support other types of detectors: high-purity germanium (HPGe), helium-3 $\left({ }^{3} \mathrm{He}\right)$, neutron arrays, and optical data. 
(a) $\mathrm{LN}_{2}$-cooled detector

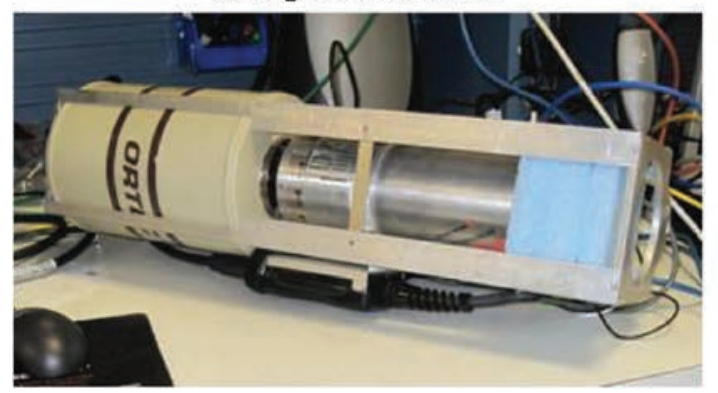

(b) Array of $2^{\prime \prime} \times 13^{\prime \prime} 3 \mathrm{He}$ detectors

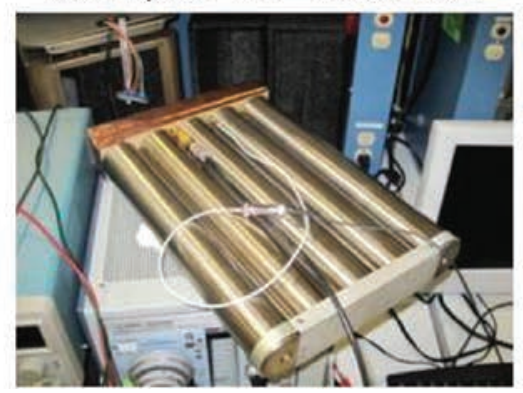

(c) ${ }^{3} \mathrm{He}$ detector counter interface

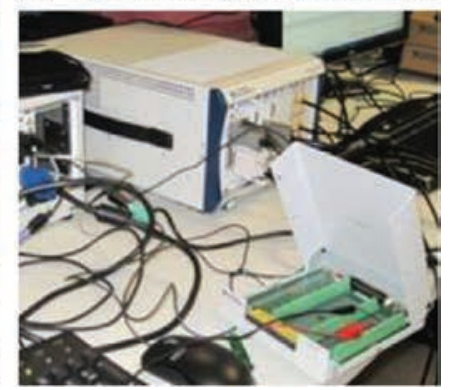

Figure 2. (a) $\mathrm{LN}_{2}$-cooled HPGe and (b) ${ }^{3} \mathrm{He}$ detectors used in the system would require a new (c) electronics interface; we chose to use a National Instruments PXI chassis

The major innovation of this project is the integration of a diverse range of detector types for use with AVID. To integrate additional detectors (Figure 2) such as liquid nitrogen $\left(\mathrm{LN}_{2}\right)$-cooled HPGe and ${ }^{3} \mathrm{He}$ neutron arrays, interface electronics are required. After reviewing available options, we determined that the best approach was to use an existing PXI chassis and PXI data acquisition boards from National Instruments (NI) and other manufacturers. The use of this unit adds further modularity to the system, as NI has already produced drivers for a wide range of data acquisition hardware and also offers users customization flexibility with software products such as LabVIEW.

The interface of the $\mathrm{LN}_{2}$-cooled HPGe unit was accomplished using an Xia Pixie-4 digitizer board capable of producing spectra of over 32,000 channels. The controller for the PXI chassis is a Windows-based processor, which has full networking capability, allowing the spectra to be transferred to AVID via TCP/IP. The interface of a GPS receiver to the chassis allows for precision timing and synchronization of data, as well as geotagging of the data.

The approach for the ${ }^{3} \mathrm{He}$ array interface with the PXI chassis was similar to that of the HPGe. The PXI chassis was configured with a counter board and external cabling interface (Figure $2 c$ ) to allow the neutron pulses to be counted on a second-by-second basis and relayed to AVID. While the demonstration system is limited to simple neutron counting, the architecture and hardware was configured to allow neutron multiplicity pulse-timing measurements with the Pixie-4 microchannel analyzer (MCA).

While many possibilities exist for adding optical sensors, the concept prototype leveraged a documentation-level camera that was on-hand: a Canon VB60 with an Ethernet interface. The CameraLink interface, part of the PXI chassis, included an industry standard that allows firing synchronization, which would allow integration of high-end aerial framing cameras in the future.

Figure 3 shows the resulting conceptualized data flow. The significance of the architecture is the location of the database, which can reside either on the PXI acquisition chassis or on the database, residing on the AVID display laptop.

Software was developed to enable the PXI chassis to digitize and process inputs from various detectors. The inter-thread and inter-process communication is managed by a multi-threaded application written in $\mathrm{C}++$. The service collects sensor data from a Xia Pixie-4 MCA in a sensor-independent way. The Pixie-4 MCA is configured with the pulse characteristics of a given sensor and provides individual pulse data, including time and energy. The software then creates a histogram of pulse energy that is ultimately passed to AVID.

The software is split into several threads (Figure 4) that read the event data from the MCA, parse the data into an event queue, collect GPS data, and 


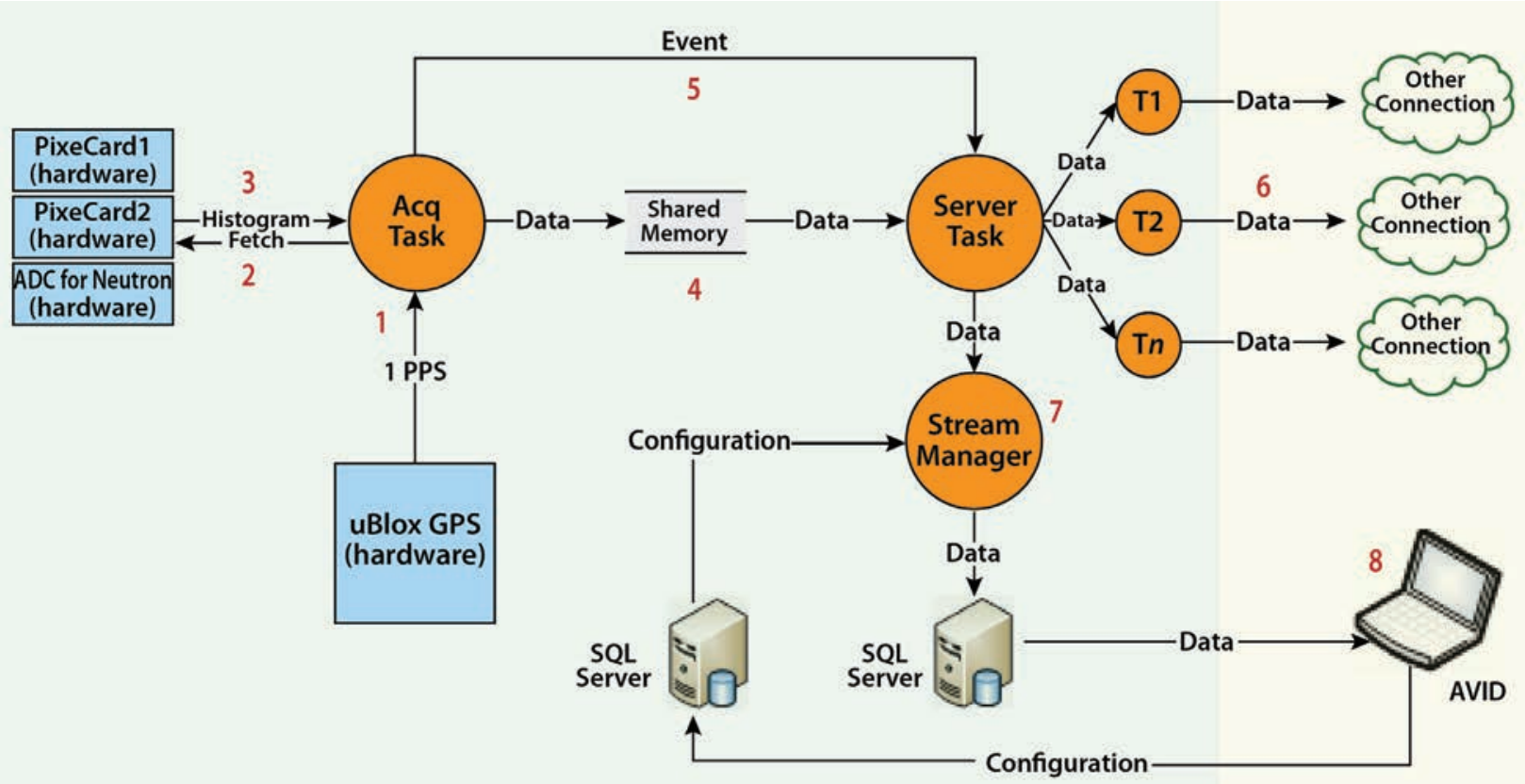

PXI Chassis (Host)

Client(s)

Figure 3. Conceptualized data flow; in this architecture the database may reside on the PXI chassis or on the laptop (red numbers indicate path of data)

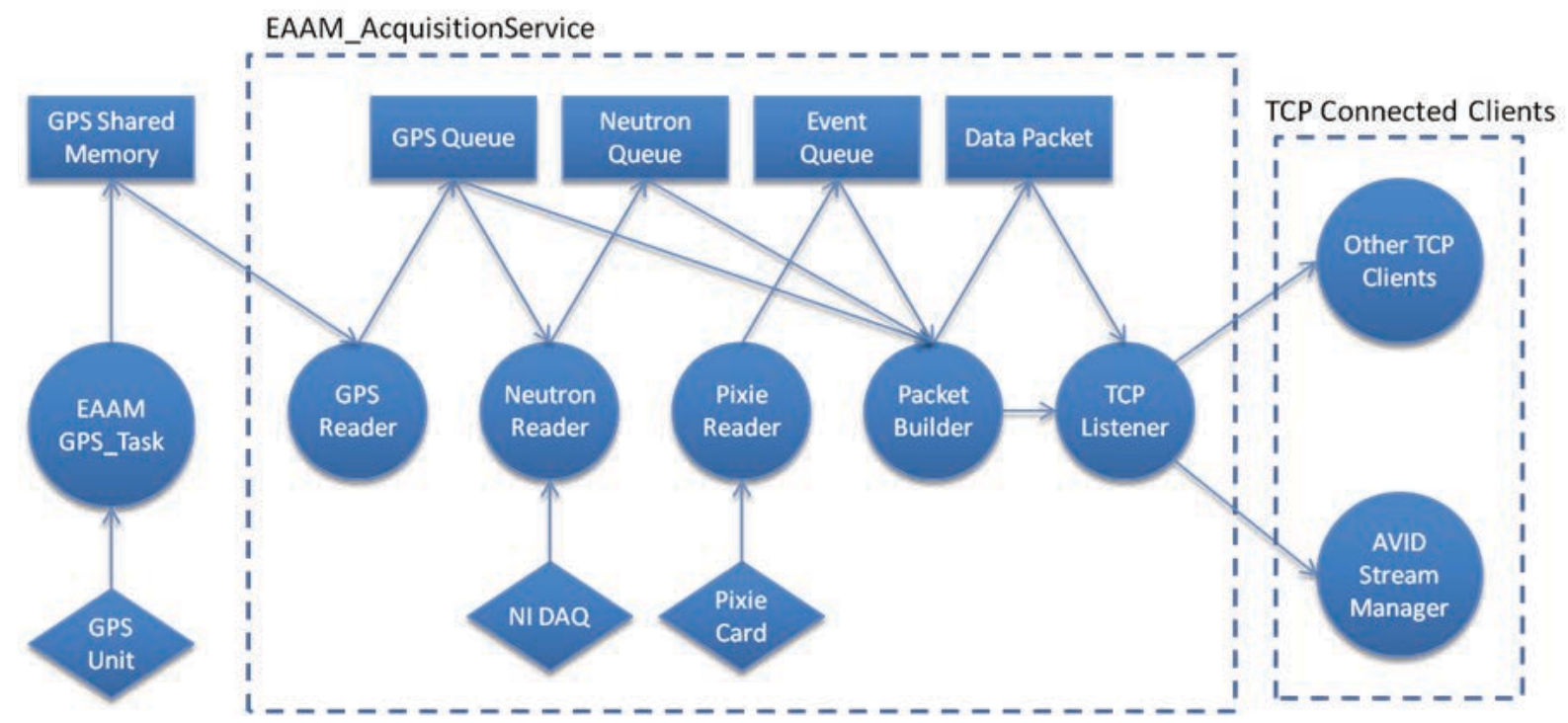

Figure 4. EAAM_Acquisition Service inter-thread and inter-process communication schematic

collect neutron data from an NI data acquisition (DAQ) device. All data are combined by the packet builder thread. The data are placed in a packet that emulates an RSL mobile system. A TCP listener thread listens for incoming network connections from external applications, and sends the packet when it is available. One of these external applications is usually the AVID Stream Manager, a Windows service that collects data from multiple sources and saves it to a database. These data are then accessible to AVID through the database. 
(a) CameraLink and Ethernet cameras

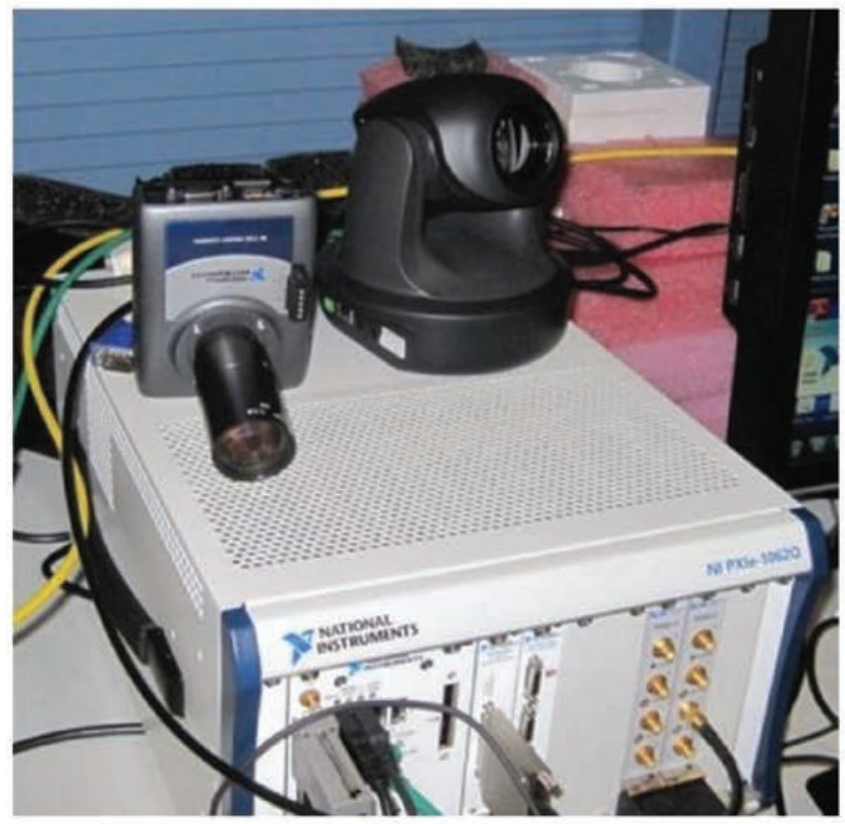

(c) Display of sample results of static vehicle testing

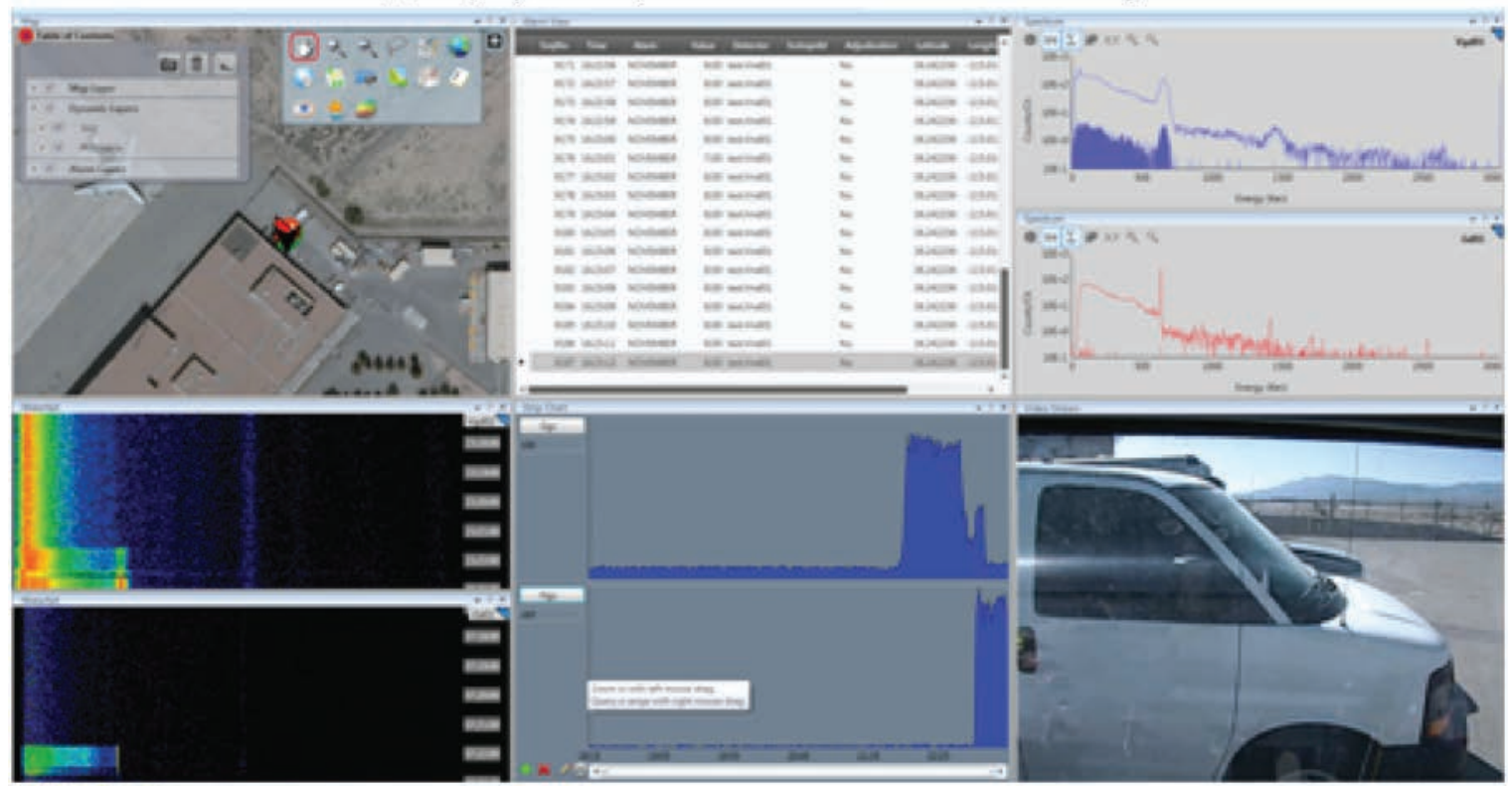

Figure 5. Initial operational testing was conducted by placing the system in a vehicle and equipping it with several detectors

\section{Implementation and Testing of the Prototype EA System}

With the acquisition software and the stream manager to push data to AVID mature, the system was placed in a vehicle and equipped with several

(b) Static testing in vehicle

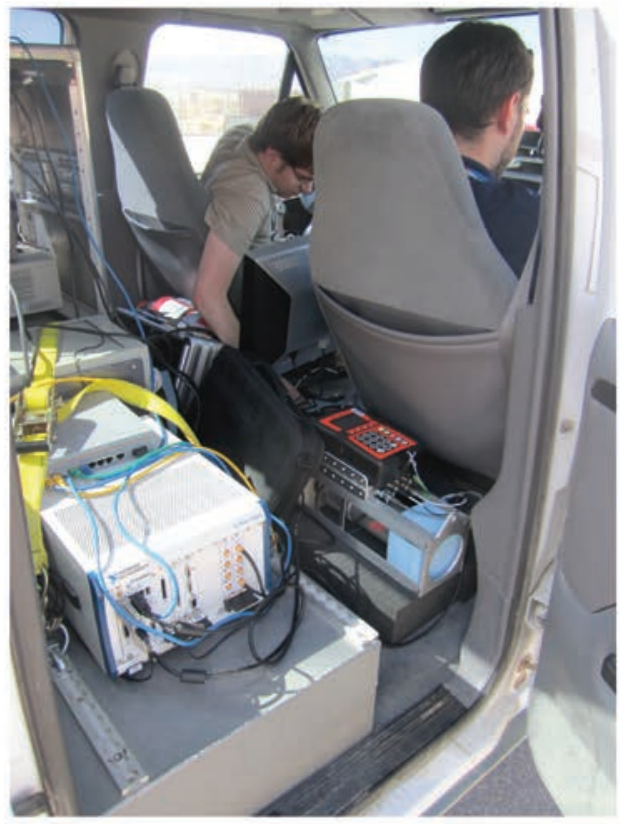

. 


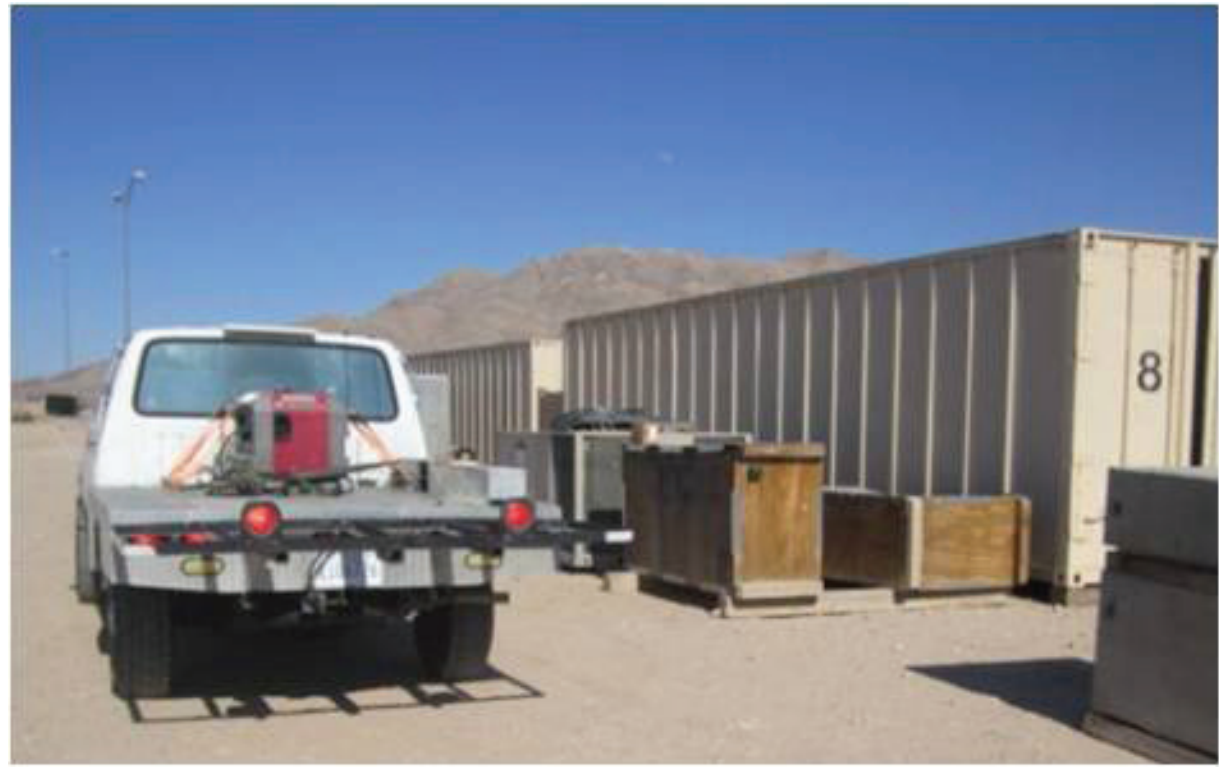

Figure 6. The system passes a package containing sources

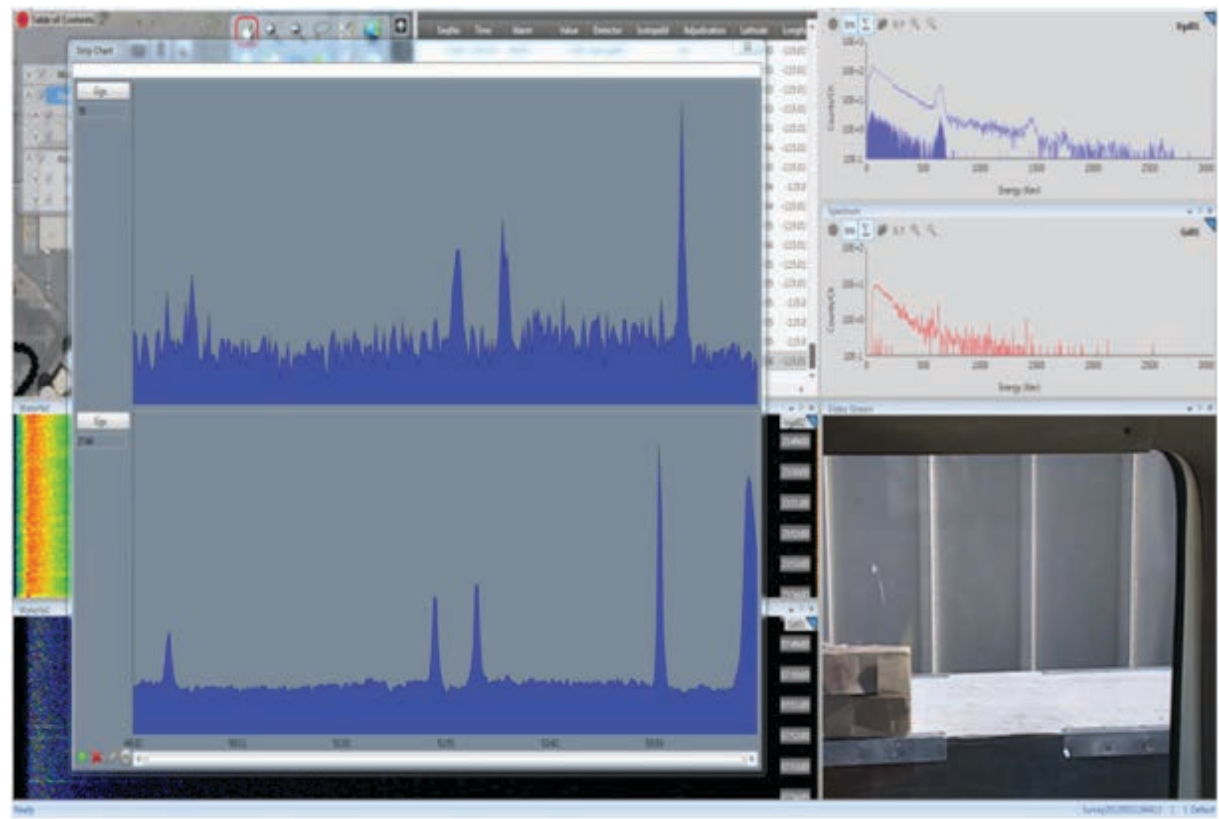

Figure 7. AVID display as vehicle passes package in Figure 6

captured video documentation of the event along with the collected HPGe, Nal, and ${ }^{3} \mathrm{He}$ data. Sample results are shown Figure 7.

Support for additional sensors was also added (Figure 8) at this time. The final concept prototype can acquire data not only with the previously mentioned detectors, but also with the 72-inch neutron array, the 13-inch backpack neutron array, and the ORTEC Detective mechanically cooled HPGe detector. The
RSL helicopter 72-inch neutron array is of particular interest for future operational use. Several arrays are available, each featuring eight tubes per array (72 inches long and 2 inches in diameter). The mechanically cooled HPGe is the type of high-resolution gamma detector that is rapidly becoming the standard for field use.

Integration was also completed for the ORTEC mobile system, another commercial detector system 

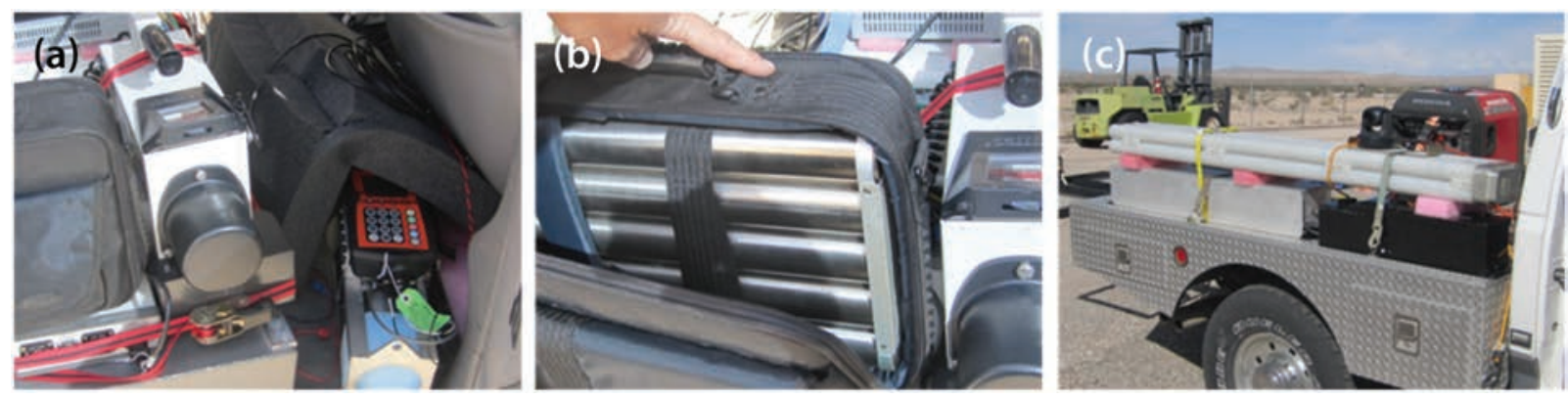

Figure 8. (a) Mechanically cooled and $\mathrm{LN}_{2}$-cooled HPGe detectors, (b) 13 -inch ${ }^{3} \mathrm{He}$ array, and (c) 36 -inch ${ }^{3} \mathrm{He}$ array configured for final operational testing

featuring 16 -inch $\mathrm{Nal}$ crystals and 36 -inch ${ }^{3} \mathrm{He}$ arrays. AVID now has the capability of gathering and managing data from any detector that can be interfaced to ORTEC's Digibase MCA. This allows future use of many different detectors with the EA system.

To demonstrate the real-time aspect of the EA system's multiple sensor display, ${ }^{137} \mathrm{Cs},{ }^{60} \mathrm{Co}$, and ${ }^{252} \mathrm{Cf}$ sources passed by the system. As shown in Figure 9, AVID was able to simultaneously display data from three neutron detector types (RSI, 6-foot array, 13-inch array), two HPGe detector types
(ORTEC Detective, $\mathrm{LN}_{2}$-cooled), and one Nal gamma detector. The display also shows photographic evidence of the cause of the increased signal.

An MCA with 1024 channels is assumed to be sufficient for the Nal data, but now that HPGe data are being used, a trade-off must be considered based on the value of increased number of recorded channels vs the burden of the increased volume of data. In this implementation, 1024 channels were used for the $\mathrm{LN}_{2}$-cooled HPGe detector, and 8192 channels were used for the mechanically cooled detector.

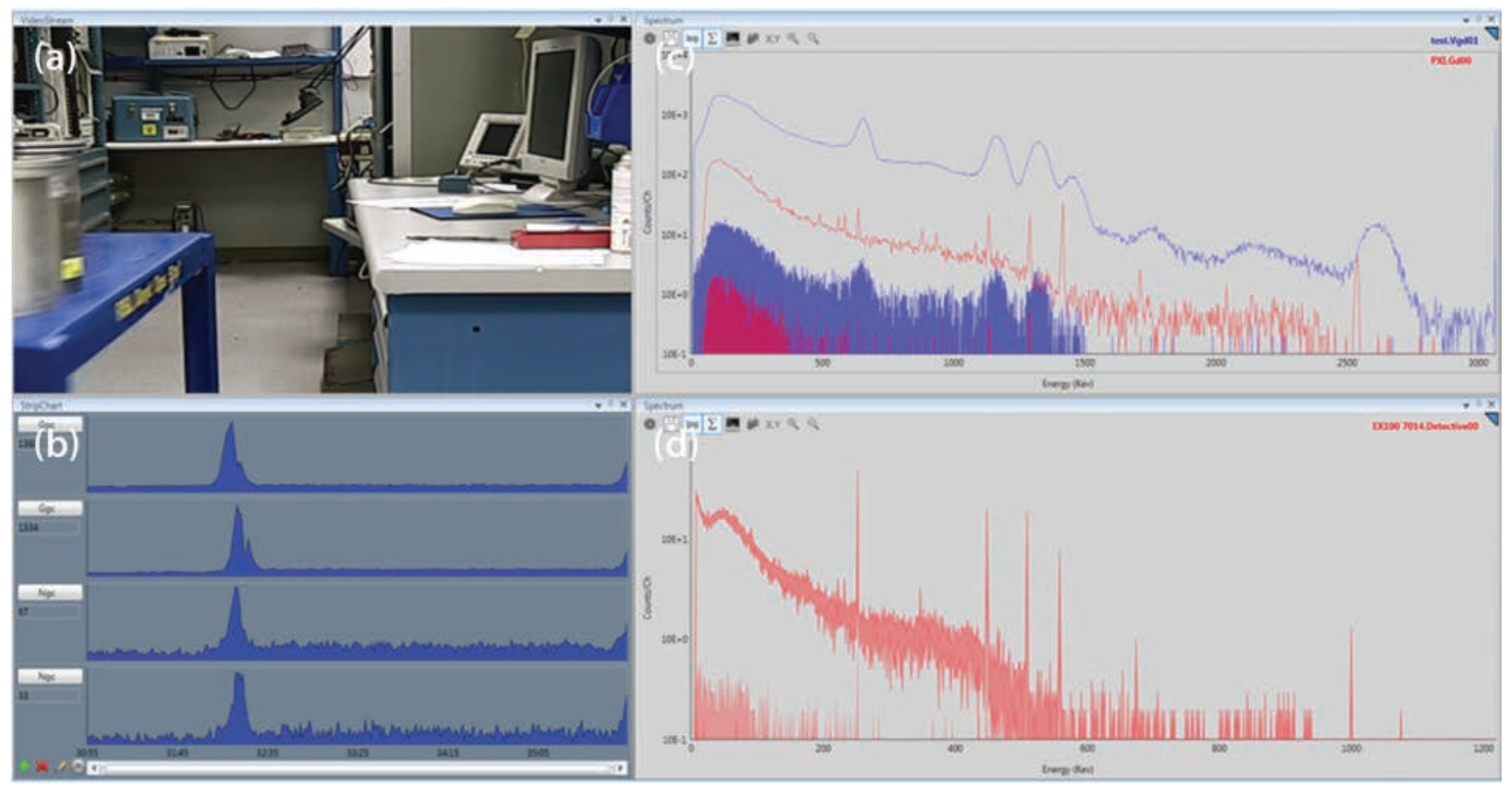

Figure 9. Screenshot of AVID software as a mix of isotopes are rolled past the prototype system. The mix includes ${ }^{137} \mathrm{Cs},{ }^{60} \mathrm{Co}$, and ${ }^{252} \mathrm{Cf}$ isotopes. (a) Image capture of photographic sensor recording video; (b) time behavior of gross gamma and gross neutron response; (c) single sample and accruing spectra from commercial HPGe and Nal detectors, and (d) single sample and accruing spectra from an HPGe detector implemented through generic MCA present on PXI chassis. 


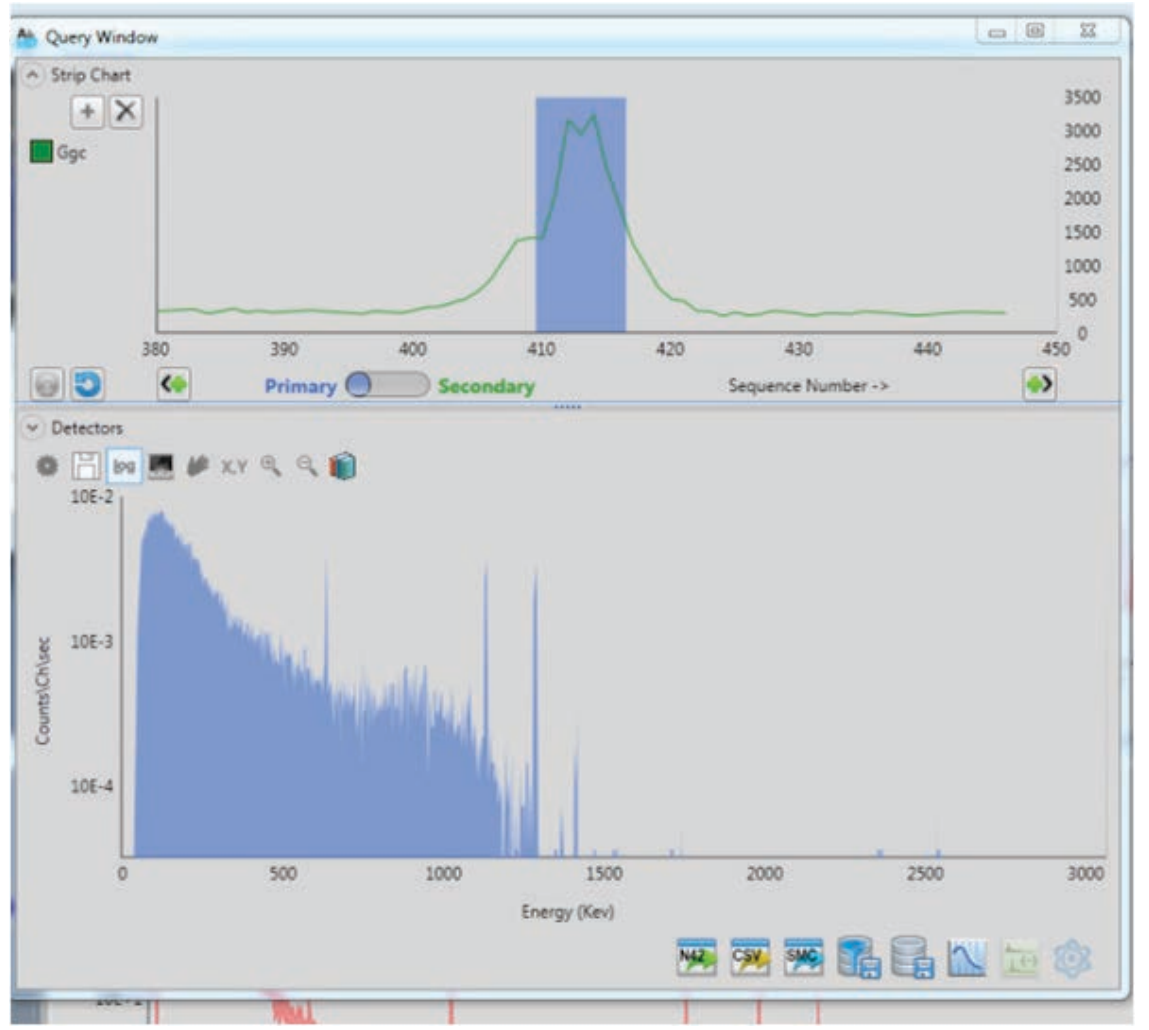

Figure 10. Spectral extraction allows several seconds of data to be selected and the summed data to be displayed in a histogram

A major benefit of leveraging the AVID software base for this system is the availability of data query and display tools, such as strip chart selection, waterfall selection, and map selection. Selected data may be viewed or exported. Figure 10 shows an example of the spectral extraction from the strip chart. Figure 11 shows the value of the waterfall display for making obvious the spectral change when the source is moved or removed entirely. The ability to notice an anomaly is valuable to an operator who is driving or flying.
Sensors other than radiation detectors can someday be easily integrated into the EA system. GPS and radar altimeter data are central to the radiological mapping mission, and can be included as well as data from light detection and ranging (lidar) instruments.

Photographic data will allow for feature recognition and documentation of what was present when the system flew or drove past the area. Lidar will provide an improved method of terrain processing for radiological mapping.
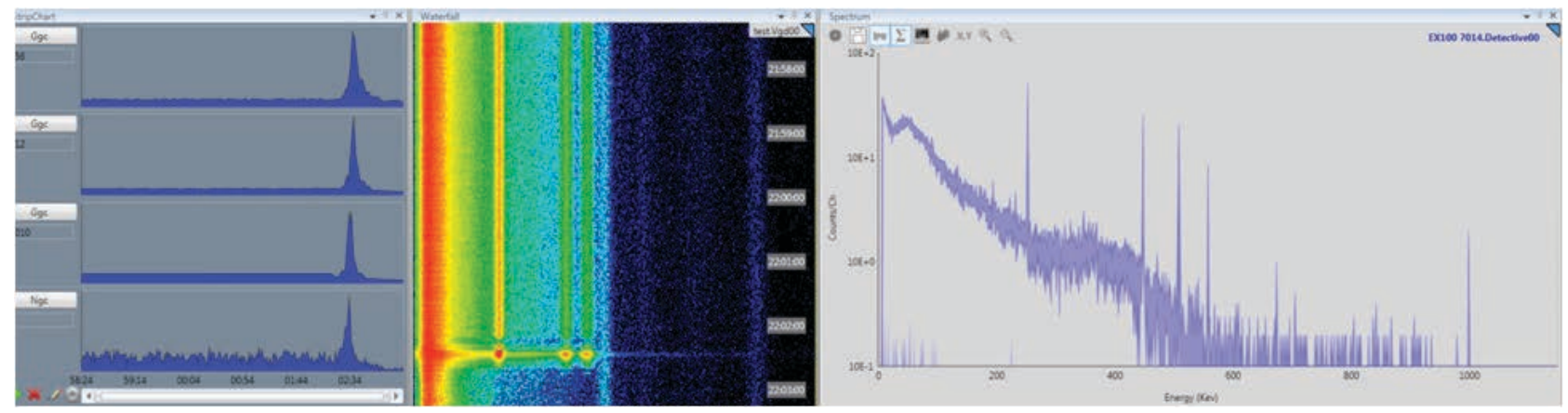

Figure 11. Partial screenshot of AVID display showing multiple neutron detector data, 1024-channel waterfall display of $\mathrm{LN}_{2}$-cooled HPGe detector data, and 4096-channel mechanically cooled HPGe detector data 
This EA concept enables new capabilities that were previously not possible:

- $\quad \mathrm{LN}_{2}$-cooled HPGe analog output can be digitized and streamed real time into AVID.

- ORTEC Detective (mechanically cooled HPGe) data can be incorporated and displayed live using AVID.

- ORTEC mobile data (Nal gamma and neutron) can be incorporated and displayed live using AVID.

- Neutron data from legacy ${ }^{3} \mathrm{He}$ arrays (most significantly the AMS 6-foot array) can be digitized and streamed real time into AVID.

- Video/still cameras and images can be integrated into the same database and display software.

- Data from these five newly added detector types are incorporated into a single database and can be displayed on a common time scale.

\section{Conclusion}

A concept, architecture, and demonstration system have been produced to prove the EA concept for radiological aerial measurements. A promising direction is the ability to interface multiple HPGe detectors to complement higher-sensitivity Nal detectors. The EA system is ideal for use as a research platform, as it can rapidly integrate new sensor types. The ability of the Pixie-4 MCA to collect pulse-time measurements will simplify neutron multiplicity research. This system is the foundation on which future acquisition systems will rest.

\section{References}

AMS Program, 2012. AMS Radiological Mapping Mission Briefing FY 2013.

AMS Program, 2012. AVID for AMS Users - Initial Training Presentation.

AMS, http://www.nnsa.energy.gov/ams, 2013. 
This page left blank intentionally 


\section{UNCERTAINTY ESTIMATION TECHNIQUES FOR HIGH-DENSITY 1-D MPDV IMAGING DIAGNOSTIC \\ NLV-21-12 | CONTINUING IN FY 2013 | YEAR 1 OF 2}

Eric Machorro, ${ }^{1, a}$ Jerome Blair, ${ }^{b}$ Aaron Luttman, ${ }^{a}$ and Ed Daykin ${ }^{b}$

The goal of this project was to refine and extend uncertainty quantification techniques developed by NSTec to high-density multiplexed photonic Doppler velocimetry (MPDV) measurements and, in particular, to see how to extend them to the 2-D measurements that are now the state of the art. This project focused on improving the methods of error estimation under high-noise conditions, providing rigorous mathematical results for analyzing errors, and taking high-quality MPDV data and high-speed video of a 1-D system to verify and evaluate the various estimation methods.

${ }^{1}$ machorea@nv.doe.gov, 702-295-5352

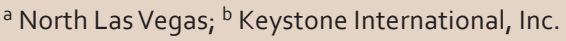

\section{Background}

The photonic Doppler velocimetry (PDV) diagnostic is based on interferometric measurements of Dopplershifted laser light. First fabricated in its current form by Strand (2006), it can measure velocities at a single point on a scale from $10 \mathrm{~m} / \mathrm{sec}$ up to $10 \mathrm{~km} / \mathrm{sec}$ on timescales that can range from $1 \mathrm{~ns}$ to $1 \mathrm{~ms}$. The robust dynamic scale in which it operates has made it an excellent diagnostic for experimenters interested in shock physics, equations of state, and dynamic material properties.

The most expensive component of the system is the oscilloscope used to record the signal. Subsequent innovations allowed multiplexing wherein several PDV measurements could be recorded nearly simultaneously with only a modest increase in hardware but at a slight loss of resolution or bandwidth. This improvement complicates the analysis of the data recorded from the system and highlights the need to quantify the uncertainties associated with this diagnostic, and presents challenges in cross-validating these measurements with other diagnostics.
This project sought to extend the error estimation techniques developed by NSTec for nuclear event analysis to quantify uncertainty in high-density multiplexed photonic Doppler velocimetry (MPDV) measurements. MPDV systems have three major signal processing problems: high noise levels, crisscrossed signals, and very large data sets (Figure 1).

The project focused on (a) improving the methods of error estimation under high-noise conditions, (b) developing mathematical proofs for several prospective alternatives for analyzing errors, and (c) taking high-quality MPDV data and video of a 1-D system that could be used to verify and evaluate the various estimation methods.

A rapidly growing number of experiments use large data sets recorded on digital oscilloscopes. The instruments generally have noise levels that are much higher than desired, so the data need to be smoothed to be useful. Increasing the amount of smoothing reduces the noise in the final result. However, 


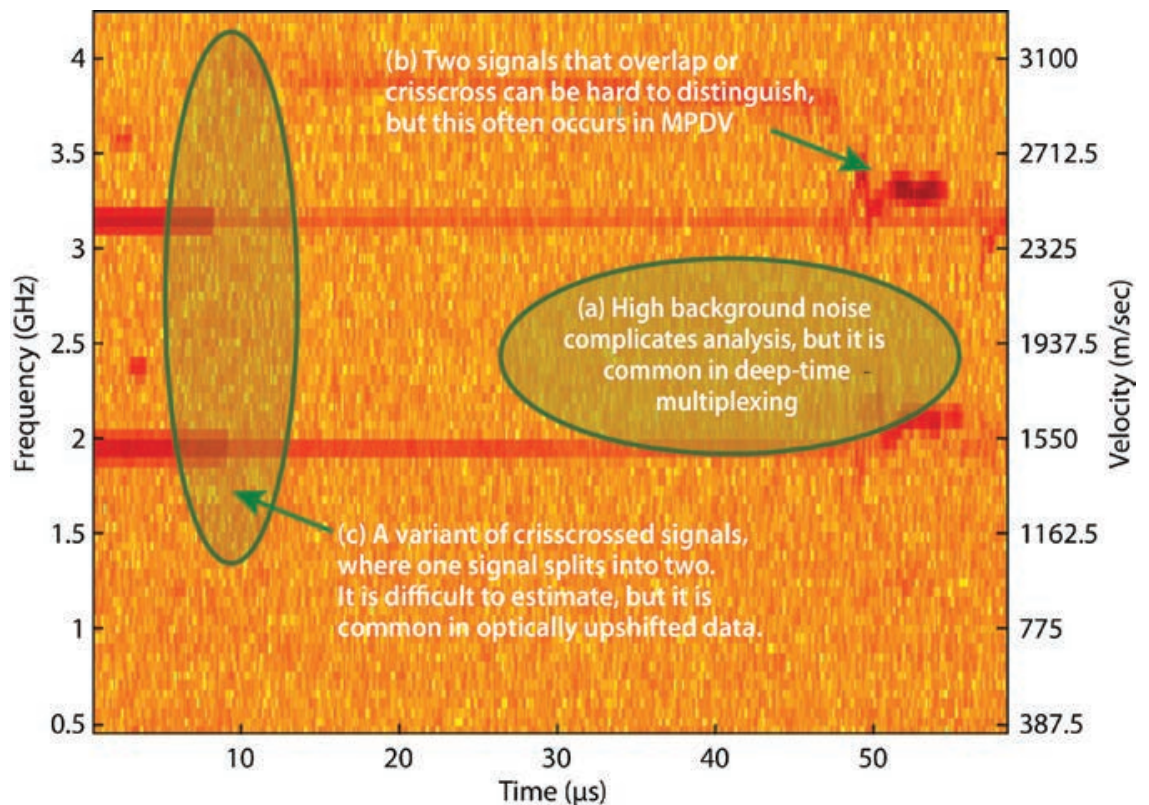

Figure 1. An example spectrogram shows the three major measurement problems identified that we addressed. The red areas indicate the magnitude of the Fourier transform of a rolling, overlapping sequence of windows. The deeper the red, the more dominant the frequency. In PDV measurements, the frequency is proportional to the velocity by a known constant. increasing the amount of smoothing increases another error called the bias error. For example, when a smoothing operation is applied to a pulse, it generally makes the amplitude of the pulse smaller and the width larger. When reporting data results, it is good to supply error bars that include major sources of error, such as noise and bias. Previously, NSTec developed a method called Statistically Based Spline Fitting that accomplishes this (Blair 2007). This method is extremely computationally intensive for the large data sets that are common for PDV data. A straightforward, and usually near optimum, approach that works for large data sets is local polynomial fitting (LPF). In this method a polynomial is fit to the phase of data in a small interval, and the derivative of the polynomial is evaluated at the center of the interval to yield one smoothed data point. This approach is repeated for every data point.

The problem to be solved is to obtain automatic estimates for the bias error in LPF smoothing of signals. Such estimates can be obtained for smoothing by least squares spline fitting, but this method can be impractical for situations with millions of data points. The estimates can also be obtained from LPF methods if one has prior knowledge of a reasonable bound on the appropriate higher order derivative of the unknown signal.

\section{Project}

Several methods for analyzing PDV data that could also provide statistically valid error estimates were investigated. Three methods were studied in great detail:

(1) A method of simultaneously estimating the bias and statistical error for least-squares fitting to cubic spline functions was adapted from techniques used to analyze reaction history data. Although the method is more amenable to small data sets, no such method has ever been reported in the peer-reviewed literature.

(2) The theory of local polynomial approximation (LPA) was explored. LPA is a statistical method of estimating an unknown signal or its derivative from noisy data. The research team was able to develop estimates for the bias error in LPA.

(3) A generalized version of the Peano kernel theorem (PKT) based on distribution theory was proven. This is a very useful result that together with some standard linearization techniques has uses in a broad range of areas that include partial-differential equations, numerical analysis, statistics, and signal processing. 


\section{Mathematical Results and Publications}

One of the goals of the project was to refine and publish some of the uncertainty estimation techniques that we have developed. This greatly facilitates finding and removing weaknesses in the methods and increases confidence in them. To this end several manuscripts were prepared, based on these concepts and are discussed next.

\section{Spline-Based Uncertainty Estimation}

The method of least-squares fitting to cubic spline functions is used in both the analysis of reaction history (RH) data and in the analysis of PDV data. The method of estimating the bias error was developed at NSTec in 2006 and reported in a conference paper (Blair 2007). This method gives a point-bypoint estimate of the bias error of the spline fit that is based entirely on the data, with no quantitative assumptions concerning the unknown signal (Figure 2). No such method is known to have been reported in the peer-reviewed literature. A greatly expanded paper (Blair "Error estimates" 2013), describes a method of estimating the bias error for least-squares fitting to cubic spline functions and justifies the method through mathematical analysis, heuristic analysis, and simulation on several examples.

\section{Uncertainty Estimation LPA-Based Methods}

LPA, a method of estimating an unknown signal or its derivative from noisy data, fits a polynomial to the data in a short interval and estimates the value of the signal or its derivative from the value of the polynomial or its derivative at the center of the interval. This method is used for PDV data analysis and was used prior to 2005 for RH data analysis. A manuscript ("Estimating the bias of local polynomial approximation methods using the Peano kernel") was written and accepted for publication (Blair "Estimating" 2013). This paper provides estimates for the bias error in LPA. The manuscript includes a table of numerical values for coefficients that can be used in bias error estimates and a new formulation of the problem that makes such a table possible and uses advanced mathematical techniques to calculate the coefficient values. The content describes an extension of LPA by Blair (2011) and presents similar results for other classic data smoothing techniques.
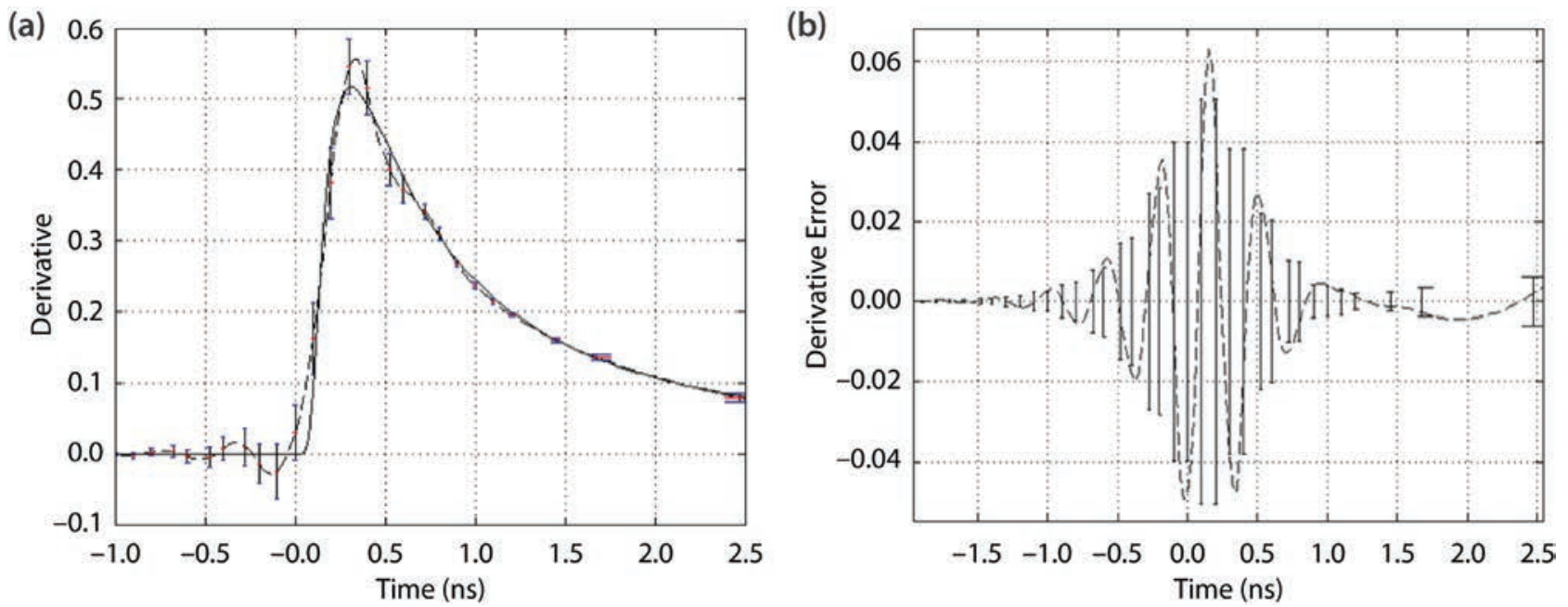

Figure 2. A typical result of the spline-based uncertainty method of estimating the bias error. This is the result of calculating the derivative of a measured signal. (a) The solid line is the true derivative of the signal, and the dashed line is the result of fitting a cubic spline function to the data and differentiating the result. The error bars calculated by our method are shown. (b) The error as a function of time along with the error bars is shown. The error bars should be at $95 \%$ confidence levels, and $95 \%$ of the results are within the bars, while the other $5 \%$ are only slightly outside of them. 
An upper bounding on the bias error by the higher order derivatives of the true signal $\mathrm{x}(\mathrm{t})$ is typically given as

$$
\sqrt{E\left[b_{v, W, n, T}^{2}\right]} \leq T_{e q}^{m-v} \gamma_{v, W, n, m} \sqrt{E\left[x^{(m)}(t)^{2}\right]}
$$

where the left-hand side is the root-mean-squared bias error, $v$ is the order of the derivative being calculated, $W$ is the weighting function used in the fitting, $n$ is the order of the polynomial fitted to the data, and $T_{e q}$ is the effective length of the time interval over which the polynomial is fit. The second factor is a constant that depends on the parameters of the method, and the third factor is the root-meansquared value of the $m^{\text {th }}$ derivative of the signal. The paper derives this result based on a generalization of the PKT and derives a method for evaluating the constants. Table 1 shows constants.

All of the constants are for $v=0$ (the bias error of the signal rather than one of its derivatives). The numbers heading each column are values of $m$, the user-selected order of derivative used in the error formula. The letters in the first column represent three common weighting functions: uniform (U), Epanechnikov (E), and Gaussian ( $G$ ). The numbers beside them are the values of $n$, the order of the polynomial fit. The missing values occur because the error formula is only valid for $m \leq n$. In any given column the method with the smallest constant will produce the smallest worst-case bias error, and this can be determined from the table.

\section{Uncertainty Analysis: Generalizing the Peano Kernel Theorem}

A more generalized version of PKT was used by Blair $(2007,2011)$ to derive useful formulas for error estimation for practical data analysis problems. The PKT was presented in its modern form by Sard (1963) and Davis (1975). However, while very useful as a starting point, Sard's results were not general enough to apply to PDV applications. The ideas and applications discussed by the authors (2007, 2011, 2012) suggested a deeper mathematical result that had much broader application. A paper entitled "A generalized Peano kernel theorem for distributions of exponential decay" was drafted. While still in draft form, the paper states and proves, mathematically rigorously, a generalized version of PKT that is very useful in a variety of statistical applications that includes PDV data analysis. In addition to the new theorems established, the proof of the generalized theorem also relies on mathematical results not generally available in the literature.

Table 1. Values for the coefficient of proportionality $\gamma_{v, w, n, m}$ described in Equation 1, which bounds the bias error in proportion to the derivative of the true signal

\begin{tabular}{|c|c|c|c|c|c|c|c|}
\hline$n \backslash m$ & 2 & 3 & 4 & 5 & 6 & 7 & 8 \\
\hline U2 & $4.2 \mathrm{e}-2$ & & & & & & \\
\hline E2 & $3.6 \mathrm{e}-2$ & & & & & & \\
\hline G2 & $4.0 e-2$ & & & & & & \\
\hline U4 & $4.3 e-2$ & $1.1 \mathrm{e}-2$ & $5.7 e-3$ & & & & \\
\hline E4 & $3.7 e-2$ & $0.95 e-2$ & $4.8 \mathrm{e}-3$ & & & & \\
\hline G4 & $3.4 \mathrm{e}-2$ & $1.0 \mathrm{e}-2$ & $6.3 e-3$ & & & & \\
\hline U6 & $5.3 e-2$ & $1.3 e-2$ & $3.5 e-3$ & $1.3 e-3$ & $8.8 e-4$ & & \\
\hline E6 & $4.5 e-2$ & $1.1 \mathrm{e}-2$ & $3.0 \mathrm{e}-3$ & $1.1 e-3$ & $7.4 \mathrm{e}-4$ & & \\
\hline G6 & $3.8 e-2$ & $1.0 \mathrm{e}-2$ & $3.2 \mathrm{e}-3$ & $1.3 e-3$ & $1.1 \mathrm{e}-4$ & & \\
\hline U8 & $6.0 \mathrm{e}-2$ & $1.4 \mathrm{e}-2$ & $3.8 \mathrm{e}-3$ & $1.1 \mathrm{e}-3$ & $3.8 \mathrm{e}-4$ & $1.7 e-4$ & $1.4 \mathrm{e}-4$ \\
\hline E8 & $5.2 \mathrm{e}-2$ & $1.2 \mathrm{e}-2$ & $3.2 \mathrm{e}-3$ & $0.94 \mathrm{e}-3$ & $3.2 \mathrm{e}-4$ & $1.4 \mathrm{e}-4$ & $1.2 \mathrm{e}-4$ \\
\hline G8 & $4.2 \mathrm{e}-2$ & $1.1 \mathrm{e}-2$ & $3.1 e-3$ & $1.0 \mathrm{e}-3$ & $3.9 e-4$ & $2.0 \mathrm{e}-4$ & $2.0 e-4$ \\
\hline
\end{tabular}

Key: $\mathrm{U}=$ Uniform, $\mathrm{E}=$ Epanechnikov, $\mathrm{G}=$ Gaussian 

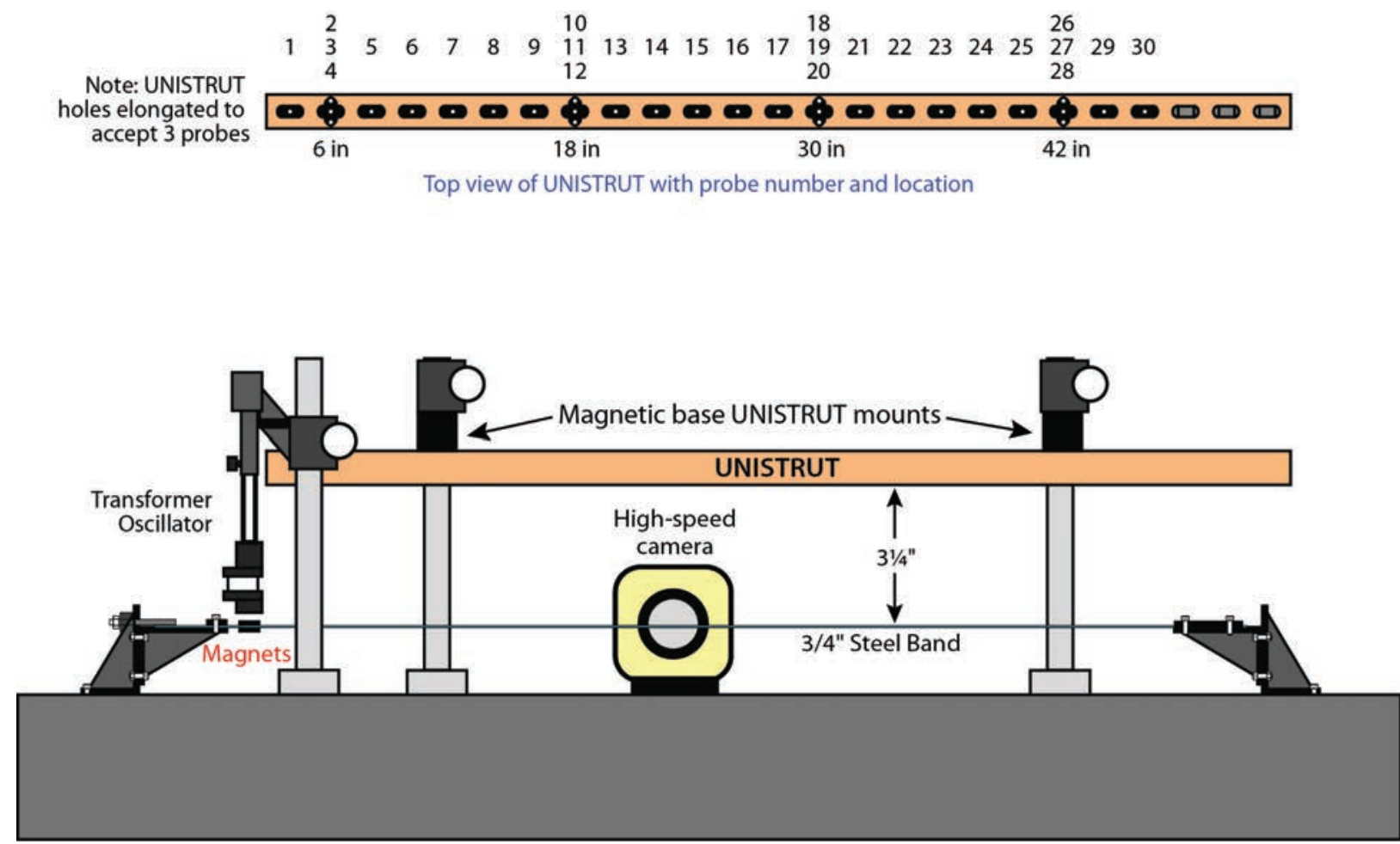

Figure 3. A schematic of the 1-D vibrational beam experiment. A metal beam is secured on each end, and a standing wave vibration is driven by neodymium magnets charged from a circuit. The standing wave is imaged with the highspeed camera, and the MPDV probes are secured in the unistrut above the beam.

\section{1-D Vibrational Beam Experiment}

In order to quantify the uncertainty in the mathematical processing of MPDV data, an experiment was designed to generate data in the lab to analyze the accuracy of computed surface positions. A flat metal beam was secured at both ends, with neodymium magnets on one side, as can be seen in the schematic in Figure 2, and 1-D oscillations in the beam were induced using an electrical current. The vibrations were measured with MPDV and with a high-speed camera, and the positions computed from the PDV data are compared to the actual positions measured with the high-speed camera. Essentially the goal is to overlay the computed positions onto the camera imagery and quantify how well the two diagnostic results align, allowing us to quantify the uncertainties in the MPDV position calculations.

In order to generate data that are appropriate for such a comparison, it is essential that the oscillations occur in one dimension, so the beam (1/16" thick, 3/4" wide, 48" long) was fixed in a way that it could only oscillate up and down, with no pitch or yaw. The high-speed camera was placed at the side of the beam, and the MPDV probes were mounted in a unistrut above the beam, pointing down normal to the surface (Figure 3). An oscillating magnetic field was generated around the magnets by running an alternating current through a coil (Figure 4). The fluctuations in the magnetic field repel the magnets, then relax, pushing the metal beam up, then allowing it to bounce back into place. The oscillations can be driven at a fixed frequency, resulting in the beam oscillating in a standing wave. The frequencies used were integer multiples between 1 and 5 of approximately $43 \mathrm{~Hz}$-the resonant frequency of the beam.

The oscillations of the beam were measured with the "prototype" MPDV system (Daykin 2011), operating at a sampling rate of 250 megasamples per second. Taking PDV measurements on a beam oscillating in 


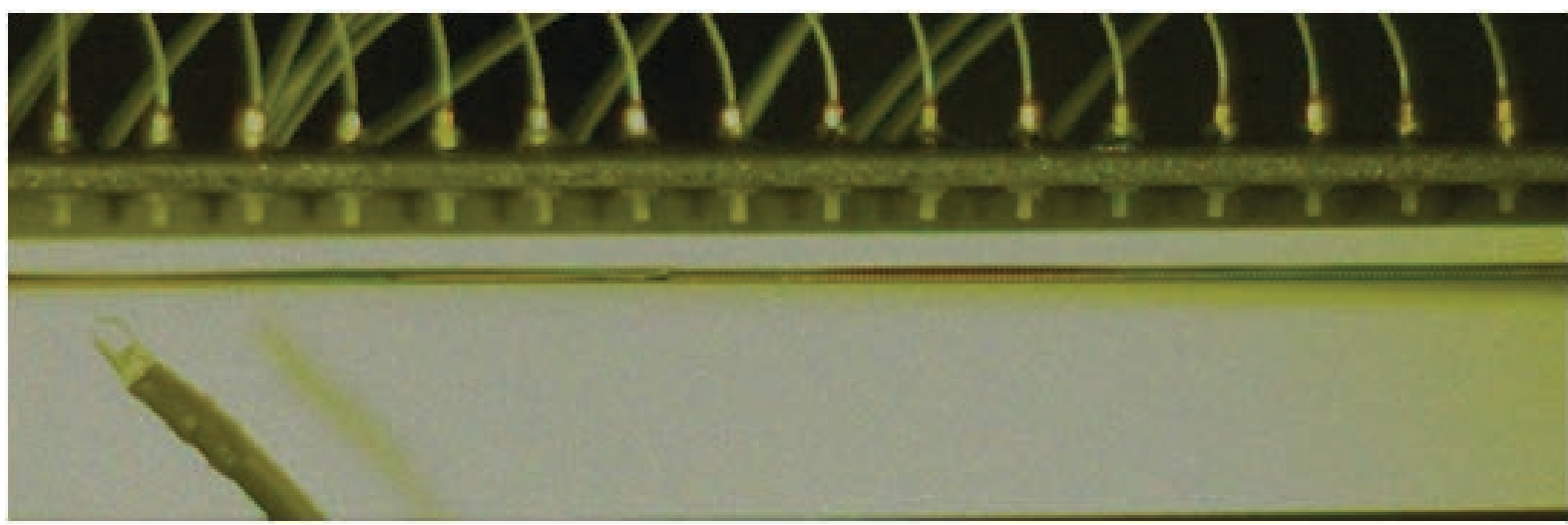

Figure 4. The oscillating metal beam with 16 MPDV probes above it, looking down. The LED (lower left) flashes when the PDV system initiates data capture.

the $10 \mathrm{~s}$ of $\mathrm{Hz}$ has a range of complications that are not seen when measuring surfaces vibrating in the $\mathrm{GHz}$ range, and the frequency upshifting is significantly more difficult, due to instabilities in the lasers. Moreover, the probes used have a narrow field of view, so the metal beam was coated with a reflective tape used on street signs that reflects light directly back in the direction from which it came. This significantly increased the intensity of the light being captured by the probes.

Five different experimental configurations were designed, each measuring a different property of the system. The configurations were as follows:

(1) Camera zoomed in on a narrow portion of the beam, in order to accurately compute the frequency of the oscillating beam, with 12 PDV probes

(2) A far-field data set, with the camera positioned to capture the entire oscillating beam, with the locations of the PDV probes marked on the unistrut for the camera to see, with 12 PDV probes

(3) Camera positioned to show 16 PDV probes evenly spaced at $1 / 2 "$

(4) Camera positioned to show 16 PDV probes evenly spaced at $1 / 4 "$

(5) Camera zoomed in on beam against a gridded background, in order to measure physical displacement of the beam
Given the beam tension and the range of possible currents, the beam could be driven into standing waves with 1, 2, 3, 4, and 5 nodes, and data were collected with each number of wave nodes at each experimental configuration. The data from configurations (1) and (2) were designed for measuring the frequency of the oscillations with the high-speed camera; configurations ( 3 ) and (4) were ideal data for benchmarking positions computed from PDV against the high-speed camera; and configuration (5) gave the physical displacement of the beam.

Figure 5 shows results of two particular measurements. The MPDV approach uses a method called optical upshifting that offsets the frequency for each PDV probe so the 0 velocity does not correspond to zero frequency, but to a selectable frequency. This allows several channels to appear in the same trace and still be distinguished from one another. In Figure $5 \mathrm{a}$, the metal beam was vibrating in a standing wave with five cycles, and the five peaks can be clearly seen in each of the four probes shown. In Figure $5 b$, the beam was oscillating with two cycles, and the peaks are more difficult to distinguish. The second image also demonstrates two of the difficulties in capturing the MPDV data. Note that the curves representing the two middle probes are quite close together, and the frequencies of the lasers for those probes had to be continually monitored and adjusted to keep the measured curves from overlapping in the spectrogram. This was a major challenge for the experimental team. 


\section{(a) Standing wave, 5 cycles}

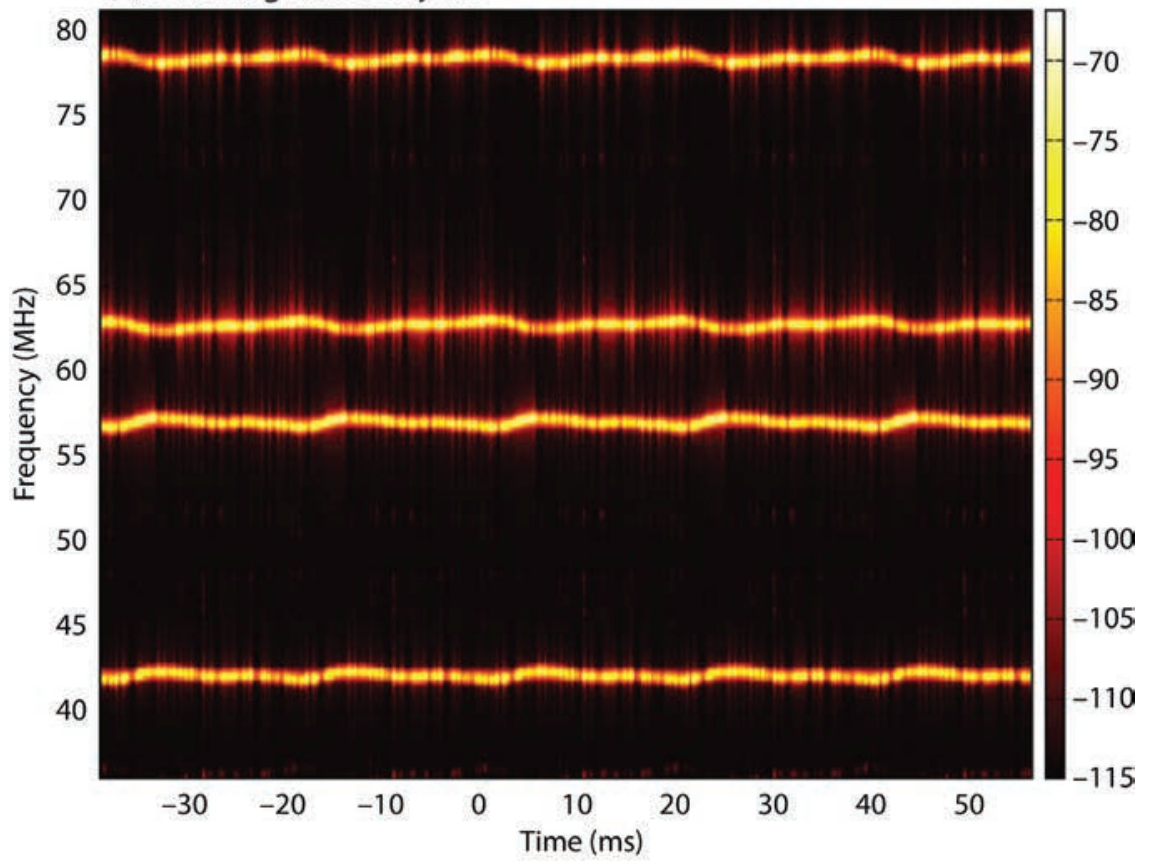

(b) Standing wave, 2 cycles

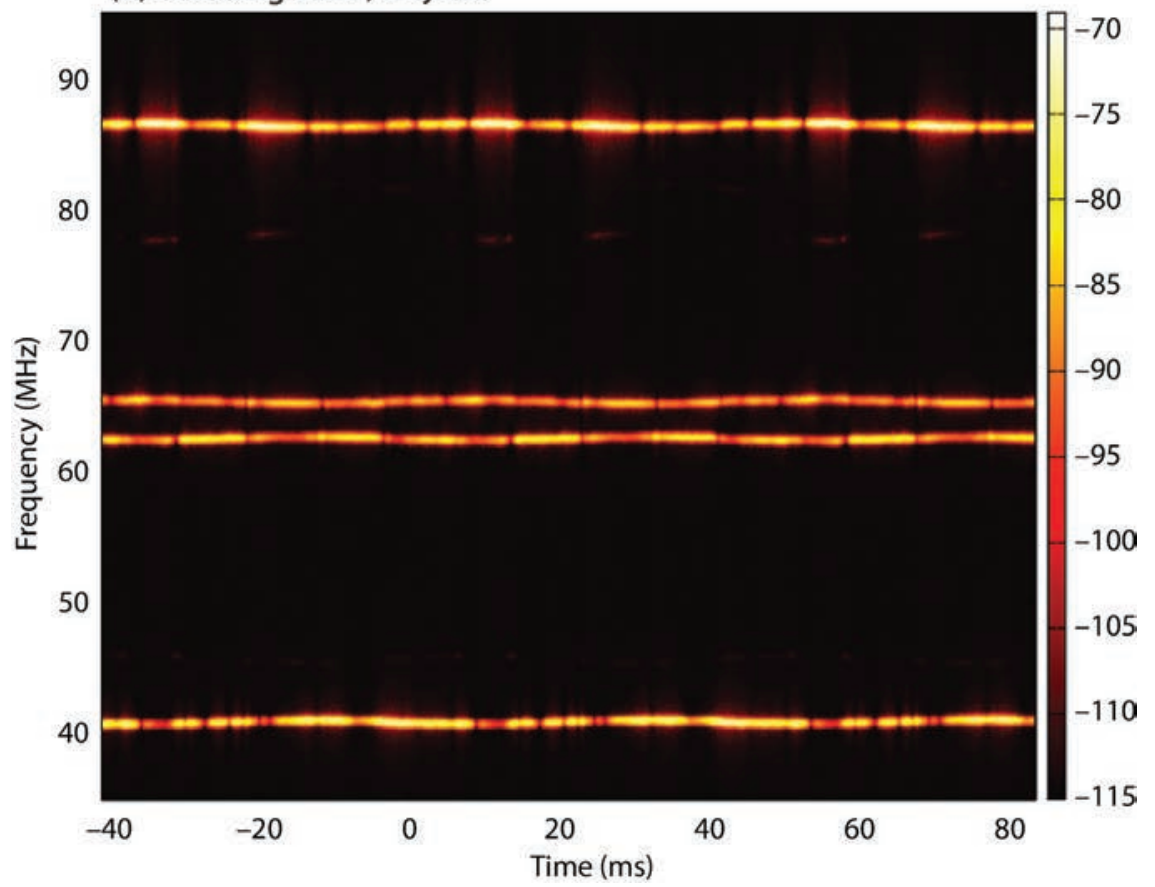

Figure 5. Spectrograms generated with the iPDV data analysis tool, showing the measurements taken from four PDV probes. (a) The beam was oscillating at a standing wave with five cycles; (b), the beam was oscillating at a standing wave with two cycles. The color scale is power spectral density.

\section{Conclusion}

The uncertainty estimation methods that have thus far been developed for MPDV measurements have been described in articles that are being readied for publication. An experimental setup has been built and tested for demonstrating the accuracy of MPDV techniques.
The analysis of the noise-related errors of the LPF method is straightforward, but the bias error is problematic (recall that bias errors occur when the data or true signal does not match up correctly with the functional form of the model [i.e., the errors expected when trying to fit a straight line to 
quadratic]). In FY 2012 we developed formulas for the bias error for LPF that are proportional to a higherorder derivative (typically the fourth) of the unknown signal. Unfortunately, estimating the higher-order derivatives in the presence of noise is quite difficult. We believe that an approach based on a variation of the short-time Fourier transform will yield estimates for the higher-order derivatives that will be adequate for making a good estimate of the bias error, which will be pursued in FY 2013 work. Recent work at Sandia National Laboratories suggests that an important portion of the crisscrossed signal problem due to circulator bleed-through may have been solved by Dolan (2012).

As this should be explored further, work will continue on this project in FY 2013 under the name "MPDV and Large Data-Set Statistics" to (a) apply various techniques researched and developed in FY 2012 to the recorded 1-D MPDV cross-section data to cross validate and compare, (b) develop a high-velocity 2-D imaging PDV system complete with an accurate mathematical estimate of the errors, and (c) address the problem of handling the extremely large data sets that often accompany MPDV measurements but that current error-estimation algorithms have difficulties handling.

\section{Acknowledgments}

We would like to acknowledge the contributions of Mike Doman who passed away this year: his gentle nature, brilliance, and elegant simplicity in problem solving will be missed.

We would like to thank Dr. Stephen Mitchell whose ideas were instrumental in simplifying the overall design of the experiment itself. We would also like to acknowledge C. Y. Tom, Steve Gardener, Nathan Sipe, Carlos Perez, and Rand Kelley for their help in setting up the experiment. Without their ingenuity, this would not have been possible.

\section{References}

Blair, J., "Error estimates derived from the data for least squares spline fitting," IEEE Instrumentation and Measurement Technology Conference Proceedings (2007) $1-6$.

Blair, J., "Optimal knot selection for least-squares fitting of noisy data with spline functions," IEEE Instrumentation and Measurement Technology Conference Proceedings (2008) 27-32.

Blair, J., "Filtering error estimates and order of accuracy via the Peano kernel theorem," Computer Standards and Interfaces 33 (2011) 122-127.

Blair, J., A. Luttman, E. Machorro, "A generalized Peano Kernel Theorem for distributions of exponential decay," ms in draft.

Blair, J., A. Luttman, E. Machorro, "Estimating the bias of local polynomial approximation methods using the Peano kernel," Proc. J. Contemp. Math, ms accepted.

Blair, J., E. Machorro, "Error estimates derived from the data for least-squares spline fitting of oscilloscope data," $m s$ in draft.

Davis, P., Interpolation and Approximation, Dover, Toronto, 1975.

Daykin, E., C. Perez, A. Rutkowski, C. Gallegos, "Advanced PDV techniques: Evaluation of photonic technologies," Nevada National Secuity Site-Directed Research and Development, FY 2010, National Security Technologies, LLC, Las Vegas, Nevada, 2011, 205-214.

Dolan, D. H., T. Ao, O. Hernandez, "Frequency-conversion photonic Doppler velocimetry with an inverted circulator," Rev. Sci. Instrum. 83 (2012) 026109.

Sard, A., Linear Approximation, American Mathematical Society, Rhode Island, 1963.

Strand, O. T., D. R. Goosman, C. Martinez, T. L. Whitworth, "Compact system for high-speed velocimetry using heterodyne techniques," Rev. Sci. Instrum. 77 (2006) 83108. 


\section{CUSTOM IP PACKET SUBSTITUTION CYBER FRAMEWORK TOOL \\ NLV-31-12 | YEAR 1 OF 1}

\section{Christopher Nivens ${ }^{1, a}$}

This SDRD work addressed the need for a custom cyber framework tool that captures, controls, and processes system network traffic and identifies vulnerabilities through the dissection of those protocol values most susceptible to disruption. The Cyber Operations Mini-Testbed uses a representative industrial control system and was selected as the proof-of-concept environment for the custom Internet protocol packet substitution tool and framework methodology developed for this project.

${ }^{1}$ nivenscm@nv.doe.gov, 702-295-8046

a Remote Sensing Laboratory-Nellis

\section{Background}

The Cyber Operations Mini-Testbed was previously developed by NSTec to provide an extensive testing environment for use by other government agencies (OGA) in exercise, training, and scenario development for real work response and mitigation of threats to critical infrastructure assets. The OGA's primary interest is in duplicating critical infrastructures such as supervisory control and data acquisition (SCADA), industrial control systems (ICS), and smart grid systems. The representative ICS node was selected to provide a demonstration of the capabilities of the custom Internet protocol packet substitution (CIPS) tool in identifying critical infrastructure vulnerabilities. The integration of the CIPS tool into an environment is the key factor that will make the CIPS useful in future OGA-sponsored exercises. The ability to modify or substitute Internet protocol (IP) packets and monitor the results aids in the identification of vulnerabilities to a particular SCADA, ICS, or smart grid system. This SDRD work addressed the need for more advanced analysis.

\section{Project}

The Cyber Operations Mini-Testbed (Figure 1) was used to develop the CIPS tool. The ICS node available on the testbed consists of the two programmable automation controllers and input/output (I/O) boards. The output to the power distribution and the

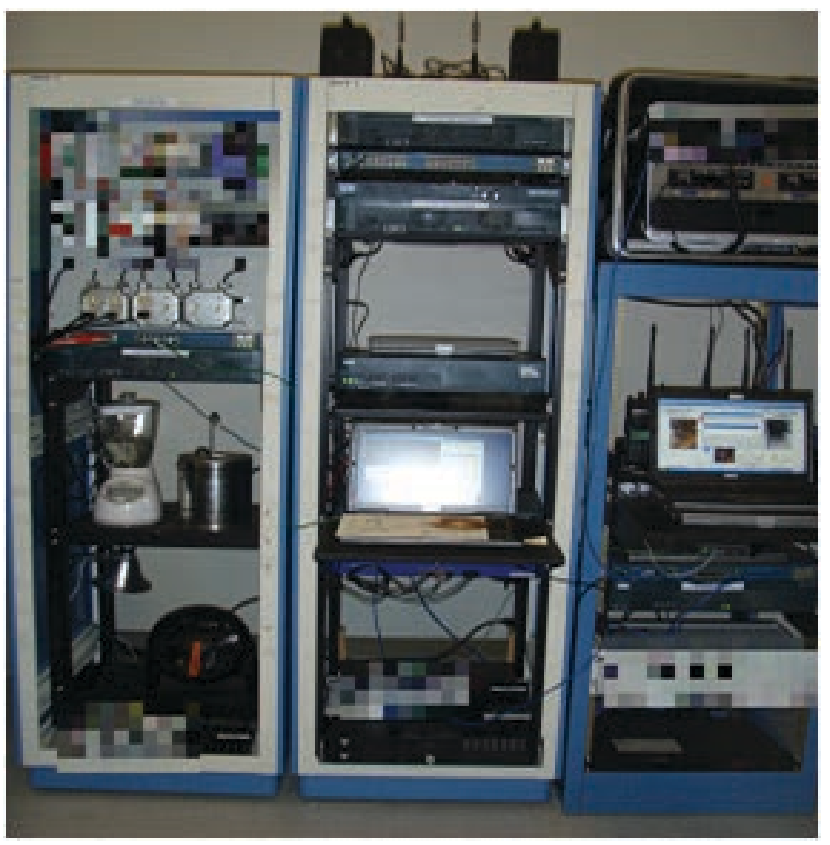

Figure 1. The Cyber Operations Mini-Testbed 
connected digital devices are a light, water pump, and fan. Network connectivity is provided through a CISCO 3500x series switch. The device state is viewed and controlled through a human-machine interface (HMI) Dell laptop.

The goals of this project were to demonstrate the ability of the CIPS tool to control and modify the ICS process control network traffic, specifically the connected digital devices (light, water pump, and fan), and monitor the results with respect to potential vulnerabilities. The devices that are connected represent various industrial systems, which are controlled via ICS controllers. In the ICS testbed node, the light represents a power system, the water pump represents a volumetric pumping system, and the fan represents an HVAC system.

The identification, control, and monitoring required a packet manipulation strategy. The methodology was derived from the nascent concept that packet crafting as a testing methodology is an art. Packet crafting methodology consists of packet assembly, editing, replay, and decoding (Poor 2011) and was used in the development of the CIPS tool.

The packet decoding step involved the capture of packets between the ICS controllers and the HMI. The methodology employed within this step involved collecting packet captures from all the various output states of the connected devices (Table 1).

Table 1. Packet captures: permutations of all connected digital device output states

\begin{tabular}{|c|c|c|}
\hline Light & Water & Fan \\
\hline On & Off & Off \\
\hline On & On & Off \\
\hline On & Off & On \\
\hline On & On & On \\
\hline Off & Off & Off \\
\hline Off & On & Off \\
\hline Off & Off & On \\
\hline Off & On & On \\
\hline
\end{tabular}

Based on their initial configurations, the two ICS controllers can interpret and use three network protocols. These protocols include a memory-mapped protocol based on the Institute of Electrical and Electronics Engineers (IEEE) 1394 standard. The other two are the Modbus/TCP and legacy serial protocols. The SCADA programmer who built the ICS portion of the testbed used the IEEE 1394 protocol. The protocol document was extensively consulted during the course of this project. The ICS devices use the IEEE 1394 specification to provide the ability to read and write data to a 48-bit memory-mapped address space. The protocol places an IEEE 1394 packet inside a TCP/IP or user datagram protocol (UDP)/IP packet (Figure 2).

Understanding the packet structure arrangement was very helpful during the initial packet decoding step; however, limitations became apparent when attempting to correlate devices' states to the analyzed packets. While the protocol breakdown is extremely helpful in determining the meaning of the bytes at various positions, they only correlate to a particular I/O point on a module in the I/O boards. An I/O point can be an $1 / 0$ operating in either digital or analog mode depending on the module. These details are left to the discretion of the SCADA/ICS programmers and depend on how they want to assign I/O points to connected devices. Therefore, the packet decoding step forced the continuous adjustment of packet capturing methods. One of the methods that worked was to apply a rapid change to each of the device's states in the permutations one at a time (Table 1). This allowed the discovery of the pattern that was causing the change in state and correlates it to the ICS packet protocol structure. An example of this correlation via the "light on" packet is given in Figure 3.

By applying this methodology to all of the remaining states given in Table 1, the packet breakdown for all the changes in state and the reading of their values were discovered. This led directly to the next step in the packet crafting methodology known as packet assembly. To accomplish this, the open source tool Scapy was chosen. Scapy is an interactive 


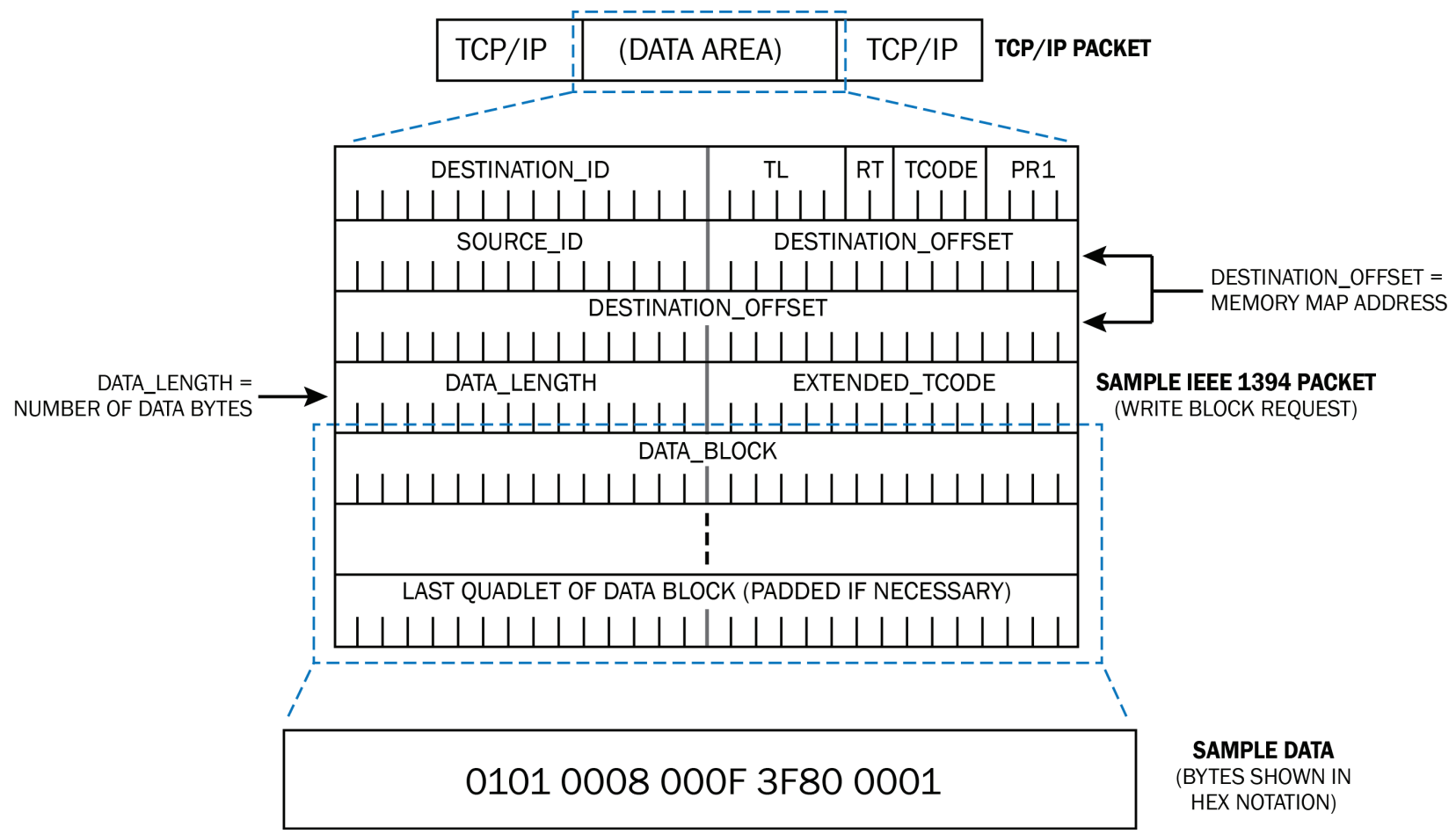

Figure 2. ICS packet protocol breakdown

packet crafting and manipulation tool based on the Python programming language (Scapy 2012). Scapy can accomplish all the goals of the packet crafting methodology and, because it is based on Python, can be written to executable code and can be used to build tools to generate the custom packets required for the CIPS tool (Hetland 2008). The code snippet in Figure 4 is the instruction sent to the ICS node controller for the light device with a change in state to ON.

Python functions using Scapy were written for each device to change their states. The user interface menus both use these functions to make unauthorized write requests to the ICS node controllers.

The final step required by the CIPS tool was to effect changes to the HMI user while keeping the user completely unaware of any changes to the state of the devices connected to the ICS node controllers. This was done in the final step in the packet crafting methodology, which combined packet editing and packet replay. The tool used for this is another open

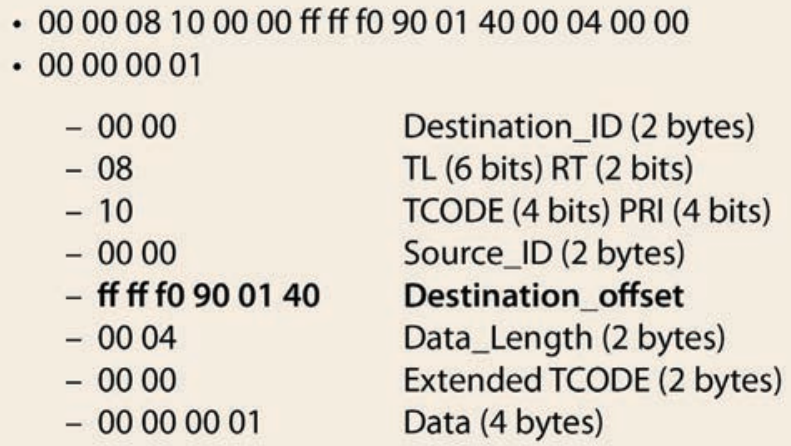

Figure 3. Packet breakdown of light to ON state

source tool known as Ettercap (Ettercap 2010). Because Ettercap is based on the C programming language, it is faster than Scapy's implementation in Python (Burns 2007). Packet editing and replay in real time requires this speed advantage if the traffic between the two hosts is significant. Packet loss was observed between the ICS node controllers and the HMI when used via a Python/Scapy implementation. There was no such loss with Ettercap. Fortunately, Ettercap also provides the ability to filter traffic in 


\section{\#Name of function}

def lighton():

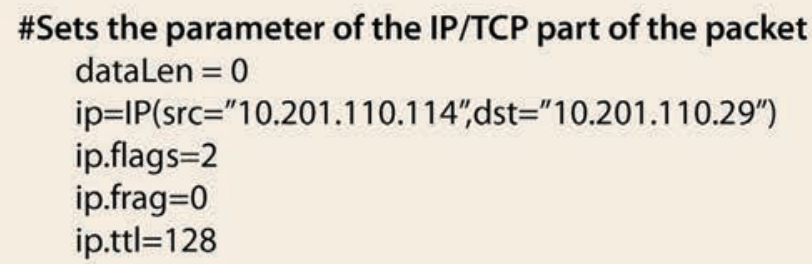

\#Sets the parameter of the IP/TCP part of the packet

dataLen $=0$

ip $=I P\left(s r c=" 10.201 \cdot 110.114 ", d s t=" 10.201 .110 .29^{\prime \prime}\right)$

ip.flags $=2$

ip. frag $=0$

ip.ttl $=128$

\section{\#Builds the SYN part of the packet}

syn=TCP(sport=1060, dport=2001, flags $=" S$ ", seq $=0$, options=[('MSS',1460),

('NOP', 1), ('NOP', 1), ('SAckOK', ")])

\section{\#Sends the SYN and saves the return to SYNACK}

synack=sr $1(i p / s y n)$

\#Sets the SEQ and ACK to the correct values and sends the correct ACK back my_seq $=$ synack[TCP].ack

my_ack $=$ synack $[T C P] . s e q+1$

$\mathrm{ACK}=\mathrm{TCP}($ sport=1060, dport=2001, flags="A", seq=my_seq, ack=my_ack) send(ip/ACK)

\#Tells the device on module 0 point 1 , which tells the light to turn on payload='MASKED'

PUSH=TCP(sport=1060, dport=2001, flags="PA", seq=my_seq, ack=my_ack) ANS $=$ sr 1 (ip/PUSH/payload)

\#If there are any data returned, computes this value for the ACK return packet if Raw in ANS == True:

$$
\text { dataLen }=\text { len(ANS[Raw]) }
$$

else:

$$
\text { dataLen }=0
$$

\section{\#Sets the SEQ and ACK to the correct values and ends the conversation}

my_seq $=$ ANS[TCP $]$.ack

my_ack $=$ ANS $[T C P] . s e q+$ dataLen

BYE $=T C P($ sport $=1060$, dport=2001, flags="RA", seq=my_seq, ack=my_ack)

send(ip/BYE)

return
Figure 4. Python

function using

Scapy to change to

the light ON state real time, based on user-developed code. This step required a similar methodology to the one used to discover the correct packets to control the connected devices' states. Using the previous data from the packet decoding, we focused on how the ICS node controllers were updating the $\mathrm{HMI}$ in a steady state and when applying the permutations in Table 1 . The ongoing conversations between the ICS node controllers and the $\mathrm{HMI}$ are shown in Figure 5.
As before, the packet patterns had to be identified, and, in this case, the packets that controlled the output of the HMI display had to be identified. Once identified, these patterns had to be edited into the original conversation and replayed. Figure 6 is an abbreviated code snippet of the filter code from Ettercap. Filter code was required for all permutations of the devices' states listed in Table 1. 
70 bytes from $\mathrm{HMI}$ to .27

582 bytes from .27 to $\mathrm{HMI}$

70 bytes from HMI to .29

582 bytes from .29 to $\mathrm{HMI}$

70 bytes from $\mathrm{HMI}$ to .27

1094 bytes from .27 to $\mathrm{HMI}$

70 bytes from HMI to .29

1094 bytes from .29 to HMI

54 bytes ACK from HMI to .27

54 bytes ACK from HMI to .29

60 bytes from .29 to $\mathrm{HMI}$

64 bytes from $\mathrm{HMI}$ to .29

54 bytes ACK from HMI to .29
Request status

Requested status updates

Request status

Requested status updates

Request status

Requested status updates

Request status

Requested status updates

Requested acknowledgment status

Requested acknowledgment status

Request for time updates

Requested time updates

Requested acknowledgment status
Figure 5. ICS

node controllers

to HMI

conversation

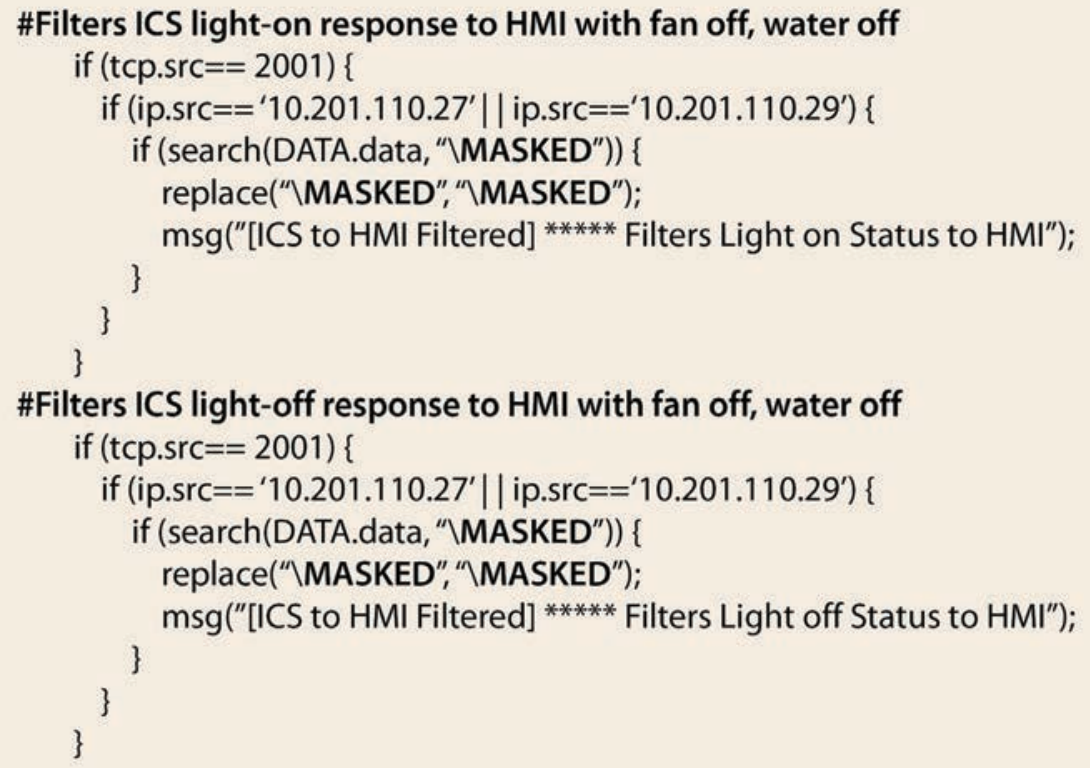

The CIPS tool uses the Python/Scapy portion of the tool to make the unauthorized changes to the ICS node and calls the Ettercap portion of the tool to edit the responses and replay the desired device states to the HMI. As stated earlier, before the correct Ettercap filter can be selected, the device states must be
Figure 6. Ettercap filter for light on/off with fan off, water off (abbreviated) known, especially if the HMI cannot be viewed or if the tool is used remotely. The read interface developed for this function uses the same structure as that shown in Figure 4 and was discovered using the same methodology. 


\section{Conclusion}

The CIPS tool accomplished its design goal as it pertains to the Cyber Operations Mini-Testbed, which uses a dingle representative ICS programmed with an IEEE 1394-based protocol. More ICS environments can be added to the tool, but due to the complexity and custom programming nature of each SCADA/ICS manufacturer and controller type, each ICS would have to be subjected to the same packet crafting methodology. The output code for each individual analysis would then be added to the tool.

This SDRD takes a very focused view on the undetected disruption and manipulation of a SCADA/ ICS. The goal was to evaluate the ability to capture, analyze, and modify, as needed, the controller traffic as it crosses the network wire without manipulating or footprinting the actual SCADA/ICS firmware. This analysis still requires observation of the output state of the system to correlate the intended effect with physical events (i.e., light on/off). Direct manipulation and disruption of the devices themselves (current hacker scenarios) is far less difficult because those unknown variables associated with the custom programming done by the SCADA/ICS engineer are removed. The direct manipulation scenarios are easily recognized through the residual data remnants or the physical destruction of the device itself.

\section{References}

Burns, B., J. Granick, S. Manzuik, P. Guersch, D. Killion, N. Beauchesne, E. Moret, J. Sobrier, M. Lynn, E. Markham, C. Lezzoni, P. Biondi, Security Power Tools, O'Reilly, Seastopol, California, 2007, 136-141.

Ettercap, Ettercap Official Documentation, 2010, http://www.ettercap.sourceforge.net/index.php, accessed July 7, 2012.

Hetland, M. L., Beginning Python, 2nd ed., Apress, New York, New York, 2008, 223-225.

Poor, M., Packet Craft for Defense-in-Depth, 2011, http://www.inguardians.com/research/docs/packetfoo.pdf, accessed January 16, 2012.
Scapy, Scapy Official Documentation, 2012, http://www.secdev.org/projects/scapy/doc, accessed May 7, 2012. 


\section{NeW HybRid TDMA/CSMA WiReless Mesh NetWORKS (WMN) FOR SECURE SMART GRID COMMUNICATION}

RSLN-01-12 | YEAR 1 OF 1

Ki Park ${ }^{1, a}$ and Yoohwan Kim ${ }^{b}$

In a smart grid environment, smart devices are installed at customers' premises. Wireless mesh network (WMN) technology is considered the most cost-effective solution for managing smart devices that communicate wirelessly with their neighbors and relay the data among each other to a control center. However, the existing WMN system has not yet advanced enough to reach its full potential. With our new algorithm, throughput and Quality of Service (QoS) performance can be greatly improved. It also reduces cyber attack risks with flexible key management based on identifier-based cryptography. The new wireless protocol for smart grid network has been studied on an OPNET Modeler network simulator. The performance results are compared against an existing mesh network protocol ad hoc on-demand distance vector.

${ }^{1}$ parkkh@nv.doe.gov, 702-295-8022

a Remote Sensing Laboratory-Nellis; ${ }^{b}$ University of Nevada, Las Vegas

\section{Background}

A smart grid contains many smart devices such as meters, thermostats, or appliances. One of the promising technologies in smart grid communication is the wireless mesh network (WMN) (Güngör 2011). The devices in a WMN communicate wirelessly with their neighbors, and they relay the data from other meters to the control center (CC) (Akyildiz 2005). The most fundamental step in this scheme is establishing a reliable path from each house to the CC. Regarding reactive and proactive algorithms, proactive algorithms are preferred due to their ability to quickly deliver data, but because they need to discover all routes between all nodes, they have high overhead. In our smart grid environment, the majority of the traffic is between the smart meters and the CC; this forms a tree-shaped network, thus eliminating the need for discovering the routes for all pairs. This research proposed to develop a WMN scheme that has the benefit of proactive routing protocol, but has a reduced overhead by utilizing the tree-shaped topology. With the augmentation of the hybrid time division multiple access/carrier sense multiple access
(TDMA/CSMA) communication approach and identifier-based cryptography (IDC), the performance and security can be further improved.

\section{Project}

\section{Wireless Mesh Network Routing Process}

Our proposed routing algorithm is named Intelligent Tree Mesh Routing (ITMR) to reflect its tree topology. A similar approach, multi-radio over multiple channels, was considered previously (Peng 2006), but our approach uses single radio over single channel, which is more cost-effective. ITMR establishes the routing paths at the Open Systems Interconnection (OSI) protocol layer 3 (network layer). It discovers and maintains only the route from all nodes to the CC, creating a tree rooted at the $\mathrm{CC}$. A routing path is set up at the $\mathrm{CC}$ in advance of the data transmission from any smart meter node. Periodically a beacon is transmitted from the CC (hop count $h=0$ ). The closest nodes receive the beacon, and they retransmit 
the beacon after incrementing the hop count to 1 . The process continues until it reaches the edge of the network. During this beacon-flooding process, each node chooses one parent node and uses it as a relay node for the uplink to the CC. The algorithm is as follows:

1. Initially every node is in receiving mode, and at the root's first beacon transmission, the route setup starts.

2. Each node chooses a parent node:

a. If the new parent is the same as the previous one, the receiving node does not acknowledge (ACK) and relays the beacon to the next hop nodes.

b. If the new parent is different, or if this is the first time, the registration process begins. For registration traffic, a normal CSMA Request-To-Send and Clear-To-Send (RTS/CTS) process is used.

3. The parent accepts the ACK from a child and assigns an identifier (ID).

4. Security registration (using IDC) is performed.

5. This process propagates outbound data.

6. If any node misses a routing round, the node will be set in the next round.

Once the ITMR process is complete, the nodes can start normal data communication in layer 2 using the 802.11 protocol. For each beacon signal, a new tree structure is reconstructed. After a tree is built, the path to the CC is immediately available, thus giving us the benefit of a proactive routing protocol. Uplink traffic (from a smart meter node to the $\mathrm{CC}$ ) can be easily handled by relaying the data from downstream nodes to the parent node. Downlink traffic (from the $\mathrm{CC}$ to individual smart meters) is handled easily by referring to the destination node ID. A node ID is assigned during the route setup process in hierarchical fashion. In ITMR, there is a unique path between the root and any node. The ID is a concatenation of the routing path with variable length. As the route is readily available from the ID itself, the need for a routing table at layer 3 is removed for downlink traffic. The ID-to-medium access control (ID-to-MAC) address conversion table at layer 2 (similar to the address resolution protocol table) is still necessary.

\section{Hybrid Communication Scheme}

The smart meters constantly transmit the electricity usage data to the CC. In a dense urban environment, the chance of simultaneous transmission is quite high, and consequently the layer 2 protocol may experience a low performance due to frame collisions. This can be reduced by adopting a TDMA-based protocol where the transmission time for each node is coordinated by time to avoid collision. However, the time-slot management in TDMA protocol in a WMN environment is not trivial and incurs high overhead (Djukic 2009). It is also shown that the time-slot scheduling is a "nondeterministic polynomial time"-complete problem, and heuristic-based algorithms may be the most feasible solution (Ergen 2010). On the other hand, ITMR can take advantage of tree-shaped topology to allocate the time slots based on the hop count information, thereby reducing the time slot management overhead.

ITMR builds a tree-based routing path, and each node has a hop count number $(h)$ from the root. Once the routing tree is built, transmission is allowed only in certain time slots that are determined by the hop count. This is controlled in layer 3 as part of ITMR. Transmission time slots are assigned in such a way to reduce collision as well as hidden terminal problems between nodes with adjacent $h$ numbers. The nodes in 3-hop distances (e.g., $h=0,3,6, \ldots$ ) can transmit simultaneously without experiencing a collision. Within the group of nodes of the same $h$, the normal 802.11 protocol is used (CSMA).

The time slots in all nodes are synchronized by a GPS clock. The beacon from the root transmits the epoch time and the time slot duration in its beacon. Each node can determine the beginning of each time slot by calculating [time slot duration $\times$ hop count] from the epoch time. 


\section{Communication Security}

The communication content should not be blocked, intercepted, tampered, or replayed by a third party. While the routing tree is generated, the node-to-node security can be achieved with IDC faster than with conventional public-key cryptography (Chatterjee 2010). The MAC address of a smart meter node serves as the public key in IDC. A private key generator can assign a private key for each device before deployment. When a child node $(c)$ receives a routing broadcast message from a parent node $(p)$, it sends a registration message to $p$ encrypted under $p$ 's public key. Since $p$ 's public key (i.e., the MAC address of $p$ ) is already known as part of the broadcast message, there is no need to ask for a public key or look up the database. To prevent a bogus node from joining the network, both nodes perform a mutual authentication in accordance with the following three steps.

Step 1. $c \rightarrow p: r_{1} P \|\left\{N_{c} \| H_{c}\right\}_{K_{a}}$

Step 2. $p \rightarrow c: r_{2} P \|\left\{N_{c}\left\|N_{p}\right\| H_{p}\right\}_{K_{c}}$

Step 3. $c \rightarrow p: \operatorname{MD5}\left(N_{p}\right)_{k 2}$
In step 1, the client sends a message encrypted with the parent's public key. Then the parent derives the common key, decrypts the message, and checks for integrity by confirming the hash value $H_{c}$. The nonce $\left(N_{c}\right)$ is a random number to confirm the proper decryption, which is sent back in step 2 along with a hash value. The child can authenticate the parent by checking whether the nonce is correct. In step 3, both have the common keys and only need to verify the common key. The child sends back the nonce and completes the handshake. Both nodes can then generate a common symmetric key ( $k$ ) that serves as a master key to derive temporal keys for future communications.

Once the peer-to-peer mutual authentication is completed among all the possible parent-client pairs, each node can start authenticating to the smart grid network. The $\mathrm{CC}$ has a remote authentication dial-in user service (RADIUS) server, and the device's credentials can be verified against the authentication database. If a device is compromised, the RADIUS does not accept the device to the network. Once the network authentication is done, normal data traffic may start.

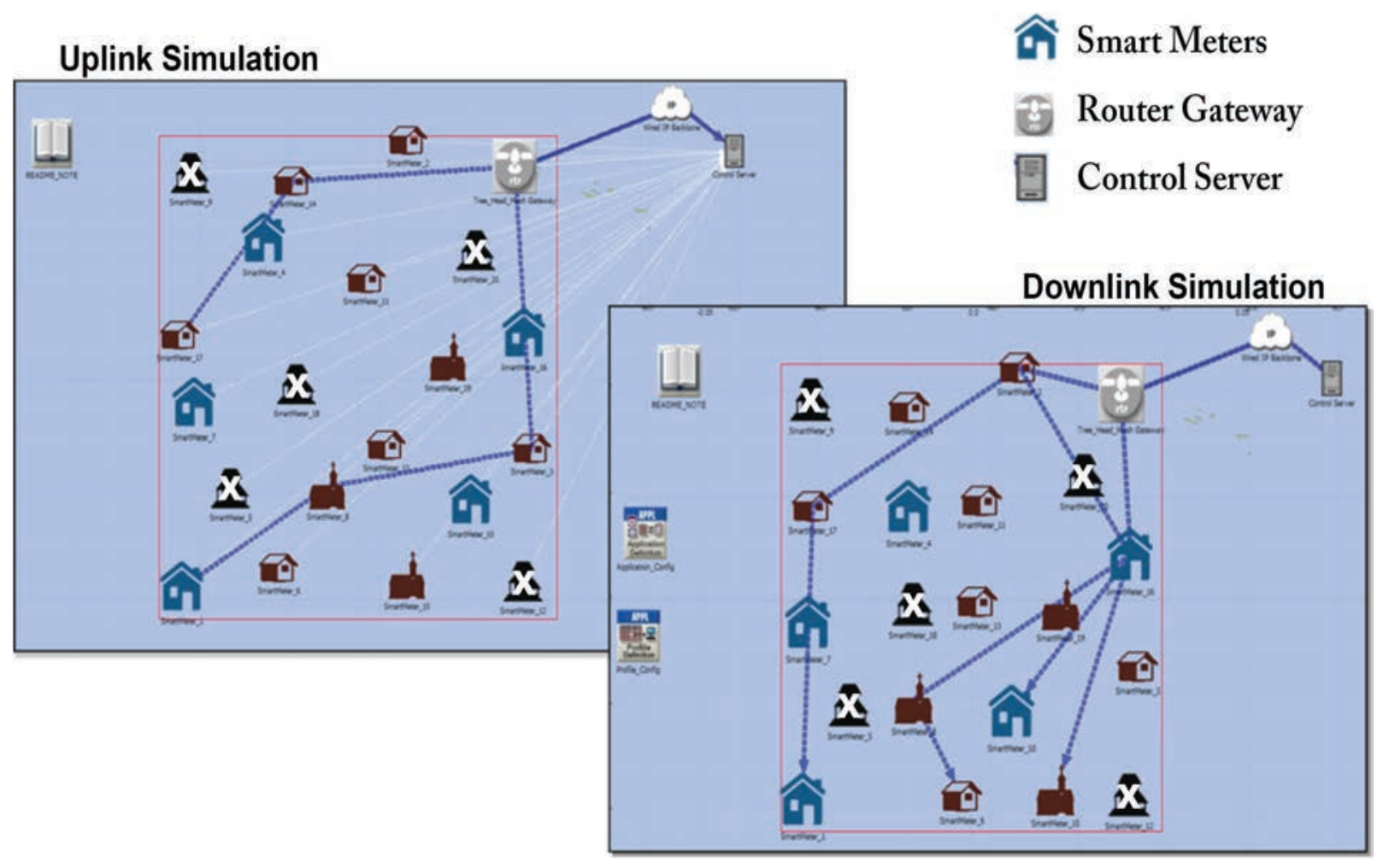

Figure 1. Network simulation showing nodes forced to fail (white Xs) 


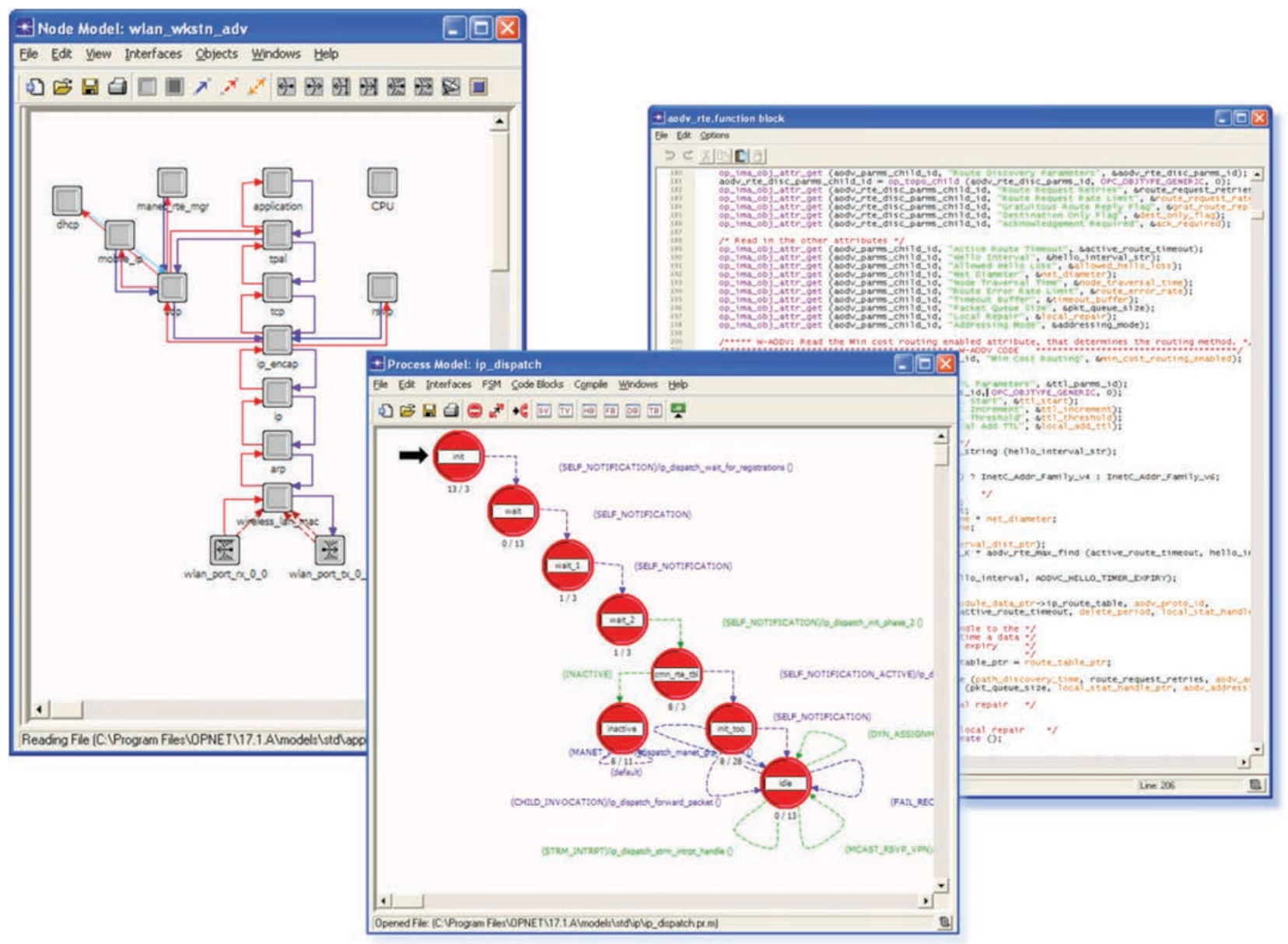

Figure 2. Network protocol design steps with OPNET Modeler

\section{Experiments}

The network models were implemented in OPNET Modeler network simulation software. We created a WMN with 20 nodes and compared the performance against ad hoc on-demand distance vector (AODV) protocol (Pirzada 2007).

Figure 1 shows a snapshot of the uplink and downlink traffic simulation. Some nodes were forced to fail (marked as a white $\mathrm{X}$ ) during simulation to measure the route recovery time. Several factors in performance measurements were defined, such as (a) route discovery time, (b) packet drop, (c) routing traffic amount, (d) network delay, and (e) network throughput.

In OPNET Modeler, network modeling is done hierarchically in progressive detail. First, the network architecture is modeled to describe the links and connectivity among various network components, such as smart meters or smart thermostats. Then the node model is created where each network component is modeled for internal data connectivity. Each node is modeled as a collection of processes to describe the behavior of the protocol. The protocol is written in

$C$ programming language. This process is described in Figure 2.

The ITMR network has been modeled following the above procedures. After the procedures are developed, the performance metrics variables are selected (Figure 3) and the discrete event simulation is performed.

The performance of AODV is measured following the same procedure. The AODV performances are then 


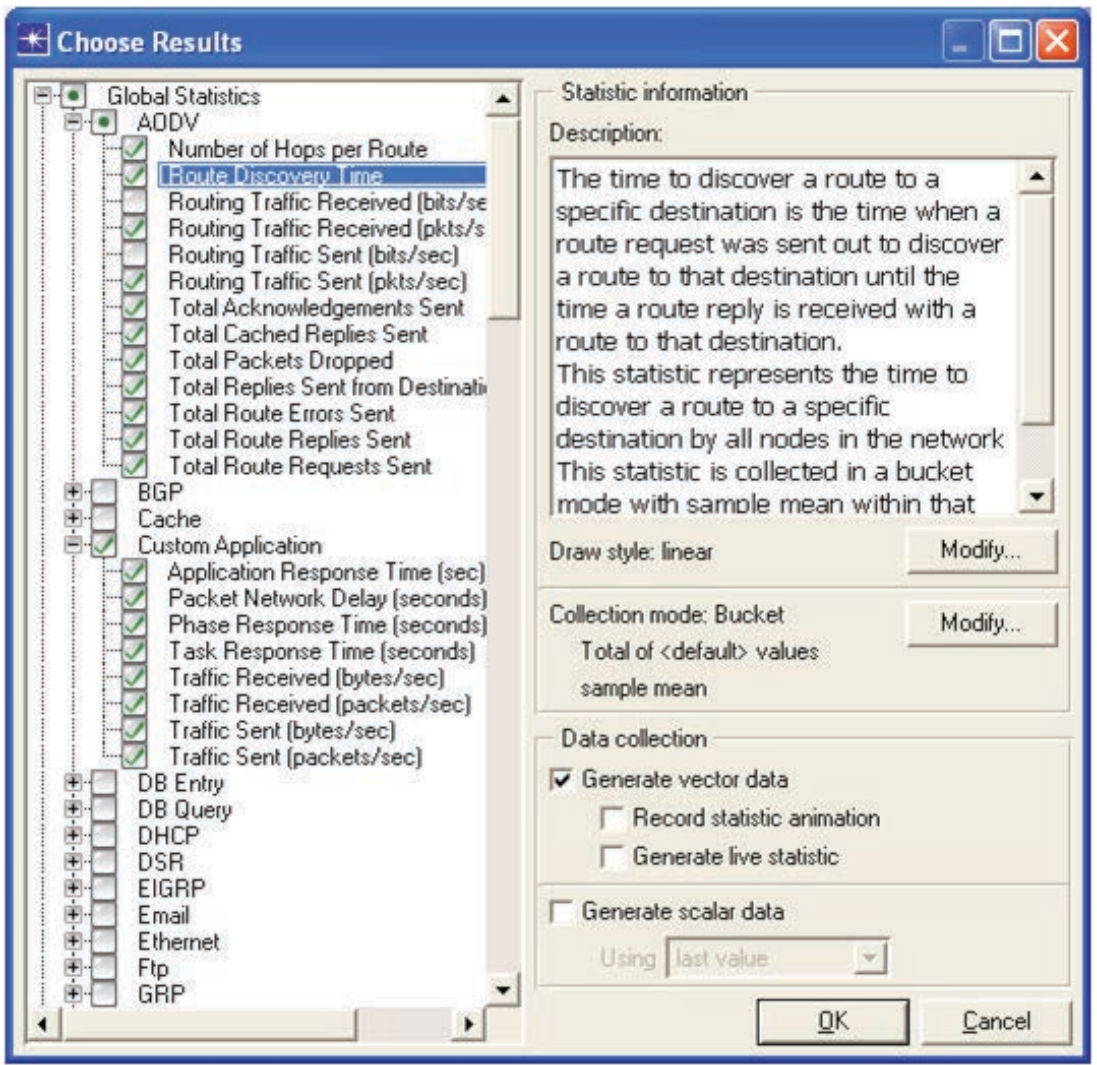

Figure 3. Choosing results

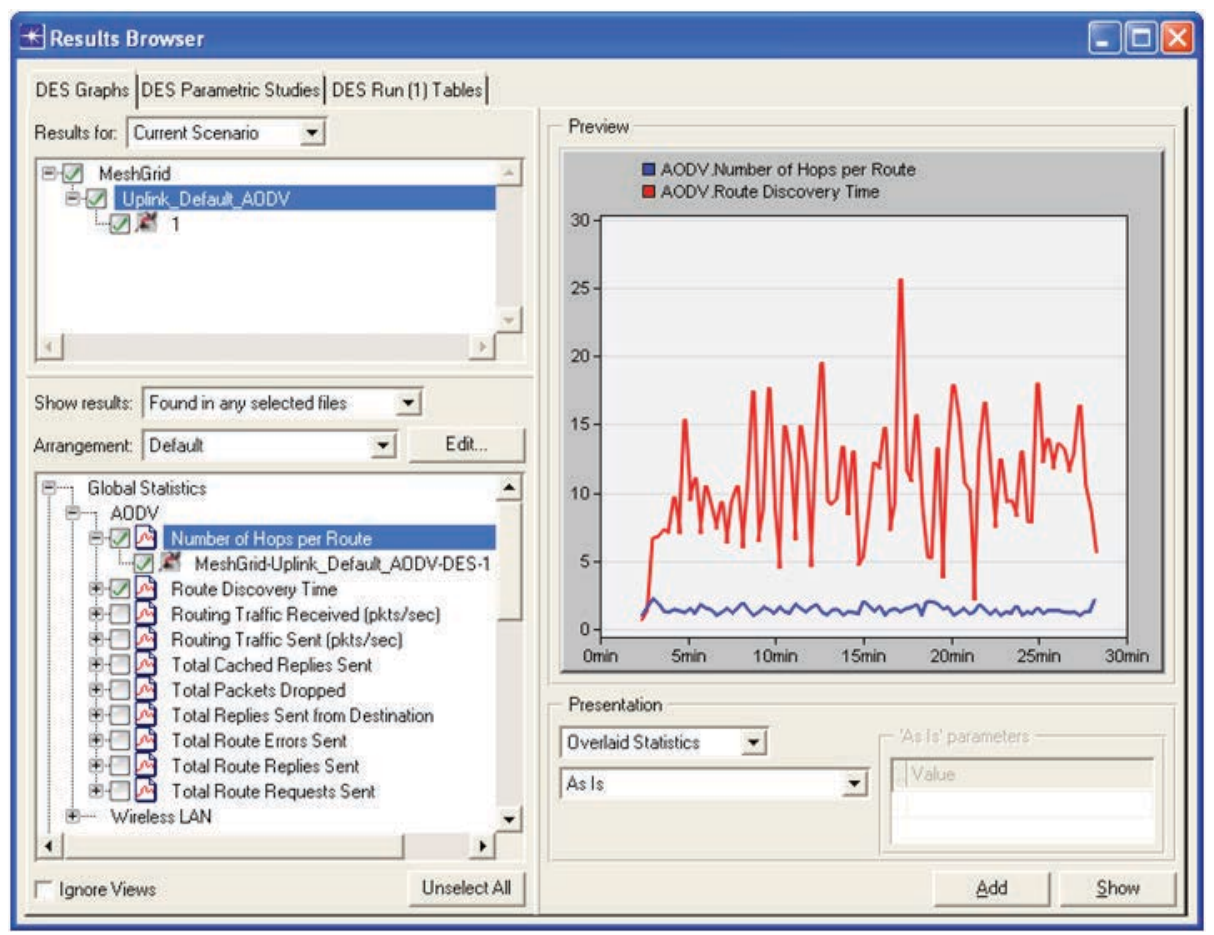

Figure 4. Browsing results 


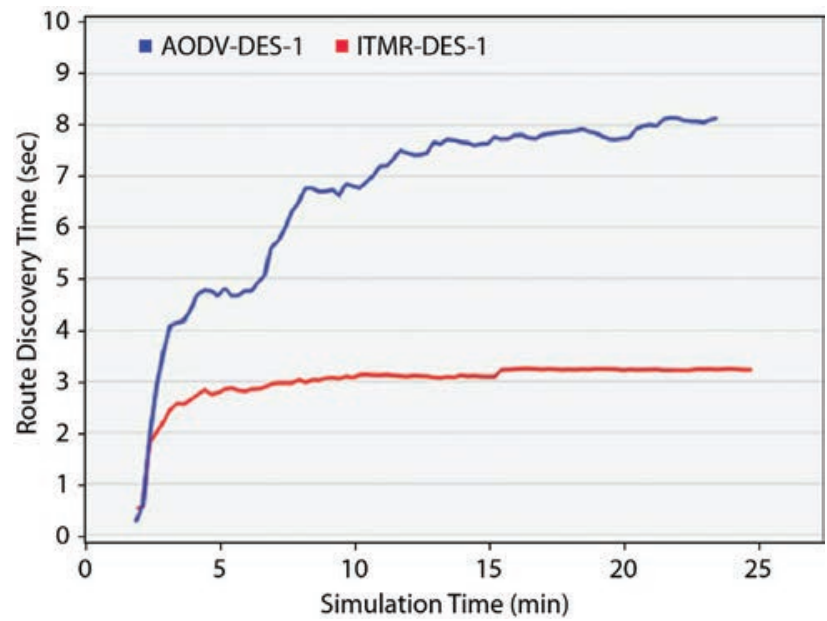

Figure 5. Route discovery time

compared in all five categories (Figure 4). The performance comparison results are discussed below.

(a) Route discovery time: The time to discover a route to a specific destination is the time a route request was sent out to that destination until the time a route reply is received. AODV attempts to discover a new route every time a route breaks down. Therefore, it spends more time discovering routes than ITMR (Figure 5).

(b) Total packets dropped: AODV may transmit over longer distances because of minimum hop criteria. When the link quality degrades, data rates drop and transmitting over longer distances becomes inefficient. ITMR builds a new tree periodically and

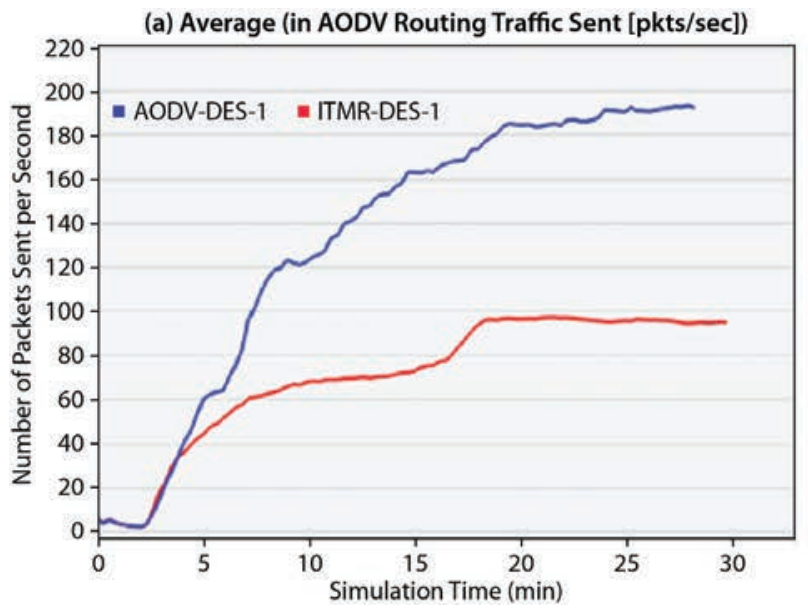

Figure 7. Routing traffic (a) sent and (b) received

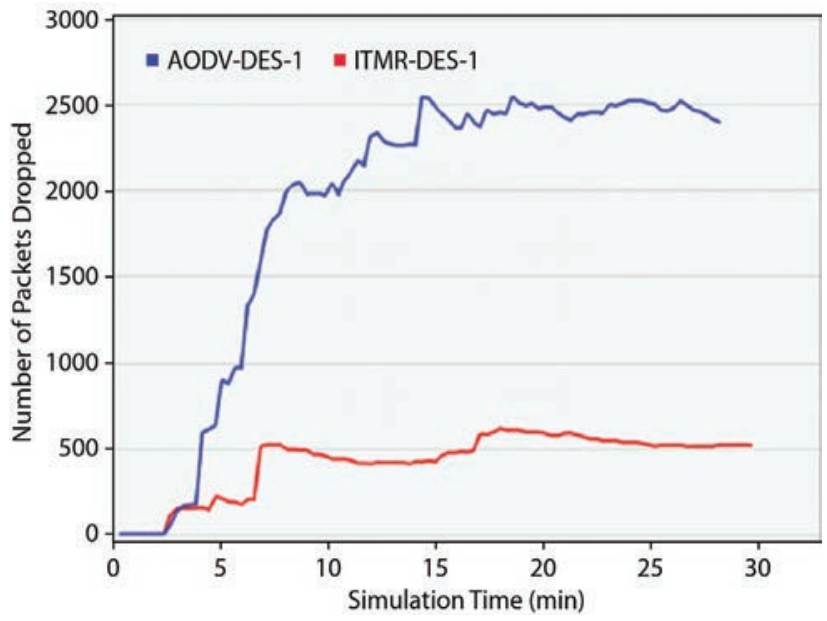

Figure 6. Number of dropped packets

maintains high quality links, leading to a reduced number of dropped packets (Figure 6).

(c) Amount of routing traffic: Figure 7 shows the routing traffic sent and received in packets/second. ITMR has far fewer routing traffic requirements.

(d) Network delay: The packet delivery delay in seconds is significantly less in ITMR (Figure 8).

(e) Network throughput: This represents the total number of bits (in bits/second) forwarded from WLAN layers to higher layers in all WLAN nodes of the network. ITMR shows a better throughput stemming from the reduced packet dropping (Figure 9).

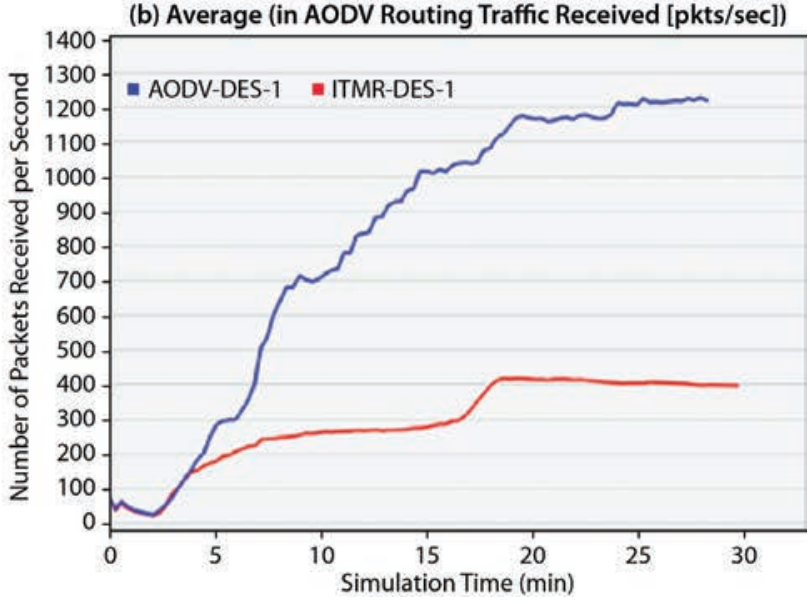




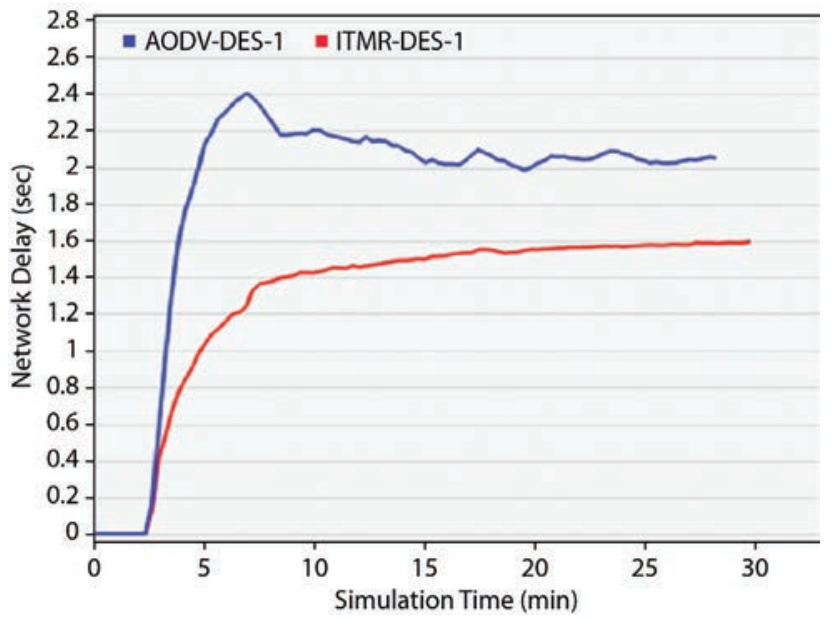

Figure 8. Network delay

\section{Conclusion}

A WMN is an important part of a smart grid. However, the current smart grid WMN faces challenges in meeting security requirements and has low throughput. This project developed low-cost, secure smart grid WMN algorithms and a demonstration platform in the OPNET Modeler.

The performance of the ITMR was compared to AODV (MANET protocol) for route discovery time, packets dropped, routing traffic amount, network delay, and network throughput. The proposed tree-based ITMR is more efficient in route selection, provides faster route discovery, prevents excessive packet loss, and ultimately increases network capacity and resource utilization. With the hybrid communication and ID-based security schemes, ITMR is considered more suitable in a smart grid communication environment.

\section{References}

Akyildiz, I. F., X. Wang, W. Wang, "Wireless mesh networks: A survey," Computer Networks 47 (2005) 445-487.

Chatterjee, S., P. Sarkar, Identity-Based Encryption, Springer (2011) 1-9.

Djukic, P., S. Valaee, "Delay aware link scheduling for multi-hop TDMA wireless networks," IEEE/ACM Trans. Networking 17, 3 (June 2009) 870-883.

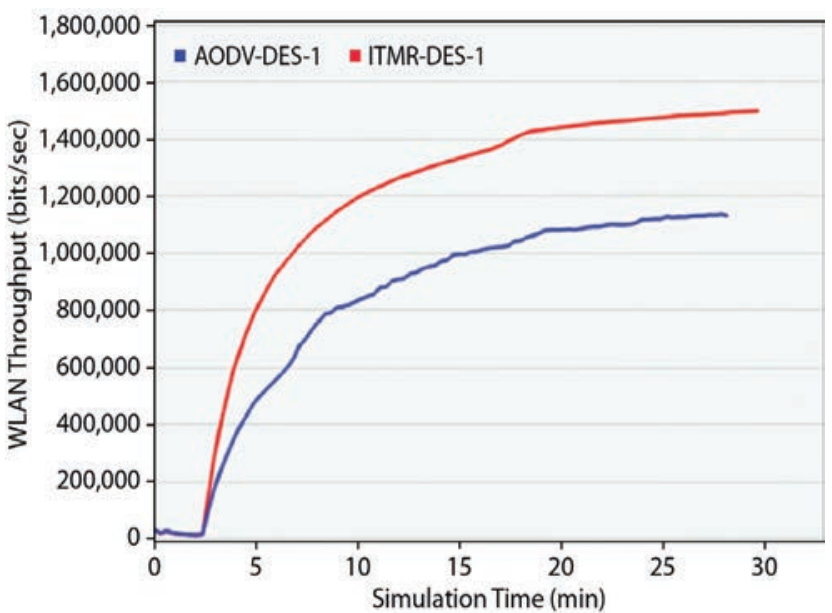

Figure 9. Network throughput

Ergen, S. C., P. Varaiya, "TDMA scheduling algorithms for wireless sensor networks," J. Wireless Networks 16, 4 (2010) 985-997.

Güngör, V. C., D. Sahin, T. Kocak, S. Ergüt, C. Buccella, C. Cecati, G. P. Hancke "Smart grid technologies: Communication technologies and standards," IEEE Trans. Industrial Informatics 7, 4 (2011) 529-539.

Peng, M., M. Li, W. Wang, "A unified architecture and the tree level based scheduling technique for multi-radio multi-channel in IEEE 802.16 standard based wireless mesh networks," First International Conference on Communications and Networking in China, 2006 (2006) 1-6.

Pirzada, A. A., M. Portmann, "High performance AODV routing protocol for hybrid wireless mesh networks," Fourth Annual International Conference on Mobile and Ubiquitous Systems: Networking \& Services, 2007 (2007) $1-5$. 
This page left blank intentionally 


\section{Photonics}
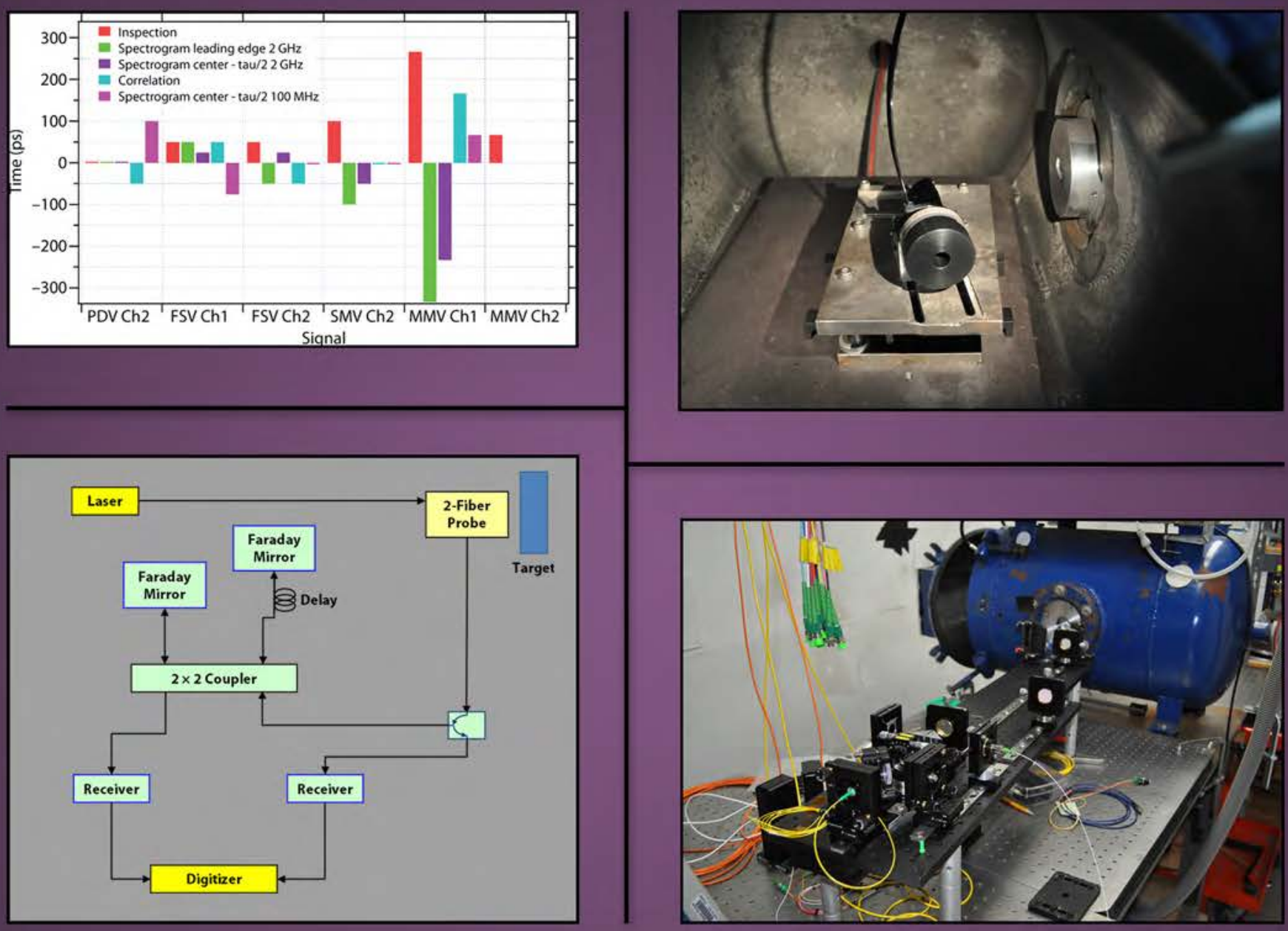


\section{ENHANCED ACCURACY OF SHOCK ARRIVAL USING PDV/HETERODYNE VISAR STL-46-12 | YEAR 1 OF 1}

Bruce Marshall, a

Ways to improve the accuracy of shock arrival time measurements using the burst of fringes that occur at shock arrival (breakout) at a free material surface during the delay time of a VISAR were investigated. We used optical mixing techniques to enhance the signal levels without success. As a compromise, we built and tested several variants of single-mode and multi-mode VISAR systems that have the required bandwidth. Breakout fringes were successfully observed in explosively driven shock experiments at the NSTec Special Technologies Laboratory Boom Box. A comparison of several measures of shock arrival time indicates that the step function that occurs in photonic Doppler velocimetry at breakout is as good as or better than any of the proposed techniques.

${ }^{1}$ marshabr@nv.doe.gov, 805-681-2266

a Special Technologies Laboratory

\section{Background}

Shock arrival time is used in shock compression measurements to determine shock velocity and for cross-timing of multiple measurements. Several methods are used to measure arrival time, including electrical pins, optical pins, photonic Doppler velocimetry (PDV), and velocity interferometer for any reflector (VISAR). In many situations, shock arrival produces an abrupt change in PDV signal level that can be readily detected. However, if offset heterodyne fringes precede the shock arrival, the transition in a PDV signal is from one frequency to another, which makes it difficult to accurately identify the transition time. To accommodate this challenge, various analysis methods are used, such as the digital downshift technique developed at NSTec Los Alamos Operations (LAO) (Tunnell 2012). VISAR is more problematic due to its intrinsic cavity delay. High-frequency fringes are always present in VISAR optical signals, but generally cannot be observed because the recording system bandwidth is inadequate and the fringe contrast is reduced due to loss of spatial coherence. Depending on the lead-in phase, the initial transition may be difficult to identify.

\section{Project}

There are two primary challenges in recording VISAR delay time fringes. First, the fringes exceed typical VISAR recording bandwidths, particularly for largearea detectors. Second, shock breakout may disrupt the spatial coherence between the prompt and delayed light, greatly reducing the fringe contrast. Operating a VISAR at $1550 \mathrm{~nm}$ reduces the bandwidth requirement by a factor of about 3 compared to operating at $532 \mathrm{~nm}$. Use of single-mode fiber allowed us to use high-bandwidth, high-gain detectors, and to some extent reduce the effect of lost spatial coherence.

The main challenge in single-mode VISAR operation is extremely low probe efficiency. Therefore, we first tried to find a way to incorporate heterodyne techniques to improve the signal level. A thorough investigation of optical mixing techniques was unsuccessful. We therefore optimized the single-mode VISAR and the experimental parameters to make measurements that would let us record breakout fringes and evaluate their usefulness under ideal conditions. Two measurements were carried out at 
the NSTec Special Technologies Laboratory Boom Box, both of which yielded usable data. An algorithm was developed to obtain the arrival time from the breakout fringe burst.

\section{Single-Mode vs Multi-Mode VISAR}

The main reasons to use a single-mode system rather than a multi-mode system for detection of breakout fringes are higher bandwidth and improved contrast. A typical multi-mode VISAR uses $200 \mu \mathrm{m}$ core fibers to collect a complex wavefront generated by multiple scattering points. The étendue of a $200 \mu \mathrm{m}, 0.22$ NA fiber is $1.936 \times 10^{-9} \mathrm{~m}^{2} \mathrm{sr}$. Multi-mode VISAR contrast will remain high as long as the wavefront does not change during the cavity delay of the VISAR. A slowly changing speckle pattern will have little effect on the coupled light level or the contrast. A rapid change in the speckle pattern will spoil the contrast but not the coupling.

In comparison, a single-mode fiber at $1550 \mathrm{~nm}$ has a mode field diameter of about $9 \mu \mathrm{m}$ and an NA of 0.12 , with étendue of $1.166 \times 10^{-12} \mathrm{~m}^{2} \mathrm{sr}$, or approximately 1660 times less than the $200 \mu \mathrm{m}$ multi-mode fiber.

A bare single-mode fiber probe may have -40 to $-60 \mathrm{~dB}$ collection efficiency depending on distance.
Also, only the component of the wavefront striking the fiber that matches its TEM00 mode will be transmitted. A rough, shocked surface generates a complex speckle pattern that produces large amplitude fluctuations ("dropouts") in the light collected by single-mode fiber probes. Loss of spatial coherence on shock breakout may cause a large change in signal level but will not affect the fringe contrast.

\section{Optical Mixing}

Our initial solution to overcome the low probe efficiency of single-mode fiber (SMF) VISAR was to try to use heterodyne detection in a manner similar to PDV. PDV overcomes the challenges of singlemode fiber by means of heterodyne detection and frequency domain analysis. The shifted light is mixed with an optical local oscillator on a square-law detector surface. As a result, the PDV signal amplitude varies as the square root of the collected optical power, doubling the dynamic range. Heterodyne detection with local oscillator gain improves the detectability by about $25 \mathrm{~dB}$ (Betti 1995) relative to homodyne detection. Frequency domain analysis further improves the signal-to-noise ratio by effectively filtering and averaging over multiple time samples. (a) SMF"VISAR" @ 1550 nm with triature outputs

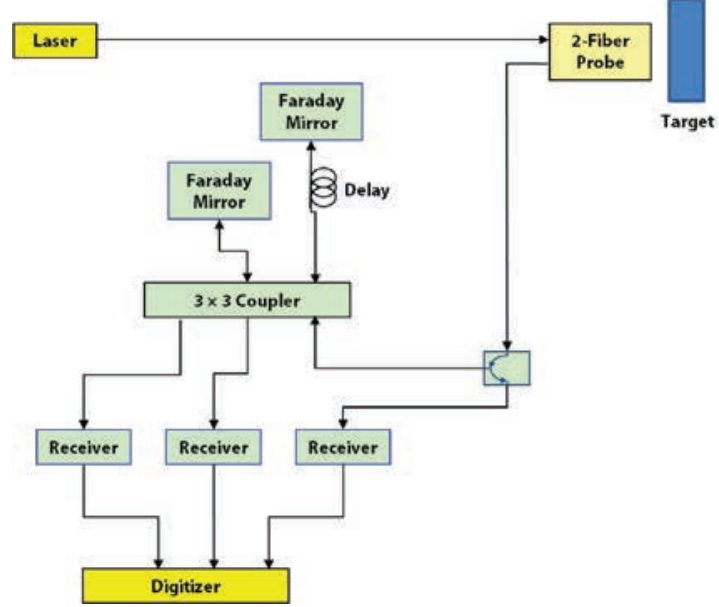

(b) SMF Heterodyne VISAR

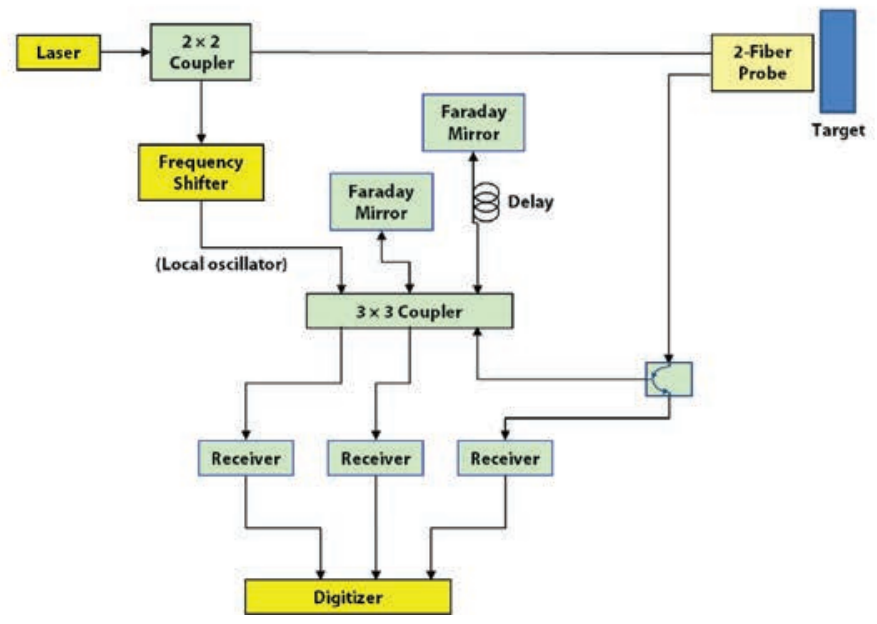

Figure 1. (a) SMF VISAR with triature outputs, and (b) SMF heterodyne VISAR 
An SMF VISAR can be built as shown in Figure 1a. Light collected from the shocked surface is delivered to an interferometer cavity via a circulator. The cavity configuration is a fiber-optic Michelson interferometer. Faraday mirrors make the interferometer insensitive to polarization. A $3 \times 3$ splitter will give three output phases separated by $120^{\circ}$ (colloquially referred to as "triature"). Fiber-optic $4 \times 4$ couplers cannot produce accurate $90^{\circ}$ phases between each of the outputs. At this time, a stable, quadrature, SMF VISAR would require a bulk optic splitter.

A local oscillator can be introduced via the unused port in the $3 \times 3$ coupler. The result is a heterodyne SMF VISAR, shown in Figure $1 \mathrm{~b}$, in which the signals consist of PDV fringes with an envelope modulated by the VISAR fringes.

Although this device has the advantage of local oscillator gain, its time resolution is limited because the PDV fringe amplitude must be determined in order to obtain the VISAR phase. Also, dropouts can result in missing VISAR fringes. This device would not be useful for extremely high velocities that cannot be measured by PDV, as the VISAR depends on recording the PDV fringes. The breakout fringe burst is actually present, but is not boosted by the local oscillator, and therefore is swamped by the PDV signals. It is important to understand that the mixing occurs at the detector, which has square-law response (Equation 1). Three waves arrive at the detector: shifted light, delayed shifted light, and the local oscillator light. They mix in pairs, and the burst fringe signal is produced by the shifted light mixing with the delayed shifted light, both of which have very small amplitude.

$$
\begin{aligned}
E= & A_{1} e^{-i \omega_{1} t}+A_{2} e^{-i \omega_{2} t}+A_{3} e^{-i \omega_{3} t} \\
I= & |E|^{2}=\left(A_{1} e^{-i \omega_{1} t}+A_{2} e^{-i \omega_{2} t}+A_{3} e^{-i \omega_{3} t}\right) \\
& \times\left(A_{1} e^{i \omega_{1} t}+A_{2} e^{i \omega_{2} t}+A_{3} e^{i \omega_{3} t}\right) \\
& =A_{1}^{2}+A_{2}^{2}+A_{3}^{2}+2 A_{1} A_{2} \cos \left(\omega_{1}-\omega_{2}\right) t \\
& +2 A_{1} A_{3} \cos \left(\omega_{1}-\omega_{3}\right) t+2 A_{2} A_{3} \cos \left(\omega_{2}-\omega_{3}\right) t,
\end{aligned}
$$

where $E$ is electric field; I is intensity; $A_{1}, \omega_{1}$ are amplitude and frequency of the local oscillator; $A_{2}, \omega_{2}$ are amplitude and frequency of the prompt shifted light; $A_{3}, \omega_{3}$ are amplitude and frequency of the delayed shifted light; and $A_{2}, A_{3}$, is $\ll A_{1}$.
The terms $2 A_{1} A_{2} \cos \left(\omega_{1}-\omega_{2}\right) t$ and $2 A_{1} A_{3} \cos \left(\omega_{1}-\right.$ $\left.\omega_{3}\right) t$ are PDV signals at high frequencies that are separated by the VISAR fringe frequency, and $2 A_{2} A_{3}$ $\cos \left(\omega_{2}-\omega_{3}\right) t$ is the VISAR signal, which is much smaller in amplitude than the PDV signals.

We investigated several alternate means of mixing the signals with a local oscillator. The ideal solution would use a nonlinear optical process to mix the shifted light with a strong local oscillator, and produce an amplified, offset mixing product. However, the efficiency of nonlinear optical processes is extremely dependent on optical power and is very low for achievable signal levels. We tried a semiconductor optical amplifier operating in its nonlinear region. The resulting heterodyned optical signals were present but too weak to use.

Another option we examined was to run the output of a PDV through an RF interferometer with an RF mixer. We tested this system as well, but again the mixer was inefficient and gave too small a signal. An integrated optic, single sideband modulator can also be used to effectively boost the PDV signal and offset its frequency, but this requires conversion from optical to electrical and back again, with a significant increase in noise.

\section{Experiment}

Because we were not successful with the heterodyne SMF VISAR or any of the optical mixing schemes, we decided to determine under what circumstances a non-heterodyne VISAR, either single-mode or multimode, could produce the breakout signal of interest, and if it has any advantage in timing accuracy. To take advantage of a $12 \mathrm{GHz}$ receiver with $50 \mu \mathrm{m}$ fiber input, we built a multi-mode fiber (MMF) VISAR operating at $1550 \mathrm{~nm}$, using concave mirrors to image the wavefront along the two paths. We also built an SMF VISAR, a PDV, and a frequency-shifted (FS) SMF VISAR. Block diagrams of the fiber interferometers are shown in Figure 2. All four interferometers are shown in the photograph in Figure 3. 
(a) PDV

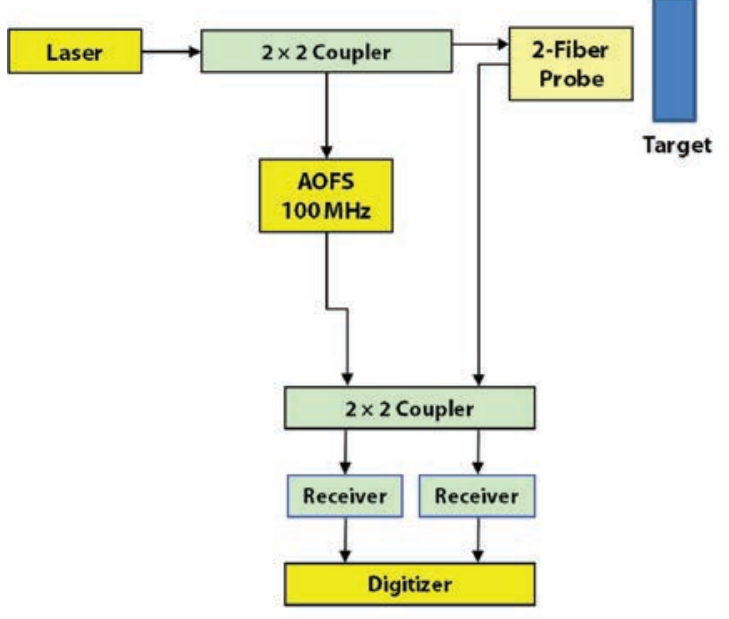

(b) Frequency-shifted SMF VISAR

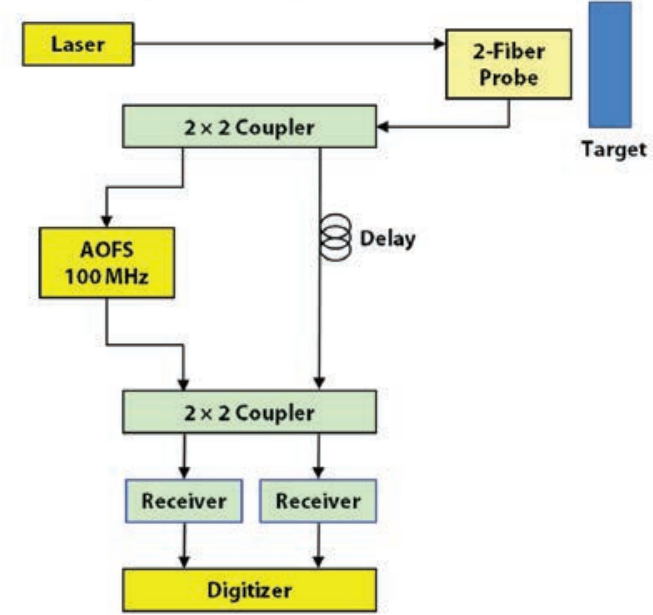

(c) SMF"VISAR"@1550nm

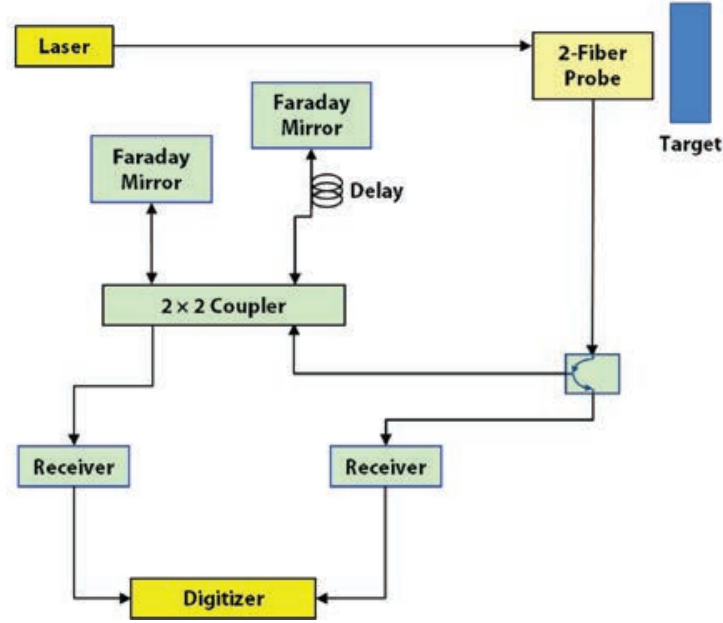

Figure 2. Fiber-optic interferometers: (a) PDV, (b) frequency-shifted SMF VISAR, and (c) SMF VISAR

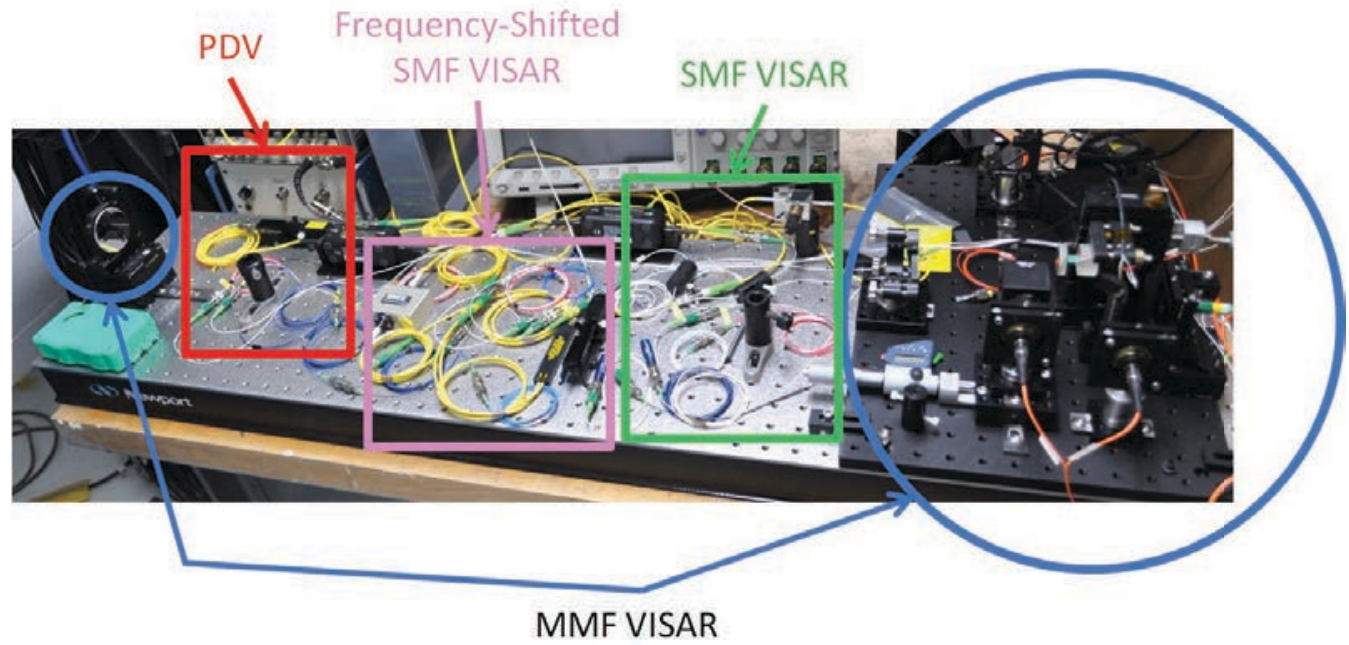

Figure 3. Interferometers at the Boom Box experiment 

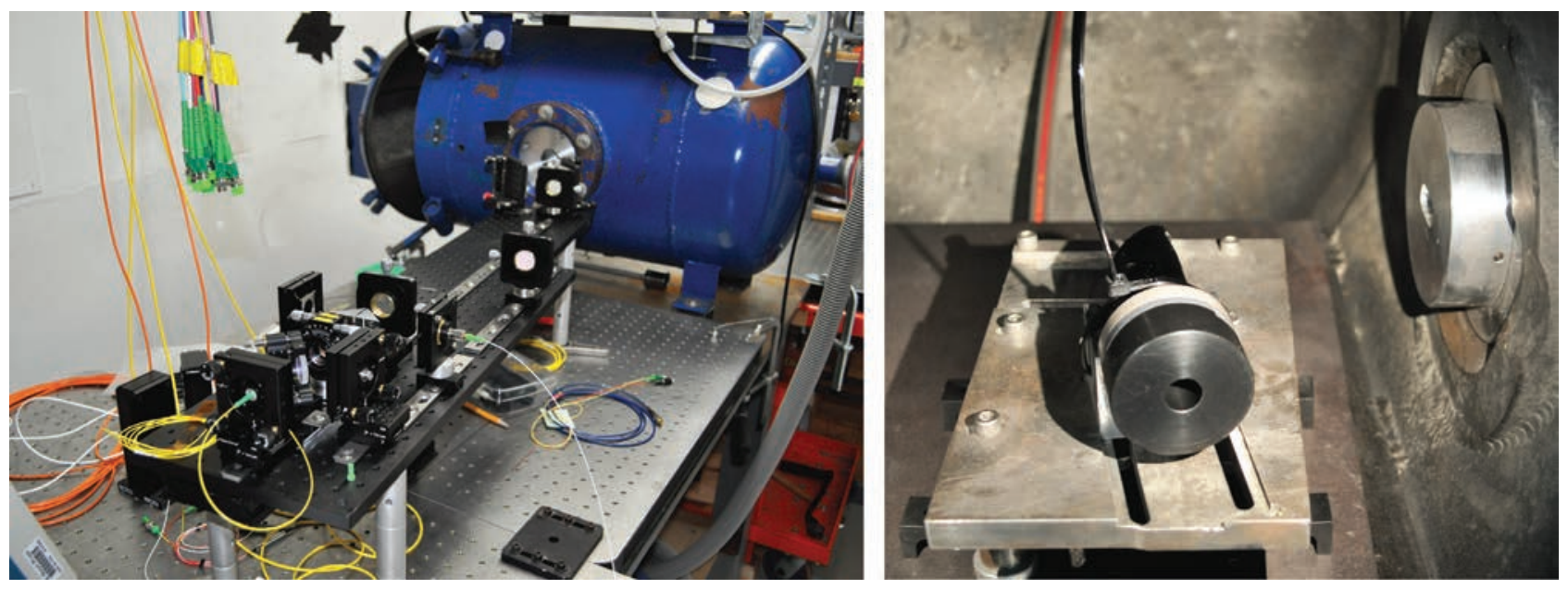

Figure 4. Probe optics with relay lenses

Delays for all VISARs were about 10 ns. All singlemode systems shared signal light from one probe fiber. Complementary signals were recorded for each system. No quadrature signals were recorded, as the emphasis was on breakout fringes rather than VISAR performance, and quadrature would have significantly complicated the optical system and required more receivers.

The target consisted of a $40 \mathrm{~mm}$ diameter by $1 \mathrm{~mm}$ thick polished disk of aluminum 6061-T6, driven by 2.9 grams of PBX 9501 with an RP-1 EBW detonator and mounted in a polycarbonate package. The first probe was optimized for the travel and tilt expected at the Boom Box, which has a convex shock front (Figure 4).

The probe optical system is diagrammed in Figure 5. Light from a $2 \mathrm{~mm}$ focal-length gradient-index (GRIN) collimator passed through a polarizing beam splitter followed by a quarter-wave retarder. An afocal relay system combined with a $40 \mathrm{~mm}$ objective lens imaged the fiber onto the target and collected the return light, which was converted to the orthogonal

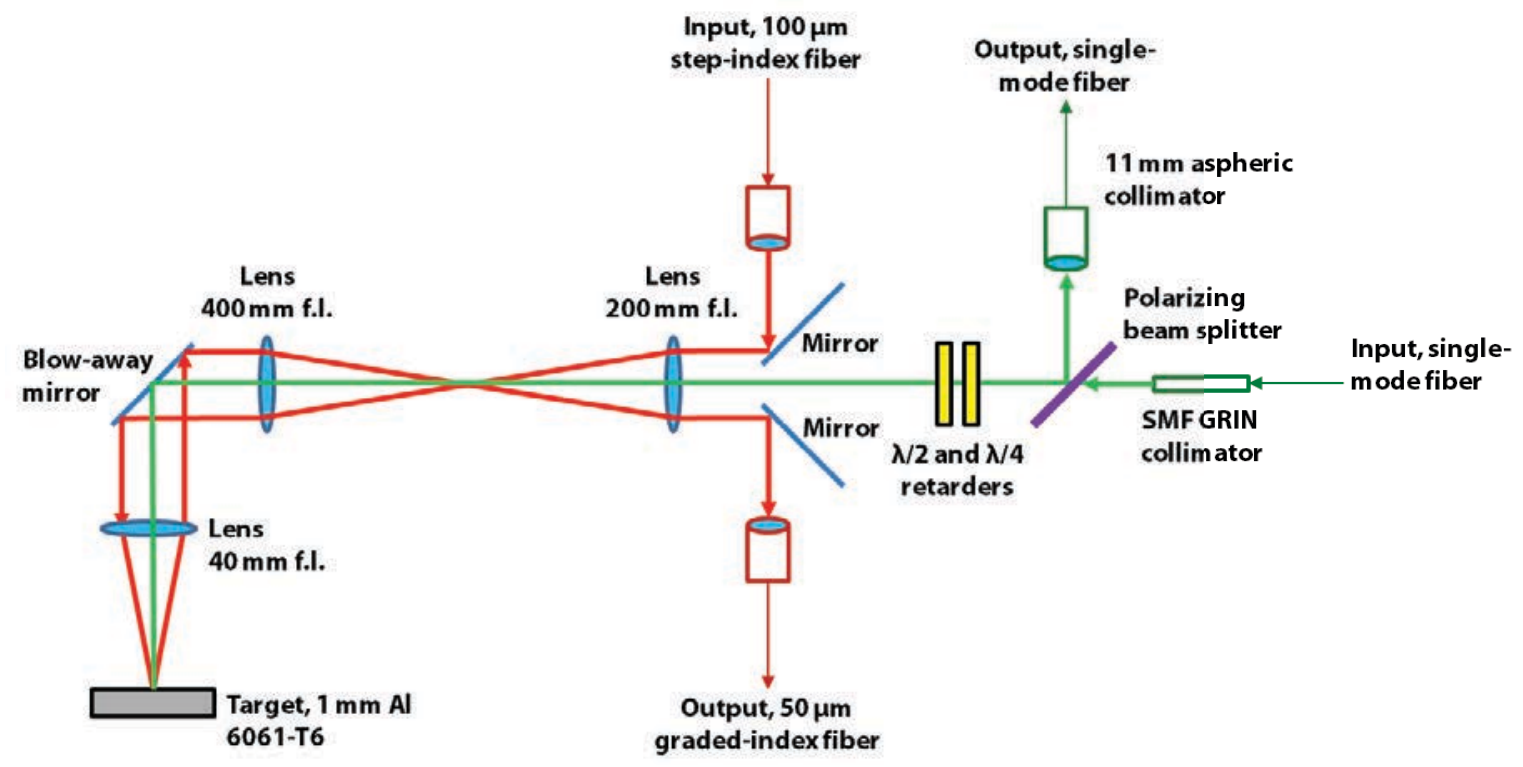

Figure 5. Probe optical system diagram 

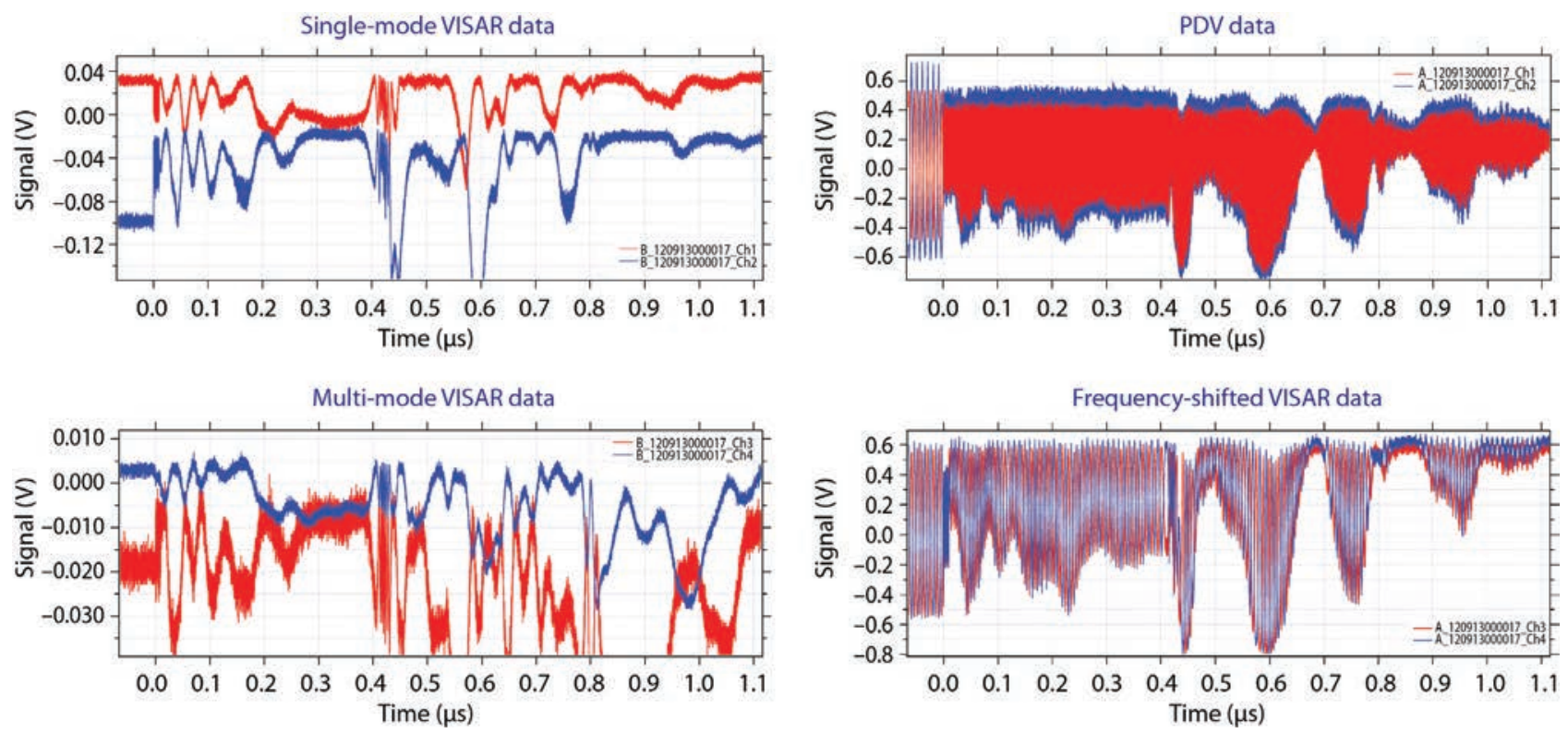

Figure 6. Raw data from explosive-driven shock experiment using a bare fiber probe

linear state by the retarder and reflected off the beam splitter to an $11 \mathrm{~mm}$ focal-length aspheric collimator with single-mode fiber output. The polarizer and waveplate formed a circulator that allowed both fibers to be on axis. The difference in magnification for the input and output fibers provided angle tolerance and extended travel range at the expense of efficiency. The limited numerical aperture reduced the speckle problem. Both single-mode systems operated with about $40 \mathrm{~mW}$ of optical power from the laser, but signal levels dropped after breakout and again after about $1.5 \mathrm{~mm}$ of travel. A multi-mode probe was included in the system with its beam running parallel to the SMF beam and offset so the light still sampled the center of the target with the angle of incidence about $12^{\circ}$. Light for the multimode probe was delivered via a mode-mixed $100 \mu \mathrm{m}$ step-index fiber and collected by a $62.5 \mu \mathrm{m}$ gradedindex fiber. Approximately $200 \mathrm{~mW}$ of optical power was used for the multi-mode system.

For comparison, we also tested a simple, bare fiber probe configuration. Light was delivered via a singlemode fiber and collected via another single-mode fiber and by a $62.5 \mu \mathrm{m}$ multi-mode graded-index fiber. The fibers were placed on axis, $4 \mathrm{~mm}$ from the

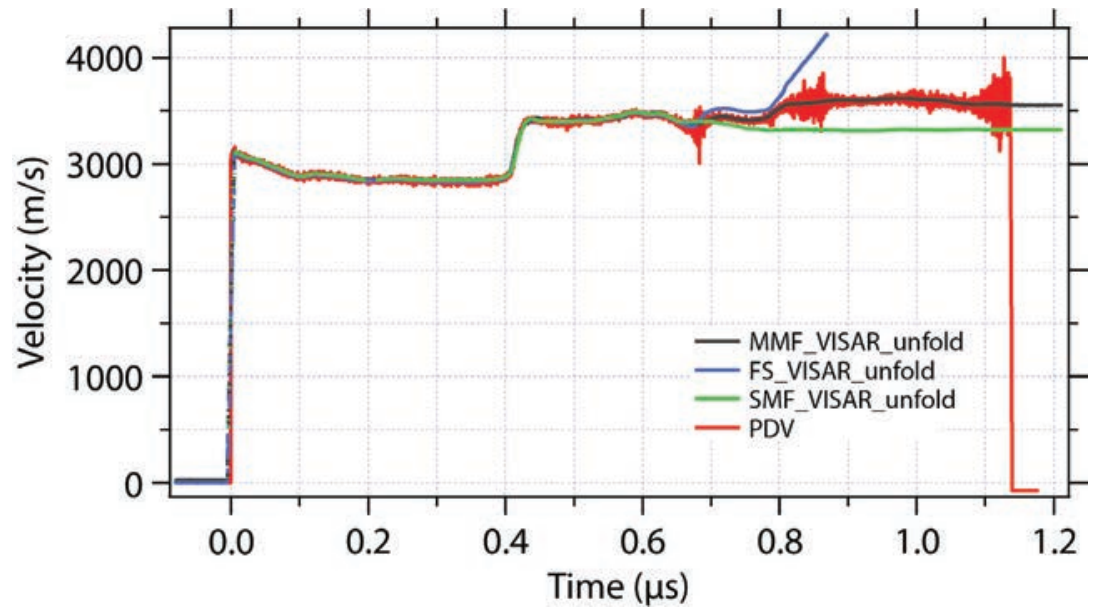

Figure 7. Velocity profiles from PDV and VISAR data. Note the good agreement between all four systems where signal levels are good, and the susceptibility of the single mode VISAR systems to dropouts starting at about $0.68 \mu \mathrm{s}$. 

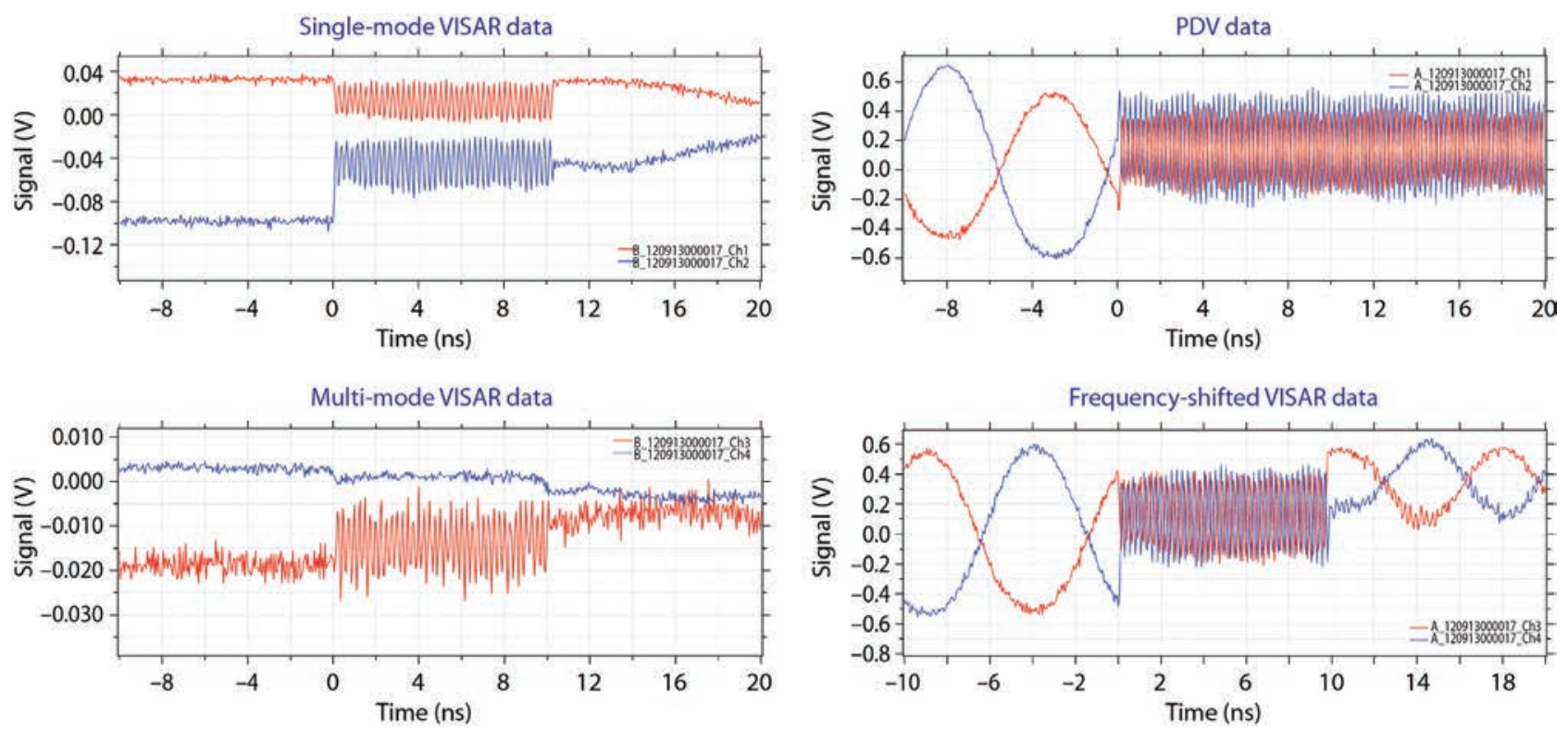

Figure 8. Raw data at shock breakout time

target surface. Using an aluminum mirror target, probe efficiencies for the single-mode and multimode fibers were about $-42 \mathrm{~dB}$ and $-30 \mathrm{~dB}$, respectively. The SMF efficiency for a bead-blasted aluminum target dropped to the range of $-55 \mathrm{~dB}$ to $-60 \mathrm{~dB}$. The target for these measurements was $1 \mathrm{~mm}$ thick polished 6061 aluminum.

An erbium fiber laser with an erbium-doped fiber amplifier supplied $400 \mathrm{~mW}$ of laser power to the probe input. An erbium fiber preamp boosted the return SMF signal to about $5 \mathrm{~mW}$, which was split and delivered to the three interferometers. The PDV and FS VISAR used MITEQ receivers. The SMF VISAR used New Focus 1544-B receivers (DC-12GHz). One output of the multi-mode VISAR used a New Focus 1544-B-50 receiver (DC-12 GHz) with a $50 \mu \mathrm{m}$ fiber input. The other MMF output used a New Focus 1611 receiver (DC-1 GHz). All channels were recorded on Tektronix TDS6124C $12 \mathrm{GHz}$ digitizers at $20 \mathrm{GS} / \mathrm{sec}$ and a $20 \mu \mathrm{sec}$ record length.

The raw data, obtained using the bare fiber probe, are shown in Figure 6. The PDV signals were analyzed using a sliding FFT algorithm with a line fitting routine. The VISAR signals were unfolded by means of an arcsine unfold routine, with the PDV signal providing the information to identify changes in the sign of acceleration. The resulting velocity profiles are shown in Figure 7.

One pitfall of the SMF VISAR is readily visible: dropouts resulting in missing fringes cause the traces to diverge after about $0.6 \mu \mathrm{s}$. Use of a second VISAR with a different velocity per fringe constant (VPF) would allow us to infer the missing fringes. Also, the VPF used in this measurement, approximately $75 \mathrm{~m} / \mathrm{s} /$ fringe, was chosen to give a large number of breakout fringes but is rather low for velocities of $\sim 3000 \mathrm{~m} / \mathrm{s}$. The first $20 \mathrm{~ns}$ of the signals are shown in Figure 8.

Time of arrival for each signal was determined in four different ways. First, we visually identified the first data point that clearly deviated from the baseline (by "inspection"). Second, we calculated a spectrogram, with $2 \mathrm{GHz}$ resolution and with $100 \mathrm{MHz}$ resolution, at each point and identified the half-maximum point on the leading edge. Third, using a profile of the spectrogram at the burst frequency and calculating the center of the burst, we then subtracted one-half of the VISAR delay time as measured with a 10-picoscond pulsed laser. Fourth, we correlated the signal with a sine wave at the burst frequency and 


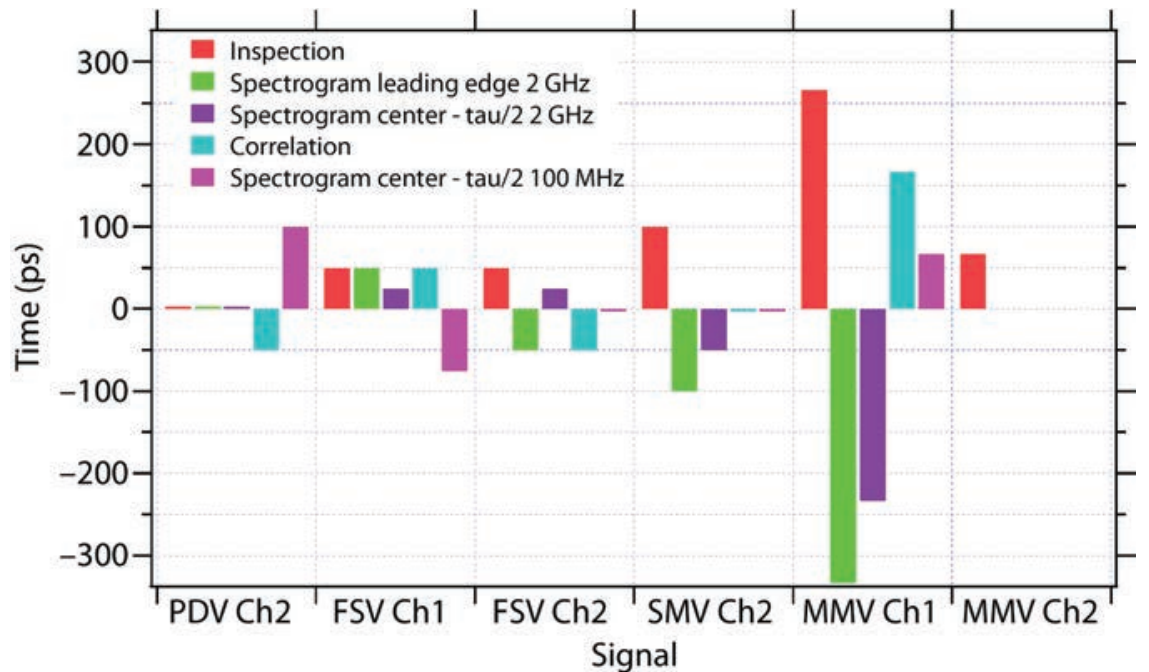

Figure 9. Shock arrival time delta between PDV channel 1 and the other channels, for each channel and method phase to determine the midpoint, and subtracted one-half of the VISAR delay time. For PDV, we used the start of the flat top instead of the peak of the correlation signal.

The correlation routine also included an algorithm to optimize the value used for the width of the VISAR delay. The purpose of this was to see if the burst width was equal to the independently measured VISAR delay, or if other factors such as off-normal shock waves affected the rise and fall times of the burst. The result confirmed that the burst width was equal to the VISAR delay time within the resolution of our system.

The resulting shock arrival time delta between channel 1 of the PDV and other channels is shown in Figure 9. Note that the $1 \mathrm{GHz}$ bandwidth of MMV Channel 2 was inadequate to detect the breakout fringes, so only the manual breakout estimate is included. Several conclusions may be drawn from Figure 9. Visual inspection of the leading edge worked as well as the more complicated procedures. The rather time-consuming correlation procedure in most cases was outperformed by the spectrogram center methods. Another notable feature is that the MMF VISAR had much larger variation than the other systems, probably due to the much lower signal-tonoise level in the data.

\section{SMF VISAR Observations}

We made a significant effort to field an SMF VISAR on an explosively driven shock measurement, and in the process we learned several things. The term "singlemode VISAR" is an oxymoron because the entire point of VISAR is to construct an interferometer that can operate with an extended source such as a multimode fiber and "any reflector." A single-mode fiber acts as a spatial filter to limit the field to a TEMOO Gaussian beam. In a multi-mode system, light from many points is processed in parallel. In comparison, the single-mode fiber spatially filters those modes and sums their amplitudes, which is why speckle has such a strong effect on the optical power collected by the probe.

Several trade-offs must be considered in designing a single-mode probe. Tilt, travel, beam diameter, and coupling efficiency are all related. The additional modes available in multi-mode fiber allow much more flexibility. The spatial filtering aspects of single-mode fiber depend on the angle over which the fiber may receive light. Speckle is a particular problem for SMF VISAR because it can cause missing fringes.

The FS VISAR is based on an idea (Tunnell 2012) that a heterodyned VISAR would only need one detector, and the velocity information would be encoded in the 
phase shift of the heterodyne signal. The FS VISAR was set up in a Mach-Zehnder configuration with an acousto-optic frequency shifter (AOFS) in the delay leg. Although the fastest AOFS on hand was only $100 \mathrm{MHz}$, including it gave us a quick look at how the concept works. AOFSs operate at up to about $3 \mathrm{GHz}$, which should be adequate if the VISAR delay is not too long. Using an AOFS appears promising, particularly if a way can be found to heterodyne the signals. Our data have been submitted to LAO for evaluation.

\section{Conclusion}

Based on our results, it appears that VISAR breakout fringes do not provide improved shock arrival time measurements when compared to homodyne or low-offset heterodyne PDV measurements. Also, VISAR systems require much higher laser power than PDVs at the same working distance. In the course of the project, we demonstrated use of a single-mode fiber VISAR on a shocked surface. SMF VISAR is possible on a relatively well-behaved target but requires high optical power and is susceptible to dropouts.

\section{References}

Betti, S., G. De Marchis, E. Iannone, Coherent Optical Communications Systems, John Wiley and Sons, New York, 1995, 11-39.

Tunnell, T., National Security Technologies, LLC, private communication, 2012. 
This page left blank intentionally 


\section{Distribution}

\section{NNSA/NA-114}

Lucille Gentry

(1) Bob Meisner

(1)

NNSA/NA-124.1

Chrysta Travers

NNSA/NSO

Charlotte Carter

(2) Steve Lawrence

(1) Carol Sohn

Laura Tomlinson

(1)

NSTec

Dennis Barker

Frank Cverna

Steve Goldstein

Steve Henry

Jim Holt

Amy Lewis

Eric Machorro

Eric Moore

PIs

Tom Waltman
(1) Howard Bender

(1) Daniel Frayer

(1) Paul Guss

(1) John Hollabaugh

(1) Raymond J. Juzaitis

(2) Wil Lewis

(2) Michael Martinez

(1) Cheryl Oar

(1 ea)

(1) Alan Will
(2) Mike Butchko

(1) Jim Gatling

(2) E. Chris Hagen

(1) A. C. Hollins

(1) Ping Lee

(1) Dana Lindsay

(1) Michael Mohar

(1) Raffi Papazian

(1) Gerald Stevens

(1)

Joint Nevada Program Office

Rick Higgs

(1)

LANL

LDRD Office

(ATTN: William Priedhorsky)

(1)

LDRD Office

(ATTN: Bill Craig)

(1) LDRD Office

(ATTN: Hank Westrich, Sheri Martinez)

OSTI

\section{Resource Centers}

Electronic copy
(1) Technical Library
Public Reading Center



